\title{
Perceptual and motor intentional processing in dorsal pulvinar
}

\author{
Dissertation \\ for the award of the degree \\ "Doctor rerum naturalium" \\ of the Georg-August-Universität Göttingen
}

within the doctoral program systems neuroscience

of the Georg-August University School of Science (GAUSS)

submitted by

Lukas Schneider

from Bregenz, Austria

Göttingen 2018 


\section{Thesis Committee}

Dr. Igor Kagan, Decision and Awareness Group, Cognitive Neuroscience Laboratory, German Primate center (DPZ)

Prof. Dr. Melanie Wilke, Department of Cognitive Neurology, University Medical center Göttingen (UMG)

Prof. Dr. Hansjörg Scherberger, Neurobiology Laboratory, German Primate Center (DPZ)

\section{Members of the Examination Board}

Referee: Dr. Igor Kagan, Decision and Awareness Group, Cognitive Neuroscience Laboratory, German Primate Center (DPZ)

$2^{\text {nd }}$ Referee: Prof. Dr. Melanie Wilke, Department of Cognitive Neurology, University Medical center Göttingen (UMG)

\section{Further members of the Examination Board}

Prof. Dr. Hansjörg Scherberger, Neurobiology Laboratory, German Primate Center (DPZ)

Prof. Dr. Stefan Treue, Cognitive Neuroscience Laboratory, German Primate Center (DPZ)

Prof. Dr. Siegrid Löwel, Systems Neuroscience Group. Johann-Friedrich-BlumenbachInstitute of Zoology and Anthropology

Dr. Arezoo Pooresmaeili, Perception and Cognition Group, European Neuroscience Institute Göttingen (ENI)

Date of oral examination: $2^{\text {nd }}$ of July 2018 
I hereby declare that this thesis has been written independently and with no other sources and aids than quoted

Lukas Schneider

\author{
Göttingen \\ $30^{\text {th }}$ of April \\ 2018
}




\section{Acknowledgements}

I thank Dr. Igor Kagan and Prof. Dr. Melanie Wilke for their supervision, which has shaped and refined my scientific thinking.

I thank my third thesis committee member Prof. Hansjörg Scherberger for insightful comments and discussions. They were always on point and helped me not to lose sight of the greater scope of my work.

I thank Prof. Stefan Treue, Prof. Siegrid Löwel, and Dr. Arezoo Pooresmaeili for participating in my doctoral examination board.

I would like to thank Adán Ulises Domínguez-Vargas, Enrico Ferrea, Danae Theodosopoulou, Kathleen Williams and Yuranny Cabral and for their invaluable company. Adán was my not only my main collaborator throughout the entire Ph.D. period and the first person to talk to when I had difficulties advancing in a project, but also my flat mate and closest friend. His views had such a positive impact on my social behavior. Enrico is an awesome flat and lab mate, and has made my life so much better. Danae and Katie are unique remarkable individuals, both in their own way. Danae's enthusiasm is contagious and Katie is always fun to be around with. I am glad I have met you guys.

I thank two master students I had the opportunity and joy to work with, Alexander Kratzenberg and Uwe Zimmermann. Their input influenced many aspects of the projects that are presented here. Alex is one of the most balanced people I have ever met and a dear friend.

I thank all my colleagues that have not been mentioned individually, in the department of Cognitive Neurology at the Klinikum Goettingen, in the decision and awareness group and other members of the cognitive neuroscience laboratory, and more generally colleagues in the German Primate Center for the fun social activities and the help and fruitful discussions regarding my projects.

I thank three main subjects of this work which have evoked great empathy in me, Cornelius, Linus, and Curius, the work with them has greatly improved my understanding of human nature.

Most importantly, I thank my family and friends for their unconditional support. 


\section{Index}

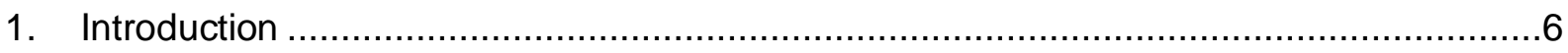

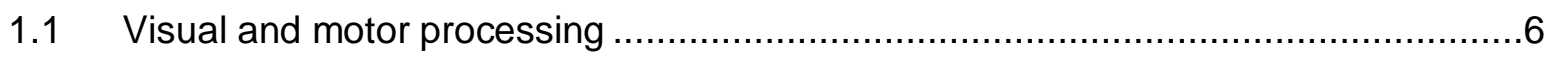

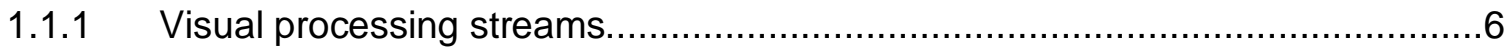

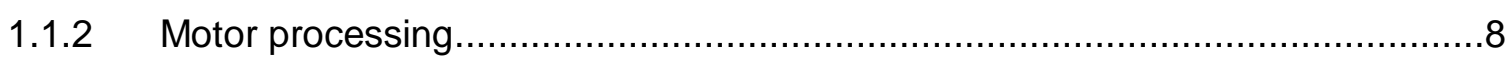

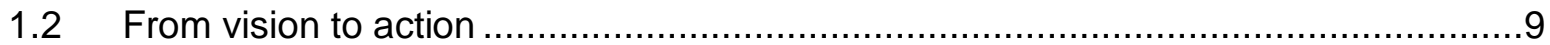

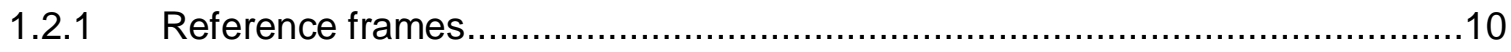

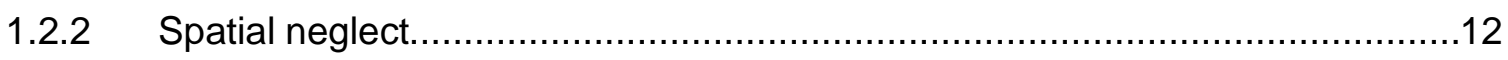

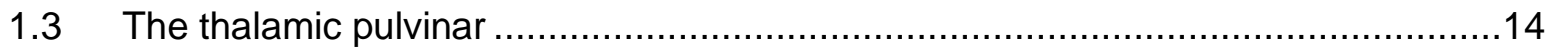

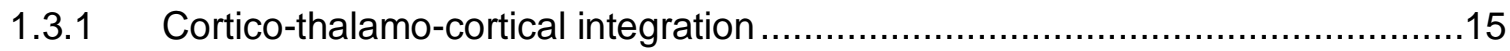

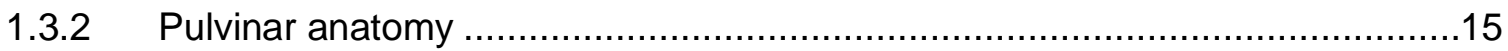

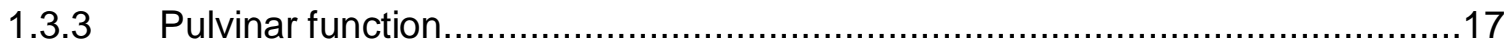

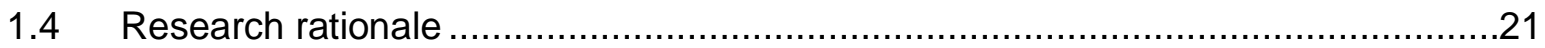

2. Visual, delay and oculomotor timing and tuning in macaque dorsal pulvinar during

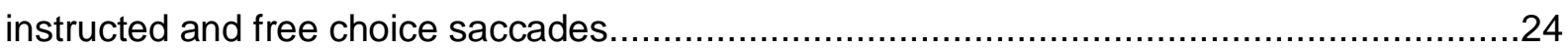

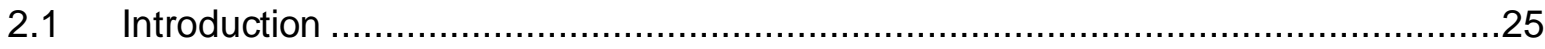

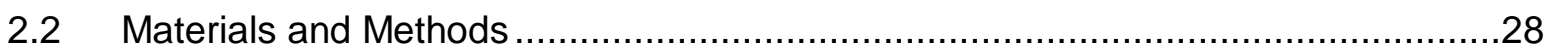

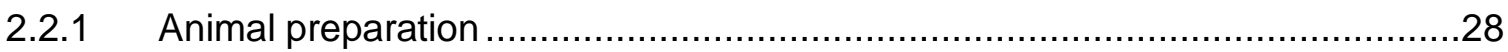

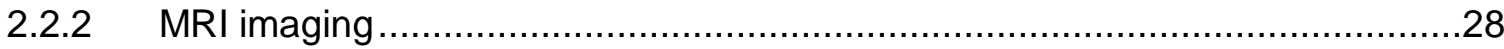

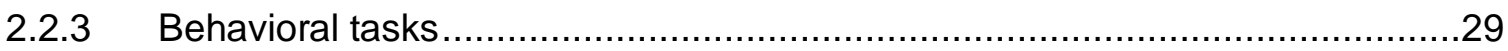

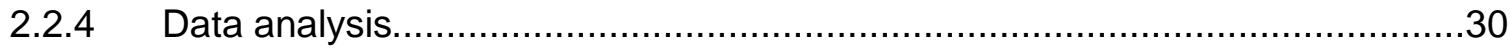

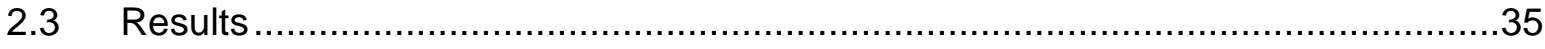

2.3.1 Response timing and tuning in memory-guided saccade task ......................35

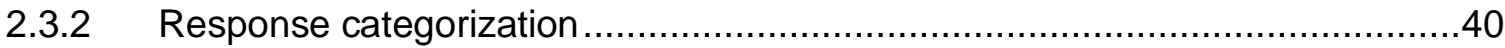

2.3.3 Comparison with visually-guided saccades ............................................

2.3.4 Delay period and pre-/peri-saccadic activity .............................................46

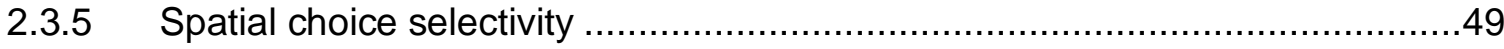

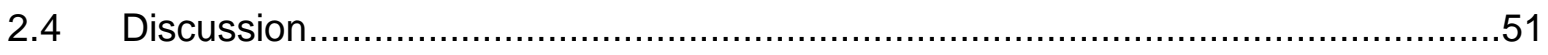

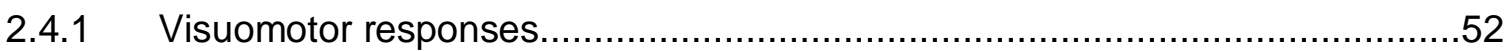

2.4.2 Delay period activity and choice selectivity ..............................................53 


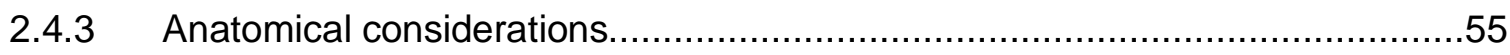

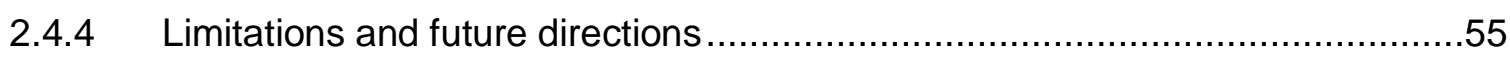

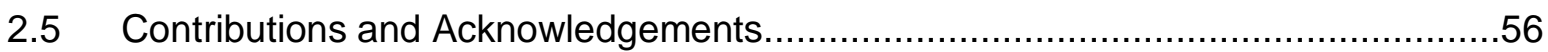

3. Eye position signals in the dorsal pulvinar during fixation and goal-directed saccades ..57

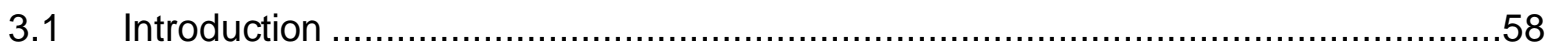

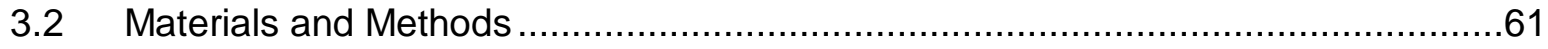

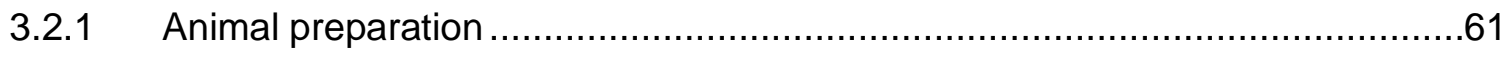

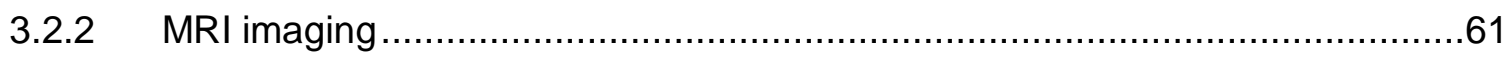

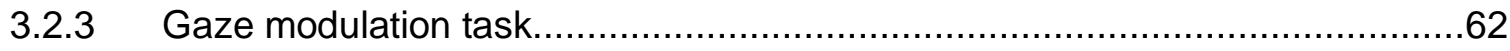

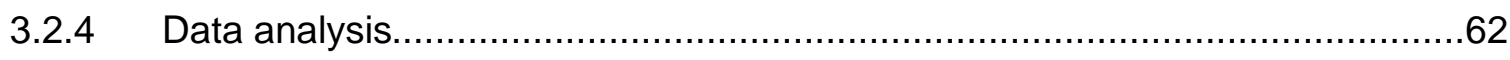

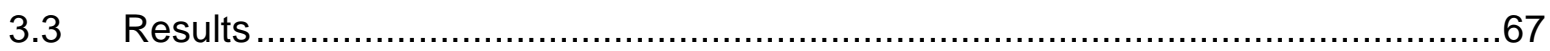

3.3.1 Gaze-dependent activity during initial fixation ...........................................69

3.3.2 Relationship between initial and final gaze effects.................................. 71

3.3.3 Gaze-dependent modulation of spatially-contingent task epochs ...................74

3.3.4 Modulation of retinocentric tuning by gaze position .................................78

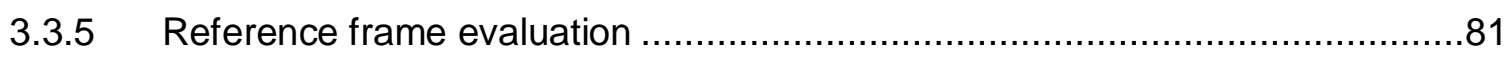

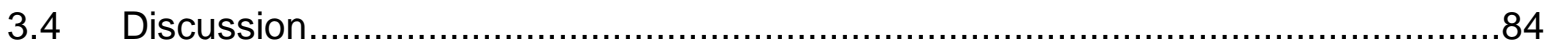

3.4.1 Comparison to previous electrophysiological studies in the thalamus.............84

3.4.2 Comparison to previous electrophysiological studies in cortex ......................85

3.4.3 Possible sources of eye position signals in dorsal pulvinar...........................88

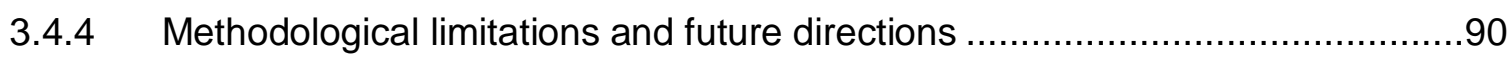

3.4.5 Functional significance of eye position signals in dorsal pulvinar..................92

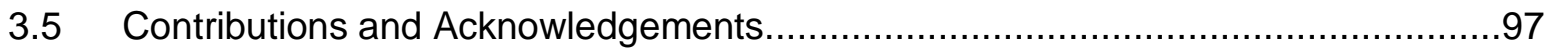

4. Dissociating perceptual and intentional aspects of neglect-like deficits......................98

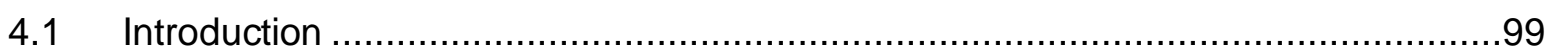

4.1.1 Novel search-to-sample task design......................................................

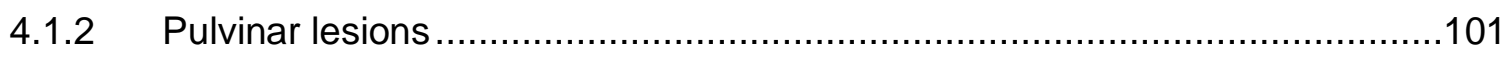

4.1.3 Control inactivation of area TPO...........................................................

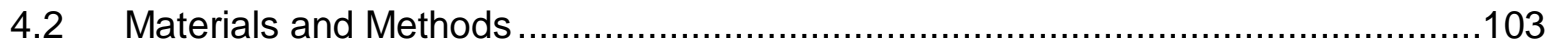

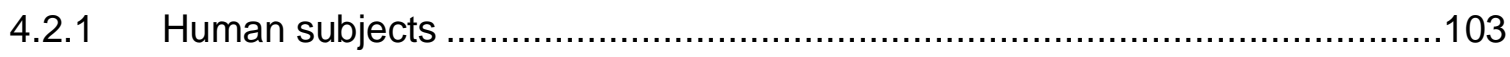




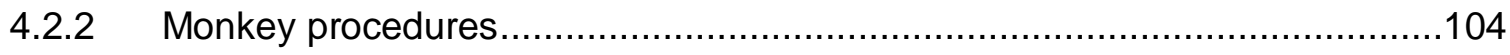

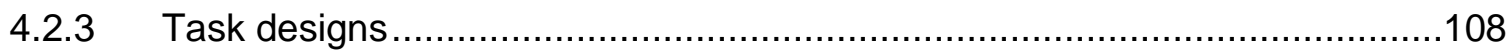

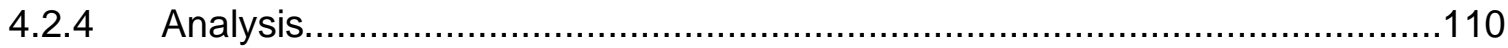

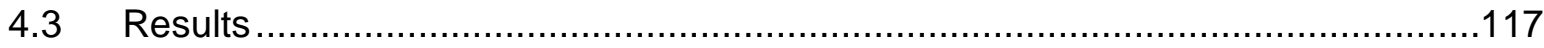

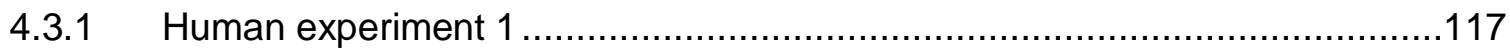

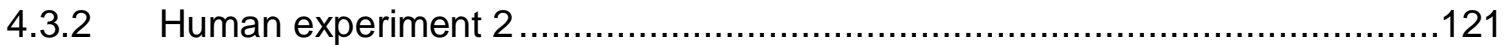

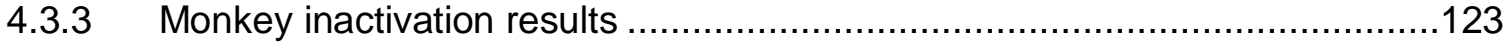

4.4 Control experiments in cortical area TPO .....................................................130

4.4.1 TPO/STS electrophysiology (memory guided saccade task) ......................130

4.4.2 TPO inactivation effects in the search-to-sample task .............................138

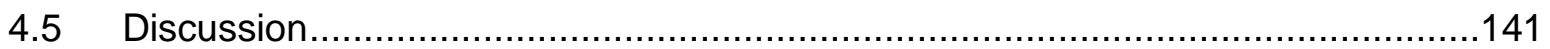

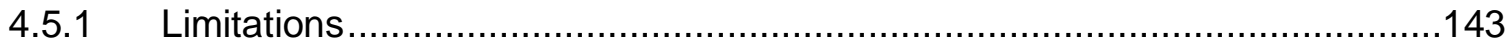

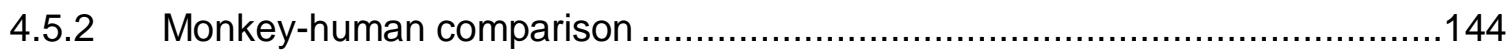

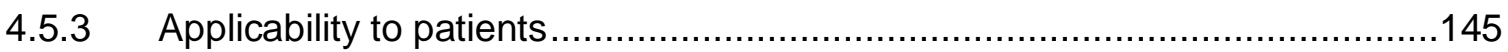

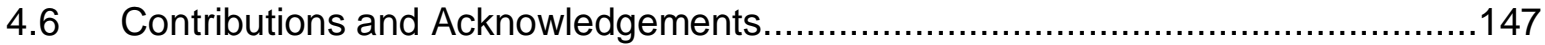

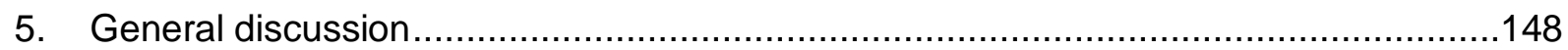

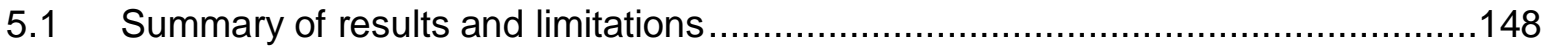

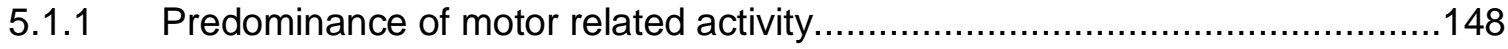

5.1.2 Non-spatial specific motor preparation activity.........................................150

5.1.3 Strong influence of gaze position on pulvinar neuronal activity ...................151

5.1.4 Target selection biasing by electrical microstimulation ..............................151

5.1.5 Pulvinar inactivation disrupts motor-intentional processing .........................153

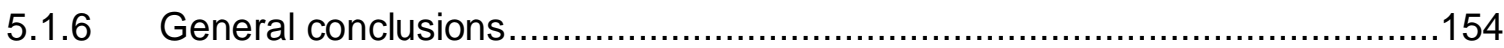

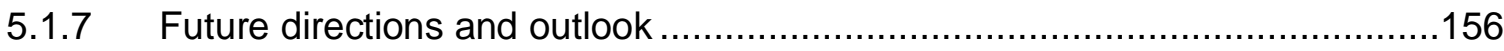

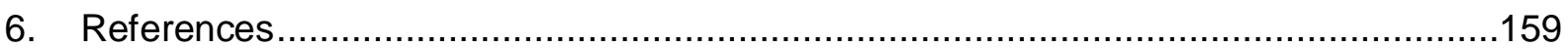

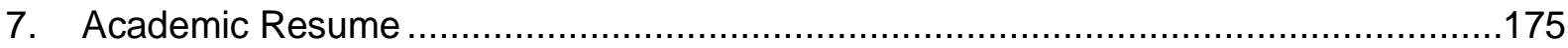

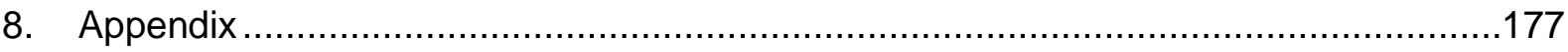

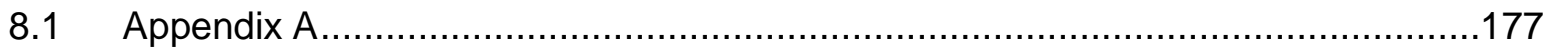

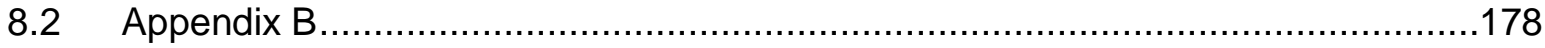




\section{Summary}

The pulvinar is the largest thalamic structure in the center of the brain, reciprocally connected to a large diversity of cortical areas. It has grown more than other subcortical structures during primate evolution, which is one of the reasons why it is thought to be crucial to primate behavior. Taking the few studies on pulvinar electrophysiology and perturbation together, it is clear that the function of pulvinar is complex, and might be involved in a wide range of cognitive processes such as allocation of spatial attention, target selection, motor coordination, and even emotional processing or confidence. What most of the recent studies agree upon is that its function is strongly linked to voluntary movements, going well beyond purely relaying sensory information to cortical areas as initially thought, and making pulvinar a particularly interesting target for understanding the neuronal substrates of goal-directed behavior in primates. Especially the non-retinotopically organized, dorsal aspect of the pulvinar remains understudied and its contribution to perceptual and motor intentional processing remains speculative. Further investigation of dorsal pulvinar function in these processes should contribute to our understanding of how the brain processes visual information in order to select and execute appropriate actions and help to elucidate the function of this mysterious structure which has puzzled researchers for the last decades. Here, we provide new evidence of dorsal pulvinar involvement in goal-directed behavior, contributing to the ongoing discussion in the field about the role of pulvinar in visuomotor processing using a battery of different approaches in behaving macaque monkeys and humans.

First, we analyzed visual and motor related neuronal processing in the dorsal pulvinar during basic oculomotor tasks, visually-guided and delayed memory-guided saccades. In the memory-guided task, visual responses were more pronounced, time-locked and space specific (predominately contralateral), but overall pulvinar exhibits more motor related activity, in some cells prior to and during saccades, but mostly in the post-saccadic period. The patterns of motor-related activity were diverse, spanning contralateral and ipsilateral spatial tuning and also motor-related enhancement and suppression. Despite strong connectivity to parietal cortex, in this task most pulvinar cells did not show classical visuomotor delay period activity or pre-saccadic ramping of firing. Instead, delay period activity was typically suppressed (relative to initial fixation period) with no spatial preference, and delay period activity did not predict the upcoming movement in free-choice trials when two saccade options were available. However, on the level of synaptic processing represented by the local field potentials, the upcoming choice was encoded, suggesting that in principle the information about target selection was present before motor execution. Moreover, we show that a subset of pulvinar neurons exhibits properties similar to gain fields 
related to gaze position, suggesting that pulvinar encodes space not only in eye-centered, retinotopic reference frame, and might also be involved in visuomotor transformations.

To further assess the role of pulvinar function in target selection we investigated effects of dorsal and ventral pulvinar microstimulation in similar oculomotor tasks. Systematic variation of stimulation times relative to the behavioral states and stimulus/saccade onset revealed space-specific, time-specific, task-specific, and site-specific involvement of pulvinar in target selection, evident in microstimulation-induced modulation of spatial decisions in free-choice trials, and effects on saccadic reaction times. These results suggest that the dorsal pulvinar influences target selection when the decision and action are in close temporal proximity, and not when the visual processing and the motor response are separated by the intervening memory delay period.

One of main outstanding questions in studying spatial choice processing in general and in pulvinar research in particular is whether behavioral effects of causal perturbations or neurological lesions stem from perception or motor intention impairments. To dissociate sensory-perceptual and motor-intentional aspects of spatial deficits, we designed a novel "search-to-sample" task and tested in in groups of young and elderly human subjects and in the macaque monkey. The comparative aspect of this work was intended to establish experimental approaches that would allow drawing parallels between studies in neglect patients and in reversible pharmacological inactivation-based macaque model of spatial deficits. Although the effects of MRI-guided pulvinar inactivation in this task were small, they further support the idea that pulvinar is involved in motor-intentional processing, especially when compared with effects after control inactivation of the cortical temporal parietal occipital area (TPO) in the same monkey. The electrophysiological characterization of the area TPO however suggested considerable similarities between encoding of visuospatial and motor contingencies between this area and the dorsal pulvinar. Given that these two regions are both anatomically and functionally connected, this shows that functional contribution of specific regions has to be considered not in isolation but within the interconnected circuitry. At the same time, these data emphasize the importance of combining "correlative" recordings with causal interference approaches.

Additionally, we tested saccadic, grasping and reaching deficits of a patient with rare bilateral pulvinar lesion. The patient exhibited mainly postural deficits, specifically when reaching with the right hand (contralateral to the more pronounced lesion), accompanied by general slowing of movements and grasping deficits. These findings further support the idea that pulvinar might be involved both in guiding goal-directed actions and more specifically in sensorimotor transformations. In combination with findings from other related projects in our 
group, the results of this thesis indicate that the function of the dorsal pulvinar goes well beyond purely visuospatial or attentional processing. 


\section{Abbreviations}

\begin{tabular}{|c|c|}
\hline AC-PC & Anterior commissure - posterior commissure \\
\hline ANG & Angular gyrus \\
\hline ANOVA & Analysis of variance \\
\hline BOLD & Blood oxygen level dependent \\
\hline BrSC & Brachium of the superior colliculus \\
\hline $\mathrm{Cd}$ & Caudate nucleus \\
\hline $\mathrm{Cl}$ & Contralaterality index \\
\hline$C L$ & Centrolateral nucleus \\
\hline $\mathrm{CM}$ & Centromedian nucleus \\
\hline dIPFC & Dorsolateral prefrontal cortex \\
\hline dPul & Dorsal pulvinar \\
\hline FDR & False discovery rate \\
\hline FEF & Frontal eye fields \\
\hline FR & Firing rate \\
\hline GABA & gamma-Aminobutyric acid \\
\hline IML & Intermediolateral nucleus \\
\hline IPL & Inferior parietal lobe \\
\hline IPul & Inferior pulvinar \\
\hline IT & Inferior temporal cortex \\
\hline LED & Light-emitting diode \\
\hline LGN & Lateral geniculate nucleus \\
\hline LIP & Lateral intraparietal area \\
\hline LFP & Local field potential \\
\hline LPul & Lateral pulvinar \\
\hline MATLAB & Matrix laboratory \\
\hline MD & Mediodorsal nucleus \\
\hline ME & Mesencephalic reticular formation \\
\hline MIP & Medial intraparietal cortex \\
\hline MRI & Magnetic resonance imaging \\
\hline MST & Medial superior temporal area \\
\hline MT & Medial temporal area \\
\hline MPul & Medial pulvinar \\
\hline OFC & Orbitofrontal cortex \\
\hline $\mathrm{Pc}$ & Paracentral nucleus \\
\hline PEEK & Polyetheretherketone \\
\hline $\mathrm{PGa}$ & area PGa in the superior temporal sulcus \\
\hline Plcl & Central lateral nucleus of the inferior pulvinar \\
\hline Plcm & Central medial nucleus of the inferior pulvinar \\
\hline PIm & Medial nucleus of the inferior pulvinar \\
\hline PIp & Posterior nucleus of the inferior pulvinar \\
\hline PLdm & Dorsomedial subdivision of lateral pulvinar \\
\hline PLvl & Ventrolateral subdivision of lateral pulvinar \\
\hline PMI: & Lateral subdivision of medial pulvinar \\
\hline PMm & Lateral subdivision of medial pulvinar \\
\hline PPC & Posterior parietal cortex \\
\hline PRF & Pontine reticular formation \\
\hline PSTH & Peri/Post-stimulus time histogram \\
\hline RARE & Rapid acquisition with relaxation enhancement \\
\hline RT & Reaction time \\
\hline RF & Response field \\
\hline S2S & "Search-to-Sample" task \\
\hline SEF & Supplementary eye fields \\
\hline SC & Superior colliculus \\
\hline SMG & Supramarginal gyrus \\
\hline SNr & Substantia nigra pars reticularis \\
\hline STG & Superior temporal gyrus \\
\hline STS & Superior temporal sulcus \\
\hline STP & Superior temporal polysensory area \\
\hline THIP & 4,5,6,7-tetrahydroisoxazolo(5,4-c)pyridin-3-ol \\
\hline
\end{tabular}


Temporo-parietal-occipital area

Temporo-parietal junction

Ventral anterior nucleus

VFC

Ventral frontal cortex

VIP

Ventral intraparietal cortex

$\mathrm{VL}$

Ventral lateral nucleus

VMI

vPul

Visuomotor index

Ventral pulvinar

$\mathrm{V} 1$

Primary visual area (striate cortex)

V2

Extrastriate visual area 2

Extrastriate visual area 3

V4

Extrastriate visual area 4 


\section{Introduction}

\section{$1.1 \quad$ Visual and motor processing}

Our brains are constantly gathering information about surrounding environment. This information comes in different modalities, for example visual or auditory. The sensory information is typically acquired by afferent nerves, then filtered and compressed and further processed in specific brain areas responsible for decoding different aspects of the sensory signals. To interact with the environment, we and other animals form decisions based on our assessment of the internal states and the state of the environment, which together determine the selection and execution of corresponding actions.

Primates in particular rely mostly on visual sensory inputs, which is reflected not only in the large extent of areas involved in processing of visual information, but also in the variety of studies focusing on visual processing in humans and monkeys. Regarding motor actions, eye and hand movements are of particular interest, since most of the goal directed behaviors involve at least one of the two effectors. Again, this is not only reflected in the comparably wide representation in the brain, but also in the extensive research on the neural basis of eye and hand movements in primates.

\subsubsection{Visual processing streams}

The sensory neurons of the visual system, photoreceptor cells, are located in the retina of the eyes, and are sensitive to electromagnetic radiation of either specific wavelength (cones) or intensity (rods). Because all inciding light is focused in one point by the lenses in the eye, the location in the retina of a firing photoreceptor cell is proportional to the angle of incidence of the light beam that activated the receptor. After initial contrast enhancement in deeper layers of the retina, the information is sent to the lateral geniculate nucleus of the thalamus (LGN) via the optic tract, where inputs from both eyes are split into two halves and recombined such that inputs from the left visual field reach right LGN and vice versa, preserving retinotopic spatial organization. LGN projects to the primary visual area (V1) in the cortex. From there, visual information travels along two major pathways with distinct features: The ventral and the dorsal stream (see Figure 1.1). This has been demonstrated by different deficits in after confined brain lesions both in humans and monkeys (Mishkin and Ungerleider, 1982; Schneider, 1969).

The ventral stream, often referred to as the "what" pathway, includes V1, V2v (ventral part of V2), V4 and the inferior temporal cortex (IT). It processes shape, orientation and size of stimuli in order to recognize objects, faces and text. At the end of the ventral pathway are the medial temporal lobe, the hippocampus and the amygdala. The idea is that ventral 
information is used for "recognition, memory and emotional content" (de Haan and Cowey, 2011).

The dorsal stream includes V1, V2d (dorsal part of V2), V3, the medial temporal area (MT), the medial superior temporal area (MST) and several areas in the posterior parietal cortex (Rokszin et al., 2010). It processes spatial information such as location and motion in order to estimate spatial relationships. It is therefore commonly referred to as the "where" pathway. However, more recently it has been shown that it is also involved in time perception (Battelli et al., 2008). The general notion is that the dorsal pathway is heavily involved in action guidance (Goodale, 2011; Goodale and Milner, 1992) and eventually feeds into motor cortex.

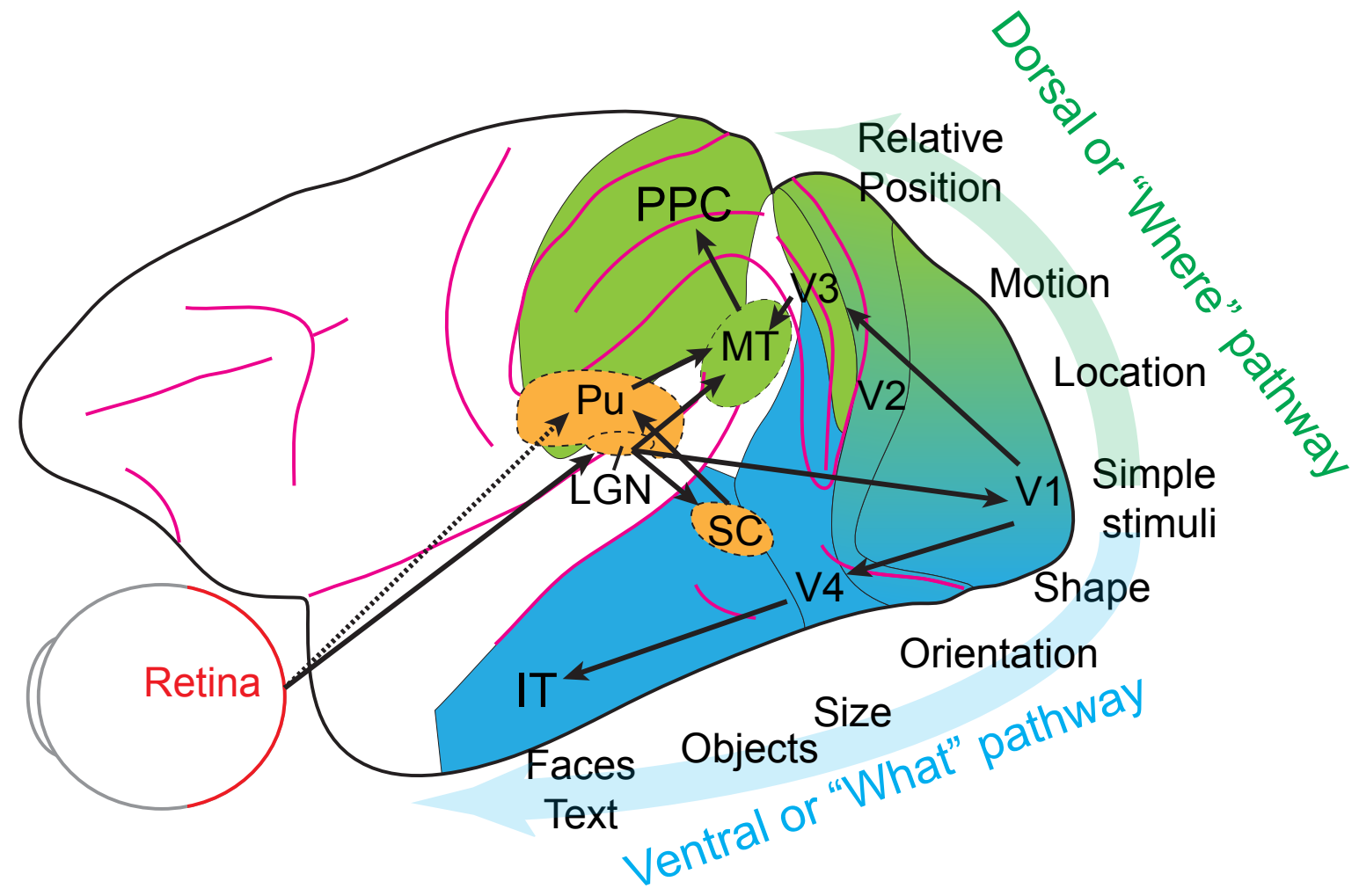

Figure 1.1: Visual pathways, ventral and dorsal stream. Composite figure from and with permission of de Haan and Cowey 2011, Goodale 2011, and Rokszin et al. 2010

Importantly, these classical pathways are simplifications. In total, more than 30 areas have been associated with visual features, and they are heavily interconnected (Ungerleider and Haxby, 1994). For example, LGN also projects to the superior colliculus (SC) in the midbrain, which is responsible for controlling fast and goal directed eye movements. SC on the other hand has afferents projecting to the thalamic pulvinar (Pul), which also is connected to MT for example. Furthermore, it has been shown recently, that the optic nerve also projects directly to the pulvinar (Maleki et al., 2012). 


\subsubsection{Motor processing}

The ultimate goal of the vast sensory data collection is widely believed to be taking the most beneficial actions possible. Action commands can be seen as the output of the brain leading to planned and coordinated movements as a reaction to the current situation. Cortical areas involved in movement shaping not only include primary motor cortex (activity mostly related to movement execution) and premotor cortex (activity related to movement planning), but also several other parietal and frontal areas. Posterior parietal cortex was found to be heavily involved in action selection and provides strong inputs to premotor areas. The frontal eye fields (FEF) can be seen as an eye movement motor area. Motor areas are responsible for sending motor commands to coordination controlling circuits, which then forward these commands to motor neurons. Coordination controlling circuits include the cerebellum, big parts of the brainstem, and the corticospinal tracts.

In the case of eye movements, different networks control different types of eye movements saccades (fast and targeted eye movements), smooth pursuit (following a moving target), vergence shifts (adapting to object distance), vestibulo-ocular movements (non-voluntary adaptation to vestibular assessment of body motion), optokinetic nystagmus (following an object passing by, then switching to the next object), and eye fixation (Goldberg, 2000; Krauzlis, 2004). In this work, a main focus will be on saccadic eye movements and the saccade network (see Figure 1.2).

The posterior parietal cortex, specifically the lateral intraparietal area (LIP) has bidirectional connections to the frontal eye field (FEF), which also receives input from the supplementary eye fields. While LIP is believed to be involved in visual guidance and target selection since the activity of LIP cells is modulated by attention, desirability and the behavioral relevance of a stimulus, FEF is seen as the cortical motor command center for voluntary saccades, although it has also been shown to respond to attentional shifts towards the response field when no saccade or a saccade to the opposite direction is performed (Schall, 2004). Although the role of SEF is not yet completely clear, current research indicates that it might contribute in visual search. Both FEF and LIP have afferent connections to superior colliculus (SC), which initiates saccades by activating mesencephalic and pontine reticular formations, responsible for forwarding coordinated commands to six extraocular muscles via three different cranial nerves, allowing rotations of eye along three different axes. It should be mentioned here that one additional pathway from FEF to SC passes the subcortical structures of caudate nucleus and the substantia nigra, which provides tonic inhibition to the SC when no saccade is to be executed (Goldberg, 2000). 


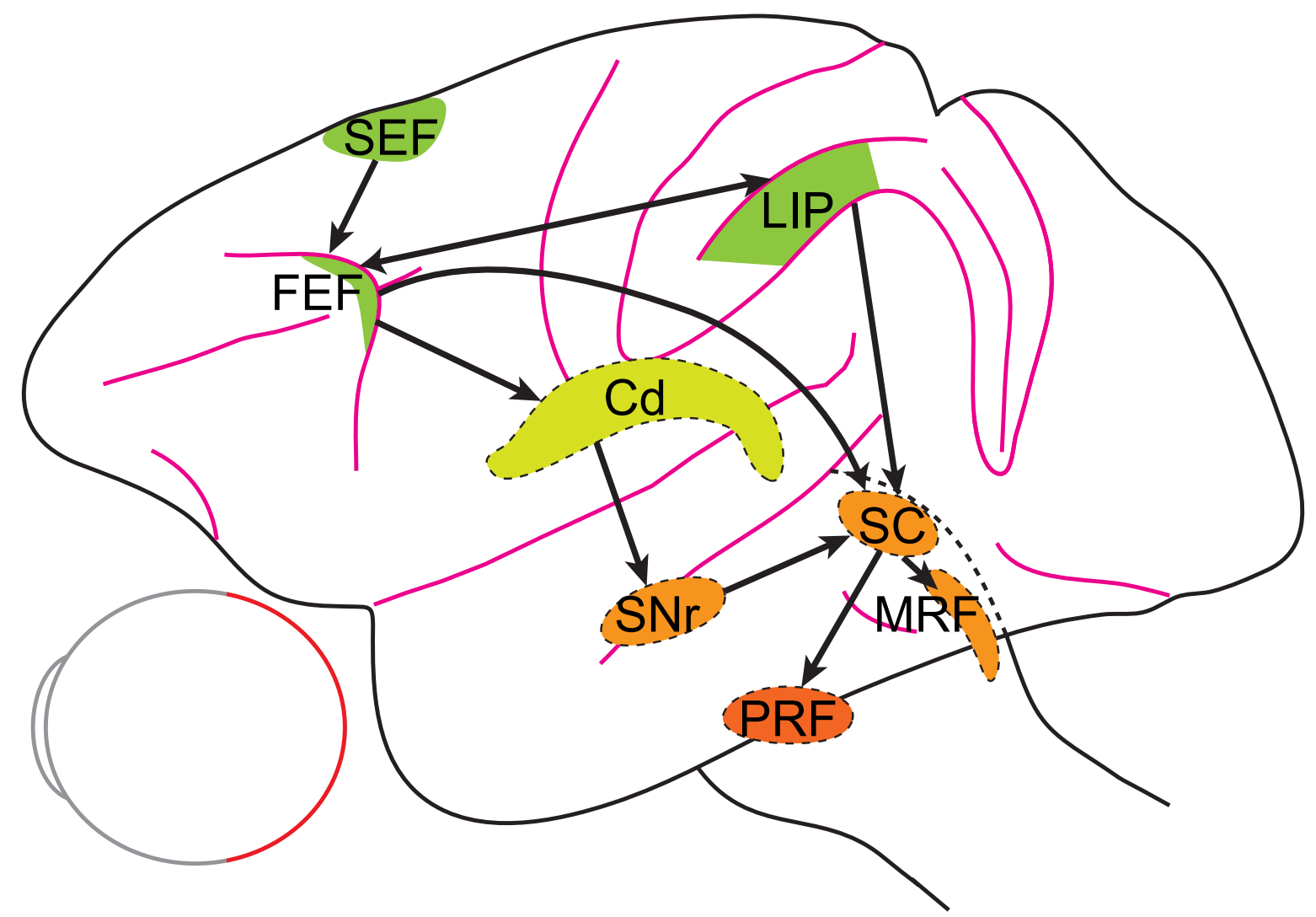

Figure 1.2: Schematic saccade network. Green regions indicate cortical areas - supplementary eye fields (SEF), frontal eye fields (FEF), and the lateral intraparietal cortex (LIP); red regions are the mesencephalic (ME) and pontine reticular formation (PRF); blue regions the subcortical structures Caudate (Cd), the substantia nigra pars reticulata (SNr) and the superior colliculus (SC). Image adapted from Krauzlis 2004.

Finally, copies of motor commands can also be fed back to the sensory processing stream, allowing the suppression of predicted changes in sensory input. The predictions of upcoming changes are also compared to the actual changes induced by the motion, and the difference in both serves as a feedback to motor areas to enable motor adaptation and hastened movement refinement. All of these mechanisms are referred to by the term "corollary discharge" (Poulet and Hedwig, 2007; Sperry, 1950). Corollary discharge is believed to be mediated by the thalamus (Bellebaum et al., 2005; Sommer and Wurtz, 2004).

\section{$1.2 \quad$ From vision to action}

The link between sensory and motor processing is yet not fully understood and therefore a main target of modern systems neuroscience. The questions that are proposed in this context are unsurprisingly widespread. Just to give a few examples: How and where are decisions for upcoming actions formed? What are the neuronal substrates for an implied cost-benefit assessment? How is sensory evidence integrated to form these decisions? How does attentional focus filtering sensory information work on a neuronal basis? How do memory and emotions affect the decision making process? 
For the current work, it is crucial to consider the different spatial representations in the brain that have been reported, and the questions arising from these observations. Importantly, visual information enters the brain via the retina, meaning that any spatial encoding at this stage is always relative to the current eye position. But for executing a goal directed arm movement for example, the target location has to be represented relative to the body and the current arm position in the respective motor area. These different relative spatial representations are commonly referred to as reference frames.

\subsubsection{Reference frames}

Several different reference frames have been reportedly found in different areas (Batista, 2002), but also within the same areas (specifically in posterior parietal cortex). The most prominent reference frames are egocentric: eye-, or gaze-centered (i.e. retinotopic), headcentered (Brotchie et al., 1995), hand-centered (Chang and Snyder, 2010) and bodycentered (Knudsen and Konishi, 1978), but there are also allocentric frames, namely objectand world-centered (Snyder et al., 1998). The difference between the latter two is that objectcentered refers to a location relative to certain object, regardless of the position of the object in space - for example the location of the C-1key relative to a Steinway piano - , whereas world-centered refers to the absolute location, regardless of the observers location or orientation - for example the location of the piano in the Metropolitan Opera House or the location of the Opera House in New York. Furthermore, several studies showed that many neurons across sensorimotor circuitry exhibit mixed reference frames, including relative encoding (e.g. relative position of gaze and hand), and even more complex nonuniform/idiosyncratic representations (Chang and Snyder, 2010).

How information is transformed from one reference frame into another remains yet unclear. These transformations can be seen as translations of inertial systems. The main difficulty here is that the mathematical operation of addition is a non-trivial task for a neuronal network, meaning that each neuron always and only fires when the sum equals certain value. However, there is at least one strong hypothesis on how the brain solves this task, namely via "gain fields" (Salinas and Abbott, 2001), a model which has been also successfully applied to explain other kinds of modulations in the brain, such as for example feature based attention (Treue and Trujillo, 1999). The gain field model for reference frame transformation has also been theoretically demonstrated in the case of transformation from eye-centered to attention-centered reference frames (Salinas and Abbott, 1997). The basic idea here is that information represented in the original reference frame from one group of neurons (input layer 1 ) is integrated with information about the relation of the two reference frames (input layer 2) in order to compute representations in the new coordinates. The integration is thought to happen on the dendritic side of an intermediate layer in a multiplicative way 
(amplification/gain) rather than by addition. The entirety of outputs in this layer represents the original information in the new reference frame.
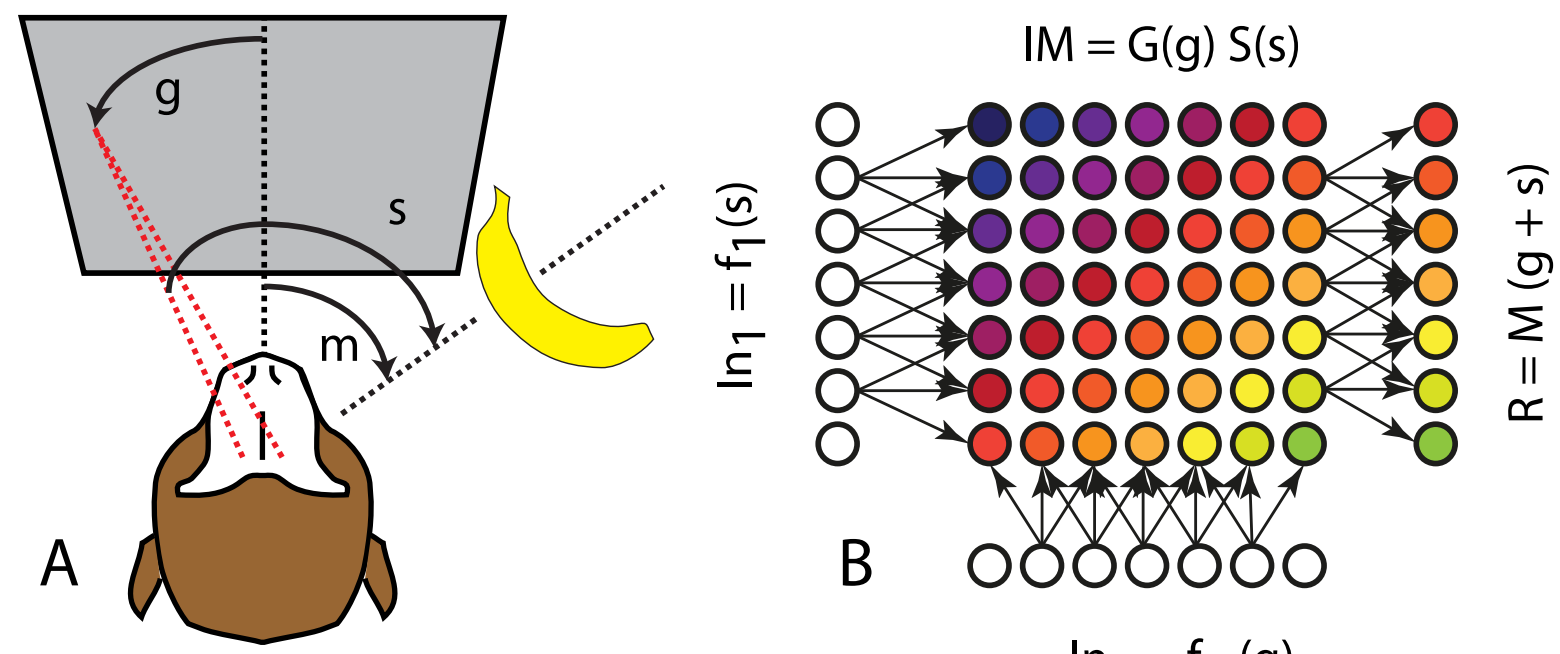

$$
\ln _{2}=f_{2}(g)
$$
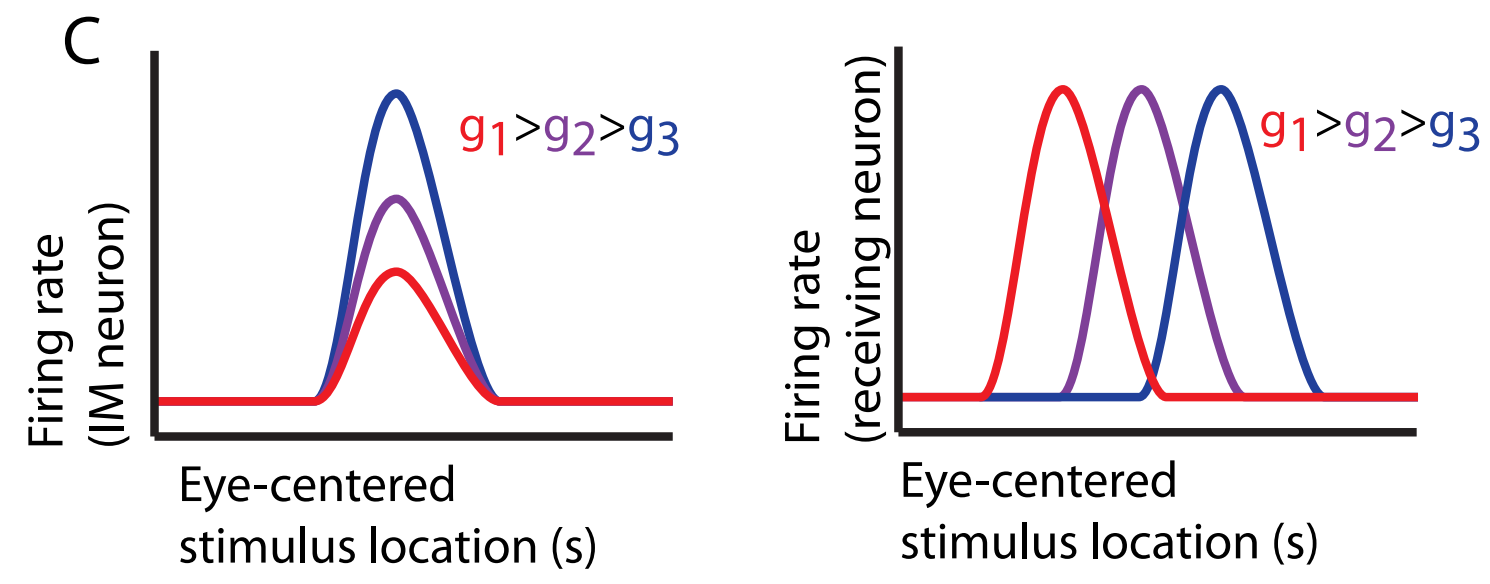

Figure 1.3: Reference frame transformation via gain field modulation. A) Illustration of a frequent reference frame transformation; $g$ = gaze position relative to the body/head, $s=$ stimulus position in eye-centered coordinates, $\mathrm{m}=\mathrm{g}+\mathrm{s}=$ direction of movement to perform in order to reach for the banana. B) Schematic of a hypothetical underlying network performing the transformation; Input layer $1\left(\ln _{1}\right)$ represents the eye-centered information s, Input layer $2\left(\ln _{2}\right)$ provides information about gaze position g, the intermediate layer contains the eye-centered information modulated by a gaze-dependent gain field, the receiving layer $R$ reads out the transformed coordinates $\mathrm{g}+\mathrm{s}$, that are relevant for the correct movement. C) Illustration of the gaze modulation for two example neurons, one from the intermediate layer (left), and one from the receiving layer (right). While the neuron on the left has an eye-centered receptive field, and its response is amplified by the gaze position, the neuron on the right responds specifically to certain egocentric stimulus location (in eye-centered coordinates, gaze shifts cause receptive field shifts in the opposite direction). Composite figure from and with permission of Salinas and Abbott 2001

As an example, if input layer 1 transfers eye-centered information and input layer 2 represents the current eye position relative to the head, combining the two inputs allows the receiving layer to transform the eye-centered information into a head-centered reference frame (see Figure 1.3). Gaze dependent gain fields for eye-centered reference frames have 
been reported for the posterior parietal cortex (Andersen and Mountcastle, 1983), but even at the level of LGN and primary visual cortex, eye-centered reference frames can be modulated by gaze position (Weyand and Malpeli, 1993). Note that areas with eye-centered reference frames do not have to be retinotopically organized, as one describes the properties of response fields, while the other describes the similarity of properties between adjacent neurons.

\subsubsection{Spatial neglect}

As mentioned above, visual information is split into two hemifields very early in the sensory processing and treated in large part independently in the contralateral hemispheres. It is therefore not surprising that unilateral lesions can lead to perceptual spatial biases. But on top of primary sensory spatial deficits, various types of non-sensory spatial awareness biases can occur, which are often summarized by the term "spatial neglect". This neurophysiological condition is most commonly observed in stroke patients $(23 \%$ of the cases (Pedersen et al., 1997)). Lesions in several areas have been associated with spatial neglect, most prominently the supramarginal gyrus (SMG) (Vallar and Perani, 1986), and angular gyrus (ANG) (Mort et al., 2003) in the inferior parietal lobe (IPL), the temporoparietal-occipital junction (TPO, often also called temporo-parietal junction, TPJ) (Leibovitch et al., 1999), the superior temporal gyrus (STG) (Karnath et al., 2001) and sub-regions of the ventral frontal cortex (VFC) (Rengachary et al., 2011). But also damages in subcortical structures, especially the thalamic pulvinar can lead to similar symptoms (Arend et al., 2008a).

One of the most puzzling findings regarding spatial neglect is that it seems to be more likely to occur following a lesion in the right hemisphere (Becker and Karnath, 2007; Behrmann et al., 2004; Bowen et al., 1999; Corbetta et al., 2005; Pedersen et al., 1997; Stone et al., 1993), while the affected side is always contralateral to the lesion. This has led to two major hypotheses trying to frame the underlying mechanisms. One is the hemispheric imbalance model, claiming that each hemisphere controls attention shifts to the contralateral side and inhibits the other hemisphere. The imbalance between right and left hemisphere lesions in this model is explained by a stronger attention shift power in the left hemisphere and a stronger contralateral hemisphere inhibition in the right hemisphere, balancing the net attention shift in healthy subjects. When one hemisphere is lesioned however, this would lead to stronger effects when the right hemisphere is affected (Kinsbourne, 1987). The second theory assumes that the right hemisphere controls attention for both sides of space, while the left hemisphere is only responsible for the right side. The imbalance is then explained by compensation in case of left hemisphere lesions, whereas the left hemisphere cannot compensate for right hemisphere lesions (Mesulam, 1981). 
In more detail, apart from contralateral spatial deficits including stimuli appearing less salient as well as reduced spatial attention and short term memory, patients often suffer from nonspatial deficits such as reduced arousal, general problems in detecting targets and slower reorienting (Corbetta and Shulman, 2011). However, the symptoms can be grouped into two main deficits: A bias in head and eye position towards the ipsilesional side and the unawareness of the perceptual shift (Hornak, 1992; Karnath and Rorden, 2012). These two aspects will be referred to in this work as intentional and perceptual deficits. Perceptual, also referred to as 'sensory-attentional' or 'perceptual-sensory' deficits include no sense of touch as well as not hearing sounds or seeing stimuli on the neglected side, while intentional, also referred to as 'motor-intentional' or 'exploratory-motor' include reduced exploration both with eyes and hands in the neglected side deficits (Liu et al., 1992; $\mathrm{Na}$ et al., 1998). The main underlying hypothesis behind this separation is that maybe the exact location of the lesion defines the type of deficits (Husain, 2000), a hypothesis that is very difficult to verify in patients with rather unique lesion sites.

Importantly, a reduced sense of touch on one arm as well as deficits in reaching towards one side already give a hint that the "neglected side" might not only be one (retinotopic) hemifield, since this happens regardless of where the patient is looking. It has been shown, that the trunk orientation can have an impact on the severity of perceptual deficits, meaning that even if stimuli are placed in the same location relative to the eye position, deficits are less apparent when the body is turned more towards the neglected stimulus (Karnath et al., 1991). Another, more complex symptom indicating that different reference frames might be affected is "object-based" neglect. Patients with object based neglect fail to consciously perceive the neglected half of an object, regardless of the side of space in which the object is presented. In addition, when asked to draw or even copy an object, they will only draw one half of it (Gainotti et al., 1972). Also object related neglect has been shown to also interfere with the egocentric position of the object (Karnath, 2015; Karnath et al., 2011), see Figure 1.4. It seems like the deficits appear in several reference frames at the same time (Driver and Halligan, 1991), and which reference frames are more affected could very plausibly be different for different patients with different lesions (Committeri et al., 2007).

However, if the lesioned area does not represent a specific reference frame, but rather provides the necessary inputs for reference frame transformations (such as the intermediate layer in the basic gain field model described earlier), this could explain the presence of deficits in multiple reference frames at the same time. The assumption here is that the lesioned area represents "basis functions", which allows receiving areas to compute representations in different reference frames, based on the same inputs. Neuronal network models have shown that a lesion in an area with such properties (e.g. posterior parietal 
cortex) would lead to the observed deficits in multiple reference frames (Pouget and Sejnowski, 1997). Furthermore, the same model can explain the different severities of hemispatial neglect after left and right hemisphere lesion, based on the asymmetric space representation between left and right hemisphere in humans (Pouget and Driver, 2000).
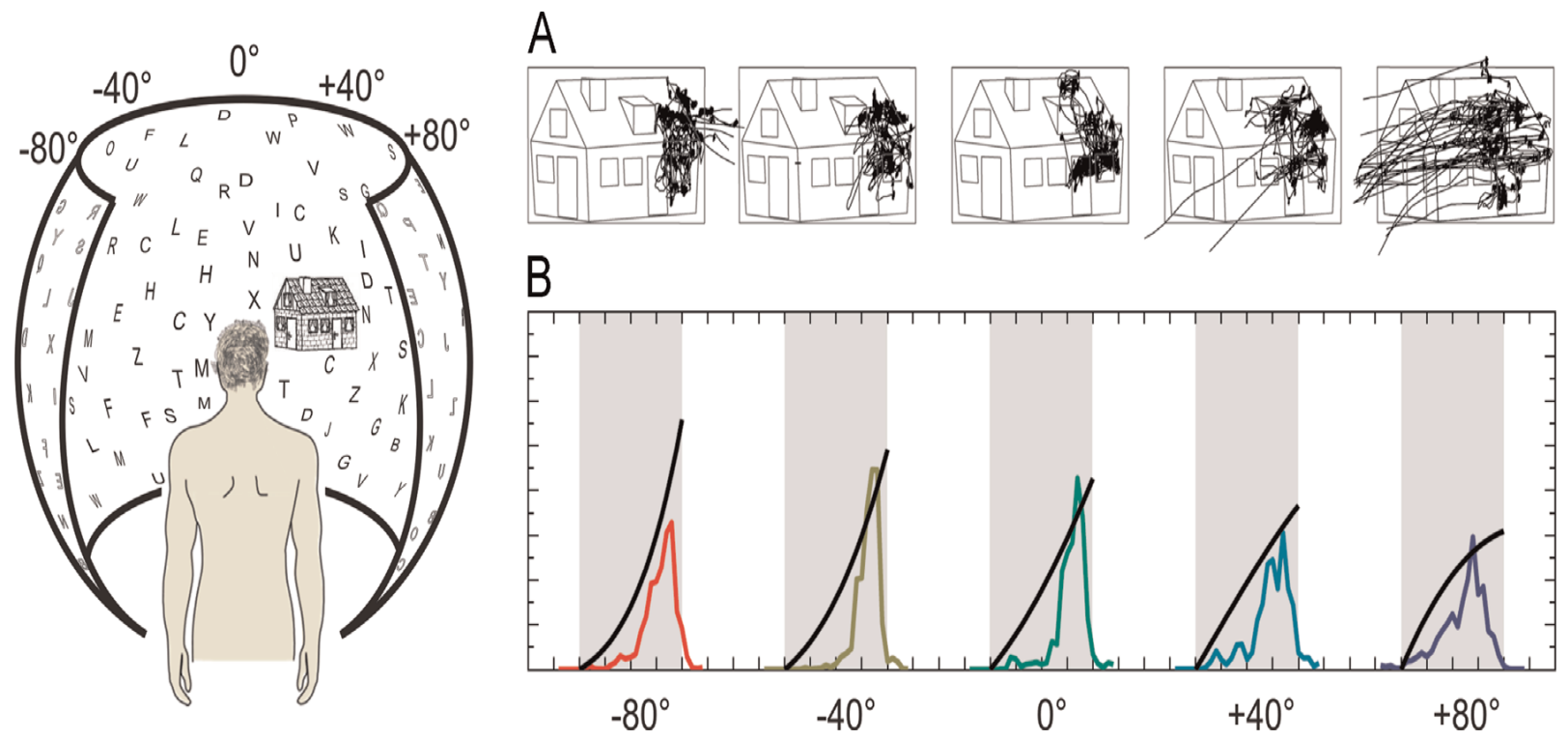

Figure 1.4: Object-based neglect is affected by egocentric object position. Exploration patterns $(A)$ and histograms of gaze positions of a neglect patient with right hemisphere lesion for the five different egocentric object positions. Figure from and with permission of Karnath, 2015

\subsection{The thalamic pulvinar}

The thalamus is the largest subcortical structure. Like the cortex, it has two symmetric hemispheres which are located close to the center of the brain on both sides of the brain and can be divided into two major components; the dorsal and the ventral thalamus, both of which consist of several distinct nuclei. Nuclei in the dorsal thalamus are also referred to as "relay nuclei", since most of the cells are "relay cells", innervating middle layers of cortex, with a few exceptions that project to upper layers. The other main cell type found in the dorsal thalamus are local interneurons (around 1 out of 4 ), mainly inhibiting relay cells. The main part of the ventral thalamus is the reticular nucleus. Reticular cells inhibit dorsal thalamus relay cells. Relay cells use glutamate as their neurotransmitter, while local interneurons and reticular cells are GABAergic. The most common ion channel types besides the conventional $\mathrm{Na}^{+}$and $\mathrm{K}^{+}$channels are $\mathrm{T}$-type $\mathrm{Ca}^{2+}$ channels, which allow the neuron to switch from tonic to bursting activity. The receptor types in relay cells are either ionotropic (fast responding) and metabotropic (slow responding) (Sherman, 2009). 


\subsubsection{Cortico-thalamo-cortical integration}

The main inputs to relay cells are either from peripheral sensory organs (mediated only by ionotropic receptors), cortical inputs, or inhibitory inputs from surrounding interneurons and reticular cells. Importantly, all except olfactory sensory information passes through thalamus. Driving afferents activate fast responding ionotropic receptors, which leads to strong correlations of firing activities of relay cells and the driving input (e.g. LGN cells have similar response fields as their retinal afferents), although relay cells receive many other inputs from "modulators", modulating firing patterns e.g. from bursting to tonic activity (Sherman and Guillery, 1998, 2002). Interestingly, the "drivers" only make up for 5\% of all inputs (Van Horn et al., 2000), indicating that only looking at the fraction of inputs could lead to severe misinterpretations of what these subcortical areas are doing.

A very general parcellation of thalamic nuclei can be made according to their connectivity to cortex. First order nuclei receive driving inputs from peripheral nerves or from other subcortical structures and are the ones which send information to cortex for the first time, whereas higher order nuclei receive driving inputs directly from cortex itself.

In the case of the visual system, the first order nucleus is the lateral geniculate nucleus (LGN), and the higher order nucleus is the pulvinar. Both LGN and pulvinar receive modulatory input from layer 6 of cortex, but the driving input to LGN origins in the retina, whereas pulvinar receives its driving inputs from layer 5 of cortical areas (Sherman and Guillery, 2002).

Note that in general, the idea that driving inputs can come from the cortex is challenging the traditional idea of thalamus being a relay area only (Purushothaman et al., 2012), even more so in combination with the fact that higher order nuclei project back to cortex. More recent evidence indicates that thalamus is heavily involved in cognitive functions as it plays an important role in communication between cortical areas (Saalmann and Kastner, 2015), and monitors and refines motor commands, which is indicated already by the fact that cortical inputs are mainly branches of fibers projecting to lower motor centers like the spinal cord and brainstem (Guillery, 2003; Guillery and Sherman, 2002).

\subsubsection{Pulvinar anatomy}

Pulvinar, the largest of the thalamic nuclei, is located on the posterior end of the thalamus. It has greatly expanded during primate evolution compared to other nuclei of the thalamus and is broadly connected to a manifold of cortical areas. Pulvinar is traditionally seen as a higher order nucleus, but more recent studies challenge this simplified distinction as pulvinar also receives direct retinal inputs (O'Brien 2001, Warner 2010). 
Although early anatomical studies suggest a segmentation of pulvinar into four sub-nuclei anterior, medial, inferior and lateral pulvinar (Olszewski, 1952) - based on slight differences in the distribution of cells of different size, more recent connectivity studies suggest to differentiate between dorsal pulvinar ( $\mathrm{dPul}$, which consists of medial pulvinar and dorsal part of lateral pulvinar, also denoted as PLdm) and ventral pulvinar (vPul, which contains ventral part of lateral pulvinar, also denoted as PLVl, and inferior pulvinar), as the more ventrolateral parts are mostly bidirectionally connected to early striate and extrastriate visual areas leading to a somewhat systematic retinotopic organization (Benevento and Miller, 1981), whereas the dorsomedial parts of pulvinar have strong bidirectional connections to higher processing areas such as the dorsolateral prefrontal cortex (dIPFC), orbitofrontal cortex (OFC), frontal eye fields (FEF) (Bos and Benevento, 1975), posterior parietal cortex (Hardy and Lynch, 1992; Schmahmann and Pandya, 1990), insular cortex (Mufson and Mesulam, 1984), cingulate cortex, superior temporal sulcus (STS), superior temporal gyrus (STG), and amygdala (Romanski et al., 1997), and show no clear topological organization. However, even though ventral pulvinar is more strongly connected to the early visual cortex and ventral stream, and dorsal pulvinar to the dorsal stream, there are also several connections outside of this scheme in both subdivisions.

Apart from cortical afferents, also superior colliculus projects to pulvinar. Interestingly, this connection is not bidirectional, and the SC inputs to the more ventral as compared to more dorsal pulvinar regions originate in different layers of SC (Grieve et al., 2000), further supporting the relevance of distinguishing between dorsal and ventral pulvinar.

A rough separation between ventral and dorsal pulvinar can be drawn by the brachium, a fiber bundle originating in the superior colliculus, but in more detail the inferior pulvinar expands a bit into the area above the brachium (Kaas and Lyon, 2007), see Figure 1.5. Using more elaborate techniques such as immunostaining and histochemistry, up to nine different sub-areas have been identified (Gutierrez et al., 1995, 2000).

It should be mentioned here, that investigating pulvinar functions in nonhuman primates and translating the results to humans is, apart from obvious behavioral differences and neural organization discrepancies (such as hemispheric lateralization and asymmetry), further challenged by differences in the ontogeny of the structure. In short, only in humans pulvinar cells of telencephalic origin were found (Rakić and Sidman, 1969) whereas in the rhesus macaque (Macaca mulatta) all pulvinar cells seem to migrate from the diencephalon (Ogren and Rakić, 1981). However, the connectivity patterns are very much comparable between these two species (Buckner and Krienen, 2013), and for a vast majority of human brain areas there is a putative monkey homologue, as demonstrated for example for several regions in parietal cortex (Culham and Kanwisher, 2001; Orban, 2016). 

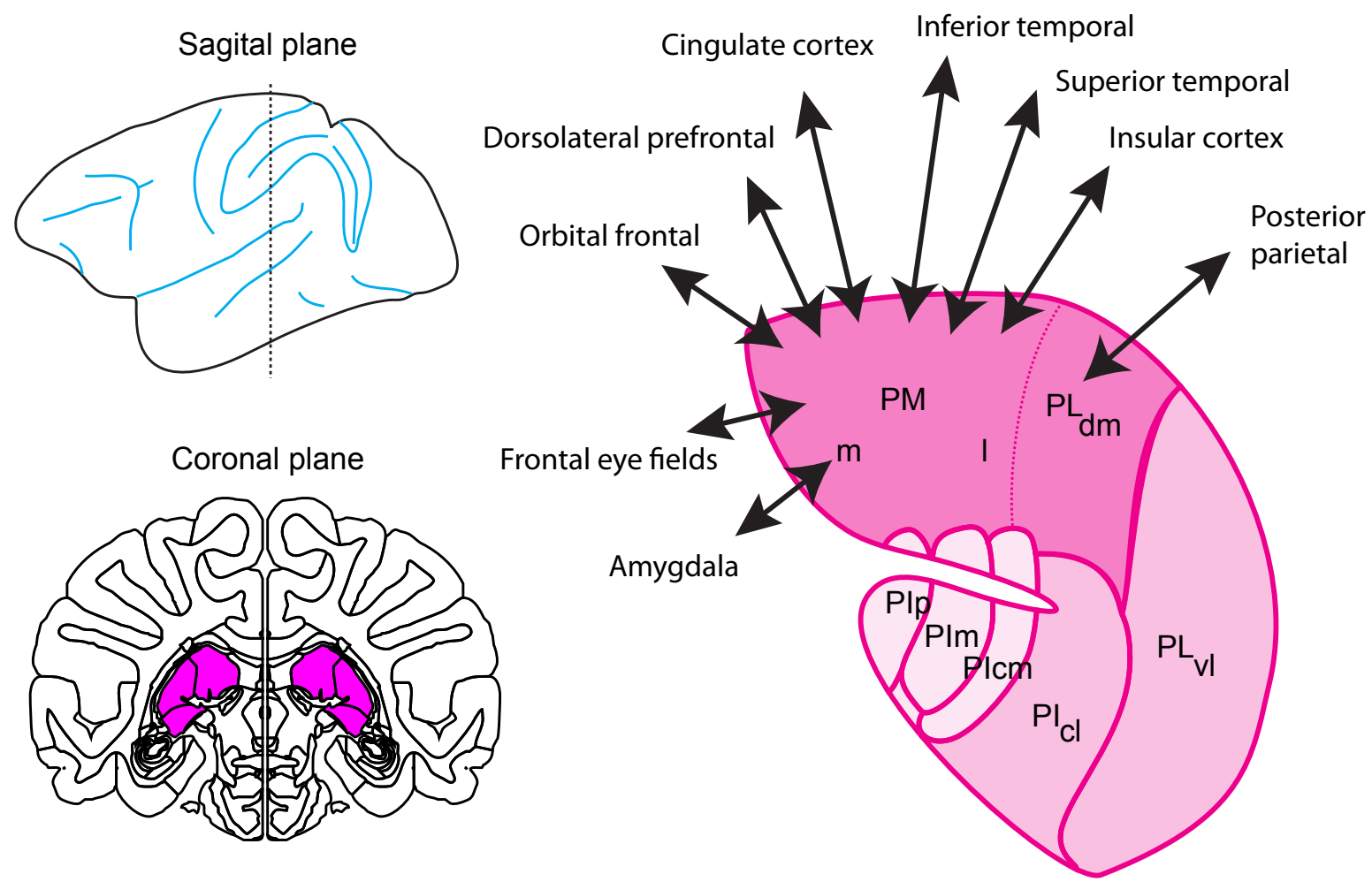

Figure 1.5: Location and subdivisions of pulvinar in the macaque brain. Left: top: sagittal view illustrating the location of the coronal slice on the bottom. Pulvinar is marked in purple. Right: Pulvinar subdivisions and dorsal pulvinar (dark pink) connectivity. Sagittal view modified from Culham and Kanwisher, 2001. Coronal template from: https://scalablebrainatlas.inforg/macaque/CBCetal15. Pulvinar subdivisions and connectivity modified from and with permission of (Kaas and Lyon, 2007). BrSC: Brachium of the superior colliculus; PMm/PMI: medial/lateral subdivision of medial pulvinar; PLdm/ PLvl: dorsomedial/ventrolateral subdivision of lateral pulvinar; $\mathrm{PIp} / \mathrm{PIm} / \mathrm{Plcm} / \mathrm{PIcl}$ : posterior/medial/central medial/central lateral nucleus of the inferior pulvinar.

\subsubsection{Pulvinar function}

Given the diversity of pulvinar connections and its complex, and not yet fully elucidated anatomical organization, it is not surprising that a wide range of functions have been attributed to pulvinar. Rather than claiming involvement in all of the attributed roles, going through different experiments that have been performed seems to be more adequate to gain a better understanding.

One of the first studies systematically recording neuronal activity from monkey pulvinar (squirrel monkeys) as a response to sensory stimulation found both the existence of mainly contralateral visual receptive fields in lateral, inferior, and medial pulvinar (around $50 \%$ of all recorded cells with receptive fields of at least 100 square degrees), as well as responses to somatosensory stimulation, mostly in lateral pulvinar (Mathers and Rapisardi, 1973). These early experiments were performed under anesthesia, which means that motor responses 
could not be tested, and one should be careful extrapolating these findings to natural conditions.

A later experiment in awake pig-tailed macaques (Macaca nemestrina), targeting specifically potential motor related responses, showed that around $60 \%$ of the recorded pulvinar cells could be classified into one of the following categories: 1) Arm and eye movement related $(\sim 15 \%) ; 2)$ Only arm movement related $(-30 \%), 3)$ Only related to saccadic eye movements $(\sim 8 \%), 4)$ Visual fixation( $7 \%)$. The latter two categories were more predominant in the inferior pulvinar, whereas anterior pulvinar contained only neurons of the second category (Acuña et al., 1983). Importantly, all of these cells were only active during intentional, goal directed movements, and not during casual exploration or somatosensory or visual stimulation. A direct comparison to parietal area 5 a neurons found that strong dependence on intention only in pulvinar cells, hypothesizing that pulvinar might reflect the attentional or motivational state (Acuña et al., 1990). Another study focusing on reach-related activity in lateral posterior pulvinar of Macaca nemestrina monkeys found few cells that start firing well before the reach onset and before reach-related activity is present in the primary motor area M1 (around 500 ms before reach onset). This reach related activity was not specific for the direction of the upcoming movement. Authors concluded that pulvinar might inform motor related areas about an upcoming intentional movement, regardless of the exact movement to be executed (Cudeiro et al., 1989).

Further underlining the importance of the behavioral state, even in visually responsive pulvinar neurons, especially in dorsal pulvinar, the activity was found to be much more pronounced when the stimuli were of behavioral relevance, giving rise to the idea that pulvinar is involved in allocating spatial attention (Petersen et al., 1985a). In the same study, retinotopical organization was attributed to ventral pulvinar regions, whereas dorsal pulvinar regions seemed to generally lack these properties, and visual responses in dorsal regions typically occurred later than in ventral pulvinar. Interestingly, this visual information seems to be modulated by the current gaze position (Robinson et al., 1990). The same group identified saccadic responses in pulvinar as being mostly post-saccadic, interpreting them as sort of a corollary discharge that might mark the end of an attentional period, signal context changes in the visual information, or simply help suppressing cortical visual information acquired during the saccade itself to maintain a stable perception (Robinson et al., 1986).

Visual responses in dorsolateral pulvinar of rhesus macaques (Macaca mulatta) have been further characterized using different colors and patterns as stimuli (Benevento and Port, 1995). $35 \%$ of the recorded neurons preferred certain color and pattern. Furthermore, 92\% 
showed increased responses when the monkey had to pay attention to the stimulus, and $58 \%$ of these cells also showed direction-specific saccade related activity during a visually guided saccade task. This suggests that both spatial (dorsal stream) and object related (ventral stream) information is processed in the same pulvinar neuron. Most interestingly, the response latency of color and pattern preferential cells was found to be around 80 milliseconds, which is well before most inferotemporal cells respond, suggesting that object related information is processed in the pulvinar before it is processed in cortex.

Visual or saccade related responses were found in almost all pulvinar neurons in freely behaving cats: $51 \%$ of the cells responded to only to a visual stimulus, $16 \%$ responded when the cat performed a saccade, and $31 \%$ responded to both visual stimuli and when a saccade was performed. Visual response fields were typically very large $\left(80^{\circ} \times 80^{\circ}\right)$, and visual neurons typically only responded to stimuli larger than $20^{\circ}$ (Sudkamp and Schmidt, 2000). However, the differences between feline and primate visual system make it hard to draw parallels to macaque and human pulvinar organization.

Comparing visual responses in LGN and pulvinar cells during a visual illusion task, it has been demonstrated that visually responsive LGN cells reflect the presence of a stimulus, while pulvinar activity was linked to the perceptual awareness of such stimuli, suggesting that pulvinar, but not LGN is involved in cognitive functions (Wilke et al., 2009).

A better understanding of the function of pulvinar can be gained by using causal interference methods. By injecting muscimol (a GABA agonist) or bicuculline (a GABA antagonist) unilaterally in a monkey dorsal pulvinar during performing a task with either congruent or incongruent spatial cue and target position, the reaction times changed significantly compared to non-inactivated trials (Petersen et al., 1987). While the monkeys were slower in reacting to targets contralateral to the injection site paired with ipsilateral cues after muscimol injection, they were faster in this task condition when bicuculline was injected. The opposite effects were found when the target was on the ipsilateral side and the cue on the contralateral side, further suggesting that the dorsomedial pulvinar plays an important role in shifting attention to the contralateral space.

When given a choice between two or more saccade or reach targets, unilateral pharmacological inactivation of the dorsal pulvinar caused an ipsilateral bias (Wilke et al., 2010). Noteworthy, the induced reaching deficits here were stronger for the hand contralateral to the injection site. However, the induced spatial bias could be compensated 
by increasing the desirability of the contralateral target (Wilke et al., 2013), suggesting a major role of dorsal pulvinar in spatial decision making rather than a purely perceptual role.

In a perceptual categorization task including an escape option ("opt out"), pulvinar activity was linked to confidence in the correct categorization, predicting the choice for categorization or opting out. Additionally, when the pulvinar was unilaterally inactivated, monkeys chose the escape option more often when the categorization stimuli were presented on the contralesional side (Komura et al., 2013).

To summarize, a lot of evidence indicates a major role of pulvinar in cognitive functions and intentional, goal-directed behavior, but due to the complexity of its anatomy, connectivity, and functional contribution, common agreement on specific functions has not been reached yet. For example, one might ask, which of the multiple connections are the most relevant. To this end, an ongoing work in our lab investigates the effects of pulvinar microstimulation on blood oxygen level dependent (BOLD) signals in monkeys performing spatial eye movement tasks in an MRI scanner. The most promising candidate so far seems to be the ipsilateral dorsal bank of the STS. This is further supported by another study focusing on effects of pulvinar inactivation during similar tasks, showing that BOLD responses in the STS decrease after inactivation, and that this decrease is mainly specific for contralesional saccade cues (Wilke, Kagan, Andersen in revision). 


\section{$1.4 \quad$ Research rationale}

The link between visual perception and goal-directed, intentional processing in the brain is yet not fully understood and the aim of many studies in current systems neuroscience. In the Introduction, I have illustrated the different spatial representations in the brain, the unanswered questions regarding visuospatial transformations, and what the occurrence of hemi-spatial neglect after unilateral brain lesions can tell us about these spatial representations. Furthermore, I described why illuminating the role of pulvinar in visuomotor behavior might be crucial in understanding how the brain uses visual information to derive desired movement plans.

Here, we provide new evidence of dorsal pulvinar involvement in goal-directed behavior, contributing to the ongoing discussion in the field about the role of pulvinar in visuomotor processing using a battery of different approaches: electrophysiology, microstimulation, pharmacological inactivation, and behavioral testing. Since the functional contribution and specificity of a particular brain region is better understood in comparison to other nodes of the interconnected circuitry, we also compared electrophysiological encoding and causal perturbation effects in two additional areas: ventral pulvinar (microstimulation) and cortical area TPO (electrophysiology and reversible inactivation)

In the first study (chapter 2) we aimed to get a deeper understanding of neuronal encoding in the dorsal pulvinar during basic oculomotor tasks, using multi-electrode electrophysiological recordings. Although there are several studies that indicate an involvement of pulvinar in the processing of visual information (Mathers and Rapisardi, 1973; Petersen et al., 1985a), generation of volitional movements (Cudeiro et al., 1989; Robinson et al., 1986), and visuomotor integration (Acuña et al., 1983; Benevento and Port, 1995), the concrete function of pulvinar in visuomotor processing remains unclear, especially regarding its lesser studied dorsal aspect. We show that visual responses are more pronounced, time-locked and space specific, but overall pulvinar exhibits more motor related, mainly post-saccadic activity. Surprisingly, and in contrast to fronto-parietal areas (Gnadt and Andersen, 1988; Rorie et al., 2010), delay period activity was found to be mostly suppressed and showed only little spatial preference for the upcoming saccade. We do not interpret this space specific delay period activity as motor preparatory, because delay period activity did not predict the upcoming movement when two saccade options were provided.

In the second study (chapter 3) we investigated gaze-dependent activity and reference frames in dorsal Pulvinar. Here we show that a vast majority of dorsal Pulvinar neurons are modulated by the gaze position, but this modulation was typically not simply monotonic, so it did not seem to reflect muscle tension or proprioceptive signals, but rather more complex integration of gaze and retinocentric visual information. Furthermore, we demonstrate the 
presence of different reference frames in dorsal Pulvinar, with a subset of pulvinar neurons exhibiting properties similar to gain fields, suggesting that pulvinar might be involved in visuomotor transformations.

In the third study (Dominguez-Vargas et al., 2017) - see Appendix - we investigated effects of pulvinar microstimulation on target selection in similar oculomotor tasks. This study was motivated by the finding that unilateral pulvinar inactivation biases target selection towards the ipsilesional side (Wilke et al., 2010, 2013), which was further modulated by the associated reward, suggesting that pulvinar processes desirability of potential contralateral movement goals. Systematic variation of stimulation times relative to the behavioral state revealed a more complex involvement of pulvinar in target selection as the effects of stimulation were space-specific, time-specific, task-specific, and specific to the stimulation site within pulvinar.

In the fourth study (chapter 3 ), we designed a novel task to dissociate sensory-perceptual and motor-intentional aspects of neglect-like deficits (Karnath and Rorden, 2012; Liu et al., 1992; Na et al., 1998), and compare the contribution of these aspects in neglect patients and monkeys with targeted unilateral lesions. It has been shown that spatial neglect can also occur after pulvinar lesions in humans (Arend et al., 2008a) and that pulvinar lesions in monkeys cause neglect-like deficits (Wilke et al., 2010). Although the effects of pulvinar inactivation in this task were small, they further support the idea that pulvinar is involved in motor-intentional processing, especially compared with effects after control inactivation of the temporal parietal occipital area in the same monkey. By adjusting the task difficulty for humans and monkeys, we achieved similar success rates in humans and in the monkey, but a comparison of other task parameters such as reaction times showed that the monkey was considerably faster, complicating the direct comparison of monkey and human performance. Unfortunately, we only had the opportunity to test one neglect patient with this task; the results of this test indicated that the task at its current stage might not be applicable to some human neglect patients.

In the fifth study (Wilke et al., 2018) - see Appendix - we had the opportunity to test saccadic, grasping and reaching deficits of a patient with a rare bilateral pulvinar lesion. Although it has been shown that pulvinar activity is related to arm movements (Acuña et al., 1990; Cudeiro et al., 1989) in monkeys and that unilateral pulvinar lesion causes reaching deficits specifically for the contralesional hand and space (Wilke et al., 2010), it was not clear how reaching and grasping would be affected in the case of bilateral lesions in humans. The results suggest that the patient exhibited mainly postural deficits, specifically when reaching with the right hand (contralateral to the more pronounced lesion), accompanied by general slowing of movements and grasping deficits. These findings further support the idea that 
pulvinar might be involved both in guiding action and more specifically in visuomotor transformations. 


\section{Visual, delay and oculomotor timing and tuning in macaque dorsal pulvinar during instructed and free choice saccades}

(This is a manuscript soon to be submitted for publication)

\section{Abstract}

Causal perturbation studies suggest that the primate dorsal pulvinar (dPul) plays a crucial role in eye and hand action selection and movement planning, but its underlying neuronal oculomotor properties are unclear. In particular, while many functional aspects of dPul and interconnected frontoparietal areas, such as ipsilesional saccade choice bias after inactivation, are similar, it is not known if dPul shares basic frontoparietal oculomotor properties. In particular, the delay period or choice related activity has not been explored. Here we investigated visuomotor timing and tuning in dPul in macaque monkeys during instructed and free choice memory saccades using multi-electrode recordings. Most units (80\%) showed significant visual (16\%), visuomotor (29\%) or motor (35\%) response patterns. Visual cue spiking responses were mainly contralaterally-tuned. Peri- and post-saccadic responses (both enhancement and suppression) were more common (64\%) than visual cuedriven responses (45\%). Pre-saccadic activity was however infrequent, and only few units exhibited classical visuomotor pattern with continuous delay period activity up to the saccade onset, or pre-saccadic ramping. Instead, activity was often suppressed during movement planning period and execution. Furthermore, even in spatially tuned neurons, spiking activity in free choice trials did not encode the upcoming decision during the delay. Prevalent postsaccadic responses imply that the dorsal pulvinar might have a role in the saccadic updating, in addition to prospective visuomotor processing, with patterns partially complementary to its frontoparietal cortical partners. 


\subsection{Introduction}

Visual information is crucial for guiding primate behavior and is represented in various cortical areas and subcortical structures. To gather this information, primates perform saccadic eye movements towards locations of interest to allow the fovea to obtain a better spatial resolution of these parts of the environment (Boi et al., 2017). Since eye movements are typically guided by visual inputs, it is not surprising that numerous cortical and subcortical regions show both visually-driven responses as well as eye movement-related activity. To study visuomotor transformations underlying conversion of visual inputs into oculomotor actions, a classical memory-guided saccade task that allows separating visual, intervening delay and motor-related activity has been used extensively (Hikosaka and Wurtz, 1983). Besides visually-evoked and motor-related responses, many neurons in oculomotor structures such as lateral intraparietal area (LIP), frontal eye fields (FFE), dorsal lateral prefrontal cortex (dIPFC) and mediodorsal thalamus (MD) show persistent delay activity that is considered a signature of cognitive signals such as working memory, motor preparation, sustained attention and evolving decisions (Barash et al., 1991a; Bruce and Goldberg, 1985; Funahashi et al., 1991; Lawrence et al., 2005; Watanabe and Funahashi, 2007).

Another challenge for the visual system is that during the normal saccade-fixation cycle, visual inputs enter the brain in eye-centered, retinotopic coordinates, but every saccade changes these representations, necessitating a mechanism maintaining visual stability (Wurtz et al., 2011a). It has been suggested that a corollary discharge (or efference copy) pathway involving superior colliculus (SC), MD and FEF is one important contributor (Wurtz et al., 2011a), enabling anticipation of movement consequences and saccade suppression; the remapping of receptive fields around saccades in FEF and LIP is a related phenomenon thought to support visual stability (Bisley and Goldberg, 2010; Mirpour and Bisley, 2015). In addition to these prospective mechanisms manifesting prior to or during saccades, postsaccadic responses might also contribute to saccadic suppression, as well as to saccade error processing (Zhou et al., 2016b).

Due to its widespread bidirectional connectivity to a host of visual and oculomotor cortical areas, the thalamic pulvinar is another likely candidate for mediating and coordinating across goal-directed eye movements (Berman and Wurtz, 2011; Grieve et al., 2000; Guillery and Sherman, 2002; Saalmann and Kastner, 2015; Wilke et al., 2010). Distinct pulvinar subdivisions might however contribute differently to these functions. Several studies have motivated a functional distinction between the ventral and the dorsal pulvinar aspects, based either on distinct patterns of connectivity (Kaas and Lyon, 2007; Stepniewska et al., 1994) or on distinct visual properties of neurons within the same anatomically-defined subdivision, e.g. leading to further division of the lateral pulvinar into dorsal and ventral parts (Baldwin et al., 
2017; Petersen et al., 1985b). The ventral pulvinar (vPul, inferior pulvinar and ventral part of lateral pulvinar) is retinotopically organized and has strong connections to visual cortices. Ventral pulvinar neurons respond to contralateral visual stimuli but show weak or no presaccadic activity - most eye movement-related responses are peri- or post-saccadic (Berman and Wurtz, 2011; Petersen et al., 1985a; Robinson et al., 1986). In particular, the inferior pulvinar has been identified as a part of pathway from superficial layers of SC to cortical area MT that carries saccadic suppression signals (Berman and Wurtz, 2011).

The dorsal pulvinar (dPul, medial pulvinar and dorsal part of lateral pulvinar) is reciprocally interconnected with 'associative' areas in posterior parietal and prefrontal cortices subserving attentional and sensorimotor functions, in particular LIP, area 7, FEF, dIPFC and posterior cingulate (PCC) (Bos and Benevento, 1975; Bridge et al., 2015; Dean et al., 2004; Gutierrez et al., 2000; Hardy and Lynch, 1992; Saalmann and Kastner, 2011). Unlike most of the ventral pulvinar, the dorsal pulvinar does not follow clear retinotopic organization (Benevento and Miller, 1981; Benevento and Port, 1995). In contrast to paucity of visuomotor neuronal studies in the dorsal pulvinar, more is known about consequences of its causal perturbation. While primary sensory and oculomotor functions are largely spared (Bender and Butter, 1987), its inactivation or microstimulation biases hemifield-specific spatial exploration and target selection, especially in conditions of a free choice (Dominguez-Vargas et al., 2017; Wilke et al., 2010, 2013). The inactivation of the lateral part of the dorsal pulvinar also impairs spatial attention (Petersen et al., 1987). It is not clear however whether the transformation from visual processing to motor actions is implemented already within the $\mathrm{dPul}$ or these transformations are only taking place in the interconnected cortical circuitry. The inactivation-induced free choice bias has been observed in "direct" visually-guided as well as in memory-guided saccade tasks (Wilke et al., 2010, 2013). Furthermore, it is not known if dPul neurons show delay period or pre-saccadic spatial choice selectivity, similar to frontoparietal cortex (Coe et al., 2002; Watanabe and Funahashi, 2007) and other higherorder thalamic nuclei such as central thalamus (Wyder et al., 2004) and MD (Watanabe and Funahashi, 2004).

Very few studies investigated visuomotor neuronal properties in the dorsal pulvinar, in particular the medial part. Robinson, Petersen and colleagues (Robinson et al., 1986) compared responses to visually-guided saccades in the ventral pulvinar and the lateral dorsal pulvinar (PLdm, denoted Pdm in the original studies), showing that a subset of neurons fires in association with eye movements, and is crudely tuned for saccade direction and amplitude. To our knowledge, only one study prior to our work investigated neurons in both medial and lateral parts of the dorsal pulvinar with the memory-guided saccade task (Benevento and Port, 1995). The main focus of this study was on color and pattern 
processing, but the comparison of visually-guided and memory-guided task responses again indicated that non-target-related activity was again post-saccadic. Surprisingly, in this sample visual target-related responses were present during visually-guided but not memory-guided guided saccades, and the delay activity was not addressed. The authors suggested that only when stimulus serves as an immediate saccade target the enhancement takes place, although it is typically not the case in the frontoparietal cortical areas.

In a previous electrical microstimulation study of the dorsal and ventral pulvinar (DominguezVargas et al., 2017), we provided initial assessment of electrophysiological recordings in $\mathrm{dPul}$ (predominately in the medial pulvinar) aimed at characterization of microstimulation sites. Most units (>80\%) were task responsive during oculomotor tasks, showing a mixture of predominately contralateral visual responses and weaker contralateral selectivity in the motor response epochs. Given the heterogeneity of observed patterns in dPul, and the lack of its comprehensive characterization in oculomotor tasks, here we further investigated its visuomotor properties in a classical memory-guided saccade task, dissociating visual responses, delay period activity, pre- and post-saccadic responses, and assessed timing and tuning of visual and motor related activity. Furthermore, we addressed the question how pulvinar activity might relate to visuomotor transformations during motor preparation by systematically analyzing the delay period activity. To explore involvement of dPul in spatial target selection, we assessed whether dPul neurons show a choice selectivity for the upcoming decision in free-choice two-target trials.

Our results demonstrate that most dorsal pulvinar neurons exhibit either enhancement or suppression at or after saccades, with or without concurrent visual responses, while the minority responded to visual stimulation only, further supporting the notion that the dorsal pulvinar might be involved in visuomotor integration. However, delay period activity was mainly characterized by a gradual suppression of firing relative to the initial fixation period, with only a small subset showing classical sustained or ramping up enhancement of activity that is frequently observed in frontoparietal areas such as LIP and FEF. On the population level, no spatial choice selectivity for the upcoming saccade was found, neither in the delay nor in pre-saccadic activity. 


\subsection{Materials and Methods}

All experimental procedures were conducted in accordance with the European Directive 2010/63/EU, the corresponding German law governing animal welfare, and German Primate Center institutional guidelines. The procedures were approved by the responsible government agency (LAVES, Oldenburg, Germany).

\subsubsection{Animal preparation}

Two adult male rhesus monkeys (Macaca mulatta) C and L weighing 8 and $9 \mathrm{~kg}$ respectively, were used. In an initial surgery monkeys were implanted with a magnetic resonance imaging (MRI) compatible polyetheretherketone (PEEK) headpost embedded in a bone cement headcap (Palacos with Gentamicin, BioMet, USA) anchored by ceramic screws (Rogue Research, Canada), under general anesthesia and aseptic conditions. MR-visible markers were embedded in the headcap to aid the planning of the chamber in stereotaxic space with the MR-guided stereotaxic navigation software Planner (Ohayon and Tsao, 2012). A separate surgery was performed to implant a PEEK MRI-compatible chamber(s) (inside diameter $22 \mathrm{~mm}$ ) allowing access to the pulvinar (Monkey $\mathrm{C}$, right hemisphere: center at $0.5 \mathrm{~A} / 14.5 \mathrm{~L} \mathrm{~mm}$, tilted -11P / 27L degrees; Monkey L, right hemisphere: center at $-3.12 \mathrm{P}$ / 20.2L mm, tilted: -18P/37L degrees; Monkey L, left hemisphere: center at $-3 \mathrm{P} / 20 \mathrm{~L}$, tilted: 18P/-38L). After confirming chamber positioning with a post-surgical MRI, a partial craniotomy was made inside the chamber. The exposed dura was covered with a silicone elastomer (Kwik-sil, World Precision Instruments, USA) to reduce the granulation tissue growth and dura thickening.

\subsubsection{MRI imaging}

Monkeys were scanned in a 3T MRI scanner (Siemens Magnetom TIM Trio). Full-head T1weighted scans (3D magnetization-prepared rapid gradient-echo, MPRAGE, $0.5 \mathrm{~mm}$ isometric) were acquired before and after chamber implantation, in awake (monkey $C$ ) or anaesthetized (monkey L) state, using either built-in gradient body transmit coil and custom single loop receive coil, or custom single loop transmit and 4-channel receive coil (Windmiller Kolster Scientific, USA).

In addition to pre- and post-implantation scans, similar T1-weighted scans as well as T2weighted (rapid acquisition with relaxation enhancement, RARE, $0.25 \mathrm{~mm}$ in plane, $1 \mathrm{~mm}$ slice thickness) scans were periodically acquired during the course of experiments, either in awake (monkey $\mathrm{C}$ ) or sedated (monkey $\mathrm{L}$ ) state, to confirm electrode positioning. T1- and T2-weighted scans were co-registered and transformed into chamber normal (aligned to the chamber vertical axis) and to anterior commissure - posterior commissure (AC-PC) space for electrode targeting and visualization. These images were acquired with the chamber and the 
grid filled with gadolinium (Magnevist, Bayer, Germany)/saline solution (proportion 1:200), with tungsten rods inserted in predefined grid holes, for alignment purposes.

\subsubsection{Behavioral tasks}

Monkeys sat in a dark room in custom-made primate chairs with their heads restrained 30 cm away from a 27 " LED display (60 Hz refresh rate, model HN274H, Acer Inc. USA). The gaze position of the right eye was monitored at $220 \mathrm{~Hz}$ using an MCU02 ViewPoint infrared eyetracker (Arrington Research Inc. USA). All stimulus presentation and behavioral control tasks were programmed in MATLAB (The MathWorks, Inc. USA) and the Psychophysics Toolbox (Brainard, 1997).

\subsubsection{Memory-guided saccade task}

A trial started with the onset of the fixation spot of $1^{\circ}$ diameter. After the monkey acquired and held fixation within a $5^{\circ}$ radius for $500 \mathrm{~ms}$, and either one peripheral cue (instructed trials) or two peripheral cues (choice trials) were displayed for $300 \mathrm{~ms}$ at the location(s) signaling the upcoming saccade target(s). Cues (also $1^{\circ}$ diameter) were presented in the left and/or right side(s) of the fixation spot, at $12^{\circ}$ or $24^{\circ}$ eccentricity, with three potential angles relative to the horizontal axis, either $0^{\circ}, 20^{\circ}$ or $-20^{\circ}\left(0^{\circ}, 4.1^{\circ},-4.1^{\circ}, 8.2^{\circ}\right.$ and $-8.2^{\circ}$ vertical eccentricity). Monkeys were required to maintain fixation throughout the cue period and also throughout the subsequent memory period (1000 ms), after which the central fixation spot disappeared, allowing monkeys to saccade to the instructed location, or make a decision to go to one of the two cued locations. This time point will be referred to as the Go signal. After a saccade to and fixation inside a $5^{\circ}$ radius window around the remembered target location for $100 \mathrm{~ms}$ to $200 \mathrm{~ms}$ the target became visible, and after additional $500 \mathrm{~ms}$ of peripheral fixation the trial was completed and the monkey received a liquid reward after a delay of 200 ms. Instructed trials were randomly interleaved with choice trials (50\%). In choice trials the monkeys were allowed to freely choose one of the two targets that were always presented simultaneously at the same height and provided equal reward. The inter-trial interval for both successful and unsuccessful trials was 2000 or 2500 ms (Figure 2.1A). All trial types and target locations were pseudo-randomized.

\subsubsection{Visually-guided saccade task}

In addition to the memory-guided saccade task, monkeys also performed visually-guided saccades in separate blocks in most of the sessions. In this task, after the initial fixation period the fixation spot disappeared and the visual target(s) appeared at the same time, serving as the Go signal. Monkeys had to saccade towards the visible peripheral target and keep fixating it for 500 ms to obtain a liquid reward. Target sizes, colors and locations were the same as for memory-guided saccades and all trial types and target locations were pseudo-randomized. 


\subsubsection{Data analysis}

\subsubsection{Saccade definition}

Saccade velocity was calculated sample by sample as the square root of the sum of squared interpolated (220 Hz to $1 \mathrm{kHz}$ ) and smoothed (12 ms moving average rectangular window) horizontal and vertical eye position traces, and then smoothed again (12 ms moving average rectangular window). Saccade onset was defined as eye position change that exceeded a starting velocity threshold of $300 \%$ s. Reaction time was defined as the time between the "Go" signal and the onset of the saccade.

\subsubsection{Datasets and unit selection criteria}

All recorded voltage drops that surpassed certain online defined threshold were defined as potential spikes. Spike sorting was done in Offline Sorter v.3.3.5 (Plexon, USA), using a waveform template algorithm after defining templates by manually clustering in $2 \mathrm{D}$ or $3 \mathrm{D}$ principle component space.

In total, 416 single and multi-units were recorded in the dorsal pulvinar in 50 sessions during the memory-guided saccade task (monkey C; right hemisphere: 235, monkey L; right hemisphere: 123; left hemisphere: 58). Out of these, 371 units (200 monkey C right, 115 monkey $L$ right, 56 monkey $L$ left) fulfilled analysis selection criteria (stable discriminability across time and reasonable SNR - assessed by inspection). Out of these 371 units, 322 units (174 monkey C right, 98 monkey L right, 50 monkey L left) were recorded for at least 4 successful instructed trials for each of the 12 targets. These 322 units were used for further analysis.

Out of these 322 units, 195 units (monkey C; right hemisphere: 101, monkey L; right hemisphere: 54; left hemisphere: 40) were also recorded during the visually-guided saccade task. 174 out of these 195 units (monkey C; right hemisphere: 88, monkey L; right hemisphere: 46; left hemisphere: 40) were recorded for at least 4 successful instructed trials for each of the 12 targets. These 174 units were used for comparison between the two tasks.

\subsubsection{Epoch definitions and modulation}

For each trial, and each epoch of interest, firing rates were computed by counting spikes within the epoch and dividing by the epoch duration. The following epochs were analyzed: inter-trial interval (400 ms to $100 \mathrm{~ms}$ before the onset of the central fixation spot, corresponding to the fixation acquire), fixation hold (last $300 \mathrm{~ms}$ of central fixation), cue onset (70 ms to $170 \mathrm{~ms}$ after cue onset), memory (last $300 \mathrm{~ms}$ of the memory period),pre-saccadic (100 to $10 \mathrm{~ms}$ before saccade onset), peri-saccadic (10 ms before to $50 \mathrm{~ms}$ after saccade onset), post-saccadic (first $150 \mathrm{~ms}$ after acquiring the invisible peripheral target), target onset 
(20 ms to $120 \mathrm{~ms}$ after the target became visible), and target hold (last $300 \mathrm{~ms}$ of fixating the peripheral target).

The cue onset epoch was defined according to findings in the response timing analysis as 50 ms before to $50 \mathrm{~ms}$ after enhancement was most common, see Figure 2.2D. We did not base our post-saccadic epoch on this analysis, for two reasons. First, the onset of postsaccadic modulation was not as synchronized across units as the cue response, see Figure 2.2F. Second, in some sessions the target became visible already $100 \mathrm{~ms}$ after saccade offset, which could lead to confounding visual responses, giving only short interval for a purely post-saccadic epoch. Instead, we defined the post-saccadic epoch simply as the first $150 \mathrm{~ms}$ after saccade offset. In those cases when the target appeared already $100 \mathrm{~ms}$ after the saccade offset, this epoch would not be contaminated by visual response because visual latency of most pulvinar neurons was $>50 \mathrm{~ms}$.

To allow comparison of firing rates in the cue onset/pre-saccadic epoch (40-140 ms after target onset/Go signal) of the visually-guided saccade task to firing rates in the memoryguided saccade task, two additional corresponding epochs were defined for the memoryguided task: cue onset 2 (40-140 ms after cue onset) and pre-saccadic 2 (40-140 ms after the Go signal, the offset of the fixation spot).

For most analyses, data from 6 left and 6 right hemifield targets were combined. For each unit, spatial tuning in each epoch was determined by unpaired t-tests comparing firing rates in ipsilateral trials to firing rates in contralateral trials. The hemifield with the higher firing rate was marked, if there was a significant difference. Enhancement or suppression of neuronal activity in each epoch was defined by paired t-tests comparing firing rates to a respective preceding baseline epoch, independently for ipsilateral and contralateral trials. For fixation hold epoch inter-trial interval served as baseline, for cue onset and memory epochs (as well as cue onset 2 and pre-saccadic 2 for visually-guided task comparison), the fixation hold epoch served as baseline. The memory epoch served as baseline for all subsequent epochs. Enhancement or suppression was reported, if either ipsilateral, contralateral, or both types of trials showed significant difference to fixation baseline. In rare cases where one hemifield would show a significant enhancement, while the other hemifield showed suppression, the unit was reported to have bidirectional response.

Units that showed enhancement or suppression for at least one hemifield in both cue onset and post-saccadic epochs were classified as visuomotor units, whereas units that only showed enhancement or suppression in one of the two epochs were classified as visual (only enhancement or suppression in cue onset) and motor (only enhancement or suppression in post-saccadic). Visuomotor index was defined independently for ipsilateral and contralateral 
trials as $(\mathrm{M}-\mathrm{V}) /(\mathrm{M}+\mathrm{V})$, where $\mathrm{M}$ is the absolute average firing rate change in post-saccadic relative to the memory epoch, and $\mathrm{V}$ is the absolute average firing rate change in cue onset relative to fixation hold (Lawrence et al., 2005).

In addition to the visuomotor index described above, we also used visuomotor categories derived using enhancement or suppression in cue onset and pre-saccadic (as opposed to post-saccadic) epochs relative to fixation hold. This was done to compare the results to previously used visuomotor categorizations (Bruce and Goldberg, 1985; Wyder et al., 2003).

To evaluate how many neurons exhibited task-related modulation, for each unit we applied a two-way ANOVA [hemifield $\times$ epoch] on firing rates across all successful trials, using hemifield of the target position and epoch as factors for determining a main effect of epoch, a main effect of the hemifield, and interaction between the two factors.

\subsubsection{Response modulation onset and tuning onset analysis}

To evaluate the time of response modulation onset for each unit and further define the cue onset epoch, spike density functions for each trial were derived by convolution of the discrete spike arrival times with a Gaussian kernel (standard deviation $20 \mathrm{~ms}$ ). For three alignments (to cue onset, saccade onset and target onset), spike densities for each unit and each bin was compared to the average firing rate in the respective baseline epoch (fixation hold for cue alignment and memory for saccade and target alignment) using paired t-tests across all trials to evaluate bin-by-bin modulation. Significant increases were reported as enhancement and significant decreases as suppression. Similarly, for each unit and each bin, the spike densities of contralateral trials and ipsilateral trials were compared using unpaired t-tests. Significantly higher spike density for contralateral trials was reported as contralateral preference and significantly higher spike density for ipsilateral trials as ipsilateral preference. To avoid overestimation of significance due to multi-comparison, significance in less than five consecutive bins was discarded. For each unit and each type of modulation, the modulation onset was taken as the first bin (out of at least 5 consecutive bins) after certain time point that showed significance. For modulation onset relative to cue onset, the first significant bin after cue onset was taken and for modulation onset relative to the saccade the first significant bin after 200 ms before saccade onset was taken. Additionally, we counted the number of units which showed significant modulation in each bin and calculated the time in which most units were modulated. Based on the finding that most units showed enhancement 120 ms after cue onset (Figure 2.2D), the cue onset epoch was selected as 50 $\mathrm{ms}$ before to $50 \mathrm{~ms}$ after that maximum. 


\subsubsection{Response fields}

To estimate the center and the extent of visual and post-saccadic response fields (RFs) for each unit, we fitted 2D Gaussian distributions on the response strengths in cue onset (Cue) and post-saccadic (Post) epoch in all trials. Response strengths were calculated relative to the respective baseline, by subtracting firing rates in fixation hold (Fhol) for visual cue responses and firing rates in the memory epoch (Mem) for post-saccadic responses. This analysis was only performed on units that showed a main effect of target position on the firing rate in the respective epoch, using a one-way ANOVA. The fitting of RFs was performed in two steps: First, a bimodal RF with two zones was fitted using a positive and a negative Gaussians, not allowing overlap of the two zones. If one of the two Gaussians did not significantly contribute to the explained variance (see below), a unimodal RF using a single Gaussian, allowing either positive or negative modulation, was fitted instead. If the unimodal RF did not significantly explain variance either, no RF was reported.

Six fitting parameters for each Gaussian response zone were determined using an iterative least squares method (400 iterations), allowing elliptic response zones with peaks (or troughs) at the center. The fitting parameters were (1) the response strength in the center of zone, (2) horizontal and (3) vertical location of the center of the response zone, (4 and 5) two standard deviations describing semi-minor and semi-major axes, and (6) an angle of rotation of these axes. Importantly, the response zone centers were always kept within the dimensions of the target array ( -24 to +24 degrees horizontally and ( -8.2 to +8.2 degrees vertically). The response strength was bounded by $-150 \%$ and $150 \%$ of the original maximum response strength, standard deviations were bounded by $1.5^{\circ}$ (a quarter of the maximum horizontal target distance) and $12^{\circ}$ (a quarter of the horizontal extend of the target array), and the rotation by $\pm 90^{\circ}$.

For bimodal response field fitting, response zone centers at $\pm 18^{\circ} / 0^{\circ}$, amplitudes of 0 , and intermediate standard deviations $\left(7.5^{\circ}\right)$ with a rotation of $0^{\circ}$ were used as starting values. For unimodal response fields, the starting value for the RF zone center was derived by weighing target positions by the respective response strengths and averaging across target positions.

The size of each elliptic zone was defined by two standard deviations in each direction (semiminor and semi-major axes). A radius $r$ approximating response zone size was calculated by taking the square root of the product of the two axes of the elliptic zones. This way, $r^{2 *} \square$ always matches with the area covered by the elliptic response zone. Response zone size is reported as diameter, $2^{*}$ r. For units with one response zone, response field size was defined equal to response zone size. For units with two response zones, we averaged response zone sizes to approximate response field size. 


\subsubsection{Peri-stimulus time histograms (PSTHs)}

To calculate population PSTHs, spike density functions of each trial were either baselinecorrected by subtracting the average ongoing firing rate in the inter-trial-interval epoch or normalized by dividing by the average firing rate (across all trials) in the fixation hold epoch. Average responses for each unit were then derived by averaging the normalized spike density for each unit across all trials for the respective condition. Means and SE of these baseline-corrected and averaged spike densities across units of a given sub-population were calculated to display population responses.

\subsubsection{Choice selectivity and instructed to choice preference}

Over all target selection was reported as the mean (left or right) target selection across sessions. To test for a target selection bias, we performed unpaired t-tests comparing averages per session. To assess if the decision to select one target or the other could be made at the very last moment, we compared saccade reaction times in instructed and choice trials. To this end, we computed mean reaction times for each spatial condition (instructed left, instructed right, choice left and choice right) per session, and compared instructed and choice session means independently for left and right saccades using unpaired t-tests. To see if information about the upcoming target selection could be derived from spiking activity, we correlated instructed and choice firing differences between contralateral and ipsilateral trials using Pearson's correlation. For this analysis, units were considered only if at least four choice trials to each hemifield were recorded. 


\section{$2.3 \quad$ Results}

\subsubsection{Response timing and tuning in memory-guided saccade task}

We recorded single- and multi-unit activity in two monkeys performing a memory-guided saccade task. Trials always started with initial fixation in the center of the screen followed by a cue or two cues, memory delay and saccade to one out of 12 peripheral instructed or chosen locations (Figure 2.1A and Materials and Methods).

A
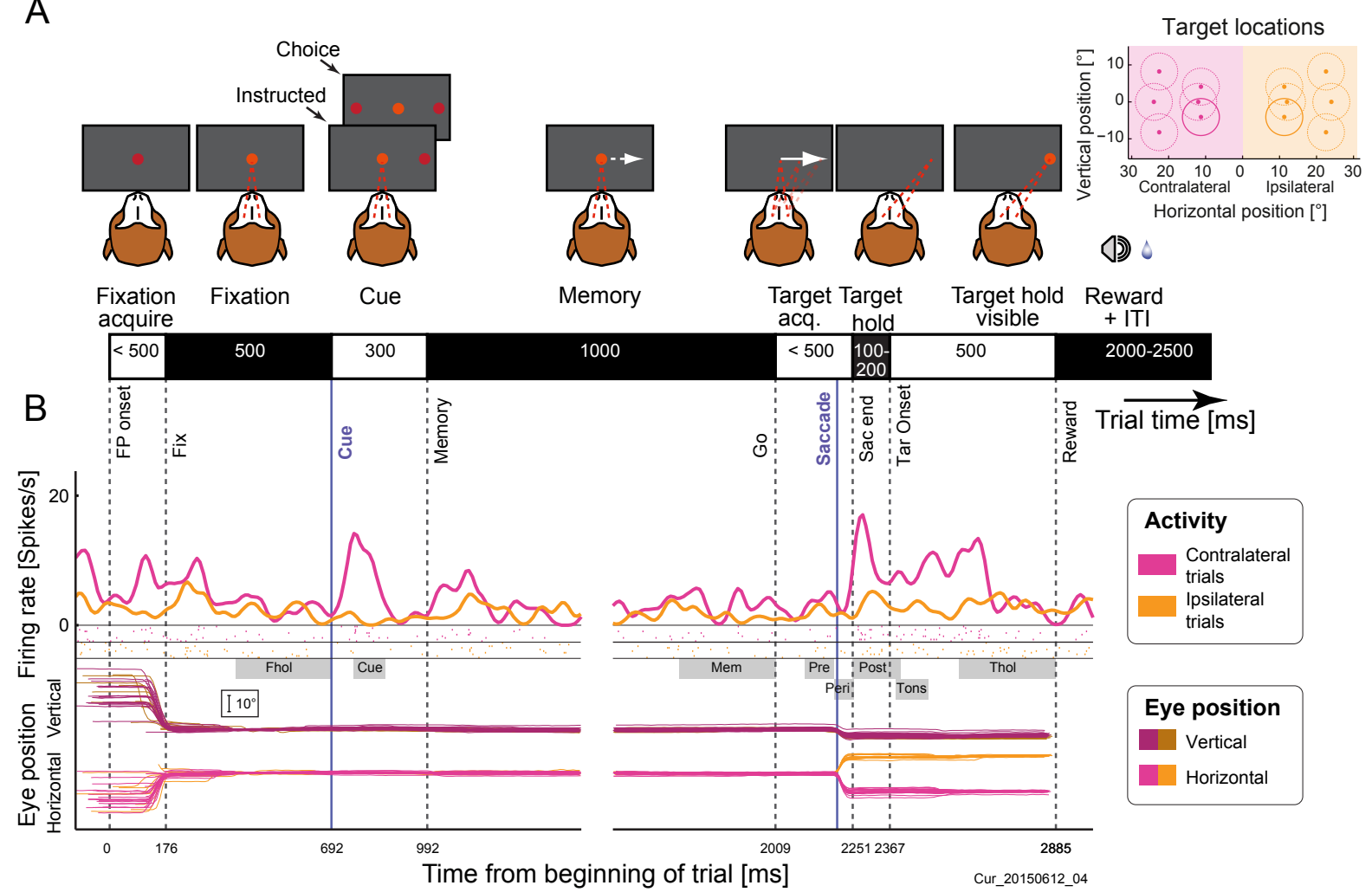

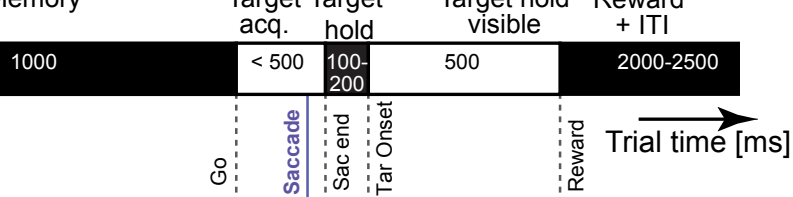

Figure 2.1. Memory-guided saccade task and example neuron activity. A) Task layout. Monkeys had to fixate in the center while one or two cues were flashed peripherally. After a $1 \mathrm{~s}$ memory period monkeys had make a saccade in the dark to (one of the) previously cued location(s), and fixate that location until and after the target became visible in order to receive reward. In instructed trials, one target was available; in choice trials two targets were available. The two targets in choice trials were always presented at the same height and eccentricity and provided equal reward. B) Raster plots and resulting spike density functions for two example positions (indicated by continuous lines on the insert showing the potential target locations), a contralateral (magenta) and the opposite ipsilateral (orange) position. Corresponding eye traces are shown below (darker lines for vertical eye position, brighter lines for horizontal eye position). Vertical lines indicate average onset of events across all trials for this recording block (including other target positions): fixation spot onset (FP onset), the monkey acquiring fixation (Fix), the cue onset, the cue offset and beginning of the memory period, the offset of the central fixation point (Go), the saccade onset, the monkey acquiring the invisible target location (Sac end), the onset of the visible peripheral target (Tar onset), and the end of the trial (Reward). Discontinuous traces indicate two different alignments to cue onset and saccade onset events (purple lines). Grey boxes mark relevant analyzed epochs: fixation hold (Fhol), cue onset (Cue), memory (Mem), pre-saccadic (Pre), peri-saccadic (Peri), post-saccadic (Post), Target onset (Tons), and target hold (Thol) (see Materials and Methods).

The activity of a representative example unit and the eye traces for one target position are shown in Figure 2.1B. Note that this unit shows an enhanced visual response after the cue presentation specifically for the contralateral position as well as a contralateral post-saccadic 
response which cannot be attributed to a target-related visual response, because it occurs before the onset of the visual target (Tar onset).

To evaluate how population activity of all 332 units selected for the analysis developed over time (and to validate the timing of analysis epochs), we estimated significant enhancement or suppression (combined across both hemifields) as well as hemifield preference for each unit in each $10 \mathrm{~ms}$ bin (Figure 2.2, see also Materials and Methods). In Figure 2.2A units are ordered by the onset of significant modulation relative to cue onset. The fixation hold epoch before cue onset was used as baseline for calculating enhancement and suppression (Figure 2.2A, top). This analysis showed a time-locked enhancement shortly after the cue onset and a predominance of suppression during the memory period (Figure 2.2A, top) combined with contralateral cue preference shortly after the cue onset and an overall lack of spatial preference during the memory epoch (Figure 2.2A, bottom). In Figure 2.2B units are ordered by the onset of significant modulation relative to saccade onset. Because of the frequent differences in firing rate between initial fixation and memory periods, we used the memory epoch as baseline for calculating enhancement and suppression around the saccade (Figure 2.2B, top). Around the saccade we observed predominantly post-saccadic enhancement (Figure 2.2B, top) and a bias to contralateral hemifield (Figure 2.2B, bottom).

A more detailed picture of the typical cue responses can be obtained by looking at the distribution of response modulation and spatial tuning onsets relative to cue (Figure 2.2C), and the number of units which showed respective property for each $10 \mathrm{~ms}$ bin after cue onset (Figure 2.2D). While onset of enhancement and contralateral preference typically occurred at around $50 \mathrm{~ms}$ after cue onset, the timing of suppression and ipsilateral preference was less systematic (Figure 2.2C,D cf. blue vs. red and orange vs. magenta). Enhancement (independent of spatial preference) relative to the cue onset was most common at $120 \mathrm{~ms}$ after cue onset which was very close to the time point where the maximum number of units showed contralateral preference (110 ms after cue onset). The same analysis for modulation relative to saccade onset (Figure 2.2E-F) showed that presaccadic and peri-saccadic modulation was rare compared to post-saccadic modulation, and that enhancement and contralateral preference were predominant in the post-saccadic period, peaking at $220 \mathrm{~ms}$ after saccade onset (or $170 \mathrm{~ms}$ after saccade end). 
A Response modulation (top) and tuning onset (bottom) per unit, relative to cue onset

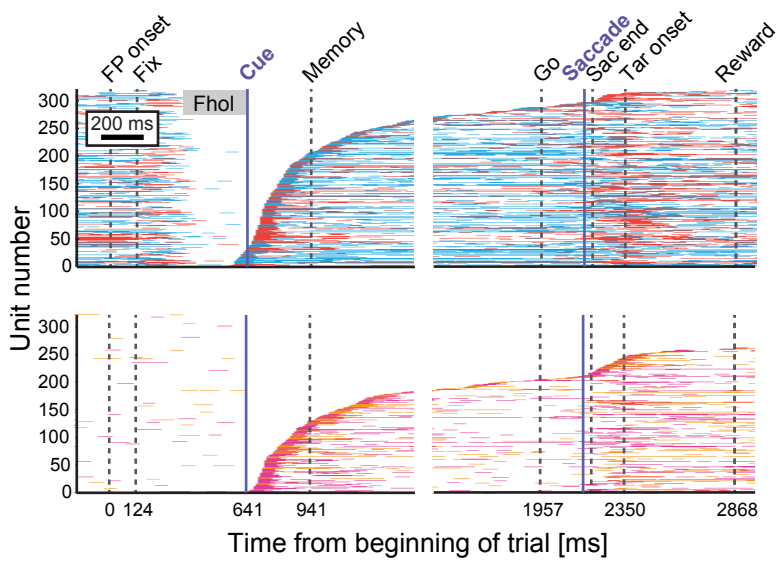

B Response modulation (top) and tuning onset (bottom) per unit, relative to saccade onset

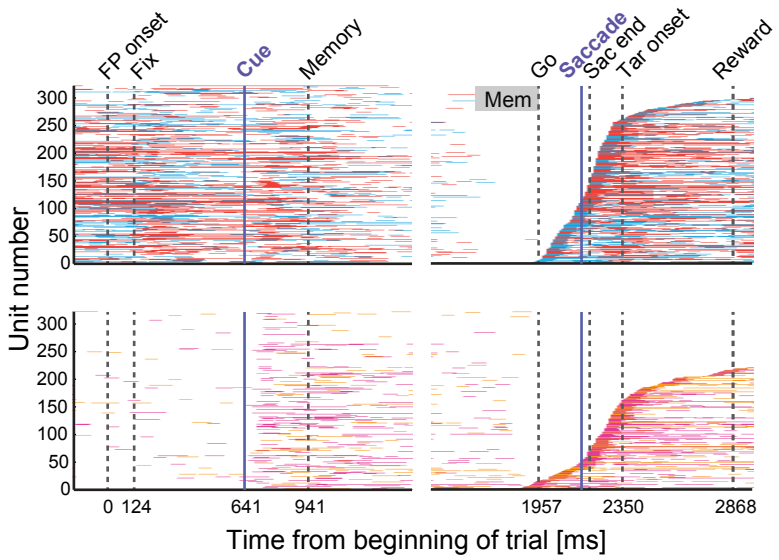

C Modulation onset

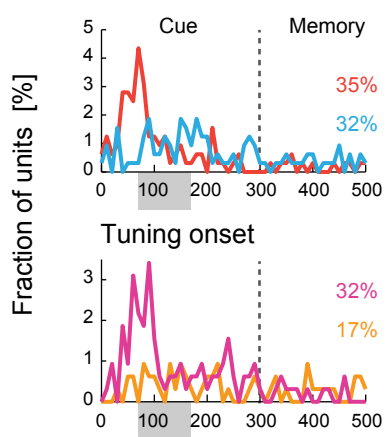

Time relative to cue onset [ms]

E

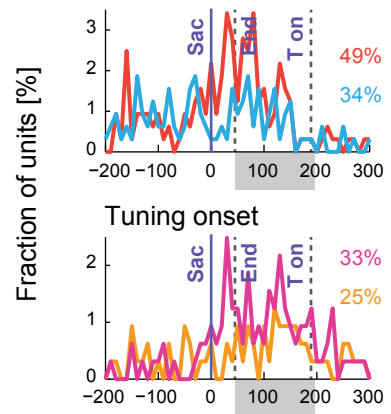

Time relative to saccade onset $[\mathrm{ms}]$
D Modulated units per bin

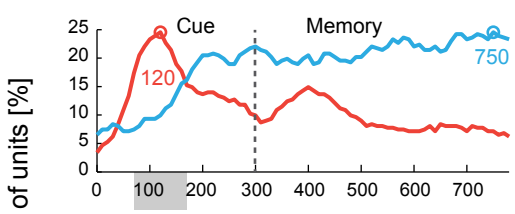

음

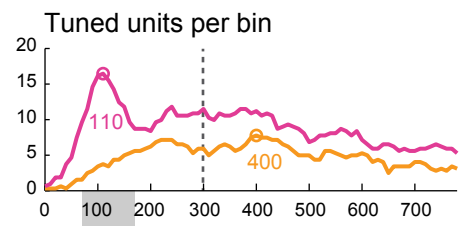

Time relative to cue onset [ms]

F $\quad$ Modulated units per bin

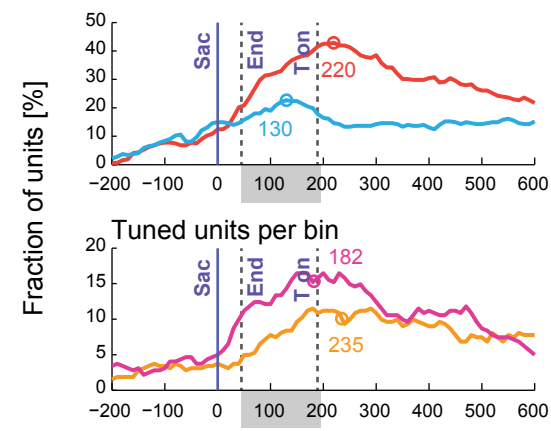

Time relative to saccade onset [ms]

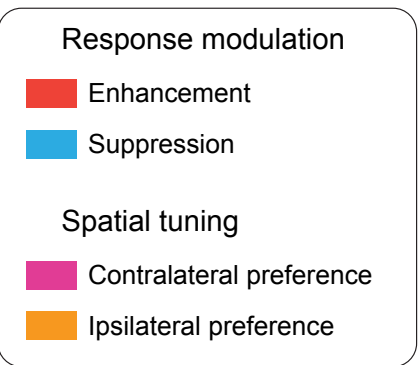

G Modulated units per bin

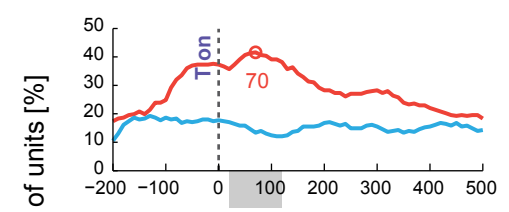

드 Tuned units per bin

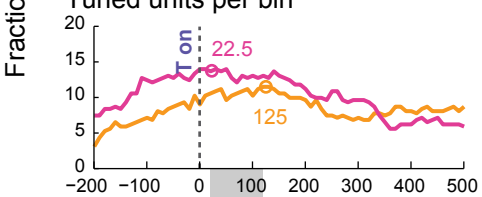

Time relative to target onset [ms]

Figure 2.2. Response modulation and spatial tuning onsets. Bin-by-bin significance of modulation for all 332 units, in 10 ms bins. A) Non-spatial specific response modulation (Top) and hemifield preference (Bottom) across time for all units recorded, ordered by modulation onset time relative to cue onset. Each horizontal line represents one unit. Non-spatial specific modulation is shown as enhancement (red) or suppression (blue) relative to the baseline epoch fixation hold (Fhol), indicated by a gray box. Hemifield preference is shown as contralateral preference (magenta) or ipsilateral preference (orange). B) Same as A, ordered by modulation onset relative to saccade onset. Here, the memory epoch (Mem) was used as the baseline epoch for non-spatial specific modulation. C) Fraction of units showing modulation and tuning onset for each $10 \mathrm{~ms}$ bin relative to cue onset. Numbers indicate the sum across all bins in the displayed time range. The grey box denotes the cue onset epoch, the dotted line denotes the cue offset. D) Fraction of units showing modulation and tuning for each $10 \mathrm{~ms}$ bin, relative to cue onset. Colored circles and numbers show the time points corresponding to the maximum number of modulated or tuned units. In case there were several maxima with the same amount of units, the respective time points were averaged. E) Same as C, relative to saccade onset. The grey box denotes the postsaccadic epoch. Vertical lines show the saccade onset (purple), the saccade end and the onset of the visible target (dotted lines). F) Same as D, relative to saccade onset. G) Same as D, relative to target onset. The grey box denotes the target onset epoch. 
Many units showed enhancement and space preference well before the target onset (Figure 2.2G), further confirming that post-saccadic responses are mainly not due to direct visual stimulation. Additionally (not shown in the plot), out of 34 units $(11 \%)$ with a significant hemifield preference both for cue and post-saccadic responses, only 11 units (3\%) preferred the opposing hemifields in the two epochs, while the majority of 23 units (7\%) showed the same hemifield preference in both epochs. This finding rules out the possibility that the postsaccadic response in those units is driven by increased luminance flux from the monitor background backlighting when the peripheral RF is shifted by the saccade from the space beyond the edge of the monitor (covering $90^{\circ}$ visual angle in the horizontal dimension) onto the monitor.

Interestingly, the fraction of units that showed enhancement after the target onset reached the maximum at $70 \mathrm{~ms}$ (Figure 2G), earlier then for cue responses (120 ms). This might indicate a difference in timing of visual responses for peripheral (cue) and foveal (target) stimuli. The post-saccadic peak is however wide and might result from confluence of postsaccadic and visual target onset-related signals.

A Response modulation

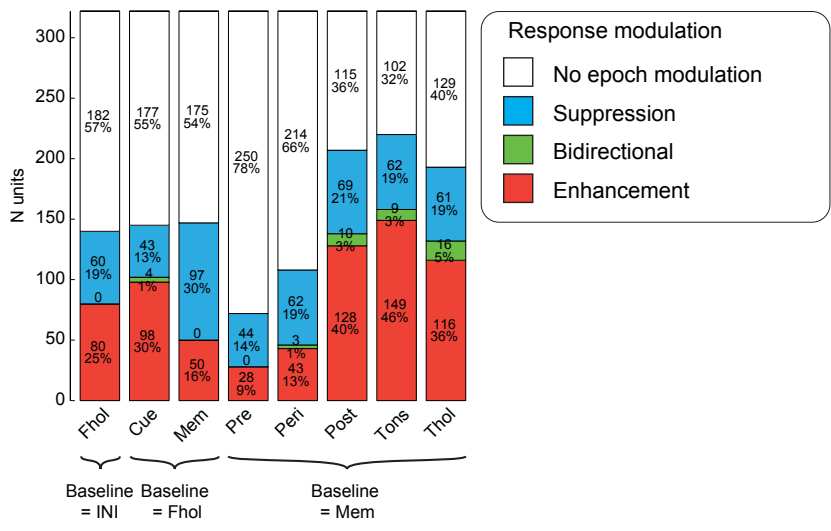

B Spatial tuning

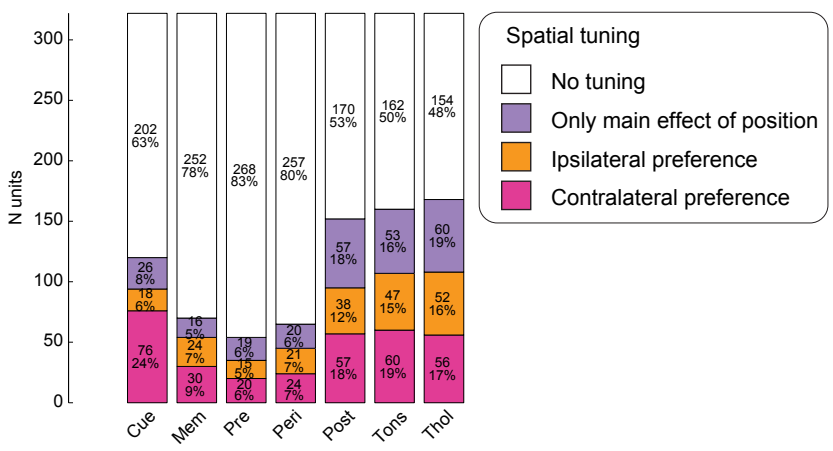

Figure 2.3. Spatial tuning and response modulation per epoch. Spatial tuning and response modulation of all 322 units recorded during the memory-guided saccade task. The analyzed epochs are fixation hold (Fhol), cue onset (Cue), memory (Mem), pre-saccadic (Pre), Peri-saccadic (Peri), Post-saccadic (Post), target onset (Tons), and target hold (Thol). A) Total number of units (and percentages) showing response modulation relative to the respective baseline, for each analyzed epochs: no enhancement nor suppression (white), only suppression (blue), bidirectional - enhancement for one hemifield and suppression for the other (green), only enhancement (red). B) Number of units that, in the respective epoch, were not tuned (white), did not prefer either hemifield but showed a main effect of position in a one-way ANOVA (purple), preferred ipsilateral hemifield (orange), preferred contralateral hemifield (magenta). 
To summarize, the predominant response patterns included contralateral cue enhancement (clear peak at $120 \mathrm{~ms}$ after the cue onset), suppression throughout the entire memory period, post-saccadic enhancement (wide peak at $220 \mathrm{~ms}$ ) and peri/post-saccadic suppression (wide peak at $130 \mathrm{~ms}$ ), often accompanied by post-saccadic hemifield preference (more contralateral than ipsilateral).

Figure 2.3 shows the summary of response modulation and hemifield tuning in all taskrelevant epochs in instructed trials. For each unit and analysis epoch, we evaluated significant enhancement or suppression (now per hemifield, as compared to across hemifields in the preceding response modulation analysis, Figure 2.2), and hemifield preference. If there was no significant hemifield preference, we also tested one-way ANOVA across 12 target positions. Majority of units (89\%) showed task-related modulation in at least one of the analyzed epochs. Contralateral hemifield preference was more common than ipsilateral preference in the cue onset epoch (20\% vs $5 \%)$. While the number of units preferring contralateral or ipsilateral hemifield was almost equalized in the subsequent epochs, hemifield preference was in general rare in memory (15\%), pre-saccadic (10\%) and peri-saccadic epoch (13\%) as compared to post-saccadic (23\%), target onset (28\%) and target hold (27\%) epochs. Suppression was more common than enhancement during the memory period ( $29 \%$ vs $14 \%$ ) as well as before and during the saccade ( $13 \%$ vs $8 \%$ in presaccadic and $19 \%$ vs $13 \%$ in peri-saccadic), while after the saccade, activity was typically enhanced (38\% enhancement vs $20 \%$ suppression in post-saccadic, $45 \%$ vs $17 \%$ in target onset and $34 \%$ vs $17 \%$ in target hold).

To compare visual and post-saccadic response fields, we computed Gaussian response field zones for both epochs and each unit that showed a main effect of target position in the respective epoch ( $\mathrm{N}=75$ for visual and $\mathrm{N}=125$ post-saccadic responses, Figure 2.4A), allowing one enhanced and one suppressed zone for each unit (Materials and Methods). Figure 2.4B shows a summary of response field zones for both epochs. While visual response fields were dominated by contralateral enhancement $(\mathrm{N}=51,68 \%)$, the centers of post-saccadic response field zones were distributed over both hemifields and the ratio of units showing unimodal suppression and enhancement zones $(\mathrm{N}=25,20 \%$ and $\mathrm{N}=74,59 \%$ respectively) was more balanced compared to visual response fields ( $N=10,13 \%$ and $N=61$, $81 \%)$. The sizes of visual and post-saccadic response fields varied across units, but were equally large $\left(26^{\circ} \pm 11^{\circ}\right.$ and $26^{\circ} \pm 12^{\circ}$ respectively) on average ( $p=0.398$, unpaired t-test). These findings are in line with the quantification of response modulation per hemifield and hemifield preference described earlier. 


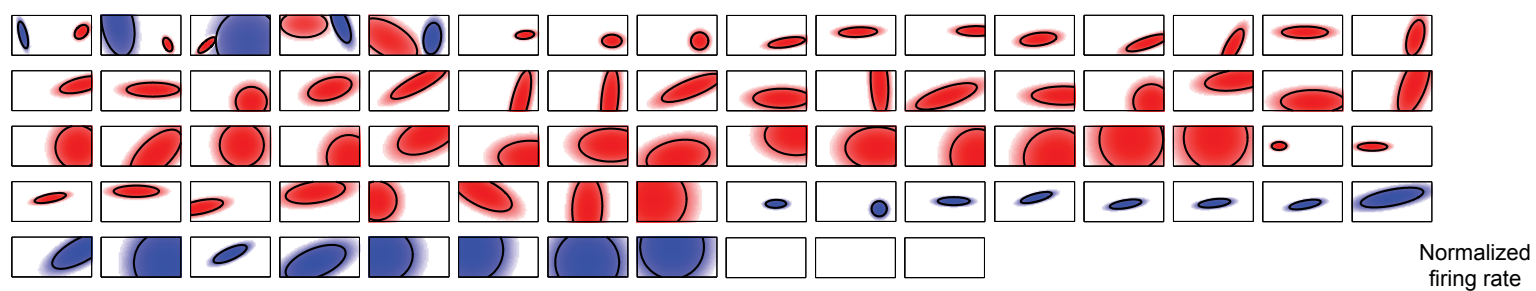

Post-saccadic response fields (Baseline $=$ MemL, $\mathrm{N}=125)$

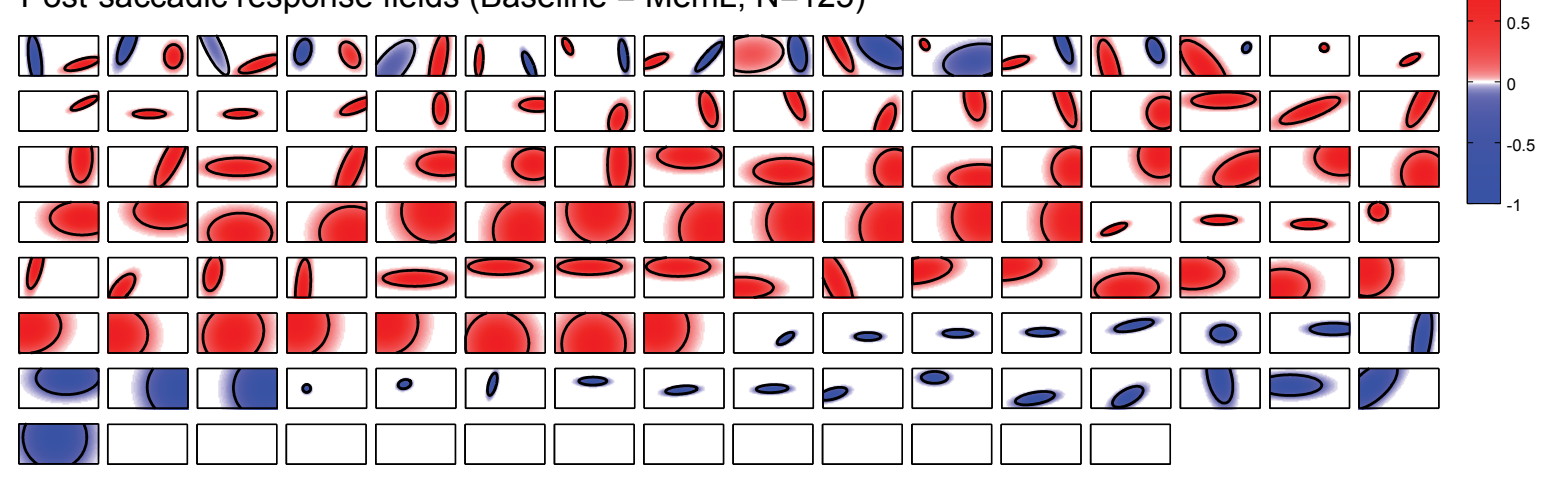

B
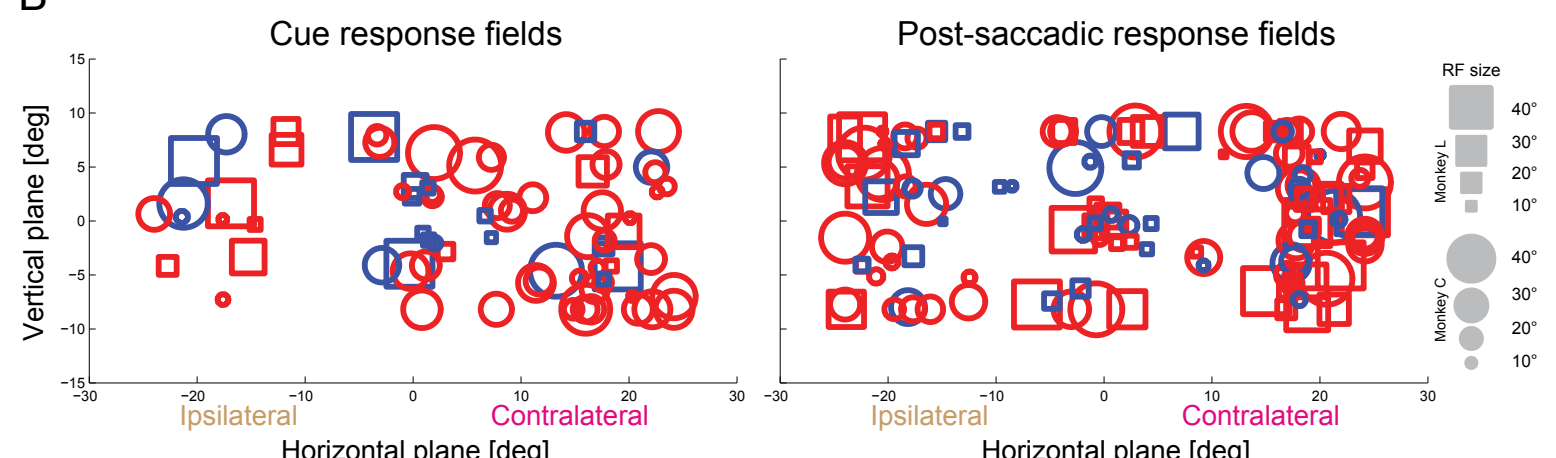

Figure 2.4. Visual and post-saccadic response fields (RFs). A) Visual RFs for units that showed a main effect of target position during cue onset (top) and post-saccadic RFs for units that showed a main effect of target position in the post-saccadic epoch. Each subplot represents one unit. Response fields were allowed to have two zones, one enhanced (red) and one suppressed (blue) relative to the respective baseline, see Materials and Methods. Only significant zones are plotted; empty subplots indicate no significant explanation of variance by the fitted response field. B) Summary of all cue (left) and postsaccadic (right) response field zones. Each marker represents one zone, circles for monkey $C$ and squares for monkey L. The location and size of the markers depicts the center and the size of the response field zone, colors indicate enhancement (red) or suppression (blue).

\subsubsection{Response categorization}

To characterize the firing patterns across the sample, and taking into account predominance cue and post-saccadic responses as compared to pre-saccadic or peri-saccadic responses, we defined three response categories based on the response modulation (enhancement or suppression) during the cue onset and post-saccadic epochs. Visual neurons were defined by statistically-significant enhancement or suppression during cue onset, but no modulation during the post-saccadic epoch, visuomotor neurons were defined by enhancement or suppression in both epochs, and motor neurons by enhancement or suppression in the postsaccadic epoch, but not during cue onset. Figure 2.5A-C shows two example units for each 
of these three categories, one example with enhancement during the respective epoch(s) on the left, and suppression on the right. The diversity of the responses is further illustrated by example units which showed enhancement or suppression during the memory epoch (Figure 2.5D) and units with spatial preference during the target hold epoch (Figure 2.5E).
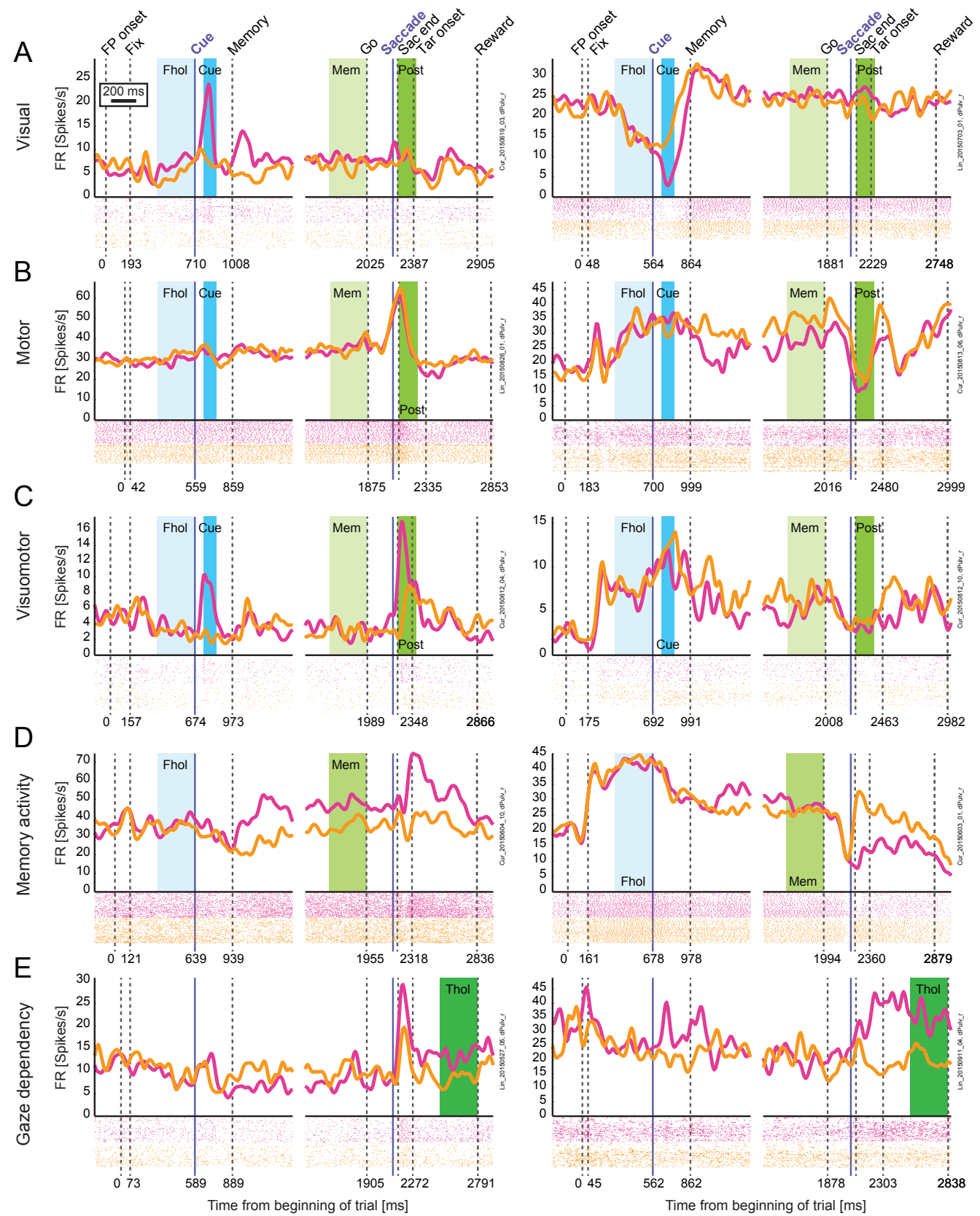

Contralateral trials Ipsilateral trials

Figure 2.5. Diversity of task-related firing modulations. Raster plots and spike density functions for contralateral (magenta) and ipsilateral (orange) trials. Vertical lines indicate the average onset of events: fixation spot onset (FP onset), the monkey acquiring fixation (Fix), the cue onset, the cue offset and beginning of the memory period, the offset of the central fixation point (Go), the saccade onset, the monkey acquiring the invisible target location (Sac end), the onset of the visible peripheral target, and the end of the trial (Reward). Discontinuous traces indicate two different alignments to cue onset and saccade onset (purple lines). Colored areas mark relevant analyzed epochs: fixation hold (Fix), cue onset (Cue), memory (Mem), post-saccadic (Post), and target hold (Thol). Examples for five different categories are shown. A) Visual units (enhancement or suppression in Cue relative to Fhol; B) Motor units (enhancement or suppression in Post relative to Mem); C) Visuomotor units (enhancement or suppression in both Cue and Post); D) Units with memory activity (left: hemifield preference in Mem, right: suppression in Mem compared to Fix); E) Gaze dependent activity (hemifield preference in Thol, in the period starting 300-400 ms after the end of the saccade, $200 \mathrm{~ms}$ after target onset). 
No systematic anatomical localization of units categorized as visual $(\mathrm{N}=50)$, motor $(\mathrm{N}=112)$, or visuomotor $(\mathrm{N}=95)$ neurons was found across the recording sites within the dorso-medial pulvinar (Figure 2.6A). To further assess the plausibility of this categorization, we computed a visuomotor index (VMI, ranging from -1 for purely visual to 1 for purely motor response) for each unit independently for the ipsilateral and the contralateral hemifield (see Materials and Methods) and tested if the mean VMls were significantly different from zero. As expected, VMIs of visual units were significantly negative and VMIs of motor units were significantly positive, see Figure 2.6B ( $p<0.001$ for all four tests, one sample t-test). Interestingly, VMls of visuomotor units were significantly positive for the ipsilateral hemifield $(p<0.01)$, while for the contralateral hemifield VMIs of visuomotor units were not different from zero $(p=0.46)$. This indicates stronger motor responses for ipsilateral saccades as compared to visual responses to ipsilateral stimuli in visuomotor units. The categories however overlapped and there were no separate modes in VMI distributions, indicating a continuum of visuomotor properties. We retain these categories for subsequent analyses as providing a tractable approach to the heterogeneity of response patterns.

To further test if specific sub-groups would be evident across the entire population, we plotted post-saccadic modulation versus cue modulation across the entire population (Figure 2.6C). Besides a noticeable cluster of units in upper right corner showing similar enhancement during the cue onset epoch and the post-saccadic epoch in contralateral trials, the units were distributed fairly uniformly, further implying a continuum of responses.

Looking at population PSTHs for each of the three categories (Figure 2.7A), the spatial preference and enhancement for contralateral cues in both visual and visuomotor units is evident, while post-saccadic enhancement without spatial preference seems to be predominant in both visuomotor and motor units. However, the latter finding might be either a consequence of combining units with different spatial preferences, or due to a contribution from few units showing unproportionally larger modulation than others.

A more detailed picture can be gained by further separating these three categories based on enhancement or suppression either in the cue onset epoch or the post-saccadic epoch, and analyzing the preferred target position for each unit (the position that exhibited the strongest response modulation in the respective epoch), see Figure 2.7A, bottom. Two observations can be made: first, visuomotor units showing suppression in the cue epoch relative to fixation hold are typically also suppressed throughout the entire memory period (although the visual conditions are identical in the fixation hold and memory periods), and second, the net enhancement of visuomotor and motor units in the post-saccadic epoch is a result of unequal proportions of enhanced and suppressed units $(\mathrm{N}=62$ and $\mathrm{N}=29 ; 65 \%$ and $31 \%$ of visuomotor units; $\mathrm{N}=66$ and $\mathrm{N}=40 ; 59 \%$ and $36 \%$ of motor units). 
A
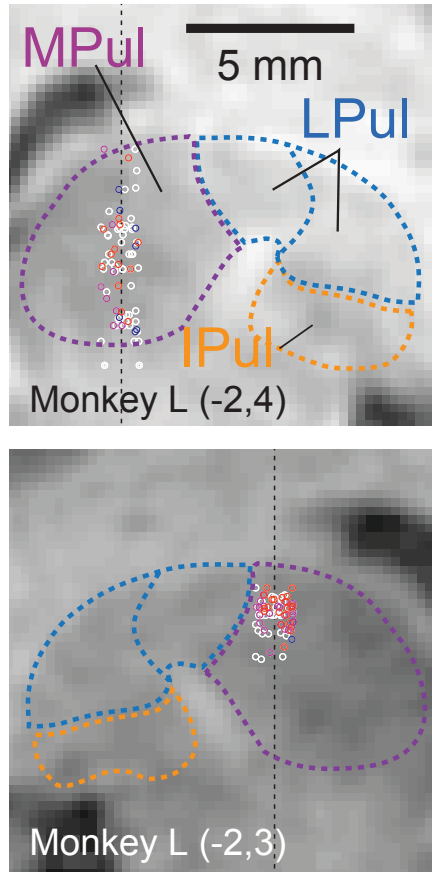

B
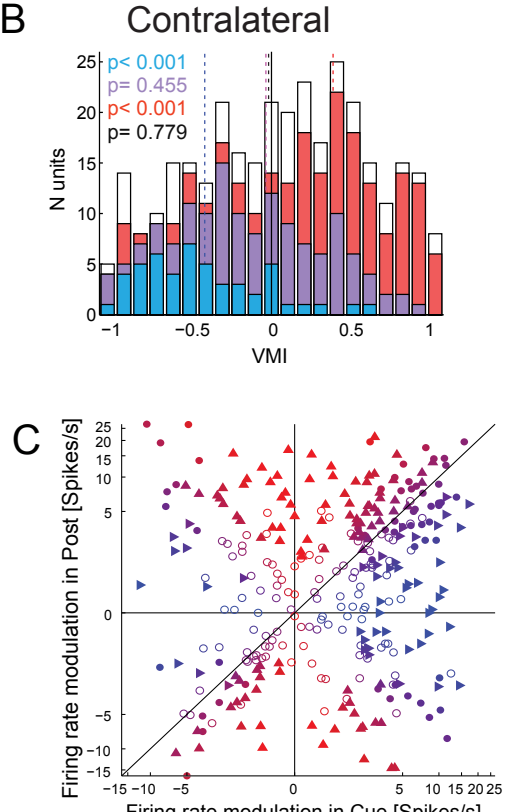
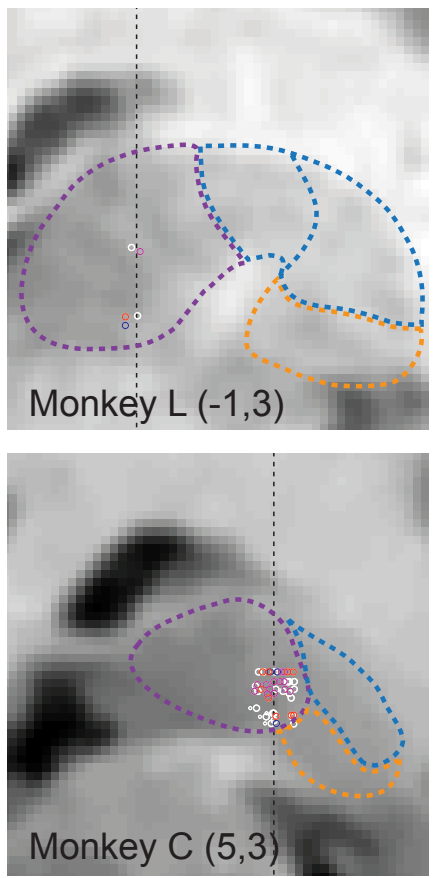

Ipsilateral
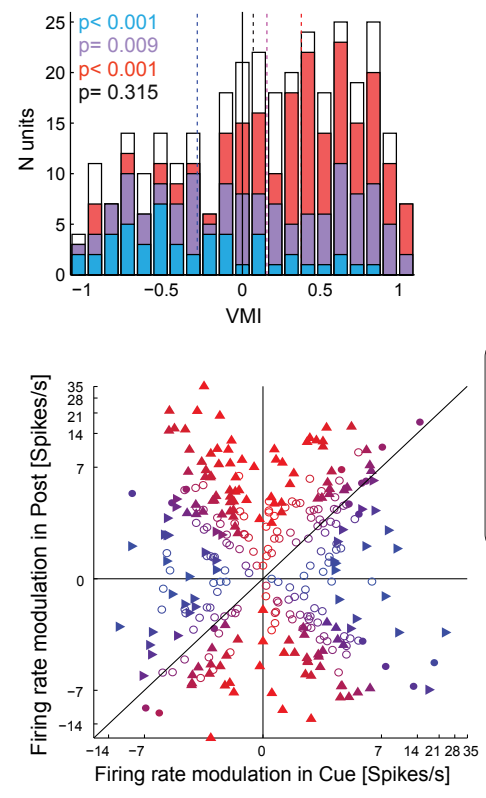
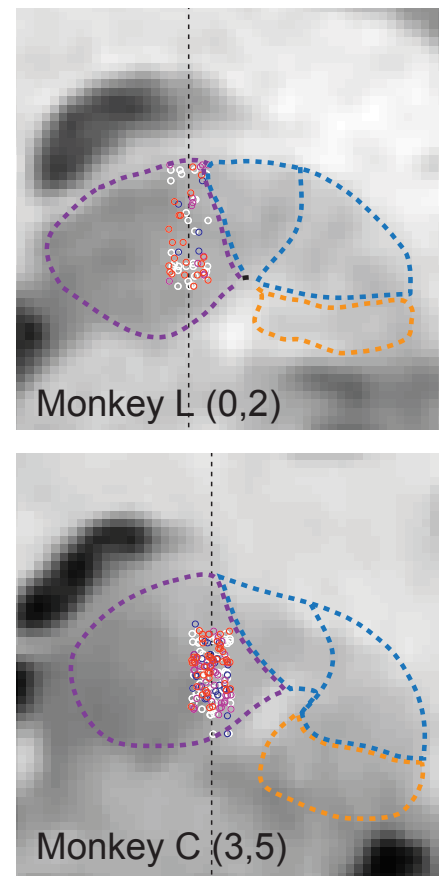

All trials
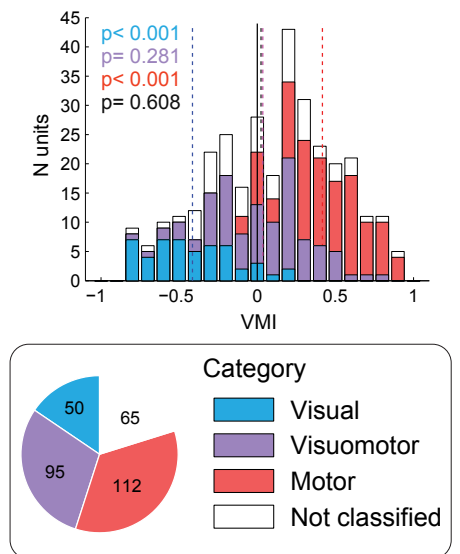

\begin{tabular}{|lr|}
\hline Significant modulation & \multicolumn{1}{c}{$\mathrm{VMI}$} \\
in Post & 0.5 \\
in Cue & 0.0 \\
in both epochs & -0.5 \\
Non significant & -1.0
\end{tabular}

Figure 2.6. Visuo-motor categories. A) Localization of recorded units in chamber-normal coronal sections in each monkey ( $\mathrm{L}$ and $\mathrm{C}$, labels on the bottom) and specific grid locations ( $\mathrm{x}, \mathrm{y}$; in parentheses). Locations were jittered along the horizontal axes for better visualization. The black dotted lines indicate the electrode tracks and mark the actual horizontal location of recorded neurons. Colors indicate the category of the unit; blue for visual, magenta for visuomotor and red for motor units. Units that did not fall within any of these categories are shown as white circles. Pulvinar nuclei outlines (MPul/LPul/IPul - medial/lateral/inferior pulvinar) were adapted from the NeuroMaps atlas (Rohlfing et al., 2012), exported via Scalable Brain Atlas, https://scalablebrainatlas.incf.org/macaque/DB09, https://scalablebrainatlas.incf.org/services/rgbslice.php, (Bakker et al., 2015), and LPul was further subdivided to dorsal (PLdm) and ventral (PLvl) parts. B) Histograms of VMls computed for contralateral trials (left), ipsilateral trials (middle), or all trials (right). Colors denote the category. P-values indicate results of one sample t-tests against zero. C) Scatter plots comparing the firing rate modulation in Cue and Post-saccadic epochs, for contralateral trials (left) and ipsilateral trials (right). Colors indicate the respective visuomotor index (legend inset on the right). For better visualization, firing rate modulation was plotted on a logarithmic scale. The pie plot on the right shows the total number of units for each of the categories. 
To further analyze the most common patterns in these three categories, and to address the contribution of units with different spatial preferences, we defined subpopulations of each of the categories based on enhancement or suppression and spatial preference either in the cue onset epoch or the post-saccadic epoch, see Table 2.1. Contralateral preference and enhancement in the cue onset epoch were predominant in both visual and visuomotor units, whereas post-saccadic enhancement and more or less balanced preference for the two hemifields was predominant in both motor and visuomotor units. Post-saccadic hemifield preference was found more commonly in visuomotor units than in motor units ( $48 \%$ vs $31 \%, p=0.015$; Fisher's exact test), suggesting that the former have a larger role in spatially-specific processing.

A Categories based on post-saccadic response

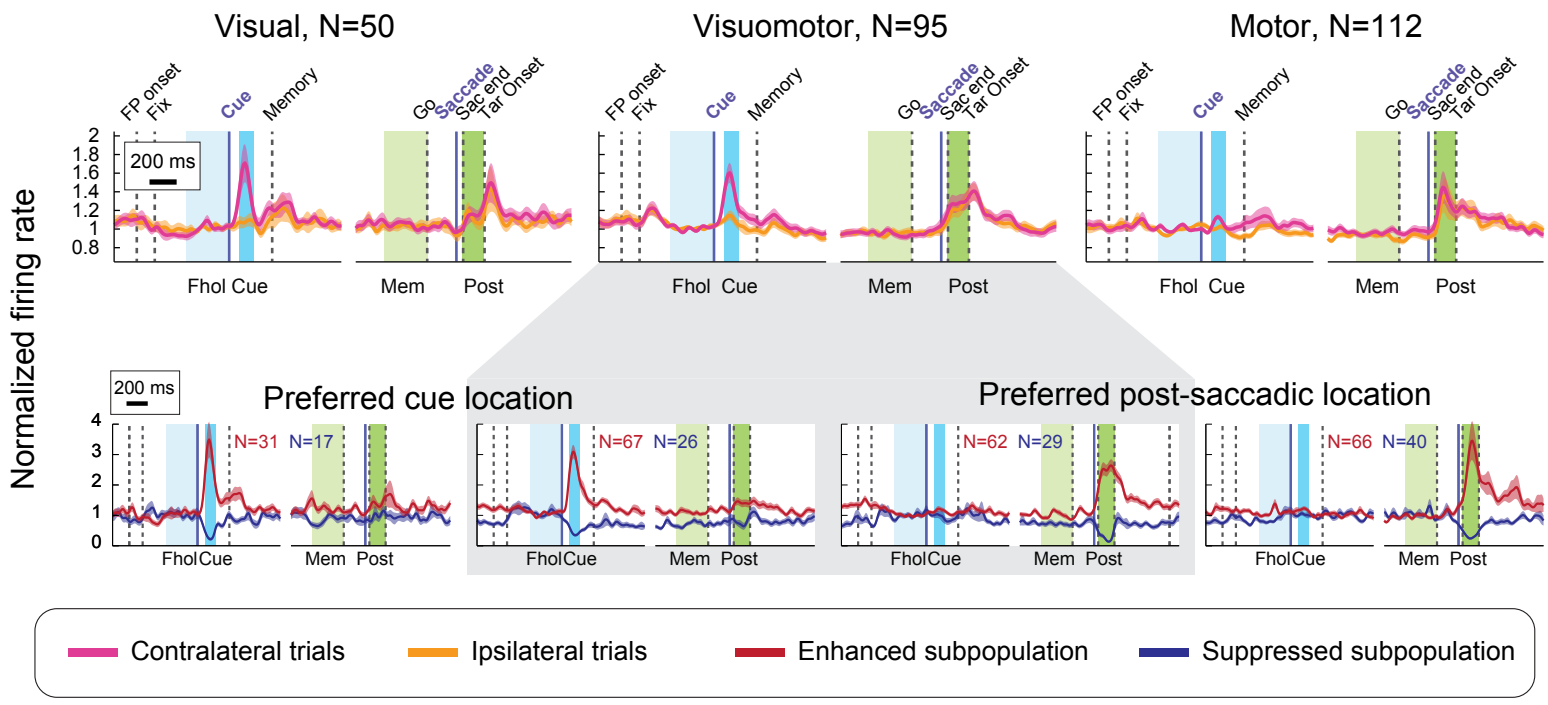

B Categories based on pre-saccadic response Visual, $\mathrm{N}=69$
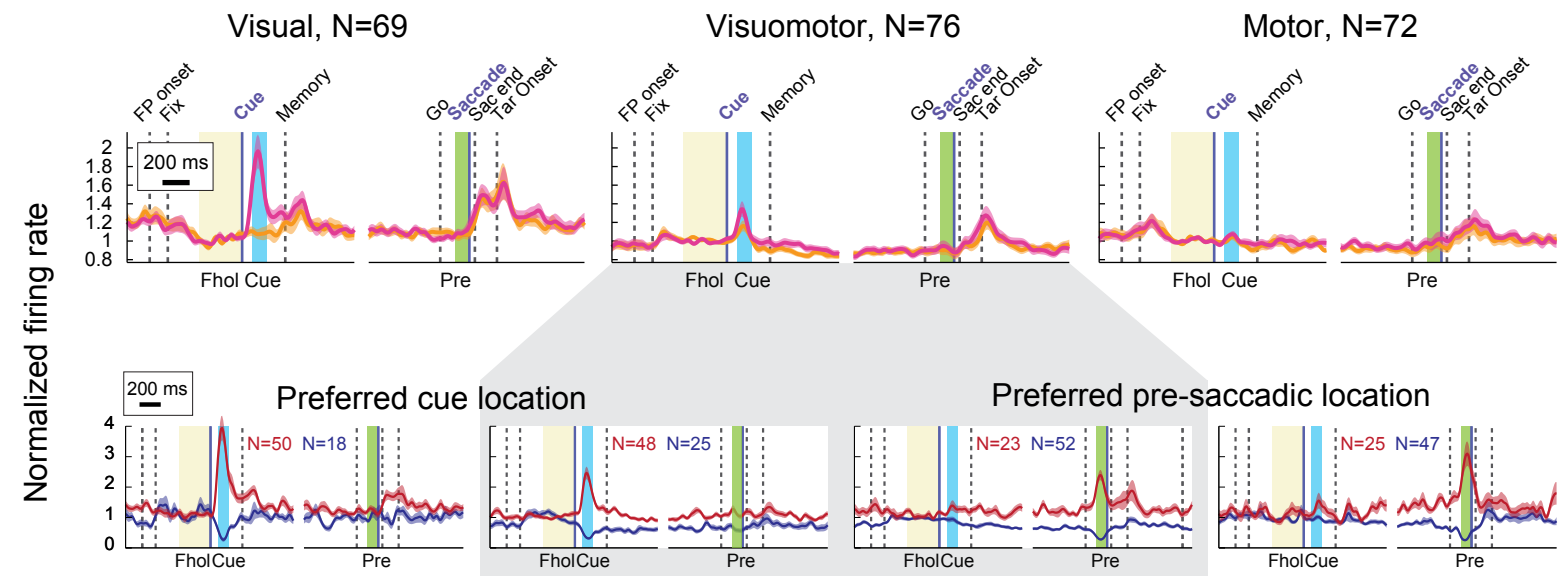

Figure 2.7. Population responses of visual, visuomotor, and motor units. Vertical lines mark events, and colored areas relevant epochs for categorization. A) Population response of the three categories: visual (enhancement or suppression in Cue relative to Fhol, but not in Post), motor (enhancement or suppression in Post relative to Mem, but not in Cue) and visuomotor units (enhancement or suppression in both epochs). Top: population responses for contralateral (magenta) and ipsilateral (orange) trials. Bottom: subpopulations showing enhancement (red) or suppression (blue) either during Cue or Post-saccadic epochs. For each unit, only the response for the most modulated target position (location with the largest firing rate difference from baseline) in the respective epoch was taken (note that few units showing enhancement for one and suppression for another location were excluded in those plots). B) same as A, for the categorization based on pre-saccadic response: visual (enhancement or suppression in Cue relative to Fhol, but not in Pre), motor (enhancement or suppression in Pre relative to Fhol, but not in Cue) and visuomotor units (enhancement or suppression in both epochs). 
Separation by response modulation and tuning in cue onset epoch

$\begin{array}{llll} & \begin{array}{l}\text { Visual } \\ (\mathrm{N}=50)\end{array} & \begin{array}{l}\text { Visuomotor } \\ (\mathrm{N}=95)\end{array} & \begin{array}{l}\text { Motor } \\ (\mathrm{N}=112)\end{array} \\ \text { Enhancement, no tuning } & 17(34 \%) & 26(27 \%) & \text { - } \\ \text { Enhancement, contralateral preference } & 14(28 \%) & 37(39 \%) & \text { - } \\ \text { Enhancement, ipsilateral preference } & 0(0 \%) & 4(4 \%) & - \\ \text { Suppression, no tuning } & 8(16 \%) & 18(19 \%) & - \\ \text { Suppression, contralateral preference } & 6(12 \%) & 4(4 \%) & - \\ \text { Suppression, ipsilateral preference } & 3(6 \%) & 4(4 \%) & - \\ \text { Contra enhancement and ipsi suppression } & 2(4 \%) & 2(2 \%) & -\end{array}$

Separation by response modulation and tuning in post-saccadic epoch

\begin{tabular}{llll} 
& \multicolumn{1}{c}{$\begin{array}{l}\text { Visual } \\
(\mathrm{N}=50)\end{array}$} & $\begin{array}{l}\text { Visuomotor } \\
(\mathrm{N}=95)\end{array}$ & $\begin{array}{l}\text { Motor } \\
(\mathrm{N}=112)\end{array}$ \\
Enhancement, no tuning & - & $32(34 \%)$ & $13(12 \%)$ \\
Enhancement, contralateral preference & - & $19(20 \%)$ & $7(6 \%)$ \\
Enhancement, ipsilateral preference & - & $11(12 \%)$ & $31(28 \%)$ \\
Suppression, no tuning & - & $17(18 \%)$ & $8(7 \%)$ \\
Suppression, contralateral preference & - & $3(3 \%)$ & $1(1 \%)$ \\
Suppression, ipsilateral preference & - & $9(9 \%)$ & $4(4 \%)$ \\
Contra enhancement and ipsi suppression & - & $3(3 \%)$ & $2(2 \%)$
\end{tabular}

Table 2.1. Visual, visuomotor, and motor subpopulations. Subpopulations were defined by response modulation and spatial tuning either in the cue onset epoch (for visual and visuomotor neurons) or in the postsaccadic epoch (for visuomotor and motor neurons). Numbers indicate the total number for each subpopulation and the percentage of the respective parent category.

Figure 2.7B shows the same analysis based on a different motor-related categorization (enhancement or suppression in the pre-saccadic epoch relative to Fhol, see Materials and Methods). Importantly, the post-saccadic enhancement is still evident in the population responses of all three categories, while enhancement and suppression as well as spatial preference in the pre-saccadic epoch balances out completely (Figure 2.7B, top), further 
supporting a classification based on post-saccadic responses. The washout of spatial preference becomes more evident when looking at preferred locations only, independently for suppressed and enhanced units (Figure 2.7B, bottom).

\subsubsection{Comparison with visually-guided saccades}

To evaluate how the presence of a visible saccade target might affect pre-saccadic activity as compared to memory-guided saccade, in some sessions we also recorded the same units while monkeys were performing a visually-guided saccade task. In the visually-guided task, monkeys were free to perform a saccade right after the onset of the targets and the targets stayed visible throughout the entire trial (see Materials and Methods).

We compared the response modulation in the target onset/pre-saccadic epoch in visuallyguided task against two partially corresponding epochs in the memory-guided task; 'cue onset 2' for visual responses and 'pre-saccadic 2' for potential motor preparation signals. These epochs were chosen as 40-140 ms after the cue onset or the Go signal to be comparable to the target onset/pre-saccadic epoch ('target-saccade') in the visually-guided task (where the interval was chosen to not overlap with the saccade onset). Response modulation was calculated by subtracting firing rates in fixation hold (Fhol) to allow for direct comparison between the two tasks, in all units recorded in the both tasks $(\mathrm{N}=174)$.

Response modulation across units were correlated in the two tasks, both when correlating visual response modulation ('cue onset 2') and pre-saccadic responses ('pre-saccadic 2') for both hemifields to 'target-saccade' ( $p=0.037$ in 'pre-saccadic 2' for ipsilateral trials, $p<0.001$ for all other correlations, Pearson's correlation), indicating that both visual and motor preparatory signals contribute to response modulations in visually-guided saccades. To assess if response modulation in the visually-guided task can be explained by the sum of visual and motor preparation signals, we compared activity in the 'target-saccade' to the sum of 'cue onset 2' and 'pre-saccadic 2' responses. The 'target-saccade' response modulation was stronger than the sum of separate epochs $(p=0.002 / 0.001$ for contralateral/ipsilateral trials, paired t-tests). This was particularly true for units showing enhancement in the visuallyguided task (sublinear summation, $\mathrm{N}=49 / 19, \mathrm{p}=0.002 / 0.009$ for contralateral/ipsilateral trials, paired t-tests).

\subsubsection{Delay period and pre-/peri-saccadic activity}

Due to the strong bidirectional connectivity between dorsal pulvinar and posterior parietal cortex (PPC), in particular area LIP (Gutierrez et al., 2000; Hardy and Lynch, 1992) as well as the suggested role of pulvinar in guiding goal-directed actions (Grieve et al., 2000; Wilke et al., 2010), we predicted that some dPul neurons show delay period and pre-/peri-saccadic 
activity - a hallmark of canonical PPC responses (Barash et al., 1991a; Premereur et al., 2011). As reported previously (Dominguez-Vargas et al., 2017), when looking at population averages for units that showed either enhancement or suppression in the peri-saccadic epoch as compared to fixation hold, it seems like a considerable subset indeed showed activity that is ramping up, similarly to LIP $(\mathrm{N}=42)$, or ramping down $(\mathrm{N}=94)$, and reaching the respective maximum or minimum during saccade execution (Figure 2.8A,B). Normalizing these two subsets using division by average firing rate in fixation hold rather than subtracting baseline inter-trial-interval activity strengthened that impression (Figure 2.8C,D). However, here we demonstrate that the ramping delay period activity culminating at the saccade is largely a result of combining different subpopulations. First, subpopulations showing perisaccadic enhancement $(\mathrm{N}=43)$ or suppression $(\mathrm{N}=62)$ relative to memory did not exhibit ramping or persistent delay period activity (Figure 2.8E,F). Second, subpopulations showing enhancement or suppression in memory relative to fixation hold ( $N=50$ and $N=97$ respectively) did not exhibit pronounced peri-saccadic responses (Figure 2.8G,H). In fact, very little overlap between these subpopulations was found; only 4 units showed consistent enhancement in memory relative to fixation hold and in peri-saccadic epoch relative to memory, and 18 units showed consistent suppression in both epochs. Besides these few ramping up/down units, subpopulations shown in Figure 2.8A,C and B,D combine units that showed enhancement or suppression either only during memory and units that showed enhancement or suppression only during the peri-saccadic epoch. Interestingly, enhanced memory period firing is constant throughout the entire period (Figure 2.8G), whereas activity was decreasing gradually throughout the memory period in the subpopulation showing suppression in the memory epoch (Figure 2.8H).

Figure $\mathbf{8} \mathbf{l}, \mathbf{J}$ shows subpopulations that were significantly tuned for contralateral or ipsilateral space in the memory delay. The contralateral spatial preference during the memory period observed in the group showing peri-saccadic enhancement (Figure 2.8A,C) is mainly due to more contribution from units with significant contralateral preference than units with ipsilateral preference during the memory period ( $\mathrm{N}=7$ and $\mathrm{N}=1$ respectively). The contralaterally- and ipsilaterally-tuned units differed in terms of response pattern: The contralaterally-tuned group (Figure 8I) showed congruent contralateral cue tuning, while the ipsilaterally-tuned group also showed contralateral cue tuning and peri-saccadic suppression, especially in ipsilateral trials.

These findings have two important implications. First, only a small minority of units showed "classical" spatially-tuned cue-delay-saccade enhancement, constraining the role of individual dPul neurons in visuomotor transformations. Second, more units showed delay period suppression and ramping down rather than enhancement, suggesting putative 

driven inhibition on the pulvinar.
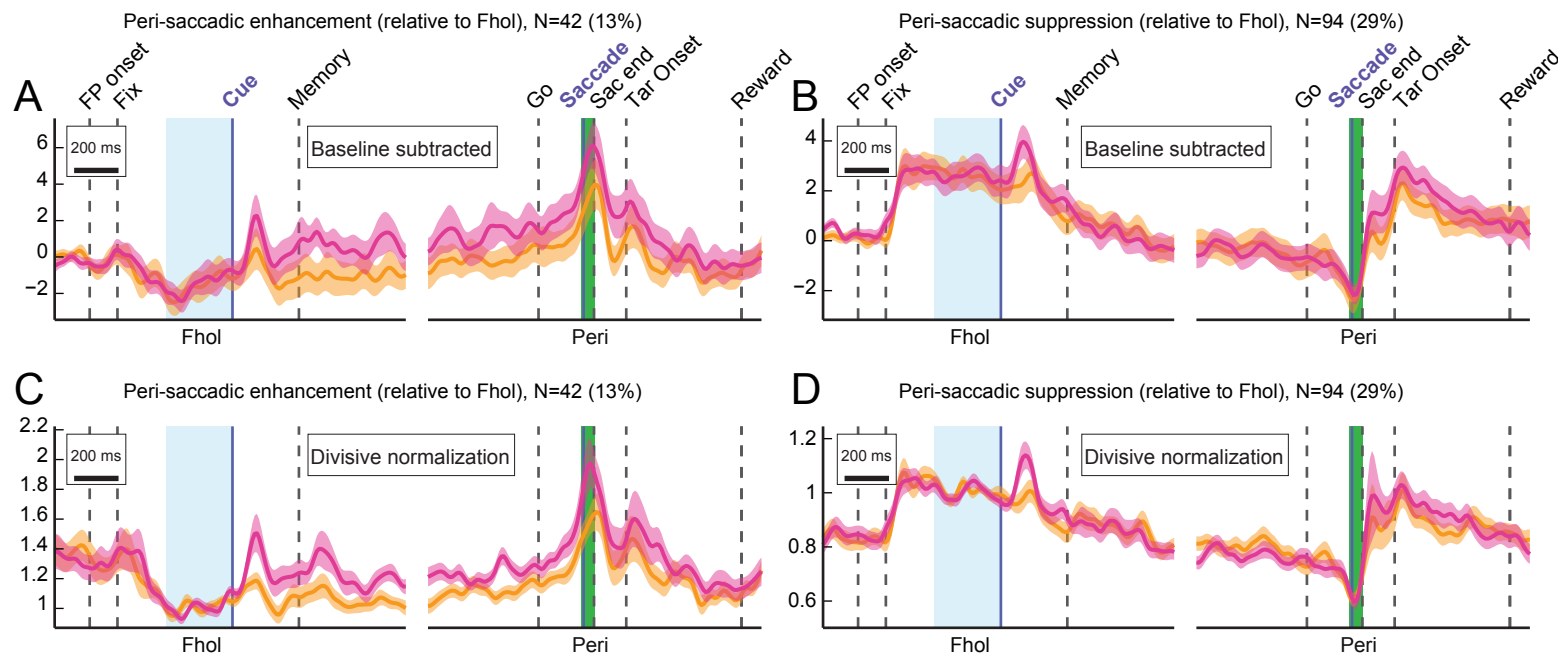

D Peri-saccadic suppression (relative to Fhol), N=94 (29\%)
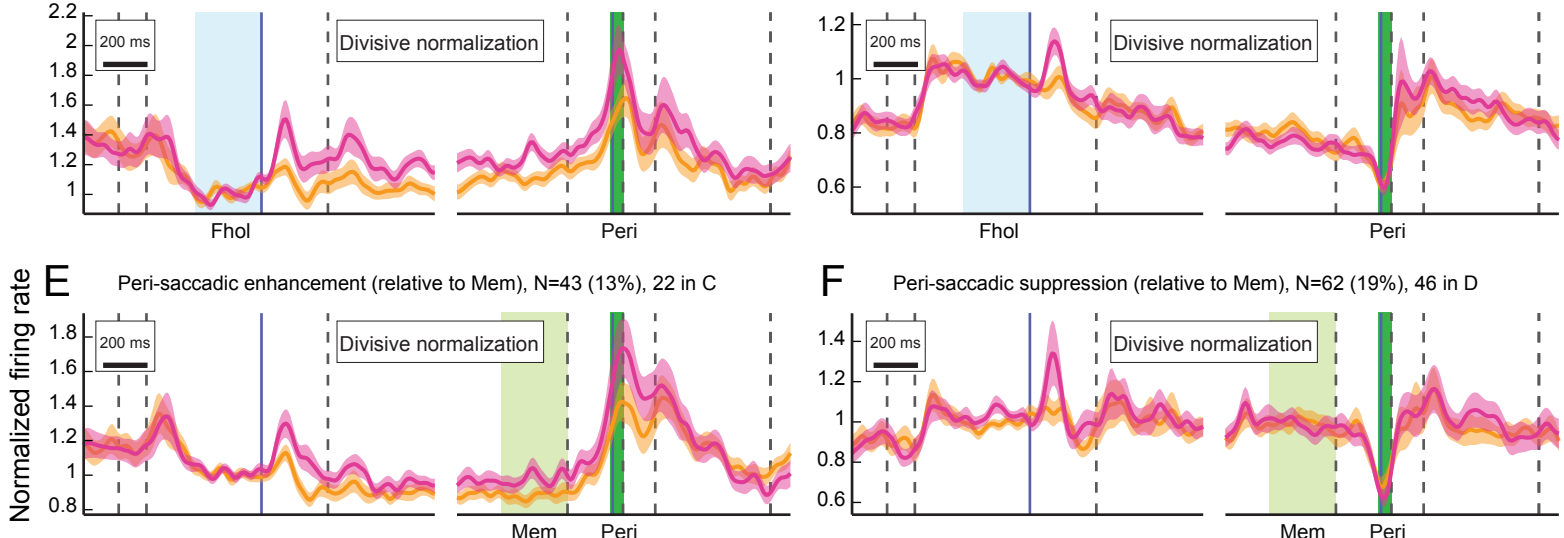

F Peri-saccadic suppression (relative to $\mathrm{Mem}), \mathrm{N}=62(19 \%), 46$ in D

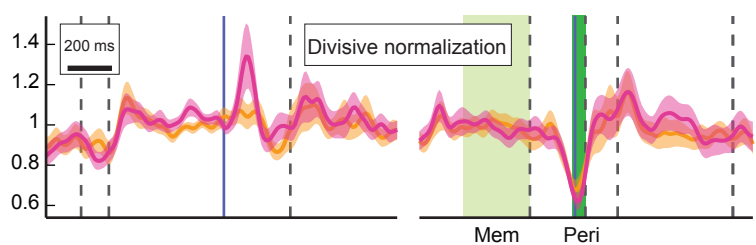

G Memory period (Mem) enhancement, $\mathrm{N}=50(16 \%), 19$ in C, 4 in E

$\mathrm{H}$ Memory period (Mem) suppression, $\mathrm{N}=97$ (30\%), 60 in D, 18 in $\mathrm{F}$
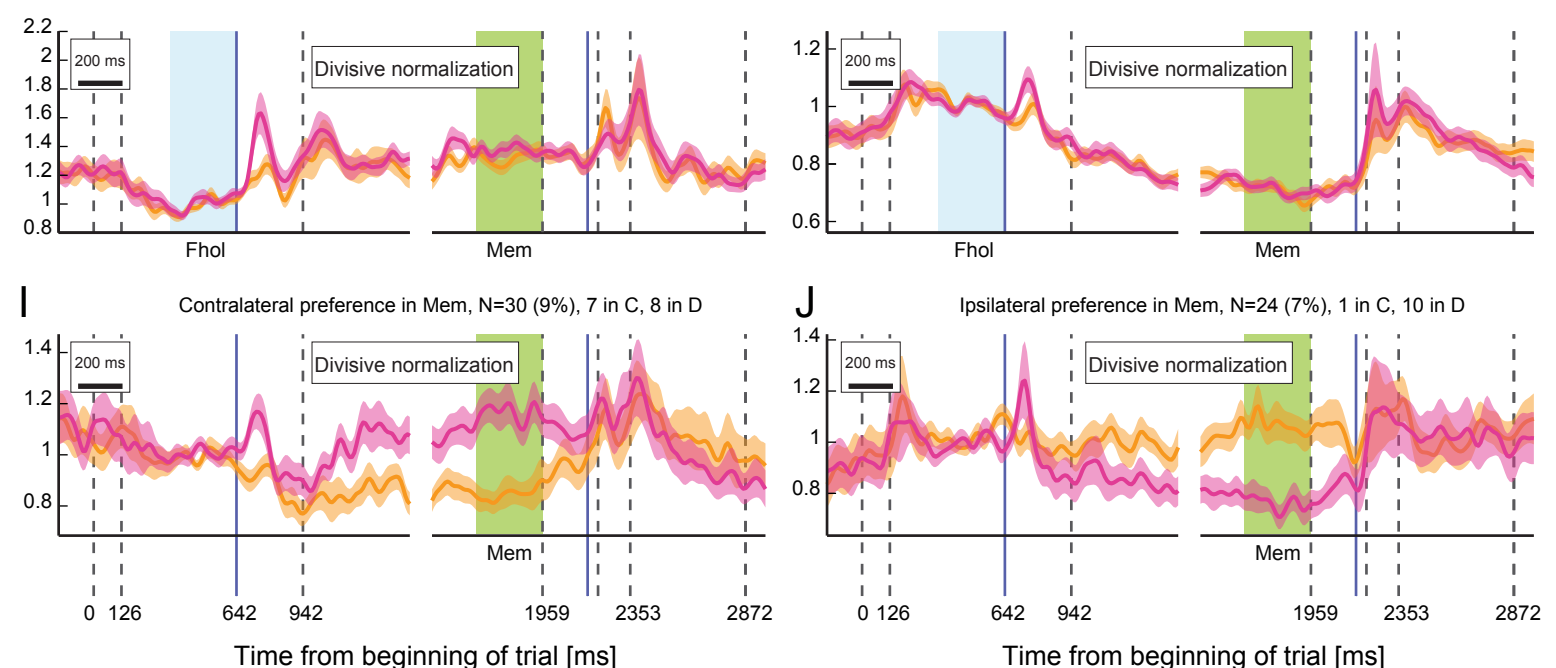

$$
\text { Contralateral trials Ipsilateral trials }
$$

Figure 2.8. Delay-period activity. Population responses for contralateral (magenta) and ipsilateral (orange) trials. Vertical lines mark events, and colored areas relevant epochs for population definition. A) Average of units showing peri-saccadic enhancement relative to fixation hold epoch (Fhol), normalized by subtracting the average firing rate in inter-trial-interval epoch. B) Same as A, for units showing peri-saccadic suppression. C) Averages of units showing peri-saccadic enhancement relative to Fhol, normalized by dividing the spike density functions by the average firing rate in Fhol. D) Same as C for units showing peri-saccadic suppression. E) Same as C for units showing peri-saccadic enhancement relative to the memory epoch (Mem). F) Same as $E$ for units showing peri-saccadic suppression. G) Same as C for units showing enhancement in Mem relative to Fhol. H) Same as G for units showing suppression. I) Same as $C$ for units showing contralateral preference in Mem. J) Same as I for units showing ipsilateral preference. Numbers in parenthesis denote the number of units that overlapped with subpopulations shown in C and D. 


\subsubsection{Spatial choice selectivity}

In half of the trials, monkeys were presented with two cues, equidistantly from the fixation point, and were free to select either of the two positions, for the same reward. There were 6 possible pairs of targets, and instructed and choice trials were randomly interleaved, discouraging monkeys to make selection before the cues were presented. It has been shown that in such conditions dPul inactivation biases the choice towards ipsilesional hemifield (Wilke et al., 2013).

Target selection in choice trials was fairly balanced between the two hemifields in both monkeys (monkey C: 60/40; monkey L: 48/52) and reaction times in choice trials were very similar to instructed trials. If anything, monkey $C$ was faster in choice trials $(p=0.01$, twosample t-test). The lack of additional time delay in choice RTs suggests that monkeys made choices before the end of delay period (see Table 2.2 for details).

$\begin{array}{rrrrccc} & & \text { Instructed RT } & \text { Choice RT } & \text { p } & \text { \% Choice } & \text { P (target selection) } \\ \text { Monkey C } & \text { Left } & 206 & 201 & 0.01 & 59.8 & <0.001 \\ & \text { Right } & 214 & 209 & 0.011 & 40.2 & \\ \text { Monkey L } & \text { Left } & 189 & 190 & 0.682 & 48.3 & 0.6274 \\ & \text { Right } & 186 & 184 & 0.139 & 51.7 & \end{array}$

Table 2.2. Saccade reaction times (RT) and target selection. $P$ values from two-sample t-tests comparing means across sessions.

To see if spatial preference during the delay period is linked to space-specific motor preparation and could therefore reflect the upcoming saccade decision, we analyzed choice trials in the subpopulations showing hemifield preference in instructed trials.

On average, there was no apparent hemifield preference in the delay period activity in choice trials, and the choice trial traces were situated between preferred and opposite hemifield instructed trials (Figure 2.9A). This might indicate that these units are not directly linked to prospective motor preparation, but instead maintain retrospective information about the visual stimuli presented earlier in the trial, or attentional allocation. 


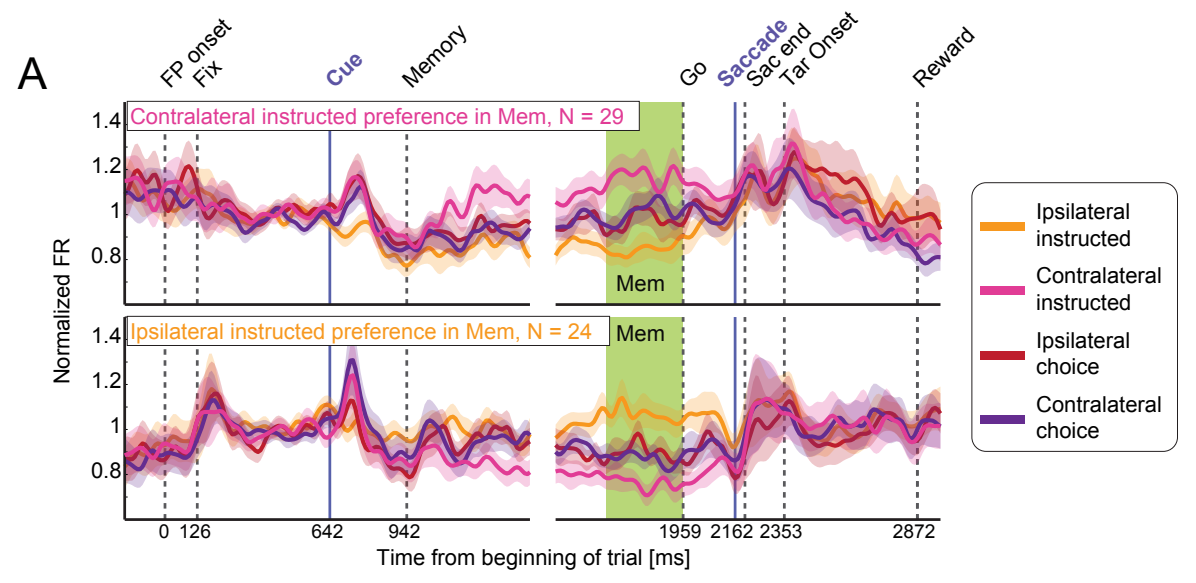

B Examples for different cue hemifield preferences in instructed and choice trials
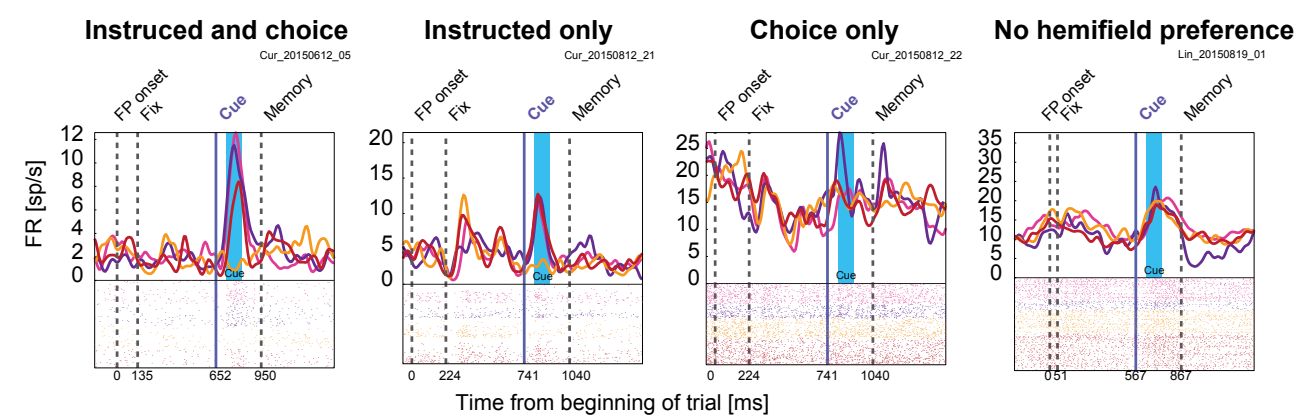

C
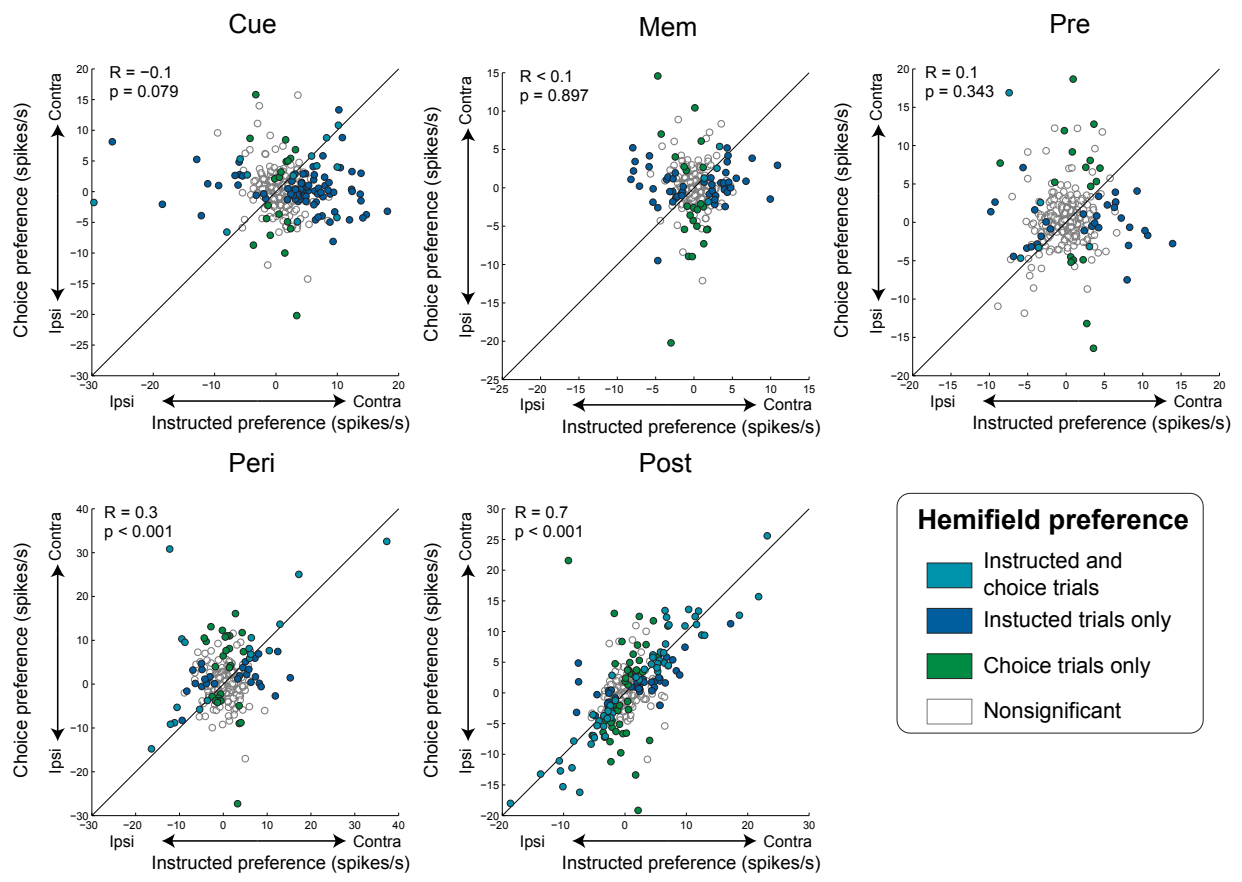

Figure 2.9. Choice preference. A) Population averages for units showing either contralateral preference (top) or ipsilateral preference (bottom) in the memory (Mem) epoch, for instructed contralateral (magenta), ipsilateral instructed (orange), contralateral choice (purple) and ipsilateral choice (dark red) trials. One unit was excluded, because it was recorded for less than 4 choice trials to each hemifield. B) Example units with different types of cue responses. From left to right: Contralateral preference in both instructed and choice, contralateral preference only in instructed, contralateral preference only in choice, no hemifield preference. C) Scatter plots comparing hemifield preference (contra - ipsi) in instructed and choice trials, for six epochs: cue onset (Cue), memory (Mem), pre-saccadic (Pre), peri-saccadic (Pre) and post-saccadic. R and $p$ values for Pearson's correlations between hemifield preference in instructed and choice trials are shown. Five units were excluded from this analysis because less than 4 choice trials for one of the two hemifields were recorded. 
Some units however showed a significant difference in firing rates prior to selection of the contralateral or ipsilateral target in choice trials, well before the saccade. The question is whether the spatial selectivity in choice trials is congruent to the spatial selectivity in instructed trials. This was only rarely the case. Figure 2.9B shows four example units with qualitatively different cue responses, i) with hemifield preference in both instructed and choice trials, ii) only in instructed trials, iii) only in choice trials, iv) no hemifield preference in both instructed and choice trials

To evaluate if pulvinar neurons can encode the upcoming choice across the population, we compared hemifield preference between instructed and choice trials in each unit in five different epochs (cue onset, memory, pre-saccadic, peri-saccadic, and post-saccadic epoch), see Figure 2.9C.

Before the saccade (cue, memory, pre-saccadic epochs) there was almost no congruency between tuning in instructed and choice trials. Only few units showed a significant congruent effect of the (selected) hemifield in both trial types (Cue: $N=12$, Mem: $N=4$, Pre: $N=5$ ), and across all units, hemifield preferences (firing rate difference contra - ipsi) were not correlated (Cue: $p=0.079$, Mem: $p=0.897$, Pre: $p=0.343$, Pearson's correlation). Additionally, in the cue the absolute response modulation (contra-ipsi) was much higher in instructed than in choice trials ( $p<0.001$, Wilcoxon's signed rank test). These findings suggest that the upcoming decision, or more specifically the spatially-specific movement planning, was not encoded in the firing rates. In other words, the spatially-selective activity during the instructed trials prior to the saccade can be explained by the visual stimulation or retrospective memory of the visual stimulus.

As a control analysis, since the movement was very similar in instructed and choice trials to the same direction, hemifield preferences were highly correlated between instructed and choice trials in peri- and post-saccadic epochs, as expected $(p<0.001$, Pearson's correlation). 


\subsection{Discussion}

The goal of this study was to characterize visual, delay and motor response properties of dorsal pulvinar. We used a classical delayed memory-guided saccade task to dissociate sensory encoding, intervening delay and motor execution phases. We found that motorrelated activity was more prevalent and mostly post-saccadic, although there was also a substantial number of neurons showing pre- and peri-saccadic activity, both enhancement and suppression.

Surprisingly, delay period activity was largely characterized mainly by non-spatial specific suppression, indicating that if the units showing delay period suppression are connected to the fronto-parietal network, they might be mainly inhibiting cortical areas (or vice versa), and do not contribute to spatial target selection. In line with this interpretation, delay period activity did not encode the upcoming choice when two saccade options were available, challenging the underlying notion that the pulvinar activity during the memory delay period is directly involved in decision making (Wilke et al., 2013). On the other hand, this result is consistent with our recent findings showing the lack of the choice bias induced by the dPul microstimulation during the memory delay, as compared to strong effect of microstimulation in the pre-saccadic epoch of visually-guided task (Dominguez-Vargas et al., 2017).

\subsubsection{Visuomotor responses}

We demonstrated that visual responses of dorsal pulvinar neurons to a small briefly presented saccade cue are typically marked by transient increases of spiking activity, with a clear preference for contralateral stimuli on a population level. The firing rate increase was found to start around 70 ms after stimulus onset, and most visually responsive units showed a significant increase in firing rate around $120 \mathrm{~ms}$ after the stimulus onset. These findings are in contrast to previous reports, where response to visual stimuli were reported only when linked to an immediate subsequent eye-movement to the visually-guided location, although with a similar response latency of 80 ms (Benevento and Port, 1995).

In contrast to findings in central oculomotor thalamus, where saccade-related responses are characterized by equally common space-specific pre- (47\%) and post-saccadic (53\%) increases and decreases (72\% and 28\%) in firing rate (Wyder et al., 2003), saccade-related responses in dorsal pulvinar neurons were found to be mainly post-saccadic (64\% of all units compared to $22 \%$ with pre-saccadic activity); but - similar to central oculomotor thalamus (Wyder et al., 2003) - twice as much enhancement as suppression in firing rates (40\% and $21 \%$ of all units) relative to the preceding period (memory delay), and no clear spatial preference on a population level. 
Many units exhibited a transient, typically non-space-specific pre-saccadic or peri-saccadic suppression (14\% with pre-saccadic suppression, $77 \%$ out of those not space-specific $19 \%$ with peri-saccadic suppression, $73 \%$ out of those not space-specific). This signal could be used to prevent self-induced stimulus motion (e.g. by suppressing visual information transfer to fronto-parietal areas during the saccade itself), in other words the pre- and peri-saccadic suppression could reflect mediated corollary discharge (Bellebaum et al., 2005; Sommer and Wurtz, 2004; Wurtz et al., 2011a, 2011b). Along these lines of interpretation, the non-spacespecific post-saccadic enhancement could further be used to signal that the saccade has ended and processing of visual input can be re-established. Another interpretation would be that post-saccadic enhancement signals the expectation of a change in visual information, namely the confirmation target onset, since it appears soon after the end of the saccade with a predictable timing, and is always located (more or less) in the fovea. This however would only be relevant for neurons with receptive fields that include fovea. Potentially, small deviations of the current eye position from the target location, or error signals, could be encoded in these post-saccadic signals, but the small saccade endpoint dispersion in fairly very stereotypical movements did not allow to systematically check for this possibility. To answer this question a task that yields larger and more variable eye position errors would be required (Mays and Sparks, 1980).

A sizable proportion of units, however, exhibited pre-saccadic and peri-saccadic enhancement $(7 \% / 13 \%)$, with an overall weak preference for the contralateral hemifield. Activity in these units resembles saccade-related responses in oculomotor thalamus and FEF, and it might depend on the behavioral demands and spatial decision urgency (Costello et al., 2015).

When trying to categorize response patterns by the type of visual and post-saccadic responses, we found very diverse patterns of activation, and no clear topographic organization of these response patterns within the dorsal pulvinar. Notably, purely visual responses were less frequent than pure motor responses or combined visual and motor responses (15\% visual units compared to $35 \%$ visuomotor and $30 \%$ motor), further supporting an involvement of dorsal pulvinar in coordinating and refining motor commands (Guillery and Sherman, 2002; Saalmann and Kastner, 2015).

\subsubsection{Delay period activity and choice selectivity}

Because of the widespread reciprocal connectivity between dorsal pulvinar and the frontoparietal network (Bos and Benevento, 1975; Hardy and Lynch, 1992; Schmahmann and Pandya, 1990) and the fact that dorsal pulvinar perturbation reliably biases saccade target selection both in visually-guided and memory-guided saccades (Dominguez-Vargas et al., 2017; Wilke et al., 2010, 2013), it is important to know if and how dorsal pulvinar activity 
might reflect spatial decision and movement planning. We found that $46 \%$ of the recorded units showed memory period activity modulation. Interestingly, suppression during the memory period was twice as common as enhancement, and the activity in units showing such suppression during the memory period was typically ramping down towards the go signal, whereas increases were typically sustained and stable throughout the entire memory period. The gradual decrease in firing rate during the memory period represents the oppositely complementary pattern to gradual ramping up, or climbing towards the saccade onset found in posterior parietal cortex (Gnadt and Andersen, 1988; Hwang and Andersen, 2012; Premereur et al., 2011; Rorie et al., 2010) and FEF (Lawrence et al., 2005), suggesting inhibitory projections either from fronto-parietal cortex to pulvinar or from pulvinar to cortex. But in contrast to cortical representations, pulvinar delay period activity was typically not space-specific, indicating that pulvinar might mediate general alertness, attention or non-specific movement preparation rather than specific movement planning.

Some pulvinar units (16\%) also showed differential memory period responses for upcoming ipsilateral and contralateral instructed saccades, indicating that they might be involved in spatially-specific motor preparation. This fraction is comparable to findings in oculomotor thalamus (Wyder et al., 2003), where 25\% of units showed spatial preferences during the delay period in a visually-guided delayed saccade task (which is likely to produce stronger delay period activity due to the presence of visual stimulus).

However, memory period and pre-saccadic activity in these spatially selective pulvinar neurons did not reflect the upcoming movement during choice trials, suggesting that the spatial modulation reflected the retrospective memory of the visual stimulus rather than the spatial decision for the upcoming movement. Looking at choice selectivity in different epochs indicated that target selection was not reflected in any of the epochs before the saccade onset, because only a very small amount of units significantly preferred the same hemifield over the other in both instructed and choice trials. This indicates that neither the upcoming saccade direction and in particular not the target selection itself was encoded in the firing rates of dorsal pulvinar neurons, suggesting that other mechanisms are responsible for creating a saccade choice bias when dorsal pulvinar is perturbed (Dominguez-Vargas et al., 2017; Wilke et al., 2010, 2013).

In addition, and not mutually exclusive to encoding visuomotor decision and motor planning, it has been shown that both dorsal and ventral pulvinar contribute to maintaining spatial attention (Petersen et al., 1987; Zhou et al., 2016a). Two recent electrophysiological studies on the ventral-lateral pulvinar and one study on the dorsal pulvinar reported slight but significantly enhanced persistent activity during sustained cued spatial attention in the receptive field (Fiebelkorn et al., 2019; Saalmann et al., 2012; Zhou et al., 2016a). In 
particular, in the studies of Saalmann and colleagues and Fiebelkorn and colleagues there was no visual stimulus during the memory period, similarly to the delay in the memory-guided saccade task. This weakly tuned persistent activity is similar to the persistent activity in the instructed memory delay in our dorsal pulvinar population. A potential pathway from deep and intermediate layers of SC via lateral pulvinar carrying preparatory movement-related activity corresponding to shifts of attention to parietal cortex has been suggested by Wurtz and colleagues (Wurtz et al., 2011a), and can be in play in both attentional and memoryguided saccade task.

\subsubsection{Anatomical considerations}

When comparing results presented in the current work with previous studies focusing on visuomotor properties of the pulvinar, it needs to be considered that previous studies in the dorsal pulvinar typically recorded from the dorsomedial division of the lateral pulvinar (now mostly denoted as PLdm (Bridge et al., 2015; Kaas and Lyon, 2007), or as Pdm in original electrophysiological studies by Robinson, Petersen and colleagues, e.g. (Robinson et al., 1986)). The notable exception is a study that covered both lateral and medial parts of the dorsal pulvinar (Benevento and Port, 1995). Our recording sites were predominately within the medial pulvinar (MPul) (see also (Dominguez-Vargas et al., 2017)). Therefore, any potential differences might indicate presence of lateral-to-medial gradients of visuomotor encoding within the subdivisions constituting the dorsal pulvinar division.

\subsubsection{Limitations and future directions}

Given the diversity of pulvinar spiking activity and its proposed involvement in multiple cognitive processes, the further insights gained by studying dorsal pulvinar activity in such basic oculomotor tasks are limited.

First, even if the current study challenges the idea that dorsal pulvinar activity is linked to target selection in delayed oculomotor tasks, it might still be involved in guiding other movements, as suggested by the apparent effect of pulvinar inactivation on target selection by reaching and grasping (Wilke et al., 2010). Furthermore, given that a considerable amount of units showed gain-field like properties, it would be interesting to see if pulvinar is involved in eye-hand coordination.

Second, richer cognitive tasks are needed to evaluate and dissociate potential involvement of dorsal pulvinar in attention, motivation and emotion processing. For example, a task similar to the reward modulation task presented in (Wilke et al., 2013), where two targets provide a different amount of reward in combination with attentional cueing of one the targets similar to (Petersen et al., 1987), either instructing the monkeys to pick the high or low reward option or letting them choose between the options, would enrich our understanding of 
the involvement of dorsal pulvinar in attentional and motivational processing and decisionmaking.

\subsection{Contributions and Acknowledgements}

LS, AUDV, MW and IK designed the experiments, AUDV and LG collected data and performed initial analyses, LS analyzed the data, LS, MW and IK wrote the paper.

Lukas Schneider (LS); Melanie Wilke (MW); Igor Kagan (IK); Adán Ulises Domínguez Vargas (AUDV), Lydia Gibson (LG).

We thank Ira Panolias, Daniela Lazzarini, Sina Plümer, Klaus Heisig, and Dirk Prüße for technical support. We also thank Stefan Treue, Alexander Gail, Hansjörg Scherberger, members of the Decision and Awareness Group, Sensorimotor Group and the Cognitive Neuroscience Laboratory for helpful discussions. Supported by the Hermann and Lilly Schilling Foundation, German Research Foundation (DFG) grants WI 4046/1-1 and Research Unit GA1475-B4, KA 3726/2-1, CNMPB Primate Platform, and funding from the Cognitive Neuroscience Laboratory. 


\section{Eye position signals in the dorsal pulvinar during fixation and goal-directed saccades}

(This is a manuscript soon to be submitted for publication)

Abstract

Most sensorimotor cortical areas contain eye position information thought to ensure perceptual stability across saccades and underlie spatial transformations supporting goaldirected actions. One pathway by which eye position signals could be relayed to and across cortical areas is via the dorsal pulvinar. Several studies demonstrated saccade-related activity in the dorsal pulvinar and we have recently shown that many neurons exhibit postsaccadic spatial preference long after the saccade execution. In addition, dorsal pulvinar lesions lead to gaze-holding deficits expressed as nystagmus or ipsilesional gaze bias, prompting us to investigate the effects of eye position. We tested three starting eye positions $\left(-15^{\circ} / 0^{\circ} / 15^{\circ}\right)$ in monkeys performing a visually-cued memory saccade task. We found two main types of gaze dependence. First, $\sim 50 \%$ of neurons showed an effect of static gaze direction during initial and post-saccadic fixation. Eccentric gaze preference was more common than straight ahead. Some of these neurons were not visually-responsive and might be primarily signaling the position of the eyes in the orbit, or coding foveal targets in a head/body/world-centered reference frame. Second, many neurons showed a combination of eye-centered and gaze-dependent modulation of visual, memory and saccadic responses to a peripheral target. A small subset showed effects consistent with eye position-dependent gain modulation. Analysis of reference frames across task epochs from visual cue to postsaccadic target fixation indicated a transition from predominantly eye-centered encoding to representation of final gaze or foveated spatial location. These results show that dorsal pulvinar neurons carry information about the orbital gaze position, which could contribute to steady gaze during postural changes and to reference frame transformations for visuallyguided eye and limb movements. 


\subsection{Introduction}

Information about eye position is ubiquitous in the primate brain and is critical for visuallyguided behavior. Neurons modulated by the position of the eyes in the orbit have been reported in brain stem nuclei (Hernández et al., 2019; Luschei and Fuchs, 1972), superior colliculus (Campos et al., 2006; Van Opstal et al., 1995), thalamus (Schlag-Rey and Schlag, 1984; Tanaka, 2007; Wyder et al., 2003), cerebellum (Noda and Warabi, 1982) and cortical regions including visual and fronto-parietal cortices (Andersen et al., 1990; Morris et al., 2013; Squatrito and Maioli, 1996; Wang et al., 2007). These eye position signals might subserve different functions. In the visual/oculomotor domain eye position signals could enable stable vision, the discrimination between self- and external motion, precise stimulus localization across eye movements, and post-saccadic updating (Sommer and Wurtz, 2008; Wurtz et al., 2011a). During inter-saccadic periods, these signals might enable stable fixation, including vergence of the eyes and smooth pursuit (Squatrito and Maioli, 1996). In the context of limb and body movements, eye position signals might serve to transform the retinal location of visual stimuli into head-, limb- or trunk-centered reference frames (Andersen et al., 1993; Colby, 1998; Pouget and Snyder, 2000). In most experimental situations, the head is immobilized, so the position of eyes in the orbit is equivalent to gaze direction, or angle.

Taking the current gaze angle into account is important for the visual guidance of movements since visual inputs enter the brain in eye-centered (retinocentric) coordinates, but control of limb movements requires locating the objects in respect to the body and the world. A common model of how the brain deals with those spatial reference frame transformations are the so called "gain fields", manifested as a modulation of sensory-evoked, motor preparation or movement-evoked responses by the current eye position on a single neuron level, such that the neural population response simultaneously represents the retinal and body-centered stimulus location (Andersen et al., 1990; Cohen and Andersen, 2002; Pouget and Snyder, 2000; Salinas and Abbott, 2001). Neurons that exhibit a modulation of visual responses by eye position were first reported in the intralaminar nuclei of the thalamus (Schlag et al., 1980) and the superior colliculus (SC) in cats (Peck et al., 1980), and later in monkeys in the ventral (retinotopically-organized) pulvinar (Robinson et al., 1990). A systematic assessment of spatial tuning at different eye positions revealed gain field properties across numerous cortical regions, including parietal areas (e.g. LIP, VIP, MIP, area 7a), the frontal eye fields (FEF), posterior cingulate and middle superior temporal area MST (Andersen et al., 1990; Bremmer et al., 1999, 2002; Caruso et al., 2018; Dean and Platt, 2006; Galletti et al., 1995; Lehky et al., 2016; Squatrito and Maioli, 1996). 
It is not entirely clear how this eye position information is distributed across cortical areas. A region that could possibly fulfill this function is the dorsal pulvinar (Arcaro et al., 2018; Sherman and Guillery, 2002). The dorsal pulvinar (dPul), consisting of the medial pulvinar and dorsal part of the lateral pulvinar, is in a good anatomical and functional position to transfer gaze-related information to and across fronto-parietal and superior temporal cortices (Bridge et al., 2015; Grieve et al., 2000; Halassa and Kastner, 2017; Wurtz et al., 2011a). The dorsal pulvinar receives direct input from the intermediate and deep layers of the superior colliculus (Baldwin and Bourne, 2017; Benevento and Standage, 1983) and is reciprocally interconnected with prefrontal (FEF, dIPFC), premotor (PMd), posterior parietal (LIP, MIP, VIP, area 7) and superior temporal sulcus regions such as MST and TPO (Romanski et al., 1997; Cappe et al., 2012; Yeterian and Pandya, 1989; Gutierrez et al., 2000; Kaas and Lyon, 2007). Beyond its anatomical connections, electrophysiological and lesion studies suggest a critical role of the dorsal pulvinar in spatial attention (Fiebelkorn et al., 2019; Petersen et al., 1987) as well as visuomotor processes including the control of eye movements. Specifically, response properties of the dorsal pulvinar in monkeys partially resemble its diverse cortical projection targets such as parietal cortex, e.g. enhancement for visual stimuli that indicate an upcoming saccade target (Robinson, 1993). While the dorsal pulvinar is not retinotopically organized and its neurons have large receptive fields, they discharge in the context of saccade tasks in visual cue and saccade execution phases, exhibiting overall preference for contralateral visual cue, peri- and post-saccadic responses (Benevento and Port, 1995; Dominguez-Vargas et al., 2017; Petersen et al., 1985a).

Unilateral pharmacological inactivation of the dorsal pulvinar results in decreased ability to shift attention into the contralesional field (Robinson and Petersen, 1992) and a saccade choice bias towards the ipsilesional field (Wilke et al., 2010, 2013). Human patient studies are largely consistent with those results, showing contralesional spatial deficits (Arend et al., 2008b; Karnath et al., 2002; Van der Stigchel et al., 2010). At the same time, inactivation or structural lesions have comparatively modest effects on basic saccade parameters, mostly consisting of decreased latencies for ipsilesional visually-guided saccades and increased latencies for contralesional memory-guided saccades (Wilke et al., 2010, 2013). In contrast to primary oculomotor regions (e.g. SC, FEF or IML/central thalamus (Schiller and Tehovnik, 2005; Tanaka and Kunimatsu, 2011)), relatively high currents (>150 $\mu \mathrm{A})$ are necessary to evoke saccades and those are small, infrequent and might depend on behavioral context (Dominguez-Vargas et al., 2017). Apart from eye movement selection, the dorsal pulvinar also plays a critical role in other visuomotor behaviors such as reaching and grasping; its neural activity correlates with reach movements (Acuña et al., 1990; Cudeiro et al., 1989) and inactivation/lesions in monkeys and humans lead to hand- and space-specific deficits in reach and grasp tasks (Wilke et al., 2010, 2018). 
Based on those anatomical and functional results it has been proposed that the dorsal pulvinar is involved in reference frame transformations critical for eye-hand behavior, although this has not been directly demonstrated (Bridge et al., 2015; Grieve et al., 2000). In fact, very basic questions such as whether dorsal pulvinar neurons carry eye position signals and how those interact with visual cue responses have not been addressed. Even the effect of static eye position on ongoing firing has not been tested, although the presence of tonic firing during initial and post-saccadic fixation (Dominguez-Vargas et al., 2017) as well as nystagmus and smooth pursuit deficits following dorsal pulvinar inactivation/lesions suggest that it might be involved in gaze stabilization as well (Ohtsuka et al., 1991; Wilke et al., 2010, 2018).

Here we investigated the effect of eye position on initial fixation, visual, memory, saccade and post-saccadic responses in monkeys performing a memory-guided saccade task. We demonstrate that about half of dorsal pulvinar cells are modulated by steady gaze position before visual cue onset and long after the saccade. We also demonstrate a gaze-dependent modulation of visual and saccadic activity, providing a possible substrate for head- or bodycentered spatial representations. 


\subsection{Materials and Methods}

All experimental procedures were conducted in accordance with the European Directive 2010/63/EU, the corresponding German law governing animal welfare, and German Primate Center institutional guidelines. The procedures were approved by the responsible government agency (LAVES, Oldenburg, Germany).

\subsubsection{Animal preparation}

Two adult male rhesus monkeys (Macaca mulatta) C and L weighing 8 and $9 \mathrm{~kg}$ respectively, were used. In an initial surgery monkeys were implanted with a magnetic resonance imaging (MRI) compatible polyetheretherketone (PEEK) headpost embedded in a bone cement headcap (Palacos with Gentamicin, BioMet, USA) anchored by ceramic screws (Rogue Research, Canada), under general anesthesia and aseptic conditions. MR-visible markers were embedded in the headcap to aid the planning of the chamber in stereotaxic space with the MR-guided stereotaxic navigation software Planner (Ohayon and Tsao, 2012). A separate surgery was performed to implant a PEEK MRI-compatible chamber(s) (inside diameter $22 \mathrm{~mm}$ ) allowing access to the pulvinar (Monkey $\mathrm{C}$, right hemisphere: center at $0.5 \mathrm{~A} / 14.5 \mathrm{~L} \mathrm{~mm}$, tilted -11P / 27L degrees; Monkey L, right hemisphere: center at $-3.12 \mathrm{P}$ / 20.2L mm, tilted: -18P/37L degrees; Monkey L, left hemisphere: center at -3P/20L, tilted: 18P/-38L). After confirming chamber positioning with a post-surgical MRI, a partial craniotomy was made inside the chamber. The exposed dura was covered with a silicone elastomer (Kwik-sil, World Precision Instruments, USA) to reduce the granulation tissue growth and dura thickening.

\subsubsection{MRI imaging}

Monkeys were scanned in a 3T MRI scanner (Siemens Magnetom TIM Trio). Full-head T1weighted scans (3D magnetization-prepared rapid gradient-echo, MPRAGE, $0.5 \mathrm{~mm}$ isometric) were acquired before and after chamber implantation, in awake (monkey $\mathrm{C}$ ) or anaesthetized (monkey L) state, using either built-in gradient body transmit coil and custom single loop receive coil, or custom single loop transmit and 4-channel receive coil (Windmiller Kolster Scientific, USA).

In addition to pre- and post-implantation scans, similar T1-weighted scans as well as T2weighted (rapid acquisition with relaxation enhancement, RARE, $0.25 \mathrm{~mm}$ in plane, $1 \mathrm{~mm}$ slice thickness) scans were periodically acquired during the course of experiments, either in awake (monkey $\mathrm{C}$ ) or sedated (monkey $\mathrm{L}$ ) state, to confirm electrode positioning. T1- and T2-weighted scans were co-registered and transformed into "chamber normal" (aligned to the chamber vertical axis) and to anterior commissure - posterior commissure (AC-PC) space for electrode targeting and visualization. These images were acquired with the chamber and the 
grid filled with gadolinium (Magnevist, Bayer, Germany)/saline solution (proportion 1:200), with tungsten rods inserted in predefined grid locations, for alignment purposes.

\subsubsection{Gaze modulation task}

Monkeys sat in a dark room in custom-made primate chairs with their heads restrained 30 cm away from a 27" LED display (60 Hz refresh rate, model HN274H, Acer Inc. USA), covering a range of 100 visual degrees. The gaze position of the right eye was monitored at $220 \mathrm{~Hz}$ using an MCU02 ViewPoint infrared eyetracker (Arrington Research Inc. USA). All stimulus presentation and behavioral control tasks were programmed in MATLAB (The MathWorks, Inc. USA) and the Psychophysics Toolbox (Brainard, 1997).

The structure of the task is shown in Figure 1A. A trial started with the onset of the fixation spot of $1^{\circ}$ diameter either in the center or $15^{\circ}$ left or right to the center of the screen. After the monkey acquired and held fixation within a $5^{\circ}$ radius for $500 \mathrm{~ms}$, a peripheral cue (also $1^{\circ}$ diameter) was displayed for $300 \mathrm{~ms}$ signaling the upcoming saccade target location. For each trial, one out of eight cue/target locations were used. These eight positions were arranged in a virtual rectangular around the initial fixation spot, at $0^{\circ}, 15^{\circ}$ or $-15^{\circ}$ horizontally and $0^{\circ}, 10^{\circ}$ or $-10^{\circ}$ vertically, resulting in 24 different spatial conditions (eight target locations for each of the three initial fixation positions). Monkeys were required to maintain fixation throughout the cue period and also throughout the subsequent memory period (1000 ms), after which the central fixation spot disappeared, allowing monkeys to saccade to the instructed target location. This time point will be referred to as the "Go signal". After a saccade and fixation inside a $5^{\circ}$ radius window surrounding the remembered target location for $200 \mathrm{~ms}$ the target became visible. After additional $500 \mathrm{~ms}$ of peripheral fixation the trial was completed and the monkey obtained a liquid reward after a delay of $200 \mathrm{~ms}$. The intertrial interval for successful and unsuccessful trials was $2500 \mathrm{~ms}$. All initial fixation positions (3) and retinocentric target locations (8) were pseudo-randomized.

\subsubsection{Data analysis}

\subsubsection{Saccade definition}

Saccade velocity was calculated sample by sample as the square root of the sum of squared interpolated ( $220 \mathrm{~Hz}$ to $1 \mathrm{kHz}$ ) and smoothed (12 ms moving average rectangular window) horizontal and vertical eye position traces, and then smoothed again (12 ms moving average rectangular window). Saccade onset was defined as the first eye position change that exceeded a starting velocity threshold of $300 \%$ s.

\subsubsection{Dataset and unit selection criteria}

All recorded voltage drops that surpassed an online visually determined threshold were defined as potential spikes. Spike sorting was done in Offline Sorter v.3.3.5 (Plexon, USA), 
using a waveform template algorithm after defining templates by manually clustering in principle component space.

In total, 325 single and multi-units were recorded in the dorsal pulvinar in 22 sessions where monkeys performed the gaze modulation task (monkey C; right hemisphere: 134, monkey L; left hemisphere: 191). Out of these, 275 units (93 monkey C, 182 monkey L) fulfilled analysis selection criteria (stable discriminability across time and reasonable signal to noise ratio assessed by inspection). 268 out of these 275 units ( 86 monkey C, 182 monkey L) were recorded for at least 4 successful trials for each of the initial gaze positions. These 268 units were used for initial gaze analysis. 238 out of these 268 units (60 monkey C, 178 monkey L) were recorded for at least 4 successful trials for each combination of initial gaze and retinocentric target location. These 238 units were used for further gaze dependent analysis.

\subsubsection{Epoch definitions and modulation}

For each trial, and each epoch of interest, firing rates were computed by counting spikes within the epoch and dividing by the epoch duration. The following epochs were analyzed: "fixation hold" (last $300 \mathrm{~ms}$ of central fixation), "cue onset" (50 ms to $150 \mathrm{~ms}$ after cue onset), "memory" (last $300 \mathrm{~ms}$ of the memory period),"pre-saccadic" (100 to $10 \mathrm{~ms}$ before saccade onset), "peri-saccadic" (10 ms before to $50 \mathrm{~ms}$ after saccade onset), "post-saccadic" (first $150 \mathrm{~ms}$ after acquiring the invisible peripheral target), "target onset" (20 ms to $120 \mathrm{~ms}$ after the target became visible), and "target hold" (last 300 ms of fixating the peripheral target).

For analysis of basic response types in the entire population (Supplementary Figure 1), trials were either grouped by retinocentric or screen-centered target location. We performed two ANOVAs on firing rates of each unit in each epoch: a two-way ANOVA with factors initial gaze position and retinocentric target location and a one-way ANOVA dependent on screencentered target location. Additionally we computed retinocentric hemifield preferences for all units that showed a main effect of retinocentric target location in the respective epoch and screen-centered hemifield preferences for all units that showed an effect of screen-centered target location. To this end, data from all contralateral and ipsilateral hemifield targets were combined. Hemifield tuning in each epoch was determined by unpaired t-tests comparing firing rates in ipsilateral trials to firing rates in contralateral trials. The hemifield with the higher firing rate was marked, if there was a significant difference.

Enhancement or suppression of neuronal activity in each epoch was defined by paired t-tests comparing firing rates to a respective preceding baseline epoch, independently for ipsilateral and contralateral trials. For the fixation hold epoch inter-trial interval served as baseline, for cue onset and memory epochs the fixation hold epoch served as baseline. The memory epoch served as baseline for all subsequent epochs. Enhancement or suppression was 
reported, if either ipsilateral, contralateral, or both types of trials showed significant difference to fixation baseline. In rare cases where one hemifield would show a significant enhancement, while the other hemifield showed suppression, the unit was reported to have bidirectional response.

\subsubsection{Peri-stimulus time histograms (PSTHs)}

Spike density functions were computed at a bin size of $10 \mathrm{~ms}$ using a Gaussian kernel $(\square=20$ ms). Example PSTHs show spike densities averaged across all trials with the same initial fixation position. For population PSTHs, we first normalized spike density functions for each unit by dividing by the average firing rate in the fixation hold epoch (across all trials), and computed average spike density and standard error (across units) for each initial gaze position and contralateral/ipsilateral retinocentric target positions individually.

\subsubsection{Gaze position dependent analysis}

To evaluate effects of gaze position on neuronal activity of each unit, we analyzed initial and final gaze position effects. An effect of initial gaze position was determined by a one-way ANOVA on firing rates during the fixation hold epoch with 3 initial gaze positions as the independent factor, and an effect of final gaze position was determined by a one-way ANOVA on firing rates during the target hold epoch with 15 final gaze positions as the independent factor. To dissociate horizontal and vertical gaze tuning, an additional two-way ANOVA was performed with factors horizontal and vertical final gaze position.

To see if gaze dependence varied systematically with horizontal and/or vertical gaze position, units with a main effect along the respective dimension were further grouped by monotonic and non-monotonic gaze-dependent activity. Monotonic gaze dependence was defined by a) a significant difference between firing rates for the most peripheral positions (unpaired t-test) and b) no significant opposite difference in firing rates between any two neighboring positions. All other units that showed an effect in the ANOVA were classified as non-monotonic. Units showing non-monotonic initial gaze preference were further grouped into central preference (highest activity for straight ahead gaze) and peripheral preference (lowest activity for straight ahead gaze). For better visualization, firing rates for each unit were normalized by dividing average responses for each of the three initial/final gaze positions by the maximum average firing rate across all positions.

To see how task contingency affected gaze dependent responses, we compared initial (fixation hold) and final (target hold) gaze contralaterality indices across all units. Contralaterality indices were computed as $(C-I) /(C+I)$, where $C$ and $\mathrm{I}$ are the average firing rates in contralateral and ipsilateral gaze positions, relative to the center of the screen. For 
final gaze contralaterality indices 6 ipsilateral and 6 contralateral gaze positions were combined. Straight ahead gaze positions were not used for this analysis.

\subsubsection{Gaze-dependent modulation of spatially-contingent task epochs}

To evaluate the presence of not purely retinocentric response fields for each unit, average firing rates in each epoch were tested with a two-way ANOVA, using the factors initial gaze position and retinocentric target location.

To evaluate the relationship of gaze and cue tuning across units, we first correlated initial gaze and retinocentric cue spatial preferences and second tested if there was a difference in response strength by comparing absolutes of gaze and cue spatial preferences using Wilcoxon's signed rank test. In total, we performed four different comparisons: Spatial preferences were either taken as raw firing rate differences (C-I, where $\mathrm{C}$ and $\mathrm{I}$ are the average firing rates in contralateral and ipsilateral trials) or contralaterality indices $(\mathrm{C}-\mathrm{I}) /(\mathrm{C}+\mathrm{I})$; and gaze preference was either derived from fixation hold (Fhol) or Cue onset (Cue) epoch. Spatial cue preference was computed using all 3 gaze positions, combining all cues contralateral or ipsilateral relative to the current gaze.

\subsubsection{Modulation of retinocentric encoding by gaze position}

To evaluate the presence of gain fields for each unit and each epoch, we used a model-free approach that does not assume any specific shape of the tuning function. First, we computed confidence intervals for tuning vector length and direction, independently for each initial gaze position using hierarchical bootstrapping. 1000 bootstrapping iterations were performed for each unit, epoch and initial gaze position, sampling 10 trials (with replacement) for each retinocentric target position. Normalized tuning vectors for each sampled trial were computed with direction equal to the direction of the retinocentric target position and the length equal to the firing rate. The average tuning vector for the current bootstrap iteration was then computed as the sum of normalized firing rate vectors across all sampled trials. To evaluate significance of gain and tuning vector shifts, the 95\% confidence intervals of differences in amplitude and direction of the bootstrapped tuning vectors for each pair of initial gaze positions was computed, independently for each epoch. Units in which at least for one of these comparisons between gaze positions, the confidence interval of amplitude or direction differences did not overlap with zero were marked as showing gain field properties or response field shifts, respectively. This analysis was performed only on units that showed a main effect of retinocentric target location in the respective epoch in the two-way ANOVA mentioned before.

To estimate gain effect size, we calculated the percent change in the amplitude of the average bootstrapped tuning vector across three initial gaze positions for each unit as 
$100 *$ (Amax-Amin)/Amax, where Amax is the amplitude of the largest and Amin the amplitude of the smallest of the three average tuning vectors.

\subsubsection{Reference frame estimation}

To see if pulvinar responses were better explained by a retinocentric or screen-centered reference frames (i.e. relative to the locations on the screen, which could signify head-, body, or world-centered representation, since the head was immobilized relative to the screen and the body orientation was not explicitly controlled), we grouped the trials either by retinocentric target location or by the target location on the screen, and compared average correlation coefficients (ACCs) in both arrangements for each unit (Mullette-Gillman et al., 2005). For each reference frame, ACCs were computed by correlating responses for two out of three initial gaze positions at a time, and then averaging the three correlation coefficients. For screen-centered encoding, we used only locations that were available for both initial gaze positions for every correlation pair. To estimate significance, we performed 1000 bootstrap iterations using $80 \%$ of the trials for each location at a time. This allowed deriving $95 \%$ confidence intervals for both retinocentric and screen-centered ACCs. Significant encoding in the respective reference frame was reported if a) the confidence interval was above zero, b) the confidence interval for this reference frame was above the ACC for the other reference frame, and c) the ACC for this reference frame was above the confidence interval for the other reference frame. In other words, both confidence intervals should be above or below the unity line diagonal. 


\subsection{Results}

We recorded single- and multi-unit activity in two monkeys performing a memory-guided saccade task with variation of initial gaze (fixation) position (Figure 3.1A). Monkeys had to fixate at one of three initial positions, hold fixation while a spatial cue was presented at one of 8 target locations and after a memory period make a saccade towards the cued target location. These 8 potential target locations were arranged in a rectangle around the initial fixation spot, with the same spatial arrangement relative to the fixation spot for each of the three initial gaze positions, see Figure 3.1A and Materials and Methods. Since the monkeys were head-fixed in the straight-ahead direction, the position of the eyes in the orbit is equivalent to gaze direction.

A total of 268 units in the dorsal pulvinar were studied in two monkeys (monkey C: 86, monkey L: 182). For most analyses beyond the effects during initial fixation we focused on the units with more than 4 trials for each combination of initial gaze and target position ( $N=238$, monkey C: 60 monkey L: 178), see Materials and Methods. Normalized population PSTHs of these 238 units are displayed in Figure 3.1B. In the population average we found no apparent dependence on the initial gaze position in the fixation hold epoch, an enhanced contralateral cue response and post-saccadic enhancement.

Neurons exhibited diverse activity patterns; a summary of response modulation and spatial preferences across task epochs is given in Supplemental Figure 3.1. In short, our sample contained $48 \%$ of units with initial fixation responses (24\% enhanced, $24 \%$ suppressed), $31 \%$ with spatial cue dependence (11\% contralateral preference, $7 \%$ ipsilateral preference, and $13 \%$ with a main effect of target location without hemifield preference), $44 \%$ with memory delay period activity (13\% enhanced, 31\% suppressed), 19\% with pre-saccadic activity (8\% enhanced, $11 \%$ suppressed), and 67\% with post-saccadic activity (47\% enhanced, $19 \%$ suppressed). A detailed analysis of dPul neuronal response properties in a more extensive non-overlapping sample will be given in a separate paper (see also (Dominguez-Vargas et al., 2017)). Here, we focus on the activity patterns pertaining to different positions of the eyes in the orbit. 
A

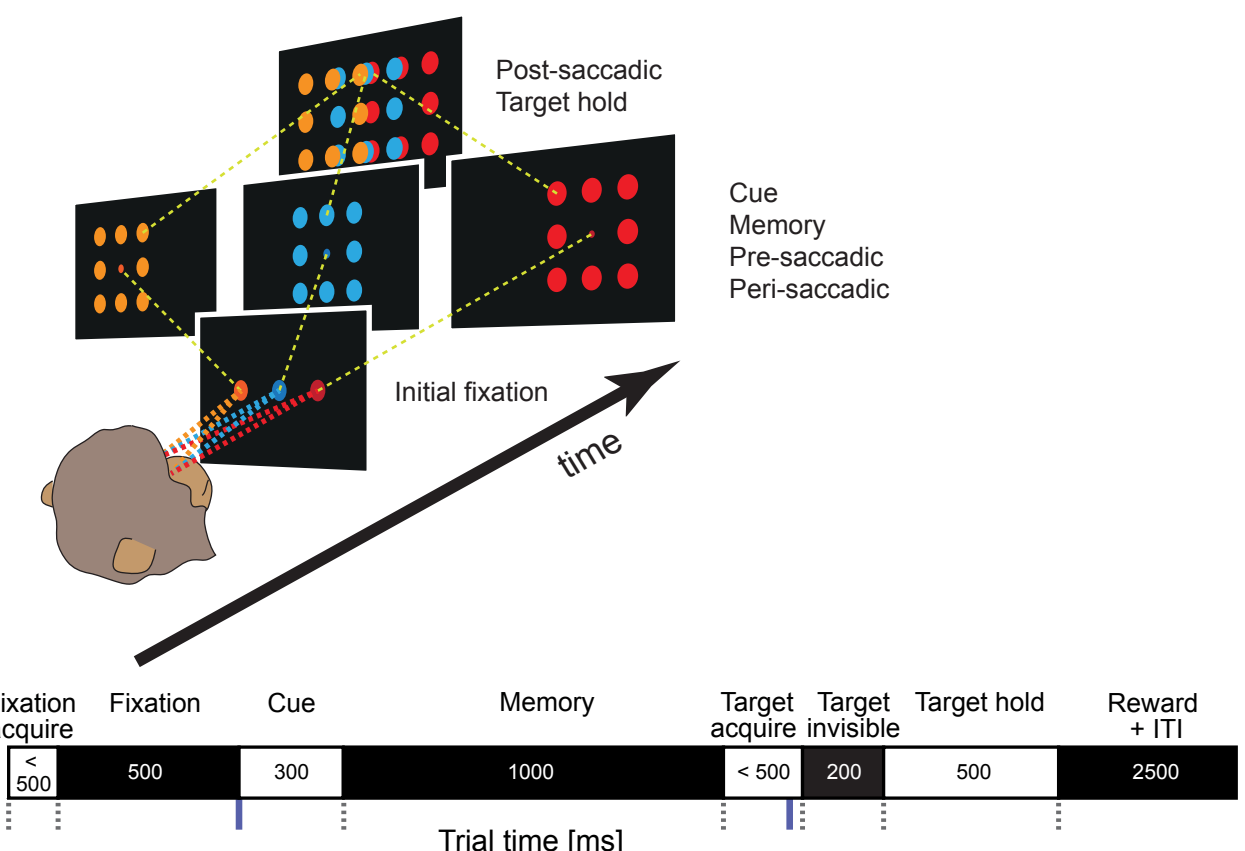

B

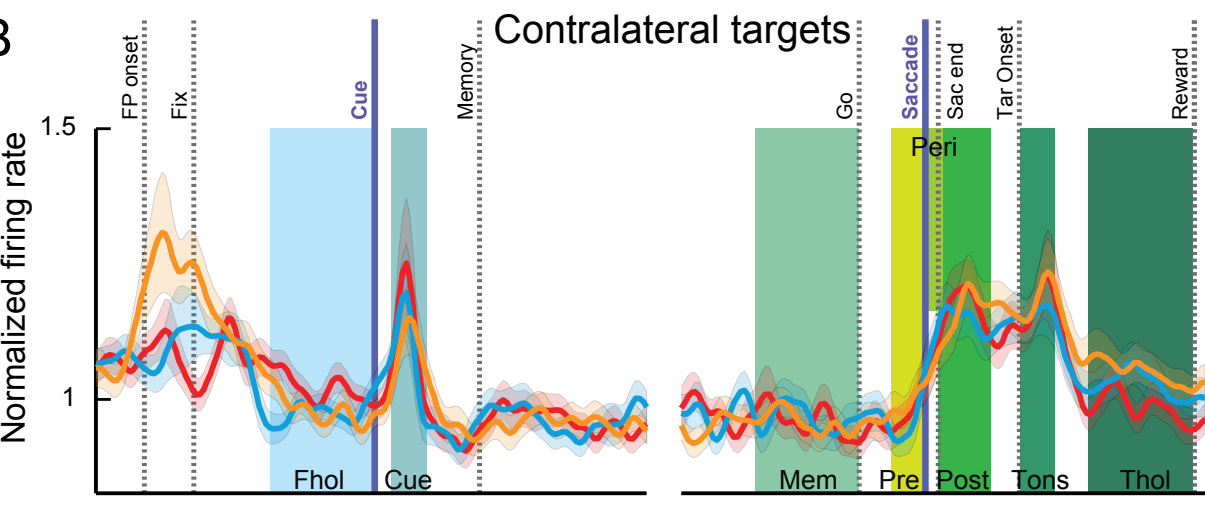

Ipsilateral targets
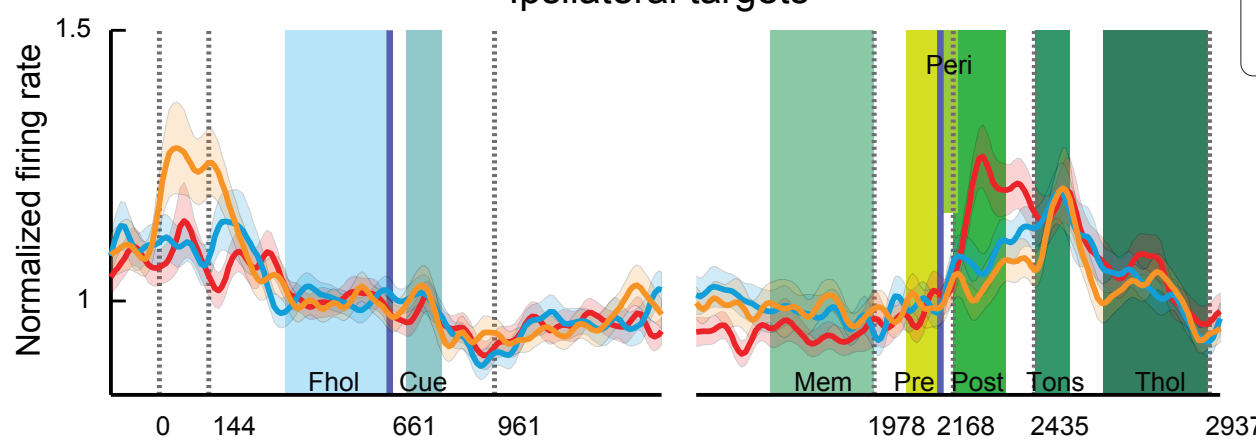

Initial gaze

Contralateral

Straight ahead Ipsilateral

Time from beginning of trial [ms]

Figure 3.1. Task and population average. A) Task conditions. A trial started at one out of three initial fixation positions (Fixation). One out of eight target positions surrounding the fixation spot was flashed (Cue). Monkeys had to remember the location while keeping fixation (Memory), until the fixation spot disappeared, prompting a saccade to the target (Target acquire). After the monkey held fixation for a short period (Target invisible), the target reappeared as a confirmation (Target hold). B) Normalized population PSTHs and standard errors across units for each initial gaze position for contralateral and ipsilateral targets (relative to initial gaze position). Vertical lines indicate average onset of events across all trials: fixation spot onset ("FP onset"), the monkey acquiring fixation ("Fix"), the cue onset, the cue offset and beginning of the memory period, the offset of the central fixation point ("Go"), the saccade onset, the monkey acquiring the invisible target location ("Sac end"), the onset of the confirmation target ("Tar Onset"), and the end of the trial ("Reward"). Discontinuous traces indicate two different alignments to cue onset and saccade onset (purple lines). Colored areas mark analysis epochs: fixation hold (Fhol), cue onset (Cue), memory (Mem), pre-saccadic (Pre), peri-saccadic (Peri), post-saccadic (Post), Target onset (Tons), and target hold (Thol) (see Materials and Methods). 


\subsubsection{Gaze-dependent activity during initial fixation}

Contralateral targets

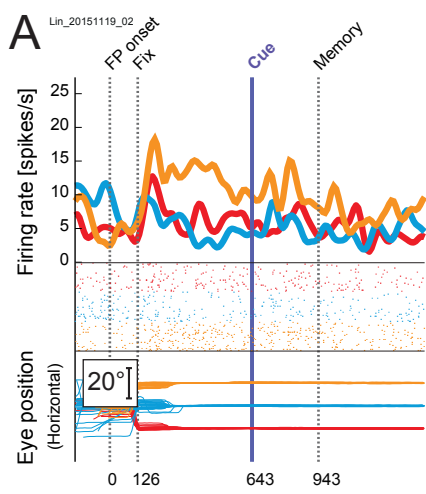

$\mathrm{B}$
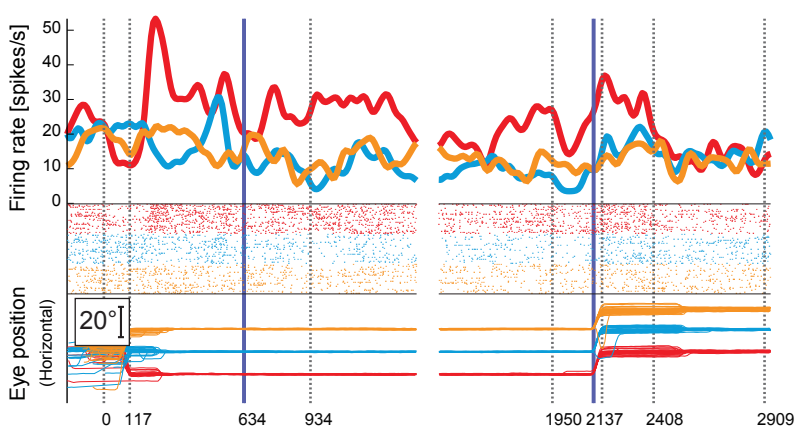

C

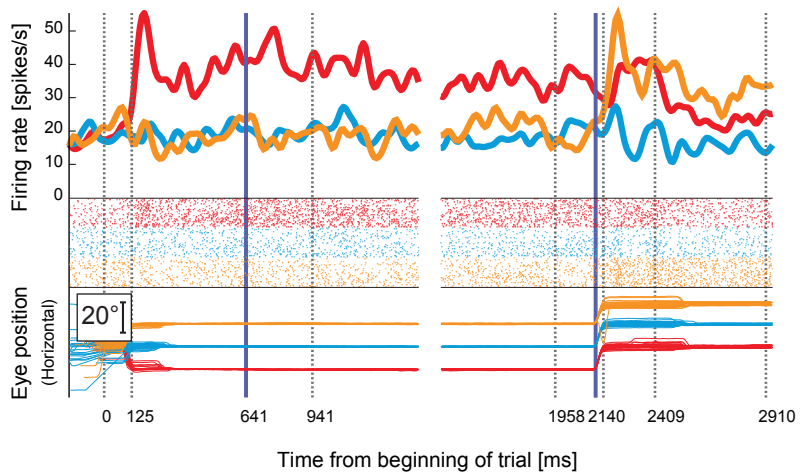

Initial gaze
Ipsilateral targets
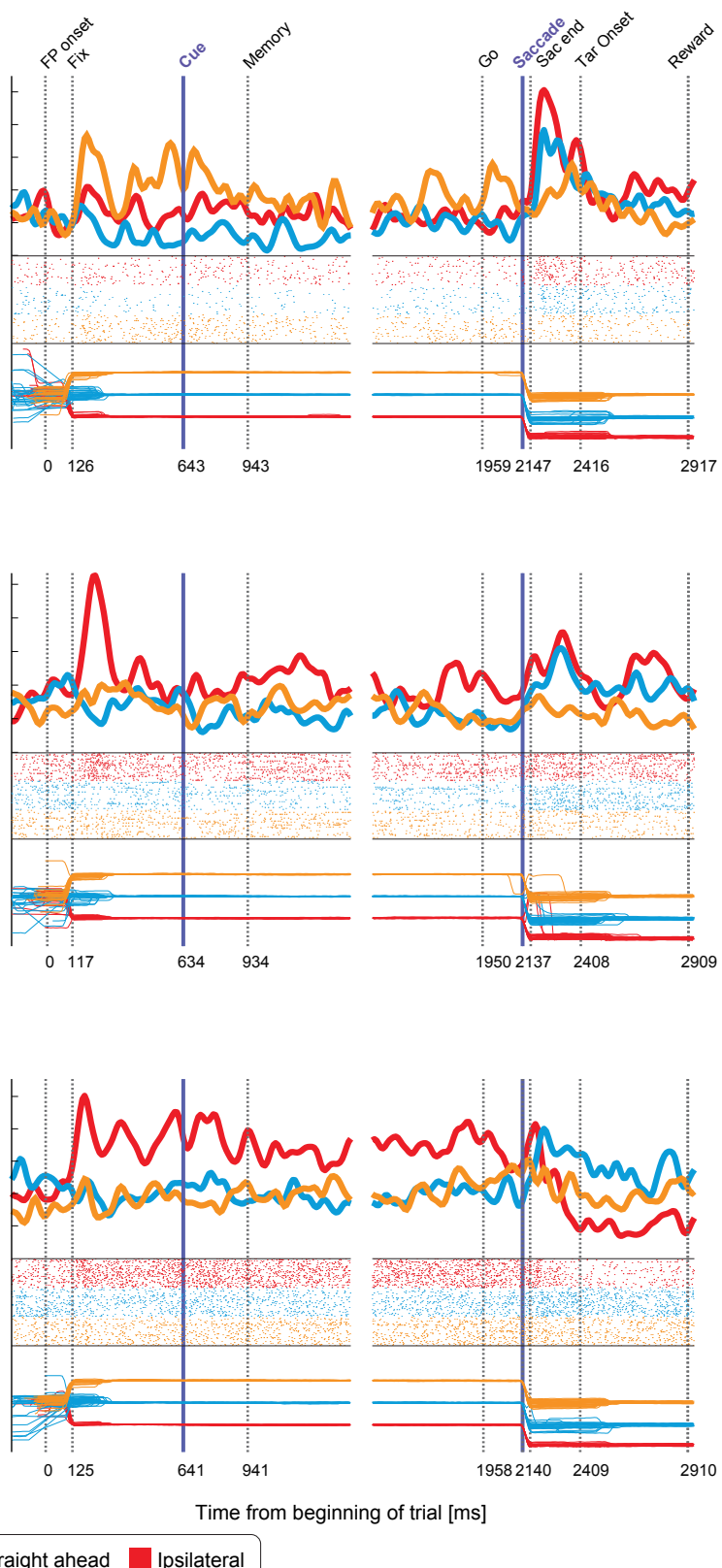

Figure 3.2. Example units with tonic gaze-dependent activity. Example unit raster plots, resulting spike density functions, and horizontal eye traces separately for each initial fixation position, for contralateral (left) and ipsilateral targets (right) relative to initial gaze position. Colors depict the three initial gaze positions (orange: contralateral, blue: central, red: ipsilateral). A) Peripheral (contralateral) gaze preference, B) Ipsilateral gaze preference, C) Ipsilateral gaze preference.

A substantial portion of units (128 out of $268,48 \%$ ) were significantly modulated by the gaze position before the cue was presented (one-way ANOVA). Many of these units maintained gaze dependence after cue presentation (47 out of 128, 37\%). Three example units shown in Figure 3.2 illustrate tonic responses during the initial fixation and subsequent trial epochs. Focusing first on the initial fixation epoch, top example (Figure 3.2A) shows contralateral 
gaze preference and two other examples (Figure 3.2B,C) show ipsilateral preference. Our first question was if such gaze-dependent activity typically increased or decreased towards peripheral contra- or ipsilateral gaze positions, or if more units prefer the straight ahead direction, as has been demonstrated in the visual cortex (Durand et al., 2010; Przybyszewski et al., 2014). To this end, we classified the units which showed a main effect of initial gaze position into units with monotonic gaze-dependent effects (showing peripheral preference), and units that showed either central or peripheral non-monotonic gaze dependence (Materials and Methods).

Figure 3.3A shows normalized firing rates during fixation hold for each unit and each of the three initial gaze positions, grouped by the monotonicity and gaze direction preference. Monotonic responses were more frequent than non-monotonic (91/128 and 37/128 units), but contralateral and ipsilateral preferences were almost balanced in monotonic units (41 and 50 units respectively). Furthermore, in non-monotonic units there was no bias towards the straight ahead direction (18 vs 19 units). If anything, we found an overall preference for peripheral positions (110 units: all monotonic and non-monotonic peripheral-preferring vs. 18 central-preferring units). MR-guided reconstruction of recording sites revealed that units with significant gaze dependence were distributed throughout the sampled locations (mostly in the dorsal medial pulvinar), without a systematic clustering of gaze-dependent patterns (Figure 3.3B). 
A Activity dependent on initial gaze position, $N=128$ out of 268
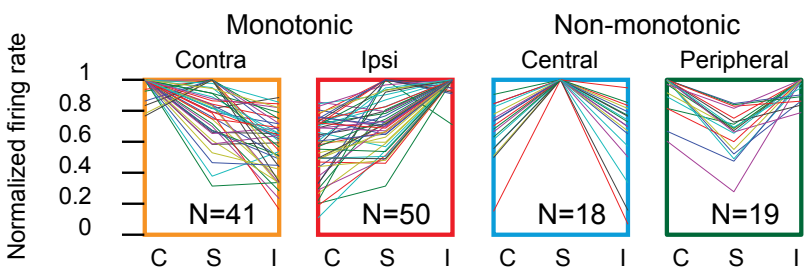

B Recording sites, $\mathrm{N}=268$
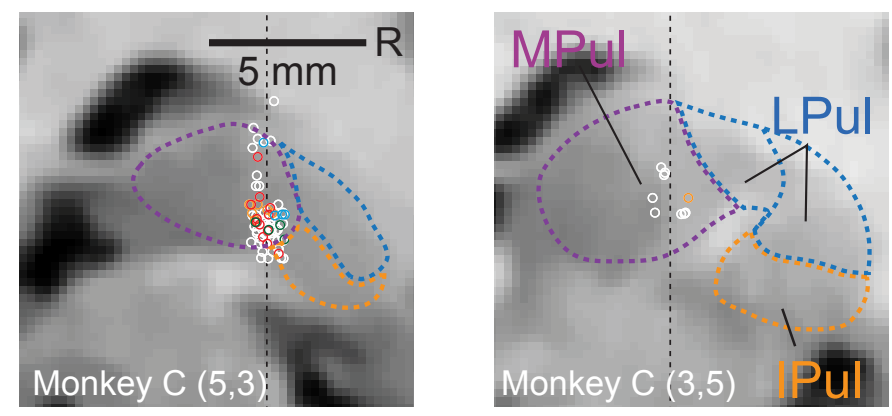

Initial gaze preference

Monotonic

Contralatera

O Ipsilateral

Non-monotonic
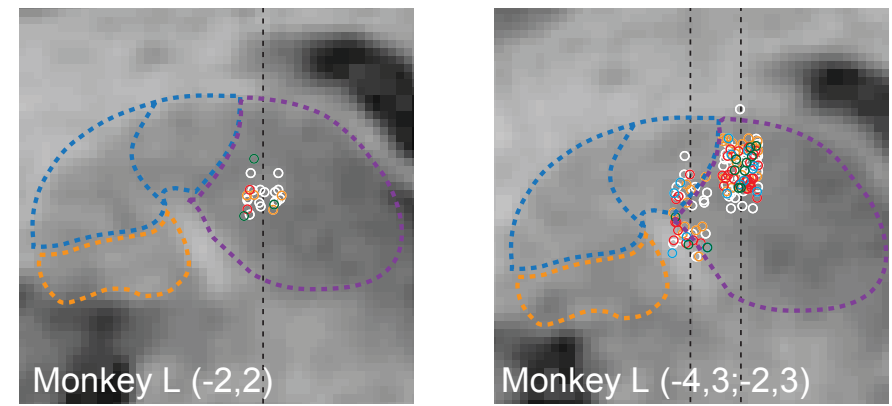

Central

O Peripheral

Nonsignificant

Figure 3.3. Gaze dependence during initial fixation and recording sites. A) Classification of initial gaze dependence. Normalized firing rates of each unit with a significant main effect of gaze on firing rates in the initial fixation epoch. Each line represents one unit. B) Localization of recorded units in chamber-normal coronal sections in each monkey ( $L$ and $C$, labels on the bottom) and specific grid locations relative to the chamber center ( $\mathrm{x}, \mathrm{y}$ in parentheses). Locations were jittered along the horizontal dimension for better visualization. The black dashed lines indicate the projection of penetration tracks and mark the actual horizontal location of recorded neurons. Each dot represents one unit; colors indicate the initial gaze effects of the unit: orange for monotonic contralateral preference, red for monotonic ipsilateral preference, blue for central gaze preference, and green for non-monotonic peripheral preference. Units that did not show a significant effect of gaze are in white. Pulvinar nuclei outlines (MPul/LPul/IPul - medial/lateral/inferior pulvinar) were adapted from the NeuroMaps atlas (Rohlfing et al., 2012), exported via Scalable Brain Atlas, https://scalablebrainatlas.incf.org/macaque/DB09, $\quad$ https://scalablebrainatlas.incf.org/services/rgbslice.php, (Bakker et al., 2015), and LPul was further subdivided to dorsal (PLdm) and ventral (PLvl) parts according to the brachium of the superior colliculus.

\subsubsection{Relationship between initial and final gaze effects}

Next we evaluated the relationship between gaze-dependent activity during initial fixation and final fixation after the saccade (during target hold). Referring back to the examples in Figure 3.2, the top example shows mostly consistent contralateral gaze preference throughout the trial. The middle example shows consistent ipsilateral gaze preference. The bottom example shows inconsistent gaze preference (contralateral for the initial gaze and ipsilateral after the 
saccade). To evaluate these patterns across the population, we compared gaze position preference in fixation hold and target hold epochs (Figure 3.4A). Gaze position preference here was computed as the difference of firing rate averages for contralateral and ipsilateral gaze positions, see schematics in Figure 3.4A and Materials and Methods. Across all units gaze position preference in the two epochs was positively correlated $(R=0.3, p<0.001$, Pearson's correlation), indicating a consistent effect of gaze direction irrespective of the task epoch. As expected, the correlation was mainly driven by units that show both an effect of initial and final gaze (significance derived from two independent one-way ANOVAs; $N=91$, $\mathrm{R}=0.4, \mathrm{p}<0.001$ ). Figure 3.4B shows the results of the two independent ANOVAs. Only 29 units showed an effect of initial gaze but not final gaze position; conversely, 65 units showed only an effect of final gaze position. The high number of units with only an effect of final gaze might be due to a wider range of (final) gaze positions in the target hold epoch.

Next, we asked whether there is any influence of the initial gaze on activity associated with the final gaze after saccade. We performed a two-way ANOVA with the factors initial gaze and final gaze on firing rates in the target hold epoch, using the seven final target positions where the gaze would arrive from multiple starting points (schematic in Figure 3.4B). The outer sectors of Figure 3.4B display the number of units that showed a main effect of the final gaze and an interaction between initial and final gaze. Only $16 \%$ of units that showed an effect of final gaze position in the one-way ANOVA also showed interaction of initial and final gaze in the two-way ANOVA (25/156), indicating that trial history (e.g. preceding saccade direction) did not have a major impact.

Since the final gaze covered more spatial locations, we assessed final gaze-dependent firing rate preferences in 2D space. Figure 3.4C shows color-coded histograms of gaze positions associated with maximum firing rates. Substantially more units preferred peripheral horizontal gaze positions as compared to central gaze positions (chi-square test, $p=0.00021$ for all units, $p=0.00235$ for significant units). This pattern is in agreement with the overall peripheral preferences during the initial fixation (cf. Figure 3.3A). 


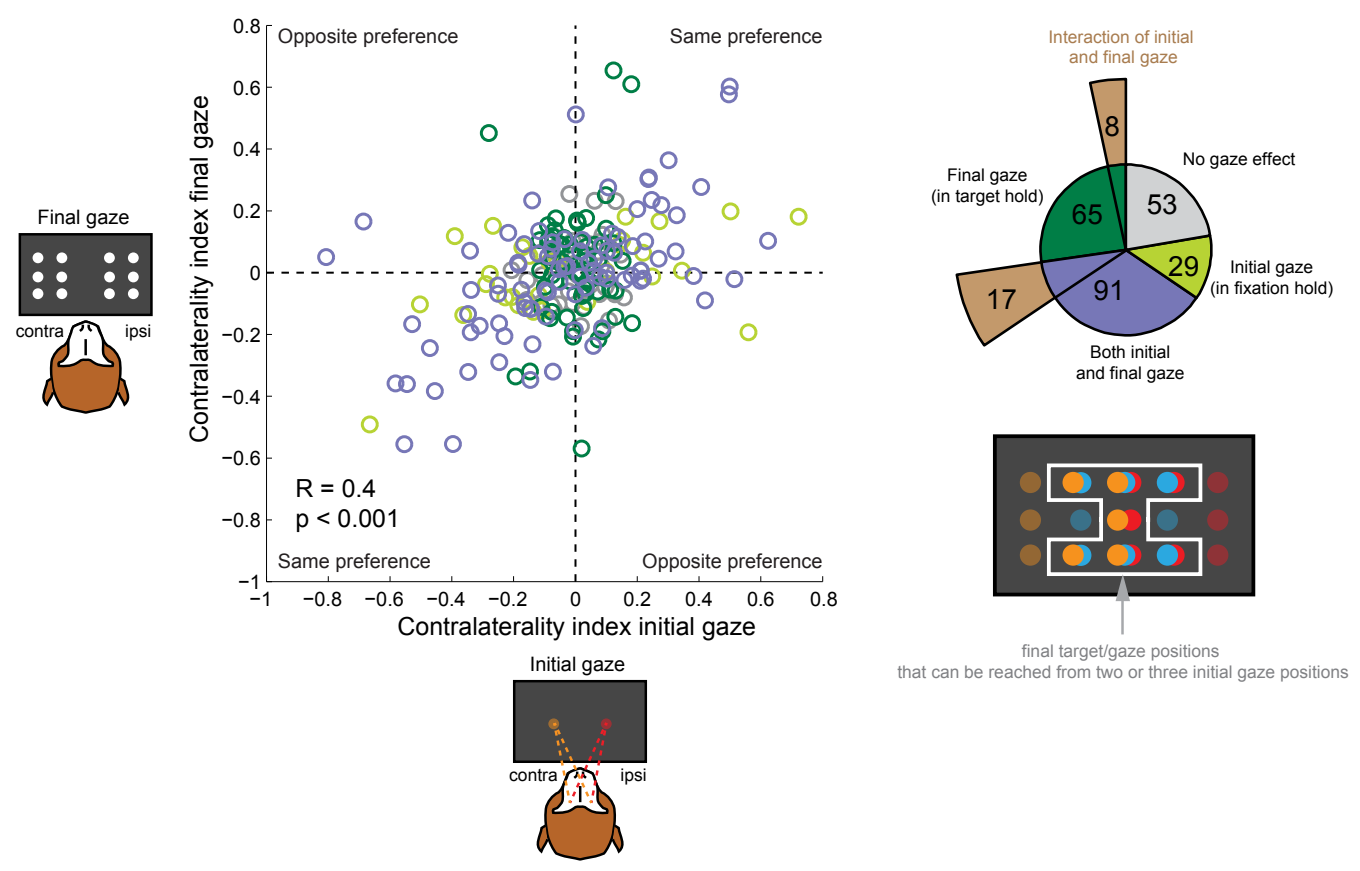

C Histogram of final gaze preference
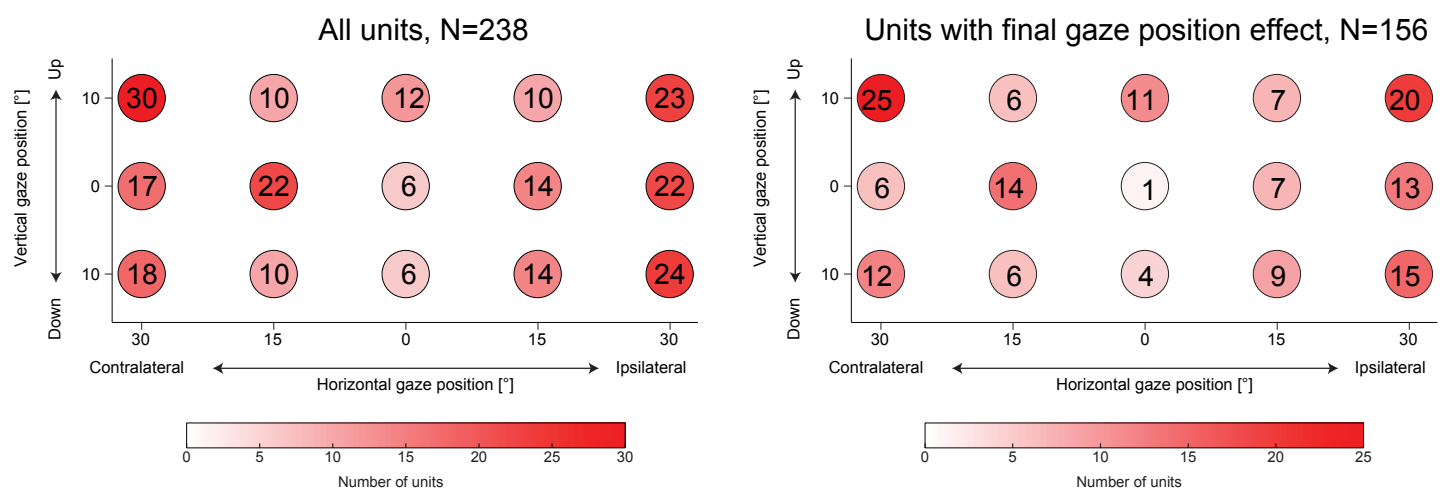

Figure 3.4. Initial vs final gaze-dependent activity. A) Scatter plot of contralaterality indices denoting gaze direction preference in initial fixation (Fhol) vs. final target hold (Thol) epochs. Each circle represents a unit with at least 4 trials for every combination of initial gaze and retinocentric target position $(\mathrm{N}=238)$. Contralaterality indices were computed as difference of firing rates for contralateral and ipsilateral gaze positions, normalized by their sum. For the final gaze positions, 6 gaze positions in each hemifield were averaged, see schematic on the left. Color of each unit depicts the combination of effects: significant initial gaze effect only (lime), significant final gaze only (dark green), both initial and final gaze (purple), or none (gray). Effects of initial and final gaze position were defined by two independent ANOVAs, testing firing rates in Fhol for initial gaze position and firing rates in Thol for final gaze position. B) ANOVA results. Inner sectors: number of units in each group (same colors as in A), derived from the two independent one-way ANOVAs (on initial and final gaze). Outer sectors: number of units showing a main effect of final gaze as well as an interaction effect of the initial and the final gaze in the additional two-way ANOVA that used only the highlighted (white border) target positions depicted in the schematic below (brown). C) 2D histogram of final gaze preferences. Red color intensity indicates the number of units which showed the strongest response for the respective final gaze position. Left panel: all units, right panel: only units showing significant effect of final gaze position. 
Given the sufficiently broad range of final gaze positions, especially in the horizontal dimension, we further quantified the monotonicity of the final gaze effects, separately for horizontal and vertical axes. We performed a two-way ANOVA with factors horizontal and vertical final gaze position on target hold firing rates, in all units that showed an effect of final gaze position in the one-way ANOVA ( $\mathrm{N}=156)$. Table 3.1 shows the number of units that exhibited main effects of horizontal/vertical gaze position, further separated into monotonic and non-monotonic gaze preference. Most units (117/156, 75\%) showed a main effect of horizontal gaze position, but only a minority showed monotonic gaze preference (32/117, $27 \%$ ). Thus, the wider range of final gaze positions (as compared to only 3 initial gaze positions) revealed predominantly non-monotonic gaze dependence. Many units (87/156, $56 \%$ ) showed a main effect of vertical gaze position, around two thirds of them showed monotonic vertical gaze preference $(62 / 87,71 \%)$. This indicates that the vertical gaze position had a strong impact on firing rates, but likely due to the smaller range of the vertical (compared to the horizontal) component less significant effects and more monotonic preference was detected. A more detailed picture of final gaze preferences for each unit can be gained from Supplemental Figure 3.2.

\begin{tabular}{|l|c|c|c|c|}
\hline \multirow{2}{*}{$\begin{array}{c}\text { Units showing an effect of final gaze } \\
\text { position (N=156) }\end{array}$} & Monotonic & Non-monotonic & Nonsignificant \\
\cline { 2 - 4 } & Monotonic & 13 & 26 & 23 \\
\hline Vertical axis & Non-monotonic & 5 & 19 & 1 \\
\cline { 2 - 5 } & Nonsignificant & 14 & 40 & 15 \\
\cline { 2 - 5 } & & & & 19 \\
\hline
\end{tabular}

Table 3.1. Monotonicity in final gaze dependence. Number of units exhibiting an effect of final gaze position, grouped by significant monotonic and non-monotonic main effects of horizontal and vertical gaze position.

\subsubsection{Gaze-dependent modulation of spatially-contingent task epochs}

So far we addressed the effect of gaze position on the neuronal firing during either initial or final (target hold) fixation. Here we ask how the retinocentric spatially-contingent encoding is affected by gaze in the visual cue, memory delay and saccadic epochs. We performed a twoway ANOVA with factors initial gaze position and retinocentric target location for each unit and each epoch of interest, (Figure 3.5A). During cue presentation purely retinocentric encoding (only main effect of retinocentric target location) was less common then some dependence on the gaze position (41/238 units vs. 82/238 units), indicating a strong 
influence of current gaze position on the response strength or tuning pattern. In the memory and peri-saccadic epoch, purely retinocentric encoding became even less common (22/238 vs. $86 / 238$ units and $25 / 238$ vs. $50 / 238$ units respectively). Finally, one third to half of the units showed interaction of initial gaze position and retinocentric target location in the postsaccadic epochs (81/238 in the "post-saccadic" epoch and 114/238 in "target hold"). For an approximate estimation of the false discovery rate due to random firing fluctuations, we performed the same analysis on firing rates in the initial fixation epoch (where no information about target location was available to the monkeys). While nearly half of the units showed only gaze-dependent activity during the initial fixation (108 out of 238), only a small fraction showed a main effect of target location only (10/238 units), both main effects (6/238 units), or any combination with [initial gaze $\times$ target position] interaction (11/238 units), as expected.

The fraction of units with gaze dependence in the cue epoch decreased as compared to initial fixation (120 vs. 82 units). Therefore we asked if the arrival of the new spatial information overrode the initial gaze encoding in some neurons. Out of 120 units that showed an effect of gaze position during initial fixation, 53 maintained gaze dependence (main effect of gaze or interaction) in the cue epoch (and additional 29 acquired gaze dependence). Out of 67 units that did not maintain gaze dependence, only 23 showed a main effect of retinocentric cue position, meaning that for the other 44 units the loss of gaze encoding cannot be explained by replacement with new spatial cue information. Conversely, 22 out of 53 units maintained gaze dependence despite additional encoding of the new retinocentric cue position. This together indicates that the decrease in gaze dependence was not related to cue responses. We also asked, more generally, if the units with the initial gaze effect are less likely to have visual cue responses. Table $\mathbf{3 . 2}$ shows that it is not the case - units with and without an effect of initial gaze position exhibited similar cue response patterns.

\begin{tabular}{|c|c|c|c|c|c|}
\hline \multicolumn{2}{|c|}{ Fixation hold } & \multicolumn{3}{|c|}{ Cue response } \\
\cline { 2 - 6 } & Enhancement & Suppression & Hemifield preference & $\begin{array}{c}\text { ANOVA position } \\
\text { effect }\end{array}$ \\
\hline $\begin{array}{c}\text { Initial gaze } \\
\text { effect } \\
\text { present }\end{array}$ & 120 & 28 & 25 & $\begin{array}{c}33(25 \text { with ANOVA } \\
\text { position effect })\end{array}$ & $45(19 / 10$ en/su $)$ \\
\hline $\begin{array}{c}\text { Initial gaze } \\
\text { effect absent }\end{array}$ & 118 & 23 & 22 & $\begin{array}{c}29 \text { (17 with ANOVA } \\
\text { position effect) }\end{array}$ & $30(13 / 9$ en/su $)$ \\
\hline
\end{tabular}

Table 3.2. Relationship between initial gaze effects and cue responses. Number of units showing enhancement, suppression, hemifield preference and an effect of retinocentric position during cue presentation, separately for units showing an initial gaze effect (in fixation hold, 120 units, cf. Figure 3.4B, 120=91+29) and units showing no effect of initial gaze (118). 
To get a better understanding of how gaze position affected visual responses, we looked into the cue epoch more closely. Example tuning curves for each category illustrate the combinations of gaze and cue dependence defined by the ANOVA (Figure 3.5B). Some units showed a strong modulation by gaze position but no directional cue tuning ("Gaze only"), some showed the similar retinocentric cue tuning regardless of gaze position ("Cue only"), some showed directional cue tuning scaled by gaze ("Gaze and Cue"), and some showed an alteration of preferred direction by gaze ("Gaze, Cue, and interaction").

It should be noted here that some units showed a clear response to the cue onset specifically for one gaze position, but no spatial cue tuning, as illustrated in the example shown in Figure 3.5C. This finding demonstrates that cue responses may be underestimated when looking at only one gaze position, and should be considered in further studies assessing functional significance of cue responses in dorsal pulvinar.

Next we tested if there is a relationship between gaze and spatial cue preference in units showing both types of dependence. We found no correlation between cue and gaze spatial preferences, neither when correlating cue contralaterality indices (Cls) to gaze Cls derived from cue epoch ( $R=-0.04, p=0.513$, Pearson's correlation; see Figure 3.5D), neither when correlating cue Cls with gaze Cls derived from the fixation hold epoch $(R=-0.05, p=0.471$, Pearson's correlation), nor when correlating raw (contra-ipsi) firing rate differences $(R=0.05$, $p=0.478$ for gaze in cue epoch; $R=-0.03, p=0.619$ for gaze in the fixation hold epoch). In fact, many units showed either hemifield preference of gaze position $(\mathrm{N}=56)$ or retinocentric cue location ( $\mathrm{N}=62)$, but only few units showed both $(\mathrm{N}=15)$, with only 6 units showing the same hemifield preference.

To compare the strength of cue and gaze tuning, we computed absolute gaze and cue Cls and raw (contra-ipsi) firing rate differences and tested if there was a difference of the mean ranks (Wilcoxon signed rank test). There was no significant difference between the strength of cue and gaze tuning in any of the comparisons mentioned above ( $p=0.103$ for cue Cls and gaze $\mathrm{Cls}$ in cue, $\mathrm{p}=0.104$ for cue $\mathrm{Cls}$ and gaze Cls in fixation hold, $\mathrm{p}=0.2$ for raw firing rate differences (gaze in cue), $\mathrm{p}=0.295$ for raw firing rate differences (gaze in fixation hold). 
A Retinocentric and gaze-dependent encoding, $N=238$

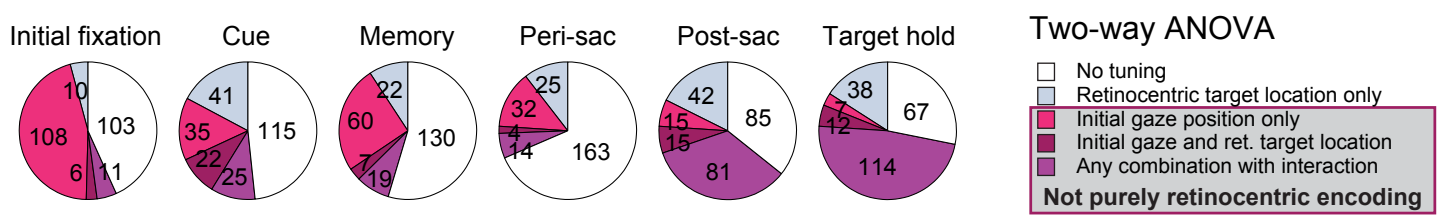

B Examples of cue tuning curves
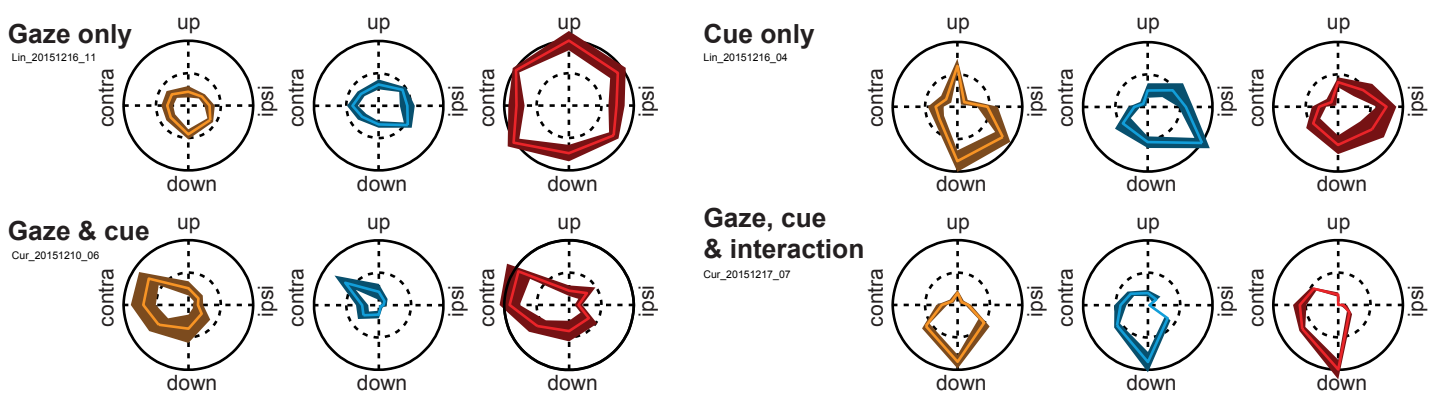

Gaze, cue \& interaction
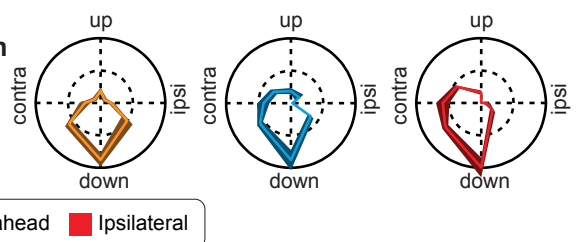

Initial gaze

Contralateral

Straight ahead Ipsilateral

C Non-retinocentric, gaze-dependent cue response (example unit)

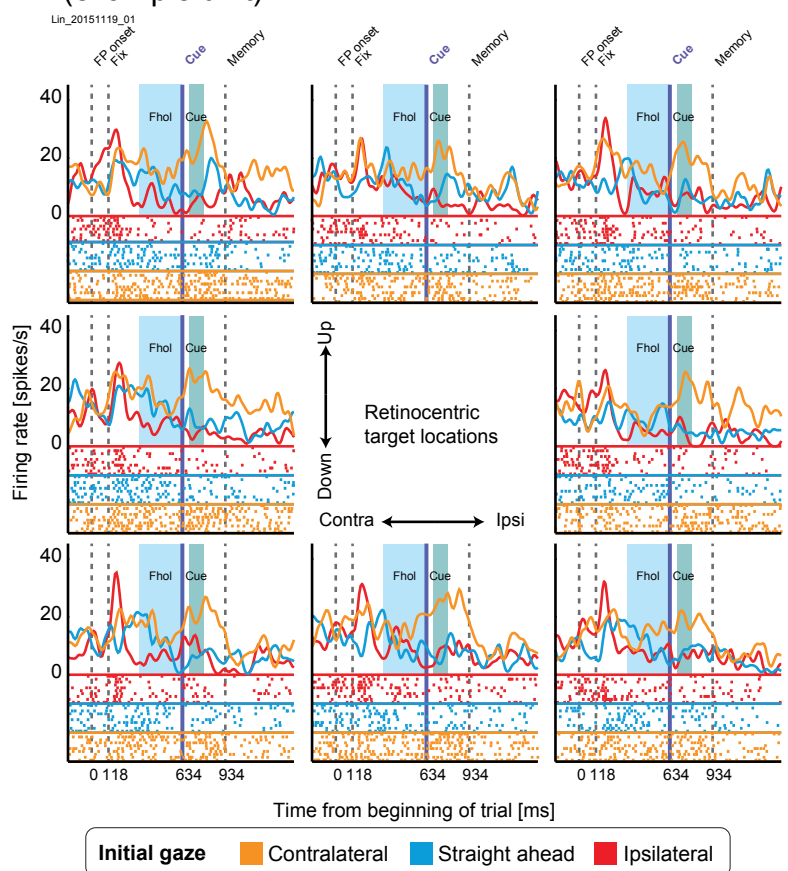

D Gaze versus cue preference
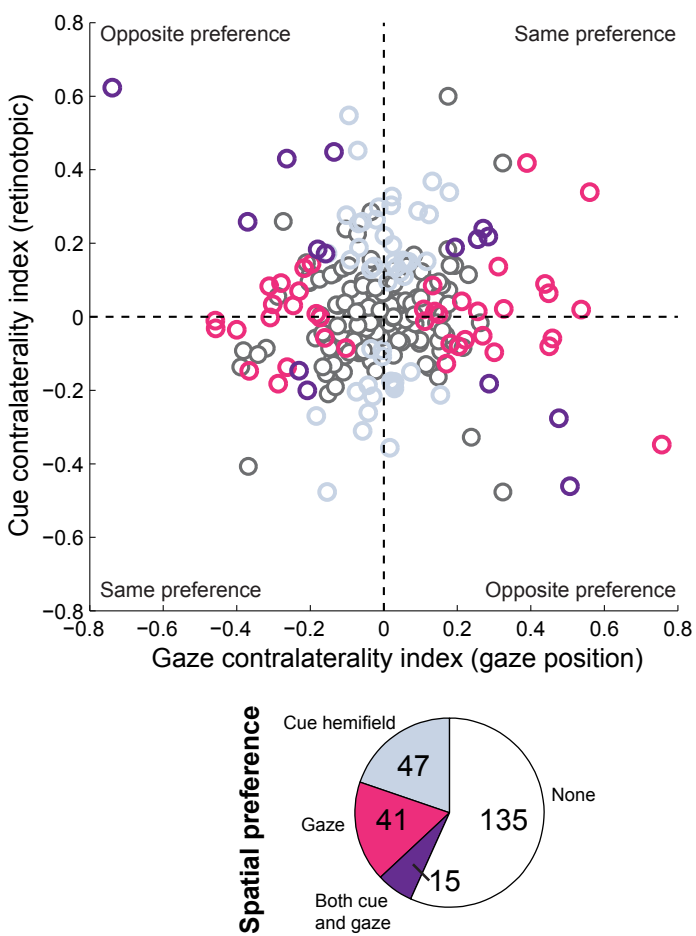

Figure 3.5. Retinocentric and gaze-dependent encoding. A) Two-way ANOVA results, with factors initial gaze position and retinocentric target location, independently for six epochs: fixation hold (Fhol), cue onset (Cue), memory (Mem), peri-saccadic (Peri), post-saccadic (Post), and target hold (Thol). Number of units showing either only a main effect of retinocentric target location, only a main effect of initial gaze position, both main effects, or any combination with [initial gaze position $\times$ retinocentric target location] interaction (plus either no main effect, both main effects, or one of the two main effects). For brevity, pre-saccadic epoch is omitted because the numbers were nearly identical to peri-saccadic epoch, target onset epoch is omitted because the numbers were nearly identical to post-saccadic epoch. B) Polar plots of retinocentric cue responses for each gaze position. One example unit from each category in the two-way ANOVA. C) PSTHs and raster plots of activity around the cue onset for one example unit showing a main effect of gaze (in the cue epoch) only, separately for each retinocentric cue location (in different subplots) and each gaze position (in different colors). D) Top: Scatter plot of cue and initial gaze contralaterality indices. Colors indicate significant hemifield preferences (light blue: cue, magenta: gaze, purple: both cue and gaze, grey: no hemifield preferences). Bottom: pie plot showing the number of units with hemifield preferences for cue, gaze, both or none. 


\subsubsection{Modulation of retinocentric tuning by gaze position}

In this section we focus on how gaze direction modulated units that showed retinocentric tuning. The motivation is to assess whether gaze-dependent modulation would be consistent with having gaze-dependent gain fields. To this end, we bootstrapped the tuning vector (dependent on retinocentric target location) for each initial gaze position, independently for each unit and each epoch. To assess if gaze position amplified or shifted response fields, we computed confidence intervals for the estimated tuning vector for each gaze position and compared the confidence intervals around the mean for vector length and direction, see Materials and Methods. This approach allowed us to assess changes in tuning without reliance on a specific tuning function. To illustrate this procedure, we modelled a neuron with a Gaussian retinocentric response field and a gaze-dependent linear multiplicative gain field and simulated recorded data for this hypothetical neuron by adding random noise for each trial (see Figure 3.6A). Using this simulated data for bootstrapping the tuning vector for each gaze position and comparing confidence intervals of estimated tuning vector end points confirmed that the gain field properties of the modelled neuron could be reconstructed (see Figure 3.6B).

A Simulated (linear) gain field

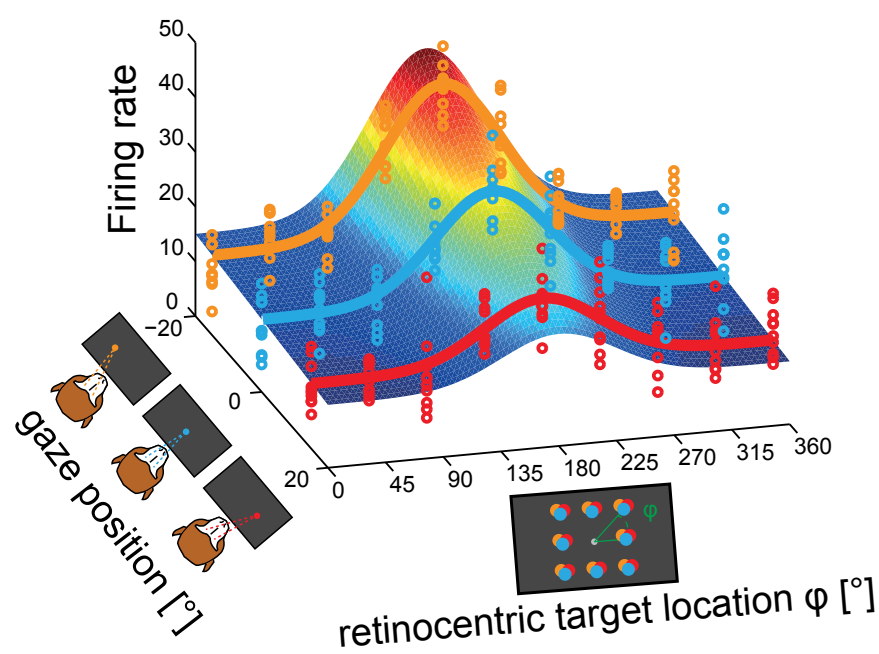

B Bootstrapped tuning vectors

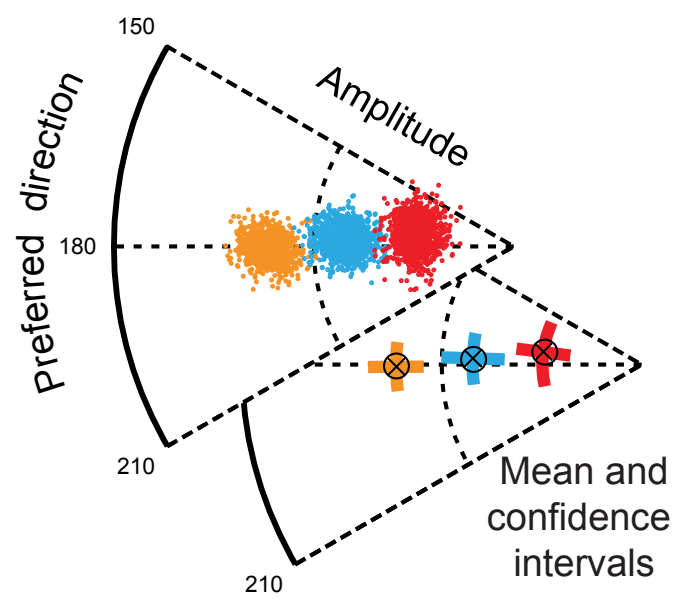

Figure 3.6. Tuning vector bootstrapping approach. A) 3D plot of a simulated gain field. Each color corresponds to one initial gaze position. Firing rates were simulated using a Gaussian tuning curve (dependent on retinocentric target location, preferred direction $=180^{\circ}$ ) which was amplified by the initial gaze position. Noise was added to create variability for each trial (colored circles). To illustrate the comparison with the results derived from two-way ANOVA on factors retinocentric location and gaze position, such gain field results in both main effects and interaction. B) Population vectors for each initial gaze position were bootstrapped (see Materials and Methods) to compute confidence intervals for amplitude and direction of the estimated population vectors for each initial gaze position.

Spatial firing rate patterns of six recorded example units exhibiting either gain field properties or response field shifts in the cue epoch are shown in Figure 3.7A. For further illustration, 
PSTHs for each combination of initial gaze and retinocentric target location as well as the distribution of bootstrapped tuning vector endpoints are shown for the two top example units. The left example shows a classical gain field, retinocentric cue response amplified by gaze position. The example on the right shows a more complex pattern: here the preferred direction is modulated by the gaze position, with more ipsilateral gaze amplifying the central and upper contralateral cue response.

Figure 3.7B shows the number of units exhibiting a main effect of retinocentric target location (derived from two-way ANOVA in Figure 3.5A), and out of those units, the number of unit showing gain, shift or both, for each epochs. Among the epochs occurring prior to the saccadic eye movement, and thus reflecting the potential effects of the static gaze, we focus on visual cue responses. Out of 75 units that showed the main effect of cue/target location, only 10 units showed a significant gain field component (7 monotonic). In those units, the gain amplitude was $66 \pm 15 \%$ (mean \pm SD, Materials and Methods). Importantly, our gain field analysis only detects multiplicative impact of gaze on retinocentric tuning, which in the twoway ANOVA analysis (cf. Figure 3.5A) would be reflected by a combination of the main effect of retinocentric target location and interaction. Indeed, 9 out of the 12 units showing this combination were identified as having gain. The 22 units which showed main effects of retinocentric location and gaze but no interaction might be attributed to additive effects which are not defined as gain. The additive effect could be illustrated by the tuning curves of the example unit in Figure 3.5B (Cue and Gaze).

The interpretation of the two post-saccadic epochs (immediately after saccade, and during target onset) is more complicated because gaze-dependent effects could also reflect the final gaze position (which is not fully dissociated from the initial gaze position). In fact, 46 out of 53 units showing gain and/or shift in the post-saccadic epoch and 42 out of 45 units showing gain and/or shift in the target onset epoch also show an effect of final gaze position in the same epoch, making gaze position encoding the most parsimonious explanation for the relatively frequent gain and shift effects in these epochs.

As a control, we also performed this analysis on firing rates during fixation hold to get an estimate for the false discovery rate due to random fluctuations (since at that point there was no difference between trials with different retinocentric target locations). Only one unit showed a spurious "shift" effect, suggesting that the findings in other epochs are not due to random noise fluctuations. 
A Example unit: gain fields in cue
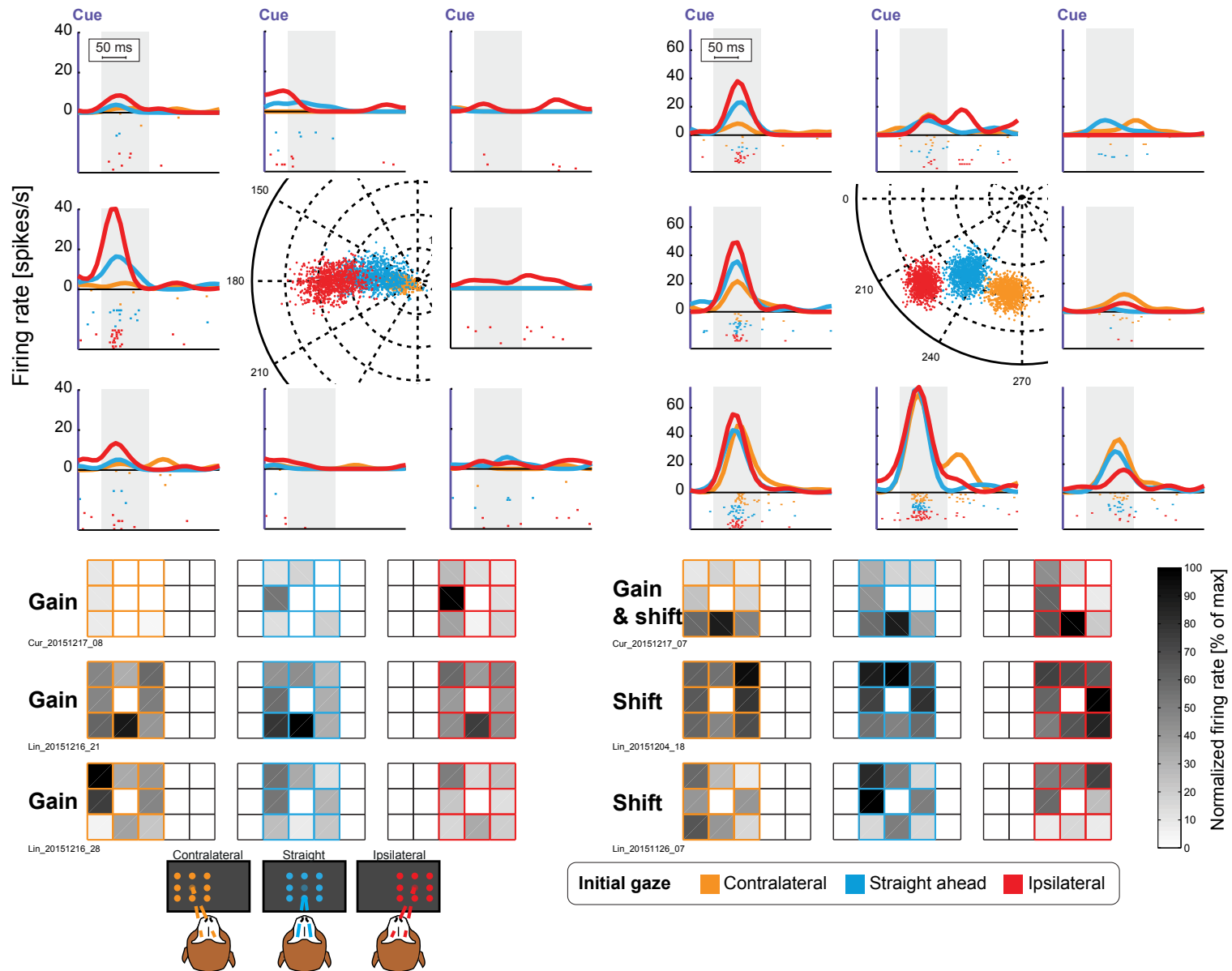

Initial gaze Contralateral Straight ahead Ipsilateral

B Population summary: gain fields and RF shifts per epoch

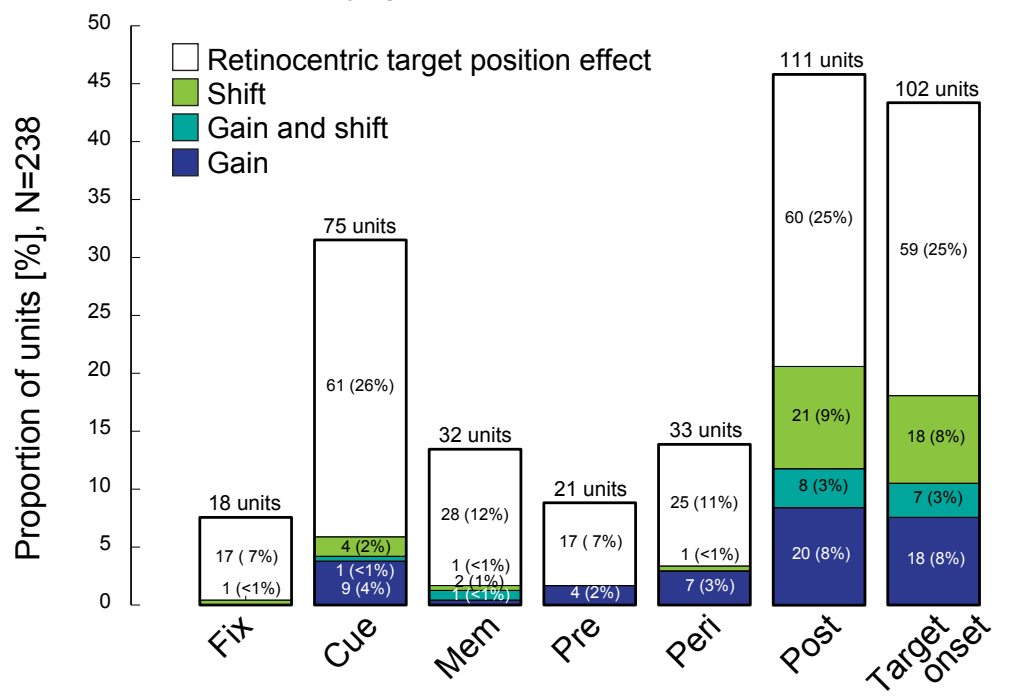

Figure 3.7. Gain field and response field (RF) shift properties. A) Example units showing gain field properties (left) or RF shifts (right) in the cue epoch. Top: PSTHs during cue presentation for each of the initial gaze positions and each of the retinocentric target locations, spatially arranged according to the retinocentric location. In the center of the PSTH plots, the corresponding bootstrapped population vector endpoints for each initial gaze position are displayed. Bottom: Heat map plots of the example units shown above (top) and two additional examples showing either gain field properties or RF shift properties. B) Number of units showing a main effect of retinocentric target position (top of the bar), and out of those, units showing gain field (blue) or RF shift properties (green) for each epoch of interest. Units showing both effects are displayed in light blue, units showing only an effect of target position are displayed in white. Fixation hold (Fix) epoch was added in order to approximate the expected statistical noise level. 


\subsubsection{Reference frame evaluation}

Finally, we evaluated in which reference frame dPul neurons encode spatial information. Since the monkey's head position was always immobilized straight ahead relative to the screen, and we did not control for the trunk rotation in the chair (although typically the trunk was facing the screen), we cannot dissociate between head-, body-, or world-centered representations. However, we can dissociate these three possibilities from retinocentric (i.e. eye-centered) representations. For simplicity, in the following text a "screen-centered" reference frame refers to a potential head-, body-, or world-centered reference frame, as opposed to a retinocentric one.

To evaluate if response patterns were better explained by retinocentric or screen-centered representations, we compared the alignment of responses in retinocentric and screencentered coordinates for seven task epochs, following a previously applied approach (Materials and Methods, (Mullette-Gillman et al., 2005)). For this analysis, we included only units that exhibited a dependence on target locations in any reference frame: i.e. only units which showed either a main effect of retinocentric target location, [retinocentric location $x$ initial gaze] interaction, a main effect of target location on the screen, or [screen location $x$ initial gaze] interaction. In Figure 3.8, each data point represents the average correlation coefficient (ACC) between the unit's responses for different initial gaze positions when locations were defined relative to the eye (retinocentric ACC, horizontal axis) and relative to the screen (screen-centered ACC, vertical axis). Units above the diagonal indicate better alignment of responses in screen-centered coordinates, and units below the diagonal indicate better alignment in retinocentric coordinates. Units showing response patterns that are significantly better explained by a screen-centered reference frame are in green, units that are better explained by a retinocentric reference frame are in red. Figure $\mathbf{3 . 8}$ shows a progression from prevalence of units compatible with retinocentric reference frames in the cue epoch to screen-centered encoding in the target hold epoch, with a balanced representation immediately after the saccade. This indicates that visual responses and/or movement preparation were encoded in retinocentric coordinates, while the new gaze position was the predominant factor encoded in post-saccadic epochs. As additional support for this interpretation, we performed paired t-tests comparing retinocentric ACCs and screencentered ACCs across all included units in each epoch, showing significantly stronger retinocentric alignment in the cue $(p<0.001)$, memory $(p=0.01)$ and peri-saccadic $(p=0.001)$ epochs and stronger screen-centered alignment in the target onset $(p=0.003)$ and target hold $(p<0.001)$ epochs. 

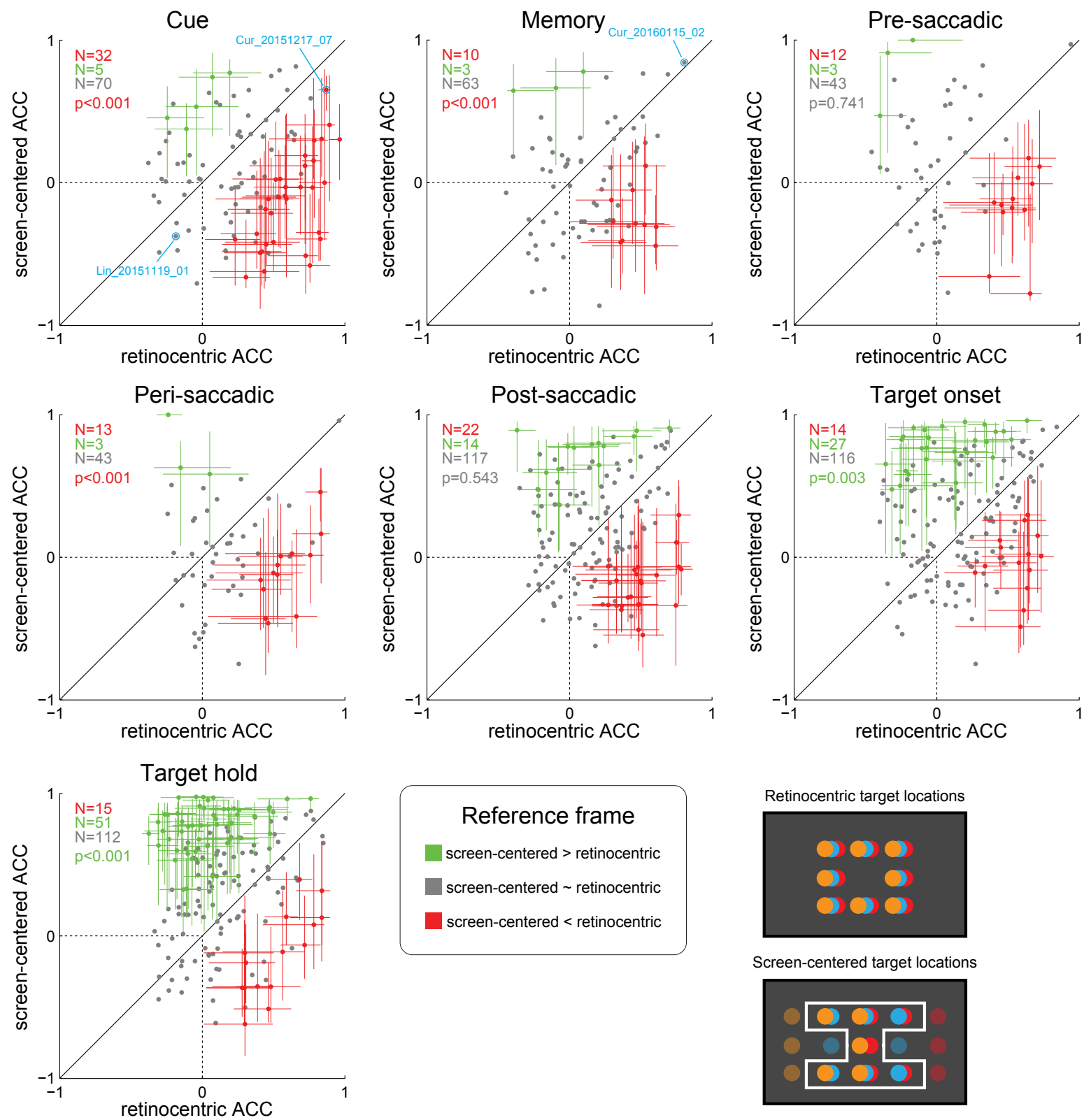

Figure 3.8. Reference frame evaluation. Average correlation coefficients (ACCs) in retinocentric coordinates (x-axis) and screen-centered coordinates (y-axis) for each unit in seven epochs of interest: cue, memory, presaccadic, peri-saccadic, post-saccadic, target onset and target hold epoch. The crosshairs indicate $95 \%$ confidence intervals derived from bootstrapping. Note that dots represent actual ACCs calculated using all data, and their location is not necessarily centered in the middle of the confidence intervals. Units where the crosshair is above the diagonal (and $>0$ on the $y$-axis) were classified as "more screen-centered than retinocentric" (green) and units where the crosshair is below the diagonal (and $>0$ in the $\mathrm{x}$-axis) were classified as "more retinocentric than screen-centered" (red), see Materials and Methods for details. For remaining units (mixed, or hybrid encoding, gray), confidence intervals were omitted for clarity. For eye-centered correlations, all 8 target locations were used, for screen-centered correlations, only overlapping target locations for each specific correlation pair were used ( 7 targets outlined by the white frame). P-values indicate significance of paired t-tests comparing screen-centered and retinocentric ACCs across all included units, the text color indicates which one was larger (in case of significant differences). For cross-referencing, we marked two units in the cue epoch; the example from Figure 5C (with main effect of gaze but no effect of retinocentric location, Lin_20151119_01) showing negative correlation in both reference frames, and the example from Figure 7A right (showing gain and shift, Cur 20151217 07), which shows strong correlation in both reference frames (but significantly more for retinocentric), as well as one unit for the memory epoch, the example from Supplemental Figure 3.3 (Cur_20160115_02), a mixed unit which shows strong correlation in both reference frames, but no significant difference between them. 
Besides the units that could be classified as retinocentric or screen-centered, in each epoch, many units showed mixed, or hybrid encoding that cannot be more strongly attributed to either of the two references frames (Caruso et al., 2018). Many of these units had high ACCs in both frames, i.e. the both reference frames could account for the observed response functions. To illustrate this, we show an example unit that responded predominantly to upper targets, and had high ACCs in both reference frames (e.g. 0.84 for screen-centered and for 0.81 retinocentric in the memory epoch), making it difficult to dissociate the two (Supplemental Figure 3.3). This and other mixed units showed response patterns that are consistent with a partial shift of response function with the eye position (Caruso et al., 2018). 


\subsection{Discussion}

Dorsal pulvinar has been implicated in spatial processing supporting goal-directed eye movements, but so far the implicit experimental assumption was that it encodes space in retinocentric (eye-centered) coordinates, even if it does not show a retinotopic organization (i.e. gradual topographic representation of visual space across the adjacent regions of neural tissue). Here we show that many dorsal pulvinar neurons are influenced by the position of the eyes in the orbit (equivalent to the gaze direction, when the animal's head is immobilized), in two different ways. First, in a half of the sample the firing during steady fixation of a foveal stimulus was influenced by the gaze direction. Second, a substantial portion of the sample showed a combination of retinocentric and gaze-dependent signals in visual and saccadic responses to a peripheral stimulus, and some of these effects could be described as gaze-depended gain modulation. The analysis of reference frames across consequent task epochs, from cue to post-saccadic target hold, indicated a transition from predominantly retinocentric encoding to coding of final gaze or target location in head/body/world-centered coordinates. Although there are limitations in the current study design (see below), these results provide electrophysiological evidence to support the notion that the dorsal pulvinar might contain neurons that reflect postural signals and represent space in non-retinocentric reference frames (Grieve et al., 2000). In the following sections, we relate our findings to the previous literature, separately discussing the eye position influence on ongoing firing during foveal fixation, and on visuomotor representations of peripheral targets.

\subsubsection{Comparison to previous electrophysiological studies in the thalamus}

Previous electrophysiological studies in central thalamus of monkeys have reported neural activity modulation by eye position in the orbit (Schlag-Rey and Schlag, 1984; Tanaka, 2007; Wyder et al., 2003). Similar to the dorsal pulvinar, the central thalamus is a 'higher-order' nucleus complex with connections to cortical regions such as frontal and supplementary eye fields and posterior parietal cortex, that are crucial for oculomotor behaviors (Tanaka and Kunimatsu, 2011). The central thalamus also receives afferent inputs from the cerebellum and oculomotor brainstem involved in eye movement control.

In respect to effects of gaze on ongoing fixation activity, a study in the central ('oculomotor') thalamus including $\mathrm{Pc}, \mathrm{CL}, \mathrm{VA}, \mathrm{VL}$ and paralaminar $\mathrm{CM}$ and $\mathrm{MD}$ nuclei reported a small subgroup of 6 neurons showing an influence of gaze on sustained post-saccadic activity, increasing or decreasing monotonically with the contralateral gaze eccentricity (Wyder et al., 2003). It is not clear if there were other gaze-dependent sustained neurons that did not show monotonic dependence. Comparing these results to the effects of the final (post-saccadic) gaze position in our sample, the majority of dPul units did not show monotonic dependence 
(e.g. for the 5 gaze positions in the horizontal axis, 32 units showed the monotonic effect and 85 non-monotonic). The number of monotonic gaze effects was much higher (71\%) for the initial fixation, but this estimate is limited by only three gaze positions covering relative narrow range $\left(30^{\circ}\right)$.

Another study that specifically focused on eye position responses in the central thalamus also found, in addition to transient post-saccadic responses, many neurons with sustained post-saccadic firing modulated by eye position (Tanaka, 2007). Most neurons in this study had positional preferences along the horizontal axis. Some neurons also showed a "memory trace" of the preceding saccade: the activity at the same final position depended on where the eye came from. Such hysteresis effect was also reported in the central thalamus during spontaneous eye movements (Schlag-Rey and Schlag, 1984). This is consistent with our subset of neurons showing an interaction between the initial and the final gaze position. But unlike our findings, the eye position effects in the central thalamus could be divided into the two main groups: the neurons that showed predictive pre-saccadic tuning corresponding to the final gaze position, and those that were modulated by the gaze only after the saccade. In our sample, the majority of neurons were similar to the latter, while the units showing presaccadic responses consistent with encoding the post-saccadic gaze were exceedingly rare.

The thalamus literature on gaze-dependent modulation of peripheral (eccentric) visual responses is sparse. The modulation of visual responses by the (passive) eye position has been reported in LGN of anesthetized cats (Lal and Friedlander, 1989). The eccentric visual responses in the cat IML were found to be related to a confluence of specific retinocentric direction and a target area defined in screen/head/body-related coordinates, determined by spontaneous gaze locations (Schlag et al., 1980). In the retinotopically organized macaque ventral pulvinar, visual responses of a subset of neurons were influenced by orbital position (Robinson et al., 1990). In this study, stimuli were briefly flashed in the receptive field at different times around visually-guided saccades, to assess the visual sensitivity dynamics. In 15 out of 41 cells, modulation of visual sensitivity by eye position has been detected.

Apart from those pioneering studies, to our knowledge there has been no systematic assessment of potential gaze-dependent gain fields in higher-order thalamus.

\subsubsection{Comparison to previous electrophysiological studies in cortex}

Eye position effects in cortex were mostly considered in the context of reference frame transformations and integration of sensory information into head- and body-centered coordinates. Nonetheless, many studies that have tested the influence of gaze on ongoing activity during fixation in task periods unrelated to the representation of peripheral targets for goal-directed movements (even in the dark) also reported eye position-dependent effects. 
Specifically, eye position signals during initial fixation have been reported in many cortical areas including visual (V1-V4, MT, MST) (Bremmer, 2000; Morris et al., 2013; Rosenbluth and Allman, 2002), posterior parietal (LIP, MIP, 7a, VIP, V6) (Andersen and Mountcastle, 1983; Andersen et al., 1990; Bremmer et al., 1997; Galletti et al., 1995; Genovesio et al., 2007; Mullette-Gillman et al., 2005; Sakata et al., 1980), dorsal premotor (Boussaoud et al., 1998) and somatosensory cortex (Wang et al., 2007).

Sakata et al. detailed eye position effects of neurons recorded from parietal area $7 \mathrm{a}$ that were clearly driven by gaze and did not show activity during passive visual stimulation ('fixation neurons'). In their sample, $\sim 10 \%$ of neurons exhibited this property (Sakata et al., 1980). The majority of the 86 reported neurons varied systematically with gaze angle in the frontal plane (39 horizontal, 38 vertical, 9 diagonal). Many of those neurons were selective for depth of fixation as well, an aspect that is important but was not measured in our current study. The results of Sakata and colleagues are broadly consistent with later studies in areas LIP and $7 \mathrm{a}$, where subpopulations of neurons were reported to exhibit tonic background activity during fixation that varied monotonically with eye position without showing any visual responsiveness (Andersen et al., 1990). Similarly, another study in LIP and 7a reported an influence of eye position during visual fixation in darkness with about a third of neurons that could be fit with a linear regression model (Bremmer et al., 1997). A comprehensive study that compared how well eye position signals during fixation can be decoded from neurons recorded from LIP, VIP or visual areas MT and MST concluded that eccentric fixations could be decoded more accurately than central ones and that LIP/VIP populations encode eye position more accurately than visual cortex (Morris et al., 2013). Eye position signals were also reported in areas that are not primarily associated with visual or saccade processing. For example, a subpopulation of neurons in premotor area PMd were reported to differentiate between gaze angles in a reach task without being tuned to visual inputs or movement directions (Boussaoud et al., 1998).

Eye position signals that were monotonically related to the eccentricity of gaze were also reported in primary somatosensory cortex S1 (Wang et al., 2007; Xu et al., 2011). This study concluded that those eye position signals in S1 had a proprioceptive origin since anesthesia of the contralateral orbital muscles nearly abolished the recorded eye position signals. Similarly, a preference for more eccentric eye positions was reported in the parietal cortex (Sakata et al., 1980). On the other hand, some studies reported a fairly balanced distribution of gaze-depending activity on a population level (Bremmer, 2000; Bremmer et al., 1998). In the dPul we found a predominance of more eccentric (peripheral) gaze preferences, both during initial fixation and during target hold. 
While the relationship between gaze-dependent effects during fixation and peripheral target encoding has not often been explicitly discussed, studies in parietal and premotor cortex report all three categories: namely neurons that are modulated by the gaze during fixation but are not responsive in subsequent task epochs, neurons that only show modulation of visual/delay/saccade responses by gaze, and neurons that show both types of gazedependence. Similarly, we found all three categories in $\mathrm{dPul}$, and no systematic relationship between presence or absence of gaze dependence and visual cue responses.

The majority of cortical studies on eye position signals focused on the second aspect of gaze dependence: modulation of visual, motor planning and saccade-related activity in response to peripheral targets (Lehky et al., 2016). Specifically, when a neuron changes its response to a stimulus depending on eye position while the retinal location remains the same, this pattern has been described as eye position gain field. Computational models applied to the response modulation of single neurons in parietal (Andersen et al., 1990) and visual cortex (Bremmer, 2000) have often modeled the gain fields as planar, or at least monotonic (Pouget and Sejnowski, 1997; Zipser and Andersen, 1988). It needs to be noted however that even in posterior parietal cortex, the proportion of neurons reported to exhibit planar gain fields was only 39\% (Andersen et al., 1985), and multidimensional scaling methods applied to neuronal populations in the lateral intraparietal cortex (LIP) and anterior inferotemporal cortex (AIT) to infer current eye position show that other, nonlinear functions (e.g. sigmoidal, elliptical or hyperbolic, or complex mixture of thereof) might fit the data as well (Lehky et al., 2016). Likewise, eye position fields of neurons in area V6 (PO) have been shown to have diverse shapes from planar to peak-shaped (Galletti et al., 1995). Similarly to those findings, the gain fields in dPul appeared to be a mixture of monotonic and peak-shaped fields (but given only three gaze positions we used here, those conclusions are tentative). This is unlike the primary visual cortex, where the straight ahead direction evokes stronger gain (Durand et al., 2010; Przybyszewski et al., 2014).

In addition to the modulation of retinocentric encoding by the eye position, several oculomotor studies also reported encoding of visual/saccade targets in other reference frames, e.g. head-centered or mixed, or hybrid coordinates (Brotchie et al., 1995; Caruso et al., 2018; Galletti et al., 1995; Mullette-Gillman et al., 2005). In addition to retinocentric reference frame, we also observed a very small subset of neurons that were more consistent with head/body/world-centered encoding prior to the saccade, and many neurons with mixed encoding. For instance, some units showed retinocentrically not tuned visual cue responses only at certain gaze positions (cf. Figure $5 \mathbf{C}$ ). Since the eye position could determine whether there was a visual response at all, it is conceivable to construe those neurons as primarily encoding eye position that is modulated by a visual response as opposed to the 
typical concept of eye position 'gain fields' where the visual response is modulated by eye position.

The prevalence of significant eye-centered encoding in cue, memory and pre-saccadic epochs is supplanted soon after the saccade by head/body/world-centered reference frame encoding, which can also be interpreted as the encoding of the current gaze. This finding is in line with ANOVA results showing the final gaze/target encoding during late post-saccadic fixation. The transition from retinocentric target to gaze position encoding in dPul resembles a recent study in FEF, with the difference that the transition happens after the saccade in $\mathrm{dPul}$, as compared to predictive pre-saccadic transition from retinocentric target code to (also retinocentric) motor goal in FEF (Sajad et al., 2016). The immediate post-saccadic epoch in $\mathrm{dPul}$ still contained units with retinocentric encoding, akin to eye movement vector postsaccadic encoding in LIP which dissipated in the late post-saccadic period (Genovesio et al., 2007). A small subset compatible with retinocentric encoding during target hold, long after the saccade, was unexpected, but the visual inspection confirmed that those units indeed retained a trace of the retinocentric saccade vector.

\subsubsection{Possible sources of eye position signals in dorsal pulvinar}

Given the extensive connectivity of the dorsal pulvinar to parietal and premotor cortex, and the resemblance of the eye position-dependent effects in those areas and in dPul, one likely possibility is that the eye position effects in $\mathrm{dPul}$ are inherited from those visuomotor areas (Cappe et al., 2007, 2009; Romanski et al., 1997; Schmahmann and Pandya, 1990; Yeterian and Pandya, 1989). The source of continuous eye position signal in the cortex is still not fully identified (Xu et al., 2012; Ziesche and Hamker, 2011). One candidate is the proprioceptive signal reflecting veridical position of the eyes in the orbit (Wang et al., 2007). The other possibility is the corollary discharge of the motor command that maintains steady eye position (Morris et al., 2012; Xu et al., 2012). Additionally, and not mutually exclusive, some of the effects in the $\mathrm{dPul}$ could represent a more direct proprioceptive signal from the primary somatosensory cortex (e.g. the trigeminal nerve representation) (Wang et al., 2007; Xu et al., 2011), given medial pulvinar connectivity to area 3a (Padberg et al., 2009). This could be investigated further by anaesthetizing the extraocular muscles (Wang et al., 2007), or inactivating the projection from somatosensory area $3 \mathrm{a}$ and recording in the $\mathrm{dPul}$.

Another possibility, which would place dPul as a potential source of eye position signals to the cortex, is the corollary discharge via superior colliculus - pulvinar - cortical pathway (Guthrie et al., 1983; Wurtz et al., 2011a). Dorsal pulvinar, including the lateral portion of the medial pulvinar where our recordings were mainly performed, receives afferents from the intermediate and deep layers of the superior colliculus (Baldwin and Bourne, 2017; Bender and Butter, 1987; Benevento and Standage, 1983; Harting et al., 1980). The intermediate 
and deep layers of SC exhibit eye position dependence, including gain fields (Campos et al., 2006; Van Opstal et al., 1995). It has been shown that besides the proprioceptive information about eye position (Andersen et al., 1990; Bremmer et al., 1997), a preparatory corollary discharge / efference copy of planned saccade, originating in the superior colliculus and routed to frontal cortex via mediodorsal thalamus (MD) can carry eye position signals (Crapse and Sommer, 2008; Wurtz et al., 2011a). Similarly, it has been suggested that the dorsal lateral pulvinar might carry preparatory movement-related signals to parietal cortex (Wurtz et al., 2011b), although those signals might only be relevant around the saccade.

Although the anatomical projections of the superior colliculus to the dPul have been reported (but see (Zhou et al., 2017)), there is a debate on specificity of projections to medial vs. lateral pulvinar (Baldwin and Bourne, 2017)). Moreover, the functional role of these connections has not been demonstrated. Conversely, the effects of superior colliculus perturbation or lesion on ventral pulvinar responses have been assessed in several studies. In particular, extensive lesions of SC caused only little effect on visual responses in ventral pulvinar, as compared to striate cortex lesions (Bender, 1983). In rabbits, however, the inactivation of SC led to a strong attenuation of responses in lateral posterior nucleus (which is a part of the LP-pulvinar complex in non-primate species: mice, rats, rabbits, cats, and tree shrews) (Casanova and Molotchnikoff, 1990). Furthermore, selective microstimulation of the superficial layers of the SC in macaques has been shown to elicit (monosynaptic) responses in the ventral pulvinar (Kinoshita et al., 2019), in agreement with an earlier work that identified the projection from SC to area MT via ventral pulvinar (Berman and Wurtz, 2011). Extrapolating from these studies, the potential influence of SC inputs to dPul (Wurtz et al., 2005) will probably be outweighed by more extensive cortical projections. To fully elucidate complex cortico-pulvinar-cortical loops and subcortical inputs to pulvinar, it would be crucial to manipulate the specific projections from and to the pulvinar, using pathway-selective techniques, such as optogenetics and/or viral vectors (Kinoshita et al., 2019; Schmitt et al., 2017).

Lastly, another possible source of eye position signals in the pulvinar is the cerebellum. The cerebellum is involved in a number of eye movement control functions including maintaining a steady fixation, smooth pursuit and binocular alignment (Krauzlis et al., 2017; Patel and Zee, 2015). To our knowledge no study in monkeys or humans has described (monosynaptic) pathways between dorsal pulvinar and cerebellum. In cats, several earlier histological labelling studies described projections from the cerebellum to the contralateral lateral-posterior nucleus of the thalamus, which is considered a putative homologue of the pulvinar (Itoh and Mizuno, 1979; Rodrigo-Angulo and Reinoso-Suarez, 1984). This cerebellopulvinar projections were found to overlap with the ones coming from the deep layers of the 
superior colliculus and brainstem structures such as the pedunculopontine tegmental nucleus. How far this homology goes is unclear as the medial portion of the dorsal pulvinar is only fully developed in primates (Jones, 2007). Some evidence (albeit not necessarily for a monosynaptic pathway) can be derived from microstimulation of the cerebellum in monkeys showing evoked BOLD fMRI activity in the dorsal pulvinar (Sultan et al., 2012). Indirect evidence for a functional connection could also be derived from a recently described human patient with a circumscribed (bilateral) lesion in the medial pulvinar who exhibited a mixture of parietal and cerebellar neurological deficits (Wilke et al., 2018). Being somewhat speculative, the anatomical and functional connectivity between pulvinar and cerebellum in primates needs to be studied in more depth.

\subsubsection{Methodological limitations and future directions}

In our experiments, as in many previous studies, the head was immobilized in the straight ahead direction relative to the body and the center of the screen. Hence, head-, body- or world (screen)-centered references cannot be dissociated in this design (Mullette-Gillman et al., 2005). Owing to the same limitation, when interpreting the modulation of responses during fixation at initial or final gaze positions, we cannot distinguish between encoding of eye position in the orbit (e.g. via a proprioceptive signal) vs. spatial representation of the fixated stimulus (in any of the above reference frames).

Furthermore, there is an intrinsic difficulty when trying to dissociate post-saccadic saccade vector encoding from effects of post-saccadic gaze position: a task cannot be designed in such way that for each final gaze position all potential saccade vectors, and vice versa, are recorded at the same time. In our design, only 7 out of 15 final gaze positions resulted from different initial gaze positions and hence different saccade vectors. Moreover, since we only varied the initial horizontal gaze position, a hypothetical extreme example of a clear retinocentric encoding with responses for all upper targets would also result in an apparent upper final gaze preference with the same 'goodness of fit' - thus, those two encoding schemes cannot be dissociated. Therefore, at least partially the final gaze encoding could be a result of "tiling" retinocentric (saccade vector) response fields originating from the three initial gaze positions. Several lines of evidence however suggest that the static position of the eyes in the orbit is the defining factors in post-saccadic tuning, especially in the later part of the post-saccadic epoch. First, the corresponding target hold epoch analyzed here started $400 \mathrm{~ms}$ after the saccade end. Second, the two-way [initial gaze $\times$ retinocentric target position] ANOVA results on firing rates in target hold indicate that purely retinocentric encoding was rare: out of 156 units with the effect of the final gaze, only 27 units showed 'purely retinocentric' effect in the separate two-way ANOVA, while most showed also the effect of initial gaze and/or the interaction. Third, only 25 out of these 156 units showed an 
interaction between the initial gaze (i.e. saccade vector) and the final gaze in the two-way ANOVA on 7 positions that were reached from more than one initial gaze position (cf. Figure 3.4B). Fourth, an independent analysis that directly compared retinocentric vs. "screencentered" (i.e. final gaze position-dependent) reference frames confirmed that most units were classified as the latter in the target hold epoch, despite relying on only a small number of overlapping target locations for calculating screen-centered correlations. Hence, the most parsimonious explanation of our results suggests that the eye position signals are indeed amply represented in the dorsal pulvinar.

In respect to encoding of cue, delay and saccade responses to the peripheral targets, our design allows dissociating between: i) purely retinocentric encoding, ii) retinocentric encoding modulated by gaze-dependent gain field, iii) head/body/world-centered, and iv) mixed reference frame. But it is important to note that with the current design (largely nonoverlapping sets of final gaze positions linked to each of only three initial gaze position, as compared e.g. to 9 starting positions in (Andersen et al., 1990), or 9 horizontal targets in (Mullette-Gillman et al., 2005)), if a unit shows not a "straightforward" gaze-specific gain effect on retinocentric encoding with but a more complex pattern (e.g. shift or other idiosyncratic pattern), we cannot always reliably identify the best fitting reference frame (Mullette-Gillman et al., 2009). Future experiments with a larger range of initial and final target positions, and rotation of the head relative to the body, and the body relative to the screen (Dean and Platt, 2006), will elucidate the functional role of these signals in postural control and guiding visually-guided actions in space (Grieve et al., 2000).

Another potential limitation was that our experiments were done in a dim light, due to background illumination of the monitor. This could be considered as a potential confound because if the neuron has a large peripheral receptive field, the initial (or final) gaze position might bring this RF outside of the illuminated monitor. This is however unlikely to explain our findings for several reasons. First, the horizontal size of the screen was large (100 degrees of visual angle). Second, a roughly equal number of units showed same or opposite hemifield preference for visual cue and initial gaze position. If the effects of gaze were due to changes in visual stimulation, we would expect these preferences to be opposite (regardless of whether a unit is showing enhancement or suppression to a bright cue) because a bigger part of the illuminated screen is always in the hemifield opposite to the direction of the gaze. Third, many units did not have a clear visual response but still showed the effect of gaze position. Finally, the two studies that tested the eye position effects in central thalamus and ventral pulvinar did not see a difference between light and darkness (Robinson et al., 1990; Schlag-Rey and Schlag, 1984). To summarize, the eye position dependence during fixation is most likely due to extraretinal signals or non-retinocentric spatial encoding. 


\subsubsection{Functional significance of eye position signals in dorsal pulvinar}

Generally, the functional significance of eye position signals in dPul is likely to be similar to other cortical and subcortical brain regions to which it connects, with the special property that the pulvinar is in a good anatomical position to distribute this information throughout the interconnected cortical circuitry (Saalmann and Kastner, 2011; Sherman and Guillery, 2002). We briefly list plausible functions before considering some of them in more detail.

In respect to eye position dependence during fixation, the putative functions could be:

1. Eye movements to auditory stimuli (Cohen and Andersen, 2002; Yao and Peck, 1997)

2. Computation of ocular vergence angle to determine depth/distance of foveated objects (Rosenbluth and Allman, 2002)

3. Maintaining stable fixation (Krauzlis et al., 2017)

In respect to influence of eye position on visual responses and activity around and after a saccade:

1. Neural computation of spatial target localization as an intermediate step in the transformation between retinal location and egocentric location in respect to the body or limbs (Andersen et al., 1993; Pouget and Snyder, 2000)

2. Modulation of cortical activity with predictive shifts of visual attention associated with saccades (Duhamel et al., 1992; Hoffman and Subramaniam, 1995; Wurtz et al., 2011a, 2011b)

3. Maintaining perceptual stability across eye movements (Mirpour and Bisley, 2015; Sommer and Wurtz, 2008)

4. Recalibration of efference copy signal after saccade (Wang et al., 2007; Xu et al., 2012)

While the functional role of gaze dependence in $\mathrm{dPul}$ can at least in part be derived from related properties in connected cortical areas and the superior colliculus, it should be constrained by known behavioral consequences of lesion and perturbation studies, as well as electrophysiological properties of dPul neurons. Given relatively coarse spatial tuning of many dorsal pulvinar responses to visual cues and saccades, and moderate effects of perturbation on saccadic precision and accuracy (Bender and Baizer, 1990; Benevento and Port, 1995; Dominguez-Vargas et al., 2017; Petersen et al., 1985a; Robinson et al., 1986; Wilke et al., 2010), it is unlikely that the main function of eye position signals in dPul is precise perceptual or saccadic localization, as might be the case for SC and FEF (Caruso et al., 2018). The basic visuomotor characteristics of many dPul neurons seem to be closer to parietal areas LIP and 7a, as well as posterior cingulate (Andersen et al., 1990; Barash et al., 1991b; Dean et al., 2004). There were however some differences between prevalent eye 
position effects in dPul and those cortical areas. In the pre-movement epochs, the encoding in dPul was mostly retinocentric or mixed, with a moderate occurrence of gaze-dependent gain fields. This is unlike more frequent gain fields in LIP (Andersen et al., 1990), or the large number of neurons with non-retinocentric encoding that has been cautiously interpreted as head-centered in parietal cortex (Caruso et al., 2018; Mullette-Gillman et al., 2005), or predominance of neurons exhibiting world-centered encoding in the posterior cingulate cortex (Dean and Platt, 2006). Nonetheless, the diverse patterns of eye position dependence in $\mathrm{dPul}$ can support at least some aspects of spatial transformation from retinocentric encoding to other coordinate frames during visuomotor planning.

The transient effects of saccadic displacement from one stable eye position to another seem to be of less relevance in the dPul given that the number of units showing the effect of the current eye position on pre-saccadic and peri-saccadic responses was small. Generally, some dPul neurons increase their firing predictively during the memory delay and/or just before the saccade and are tuned to specific retinocentric location, and might hypothetically participate in the transmission of the corollary discharge of the upcoming movement to the cortex, from the intermediate/deep layers of the superior colliculus or from one cortical area to another (Sherman and Guillery, 2002; Wurtz et al., 2011b). More neurons show pre/perisaccadic decrease of firing, consistent with the contribution to the saccadic suppression (Wurtz et al., 2011b). The effect of the eye position on the immediate post-saccadic responses is stronger, and this epoch shows a mixture between retinocentric encoding of the preceding saccade vector (with or without modulation by the pre-saccadic eye position), and the encoding of the current (post-saccadic) eye position. Post-saccadic eye position-related gain fields have been shown to provide unreliable localization signal contaminated by the pre-saccadic eye position, and their functional role should be interpreted with caution (Xu et al., 2012). A more detailed time-resolved analysis of the dynamics of eye position effects and reference frame transitions in the course of visual, memory delay, pre-saccadic and postsaccadic epochs is needed to elucidate the potential role of dPul eye position signals in preparation of the movements and post-movement updating.

Coming back to the eye position effects during fixation, the fact that the preference for a given steady gaze direction was also found in dPul neurons that do not have a visual cue response suggests that these signals are not only used to gate peripheral visuospatial attention (Fiebelkorn et al., 2019; Petersen et al., 1985a). On the other hand, the modulation of firing during steady fixation might still represent attention to relevant spatial targets, encoded either foveally or in a non-retinocentric reference frame. Further work is required to dissociate these possibilities from more mechanistic orbital eye position signals. 
It is important to note that the effects of initial vs. final gaze position were largely congruent (with a caveat of more extensive range for the final positions), and the influence of the preceding large saccade during the late part of the post-saccadic fixation was small. This implies that the cognitive, task-contingent aspects were not a major factor in gaze-dependent signals during initial fixation and target hold. We did not explicitly test for potential influences of small corrective saccades or preparation of return saccades after the end of the trial, but we did not observe any systematic relationship between patterns of the final gaze position dependence and retinocentric visual/saccade tuning in those units that showed such tuning.

It is also conceivable that the eye position signals in dPul are relevant for actions in depth. It might be possible that dPul neurons are sensitive for depth of fixation similar to fixation neurons in area $7 \mathrm{a}$ (Sakata et al., 1980). Indeed, the lesion of pulvinar can lead to stereoacuity deficits (Takayama et al., 1994). Since pulvinar neurons are binocular and are sensitive to relative retinal disparity, these cells may contribute to binocular depth perception (Casanova et al., 1991; Wilke et al., 2009).

Going beyond the oculomotor scope of this study, there are strong indications that the $\mathrm{dPul}$ is important for visually-guided reach and grasp movements (Acuña et al., 1990; Wilke et al., 2010, 2018), and likely the eye-hand coordination (Grieve et al., 2000). In particular, the perturbation effects are not only space-specific (Dominguez-Vargas et al., 2017; Wilke et al., 2013), but also limb-specific, with deficits stronger for the contralesional hand (Wilke et al., 2010, 2018), and the dPul neurons respond differently when reaches are made with the left or the right hand (unpublished observations, (Domínguez-Vargas, 2017)). In this context, the integration of eye position is a crucial step in spatial transformations from retinocentric to trunk-, limb-, or world-centered reference frames (Batista et al., 2007; Battaglia-Mayer et al., 2003; Bosco et al., 2015; Chang and Snyder, 2010; Marzocchi et al., 2008; McGuire and Sabes, 2011). In addition to limb movement aspects, gaze position has also been identified as an essential variable for stabilizing posture during upright standing (Ustinova and Perkins, 2011). Although this variable has not been explicitly addressed in monkey research, we have recently reported a patient with selective bilateral medial pulvinar lesions with difficulties to stand upright and walk, as well as nystagmus and hypometric saccades, without primary motor, vestibular or sensory deficits (Wilke et al., 2017, 2018). It is thus conceivable that the dorsal pulvinar contribution to integration of postural signals goes beyond oculomotor and reach/grasp movements, a possibility that should be explored in neurophysiological experiments. 


\subsection{Supplement}

\section{Retinocentric}

A

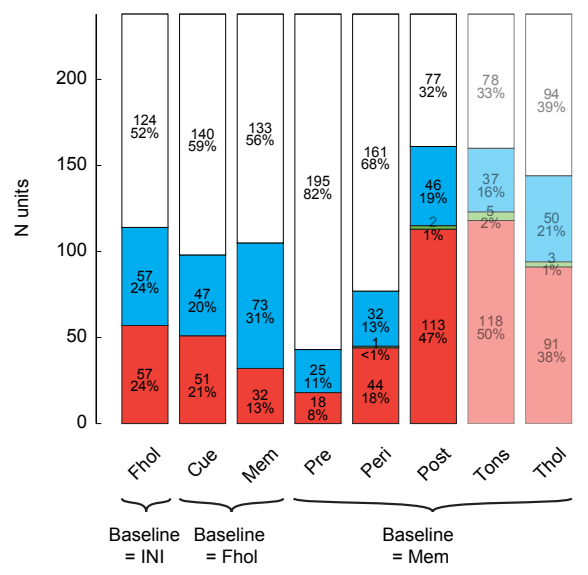

B

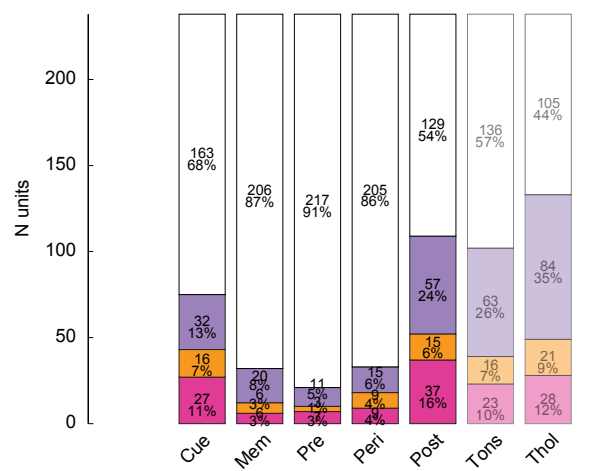

Absolute target location (relative to screen)
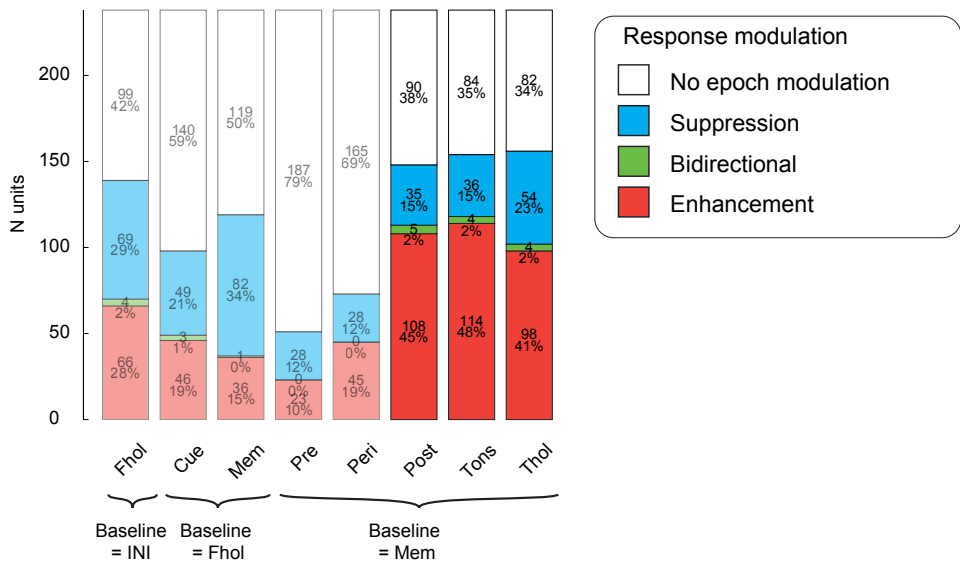

$\mathrm{D}$

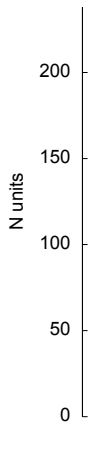

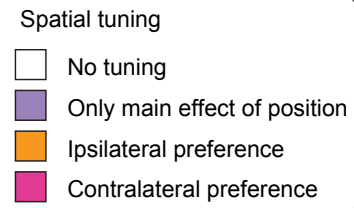

Supplemental Figure 3.1. Spatial tuning and response modulation per epoch. Spatial tuning and response modulation of all 238 units with more than 4 trials for each combination of initial gaze and retinocentric target location. The analyzed epochs are fixation hold (Fhol), cue onset (Cue), memory (MemE), pre-saccadic (Pre), Peri-saccadic (Peri), Post-saccadic (Post), target onset (Tons), and target hold (Thol). A) Total number of units (and percentages) showing response modulation relative to the respective baseline, for each analyzed epochs: no enhancement nor suppression (white), only suppression (blue), bidirectional - enhancement for one hemifield and suppression for the other (green), only enhancement (red). Hemifields relative to the gaze position (retinocentric) B) Number of units that, in the respective epoch, were not tuned (white), did not prefer either hemifield but showed a main effect of position in a one-way ANOVA (purple), preferred ipsilateral hemifield (orange), preferred contralateral hemifield (magenta). Hemifields relative to the gaze position (retinocentric). C) Same as A, but with hemifields relative to the head position (absolute target position on the screen). D) Same as $B$, but with hemifields relative to the head position. 
Activity dependent on final gaze position in 2D, $N=156$

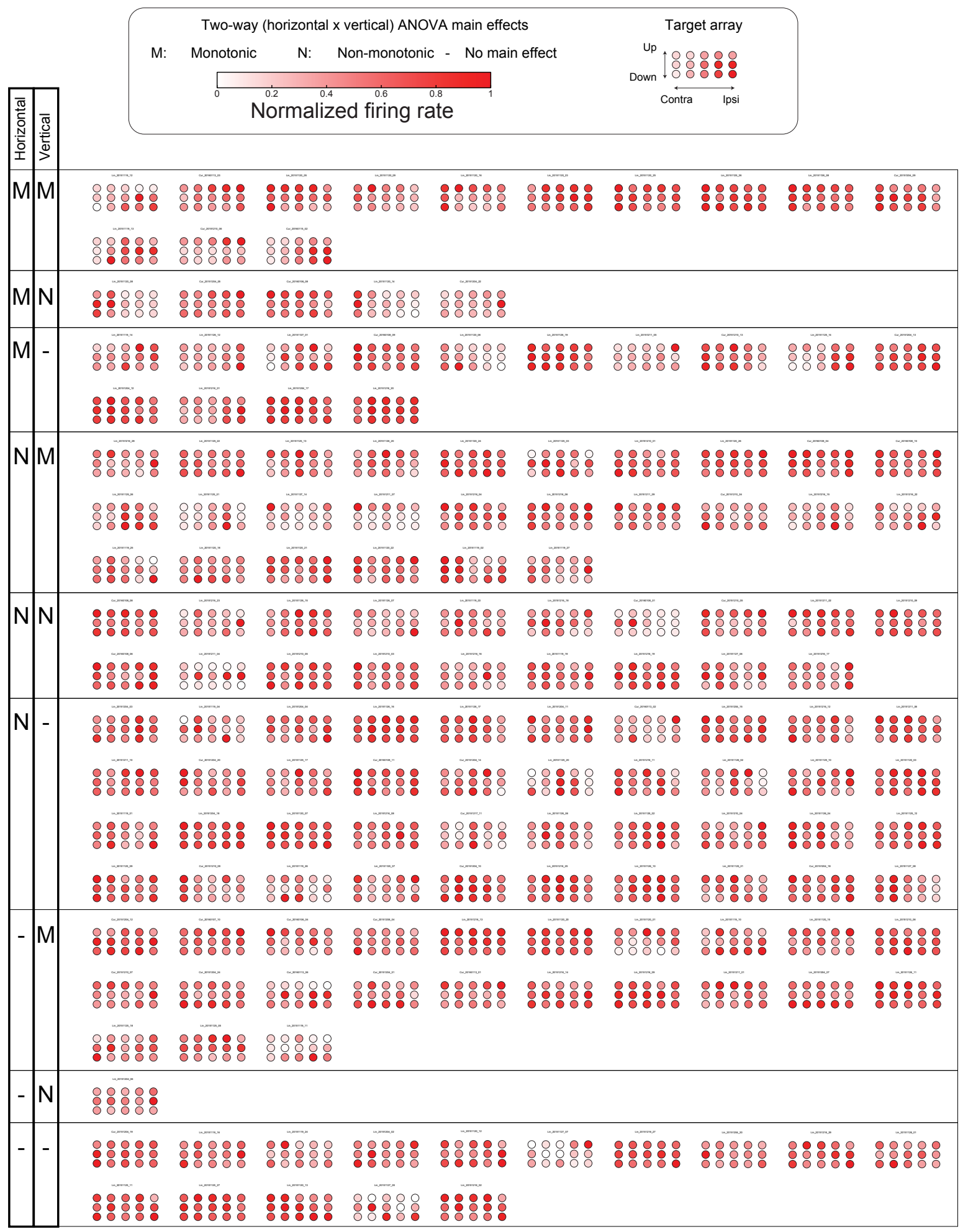

Supplemental Figure 3.2. Final gaze tuning. Heat map plots of firing rates for all 156 units that showed an ANOVA effect of final gaze position, normalized to the maximum firing rate. Units are grouped by monotonicity of vertical and horizontal gaze tuning: Monotonic $(\mathrm{M})$, non-monotonic $(\mathrm{N})$, and no main effect in the respective direction (-). Main effects of horizontal and vertical gaze position from 2-way ANOVA results. 


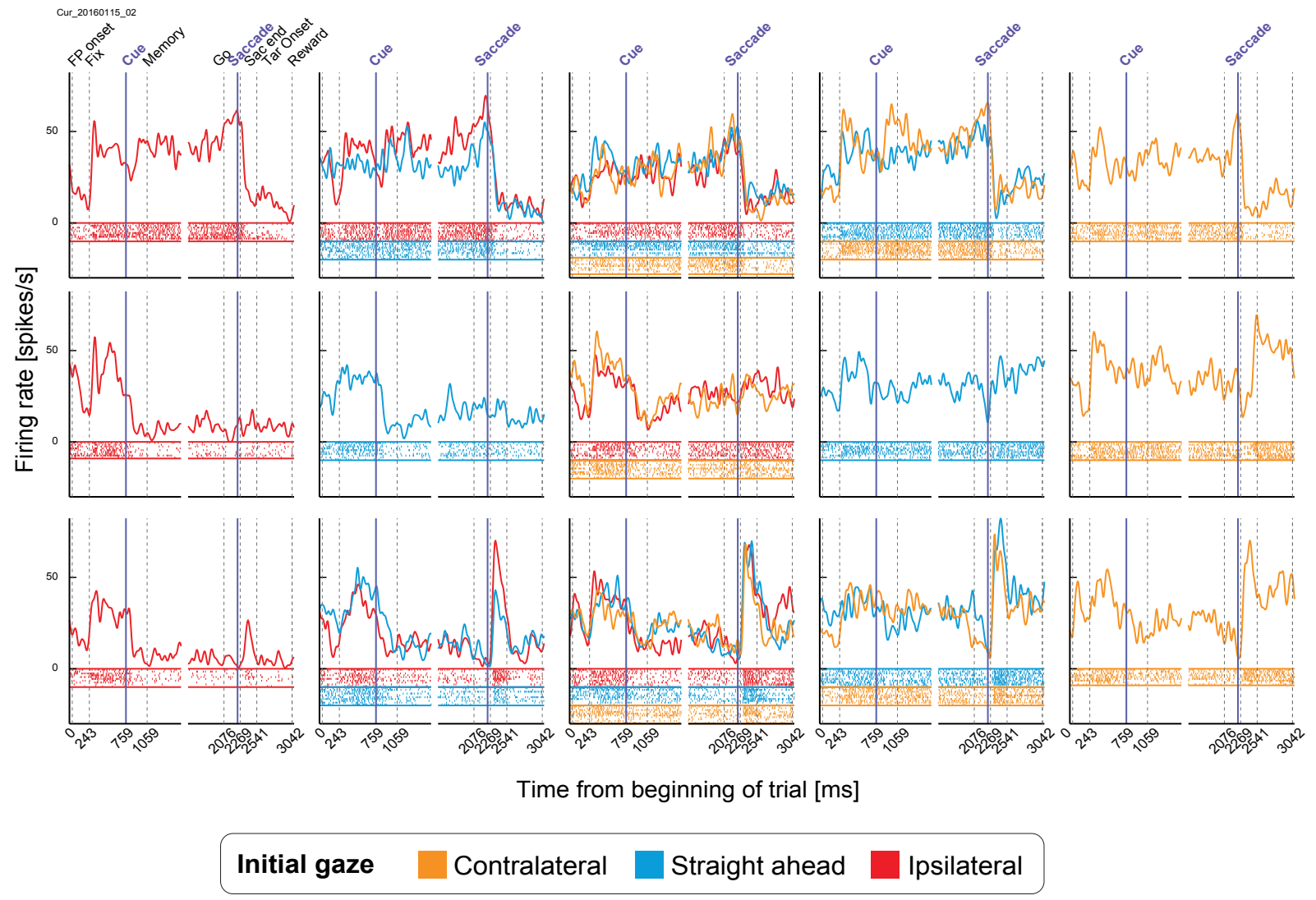

Supplemental Figure 3.3. Example unit with mixed reference frame. Example unit raster plots, spike density functions, and horizontal eye traces separately for each initial fixation position, for each of the 15 target locations relative to the screen. Colors depict the three initial gaze positions (orange: contralateral, blue: central, red: ipsilateral). This unit had high ACCs for both retinocentric and "screen-centered" references frames, from cue to pre-saccadic epoch, resulting in mixed reference frame classification.

\subsection{Contributions and Acknowledgements}

LS, AUDV, MW and IK designed the experiments, AUDV and LG collected data and performed initial analyses, LS analyzed the data, LS, MW and IK wrote the paper.

Lukas Schneider (LS); Melanie Wilke (MW); Igor Kagan (IK); Adán Ulises Domínguez Vargas (AUDV), Lydia Gibson (LG).

We thank Ira Panolias, Daniela Lazzarini, Sina Plümer, Klaus Heisig, and Dirk Prüße for technical support. We also thank Stefan Treue, Alexander Gail, Hansjörg Scherberger, members of the Decision and Awareness Group, Sensorimotor Group and the Cognitive Neuroscience Laboratory for helpful discussions. Supported by the Hermann and Lilly Schilling Foundation, German Research Foundation (DFG) grants WI 4046/1-1 and Research Unit GA1475-B4, KA 3726/2-1, CNMPB Primate Platform, and funding from the Cognitive Neuroscience Laboratory. 


\section{Dissociating perceptual and intentional aspects of neglect- like deficits}

\section{Abstract}

Hemi-spatial neglect in humans is a complex syndrome often characterized by sensoryperceptual and/or motor-intentional spatial deficits. However, the elucidation and characterization of these deficits is not trivial, and typically relies on combination of several independent and not mutually consistent tests. Here, we designed a novel "search-tosample" task sought to dissociate these two aspects in one task and allow inter-species comparisons in order to systematically evaluate the contribution of these two aspects in spatial deficits caused by lesions in different brain regions. We established task parameters that are suited for monkey-human comparisons, young and elderly human subjects, and tested the effect of reversible pharmacological inactivation on this novel task in two monkey brain regions: the dorsal pulvinar and cortical area TPO in superior temporal sulcus. In the preliminary electrophysiological analysis, single cell spiking activity and local field potentials (LFPs) of area TPO during a memory-guided saccade task showed overall similar patterns as dorsal pulvinar. The main difference was that visual responses were more pronounced and more frequent in TPO, although the majority of neurons exhibited both visual and motor related activity, and some cells showed delay period activity, also evident in the analysis of LFPs. The inactivation results in both regions suggest that area TPO inactivation disrupted contralesional stimulus perception, whereas pulvinar inactivation disrupted motor planning or execution and exploration behavior in a more general, non-spatial specific manner. Control inactivation experiments in dorsal pulvinar however revealed a space specific effect on contralesional saccade accuracy and a target selection bias towards the ipsilesional side when the monkey was allowed to immediately look at the visual target (visually-guided saccade), consistent with previous pulvinar inactivation studies. Furthermore, the pulvinar inactivation but not TPO inactivation caused late onset of the horizontal ocular nystagmus with slow drift phase into contralesional direction (fast beat ipsilesional nystagmus). These results indicate that causal perturbation in different brain regions can result in dissociable behavioral deficits even if the electrophysiological characterization of these regions does not indicate clear-cut differences. More generally, this work provides evidence that the novel task design is in principle applicable to studies of spatial deficits in monkeys and humans, but also highlights limitations due to training effects and idiosyncratic behavioral patterns in both species, as well as concerns about reliable task performance in neurological patients. 


\subsection{Introduction}

As mentioned in section 0 , several types of not primary sensory spatial deficits are summarized by the term "spatial (hemi)neglect". The symptoms can be grouped into 'sensory-perceptual' and 'motor-intentional', or motivational deficits (Hornak, 1992; Karnath and Rorden, 2012; Liu et al., 1992; Na et al., 1998). Perceptual deficits include reduced saliency, reduced spatial attention and more general the unawareness of stimuli on the neglected side, although stimuli are perceived when pointed at. Intentional deficits include a default bias in eye position and reduced exploration on the neglected side with eyes and hands, although patients are physically able to look at that side, and deficits can be compensated by increased desirability of targets on the neglected side (Malhotra et al., 2013).

Another syndrome which occurs predominantly after unilateral right hemisphere lesions is extinction (Becker and Karnath, 2007). Extinction is often described as a milder version of neglect, where the contralateral deficits only become apparent when two stimuli are presented simultaneously, one on each side (Milner and Mclntosh, 2005). Although the two syndromes often occur together, the associated lesion sites seem to be different for neglect and extinction (Karnath et al., 2003).

Typically, a battery of tests is used to evaluate spatial extinction and neglect. Most of these tests are more sensitive for assessing either perceptual or intentional deficits. One classical example is the line-bisection test. Subjects are asked to cross horizontal lines on a sheet of paper in the middle of the line. The lines have different lengths and are distributed over both left and right side of the paper. The severity of deficits is assessed by the offset of the intersection points from the actual centers of the lines. This indicates a perceptual bias, as the lines were perceived as not extending as much in one direction as in the other. Another classical example is the cancellation test. Subjects are asked to mark certain symbols which are distributed on a sheet of paper. In some cases, other symbols serving as distractors are also distributed throughout the sheet of paper. The severity of deficits is assessed by the number of missed symbols on the neglected side. This indicates reduced exploration on that side of space. So the line-bisection test is better suited to assess perceptual spatial deficits, while the cancellation test is more suitable to reveal intentional spatial deficits (Liu et al. 1992; Na et al. 1998). Importantly, both tasks require the perception of stimuli on both sides of space as well as the execution of an action towards these stimuli, which limits the comparability of the two aspects of spatial neglect. 


\subsubsection{Novel search-to-sample task design}

Here we designed a novel computer based task that sought to combine sensory and motor aspects of the performance, and thus dissociate potential perceptual and intentional deficits (see Figure 4.1). We also intended this task to be applicable to humans and monkeys, to allow inter-species comparisons and evaluation of deficits caused by targeted, region confined lesions in monkeys. This task will be referred to as "Search-to-Sample" (S2S) task.

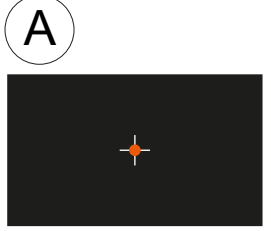

Fixation

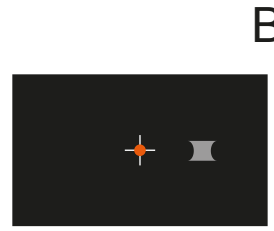

Sample (S) presentation

Basic task layout

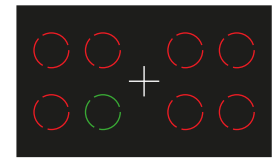

Exploration period starts

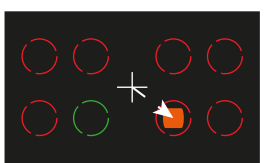

Distractor revealed

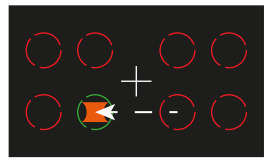

Match (M)

revealed

B

Predictions and simulated data
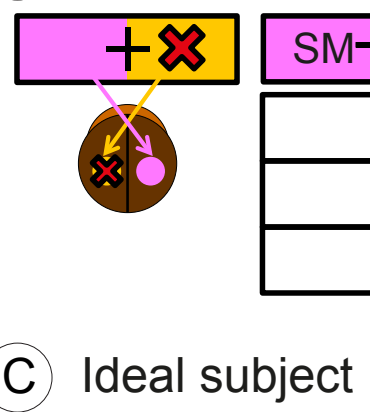

(C) Ideal subject

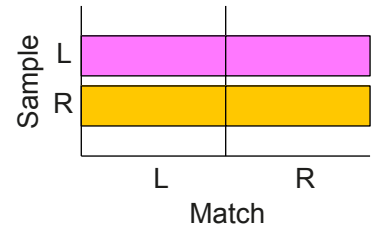

Perceptual deficits

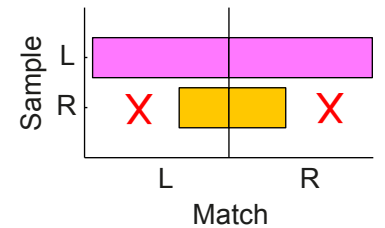

Intentional deficits

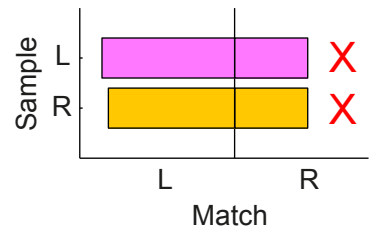

Figure 4.1: Search-to-Sample task design and outcome predictions A) Task layout. While subjects are asked to maintain fixation in the center of the screen (red dot), a sample object will appear on either the left or right side of the screen. After the fixation sample (S) disappears, subjects can freely explore the screen. At certain locations (indicated by dotted circles, which are not visible to the subject), target objects will appear when subjects look at the respective location. These objects can either be distractors or the match (M) - the same object as displayed during sample presentation. When the gaze position leaves a target area, the respective object disappears again from the screen. This way, target objects are never presented in the periphery, Final selection is indicated by keeping fixation on the target for a certain amount of time. The selected target can be either the match or a distractor. B) Expected impairments in the four main spatial conditions (combinations of match and sample locations) dependent on the type of deficit, simulated data. Red crosses indicate impaired conditions. C) Comparison of behavioral readouts for simulated deficits, demonstrated in the example of Success rate. While ideal subjects would always find the match, subjects with perceptual deficits would fail to identify the sample on the neglected side and subjects with intentional deficits would fail to explore the neglected side. This can be reflected in the success rate for the respective conditions

The basic idea is that subjects have to perceive a sample presented on the left or right side in order to find the matching target object among distractor objects, which can also be placed on both sides. Subjects have to explore the screen in order to reveal target objects one by one, and the target objects are always only presented at the current gaze position, minimizing the influence of perceptual deficits during the exploration period. Impairments in 
the different spatial conditions (dependent on sample and match position) can reveal the presence of perceptual and/or intentional deficits, meaning either the sample was not perceived correctly when presented on one side or the subject had difficulties exploring one side and to find the match located on that side. Task details will be further mentioned in the respective result sections, as several versions of the task were tested.

To give an idea of how this task can be modified, one option is to add another (distractor) stimulus during sample presentation in order to test for extinction. Another option is to introduce only unilateral difference in the stimulus shapes to test for object based neglect, which was the main motivation behind choosing this kind of stimuli (concave or convex on the left and/or right side).

\subsubsection{Pulvinar lesions}

As mentioned before, unilateral pulvinar lesions have been associated with spatial neglect (Arend et al., 2008a; Karnath et al., 2002). Furthermore, it has been hypothesized that pulvinar plays an important role in a cortico-subcortical spatial awareness network (Karnath et al., 2001). This view is supported by pulvinar perturbation studies in monkeys, leading to symptoms similar to spatial neglect or extinction (Petersen et al., 1987; Wilke et al., 2010, 2013). Importantly, after unilateral pulvinar perturbation, monkeys showed no deficits related to executing eye movements to both hemifields in single target instructed tasks (DominguezVargas et al., 2017; Wilke et al., 2010). Furthermore, target selection biases were compensated by the amount of expected reward (Wilke et al., 2013), suggesting a not purely mechanistic or perceptual, but rather motivational influence of pulvinar disruption.

In a recent study performed in our lab, we had the opportunity to examine a patient with a bilateral pulvinar lesion, see Appendix B ). General observations included postural, reach and grasp deficits with both left and right hand, a down-beat nystagmus, and saccade hypometria, but no optic ataxia. More relevant for the current context, despite the fact that the lesion was more pronounced in the left hemisphere, no neglect like deficits were found in any of the grasping, reaching, stimulus detection, or two alternative forced choice saccade tasks. This could either be explained by either bilaterality of the lesion, or by the lateralization of the human brain, as the right hemisphere is more involved in spatial processing (compared to the left hemisphere which is more involved in language processing), and neglect is in general more often observed after right hemisphere lesions. Importantly, this type of lateralization does not seem to be present, or at least present to a lesser extent in monkeys (Kagan et al., 2010).

\subsubsection{Control inactivation of area TPO}

The temporo-parietal-occipital (TPO) area in the macaque monkey, also known as STP, spans almost the entire dorsal bank of the superior temporal sulcus (STS). This area was 
selected for control inactivation experiments, based on several criteria. First, TPO is supposedly a primarily sensory, rather than sensorimotor area. Still, it has also been shown to be involved in action execution (Kilintari et al., 2014). Although it was originally assigned to be part of the dorsal stream (Ungerleider and Haxby, 1994), most recent studies targeting TPO mainly focus on object encoding and multi-sensory integration (Dahl, 2010; Hein and Knight, 2008; Hoffman, 2008). This potential interplay of perceptual ventral stream characteristics and goal-directed dorsal stream processing already makes TPO an interesting target for attempting to dissociate perceptual and intentional aspects. Second, fMRI studies suggest a space specific causal influence of dorsal pulvinar inactivation on the BOLD fMRI activity in area TPO (Wilke, Kagan, Andersen in revision), which makes TPO specifically interesting for study. And finally, lesion studies in macaque monkeys showed that ablation of area TPO also causes neglect-like symptoms (Luh et al., 1986; Scalaidhe et al., 1995). The latter finding suggests that TPO might be the monkey homologue of the superior temporal gyrus (STG) in humans, an area that is one of the major lesion foci in neglect patients (Karnath, 2001). 


\subsection{Materials and Methods}

\subsubsection{Human subjects}

All human experiments were conducted in accordance with the local ethic committees (Ethikkommission der Universitätsmedizin Göttingen for experiment 1 and Ethikkommission des Georg-Elias-Müller-Instituts für Psychologie for experiment 2).

\subsubsection{Experiment 1}

Two groups of subjects participated in this part of the study.

Group 1 (young, healthy): eight subjects (4 female), mean age $25.1+-3.4$.

Group 2 (elderly, healthy): six subjects (1 female), mean age $58.3+-6.2$.

Group 3 (neglect patients): one neglect patient (male) aged 62.

Subjects performed three to four sessions at different days. A session lasted up to two hours. Subjects were paid 8 or 10 Euro per hour for their participation (young and elderly subjects, respectively). The patient only performed two sessions of around one hour each. He was paid 20 Euro per hour.

At the beginning of the first session, all participants received the same written task instructions. At the beginning of each session, subjects performed training trials in their respective Search-to-Sample task. Training in the first session lasted as long as subjects needed to get comfortable with the task. For training, the difficulty (e.g. difference in target objects) was decreased to allow a faster understanding of the concept of the task. As soon as they were comfortable with the task, the difficulty was increased to the values to be used during the following runs. In the other sessions, training usually lasted only a couple of trials (20-30 trials compared to 70-90 trials in the first training run) until subjects were comfortable again with the task and the difficulty from the previous session (see below). Auditory feedback signaled correct and incorrect target selections. A session had the following sequence:

1: Setup adjustment, including calibration

2: Training (difficulty of previous session)

3: Difficulty adjustment run (staircase procedure to adjust success rate to $\sim 80 \%$ )

4: One to several runs with the adjusted difficulty, 96 or 128 completed trials

\subsubsection{Experiment 2}

Two groups of subjects participated in this part of the study.

Group 1 (young, healthy): eleven subjects (6 female), mean age $24.5+-8.3$.

Group 2 (elderly, healthy): six subjects (all male), mean age $62.3+-3.7$. 
Subjects performed one session each. A session lasted up to two hours. Subjects were paid 10 Euro per hour for their participation.

At the beginning of the session, all participants received the same written task instructions and performed training trials for the Search-to-Sample task. Training lasted as long as subjects needed to get comfortable with the task. For training, the difficulty was decreased, to allow a faster understanding of the concept of the task. As soon as they were comfortable with the task, the difficulty was increased to fixed values estimated from the previous experiment. No auditory feedback was used in this experiment, because when testing patients, error feedbacks could lead to additional demotivation. A session had the following sequence:

1: Setup adjustment, including calibration

2: Training (difficulty of previous session)

3: Several test runs of 48 completed trials each, typically 4 runs. Calibration was re-established before each test run

\subsubsection{Monkey procedures}

All animal experimental procedures were conducted in accordance with the European Directive 2010/63/EU, the corresponding German law governing animal welfare, and German Primate Center institutional guidelines. The procedures were approved by the responsible government agency (LAVES, Oldenburg, Germany).

\subsubsection{Animal preparation}

One adult male rhesus monkey (Macaca mulatta) weighing $9 \mathrm{~kg}$ was used. In an initial surgery the monkey was implanted with a magnetic resonance imaging (MRI) compatible polyetheretherketone (PEEK) headpost embedded in a bone cement headcap (Palacos with Gentamicin, BioMet, USA) anchored by ceramic screws (Rogue Research, Canada), under general anesthesia and aseptic conditions. MR-visible markers were embedded in the headcap to aid the planning of the chambers in stereotaxic space (right hemisphere: center at 4.4P / 23.5L mm, tilted: $5 \mathrm{P} / 20 \mathrm{~L}$ degrees; left hemisphere: center at $4.2 \mathrm{P} /-24 \mathrm{~L} \mathrm{~mm}$, tilted: 5P / -20L degrees) with the MR-guided stereotaxic navigation software Planner (Ohayon and Tsao, 2012). A separate surgery was performed to implant a PEEK MRI-compatible chamber (inside diameter $22 \mathrm{~mm}$ ) allowing access to the left and right pulvinar as well as to the left and right STS (although in all monkey experiments shown in this chapter, only the left chamber was used). A partial craniotomy was made inside each chamber. The exposed dura was covered with a silicone elastomer (Kwik-sil, World Precision Instruments, USA) to reduce the granulation tissue growth and dura thickening. 


\subsubsection{MR imaging}

The monkey was scanned in a 3T MRI scanner (Siemens Prisma). Full-head T1-weighted scans (3D magnetization-prepared rapid gradient-echo, MPRAGE, $0.5 \mathrm{~mm}$ isometric) were acquired before and after chamber implantation, in anaesthetized state, using either built-in gradient body transmit coil and custom single loop receive coil, or custom single loop transmit and 4-channel receive coil (Windmiller Kolster Scientific, USA).

In addition to pre- and post-implantation scans, similar T1-weighted scans as well as T2weighted (rapid acquisition with relaxation enhancement, RARE, $0.25 \mathrm{~mm}$ in plane, $1 \mathrm{~mm}$ slice thickness) scans were periodically acquired during the course of experiments, in sedated state, to confirm injection cannula positioning. T1- and T2-weighted scans were coregistered and transformed into "chamber normal" (aligned to the chamber vertical axis) and to AP-PC space for targeting and visualization. These images were acquired with the chamber and the grid filled with gadolinium (Magnevist, Bayer, Germany)/saline solution (proportion 1:200), with tungsten rods inserted in predefined grid locations, for alignment purposes.

\subsubsection{Pharmacological inactivation}

Two different $\mathrm{GABA}_{\mathrm{A}}$ agonists were used for pharmacological inactivation, Muscimol and 4,5,6,7-tetrahydroisoxazolo[5,4-c]pyridin-3-ol (THIP) (Tocris Bioscience). Solutions were prepared under sterile conditions, mixing the active substances with phosphate-buffered saline (PBS) to reach a concentration of $6.6 \mathrm{mg} / \mathrm{ml}$. To minimize structural damage caused by the injected fluids, the $\mathrm{pH}$ value was checked with either an electronic $\mathrm{pH}$-meter (HANNA Instruments, Italy) or $\mathrm{pH}$-measuring stripes (Macherey-Nagel, Germany) and adjusted to reach 7.3-7.7 by adding drops of sodium hydroxide $(\mathrm{NaOH})$ solution $(0.04 \mathrm{~g} / \mathrm{ml})$ to lower the $\mathrm{pH}$ value or hydrogen chloride $(\mathrm{HCl})$ solution to increase it (the latter step has not been used so far). The prepared injection fluids were filled into a gas-tight glass syringe (Hamilton, USA) avoiding any air bubbles. The syringe which was connected to the injection cannula (31 gauge, $60 \mathrm{~mm}$ long) via a $1 \mathrm{~m}$ tubing line attached to the syringe hub needle and placed into a high precision microinjection pump (Harvard Apparatus, USA). Unhindered liquid flow was ensured before inserting the cannula in the brain by running the microinjection pump until the liquid dripped out on the cannula side.

The location of the injection cannula was estimated for every inactivation session based on anatomical MRI. A custom-made MR-compatible polyetherimide (Ultem) grid $(0.8 \mathrm{~mm}$ hole spacing, $0.45 \mathrm{~mm}$ hole diameter) was used to position the injection cannula in the corresponding grid hole. A custom-made stainless steel guide tube (450 $\mu \mathrm{m}$ outer diameter, 27 gauge Spinocan, Braun Melsungen AG, Germany) with a stopper (530 $\mu \mathrm{m}$ inner diameter, $665 \mu \mathrm{m}$ outer diameter, 23 gauge MicroFil, World Precision Instruments, USA) was used to 
penetrate the dura and ensured that the injection cannula went straight and ended up in the estimated depth.

\begin{tabular}{|c|c|c|c|c|c|c|}
\hline Session & $\begin{array}{l}\text { Injection } \\
\text { target }\end{array}$ & $\begin{array}{l}\text { Injected } \\
\text { substance }\end{array}$ & $\begin{array}{l}\text { Injected } \\
\text { volume }\end{array}$ & $\begin{array}{l}\text { Visually } \\
\text { Guided }\end{array}$ & $\begin{array}{l}\text { Memory } \\
\text { guided }\end{array}$ & $\begin{array}{l}\text { Search-to- } \\
\text { sample }\end{array}$ \\
\hline 20170119 & TPO & Muscimol & $3.6 \mu \mathrm{l}$ & - & - & 8 locations \\
\hline 20170301 & TPO & Muscimol & $4.5 \mu \mathrm{l}$ & - & Short mem & 8 locations \\
\hline 20170329 & TPO & Muscimol & $4 \mu \mathrm{l}$ & - & - & 8 locations \\
\hline 20170407 & TPO & Muscimol & $5 \mu \mathrm{l}$ & - & Short mem & 8 locations \\
\hline 20170412 & TPO & Muscimol & $5 \mu \mathrm{l}$ & - & Short mem & 8 locations \\
\hline 20170421 & TPO & Muscimol & $6.5 \mu \mathrm{l}$ & - & Short mem & 8 locations \\
\hline 20170518 & Pulvinar & THIP & $2.5 \mu \mathrm{l}$ & - & Short mem & 8 locations \\
\hline 20170526 & Pulvinar & THIP & $3 \mu l$ & - & Short mem & 8 locations \\
\hline 20170601 & Pulvinar & THIP & $4.5 \mu \mathrm{l}$ & - & Short mem & 8 locations \\
\hline 20170609 & Pulvinar & Muscimol & $2.5 \mu \mathrm{l}$ & - & Short mem & 18 locations \\
\hline 20170616 & Pulvinar & THIP & $5.5 \mu \mathrm{l}$ & - & Short mem & 18 locations \\
\hline 20170629 & Pulvinar & THIP & $5.5 \mu \mathrm{l}$ & - & Short mem & 18 locations \\
\hline 20170707 & Pulvinar & THIP & $5.5 \mu \mathrm{l}$ & $y$ & Long mem & - \\
\hline 20170713 & Pulvinar & THIP & $5.5 \mu \mathrm{l}$ & $y$ & Short mem & 18 locations \\
\hline 20170721 & Pulvinar & Muscimol & $3 \mu l$ & $y$ & Long mem & 18 locations \\
\hline 20170727 & Pulvinar & Muscimol & $3 \mu \mathrm{l}$ & $y$ & - & 18 locations \\
\hline 20170804 & Pulvinar & THIP & $3 \mu l$ & $y$ & Long mem & 18 locations \\
\hline 20170811 & TPO & THIP & $4.5 \mu \mathrm{l}$ & - & Long mem & 18 locations \\
\hline
\end{tabular}

Table 4.1: Details for all inactivation sessions. "Long mem" and "Short mem" as well as "8 locations" and "18 locations" indicate different variations of the two tasks, memory guided saccades and the search-to-sample task respectively, see Task below. "-" denotes that the respective task was not performed in this session. 
In the pulvinar, $2.5-3 \mu \mathrm{l}$ of muscimol (3 sessions) or $2.5-5.5 \mu \mathrm{l}$ of THIP (8 sessions) were injected and in different sessions 3.6-6.5 $\mu \mathrm{l}$ of muscimol (6 sessions) or $4.5 \mu \mathrm{l}$ of THIP (1 session) was injected in the TPO, always at a rate of $0.25 \mu \mathrm{l} / \mathrm{min}$. For details see Table 4.1.

\section{Pulvinar injection}
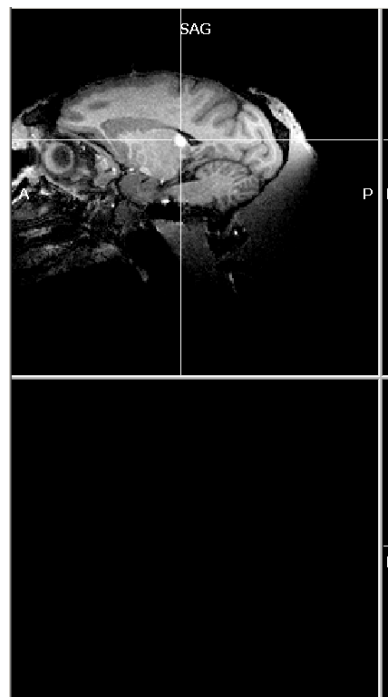

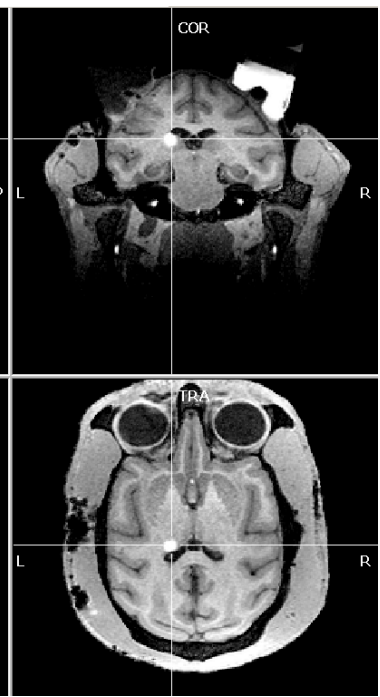

TPO injection

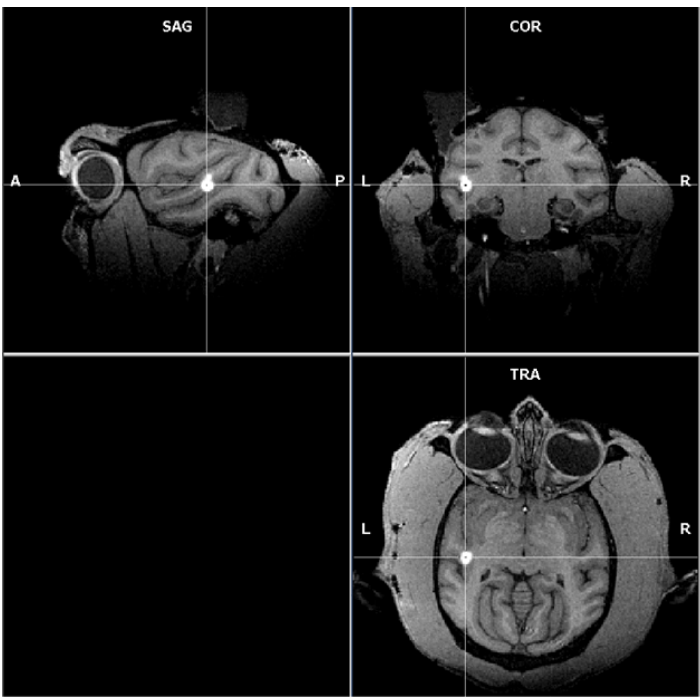

Figure 4.2: Gadolinium injection sites T1-weighted images.

Figure 4.2 shows MRI images of the injection sites for pulvinar and TPO, taken before the experiments to assess correct positioning of injection cannulas. Images were taken 30 minutes after injection of $2 \mu \mathrm{l}$ (pulvinar) and $3 \mu \mathrm{l}$ (TPO) of a gadolinium (Magnevist, Bayer, Germany)/saline solution (proportion 1:200). Control sessions were recorded the day before each inactivation session with the same tasks and the same task parameters.

\subsubsection{Electrophysiological recordings}

Neuronal activity was recorded in 9 sessions in left dorsal bank of the superior temporal sulcus (STS) with up to five individually-movable single platinum-tungsten (95\%-5\%) quartz glass-insulated electrodes with impedance ranging from $1 \mathrm{M} \Omega$ to $2 \mathrm{M} \Omega$, using chambermounted 5-channel Mini Matrix microdrive (Thomas Recording, Germany). The recording target locations were estimated similarly to the inactivation sessions, using the same grids. Similar to inactivation experiments, single custom-made stainless steel guide tubes (27 gauge) filled with the silicone oil (Thomas Recording), with a Spinocan funnel attached to the drive nozzle were used to protect electrodes during dura penetration. A reference tungsten rod was placed in the chamber filled with saline, and was connected to the chassis of the drive. Neuronal signals were amplified (x20 headstage, Thomas Recording; x5, 32 channel PZ5 preamplifier, Tucker-Davis Technologies, USA), digitized at $24 \mathrm{kHz}$ and 16 bit resolution, and sent via fiber optics to an RZ2 BioAmp Processor (Tucker-Davis 
Technologies, USA) for online filtering, display and storage on a hard drive together with behavioral and timing data streams.

\subsubsection{Task designs}

The monkey sat in a dark room in a custom-made primate chair with its heads restrained 30 cm away from a 27" LED display (120 Hz refresh rate, model HN274H, Acer Inc. USA) or a 24" LED display (120 Hz refresh rate, model VG248QE, ASUSTeK COMPUTERS INC., Taiwan). The gaze position of the right eye was monitored at $220 \mathrm{~Hz}$ using an MCU02 ViewPoint infrared eyetracker (Arrington Research Inc. USA).

Human subjects were sitting in a darkened room on a chair that was aligned to the center of the monitor with head and eyes facing straight ahead and an eye-to-screen distance of 30 $\mathrm{cm}$. Their head was stabilized by a chin rest and locked tight into the position with bars pressing against both sides of the head (HeadLock ${ }^{\mathrm{TM}}$ Ultra Precision Head Positioner TM, ViewPoint, Arrington Research, USA). Stimuli were presented on a 27" LED display (resolution of $1920 \times 1080,60 \mathrm{~Hz}$, BenQ XL2720T). Real-time eye tracking was performed with a ViewPoint system (Arrington Research Inc. USA), where a mini-IR sensitive camera placed below subjects' right eye continuously sampled their gaze position at $60 \mathrm{~Hz}$.

Before the start of each experiment, the eye tracker was calibrated using the $4 \times 5$ point matrix from the ViewPoint software (only for human subjects). An additional linear calibration implemented in the task controller guaranteed fast offset correction and gain refinement in case of slight head movements. All stimulus presentation and behavioral control tasks were programmed in MATLAB (The MathWorks, Inc. USA) and the Psychophysics Toolbox (Brainard, 1997).

\subsubsection{Visually guided saccade task}

A trial started with the onset of the fixation spot. After the monkey acquired and held fixation within a $5^{\circ}$ radius for a randomized period ranging from 500 to $1000 \mathrm{~ms}$, the fixation spot $\left(1^{\circ}\right.$ diameter) was extinguished and either one target (instructed trials) or two targets (choice trials) appeared synchronously (for an illustration see chapter 2). This time point will be referred to as the "Go signal". Targets (also $1^{\circ}$ diameter) were presented in the left and/or right side(s) of the fixation spot, at $12^{\circ}$ or $24^{\circ}$ eccentricity, with three potential angles relative to the horizontal axis, either $0^{\circ}, 20^{\circ}$ or $-20^{\circ}\left(0^{\circ}, 4.1^{\circ},-4.1^{\circ}, 8.2^{\circ}\right.$ and $-8.2^{\circ}$ vertical eccentricity). The monkey had to make a saccade within $500 \mathrm{~ms}$ and keep his gaze position for $500 \mathrm{~ms}$ inside a $5^{\circ}$ radius window surrounding the target to successfully complete a trial and obtain a liquid reward after a delay of $200 \mathrm{~ms}$. In choice trials the monkey was allowed to freely choose one of the targets, both choice targets were always presented at the same height and provided equal reward. The inter-trial interval was $1000 \mathrm{~ms}$ or $2000 \mathrm{~ms}$. All trial 
types and target locations were pseudo-randomized. A minimum of 10 instructed trials per instructed target location per session were collected.

\subsubsection{Memory guided saccade task}

Similarly to the visually guided saccade task, the monkey had to acquire and hold fixation for either $500 \mathrm{~ms}$. Next, one or two peripheral cues were displayed for $280 \mathrm{~ms}$ at the location(s) signaling the upcoming saccade target(s). These cues had the same spatial characteristics as the targets in the visually guided task. The monkey was required to maintain fixation throughout the cue period and also throughout the subsequent memory period (ranging from 500 to $1000 \mathrm{~ms}$ ), after which the central fixation spot disappeared (Go signal), allowing the monkey to saccade to the instructed location, or make a decision to go to one of the two cued locations. After a saccade to and fixation of the remembered target location for $300 \mathrm{~ms}$ the target became visible and after additional $500 \mathrm{~ms}$ of peripheral fixation the trial was completed. In the first recording session, cue presentation time was variable (200 ms to 280 $\mathrm{ms}$ ) and the required time to fixate the remembered location before the target became visible was only $100 \mathrm{~ms}$. In 4 inactivation sessions, fixation hold time was variable (500 to $1000 \mathrm{~ms}$ ), and the memory period was extended to 2000 to 2500 ms, "long mem" sessions in Table 4.1. The inter-trial interval was $1000 \mathrm{~ms}$ or $2000 \mathrm{~ms}$. All trial types and target locations were pseudo-randomized. A minimum of 10 instructed trials per instructed target location per session were collected.

\subsubsection{Task development and monkey training for the Search-to-sample task}

Since the search-to-sample task is a novel task design, several human pilot studies have been conducted and the task was modified according to the results. The adjustments were done in parallel to monkey training, so the steps of adjustment also reflect the training procedure.

In the initial training version, after fixating in the center for $200 \mathrm{~ms}$ to $300 \mathrm{~ms}$, the sample was presented for 1 second 10 degrees horizontally either on the left or on the right side. The sample and the fixation spot were turned off at the same time, and four target symbols would appear on the screen. The monkey could select by keeping fixation at one of them for one second.

In the first adjustment, instead of directly revealing the target symbols, four red dots would appear at fixed positions at the same time the fixation spot was turned off. By looking at one of the four spots, one out of four target symbols appeared instead of the red dot. By leaving the allowed radius, the target symbol was replaced again by a red dot. Final target selection was indicated by keeping the gaze at a target for one second. This was the first version of the task tested in a human pilot study, which showed that a) quite often the first saccade was related to the position of the sample (either towards the sample or in the opposite direction, 
but very consistent within subject) and b) exploration patterns were very stereotypical within subject. To counteract the a), a memory period where subjects still had to fixate in the center was introduced between sample offset and the exploration period. To counteract $b$ ) target positions were varied from trial to trial.

Another pilot study was conducted with 7 targets per trial at random positions. Since the idea was to have a continuous difference of target symbols (in curvature) while keeping them discriminable, only four different target shapes were used in this version of the task. This lead to the theoretical problem that subjects could solve the task by ignoring the sample and just select the target which was unique. Additionally, trial duration increased substantially due to extended exploration periods.

In the first human study presented here, in each trial three out of four target symbols were arranged in one out of four spatial configurations, see Figure 4.4. The location was not indicated by place holders (red dots), but instead subjects explored on a black screen until they revealed a target. Additionally, a modification of the task was tested, where synchronously with the sample a distractor cue was presented on the opposite side of the screen (double cue task). For the distractor cue, the fourth target symbol which was not present in the target array was used. Sample and distractor shapes were randomized for each trial.

Still with this target arrangement, exploration patterns of both humans and monkey became stereotypical after a short time, which is why for the final monkey version of the task, targets were randomly dispersed in three out of eight possible positions, for each trial, see Figure 4.11. In the later sessions the number of possible target locations was increased to 18 , see "18 locations" sessions in Table 4.1.

For the second human study presented here, the sample presentation was shortened to 250 ms and the memory period was again removed, allowing subjects to perform reflexive saccades (without directly looking at the sample), because from our previous finding it was expected that neglect patients would have trouble to keep fixating when a stimulus appears abruptly in the periphery, see Figure 4.8. Also, the final selection was indicated by a button press to avoid affecting the performance by difficulties keeping the eye position stable in the periphery.

\subsubsection{Analysis}

\subsubsection{Saccade definitions}

Since the sampling rate differed for monkey and human experiments $(220 \mathrm{~Hz} / 60 \mathrm{~Hz}$ respectively), saccades were defined slightly different for both species. Saccade velocity was 
calculated sample by sample as the square root of the sum of squared interpolated $(220 \mathrm{~Hz} /$ $60 \mathrm{~Hz}$ to $1 \mathrm{kHz}$ ) and smoothed (12 ms / $30 \mathrm{~ms}$ moving average rectangular window) horizontal and vertical eye position traces, and then smoothed again (12 ms / 30 ms moving average rectangular window). Saccade onset was defined as an eye position change that exceeded $300 \% \mathrm{~s}$ (40\% for humans) and the saccade offset as reaching a velocity of $50 \% \mathrm{~s}$ (25\% for humans). Saccade endpoint was defined as the eye position when the saccade velocity reached the ending threshold. For the control tasks, in cases when several consecutive eye movements in the time interval from the Go signal until the target acquisition fitted the above criteria, the first saccade was selected for the reaction time analysis and the last one for the end-point inaccuracy/imprecision analysis.

\subsubsection{Inaccuracy and imprecision}

For horizontal and vertical axes, the saccade offset was calculated as signed offset between the saccade endpoint and the target center for each trial. Inaccuracy was defined as the square root of the sum of squared mean offsets (across trials) for each axis. Similarly, saccade imprecision (endpoint scatter) was defined as the square root of the sum of squared standard deviations (across trials) of the signed saccade offsets for each axis.

\subsubsection{Performance readouts in the Search-to-Sample task}

Due to the complexity of this novel task, there are numerous potential readouts. However, initial pilot studies showed that the most consistent and easiest to interpret are reaction times, exploration times, dwell times and success rates.

Reaction time was defined for the first saccade in each trial as the time from fixation point offset until the beginning of the saccade. For comparisons across sessions, trials were grouped into four conditions, either based on the position of the sample (left or right) or the horizontal component of direction the saccade direction (left or right).

Dwell time was calculated for each target individually, based on the maximum continuous time points in which the eye position was inside the target radius (excluding the last, which was forced to be equal to selection time). For comparisons across sessions, dwell times per target were grouped into four conditions, either based on the position of the sample (left or right) or the horizontal component of direction the target position (left or right).

Exploration times were calculated for each trial and side of the screen (left or right) independently as the total time spent exploring the respective side of the screen. For comparisons across sessions, exploration times were also grouped based on the position of the sample (left or right), resulting in four conditions. 
- Reaction time

- First saccade

- Dwell time

- Exploration time

- Success rate

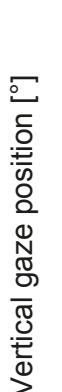

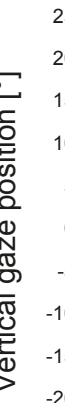

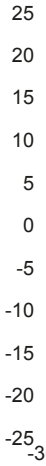

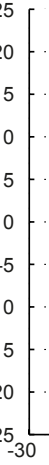

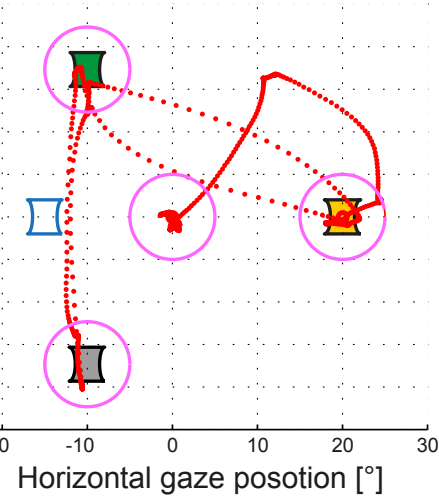

- gaze position eye allowed radius Sample Match Selected target not selected targets

Figure 4.3: Example Search-to-Sample trial. During sample presentation on the left side, the eye position (red dots, one for each millisecond) remained within the allowed radius around the fixation spot. After the fixation spot vanished and the subject registered that change (reaction time), he made his initial saccade, in this case to the right side. Since no target was revealed in this position, he continued exploring and revealed all the three targets, one at a time. Two of them were revealed even twice. The dwell time for each target was taken as the maximum time that the respective target was revealed continuously. The exploration time was defined as the total time spent exploring after the initial saccade. Note that this subject knew exactly where the targets were to be found after the first saccade, even though the locations were not indicated.

Success rate was defined for each session as the number of trials in which the match was selected divided by the number of completed trials, and computed independently for four trial types, dependent on the location of the sample (left or right) or the location of the match (left or right).

Mean values per session were computed for each of the four conditions, for each parameter (except for success rate, since that already represents one value per session per condition). These mean values were compared either between groups of subjects, tasks with different cognitive demand, or control and inactivation sessions.

\subsubsection{Statistical analysis of behavioral data}

All data analysis was performed using MATLAB R2012b. In control tasks (visually guided and memory guided saccade task), Mann-Whitney-U test was used for all comparisons between two spatial conditions and to compare inactivation with control sessions within the same spatial condition. In the search-to-sample task, paired t-tests were used to compare means per session between inactivation and control sessions and to compare means per subject in the two different tasks in the human experiment 1. For comparisons across groups of subjects, unpaired t-tests were used. To evaluate significant differences within subject or paired control/inactivation sessions, unpaired t-tests were used, except for success rate comparisons, where Fisher's exact test was used. Statistical significance was reported at $p<0.05\left(^{*}\right), p<0.01\left(^{(*}\right)$ and $\left.p<0.001{ }^{(* *}\right)$. Specific statistical tests are listed for each 
individual analysis. In the figures and in the text, standard deviation (SD) was used when averaging across trials and standard error of mean (SE) when averaging across sessions.

\subsubsection{Spike analysis}

Broadband data was preprocessed using an adapted version of wave_clus (Kraskov et al., 2009; Michaels et al., 2015; Quiroga et al., 2004) in order to isolate spikes. The preprocessing steps included filtering (333 Hz $-5000 \mathrm{~Hz}$ bandpass Butterworth), automatic thresholding ( 3 times the median of the absolute deviations from 0 in the filtered data, divided by 0.6745 - which equals 3 standard deviations assuming normal distribution) and discarding spikes that were detected within $1 \mathrm{~ms}$ of another, larger spike. Spike sorting was done in Plexon Offline Sorter v.3.3.5, using a waveform template algorithm after defining templates by manually clustering in principle component space.

150 single and multi-units recorded in the dorsal bank and fundus of STS during the memory guided saccade task. Out of these, 125 cells fulfilled analysis selection criteria (stable discriminability across time and reasonable SNR - assessed by inspection). 97 out of these 125 cells were recorded for at least 5 successful instructed trials for each of the 12 targets. These 97 cells were used for further analysis.

For each trial, and each epoch of interest, firing rates were computed by counting spikes within the epoch and dividing by the epoch duration. The analyzed epochs were "fixation hold" (last $300 \mathrm{~ms}$ of central fixation), "cue onset" (40 ms to $140 \mathrm{~ms}$ after onset of the cue), "early memory" (40 ms to 140 ms after cue offset), "late memory" (last 300 ms of the memory period), "pre-saccadic" (100 to $10 \mathrm{~ms}$ before saccade onset), "peri-saccadic" (10 ms before to 50 ms after saccade onset), "post-saccadic" (first 150 ms of fixating the invisible peripheral target), "target onset" (first $100 \mathrm{~ms}$ after target onset), and "target hold" (last $300 \mathrm{~ms}$ of fixating the peripheral target).

For population analysis, data from 6 left and 6 right hemifield targets were combined. Spatial tuning in each epoch was determined by unpaired t-tests comparing firing rates in ipsilateral trials to firing rates in contralateral trials. The hemifield with the higher firing rate was marked, if there was a significant difference. Enhancement or suppression of neuronal activity in each epoch was defined by paired t-tests comparing firing rates to a respective baseline epoch, for ipsilateral and contralateral trials independently. For "cue onset", "early memory" and "late memory" epochs "fixation hold" served as baseline, and "late memory" epoch served as baseline for subsequent epochs. Enhancement or suppression was reported, if either ipsilateral, contralateral, or both types of trials showed significant difference to fixation baseline. In rare cases where one hemifield would show a significant enhancement, while the other hemifield showed suppression, the unit was reported to have bidirectional response. 
Units that showed enhancement or suppression for at least one hemifield in both "cue onset" and "post-saccadic" epochs were classified as visuomotor units, whereas units that only showed enhancement or suppression in one of the two epochs were classified as visual (only enhancement or suppression in "cue onset") and motor (only enhancement or suppression in "post-saccadic").

For each unit, a two-way ANOVA was performed across all firing rates in each of the respective epochs from successful trials, using hemifield of the target position and epoch as factors for determining a main effect of epoch, a main effect of the hemifield, and interaction between the two. In the data presented in Figure 4.20, spatial tuning is only displayed for units showing either a main effect of hemifield or [hemifield $\times$ epoch] interaction, and enhancement or suppression is only displayed for units that showed either a main effect of epoch or [hemifield $\times$ epoch] interaction.

To evaluate the time of modulation onset for each unit and further select the "cue onset" epoch, spike density functions for each trial were derived by convolution of the discrete spike arrival times with a Gaussian kernel (SD $20 \mathrm{~ms}$ ). For three alignments (to cue onset, saccade onset and target onset), spike densities for unit and each bin was compared to the average firing rate in the respective baseline epoch ("fixation hold" for cue alignment and "late memory" for saccade and target alignment) using paired t-tests across all trials to evaluate bin-by-bin modulation. Significant increases were reported as enhancement and significant decreases as suppression. Similarly, for each unit and each bin, the spike densities of contralateral trials and ipsilateral trials were compared using unpaired t-tests. Significantly higher spike density for contralateral trials was reported as contralateral preference and significantly higher spike density for ipsilateral trials as ipsilateral preference. To avoid overestimation of significance due to multi-comparison, significance in less than five consecutive bins was discarded. For each unit and each type of modulation, the modulation onset was taken as the first bin (out of at least 5 consecutive bins) after certain time point that showed significance. For modulation onset relative to cue onset, the first significant bin after cue onset was taken and for modulation onset relative to the saccade the first significant bin after 200 ms before saccade onset was taken. Additionally, we counted the number of units which showed significant modulation in each bin and calculated the time in which most units were modulated. Based on the finding that most units showed enhancement 90 ms after cue onset (Figure 4.18), the "cue onset" epoch was selected as 50 $\mathrm{ms}$ before to $50 \mathrm{~ms}$ after that maximum.

Spike density functions for each trial were derived by convolution of the discrete spike arrival times with a Gaussian kernel (SD 20 ms). Significant enhancement or suppression across time was tested comparing spike density in each $10 \mathrm{~ms}$ bin with the firing rate in the 
respective baseline epoch ("fixation hold" for cue aligned activity and "late memory" for saccade onset aligned activity) using paired t-tests across all trials. Significant spatial preference across time was tested comparing spike density in each $10 \mathrm{~ms}$ bin between the two spatial conditions (ipsilateral and contralateral) using t-tests. Significant bins for both comparisons were only reported if at least five consecutive bins were significant.

To calculate population PSTHs, spike density functions of each trial were normalized dividing by the firing rate during "fixation hold" epoch. Average responses for each unit were then derived by averaging the normalized spike density for each unit across all trials for the respective condition. Means and SE of these normalized and averaged spike densities across units of a given sub-population were calculated to display population responses.

\subsubsection{LFP power analysis}

To obtain LFP signal, we applied a median filter on the broad band signal with the window size of 250 ms which reliably gave us LFP signal for the frequencies up to $150 \mathrm{~Hz}$. To remove the $50 \mathrm{~Hz}$ AC line noise, band-stop Butterworth filter (Matlab "butter" and "filtfilt" functions) for the range of $49.9-50.1 \mathrm{~Hz}$ and also 99.9-100.1 Hz was applied. LFP signal power was computed using Fieldtrip toolbox (Oostenveld et al., 2011). LFP power was obtained for each of the frequency bins between 2 and $120 \mathrm{~Hz}$ in logarithmic steps ("logscale" function of MATLAB) by tapering data using "Hanning window" in a cycle-based time-window manner and then applying Fourier transform.

To get the power time-frequency representation (TFR or spectrogram), we used a cyclebased time window for each frequency ( $\mathrm{N}=4$ cycles) with the step of $50 \mathrm{~ms}$ through one trial. This means for lower frequencies the time window was longer than higher frequencies. Since for a typical length of trial (about 6 seconds), the full power distribution for the whole trial in lower frequencies was not possible, zero-padding was done such that the length of a trial was enough for power calculations for all frequency bins.

We normalized spectrograms within each block to bring all frequencies to a comparable level (to avoid 1/f nature of power spectrum) and also to be able to compare task conditions representations across blocks and sessions. To this end, LFP power in each frequency was normalized by a z-score approach: $P_{\text {zscore }}=(P-\operatorname{mean}(P)) / \operatorname{std}(P)$.

The statistical measures (mean and standard deviation) were computed across all successful trials in a block. We also computed the average LFP power for separate frequency-bands (Delta [2 4) Hz, Theta [4 8) Hz, Alpha [8 12) Hz, Beta1 [12 18) Hz, Beta2 [18 30) Hz, Gamma1 $[3070) \mathrm{Hz}$, Gamma2 [70 120) Hz), by averaging the power across all frequency bins within each band. We only analyzed a trial if it was not detected as noisy. To detect noisy trials we computed first the raw LFP power spectrogram and then mean and standard 
deviation of power across time-bins within each frequency. If at any time bin more than $50 \%$ of the frequency bins had power more than mean $(f)+2 * \operatorname{std}(f)$, that trial was detected as noisy. This is because we observed that unnecessary movements caused sharp increase in power across many frequencies.

To compare if there is significant difference between contra versus ipsilateral space representations at the population level, we applied paired t-test across sites with FDR multiple comparison correction ((Benjamini and Hochberg, 1995); "fdr_bh" function downloaded from https://de.mathworks.com/matlabcentral/fileexchange/27418-fdr-bh) to control for false discovery rate across all time-frequency bins. In each bin with a significant difference we show the actual normalized power value and in the rest the value set to zero. In other words we show the strength of the power only for the bins with a significant difference. 


\subsection{Results}

\subsubsection{Human experiment 1}

Two groups of subjects (young and elderly, see Materials and Methods) performed two different tasks, single cue task and double cue task with a distractor cue, see Figure 4.4A. The difficulty was adjusted for each subject individually to reach a success rate of around $80 \%$. The difficulty adjustment was achieved using a staircase procedure to modify the larger of the two curvatures used for generating the target shapes, while the smaller curvature was kept constant (at 0.3 ), see Figure $4.4 \mathrm{C}$. The staircase adjustment resulted in different curvature ranges for both groups of subjects $(0.42$ to 0.83 for elderly and 0.37 to 0.49 for young subjects). This procedure was meant to give an idea of how different the shapes need to be to make the task difficult enough to able to observe differences in performance, but not frustrating. For neglect patients, adjusting the difficulty to performance does not seem feasible.

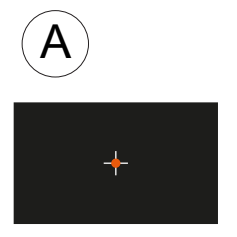

Fixation

$500 \mathrm{~ms}$

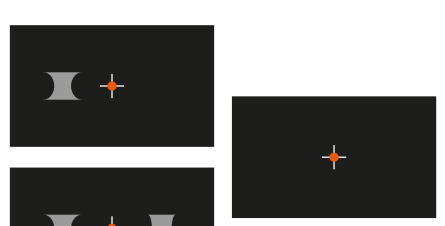

Memory period $300-800 \mathrm{~ms}$
Sample (S) presentation $600 \mathrm{~ms}$

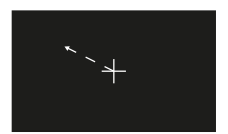

Exploration

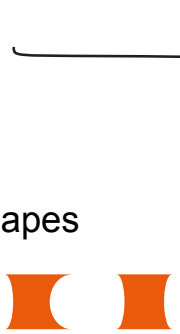

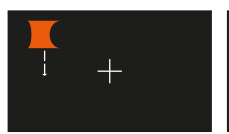

Distractor revealed maximum $15 \mathrm{~s}$

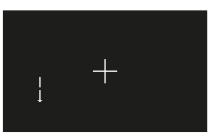

Exploration

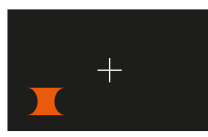

Match (M) revealed
D Example confusion matrixes

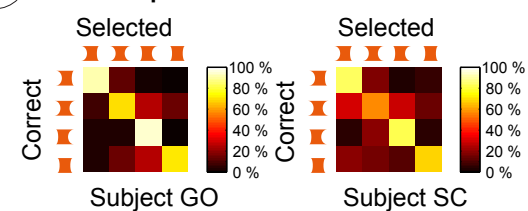

Figure 4.4: S2S task design(s) for human experiment 1. A) Task contingency. Subjects had to fixate in the center (red dot) for $500 \mathrm{~ms}$, until the sample would appear for $600 \mathrm{~ms}$ either on the right or left at 15 degrees horizontal distance. In another block, a distractor cue was presented at the same time as the sample on the opposite side (double cue task). After a short memory period of $300-800 \mathrm{~ms}$, in which subjects had to keep fixating, they were allowed to freely explore the black screen for up to 15 seconds in order to reveal one target at a time. A trial ended when a target was selected by fixating on it for $1200 \mathrm{~ms}$ and an auditory cue indicated if the selection as correct or incorrect. After $1000 \mathrm{~ms}$, the next trial started. Neither the white cross nor the arrows illustrating an example exploration path were visible to the subjects. B) For each trial, one out of four target position configurations was used. The colors indicate sets of positions for one configuration. Targets were located at an eccentricity of $10^{\circ}$ or $20^{\circ}$ with three potential angles relative to the horizontal axis of $0^{\circ}, 60^{\circ}$ or $-60^{\circ}$. C) Set of four target shapes. In each trial, one out of these four shapes was drawn randomly to be the sample/match, 2 were used as distractor targets and the fourth one as distractor cue (in the double cue task). D) Confusion matrixes for two example subjects (left elderly, right young). Colors indicate how often each shape was selected (columns) when the shape in the respective row was presented as a sample. The diagonal represents correct selections. 
The idea behind this set of target shapes was to allow the possibility to test for object based spatial deficits (i.e. specific confusion of target shapes with differences only on one side). Unfortunately, this set of targets leads inevitably to nonsymmetrical confusions, even in healthy subjects (see Figure 4.4D) and the monkey had problems learning to dissociate shapes with different curvatures on each side, which is why for further experiments (human experiment 2 and monkey experiments) only symmetrical target shapes were used.

To allow the assessment of neglect and/or extinction syndromes using the two task types (single or double cue including a distractor cue), it is important to compare the performance between these two tasks in healthy subjects in order to establish a baseline. This is necessary, because obviously, the cognitive demand is higher with a distractor cue, as subjects do not know in advance which of the two cues will be relevant for finding the match. Therefore they have to pay attention to both cues and memorize the shapes.

Importantly, the results of this comparison (see Figure 4.5) suggest that the performance readouts are informative, as the higher cognitive demand in the double cue task lead to a decrease in success rate and an increase in total exploration time as well as in dwell time per target, for all task conditions. Even reaction times increased, although not significantly. The same trend was observed in the elderly group, although the differences only reached significance for success rates (results not shown).

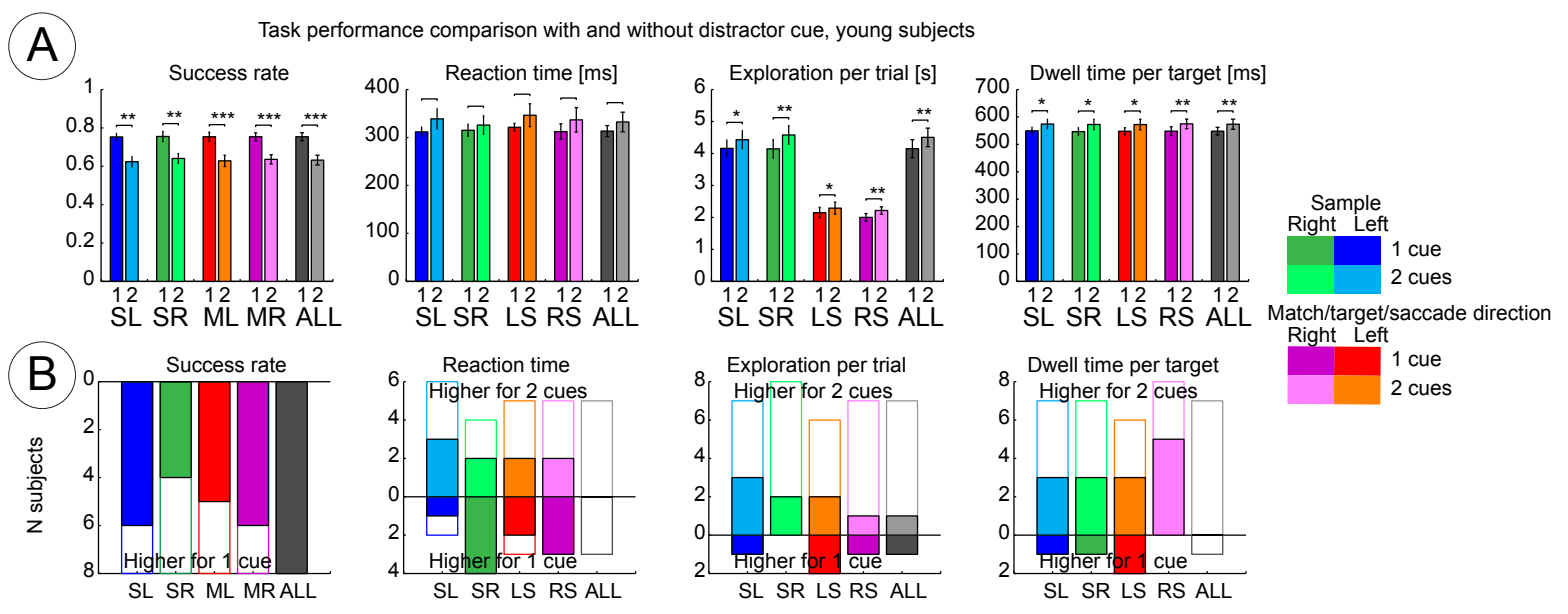

Figure 4.5: Task performance comparison with and without distractor cue. Comparison of success rate, Reaction time, exploration time and dwell time in the two tasks, single and double cue (with distractor cue). Values were grouped either by sample position (SL/SR), by the location of the match (ML/MR), the direction of the saccade or the explored hemifield (LS/RS). A) Mean and SE across means per subject, paired t-tests. B) Number of subjects for which the respective value was higher (upper part) or lower (lower part) in the double cue task. Filled bars mark subjects for whom the difference between tasks was significant (see Materials and Methods for details).

Because neglect patients are typically 50 years and older (as stroke occurs more likely at higher age), baseline behavior needs to be established in a matched-age group of healthy 
subjects. The main question was, if the task also works for the group of elderly subjects. To evaluate the effect of age on the task performance, results from the young and elderly group were compared, see Figure 4.6. Although differences were not significant, the results indicate that elderly subjects were slower to react to the "Go"-cue, and took longer to explore and inspect the targets, although they reached higher success rates. The latter finding seems counterintuitive at first glance, but one needs to remember that the difficulty was adjusted for each subject individually, leading to much easier discrimination for elderly subjects. The deviation from aimed $80 \%$ success rate however indicates that the staircase procedure did not work perfectly to adjust difficulty, or that elderly subjects still got better in the task, while for the young subjects, fatigue decreased the performance after the initial runs. The same trend was observed when comparing task performance between the two groups in double cue task (results not shown).

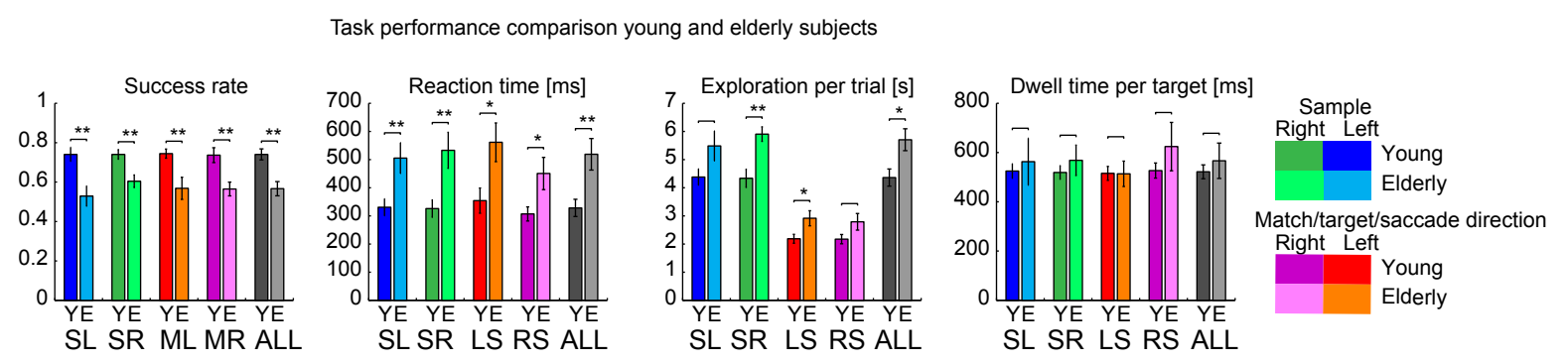

Figure 4.6: Task performance comparison between young and elderly subjects in the single cue task. Comparison of success rate, Reaction time, exploration time and dwell time in the two groups of subjects. Values were grouped either by sample position (SL/SR), by the location of the match (ML/MR), the direction of the saccade (LS/RS), or the explored hemifield (LS/RS). Top: Mean and SE across means per subject, unpaired ttests.

Because one of the findings in monkeys with pulvinar lesions was that the ipsilesional side was preferred for a first, exploratory saccade, while the ability to perform contralesional saccades was not affected (Wilke et al., 2010), one interesting readout of the search-tosample task are exploration patterns. This proved to be more difficult than expected, because exploration patterns differed drastically in different subjects, see Figure 4.7. While some subjects (for example Subject SC) explored rather randomly, others showed very stereotypical exploration (Subjects MA and GO). Furthermore, a few (especially young) subjects seemed to understand very soon which target location configurations were used, and explored only the currently present locations, after they had revealed the first target (see the different exploration patterns of subject TL for two different groups of target location configurations). 
A similar behavior was observed in the monkey when tested on this set of target locations (results not shown). Due to these findings, another approach for defining target locations was tested in the second human experiment presented here.
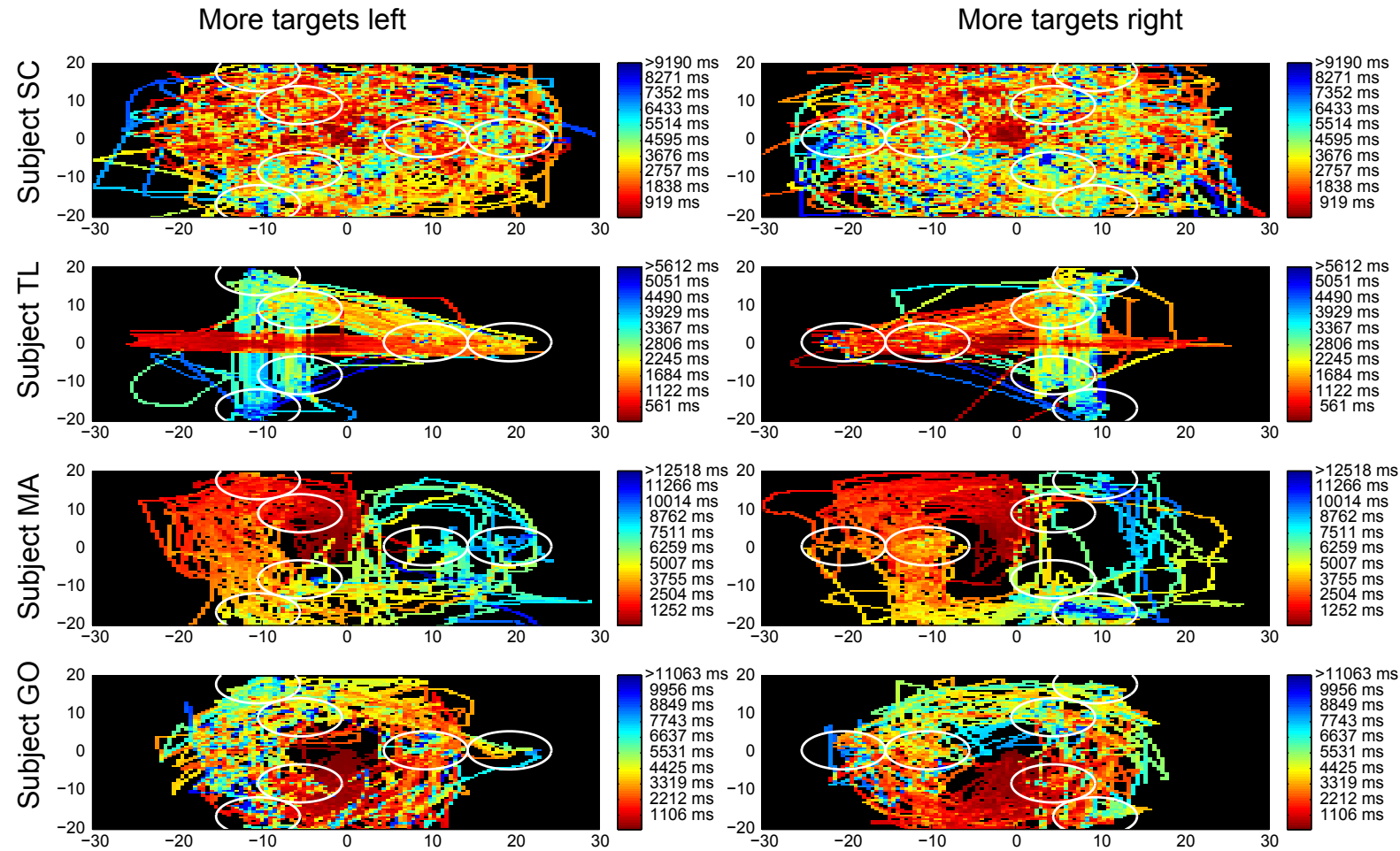

Figure 4.7: Example exploration patterns of four subjects. Exploration patterns shown were computed for the single cue task, averaging across all successful trials in which the sample was presented on the left side, independently for two task conditions (more targets on the left/right side - columns) and four subjects (rows). Eye traces were first binned to $1 \times 1$ degree pixels, and the average inspection time relative to beginning of exploration was computed across all time points in which the eye position landed in the respective pixel, independently for each pixel. Colors red (early) to blue (late) indicate the average inspection time for each pixel. 


\subsubsection{Human experiment 2}

Two groups of subjects (young and elderly, see Materials and Methods) performed another version of the search-to-sample task, see Figure 4.8. Only symmetrical, concave shapes were used. The difficulty was defined by the difference in curvature between the most similar target shapes, and fixed for both groups (with small adjustments, 0.17-0.18 for young, 0.20.22 for elderly subjects). Although using these target shapes lead to more or less predictable confusions (mainly between the most similar shapes), it should be noted that a linear spacing of curvature differences did not result in the same discrimination difficulty for each shape, see Figure 4.8. A second major modification was that for each trial, three target locations were pseudo-randomly drawn out of eight possible locations. This allowed conditions where all targets were located on one side of the screen.

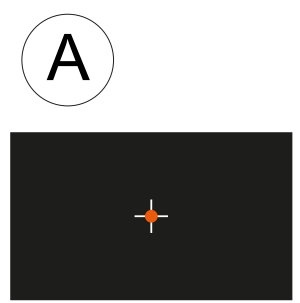

Fixation

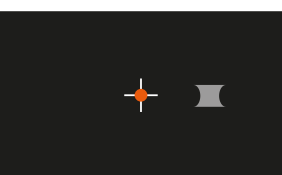

Sample (S) presentation

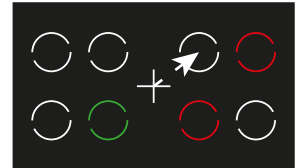

No target revealed

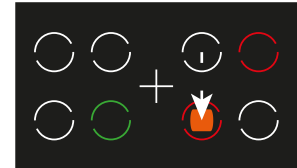

Distractor revealed

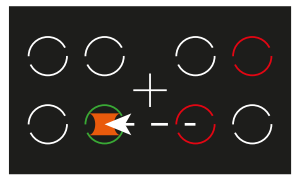

Match (M) revealed

$$
200-300 \mathrm{~ms} \quad 250 \mathrm{~ms}
$$

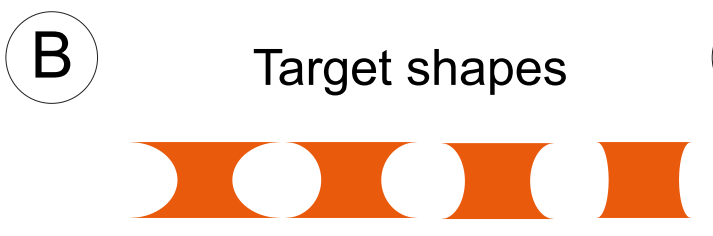

maximum $20 \mathrm{~s}$

\section{Example confusion matrixes}

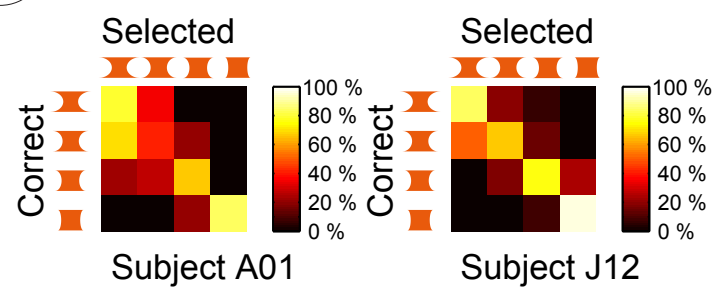

Figure 4.8: S2S task design for human experiment 2. A) Task contingency. Subjects had to fixate in the center (red dot) for $200-300 \mathrm{~ms}$, until the sample would appear for $250 \mathrm{~ms}$ either on the right or left at $15^{\circ}$ horizontal distance. After the sample and the fixation spot disappeared simultaneously, they were allowed to freely explore the black screen for up to 20 seconds in order to reveal one target at a time. A trial ended when a target was selected by pressing the space bar while fixating on the target. After $1000 \mathrm{~ms}$, the next trial started. Dashed circles indicate possible target locations, red circles indicate the locations for distractor targets in this trial and the green circle indicates the location of the match in this trial. The white cross, arrows illustrating an example exploration path, and the circles were not visible to the subjects. B) Set of four target shapes. In each trial, one out of these four shapes was drawn randomly to be the sample/match and 2 were used as distractor targets. Targets were located at a horizontal eccentricity of $8^{\circ}$ or $24^{\circ}$ and vertical eccentricities of $+8^{\circ}$ or $-8^{\circ}$. C) Confusion matrixes for two example subjects (left elderly, right young). Colors indicate how often each shape was selected (columns) when the shape in the respective row was presented as a sample. The diagonal represents correct selections. 
Figure 4.9 shows the comparison between two groups. Elderly subjects were slower to respond, took more time to explore and selected the correct target less often, even though the discrimination difficulty was set easier. The main problem here was a big difference between subjects of the elderly group. While some reached similar success rates as the subjects in the young group, others had big difficulties finding the correct targets. The difficulty here however was rather the exploration then the discrimination itself, since the correct target was typically selected when revealed.

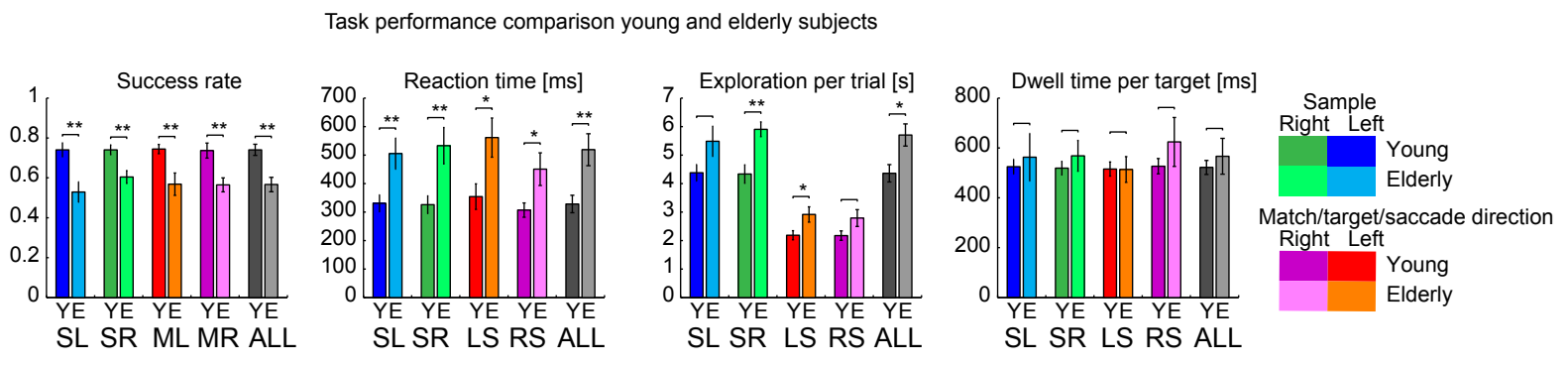

Figure 4.9: Task performance comparison between young and elderly subjects. Conventions according to Figure 4.6.

Examining exploration patterns revealed again big differences between subjects, see Figure 4.10. While some subjects explored rather randomly (e.g. subject J16), others showed very stereotypical exploration behavior (e.g. Subject A04 and A06). A few subjects seemed to have learned the target locations within a few trials, as their exploration path only crossed potential target locations (e.g. Subject 14).

Subject A04

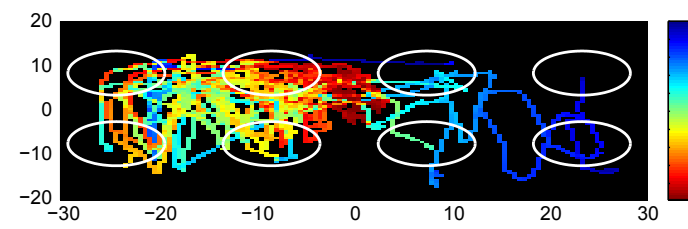

Subject A06

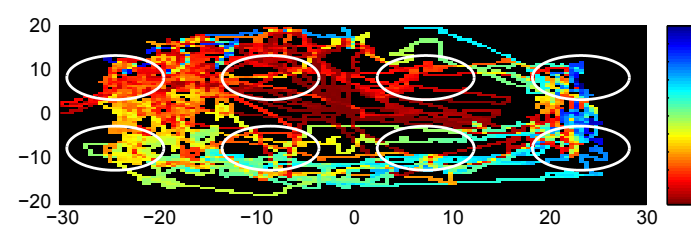

Subject J14

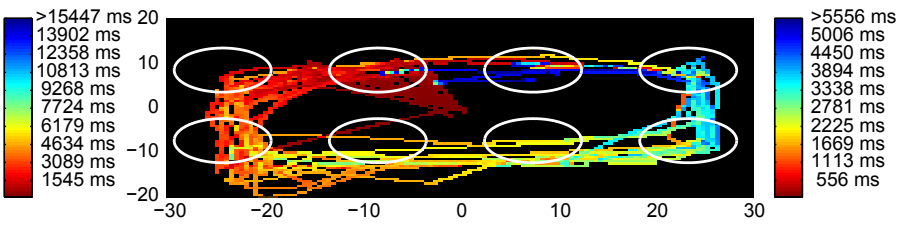

Subject J16

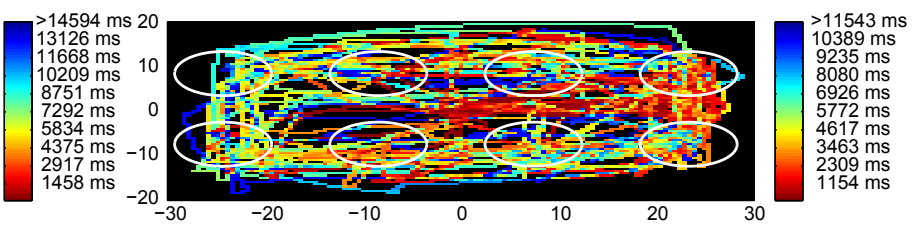

Figure 4.10: Example exploration patterns of four subjects. Exploration patterns shown were computed across all successful trials in which the sample was presented on the right side and more targets were located on the left side, independently for the four subjects (rows). Calculations according to Figure 4.7. 


\subsubsection{Monkey inactivation results}

For monkey inactivation experiments, a similar version of the task as in human experiment 2 was used, see Figure 4.11. The main difference was the memory period between sample presentation and exploration phase. Besides that, the monkey received an auditory feedback (as well as a reward feedback), and convex target shapes were included. Since target shapes were adjusted over several training sessions, confusions were nearly equally likely between the two most similar target shapes, see Figure 4.11. Surprisingly, confusions between convex and concave shapes were equally likely as (if not even more likely than) confusions within convex or concave object pairs. This was not observed in the first human pilot study (where similar target shapes were tested), presumably because humans differentiate categorically between convex and concave.

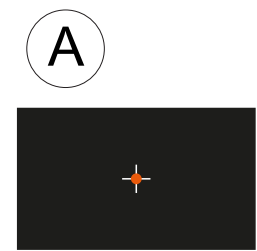

Fixation

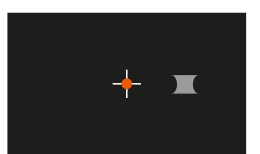

Sample (S) presentation

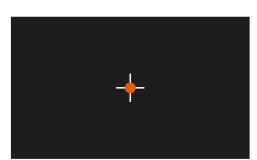

Memory period

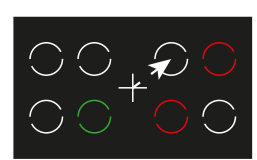

No target revealed

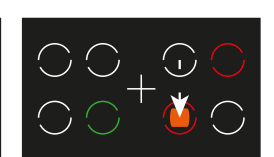

Distractor revealed

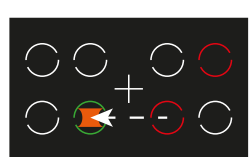

Match (M) revealed

$$
300-500 \mathrm{~ms} \quad 1000 \mathrm{~ms} \quad 300-800 \mathrm{~ms}
$$
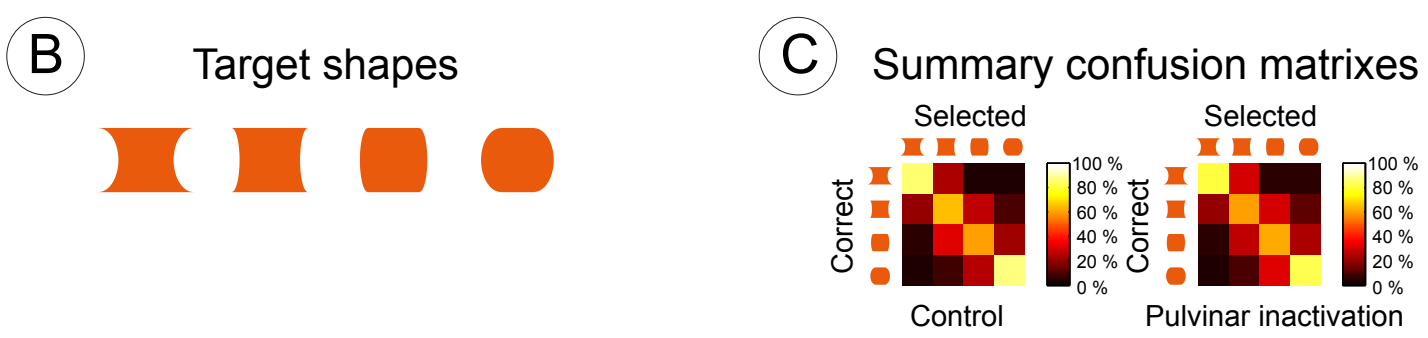

Figure 4.11: S2S task design for monkey experiments. Top: Task contingency. The monkey had to fixate in the center (red dot) for $300-500 \mathrm{~ms}$, until the sample would appear for $1000 \mathrm{~ms}$ either on the right or left at $15^{\circ}$ horizontal distance. After a short memory period of $300-800 \mathrm{~ms}$, in which the monkey had to keep fixating, he was allowed to freely explore the black screen for up to 20 seconds in order to reveal one target at a time. A trial ended when a target was selected by fixating on the target for one second, and an auditory cue indicated if the selection was correct. A drop of reward was dispensed only for correct trials. After $1000 \mathrm{~ms}$, the next trial started. Dashed circles indicate possible target locations, red circles indicate the locations for distractor targets in this trial and the green circle indicates the location of the match. The white cross, arrows illustrating an example exploration path, and the circles were not visible to the monkey. Bottom, left: Set of four target shapes. In each trial, one out of these four shapes was drawn randomly to be the sample/match and 2 were used as distractor targets. Targets were located at a horizontal eccentricity of $8^{\circ}$ or $24^{\circ}$ and vertical eccentricities of $+8^{\circ}$ or $-8^{\circ}$. In the last 7 sessions sample eccentricity was increased to $18^{\circ}$ and target eccentricities to $9^{\circ}$ or $27^{\circ}$ horizontally and $+9^{\circ}$ or $-9^{\circ}$ vertically. Bottom, right: Confusion matrixes across all sessions (left control, right inactivation). Colors indicate how often each shape was selected (columns) when the shape in the respective row was presented as a sample. The diagonal represents correct selections.

Figure 4.12 shows the effects of pulvinar inactivation on the task performance. It has to be noted, that exploration starting on the left side was very rare even in control sessions, so the reaction times to the left (ipsilesional) side have to be disregarded. Over all, reaction times 
increased, and specifically in trials where the sample was presented on the contralesional side. It is questionable, if this is meaningful, because the memory period was supposedly long enough to dissociate the first response from the offset of the sample. Interestingly, while dwell times per target decreased (not significantly though, and not consistently across sessions), total exploration times increased. This could indicate general motor impairments, since it took longer to reach the correct locations while the monkey seemed to be more impatient to reveal the next target. Unexpectedly, exploration time increased significantly on the contralesional side, whereas the increase of exploration time was not significant for the ipsilesional side. If the induced deficits were neglect like, the opposite would be expected (Karnath, 2015). This result could be linked to increased saccade inaccuracy, an interpretation which is supported by the findings in control tasks, see Figure 4.16. In contrast, looking at the exploration patterns it does not seem like the monkey had big troubles reaching the target locations, see Figure 4.14. The most surprising result however was that success rates decreased significantly for trials in which the sample was presented on the ipsilesional side, but not when it was presented on the contralesional side.
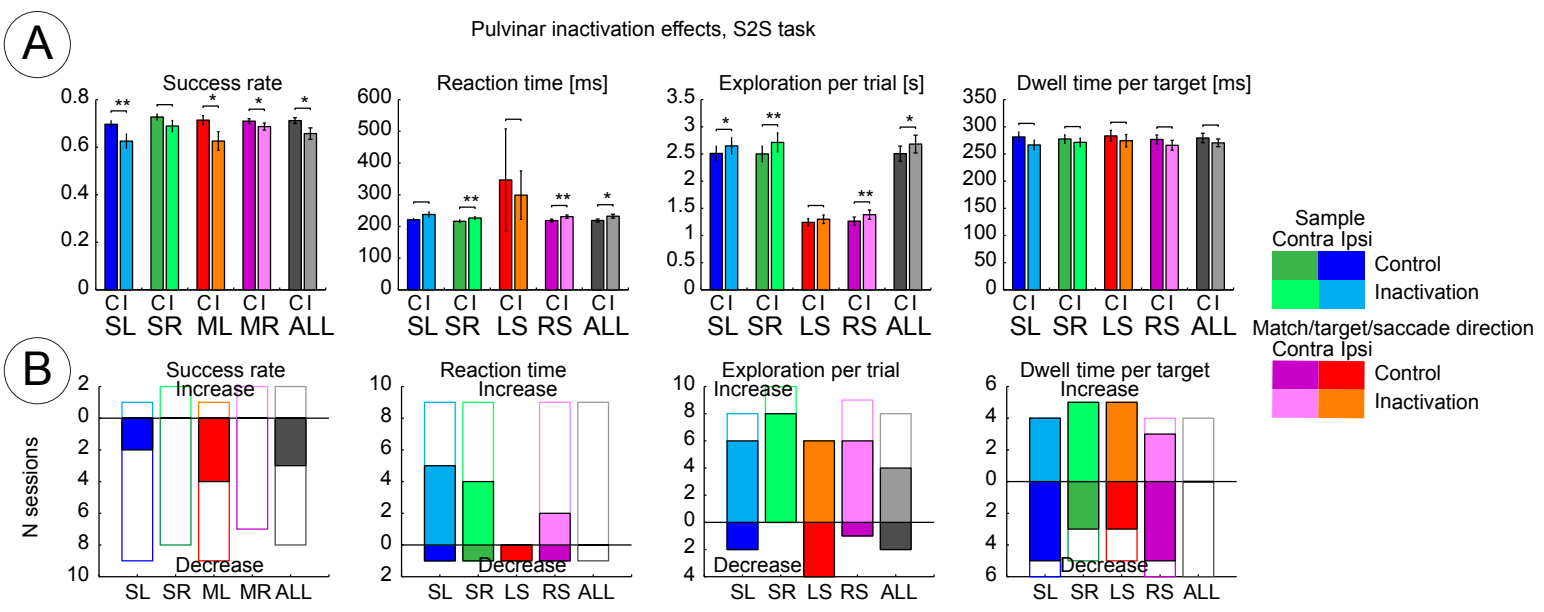

Contra Ipsi
Control
Inactivatio

Figure 4.12: Task performance comparison between control and inactivation sessions. Conventions according to Figure 4.5.

The most straight forward interpretation that pulvinar is important for the processing of ipsilateral stimuli, has to be disregarded, because that would contradict everything we believe to know about pulvinar. However, not many alternative explanations are feasible. One possible option would be that the monkey focused so much on the affected side in order to be able to reach targets there, that he neglected the ipsilesional sample. Another, maybe more likely explanation would be that the contralesional sample served as some sort of anchor point in a distorted contralesional space representation, facilitating the accustomed exploratory behavior, which typically started on the right (contralesional) side, see Figure 
4.14. A third, and maybe the most convincing explanation is linked to another observation, namely induced rightward eye position slow drift (left-beat, i.e. ipsilesional, nystagmus, see Figure 4.13). The nystagmus was not analyzed systematically, but drifts towards the right (contralesional) side during fixation would result in an overall shifted fixation position (inside the allowed window) towards the right, which could facilitate the perception of stimuli presented on that (right/contralesional) side of space, simply because they were on average closer to the fovea. Similar effects of pulvinar inactivation have been reported previously (Wilke et al., 2010).

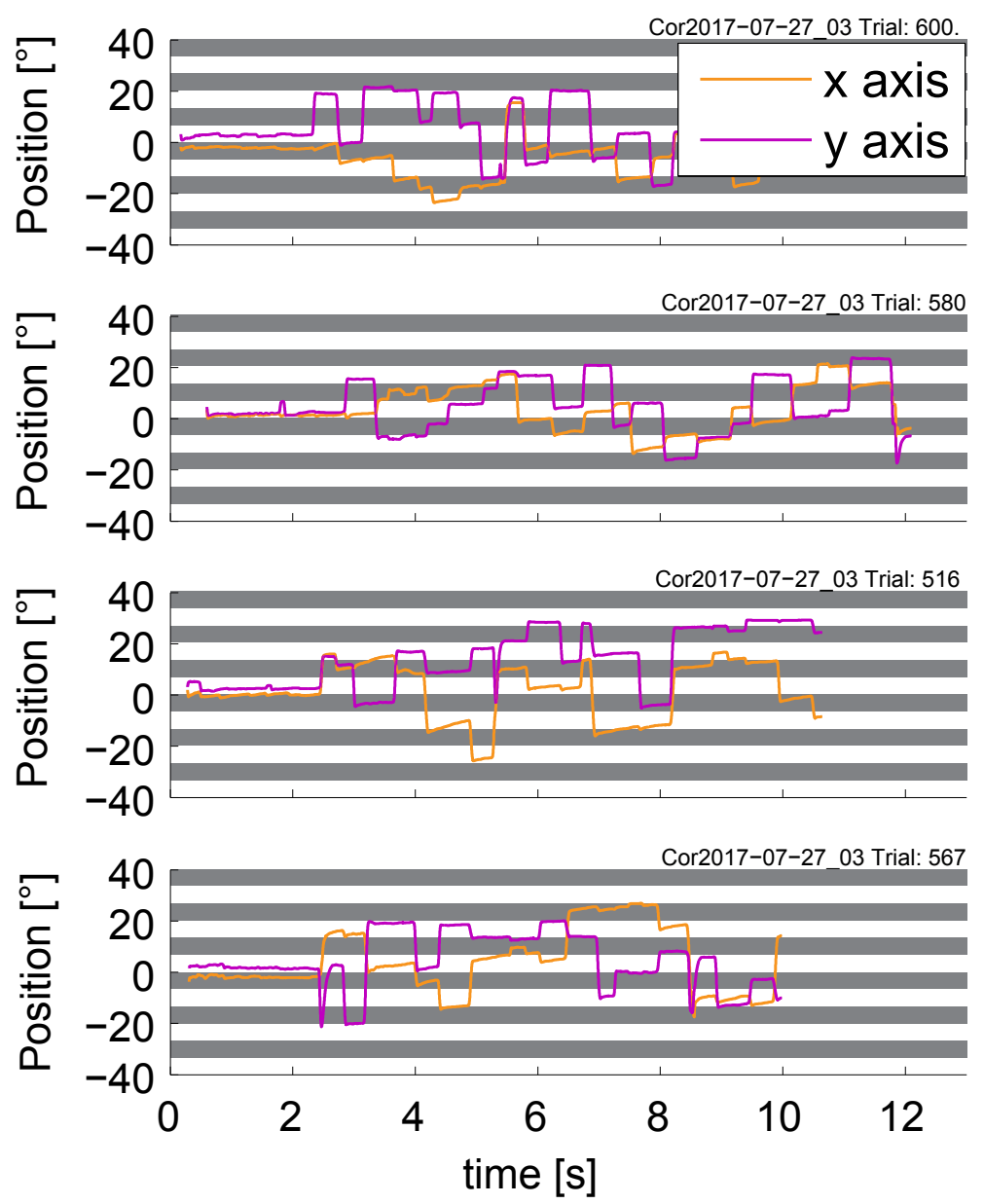

Figure 4.13: Rightward eye position drifts after pulvinar inactivation. Horizontal (X, orange) and Vertical ( $Y$, purple) eye positions for four example trials after pulvinar inactivation. Positive eye position values correspond to above (for $\mathrm{Y}$ ) and the right side (for $\mathrm{X}$ ) of the fixation point, negative values correspond to below (for $Y$ ) and the left side (for $X)$.

Looking at the effects per session on the other hand shows that a decrease of success rate was very common for all conditions. Significant effects on success rate within sessions suggest that the most affected condition was when the match was to be found on the ipsilesional side. This result could be linked to the stereotypical exploration (which typically started on the contralesional side), indicating a general decrease in motivation or reduced 
memory, since the time that passed after sample presentation was usually longer when exploring the ipsilesional side as compared to exploring the contralesional side. 
Control session
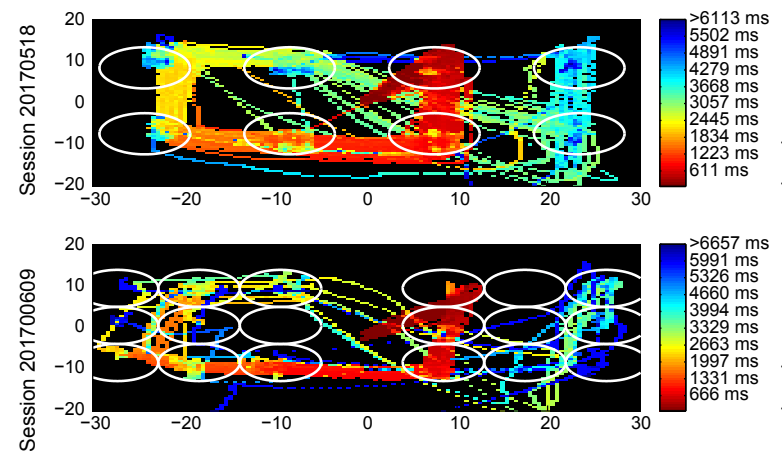

Inactivation session
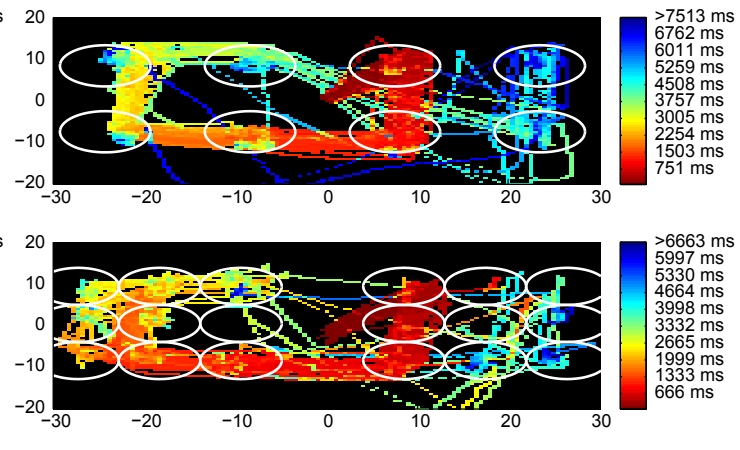

Figure 4.14: Example exploration patterns of four sessions. Exploration patterns shown were computed averaging across all successful trials in which the sample was presented on the left side and more targets were located on the left side, independently for two inactivation sessions (right) and the matching control sessions (left). These exploration patterns are representative for all task conditions and sessions. Calculations according to Figure 4.7.
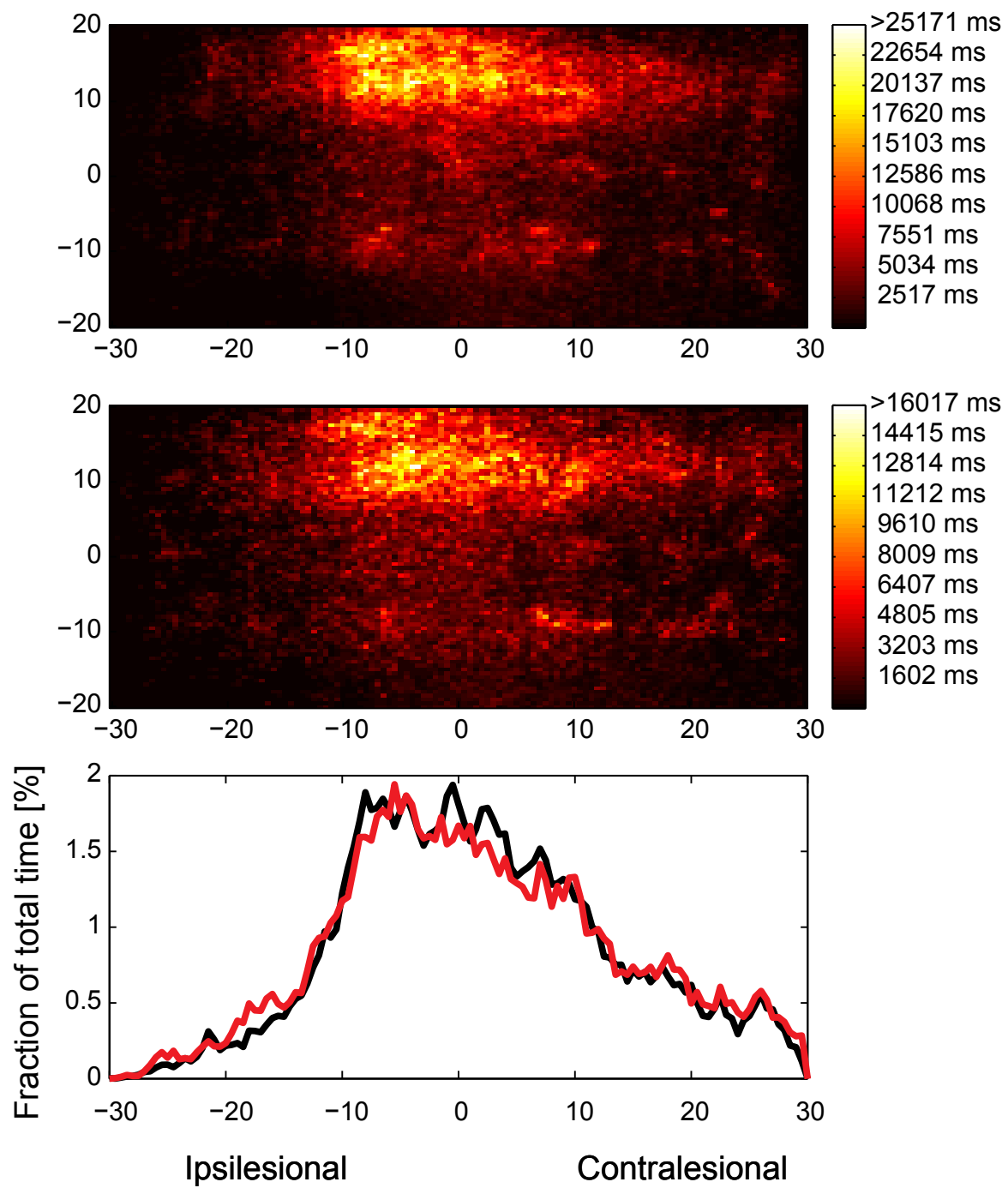

Horizontal position $\left[{ }^{\circ}\right]$

Figure 4.15: "Free exploration" in the inter-trial interval. Eye position heat maps for the inter-trial-interval across all trials in control sessions (top) and inactivation sessions (middle). Colors indicate the total time the eye position was recorded in the respective bin, starting after reward and until the next trial started. Bottom: Histogram of eye positions across the horizontal axes for control (black) and inactivation sessions (red). 
It should be mentioned here that one hope was to disrupt the stereotypical exploration patterns, as they typically started in the contralesional space. Because that was not achieved, target locations were modified during the experiments, introducing 18 possible target locations. This did not alter the stereotypical exploration pattern either, as the monkey adjusted very fast to the new conditions, see Figure 4.14. To see if the lack of effect exploration is related to this acquired and "over trained" exploration path, eye positions were also recorded during the inter-trial-interval, where no specific exploration pattern was required or beneficial and therefore exploration should be even more free. No difference was found between control and inactivation sessions for the inter-trial-interval either (see Figure 4.15).

For better understanding of the induced effects, and linking them to effects found in previous studies, two control tasks were performed - visually guided saccades and memory guided saccades including free choice trials, see Materials and Methods for details. The results are shown in Figure 4.16. While we failed to reproduce an induced bias to select ipsilesional targets in the memory guided task, inactivation affected the target selection in the visually guided task in the expected way. However, reaction times were unaffected in both tasks. Interestingly, inactivation caused an increase in imprecision, specifically for targets on the contralateral side. The fact that this is reflected in imprecision and not inaccuracy is probably linked to pooling endpoint differences for all targets on one side, see Materials and Methods. When saccade endpoint distributions are compared for each target separately, it seems more like the scatter (imprecision) for each target on the contralesional (right) side is reduced after inactivation, whereas the mean offset (inaccuracy) is increased, but in different directions for each target; overshooting for close targets, and undershooting for far targets. For the ipsilesional space, saccade endpoints seem unaffected. This indicates a distorted internal contralesional space representation. It seems plausible that this increased inaccuracy could lead to a decreased preference for targets on the affected side, which was potentially just not strong enough to cause a systematic choice bias in the memory saccade task, as it did not affect the success rate. 

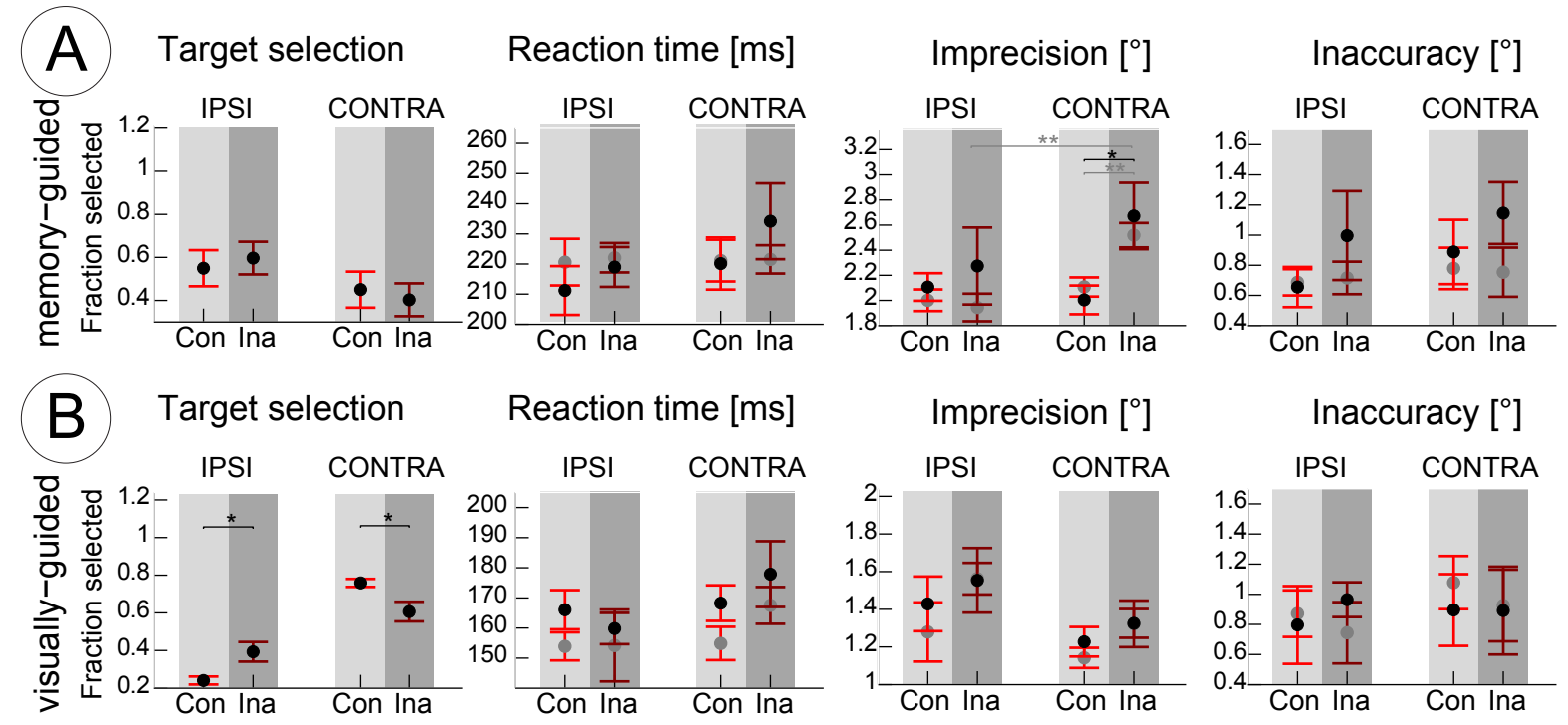

Reaction time [ms]
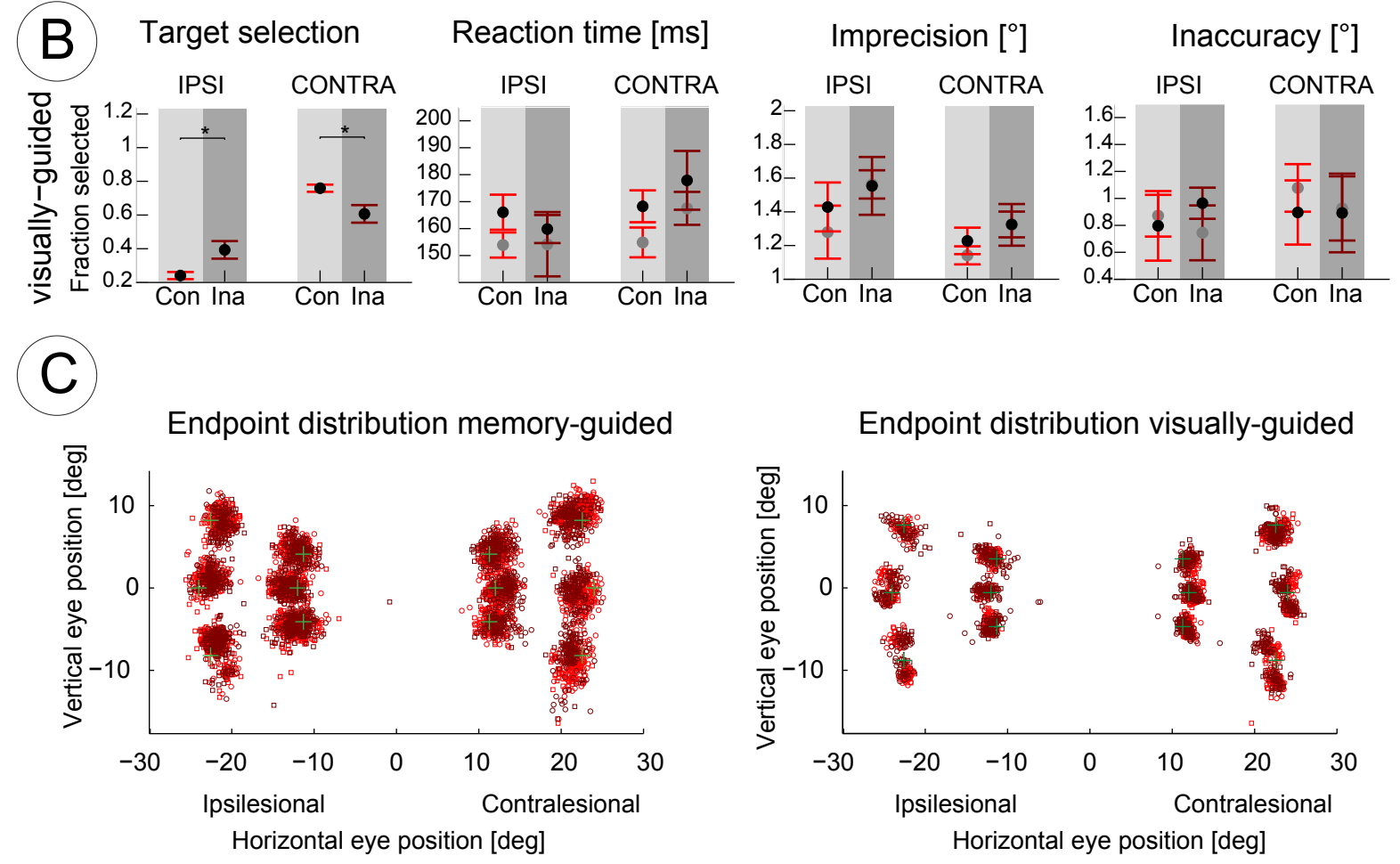

$$
\text { - Control - Inactivation - Instructed C Choice }
$$

Figure 4.16: Pulvinar inactivation effects in the two control tasks (visually guided and memory guided saccades). A) Memory guided saccades. B) Visually guided saccades. C) Endpoint distributions. Dark red: inactivation sessions, bright red: Control sessions. Black dots: Mean values for choice trials, grey dots: mean values for instructed trials. Error bars indicate SE. Comparisons between mean values per session, MannWhitney-U test. 


\subsection{Control experiments in cortical area TPO}

\subsubsection{TPO/STS electrophysiology (memory guided saccade task)}

To assess similarities and differences of neuronal encoding in pulvinar and TPO, we recorded neuronal activity in TPO (including the medial part of the dorsal bank often referred to as area PGa) during a similar memory guided saccade task as used in Chapter 2, see Materials and Methods. The recording sites are shown in Figure 4.17. Note that a few recording sites also include the fundus of the superior temporal sulcus (FST), but we did not see any qualitative differences in the activity in FST and TPO neurons. Therefore, data from all sites were combined in this analysis.
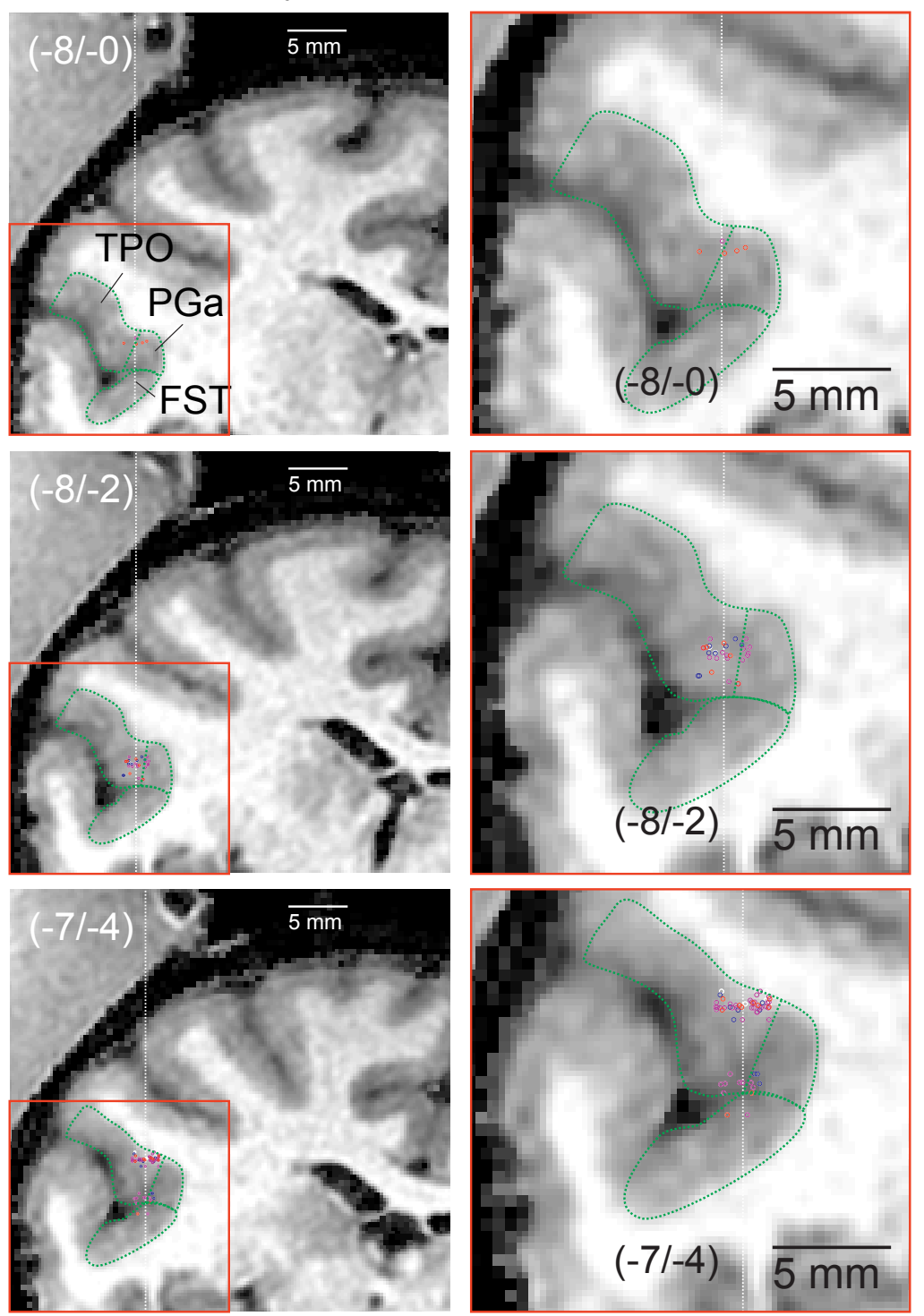

Figure 4.17: STS recording sites. Rows: Three slices of chamber-aligned T1-weighted MR images, one for each grid hole used (-8/0, -8/-2, -7/-4). Anterior to posterior slices from top to bottom. Dots indicate the locations of cells, colors for 4 different categories: Visual (blue), Visuomotor (green), Motor (red), and cells that fall in none of these categories in white (see Figure 4.20 for details on categorization). Actual locations were jittered in the horizontal direction to allow better visualization. Dashed white lines indicate the electrode tracks. Right column: Close-ups of the area indicated by the red frame in the left column. 
As a first step of analysis, activity of each cell was binned at $10 \mathrm{~ms}$ and compared to baseline for evaluating enhancement and suppression across time. At the same time, activity in each bin was also compared between ipsilateral and contralateral conditions to evaluate spatial preference, see Figure 4.18. Most cells showed a certain pattern of tuning (enhancement and/or contralateral preference) very close to cue onset, while delay period activity (during memory) was sparse. Alignment to saccade onset showed a less consistent activity pattern with both contralateral and ipsilateral tuning, and nearly equal proportions of enhancement and suppression.

Since the cue response happened earlier then in pulvinar (compare chapter 2 and Figure 4.19), the epoch for evaluating cue response in TPO activity was adjusted to $50 \mathrm{~ms}$ before to $50 \mathrm{~ms}$ after the bin where the most cells showed enhancement (40 ms - $140 \mathrm{~ms}$ after cue onset), see Figure 4.19. The cue response timing estimated by enhancement matched very well with timing estimated by contralateral preference. Tuning timing relative to saccade onset was less consistent, which is why the same analysis epoch as in for the analysis of pulvinar electrophysiology was taken to evaluate post-saccadic responses.

A Ordered by tuning onset relative to cue

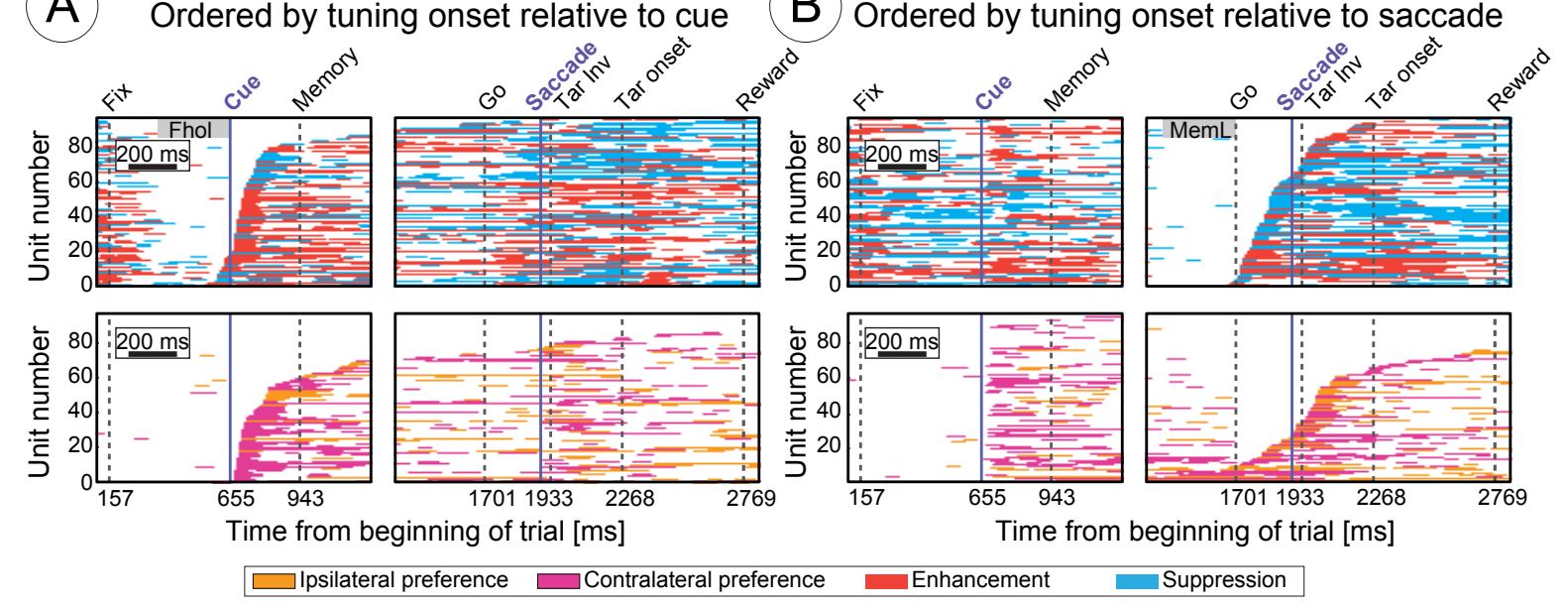

B Ordered by tuning onset relative to saccade

Figure 4.18: Tuning across time for all cells. For each window (black frames), tuning was aligned to either Cue or Saccade onset (pale blue lines in each window). Grey lines indicate average event onsets, grey rectangles indicate baseline epochs: "fixation hold" (Fhol), and "late memory" (MemL). A) Top: Enhancement (red) and suppression (blue) relative to fixation hold (Fhol) epoch. Bottom: Contralateral (pink) and ipsilateral (orange) preference, aligned to cue. B) Top: Enhancement (red) and suppression (blue) relative to late memory (MemL) epoch. Bottom: Contralateral (magenta) and ipsilateral (orange) preference, aligned to saccade onset. See Materials and Methods for details on significance calculations on a bin-by-bin level. 
A

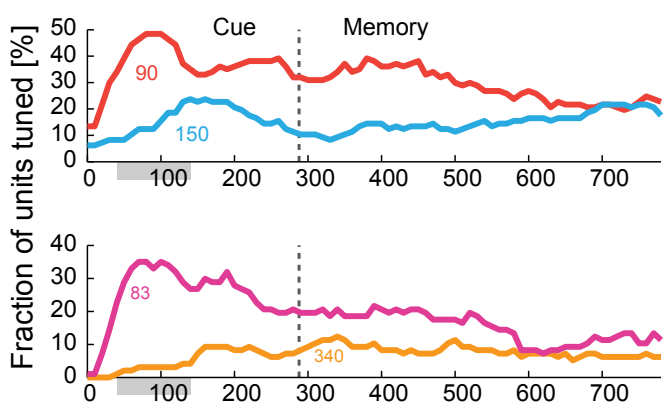

B

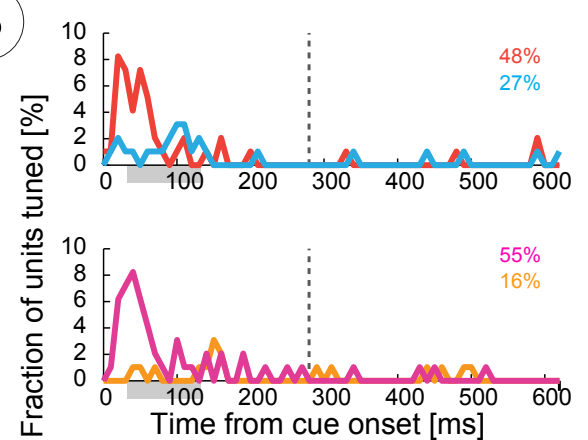

Aligned to Saccade
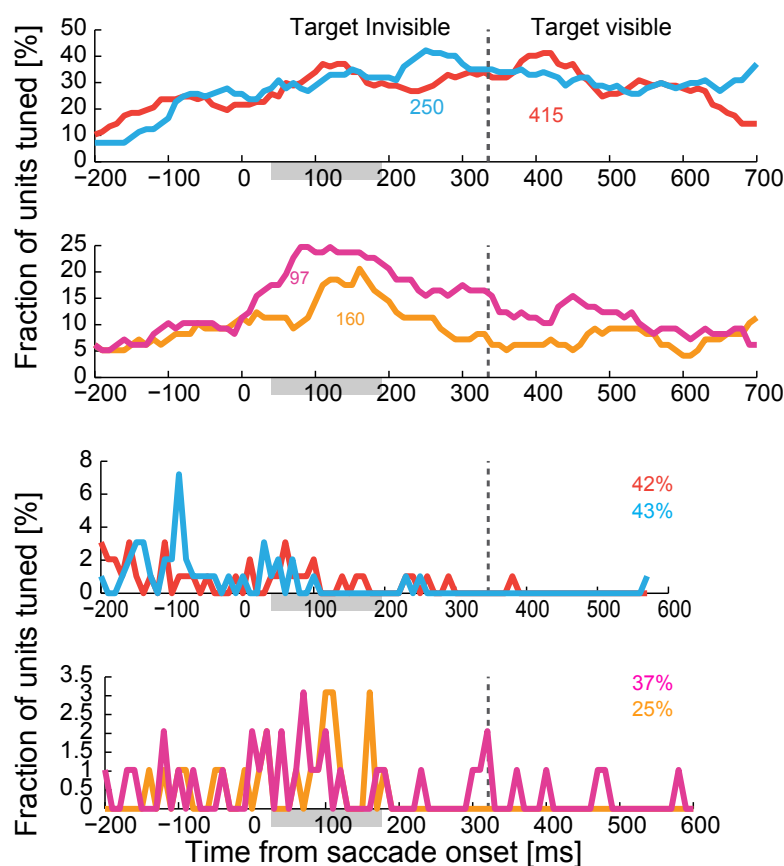

Ipsilateral preference

Figure 4.19: Epoch and tuning onset evaluation. Enhancement in red, suppression in blue, contralateral preference in pink and ipsilateral preference in orange. A): Fraction of tuned cells for each bin. Lines indicate the time where most cells showed respective tuning. B): fraction of cells showing tuning onset in the respective bin. Left: Aligned to cue onset, using Fhol as baseline for enhancement and suppression. Right: Aligned to saccade onset, using MemL as baseline.

Analysis of specific epochs (see Materials and Methods) confirmed that around half the cells showed very consistent visual responses (enhancement and contralateral preference), see Figure 4.20. The predominance of visually-driven responses is further supported by the comparably few cells that show pre-saccadic and peri-saccadic activity, whereas most cells responded to the onset of the target after the monkey completed memory saccade. However, post-saccadic responses prior to the target onset were also not uncommon, similar to neuronal activity we observed in dorsal pulvinar (chapter 2). Classification of responses was performed by grouping response patterns into three categories: Visual cells were defined by enhancement or suppression in Cue epoch, but not in "post-saccadic" (Post) epoch, visuomotor cells were defined by enhancement or suppression in Cue and Post epoch, motor cells were defined by enhancement or suppression in Post epoch, but not in Cue. Nearly all cells (90/97) fell within one of the categories. Surprisingly, most cells (53/97) were classified as visuomotor cells, suggesting considerable involvement of TPO in motor processing. 

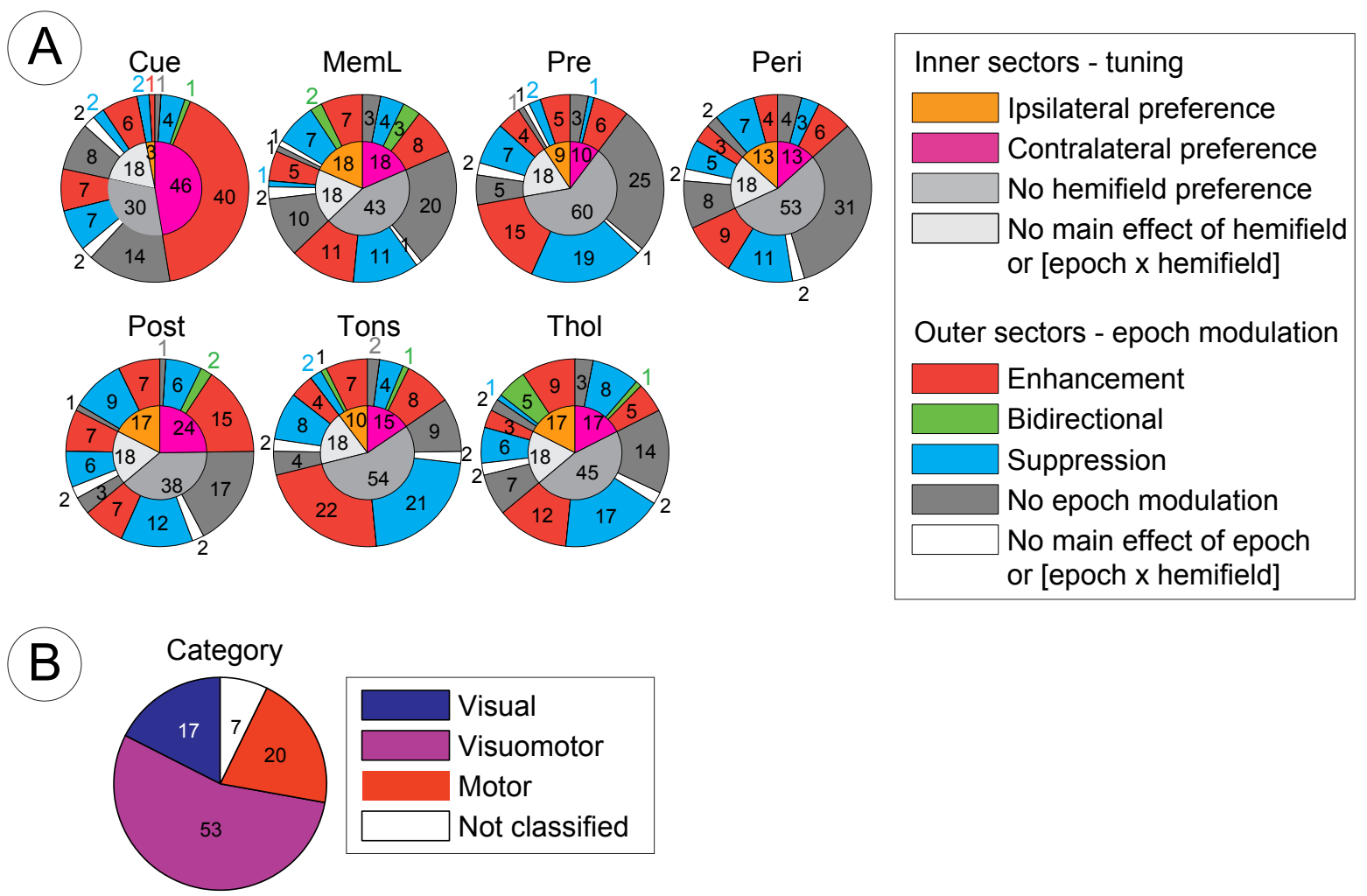

Figure 4.20: Response categorization. A) Tuning in each analysis epoch. Sector size and numbers indicate the number of cells with the respective response. Inner circles display the number of cells with spatial preference; contralateral preference in pink and ipsilateral preference in orange. Outer circles display the number of cells that showed enhancement or suppression relative to the respective baseline epoch, see Materials and Methods. Green sectors for cells that showed enhancement for one and suppression for the other hemifield, $\mathrm{red} / \mathrm{blue}$ sectors for cells that showed enhancement/suppression in at least one hemifield. Grey sectors display areas that did not show tuning in the respective epoch, and white sectors display cells that were not tested for the respective tuning, because ANOVA did not reach significance, see Materials and Methods. B) Categorization of cells according to Cue activity and post-saccadic activity (Post); Visual cells showed enhancement or suppression in Cue epoch, but not in Post, visuomotor cells showed enhancement or suppression in Cue and Post epoch, Motor cells showed enhancement or suppression in Post epoch, but not in Cue.

The population activity across the three classed was dominated by visual responses and reflects mostly the activity found in visual and visuomotor cells, see Figure 4.21. Note that the cue off response is more predominant in visuomotor cells, and that the activity in motor cells drops on average (relative to memory period) for the ipsilateral trials, and for the contralateral trials in the later part of post-saccadic (Post) epoch. 


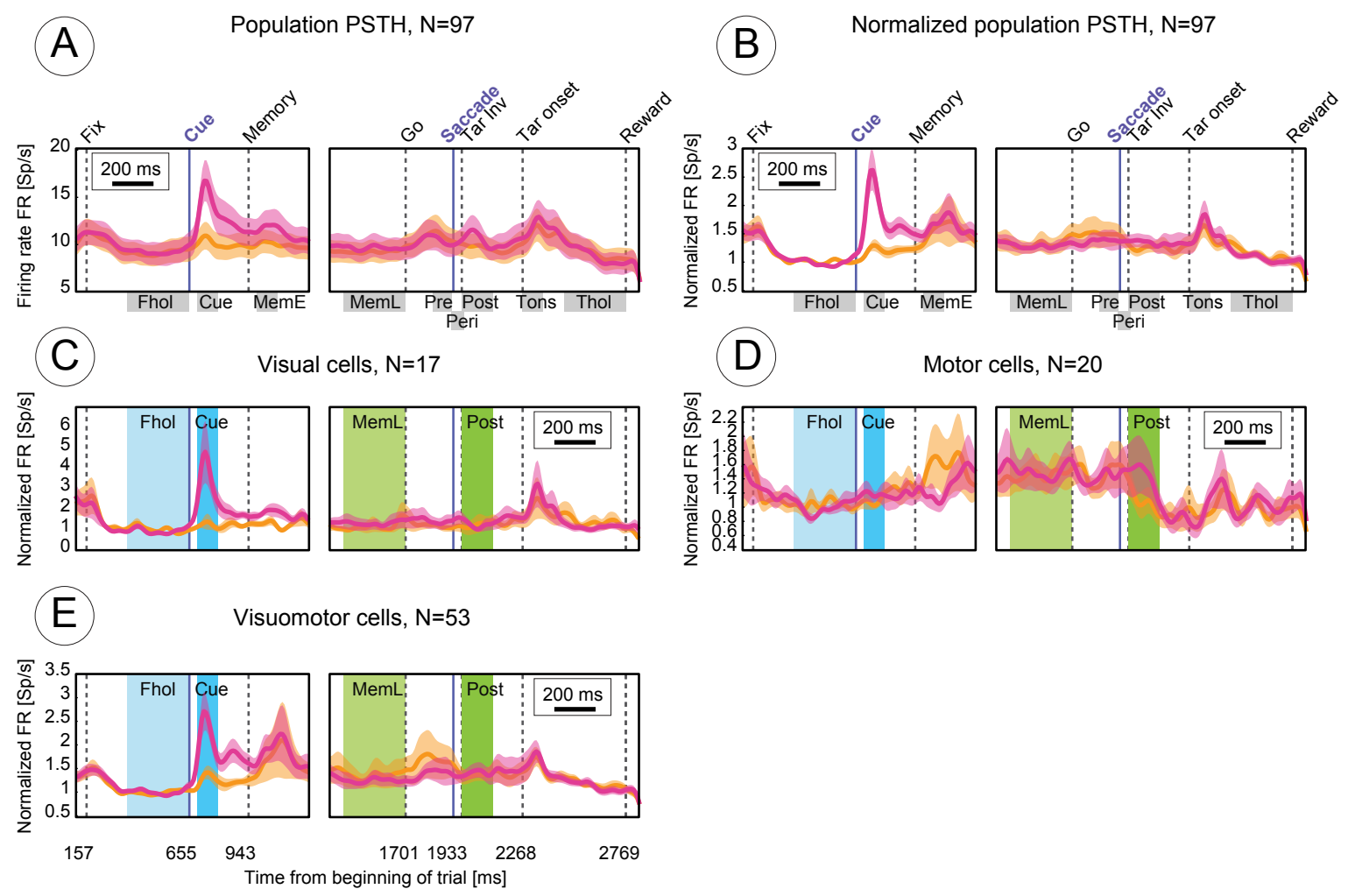

Figure 4.21: Population PSTHs. Average spike densities shown independently for contralateral (magenta) and ipsilateral (orange) trials. Grey rectangles indicate analysis epochs; "fixation hold" (Fhol), "cue onset" (Cue), "early memory" (MemE), "late memory" (MemL), "pre-saccadic" (Pre), "peri-saccadic" (Peri), "post-saccadic" (Post), "target onset" (Tons), and "target hold" (Thol), see Materials and Methods. Blue and green colored areas mark relevant baseline and response epochs for the respective category. A) Raw PSTHs for all cells. B) Normalized PSTHs for all cells. C) Subpopulation of cells categorized as visual. D) Subpopulation of cells categorized as motor. E) Subpopulation of cells categorized as visuomotor.

To evaluate the consistency of activation patterns within each of these categories, subcategories were defined based on conjunction of enhancement/suppression and spatial preference. The activation pattern was very consistent for visual neurons: 12/17 showed enhancement and contralateral preference during cue, 1/17 showed enhancement for contralateral cues and suppression for ipsilateral cues, 1/17 showed suppression and contralateral preference, 1/17 showed enhancement but no spatial preference, 2/17 showed suppression but no spatial preference. For motor cells, the activation patterns were less consistent: 1/20 showed contralateral enhancement and ipsilateral suppression in the postsaccadic epoch (Post), 3/20 showed enhancement and contralateral preference, 4/20 showed enhancement but no spatial preference, $2 / 20$ showed suppression and contralateral preference, 4/20 showed suppression and ipsilateral preference, 6/20 showed suppression but no spatial preference.

For visuomotor cells, subcategories were defined based on either cue response or postsaccadic response. Grouping visuomotor cells by cue response confirmed the predominance 
of contralateral enhancement (29/53 cells), whereas other sub-categories were less common (10/53 showed enhancement but no spatial preference, 2/53 showed enhancement and ipsilateral preference, 3/53 showed suppression and contralateral preference, 2/53 showed suppression and ipsilateral preference, $7 / 53$ showed suppression but no spatial preference). Grouping visuomotor cells by post-saccadic responses showed less consistent patterns. The four most common ones are shown in Figure 4.22. Besides the subpopulations shown, 1/53 cells showed post-saccadic contralateral enhancement and ipsilateral suppression, 5/53 showed suppression and contralateral preference and 6/53 showed suppression and ipsilateral preference. Note that besides the 23 visuomotor cells that showed post-saccadic enhancement and spatial preference, motor responses are in general small compared to visual responses. Interestingly, the cue off response seems to be mainly present in the subset of cells with non-spatially specific post-saccadic suppression.

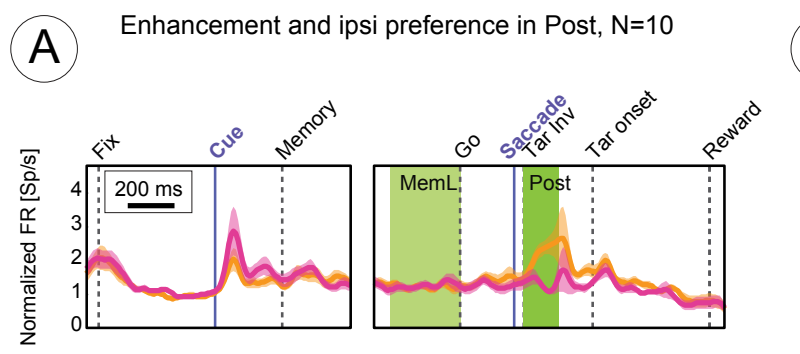

B Enhancement and contra preference in Post, $\mathrm{N}=13$
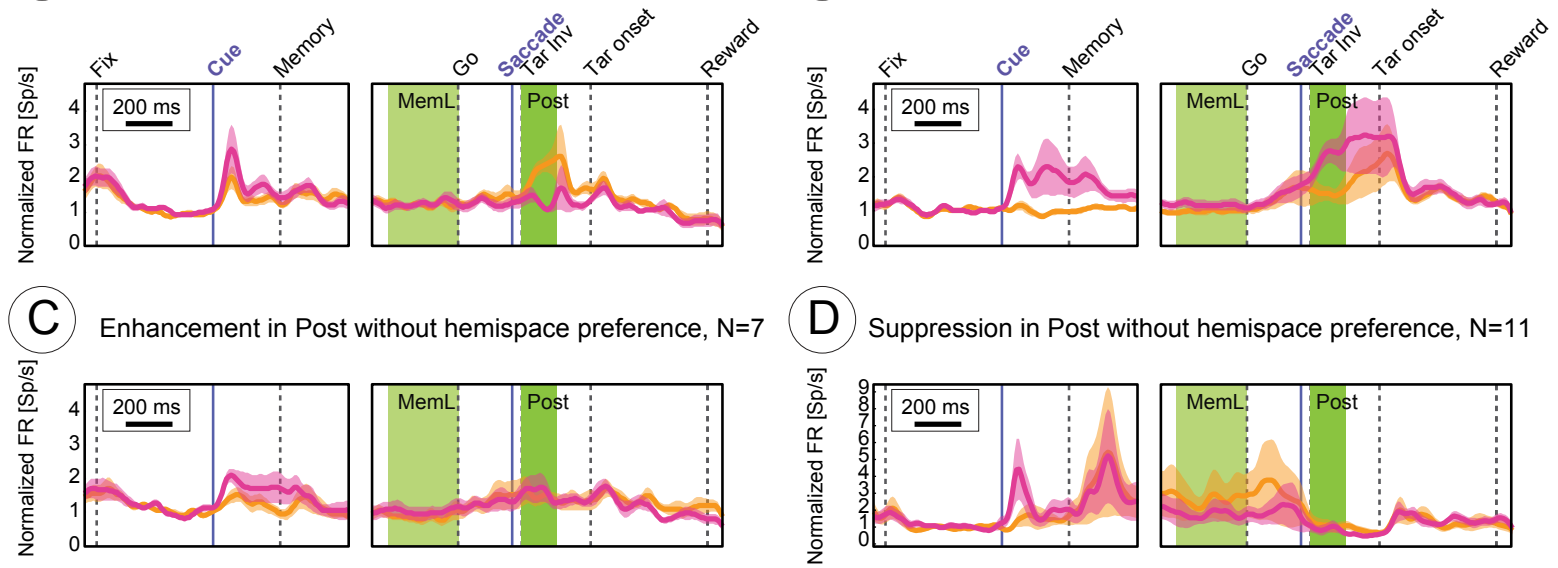

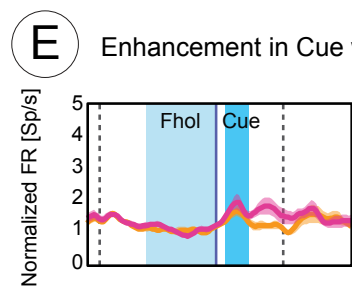

157 655943

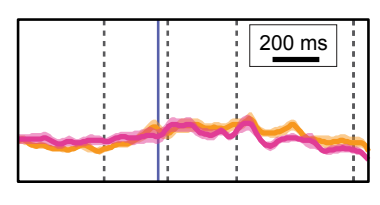

$17011933 \quad 2268 \quad 2769$ Time from beginning of trial [ms]

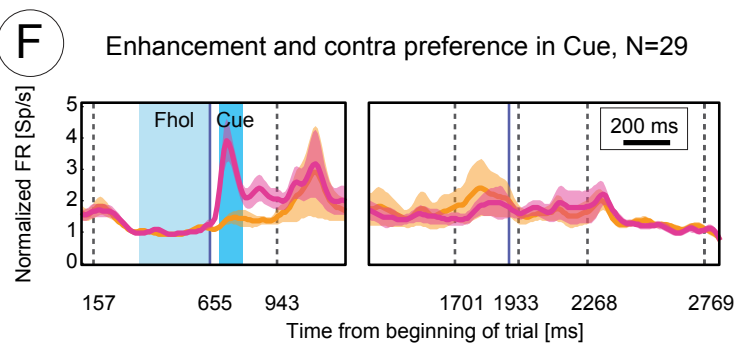

Figure 4.22: Subpopulations of visuomotor cells. Average spike densities shown independently for contralateral (magenta) and ipsilateral (orange) trials for six example subpopulations of cells categorized as visuomotor, grouped either by post-saccadic (Post) response, (A-C), or Cue response, (E-F).

To assess potential involvement of TPO in motor planning, and gaze-dependent activity, four additional subpopulations were defined based on spatial preference either during the memory period (MemL) or target hold period (Thol), see Figure 4.23. Memory period activity was not uncommon, but the firing rate was typically not ramping up toward the upcoming movement and did not reach its maximum right before or during movement execution. In 
total, only 10/98 cells showed enhancement in the late memory epoch compared to fixation hold and in the peri-saccadic epoch compared to late memory (the pattern indicating ramping that is likely related to motor preparation), and 6/98 cells showed suppression in both epochs. This suggests that memory period activity in TPO/FST mostly reflects the retrospective memorization of the upcoming saccade target location rather than prospective movement planning. However, it has been shown that ramping activity on a population level in LIP for example can be explained by step-like increase of firing rates in individual neurons and different onset of the increase in different cells (Latimer et al., 2015), hence challenging this interpretation of lack of ramping activity in individual neurons.

Spatial preference in the target hold period (when the visual target was foveated) was not uncommon either, but the firing rate difference between contralateral and ipsilateral gaze positions was typically very small compared to visual responses. 14/40 cells which showed spatial preference during target hold epoch, showed preference for the opposite hemifield in cue onset (12 preferred contralateral hemifield in cue and the ipsilateral hemifield in target hold, and 2 preferred ipsilateral hemifield in cue and the contralateral hemifield in target hold), whereas $7 / 40$ showed the same hemifield preference (5 preferred the contralateral hemifield in both epochs, and 2 preferred the ipsilateral hemifield). This could indicate that the difference between spatial target hold conditions was mostly related to small differences in visual input, since after the saccade, the receptive field covered a larger part of the screen, which was not entirely darkened due to background illumination. However, this explanation cannot be applied for the 7 cells which showed the same spatial preference in cue and target hold epochs. 

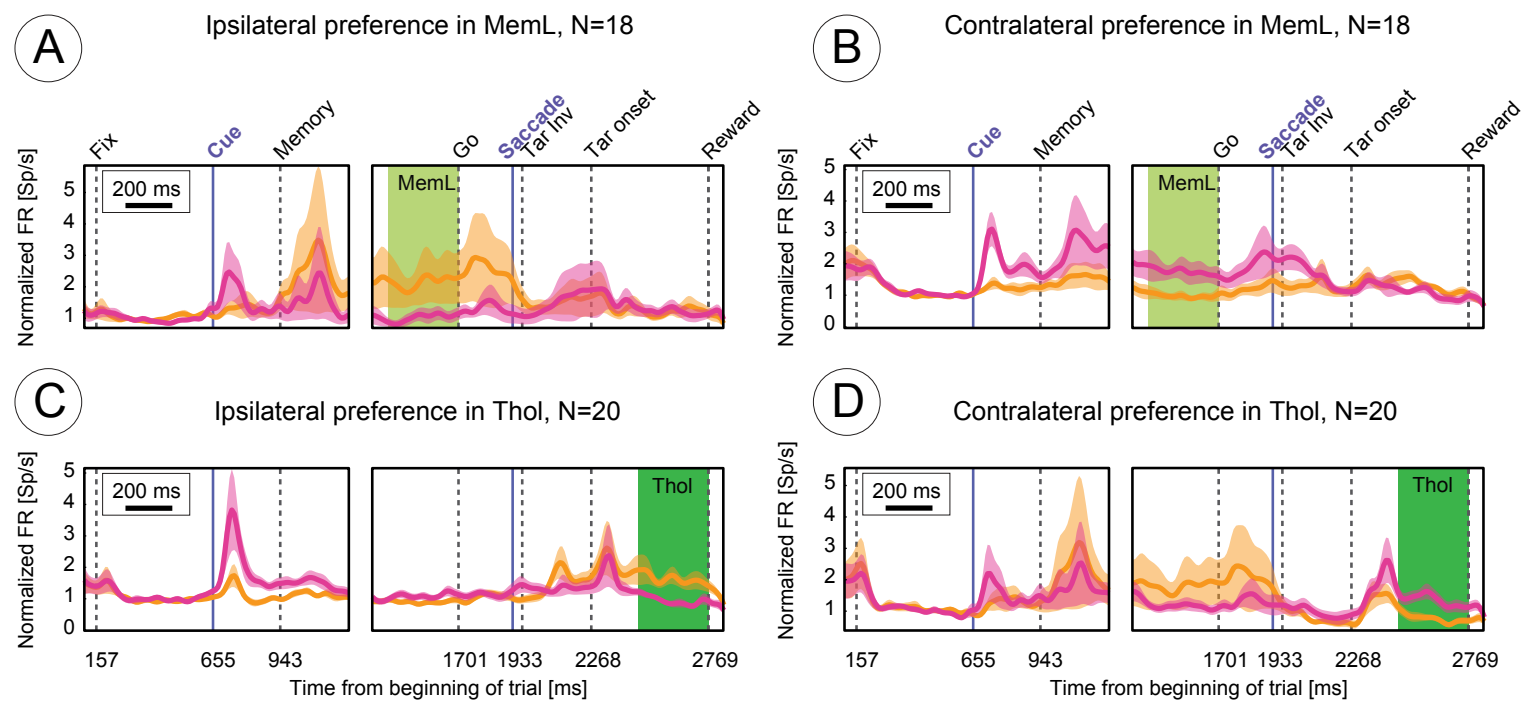

Figure 4.23: Delay period activity and gaze-dependent activity. Average spike densities shown independently for contralateral (pink) and ipsilateral (orange) trials. Top: Subpopulations defined by spatial preference in target hold epoch (Thol), ipsilateral preference left and contralateral preference right. Bottom: Subpopulations defined by spatial preference in memory epoch (MemL), ipsilateral preference left and contralateral preference right.

Finally, local field potentials for 65 recording sites were compared between ipsilateral and contralateral trials, see Figure 4.24. Transient power increase in the low frequencies, delta and theta, and gamma frequency bands after cue onset was much stronger for contralateral cues, as compared to ipsilateral cues, and this tuning extended into the early memory period. In addition, there was more transient $(\sim 100 \mathrm{~ms})$ contralateral increase of alpha and beta power shortly after the cue, but this might be an artifact of transient evoked response that would contain all frequencies. Importantly, the low gamma power was stronger for contralateral trials during the memory delay period. Conversely, low beta, alpha and theta power was more suppressed during contralateral delay and saccadic/post-saccadic periods. A small, contralaterally-tuned increase in delta power can also be seen after the movements. The tuning in delay and movement periods suggest that on the level of population synaptic activity, areas TPO and FST are involved not only in the processing of visual stimuli, but also carry action-related signals. 

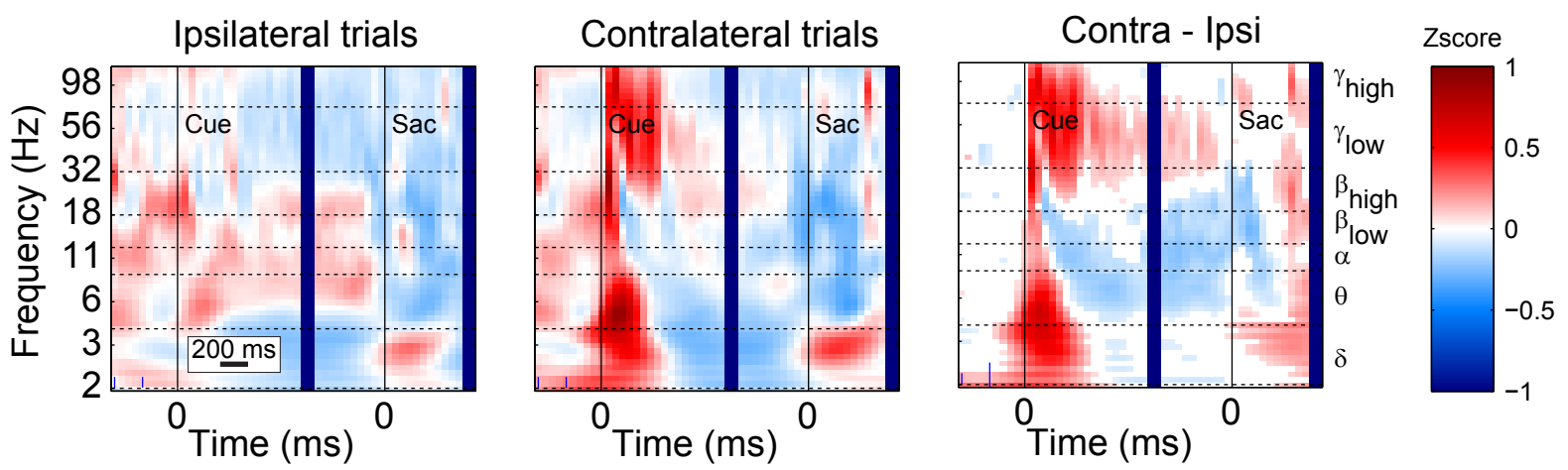

Figure 4.24: Local field potentials. Z-scored power per frequency and time bin, averaged across all LFP sites, see Materials and Methods for details. Left: Ipsilateral trials. Middle: contralateral trials. Right: Difference between contralateral and ipsilateral trials, shown only for bins with significant difference, see Materials and Methods.

Taken together, the results presented in this section suggest that neuronal activity found in TPO strongly depends on contralateral visual inputs, whereas motor preparatory activity was rare and post-saccadic activity showed to be inconsistent within motor and visuomotor subpopulations, with nearly the equal amount of cells being enhanced or suppressed and preferring either contralateral or ipsilateral hemifields. These findings agree with the common notion that TPO/FST is mainly involved in sensory processing, although our spiking and LFP results also indicate presence of visuomotor encoding.

\subsubsection{TPO inactivation effects in the search-to-sample task}

To compare dorsal pulvinar inactivation effects with a predominantly visual area, inactivation was additionally performed in area TPO, using the same search-to-sample task. The effects are shown in Figure 4.25. The apparent drop in success rate for samples presented on the contralesional (right) side compared to ipsilesional sample presentation is expected from inactivating a visual area, although the effect is very small. This can be seen as a general confirmation of the hypothesis underlying the task design, especially because this effect was very consistent across sessions. The small size of the effect might be due to a limited extent of the inactivated area after a single injection, as compared to the full size of area TPO. Additionally, TPO is not a primary visual area, so the inactivation might be partially compensated by other pathways.

The more surprising effect is that dwell times were increased specifically for targets on the contralesional side. This might indicate some minor involvement of STP in updating motor commands. But looking at the effects per session, it seems more like the dwell time increase is not space specific and potentially indicates a general reduction of confidence when selecting the target due to perceptual deficits. 

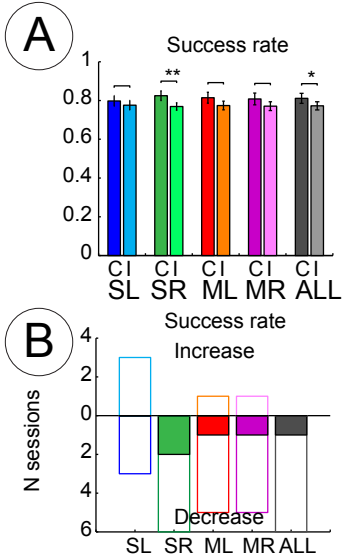
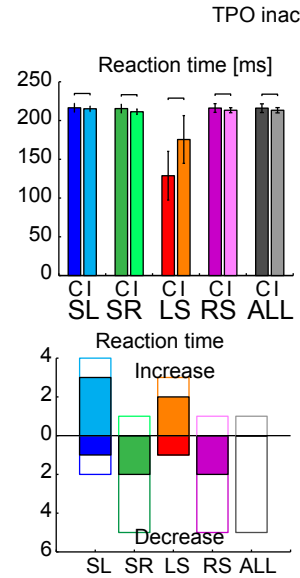
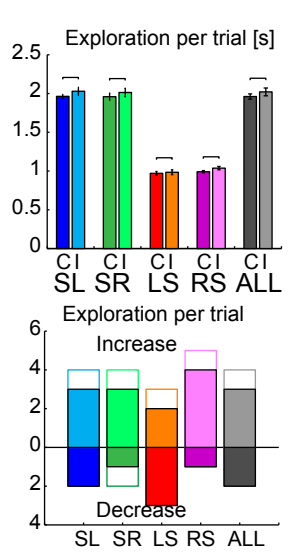

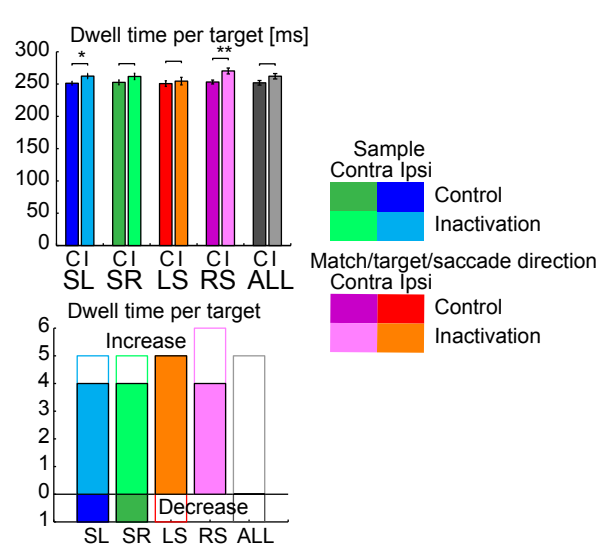

Figure 4.25: Task performance comparison between control and inactivation sessions. Comparison of success rate, Reaction time, exploration time and dwell time in in control and inactivation sessions. Values were grouped either by sample position (SL/SR), by the location of the match (ML/MR), the direction of the saccade or the explored hemifield (LS/RS). A) Mean and SE across means per subject, paired t-tests. B) Number of sessions for which the respective value was higher (upper part) or lower (lower part) after inactivation. Filled bars mark sessions in which the difference between tasks was significant (see Materials and Methods for details).

To further validate that exploration behavior was not much affected by TPO inactivation, eye positions were also recorded in the inter-trial-interval, see Figure 4.26. Similar to pulvinar inactivation, TPO inactivation did not show evident bias for exploring either hemifield under non-task related conditions. If anything, exploration in the contralesional hemifield was more common after inactivation compared to control sessions. Taken together, the results indicate perceptual impairments induced by STP inactivation. This agrees with the predominance of visual responses seen in the neuronal activity during memory guided saccades. However, the effect size is rather small. 

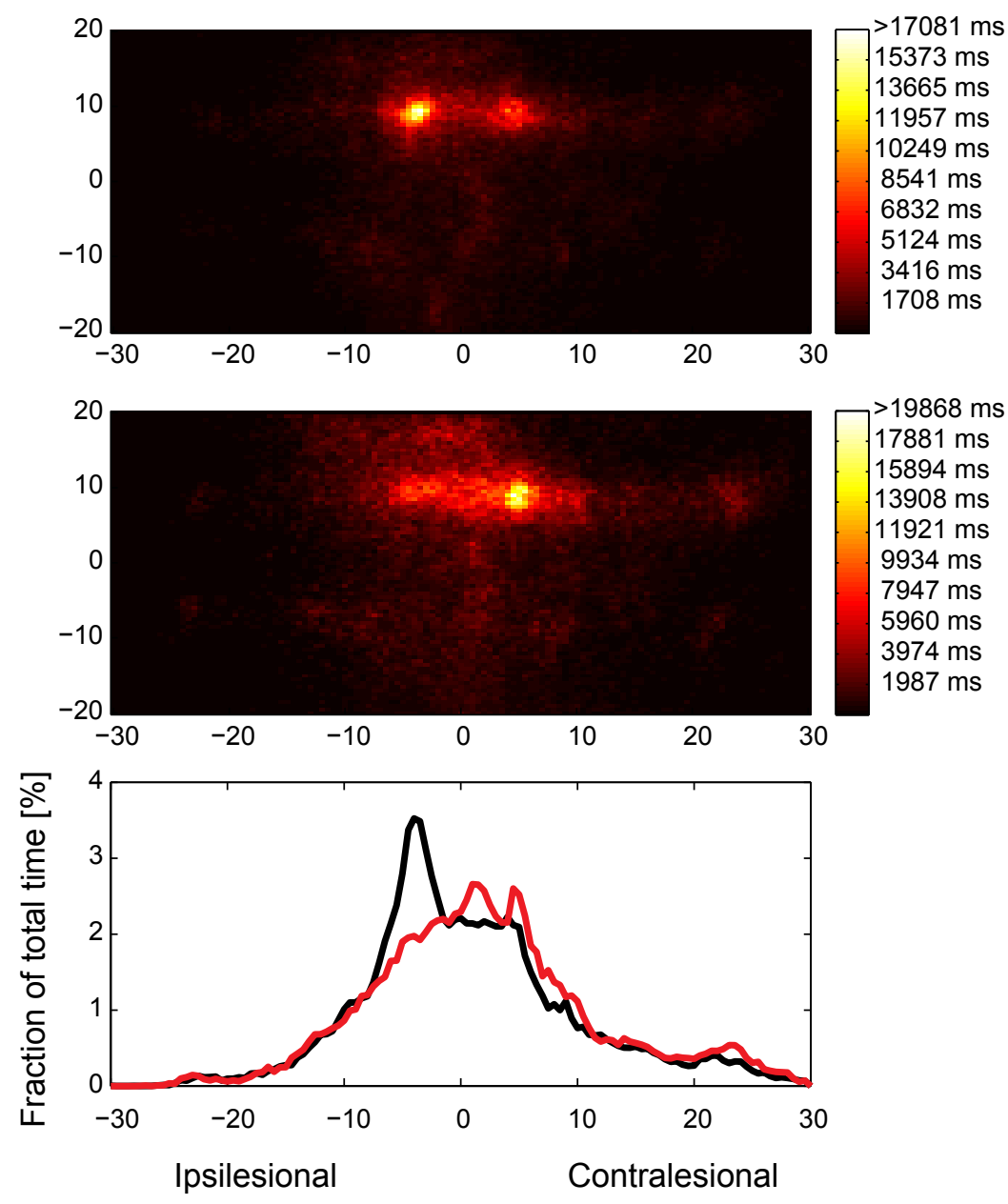

Horizontal position $\left[^{\circ}\right]$

Figure 4.26: "Free exploration" in the inter-trial interval. Eye position heat maps for the inter-trial-interval across all trials in control sessions (top) and inactivation sessions (middle). Colors indicate the total time the eye position was recorded in the respective bin, starting after reward and until the next trial started. Bottom: Histogram of eye positions across the horizontal axes for control (black) and inactivation sessions (red). 


\subsection{Discussion}

Delayed match-to-sample tasks are traditionally used to study working memory (Jackson et al., 1989; Rodriguez and Paule, 2009), whereas visual search tasks are typically used to evaluate motor-intentional spatial deficits (Ellison, 2004; Hornak, 1992; Karnath and Fetter, 1995). Here we designed a novel task, combining delayed match-to-sample and visual search aspects, a "search-to-sample" task. The aim was to combine sensory and motoric demands in one task in order to allow dissociation of sensory-perceptual and motorintentional aspects of spatial deficits. It should be noted, that due to the match-to-sample aspect, working memory deficits are expected to be reflected in task performance as well, and spatial working memory deficits have been hypothesized to contribute to spatial neglect (Husain, 2001; Malhotra, 2004), since neglect patients might forget that they already explored the ipsilesional side of space. Also, since the distractors in the search-to-sample task differ from the sample in only one feature but the exploration of potential targets is forced to be serial, it does not correspond to either of the two classical categories of search tasks, and rather represents a mixture of "parallel" or "disjunctive" and "serial" or "conjunctive" search (Schindler et al., 2008), further complicating comparisons between the search-to-sample task and studies using more classical visual search tasks.

Human experiment 1 demonstrated the general applicability of the novel search-to-sample task design to humans and established reasonable perceptual discrimination difficulties for both young and elderly subjects. The latter was chosen as a matched-age group for potential neglect patients to be tested. A staircase procedure to adjust the difficulty per subject established a more or less equal success rate between subjects. Furthermore, the comparison between two task variants showed that task readouts reflect cognitive demand. However, exploration patterns differed drastically between subjects and confusions between distractor targets and the correct target seemed rather random and not easy to control.

In the second human experiment, target shapes were symmetrical and fixed for both tested groups of subjects with linearly spaced curvatures (different spacing for the two groups). Confusion matrixes showed that this set of target shapes lead to confusions mainly between the most similar shapes. Unfortunately, one of the shapes was nearly always discriminated correctly, indicating that ideally the curvatures should not be linearly spaced. As an additional modification, the target locations were randomized taking three out of eight possible locations (compared to four fixed sets of target locations in the previous experiment). Still the exploration patterns differed between subjects, and most subjects showed very stereotypical exploration.

Although the effects of both monkey pulvinar and TPO inactivation were small, the results indicate a qualitative difference of impairments caused by the two inactivated regions. TPO 
inactivation caused a consistent drop in success rate for contralesional samples, indicating that neglect-like deficits previously reported in monkeys after TPO ablation (Luh et al., 1986; Scalaidhe et al., 1995) are primarily perceptual.

Success rate also dropped after pulvinar inactivation, but less dependent on the spatial condition as compared to TPO inactivation. Additionally, after pulvinar inactivation the monkey exhibited longer reaction and exploration times. In contrast to previously reported effects of pulvinar inactivation on saccade latency (Wilke et al., 2013), these increases were not specific for any spatial condition. But one should be careful evaluating the difference in reaction time of initial saccades to ipsilesional and contralesional hemifield, as ipsilesional saccades were in principle very rare.

However, in control experiments (memory guided and visually guided saccades), the only two parameters affected by pulvinar inactivation were target selection in the visually guided saccade task and accuracy/precision in the memory guided saccade task. We could not reproduce the increased latency for contralesional saccades and the target selection bias towards ipsilesional targets in memory saccades reported in previous studies (Wilke et al., 2013). This finding is especially surprising, because these studies used very similar oculomotor tasks. Although this remains pure speculation, it might be that the exact injection site is crucial for causing this type of spatial impairments, since the effects of pulvinar microstimulation also strongly depended on the exact location of the stimulation electrode tip (Dominguez-Vargas et al., 2017). On the other hand, the large extent of the inactivation spread argues against this interpretation. An alternative explanation could be that the established and idiosyncratic behavioral patterns that emerged after long training in this particular monkey counteracted the effects of inactivation. For example, this monkey did not show a typical increase of choice memory saccade reaction times, as compared to instructed trials, due to additional decision process resolving spatial competition between the response options. If anything, the relationship was the opposite (RT instructed > RT choice) for ipsilesional trials in control sessions. This might suggest that the monkey often decided on a hemifield before the onset of visual cues, and changed the plan if it was an instructed trial. In this line of reasoning, the pulvinar inactivation might only affect target selection when the decision is made at or after the onset of visual information. This resembles, at a conceptual level, the observed lack of microstimulation effect on saccade choices in memory guided saccade task, as compared to the visually guided task where stimuli, decision and actions are in close temporal proximity (see Appendix A).

Overall, the results suggest that TPO inactivation caused contralesional perceptual deficits, whereas pulvinar inactivation caused more general, non-spatially specific motor-intentional deficits. This agrees with the more dominant activation for contralateral visual stimuli in TPO 
as well as with the notion that TPO is a primarily sensory, rather than sensorimotor area (Dahl, 2010; Hoffman, 2008). However, the finding that most cells were classified as visuomotor and the presence of pre-saccadic and peri-saccadic activity, as well as delay period activity in spiking and in LFP challenges this view, and is more in line with dorsal stream processing, as suggested by earlier studies focusing on motion processing in TPO (Bruce et al., 1981; Desimone and Gross, 1979; Oram et al., 1993). Overall, although the proportions differed, TPO exhibited similar activation patterns as dorsal pulvinar, suggesting that TPO-pulvinar communication is relevant for visuomotor behavior (Wilke, Kagan, Andersen in revision).

\subsubsection{Limitations}

The main question for this task remains if there is any target location configuration which would result in non-stereotypical exploration patterns. The problem is, that probably even with completely randomized target locations, developing a fixed exploration strategy is beneficial as it reduces the time spent planning the next eye movement. This raises the question, if it even makes sense to compare exploration patterns between healthy subjects and neglect patients with this task.

Especially after pulvinar inactivation we expected to see a major impact on exploratory behavior (Wilke et al., 2010). This suggests that the impact of inactivation was not strong enough to overrule the acquired, very stereotypical exploration pattern during searching for the match. However, exploration in the inter-trial-interval without any task context was not affected either.

Additionally, we typically observed that subjects and also the monkey got better in the task within a session. Even after thousands of repetitions, it seemed like the monkey still needed a certain amount of trials to adapt to either the target shapes or the target locations or both. This is probably related to the fact, that not all shapes and positions are present in each trial, so it takes several trials until all potential shapes and target locations are explored. Because of this observation, we used control sessions from the previous day as baseline for the inactivation experiments, accepting day by day differences in performance just not to bias our results by comparing inactivation runs to earlier runs acquired in the same session, which would be influenced by the day by day learning curve.

Another problem is a tradeoff between task complexity and statistical validity of sample sizes (number of trials). Hypothetically, more targets, more potential target locations and target shapes result in more homogenous and comparable data, but the duration of each trial becomes longer, as it gets more difficult to find and identify the match. Longer trials means less trials per session, and the number of sessions is also limited, especially for testing 
neglect patients. It should also be mentioned that the number of targets and target locations is limited by the space on the screen, the maximum visual angle and the eye tracking accuracy. While targets have to have a minimum size in order to be distinguishable, the allowed radius for each target has to be liberal enough to guarantee that the targets are revealed when eye position lands on the respective location. Moreover, due to the stochastic nature of task conditions and especially target location distribution, exploration times differed considerably across trials, which is another consideration to be taken into account when talking about statistical validity.

As seen in human experiment 2, the subjective difficulty of this task also differs quite substantially between subjects, especially in the elderly group. This finding is somewhat concerning as it complicates comparisons between healthy subjects and neglect patients. Finally, in all results shown here, four task conditions (dependent on sample position and target position or explored side) were always treated separately. The problem is, that even if a tested subject had a strong perceptual deficit (e.g. identifying samples on the left side) without any intentional impairment or bias, the success rate for example would not only be lower for trials where the sample was presented on the left, but also for trials where the match was on the left (or right), because half of these trials overlap. The comparison therefore should happen between differences (control-inactivation or healthy-patient) in the two perceptual conditions and between differences in the two exploratory conditions. This adds another layer of analytical complexity, which is also why it has not been done systematically yet.

\subsubsection{Monkey-human comparison}

Another question is if results in monkeys and humans are in principle comparable. One difference mentioned earlier was that shape discrimination seems to be different in humans and monkeys. Humans seem to be better for categorizing target shapes, which is why for human experiments only concave shapes were used, whereas in the monkey experiments both concave and convey shapes were used. This still leads to comparable levels of confusions between monkey and human experiments. However, in terms of timing, there seem to be big differences humans and monkeys, see Figure 4.27. Whether these differences are due to overtraining the monkey or just species-specific differences remains unclear, but comparisons between deficits in human neglect patients and in monkeys caused by inactivation will have to be normalized in order to be comparable. 


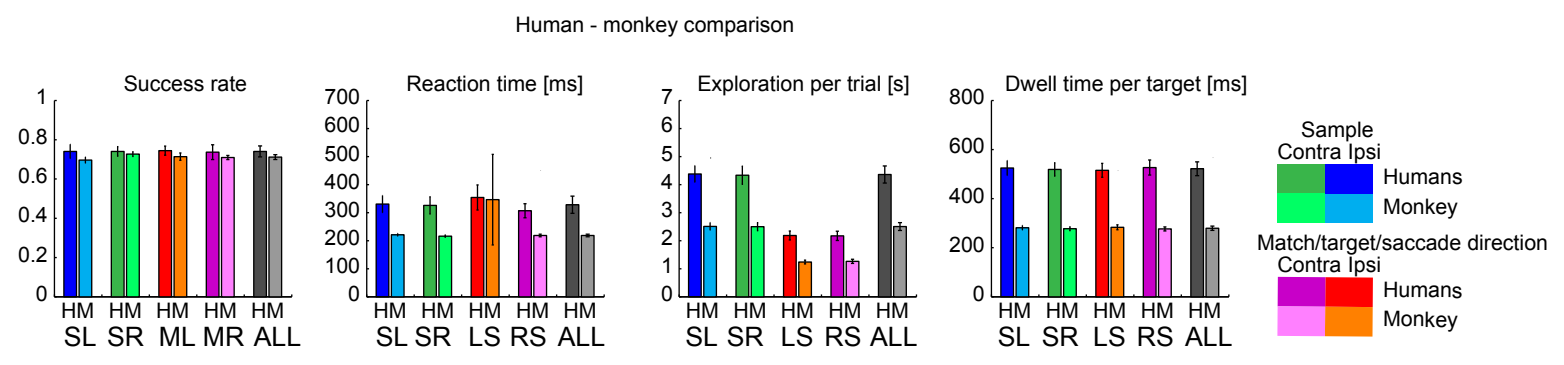

Figure 4.27. Human-monkey comparison of performance in the search-to-sample task. Human results from young human subjects for experiment $2(\mathrm{H})$ and monkey baseline behavior $(\mathrm{M})$ compared. While similar success rates were achieved by adjusting task difficulty, the monkey's reaction times, exploration times and dwell times were in general much lower.

\subsubsection{Applicability to patients}

As briefly mentioned in Materials and Methods, we had the opportunity to test the first version of the search-to-sample task (human experiment 1) on a patient (JA), who exhibited a right hemisphere lesion and had been attested with symptoms of left hemifield neglect by paper based tests. Unfortunately, JA had considerable difficulties with this task. Therefore, only the task without distractor was tested. Over the course of two sessions, each lasting more than one hour, he only completed 38 trails. In these trials he typically selected the first revealed target. The biggest problem was keeping fixation during sample presentation, as he typically exhibited saccades towards the sample, see Figure 4.28.

However, while the latencies of towards samples on the right were consistent across trials, saccades towards the sample on the left happened at rather random moments. This could indicate that the perception of samples on the left was impaired. Furthermore, the success rate was lower for samples presented on the left (although the number of trials is far from allowing any sort of statistical comparison). Importantly, in the few trials that he managed to keep fixating, the exploration patterns did not indicate any spatial exploratory impairment. It was mainly due to these findings, that the memory period was removed and the sample period was shortened to match the observed fixation break saccade latency. This way, subjects are allowed to saccade towards the sample, but it will not be displayed any more once they reach there. In an ongoing collaboration with Prof. Dr. Hans Otto Karnath (Department of Cognitive Neurology, Eberhard Karls Universität Tübingen), the task has been further refined to account for potential difficulties neglect patients might exhibit which we have not considered yet. However, it remains to be seen if the adapted version (human experiment 2 ) is applicable to neglect patients. 


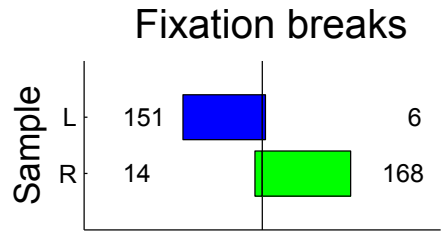

to the left to the right

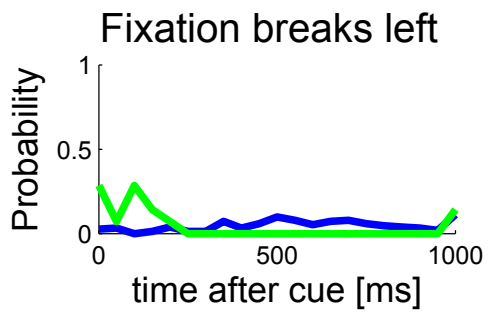

Success rate
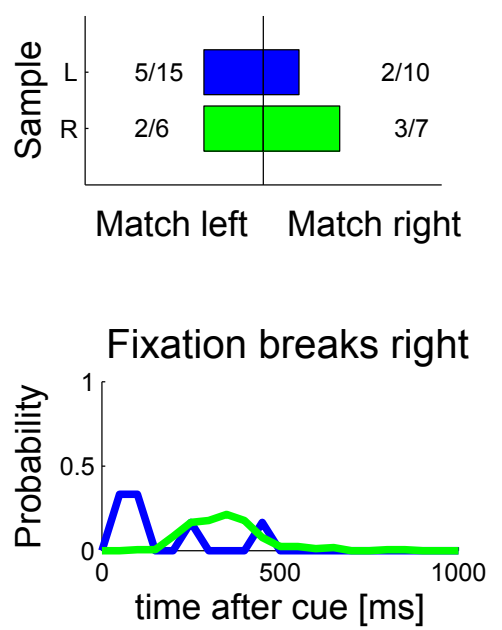
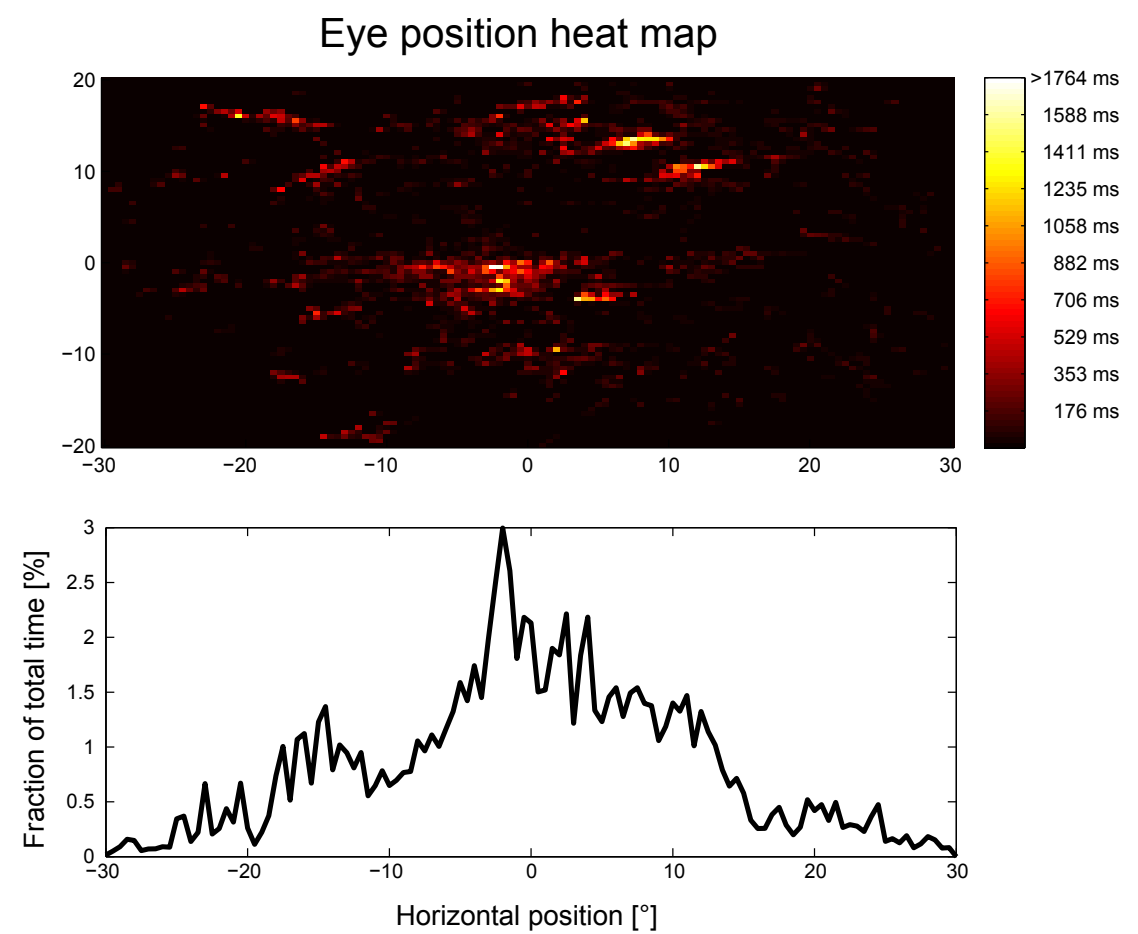

Figure 4.28: Task performance of patient JA in human experiment 1. Top, left: Number of fixation breaks towards the left and right side, independently for trials in which the sample was presented on the left (blue) or right (green). Top, right: Success rate in the four main task conditions, sample left (blue) or right (green) and match left or right. Middle: Histograms of fixation break times for leftward saccades (left) and rightward saccades (right), independently for trials in which the sample was presented on the left (blue) or right (green). Bottom: Eye position heat map and histogram of eye positions across the horizontal axes from the few trials that were not aborted during fixation. Colors indicate the total time the eye position was recorded in the respective bin, starting after the "Go" cue. 


\subsection{Contributions and Acknowledgements}

LS, MW and IK designed the experiments. AK collected the initial pilot data, data for human experiment 1 , and was heavily involved in task refinements. HL collected the data for human experiment 2. UZ and LS performed the monkey inactivation experiments. AUDV wrote the behavioral analysis scripts for memory guided saccades and visually guided saccades. DA wrote and performed the LFP analysis. LS wrote the S2S and electrophysiological analysis scripts and analyzed the data. IK supervised the projects.

Lukas Schneider (LS); Melanie Wilke (MW); Igor Kagan (IK); Alexander Kratzenberg (AK); Hannah Lönneker (HL); Uwe Zimmermann (UZ); Adán Ulises Domínguez Vargas (AUDV), Danial Arabali (DA).

We thank Ira Panolias, Daniela Lazzarini, Sina Plümer, Klaus Heisig, and Dirk Prüße for technical support. We also thank Stefan Treue, Alexander Gail, Hansjörg Scherberger, members of the Decision and Awareness Group, Sensorimotor Group and the Cognitive Neuroscience Laboratory for helpful discussions. Special thanks to Benjamin Dann for helpful discussions on spike and LFP analysis. Special thanks also to Hans-Otto Karnath, Daniel Wiesen, and Sophia Nestmann for sharing their experience with neglect patients and fruitful discussions for adapting the S2S task towards applicability to patients. Supported by the Hermann and Lilly Schilling Foundation, German Research Foundation (DFG) grants WI 4046/1-1 and Research Unit GA1475-B4, KA 3726/2-1, CNMPB Primate Platform, and funding from the Cognitive Neuroscience Laboratory. 


\section{General discussion}

The goal of this thesis was to elucidate perceptual and motor intentional processing in the pulvinar, a subcortical thalamic structure, which due to its widespread reciprocal connectivity to cortical areas and the diversity of neuronal activation patterns has been attributed to various cognitive functions such as allocation of spatial attention (Petersen et al., 1985a; Saalmann et al., 2012; Zhou et al., 2016a), distractor filtering (Strumpf et al., 2013), emotion control (Almeida et al., 2015), confidence rating (Komura et al., 2013) and coordination of motor commands (Guillery, 2003). Especially the function of the dorsal aspect of pulvinar, the thalamic structure that expanded most during primate evolution, is understudied and remains unclear, despite ongoing attempts to elucidate its significance (Bridge et al., 2015). Using electrophysiological recordings, electrical microstimulation and reversible pharmacological inactivation in the dorsal pulvinar of rhesus macaque monkeys performing various types of visuomotor tasks (namely visually guided, memory guided), we provide new evidence for a contribution of dorsal pulvinar in target selection, goal-directed actions, visuomotor integration and visuomotor transformations.

\subsection{Summary of results and limitations}

\subsubsection{Predominance of motor related activity}

In agreement with previous findings (Mathers and Rapisardi, 1973; Petersen et al., 1985a), visual responses to contralateral stimuli were typically marked by excitation starting around $50 \mathrm{~ms}$ after cue onset and reaching its maximum at $120 \mathrm{~ms}$ after cue onset, whereas ipsilateral stimuli had less influence on neuronal activity. A similar space dependency was found in theta, alpha and low beta frequency bands of local field potentials, indicating that visual information in the pulvinar is lateralized. Motor responses were typically post-saccadic with not very consistent response latencies, but a few cells also exhibited pre- and perisaccadic activity, consistent with previous reports (Benevento and Port, 1995). Interestingly, in this study authors concluded that the pre-saccadic activity is linked to the expectation of a change in visual input, because they found pre-saccadic responses only in visually guided saccades, not in memory guided saccades. In contrast, we found pre-saccadic activity also during memory saccades, which are performed in absence of direct visual input (except the visual stimulation due to background screen illumination and self-induced motion signal).

The post-saccadic activity of the population was characterized by non-spatial specific enhancement or suppression, which further supports the idea that pulvinar mediates corollary discharge and might signal context changes in the visual information or suppress cortical processing of visual information after the saccade that was acquired during the 
saccade itself to facilitate a stable perception (Robinson et al., 1986). Due to the short (100 or $200 \mathrm{~ms}$ ) post-saccadic period without visual stimulation (i.e. prior to target onset) however, we cannot rule that out post-saccadic activity reflected the expectation of the upcoming visual target rather than pure post-saccadic activity. For comparison, the corresponding period in (Benevento and Port, 1995) was about 300 ms.

Categorizing the cells into visual, visuomotor and motor cells dependent on visual and postsaccadic modulation showed that almost all cells fell into one of these categories, with most cells being motor (35\%), followed by visuomotor (30\%) and visual cells (15\%), suggesting that pulvinar is more involved in motor then visual processing. However, looking at the distribution of visuomotor indexes showed a smooth transition between categories, indicating that the classification is not clear cut. Additionally, there was no apparent organization of these cell categories within the pulvinar, and further splitting into subcategories by the type of visual and post-saccadic response (enhancement/suppression and spatial preference) revealed very diverse patterns within each of these categories.

In general, to get further insight in population dynamics of pulvinar activity, the relationship of firing patterns of cells recorded simultaneously has to be analyzed. However, since we typically did not record more than a handful of neurons at the same time, the interpretations that we could derive from this analysis on the given dataset would be very limited. In order to record from a larger set of neurons and analyze network dynamics, typically multi-electrode arrays are implanted in the region of interest. Implanting multi-electrode arrays in subcortical structures is technically very challenging and we are not aware of any study that has employed multi-electrode array recodings in subcortical structures. A more viable approach would be to use multi-contact probes, i.e. one probe with multiple electrodes, such as Vprobe or S-probe from Plexon. This would also improve the assessment of spatial relationships between the adjacent recording sites, since they would be pre-defined by the probe dimensions.

Given the current recording approach (several individually moveable, flexible floating electrodes), the precision of assessment of exact electrode tip locations and therefore the spatial relationship of recorded cells is very limited. To make sure we are only comparing cells which are in close vicinity, we could compare the firing patterns of neurons recorded from the same electrode at the same time. However, we typically were not able to cleanly isolate more than two cells recorded from the same electrode in the same recording site, thus we did not try to investigate this aspect further.

Additionally, a more elaborate analysis of population dynamics like state space analysis (for example using demixed principle component analysis, dPCA) could improve our 
understanding of the response patterns in population activity (Kobak et al., 2016). Although this method is typically applied when activity in the population is recorded simultaneously, using multi-electrode arrays, there are also dPCA approaches that would work for our data that was recorded sequentially (Michaels and Scherberger, 2018).

In general, it also has to be noted that we included well isolated single-unit activity as well as multi-unit activity in our analysis. It remains to be seen if visuomotor responses are more commonly found in multi-units, which would suggest that this response pattern is merely a result of combining activities of multiple cells in one response pattern. One possibility is to perform analyses separately for the units that were assigned good isolation ranking with multi-units, and compare the results. However, we cannot fully resolve this issue, since perfect isolation of single units is difficult for the extracellular recordings and limited by the level of randomly recorded voltage fluctuations due to neuronal and instrumental noise, a problem that is inherent to all electrophysiological recordings.

\subsubsection{Non-spatial specific motor preparation activity}

Taking a closer look at potential involvement of dorsal pulvinar in motor preparation, we also found a considerable amount of cells (17\%) predicting the direction of the upcoming movement in instructed trials when only one option was available. When two options were available, the activity of these cells was not different for the two selected options, which could either indicate that the decision only happened right before the movement or (and more likely) that the spatial preference in these cells reflected retrospective stimulus processing rather than prospective movement planning. On the level of local field potentials however, there was a considerable delay period activity modulation in the alpha and beta band, which differed when the monkeys selected the contralateral side as compared to when they selected the ipsilateral side, indicating that the upcoming choice is in fact reflected in dorsal pulvinar, but maybe not to an extend where it reliably affects single cell activity. Importantly, another subset of cells that showed spatial specific responses during the early memory period in instructed conditions, right after cue offset, did predict the upcoming target selection, but these cells showed increased responses when the non-preferred target was selected afterwards, consistent with the idea that pulvinar allocates spatial attention towards the preferred location when attention is not already focusing that location (Petersen et al., 1987).

Interestingly, spiking activity typically decreased in the memory period, exhibiting a population response that resembles complementary patterns of gradual ramping up found in posterior parietal cortex, for example in area LIP and the so called parietal reach region 
(PRR) (Gnadt and Andersen, 1988; Hwang and Andersen, 2012), but is inverted, hinting at a potential inhibitory influence of these cells on fronto-parietal cortical areas or vice versa. Simultaneous recording in both regions would certainly provide a better understanding of the underlying mechanisms.

Further comparing neuronal responses in trials where the monkeys were instructed to perform a movement towards the preferred (from the cells perspective) location to responses when an additional saccade option was available indicated that the additional option has a suppressing effect on pulvinar activity. This result has to be taken with caution, because the estimation of preferred direction was based on single target trials in the first place, potentially leading to a bias for preference of single targets.

\subsubsection{Strong influence of gaze position on pulvinar neuronal activity}

Gaze-dependent activity has been shown before for ventral pulvinar (Robinson et al., 1990). In dorsal pulvinar, we found many cells (77\%) that exhibited gaze dependent activity, and the differences in cells showing an effect of gaze during initial fixation and final target fixation suggest an impact of the behavioral state on gaze dependent encoding in dorsal pulvinar, although it has to be considered that the set of final gaze positions included additional locations. We could neither find systematic spatial preference for gaze position nor a conclusive co-variation of activity with gaze position. Importantly, not purely retinotopic representations were found more frequently compared to purely retinotopic representations, even during the visual stimulation epoch.

A considerable amount of cells exhibited gain-field properties (Andersen and Mountcastle, 1983) and response field shifts not only during the post-saccadic epoch, but also during visual stimulation and the memory period. These findings suggest that gaze dependent activity is crucial for understanding the function of pulvinar and support the idea that pulvinar might be involved in visuomotor transformations. However, we cannot confirm that gain fields and response field shifts in the classical sense (Salinas and Abbott, 2001) are present in the dorsal pulvinar or if our findings are mainly a result of head-/body-/hand- or world-centered encoding in the pulvinar, because we did not include enough initial gaze positions and the sets of final gaze positions were largely non-overlapping for the linked initial gaze positions.

\subsubsection{Target selection biasing by electrical microstimulation}

In agreement with previously reported findings of dorsal pulvinar inactivation biasing saccade target selection towards the ipsilesional side (Wilke et al., 2010, 2013), we found that electrical stimulation of dorsal pulvinar can bias target selection towards to contraversive side in a visually-guided saccade task, indicating that stimulation had the opposite effect as inactivation. 
However, this was only true when stimulation was applied after the monkeys were free to go; when stimulation was applied during initial fixation, we observed the opposite effect. A similar impact of early electrical stimulation on target selection dependent has been shown for the caudate nucleus (Ding and Gold, 2012), a structure that is considerably close to pulvinar. More importantly, stimulation had no significant effect on target selection in a memory-guided saccade task, neither when applied during the visual period nor when applied during saccade execution. This indicates that the effects of microstimulation cannot be explained by purely mechanistic perceptual or motor execution perturbation, but suggests that pulvinar function is crucial for making fast decisions based on visual information (Soares et al., 2017).

Furthermore, electrical microstimulation had a similar bimodal effect on saccade latencies. While late stimulation increased saccade latency to both sides (but more so for ipsiversive saccades), early stimulation facilitated ipsiversive saccades. Prolonged saccade latency has been reported in microstimulation studies in several cortical oculomotor areas such as frontal eye fields (Izawa et al., 2004) and supplementary eye fields (Yang et al., 2008), and subcortical structures such as caudate nucleus (Watanabe and Munoz, 2013) and superior colliculus (Munoz and Wurtz, 1993), where stimulation of cortical areas typically leads to stronger delays for ipsiversive saccades and subcortical stimulation typically causes stronger delays for contraversive saccades. This further illustrates that pulvinar function is closely linked to the fronto-parietal network.

Importantly, effects on reaction times were found in visually-guided as well as when stimulation was applied close to motor execution in memory-guided saccades, where we did not see an effect on target selection, thus indicating that the two effects are not causally linked.

One limitation of this study was that we do not know about the current spread, potentially activating other regions as well. Especially antidromic activation of superior colliculus via the brachium cannot be ruled out. However, stimulation effects were specific to the stimulation site within the pulvinar, meaning that more ventral stimulation exhibited effects on target selection only when stimulation was applied at the same time as the targets appeared, suggesting that stimulation effects were fairly localized and distinct visuomotor processing in ventral and dorsal pulvinar, and stimulation effects did not systematically change depending on the distance of the stimulation electrode tip and the brachium.

A more critical factor was the stimulation protocol. The stimulation parameters used in this study were based on a simultaneous neuroimaging study investigating pulvinar connectivity. Therefore, the amplitudes were large compared to other studies employing microstimulation for effects on behavior for example in oculomotor output structures such as FEF (Izawa et 
al., 2004) or superior colliculus (Munoz and Wurtz, 1993). However, in more visuomotor regions, which are more remote to motor output, such as posterior parietal cortex, posterior cingulate, dorsal premotor or dorsomedial prefrontal cortex higher currents similar to ones applied here have been used to investigate behavioral effects (Churchland and Shenoy, 2007; Cutrell and Marrocco, 2002; Hayden et al., 2008; Yang et al., 2008). However, lower amplitudes would potentially provide a better spatial and further clarify the site specificity of stimulation effects, or might show no effects at all. Another problem in interpreting microstimulation results arises from the constant duration of the stimulation trains, so we do not know if the behavioral effects were linked to stimulation onset or stimulation offset. Shorter and varying train durations, if proven efficient for eliciting the behavioral effects, would improve the temporal resolution and allow a better evaluation of both the critical behavioral period and if the observed effects are more linked to stimulation onset or offset.

\subsubsection{Pulvinar inactivation disrupts motor-intentional processing}

Unilateral pulvinar lesions and have been associated with hemi-spatial neglect (Arend et al., 2008a; Karnath et al., 2002), and pulvinar inactivation in monkeys caused symptoms similar to spatial neglect (Petersen et al., 1987; Wilke et al., 2010, 2013). Similar to pulvinar lesions, ablation of the temporal parietal occipital area (TPO) also caused neglect like symptoms (Luh et al., 1986; Scalaidhe et al., 1995). Since neglect is characterized by two main spatial deficits - sensory-perceptual and motor-intentional (Hornak, 1992; Karnath and Rorden, 2012; Liu et al., 1992; Na et al., 1998) - and the neglect-like symptoms in monkeys after pulvinar inactivation could be a manifestation of both of these aspects, we designed a novel "Search-to-Sample" task sought to dissociate sensory-perceptual and motor-intentional deficits caused by pulvinar and TPO lesions and allow inter-species comparisons of spatial deficits.

Although neuronal activity in TPO was somewhat comparable to pulvinar activity - which further underlines the strong reciprocal connectivity of these two regions - inactivation effects were considerably different. While pulvinar inactivation affected exploration duration and saccade latencies in a non-spatial specific manner (suggesting general motor-intentional deficits), TPO inactivation caused reduced perception of contralateral stimuli. However, effect sizes were small and we could not reproduce the target selection bias reported in a previous study using the same memory guided task as in our control condition (Wilke et al., 2013).

Furthermore, and in contrast to previous reports (Wilke et al., 2010) the spatial eye movement exploration patterns were unaltered by pulvinar inactivation. For exploration during the task, the explanation for the lack of affected exploratory behavior could be that the inactivation effects were too small to overrule the acquired stereotypical search pattern, but we did not observe any changes in not-task related periods either. 
Task performance typically increased during each session, which would bias the pre- and post-injection comparisons, which are typically used for inactivation studies. Here we used control session from the day prior to inactivation sessions. This has the disadvantage, that day by day performance differences add additional variance.

Stereotypical exploration behavior during the task was not uncommon in healthy human subjects either, and differed drastically between subjects, further limiting the hypothetical interpretations that can be made comparing exploration behavior in neglect patients with healthy human subjects and inactivated monkeys.

Another hypothetical problem with analyzing differences in healthy human subjects and neglect patients became apparent: Even in healthy subjects, subjective difficulty differed substantially across subjects. While it is possible to adjust difficulty to the individual subject's performance, this approach does not work when we want to study expected performance differences in healthy subjects and patients.

However, we only had the opportunity to test one neglect patient with the "Search-to-Sample" task, and the results clearly suggest that the task needs to be modified in order to be applicable to patients, since over the course of several sessions he only completed a very limited amount of trials.

\subsubsection{General conclusions}

Pulvinar activity patterns during a memory guided saccade task were diverse, but still a few general conclusions can be drawn. First, motor related activity was more commonly found than visual response, hence supporting in principle the general idea of pulvinar involvement in motor processing. However, most pulvinar cells did not show classical visuomotor delay period or pre-saccadic activity, but instead a non-specific suppression during delay, which did not predict the upcoming movement in free-choice conditions. This might be construed as challenging the idea of a major role of pulvinar in target selection proposed by our microstimulation experiments, where stimulation of dorsal pulvinar consistently biases saccadic target selection in free choice trials. Importantly, stimulation effects on target selection were only present in a visually guided saccade task and not in a memory guided saccade task, indicating that pulvinar only contributes to target selection when fast decisions are required, or when the decisions are temporally coupled to onset of visual information. This would also explain the lack of encoding of the upcoming movement in memory guided conditions based on electrophysiological recordings and is consistent with the finding from the current work (in one monkey) that pulvinar inactivation caused a target selection bias towards the ipsilesional side only in the visually guided saccade task and not in the memory guided saccade task. However, these results do not agree with previous findings (Wilke et 
al., 2013). One behavioral difference between this study and our present work however is that monkeys already exhibited a bias towards contralesional targets in control conditions in the memory guided saccade task, while in our studies, target selection was fairly balanced in the memory guided task, suggesting that pulvinar disruption might in general cancel acquired preferences and subjective value attributed to the targets.

Delay period activity of dorsal pulvinar neurons was characterized mainly by non-spatial specific decreases as compared to the initial fixation period. This could indicate that pulvinar is informing fronto-parietal cortical areas about an upcoming movement, regardless of the direction, via decrease of inhibition (assuming inhibitory projections from pulvinar to cortex). This delay period activity could signal general alertness, or simply help cortex to suppress visual processing during the saccade itself to maintain stable perception. However, it could also indicate that these cells are similar to "fixation cells" found in the frontal eye fields (Izawa et al., 2009) and superior colliculus (Munoz and Wurtz, 1993), and the decrease observed during delay is merely due to the expected release of fixation. This interpretation could also explain the prolonged saccade latencies to both sides observed after pulvinar microstimulation in late periods (by potential recruitment of these fixation cells) and the fact that a majority of cells was modulated by the gaze direction.

Moreover, we show that a subset of pulvinar neurons exhibits properties similar to gain fields related to gaze position, suggesting that pulvinar encodes space not only in eye-centered, retinotopic reference frame, and might also be involved in sensorimotor transformations. This interpretation is further supported by the findings in a patient with bilateral pulvinar lesions who exhibited mainly postural deficits, specifically when reaching with the right hand (contralateral to the more pronounced lesion), accompanied by general slowing of movements and grasping deficits.

Although the effects of pulvinar inactivation in the search-to-sample task were small, they further support an involvement of pulvinar in motor-intentional processing. Although the electrophysiological characterization of cortical temporal parietal occipital area (TPO) revealed considerable similarities between dorsal pulvinar and TPO in visuomotor properties, effects of inactivation of area TPO were considerably different in the same monkey.

Given that these two regions are both anatomically and functionally connected, further insight in the separate participation in visuomotor behavior and the relevance of communication between these regions in processing visuomotor behavior could be gained by simultaneous recordings or by studying the effects of inactivating one on the neuronal activity in the other region. 
Taken together, the results of this thesis illustrate that the function of pulvinar cannot be explained by visuospatial or attentional processing alone, and indicate an involvement of pulvinar in target selection, motor processing and sensorimotor integration.

\subsubsection{Future directions and outlook}

It's in the nature of things that answers always motivate new questions. Here, we demonstrated different aspects of dorsal pulvinar involvement in purposeful oculomotor behavior.

\subsubsection{Different cell classification approaches}

Given the observed diversity of response patterns, the question if different response patterns are more frequently found in different cell types, or in populations with different connectivity, arises. One possibility is that since action potentials of excitatory cells typically show longer peak-to-through times then action potentials of inhibitory neurons, a putative cell classification based on spike shapes might elucidate different contributions of different cell types. Another cell classification could be based on coherency of their spiking activity with the local field potential recorded in the same site or in different brain regions, hence separating between cells that show specific patterns of intra- or inter-areal "functional connectivity" and cells that have a more localized function. For example, it has been demonstrated that only coherent spiking cells in PPC contribute to coordinating saccade and reaching responses (Dean et al., 2012). Both of these classification approaches could potentially further improve our understanding of the underlying thalamo-cortical neuronal organization.

\subsubsection{Simultaneous recording from cortex}

Since pulvinar is thought to coordinate information transmission between cortical areas, it is clear that we will not understand the integrative function of pulvinar in isolation. For example, simultaneous recordings in area TPO and pulvinar would help to interpret the different aspects of disrupted sensory-motor function observed after TPO and pulvinar lesion. In addition, simultaneous recordings in parietal areas and dorsal pulvinar would provide further insight in potential motor preparation functions of the dorsal pulvinar, and its postulated role in shaping cortical responses. Simultaneous recordings in different regions would also allow assess the inter-regional connectivity of individual cells based on spike-spike or spike-field correlations and evaluate if cells with different connectivity exhibit different firing patterns.

\subsubsection{Validation of Search-to-Sample task readouts}

Regarding the Search-to-Sample task, it needs to be seen if we can find a variant that is applicable to neglect patients. This data would be extremely valuable to determine if this task can be used in principle to read out clear spatial deficits and if those readouts agree with 
commonly used paper based tests for assessing hemi-spatial neglect. Only then can we be sure that our research on targeted inactivations and their effects on sensory-perceptual and motor-intentional aspects of spatial deficits are interpretable.

\subsubsection{Dissociating microstimulation effects}

We showed that pulvinar microstimulation had a bimodal effect on target selection dependent on the time of stimulation in free choice conditions, when both response options are equally rewarded. However, it is not clear if these time-specific effects reflect perceptual-attentional or motor-intentional processes, or even both but at different time points. To this end, one project in our lab investigated specifically how microstimulation at different time points and behavioral states affects target selection in a perceptual discrimination task, i.e. similar to the visually-guided task presented in our study, but with a correct (rewarded) target and a difficult or easy (not rewarded) distractor target instead of two correct targets. These experiments showed that pulvinar microstimulation biases choices even when only one response option is rewarded. The interaction of stimulation effects with the perceptual difficulty suggested that the microstimulation of the dorsal pulvinar contributes to response selection not via "automatic" motor engagement, but via modulation of developing decision signals (Gibson, Kaduk, Wilke and Kagan, in preparation). In line with previously discussed limitations of characterizing the complex cognitive brain structure with simple visuomotor tasks, electrophysiological investigation of neuronal encoding during such perceptual decision tasks, as well as the Search-to-Sample task introduced in chapter 3, will potentially lead to further insights into the functioning of pulvinar.

\subsubsection{Effector specificity}

This thesis focused on the saccadic eye movements as the motor response effector. Saccades are undoubtedly of primary importance for vision and action in humans and monkeys, but purposeful primate behavior is not limited to eye movements. As previous studies suggested, pulvinar might play a crucial role especially in reaching and grasping movements (Wilke et al., 2010, 2018). To further elucidate the role of pulvinar in volitional behavior, it would be interesting to see how neuronal activity in the dorsal pulvinar relates to arm and hand movements, and how it might mediate coordinated eye-hand actions.

Additionally, expanding the study to manual response contingencies could help to address the question if pulvinar is involved in visuomotor transformations. However, to address and further clarify the postural deficits observed in the patient, which were partially hand specific, and illuminate if this relates to a distortion of body- or hand-centered representations, experiments on monkeys with varying head and hand position relative to the screen would provide further insight. This would also allow investigating the putative neural basis of 
behavioral coupling between hand and eye movements, and potential modulation of visual and saccadic responses by the static hand position.

\subsubsection{Combining electrophysiological recordings and causal interference}

In this work, the microstimulation was used to causally perturb the activity in the target area. However, microstimulation can be used to identify the effective connectivity between the two recording sites, e.g. in pulvinar and in the cortex. So far, in our lab we utilized functional imaging in combination with electrical microstimulation to map out the effective connectivity patterns (Gibson, Wilke, Kagan in preparation). While being a powerful approach for the whole-brain assessment, this method does not allow using precise temporal patterns and low currents that would help to identify evoked and induced response patterns due to remote stimulation on the neuronal level. Utilizing microstimulation with simultaneous electrophysiological recordings might help elucidating connectivity patterns of specific neurons, hopefully leading to explanatory power concerning diverse response patterns in the pulvinar.

Finally, another ongoing work in our lab that was motivated by the question how pulvinar investigates the influence of pulvinar on posterior parietal cortex (PPC) activity using a combination of causal interference with recordings, i.e. inactivating pulvinar, evaluating behavioral changes and correlating them to activity changes in medial intraparietal area of the PPC during reaching behavior. Ideally, these experiments would include inhibition or excitation of a specific subset of pulvinar neurons which are directly projecting to posterior parietal cortex. This could be achieved by more advanced techniques employing optogenetics which has a become a popular and frequently applied technique for studying inter-areal or thalamo-cortical communication (Schmitt et al., 2017). However, optogenetical approaches in the monkey, especially cell type-specific targeting, are not yet fully established and will likely be a focus of the research in the next decade, due to a high promise for enabling systematic dissection of complex thalamo-cortical circuitry. 


\section{References}

Acuña, C., Gonzalez, F., and Dominguez, R. (1983). Sensorimotor unit activity related to intention in the Pulvinar of behaving Cebus Apella monkeys. Exp. Brain Res. 52.

Acuña, C., Cudeiro, J., Gonzalez, F., Alonso, J.M., and Perez, R. (1990). Lateral-posterior and pulvinar reaching cells- comparison with a parietal area 5a: a study in behaving Macaca nemestrina monkeys. Exp. Brain Res. 82, 158-166.

Almeida, I., Soares, S.C., and Castelo-Branco, M. (2015). The Distinct Role of the Amygdala, Superior Colliculus and Pulvinar in Processing of Central and Peripheral Snakes. PLOS ONE 10, e0129949.

Andersen, R.A., and Mountcastle, V.B. (1983). The Influence of the Angle of Gaze upon the Excitability of the light-sensitive Neurons of the Posterior Parietal Cortex. J. Neurosci. 3, 532-548.

Andersen, R.A., Essick, G.K., and Siegel, R.M. (1985). Encoding of spatial location by posterior parietal neurons. Science 230, 456-458.

Andersen, R.A., Bracewell, R.M., Barash, S., Gnadt, J.W., and Fogassi, L. (1990). Eye Position Effects on Visual, Memory, and Saccade-Related Activity in Areas LIP and 7a of Macaque. J Neurosci 10, 1176-1196.

Andersen, R.A., Snyder, L.H., Li, C.S., and Stricanne, B. (1993). Coordinate transformations in the representation of spatial information. Curr Opin Neurobiol 3, 171-176.

Arcaro, M.J., Pinsk, M.A., Chen, J., and Kastner, S. (2018). Organizing principles of pulvinocortical functional coupling in humans. Nat. Commun. 9, 5382.

Arend, I., Rafal, R., and Ward, R. (2008a). Spatial and temporal deficits are regionally dissociable in patients with pulvinar lesions. Brain 131, 2140-2152.

Arend, I., Machado, L., Ward, R., McGrath, M., Ro, T., and Rafal, R.D. (2008b). The role of the human pulvinar in visual attention and action: evidence from temporal-order judgment, saccade decision, and antisaccade tasks. Prog Brain Res 171, 475-483.

Bakker, R., Tiesinga, P., and Kötter, R. (2015). The Scalable Brain Atlas: Instant Web-Based Access to Public Brain Atlases and Related Content. Neuroinformatics 13, 353-366.

Baldwin, M.K.L., and Bourne, J.A. (2017). The Evolution of Subcortical Pathways to the Extrastriate Cortex. In Evolution of Nervous Systems, (Elsevier), pp. 165-185.

Baldwin, M.K.L., Balaram, P., and Kaas, J.H. (2017). The evolution and functions of nuclei of the visual pulvinar in primates. J. Comp. Neurol. 525, 3207-3226.

Barash, S., Bracewell, R.M., Fogassi, L., Gnadt, J.W., and Andersen, R.A. (1991a). Saccade-related activity in the lateral intraparietal area. I. Temporal properties; comparison with area 7a. J. Neurophysiol. 66, 1095-1108.

Barash, S., Bracewell, R.M., Fogassi, L., Gnadt, J.W., and Andersen, R.A. (1991b). Saccade-related activity in the lateral intraparietal area. II. Spatial properties. J Neurophysiol $66,1109-1124$.

Batista, A. (2002). Inner space: Reference frames. Curr. Biol. 12, 380-383. 
Batista, A.P., Santhanam, G., Yu, B.M., Ryu, S.I., Afshar, A., and Shenoy, K.V. (2007). Reference Frames for Reach Planning in Macaque Dorsal Premotor Cortex. J. Neurophysiol. 98, 966-983.

Battaglia-Mayer, A., Caminiti, R., Lacquaniti, F., and Zago, M. (2003). Multiple Levels of Representation of Reaching in the Parieto-frontal Network. Cereb. Cortex 13, 1009-1022.

Battelli, L., Walsh, V., Pascual-Leone, A., and Cavanagh, P. (2008). The 'when' parietal pathway explored by lesion studies. Curr. Opin. Neurobiol. 18, 120-126.

Becker, E., and Karnath, H.O. (2007). Incidence of Visual Extinction After Left Versus Right Hemisphere Stroke. Stroke 38, 3172-3174.

Behrmann, M., Ebert, P., and Black, S.E. (2004). Hemispatial neglect and visual search: a large scale analysis. Cortex 40, 247-263.

Bellebaum, C., Daum, I., Koch, B., Schwarz, M., and Hoffmann, K.-P. (2005). The role of the human thalamus in processing corollary discharge. Brain 128, 1139-1154.

Bender, D.B. (1983). Visual activation of neurons in the primate pulvinar depends on cortex but not colliculus. Brain Res 279, 258-261.

Bender, D.B., and Baizer, J.S. (1990). Saccadic eye movements following kainic acid lesions of the pulvinar in monkeys. Exp Brain Res 79, 467-478.

Bender, D.B., and Butter, C.M. (1987). Comparison of the effects of superior colliculus and pulvinar lesions on visual search and tachistoscopic pattern discrimination in monkeys. Exp Brain Res 69, 140-154.

Benevento, L.A., and Miller, J. (1981). Of Single Neurons in the Caudal of the Macaque Monkey. J. Neurosci. 1, 1268-1278.

Benevento, L.A., and Port, J.D. (1995). Single neurons with both form/color differential responses and saccade-related responses in the nonretinotopic pulvinar of the behaving macaque monkey. Vis. Neurosci. 12, 523-544.

Benevento, L.A., and Standage, G.P. (1983). The organization of projections of the retinorecipient and nonretinorecipient nuclei of the pretectal complex and layers of the superior colliculus to the lateral pulvinar and medial pulvinar in the macaque monkey. $\mathrm{J}$. Comp. Neurol. 217, 307-336.

Benjamini, Y., and Hochberg, Y. (1995). Controlling the False Discovery Rate: A Practical and Powerful Approach to Multiple Testing. J. R. Stat. Soc. Ser. B Methodol. 57, 289-300.

Berman, R.A., and Wurtz, R.H. (2011). Signals Conveyed in the Pulvinar Pathway from Superior Colliculus to Cortical Area MT. J. Neurosci. 31, 373-384.

Bisley, J.W., and Goldberg, M.E. (2010). Attention, intention, and priority in the parietal lobe. Annu Rev Neurosci 33, 1-21.

Boi, M., Poletti, M., Victor, J.D., and Rucci, M. (2017). Consequences of the oculomotor cycle for the dynamics of perception. Curr. Biol.

Bos, J., and Benevento, L.A. (1975). Projections of the medial pulvinar to orbital cortex and frontal eye fields in the rhesus monkey (Macaca mulatta). Exp. Neurol. 49, 487-496. 
Bosco, A., Breveglieri, R., Reser, D., Galletti, C., and Fattori, P. (2015). Multiple Representation of Reaching Space in the Medial Posterior Parietal Area V6A. Cereb. Cortex 25, 1654-1667.

Boussaoud, D., Jouffrais, C., and Bremmer, F. (1998). Eye position effects on the neuronal activity of dorsal premotor cortex in the macaque monkey. J. Neurophysiol. 80, 1132-1150.

Bowen, A., McKenna, K., and Tallis, R.C. (1999). Reasons for variability in the reported rate of occurrence of unilateral spatial neglect after stroke. Stroke 30, 1196-1202.

Brainard, D.H. (1997). The Psychophysics Toolbox. Spat. Vis. 10, 433-436.

Bremmer, F. (2000). Eye position effects in macaque area V4. Neuroreport 11, 1277-1283.

Bremmer, Pouget, and Hoffmann, K. -P. (1998). Eye position encoding in the macaque posterior parietal cortex. Eur. J. Neurosci. 10, 153-160.

Bremmer, F., Distler, C., and Hoffmann, K.P. (1997). Eye position effects in monkey cortex. II. Pursuit- and fixation-related activity in posterior parietal areas LIP and 7A. J. Neurophysiol. 77, 962-977.

Bremmer, F., Graf, W., Ben Hamed, S., and Duhamel, J.R. (1999). Eye position encoding in the macaque ventral intraparietal area (VIP). Neuroreport 10, 873-878.

Bremmer, F., Duhamel, J.R., Ben Hamed, S., and Graf, W. (2002). Heading encoding in the macaque ventral intraparietal area (VIP). Eur J Neurosci 16, 1554-1568.

Bridge, H., Leopold, D.A., and Bourne, J.A. (2015). Adaptive Pulvinar Circuitry Supports Visual Cognition. Trends Cogn. Sci.

Brotchie, P.R., Andersen, R.A., Snyder, L.H., and Goodman, S.J. (1995). Head position signals used by parietal neurons to encode locations of visual stimuli. Nature 375, 232-235.

Bruce, C.J., and Goldberg, M.E. (1985). Primate frontal eye fields. I. Single neurons discharging before saccades. J Neurophysiol 53, 603-635.

Bruce, C., Desimone, R., and Gross, C.G. (1981). Visual properties of neurons in a polysensory area in superior temporal sulcus of the macaque. J. Neurophysiol. 46, 369-384.

Buckner, R.L., and Krienen, F.M. (2013). The evolution of distributed association networks in the human brain. Trends Cogn. Sci. 17, 648-665.

Campos, M., Cherian, A., and Segraves, M.A. (2006). Effects of eye position upon activity of neurons in macaque superior colliculus. J. Neurophysiol. 95, 505-526.

Cappe, C., Morel, A., and Rouiller, E.M. (2007). Thalamocortical and the dual pattern of corticothalamic projections of the posterior parietal cortex in macaque monkeys. Neuroscience 146, 1371-1387.

Cappe, C., Morel, A., Barone, P., and Rouiller, E.M. (2009). The thalamocortical projection systems in primate: an anatomical support for multisensory and sensorimotor interplay. Cereb. Cortex 19, 2025-2037.

Cappe, C., Rouiller, E.M., and Barone, P. (2012). Cortical and Thalamic Pathways for Multisensory and Sensorimotor Interplay. In The Neural Bases of Multisensory Processes, M.M. Murray, and M.T. Wallace, eds. (Boca Raton (FL): CRC Press/Taylor \& Francis), p. 
Caruso, V.C., Pages, D.S., Sommer, M.A., and Groh, J.M. (2018). Beyond the labeled line: variation in visual reference frames from intraparietal cortex to frontal eye fields and the superior colliculus. J. Neurophysiol. 119, 1411-1421.

Casanova, C., and Molotchnikoff, S. (1990). Influence of the superior colliculus on visual responses of cells in the rabbit's lateral posterior nucleus. Exp. Brain Res. 80, 387-396.

Casanova, C., Nordmann, J.P., and Molotchnikoff, S. (1991). [Pulvina-lateralis posterior nucleus complex of mammals and the visual function]. J. Physiol. (Paris) 85, 44-57.

Chang, S.W.C., and Snyder, L.H. (2010). Idiosyncratic and systematic aspects of spatial representations in the macaque parietal cortex. Proc. Natl. Acad. Sci. 107, 7951-7956.

Churchland, M.M., and Shenoy, K.V. (2007). Delay of Movement Caused by Disruption of Cortical Preparatory Activity. J. Neurophysiol. 97, 348-359.

Coe, B., Tomihara, K., Matsuzawa, M., and Hikosaka, O. (2002). Visual and anticipatory bias in three cortical eye fields of the monkey during an adaptive decision-making task. J Neurosci 22, 5081-5090.

Cohen, Y.E., and Andersen, R.A. (2002). A common reference frame for movement plans in the posterior parietal cortex. Nat Rev Neurosci 3, 553-562.

Colby, C.L. (1998). Action-oriented spatial reference frames in cortex. Neuron 20, 15-24.

Committeri, G., Pitzalis, S., Galati, G., Patria, F., Pelle, G., Sabatini, U., CastriotaScanderbeg, A., Piccardi, L., Guariglia, C., and Pizzamiglio, L. (2007). Neural bases of personal and extrapersonal neglect in humans. Brain 130, 431-441.

Corbetta, M., and Shulman, G.L. (2011). Spatial Neglect and Attention Networks. Annu. Rev. Neurosci. 34, 569-599.

Corbetta, M., Kincade, M.J., Lewis, C., Snyder, A.Z., and Sapir, A. (2005). Neural basis and recovery of spatial attention deficits in spatial neglect. Nat. Neurosci. 8, 1603-1610.

Costello, M.G., Zhu, D., May, P.J., Salinas, E., and Stanford, T.R. (2015). Task dependence of decision- and choice-related activity in monkey oculomotor thalamus. J. Neurophysiol. $115,581-601$.

Crapse, T.B., and Sommer, M.A. (2008). Corollary discharge circuits in the primate brain. Curr. Opin. Neurobiol. 18, 552-557.

Cudeiro, J., González, F., Pérez, R., Alonso, J.M., and Acuna, C. (1989). Does the pulvinarLP complex contribute to motor programming? Brain Res. 484, 367-370.

Culham, J.C., and Kanwisher, N.G. (2001). Neuroimaging of cognitive functions in human parietal cortex. Curr. Opin. Neurobiol. 11, 157-163.

Cutrell, E.B., and Marrocco, R.T. (2002). Electrical microstimulation of primate posterior parietal cortex initiates orienting and alerting components of covert attention. Exp. Brain Res. $144,103-113$.

Dahl (2010). Modulation of visual responses in the superior temporal sulcus by audio-visual congruency. Front. Integr. Neurosci.

Dean, H.L., and Platt, M.L. (2006). Allocentric spatial referencing of neuronal activity in macaque posterior cingulate cortex. J. Neurosci. Off. J. Soc. Neurosci. 26, 1117-1127. 
Dean, H.L., Crowley, J.C., and Platt, M.L. (2004). Visual and saccade-related activity in macaque posterior cingulate cortex. J Neurophysiol 92, 3056-3068.

Dean, H.L., Hagan, M.A., and Pesaran, B. (2012). Only Coherent Spiking in Posterior Parietal Cortex Coordinates Looking and Reaching. Neuron 73, 829-841.

Desimone, R., and Gross, C.G. (1979). Visual areas in the temporal cortex of the macaque. Brain Res. 178, 363-380.

Ding, L., and Gold, J.I. (2012). Separate, Causal Roles of the Caudate in Saccadic Choice and Execution in a Perceptual Decision Task. Neuron 75, 865-874.

Domínguez-Vargas, A.U. (2017). The role of thalamic pulvinar in eye-hand coordination for goal-directed actions.

Dominguez-Vargas, A.-U., Schneider, L., Wilke, M., and Kagan, I. (2017). Electrical Microstimulation of the Pulvinar Biases Saccade Choices and Reaction Times in a TimeDependent Manner. J. Neurosci. 37, 2234-2257.

Driver, J., and Halligan, P.W. (1991). Can Visual Neglect Operate in Object-centred Coordinates? An Affirmative Single-case Study. Cogn. Neuropsychol. 8, 475-496.

Duhamel, J.R., Colby, C.L., and Goldberg, M.E. (1992). The updating of the representation of visual space in parietal cortex by intended eye movements. Science $255,90-92$.

Durand, J.-B., Trotter, Y., and Celebrini, S. (2010). Privileged Processing of the StraightAhead Direction in Primate Area V1. Neuron 66, 126-137.

Ellison, A. (2004). An exploration of the role of the superior temporal gyrus in visual search and spatial perception using TMS. Brain 127, 2307-2315.

Fiebelkorn, I.C., Pinsk, M.A., and Kastner, S. (2019). The mediodorsal pulvinar coordinates the macaque fronto-parietal network during rhythmic spatial attention. Nat. Commun. 10, 215.

Funahashi, S., Bruce, C.J., and Goldman-Rakic, P.S. (1991). Neuronal activity related to saccadic eye movements in the monkey's dorsolateral prefrontal cortex. J Neurophysiol 65, 1464-1483.

Gainotti, G., Messerli, P., and Tissot, R. (1972). Qualitative analysis of unilateral spatial neglect in relation to laterality of cerebral lesions. J. Neurol. Neurosurg. Psychiatry $35,545-$ 550.

Galletti, C., Battaglini, P.P., and Fattori, P. (1995). Eye Position Influence on the Parietooccipital Area PO (V6) of the Macaque Monkey. Eur. J. Neurosci. 7, 2486-2501.

Genovesio, A., Brunamonti, E., Giusti, M.A., and Ferraina, S. (2007). Postsaccadic Activities in the Posterior Parietal Cortex of Primates Are Influenced by both Eye Movement Vectors and Eye Position. J. Neurosci. 27, 3268-3273.

Gnadt, J.W., and Andersen, R.A. (1988). Memory related motor planning activity in posterior parietal cortex of macaque. Exp. Brain Res. 70, 216-220.

Goldberg, M.E. (2000). The Control of Gaze. In Principles of Neural Science, (McGraw-Hill Medical), pp. 783-800.

Goodale, M.A. (2011). Transforming vision into action. Vision Res. 51, 1567-1587. 
Goodale, M.A., and Milner, A.D. (1992). Separate visual pathways for perception and action. Trends Neurosci. 15, 20-25.

Grieve, K.L., Acuña, C., and Cudeiro, J. (2000). The primate pulvinar nuclei: vision and action. Trends Neurosci. 23, 35-39.

Guillery, R.W. (2003). Branching Thalamic Afferents Link Action and Perception. J. Neurophysiol. 90, 539-548.

Guillery, R.W., and Sherman, S.M. (2002). The thalamus as a monitor of motor outputs. Philos. Trans. R. Soc. B Biol. Sci. 357, 1809-1821.

Guthrie, B.L., Porter, J.D., and Sparks, D.L. (1983). Corollary discharge provides accurate eye position information to the oculomotor system. Science 221, 1193-1195.

Gutierrez, C., Yaun, A., and Cusick, C.G. (1995). Neurochemical subdivisions of the inferior pulvinar in macaque monkeys. J. Comp. Neurol. 363, 545-562.

Gutierrez, C., Cola, M.G., Seltzer, B., and Cusick, C. (2000). Neurochemical and connectional organization of the dorsal pulvinar complex in monkeys. J. Comp. Neurol. 419, 61-86.

de Haan, E.H.F., and Cowey, A. (2011). On the usefulness of 'what' and 'where' pathways in vision. Trends Cogn. Sci. 15, 460-466.

Halassa, M.M., and Kastner, S. (2017). Thalamic functions in distributed cognitive control. Nat. Neurosci. 20, 1669-1679.

Hardy, S.G.P., and Lynch, J.C. (1992). The Spatial Distribution of Pulvinar Neurons That Project to Two Subregions of the Inferior Parietal Lobule in the Macaque. Cereb. Cortex 2, 217-230.

Harting, J.K., Huerta, M.F., Frankfurter, A.J., Strominger, N.L., and Royce, G.J. (1980). Ascending pathways from the monkey superior colliculus: An autoradiographic analysis. J. Comp. Neurol. 192, 853-882.

Hayden, B.Y., Nair, A.C., McCoy, A.N., and Platt, M.L. (2008). Posterior Cingulate Cortex Mediates Outcome-Contingent Allocation of Behavior. Neuron 60, 19-25.

Hein, G., and Knight, R.T. (2008). Superior Temporal Sulcus—It's My Area: Or Is It? J. Cogn. Neurosci. 20, 2125-2136.

Hernández, R., Calvo, P.M., Blumer, R., de la Cruz, R.R., and Pastor, A.M. (2019). Functional diversity of motoneurons in the oculomotor system. Proc. Natl. Acad. Sci. U. S. A. 116, 3837-3846.

Hikosaka, O., and Wurtz, R.H. (1983). Visual and oculomotor functions of monkey substantia nigra pars reticulata. III. Memory-contingent visual and saccade responses. J. Neurophysiol. $49,1268-1284$.

Hoffman, K.L. (2008). -specific responses to faces and objects in primate auditory cortex. Front. Syst. Neurosci. 1.

Hoffman, J.E., and Subramaniam, B. (1995). The role of visual attention in saccadic eye movements. Percept. Psychophys. 57, 787-795. 
Hornak, J. (1992). Ocular exploration in the dark by patients with visual neglect. Neuropsychologia 30, 547-552.

Husain, M. (2000). Distinguishing sensory and motor biases in parietal and frontal neglect. Brain 123, 1643-1659.

Husain, M. (2001). Impaired spatial working memory across saccades contributes to abnormal search in parietal neglect. Brain 124, 941-952.

Hwang, E.J., and Andersen, R.A. (2012). Spiking and LFP activity in PRR during symbolically instructed reaches. J. Neurophysiol. 107, 836-849.

Itoh, K., and Mizuno, N. (1979). A cerebello-pulvinar projection in the cat as visualized by the use of anterograde transport of horseradish peroxidase. Brain Res. 171, 131-134.

Izawa, Y., Suzuki, H., and Shinoda, Y. (2004). Suppression of Visually and Memory-Guided Saccades Induced by Electrical Stimulation of the Monkey Frontal Eye Field. II. Suppression of Bilateral Saccades. J. Neurophysiol. 92, 2261-2273.

Izawa, Y., Suzuki, H., and Shinoda, Y. (2009). Response Properties of Fixation Neurons and Their Location in the Frontal Eye Field in the Monkey. J. Neurophysiol. 102, 2410-2422.

Jackson, W.J., Elrod, K., and Bucafusco, J.J. (1989). Delayed Matching-to-Sample in Monkeys as a Model for Learning and Memory Deficits: Role of Brain Nicotinic Receptors. In Novel Approaches to the Treatment of Alzheimer's Disease, E.M. Meyer, J.W. Simpkins, and J. Yamamoto, eds. (Springer, Boston, MA), p.

Jones, E.G. (2007). The lateral posterior and pulvinar nuclei. In The Thalamus, (Cambridge: Cambridge University Press), pp. 1009-1071.

Kaas, J.H., and Lyon, D.C. (2007). Pulvinar contributions to the dorsal and ventral streams of visual processing in primates. Brain Res. Rev. 55, 285-296.

Kagan, I., Iyer, A., Lindner, A., and Andersen, R.A. (2010). Space representation for eye movements is more contralateral in monkeys than in humans. Proc. Natl. Acad. Sci. 107, 7933-7938.

Karnath, H.-O. (2001). New insights into the functions of the superior temporal cortex. Nat. Rev. Neurosci. 2, 568-576.

Karnath, H.-O. (2015). Spatial attention systems in spatial neglect. Neuropsychologia 75, 6173.

Karnath, H.-O., and Fetter, M. (1995). Ocular space exploration in the dark and its relation to subjective and objective body orientation in neglect patients with parietal lesions. Neuropsychologia 33, 371-377.

Karnath, H.-O., and Rorden, C. (2012). The anatomy of spatial neglect. Neuropsychologia 50, 1010-1017.

Karnath, H.O., Schenkel, P., and Fischer, B. (1991). Trunk Orientation as the Determining Factor of the 'Contralateral' Deficit in the Neglect Syndrome and as the Physical Anchor of the Internal Representation of Body Orientation in Space. Brain 114, 1997-2014.

Karnath, H.-O., Ferber, S., and Himmelbach, M. (2001). Spatial awareness is a function of the temporal not the posterior parietal lobe. Nature 411, 950-953. 
Karnath, H.O., Himmelbach, M., and Rorden, C. (2002). The subcortical anatomy of human spatial neglect: putamen, caudate nucleus and pulvinar. Brain 125, 350-360.

Karnath, H.O., Himmelbach, M., and Küker, W. (2003). The Cortical Substrate of Visual Extinction. Neuroreport 14, 437-442.

Karnath, H.O., Mandler, A., and Clavagnier, S. (2011). Object-based Neglect Varies with Egocentric Position. J. Cogn. Neurosci. 23, 2983-2993.

Kilintari, M., Raos, V., and Savaki, H.E. (2014). Involvement of the Superior Temporal Cortex in Action Execution and Action Observation. J. Neurosci. 34, 8999-9011.

Kinoshita, M., Kato, R., Isa, K., Kobayashi, K., Kobayashi, K., Onoe, H., and Isa, T. (2019). Dissecting the circuit for blindsight to reveal the critical role of pulvinar and superior colliculus. Nat. Commun. 10, 135.

Kinsbourne, M. (1987). Mechanisms of Unilateral Neglect. In Neurophysiological and Neuropsychological Aspects of Spatial Neglect, (Elsevier Science Publishers B.V.), pp. 6986.

Knudsen, E., and Konishi, M. (1978). A neural map of auditory space in the owl. Science 200, 795-797.

Kobak, D., Brendel, W., Constantinidis, C., Feierstein, C.E., Kepecs, A., Mainen, Z.F., Qi, X.L., Romo, R., Uchida, N., and Machens, C.K. (2016). Demixed principal component analysis of neural population data. ELife 5 .

Komura, Y., Nikkuni, A., Hirashima, N., Uetake, T., and Miyamoto, A. (2013). Responses of pulvinar neurons reflect a subject's confidence in visual categorization. Nat. Neurosci. 16, 749-755.

Kraskov, A., Dancause, N., Quallo, M.M., Shepherd, S., and Lemon, R.N. (2009). Corticospinal Neurons in Macaque Ventral Premotor Cortex with Mirror Properties: A Potential Mechanism for Action Suppression? Neuron 64, 922-930.

Krauzlis, R.J. (2004). Recasting the Smooth Pursuit Eye Movement System. J. Neurophysiol. 91, 591-603.

Krauzlis, R.J., Goffart, L., and Hafed, Z.M. (2017). Neuronal control of fixation and fixational eye movements. Philos. Trans. R. Soc. Lond. B. Biol. Sci. 372.

Lal, R., and Friedlander, M.J. (1989). Gating of retinal transmission by afferent eye position and movement signals. Science 243, 93-96.

Latimer, K.W., Yates, J.L., Meister, M.L.R., Huk, A.C., and Pillow, J.W. (2015). Single-trial spike trains in parietal cortex reveal discrete steps during decision-making. Science 349, 184-187.

Lawrence, B.M., White, R.L., and Snyder, L.H. (2005). Delay-Period Activity in Visual, Visuomovement, and Movement Neurons in the Frontal Eye Field. J. Neurophysiol. 94, 1498-1508.

Lehky, S.R., Sereno, M.E., and Sereno, A.B. (2016). Characteristics of Eye-Position Gain Field Populations Determine Geometry of Visual Space. Front. Integr. Neurosci. 9. 
Leibovitch, F.S., Black, S.E., Caldwell, C.B., Mclntosh, A.R., Ehrlich and, L.E., and Szalai, J.P. (1999). Brain SPECT imaging and left hemispatial neglect covaried using partial least squares: The sunnybrook stroke study. Hum. Brain Mapp. 7, 244-253.

Liu, G.T., Bolton, A.K., Price, B.H., and Weintraub, S. (1992). Dissociated perceptualsensory and exploratory-motor neglect. J. Neurol. Neurosurg. Psychiatry 55, 701-706.

Luh, K.E., Butter, C.M., and Buchtel, H.A. (1986). Impairments in orienting to visual stimuli in monkeys following unilateral lesions of the superior sulcal polysensory cortex. Neuropsychologia 24, 461-470.

Luschei, E.S., and Fuchs, A.F. (1972). Activity of brain stem neurons during eye movements of alert monkeys. J. Neurophysiol. 35, 445-461.

Maleki, N., Becerra, L., Upadhyay, J., Burstein, R., and Borsook, D. (2012). Direct optic nerve pulvinar connections defined by diffusion MR tractography in humans: Implications for photophobia. Hum. Brain Mapp. 33, 75-88.

Malhotra, P. (2004). Spatial working memory capacity in unilateral neglect. Brain 128, 424435.

Malhotra, P.A., Soto, D., Li, K., and Russell, C. (2013). Reward modulates spatial neglect. J. Neurol. Neurosurg. Psychiatry 84, 366-369.

Marzocchi, N., Breveglieri, R., Galletti, C., and Fattori, P. (2008). Reaching activity in parietal area $\mathrm{V} 6 \mathrm{~A}$ of macaque: eye influence on arm activity or retinocentric coding of reaching movements? Eur. J. Neurosci. 27, 775-789.

Mathers, L.H., and Rapisardi, S.C. (1973). Visual and somatosensory receptive fields of neurons in the squirrel monkey pulvinar. Brain Res. 64, 65-83.

Mays, L.E., and Sparks, D.L. (1980). Dissociation of visual and saccade-related responses in superior colliculus neurons. J. Neurophysiol. 43, 207-232.

McGuire, L.M.M., and Sabes, P.N. (2011). Heterogeneous Representations in the Superior Parietal Lobule Are Common across Reaches to Visual and Proprioceptive Targets. J. Neurosci. 31, 6661-6673.

Mesulam, M.M. (1981). A cortical network for directed attention and unilateral neglect. Ann. Neurol. 10, 309-325.

Michaels, J.A., and Scherberger, H. (2018). Population coding of grasp and laterality-related information in the macaque fronto-parietal network. Sci. Rep. 8.

Michaels, J.A., Dann, B., Intveld, R.W., and Scherberger, H. (2015). Predicting Reaction Time from the Neural State Space of the Premotor and Parietal Grasping Network. J. Neurosci. 35, 11415-11432.

Milner, A.D., and McIntosh, R.D. (2005). The neurological basis of visual neglect: Curr. Opin. Neurol. 18, 748-753.

Mirpour, K., and Bisley, J.W. (2015). Remapping, Spatial Stability, and Temporal Continuity: From the Pre-Saccadic to Postsaccadic Representation of Visual Space in LIP. Cereb. Cortex bhv153. 
Mishkin, M., and Ungerleider, L.G. (1982). Contribution of striate inputs to the visuospatial functions of parieto-preoccipital cortex in monkeys. Behav. Brain Res. 6, 57-77.

Morris, A.P., Kubischik, M., Hoffmann, K.-P., Krekelberg, B., and Bremmer, F. (2012). Dynamics of Eye-Position Signals in the Dorsal Visual System. Curr. Biol. 22, 173-179.

Morris, A.P., Bremmer, F., and Krekelberg, B. (2013). Eye-position signals in the dorsal visual system are accurate and precise on short timescales. J. Neurosci. Off. J. Soc. Neurosci. 33, 12395-12406.

Mort, D.J., Malhotra, P., Mannan, S.K., Rorden, C., Pambakian, A., Kennard, C., and Husain, M. (2003). The anatomy of visual neglect. Brain 126, 1986-1997.

Mufson, E.J., and Mesulam, M.M. (1984). Thalamic connections of the insula in the rhesus monkey and comments on the paralimbic connectivity of the medial pulvinar nucleus. $\mathrm{J}$. Comp. Neurol. 227, 109-120.

Mullette-Gillman, O.A., Cohen, Y.E., and Groh, J.M. (2005). Eye-centered, head-centered, and complex coding of visual and auditory targets in the intraparietal sulcus. J Neurophysiol $94,2331-2352$.

Mullette-Gillman, O.A., Cohen, Y.E., and Groh, J.M. (2009). Motor-Related Signals in the Intraparietal Cortex Encode Locations in a Hybrid, rather than Eye-Centered Reference Frame. Cereb. Cortex 19, 1761-1775.

Munoz, D.P., and Wurtz, R.H. (1993). Fixation cells in monkey superior colliculus. I. Characteristics of cell discharge. J. Neurophysiol. 70, 559-575.

Na, D.L., Adair, J.C., Williamson, D.J.G., Schwartz, R.L., Haws, B., and Heilman, K.M. (1998). Dissociation of sensory-attentional from motor-intentional neglect. J. Neurol. Neurosurg. Psychiatry 64, 331-338.

Noda, H., and Warabi, T. (1982). Eye position signals in the flocculus of the monkey during smooth-pursuit eye movements. J. Physiol. 324, 187-202.

Ogren, M.P., and Rakić, P. (1981). The Prenatal Development of the Pulvinar in The Monkey: $3 \mathrm{H}$-thymidine Audiographic and Morphometric Analyses. Anat. Embryol. (Berl.) 120.

Ohayon, S., and Tsao, D.Y. (2012). MR-guided stereotactic navigation. J. Neurosci. Methods 204, 389-397.

Ohtsuka, K., Igarashi, Y., Maekawa, H., and Nakagawa, T. (1991). Pursuit deficits in bilateral pulvinar lesions. Ophthalmol. J. Int. Ophtalmol. Int. J. Ophthalmol. Z. Augenheilkd. 203, 196202.

Olszewski, J. (1952). The Thalamus of Macaca mulatta (Karger).

Oostenveld, R., Fries, P., Maris, E., and Schoffelen, J.-M. (2011). FieldTrip: Open Source Software for Advanced Analysis of MEG, EEG, and Invasive Electrophysiological Data. Comput. Intell. Neurosci. 2011, 1-9.

Oram, M.W., Perrett, D.I., and Hietanen, J.K. (1993). Directional tuning of motion-sensitive cells in the anterior superior temporal polysensory area of the macaque. Exp. Brain Res. 97. 
Orban, G.A. (2016). Functional definitions of parietal areas in human and non-human primates. Proc. R. Soc. B Biol. Sci. 283, 20160118.

Padberg, J., Cerkevich, C., Engle, J., Rajan, A.T., Recanzone, G., Kaas, J., and Krubitzer, L. (2009). Thalamocortical Connections of Parietal Somatosensory Cortical Fields in Macaque Monkeys are Highly Divergent and Convergent. Cereb. Cortex 19, 2038-2064.

Patel, V.R., and Zee, D.S. (2015). The cerebellum in eye movement control: nystagmus, coordinate frames and disconjugacy. Eye 29, 191-195.

Peck, C.K., Schlag-Rey, M., and Schlag, J. (1980). Visuo-oculomotor properties of cells in the superior colliculus of the alert cat. J. Comp. Neurol. 194, 97-116.

Pedersen, P.M., Jorgensen, H.S., Nakayama, H., Raaschou, H., and Olsen, T.S. (1997). Hemineglect in acute stroke - incidence and prognostic implications: The Copenhagen Stroke Study. Am. J. Phys. Med. Rehabil. 76, 122-127.

Petersen, S.E., Robinson, D.L., and Keys, W. (1985a). Pulvinar nuclei of the behaving rhesus monkey: visual responses and their modulation. J. Neurophysiol. 54, 867-886.

Petersen, S.E., Robinson, D.L., and Keys, W. (1985b). Pulvinar nuclei of the behaving rhesus monkey: visual responses and their modulation. J. Neurophysiol. 54, 867-886.

Petersen, S.E., Robinson, D.L., and Morris, J.D. (1987). Contributions of the pulvinar to visual spatial attention. Neuropsychologia 25, 97-105.

Pouget, A., and Driver, J. (2000). Relating unilateral neglect to the neural coding of space. Curr. Opin. Neurobiol. 10, 242-249.

Pouget, A., and Sejnowski, T.J. (1997). A new view of hemineglect based on the response properties of parietal neurones. Philos. Trans. R. Soc. B Biol. Sci. 352, 1449-1459.

Pouget, A., and Snyder, L.H. (2000). Computational approaches to sensorimotor transformations. Nat. Neurosci. 3, 1192-1198.

Poulet, J.F.A., and Hedwig, B. (2007). New insights into corollary discharges mediated by identified neural pathways. Trends Neurosci. 30, 14-21.

Premereur, E., Vanduffel, W., and Janssen, P. (2011). Functional Heterogeneity of Macaque Lateral Intraparietal Neurons. J. Neurosci. 31, 12307-12317.

Przybyszewski, A.W., Kagan, I., and Snodderly, D.M. (2014). Primate area V1: largest response gain for receptive fields in the straight-ahead direction. NeuroReport 25, 11091115.

Purushothaman, G., Marion, R., Li, K., and Casagrande, V.A. (2012). Gating and control of primary visual cortex by pulvinar. Nat. Neurosci. 15, 905-912.

Quiroga, R.Q., Nadasdy, Z., and Ben-Shaul, Y. (2004). Unsupervised Spike Detection and Sorting with Wavelets and Superparamagnetic Clustering. Neural Comput. 16, 1661-1687.

Rakić, P., and Sidman, R.L. (1969). Telencephalic origin of pulvinar neurons in the fetal human brain. Z. Für Anat. Entwicklungsgeschichte 129, 53-82.

Rengachary, J., He, B.J., Shulman, G.L., and Corbetta, M. (2011). A Behavioral Analysis of Spatial Neglect and its Recovery After Stroke. Front. Hum. Neurosci. 5. 
Robinson, D.L. (1993). Functional contributions of the primate pulvinar. Prog Brain Res 95, 371-380.

Robinson, D.L., and Petersen, S.E. (1992). The pulvinar and visual salience. Trends Neurosci 15, 127-132.

Robinson, D.L., Petersen, S.E., and Keys, W. (1986). Saccade-related and visual activities in the pulvinar nuclei of the behaving rhesus monkey. Exp. Brain Res. 62.

Robinson, D.L., McClurkin, J.W., and Kertzman, C. (1990). Orbital position and eye movement influences on visual responses in the pulvinar nuclei of the behaving macaque. Exp. Brain Res. 82.

Rodrigo-Angulo, M.L., and Reinoso-Suarez, F. (1984). Cerebellar projections to the lateral posterior-pulvinar thalamic complex in the cat. Brain Res. 322, 172-176.

Rodriguez, J., and Paule, M. (2009). Working Memory Delayed Response Tasks in Monkeys. In Methods of Behavior Analysis in Neuroscience, J. Buccafusco, ed. (CRC Press/Taylor \& Francis), $p$.

Rohlfing, T., Kroenke, C.D., Sullivan, E.V., Dubach, M.F., Bowden, D.M., Grant, K., and Pfefferbaum, A. (2012). The INIA19 template and NeuroMaps atlas for primate brain image parcellation and spatial normalization. Front. Neuroinformatics 6, 27.

Rokszin, A., Márkus, Z., Braunitzer, G., Berényi, A., Benedek, G., and Nagy, A. (2010). Visual Pathways Serving Motion Detection in the Mammalian Brain. Sensors 10, 3218-3242.

Romanski, L.M., Giguere, M., Bates, J.F., and Goldman-Rakic, P.S. (1997). Topographic organization of medial pulvinar connections with the prefrontal cortex in the rhesus monkey. J. Comp. Neurol. 379, 313-332.

Rorie, A.E., Gao, J., McClelland, J.L., and Newsome, W.T. (2010). Integration of Sensory and Reward Information during Perceptual Decision-Making in Lateral Intraparietal Cortex (LIP) of the Macaque Monkey. PLoS ONE 5, e9308.

Rosenbluth, D., and Allman, J.M. (2002). The effect of gaze angle and fixation distance on the responses of neurons in V1, V2, and V4. Neuron 33, 143-149.

Saalmann, Y.B., and Kastner, S. (2011). Cognitive and perceptual functions of the visual thalamus. Neuron 71, 209-223.

Saalmann, Y.B., and Kastner, S. (2015). The cognitive thalamus. Front. Syst. Neurosci. 9.

Saalmann, Y.B., Pinsk, M.A., Wang, L., Li, X., and Kastner, S. (2012). The Pulvinar Regulates Information Transmission Between Cortical Areas Based on Attention Demands. Science 337, 753-756.

Sajad, A., Sadeh, M., Yan, X., Wang, H., and Crawford, J.D. (2016). Transition from Target to Gaze Coding in Primate Frontal Eye Field during Memory Delay and Memory-Motor Transformation. ENeuro 3, ENEURO.0040-16.2016.

Sakata, H., Shibutani, H., and Kawano, K. (1980). Spatial properties of visual fixation neurons in posterior parietal association cortex of the monkey. J. Neurophysiol. 43, 16541672. 
Salinas, E., and Abbott, L.F. (1997). Invariant Visual Responses From Attentional Gain Fields. J. Neurophysiol. 77, 3267-3272.

Salinas, E., and Abbott, L.F. (2001). Coordinate Transformation in the Visual System: How to Generate Gain Fields and What to Compute with them. In Advances in Neural Population Coding, (Elsevier), pp. 175-190.

Scalaidhe, S.P.Ó., Albright, T.D., Rodman, H.R., and Gross, C.G. (1995). Effects of Superior Temporal Polysensory Area Lesions on Eye Movements in the Macaque Monkey. J. Neurophysiol. 73, 1-19.

Schall, J.D. (2004). On the role of frontal eye field in guiding attention and saccades. Vision Res. 44, 1453-1467.

Schiller, P.H., and Tehovnik, E.J. (2005). Neural mechanisms underlying target selection with saccadic eye movements. Prog Brain Res 149, 157-171.

Schindler, I., Ellison, A., and Milner, A.D. (2008). Contralateral visual search deficits following TMS. J. Neuropsychol. 2, 501-508.

Schlag, J., Schlag-Rey, M., Peck, C.K., and Joseph, J.P. (1980). Visual responses of thalamic neurons depending on the direction of gaze and the position of targets in space. Exp. Brain Res. 40, 170-84.

Schlag-Rey, M., and Schlag, J. (1984). Visuomotor functions of central thalamus in monkey. I. Unit activity related to spontaneous eye movements. J. Neurophysiol. 51, 1149-1174.

Schmahmann, J.D., and Pandya, D.N. (1990). Anatomical investigation of projections from thalamus to posterior parietal cortex in the rhesus monkey: A WGA-HRP and fluorescent tracer study. J. Comp. Neurol. 295, 299-326.

Schmitt, L.I., Wimmer, R.D., Nakajima, M., Happ, M., Mofakham, S., and Halassa, M.M. (2017). Thalamic amplification of cortical connectivity sustains attentional control. Nature 545, 219-223.

Schneider, G.E. (1969). Brain mechanisms for localization and discrimination are dissociated by tectal and cortical lesions. Science 163, 9.

Sherman, S.M. (2009). Thalamocortical Relations. In Handbook of Neuroscience for the Behavioral Sciences, (John Wiley \& Sons Inc.), p. 23.

Sherman, S.M., and Guillery, R.W. (1998). On the actions that one nerve cell can have on another: Distinguishing "drivers" from "modulators." Proc. Natl. Acad. Sci. 95, 7121-7126.

Sherman, S.M., and Guillery, R.W. (2002). The role of the thalamus in the flow of information to the cortex. Philos. Trans. R. Soc. B Biol. Sci. 357, 1695-1708.

Snyder, L.H., Grieve, K.L., Brotchie, P., and Andersen, R.A. (1998). Separate body- and world-referenced representations of visual space in parietal cortex. Nature 394, 887-891.

Soares, S.C., Maior, R.S., Isbell, L.A., Tomaz, C., and Nishijo, H. (2017). Fast Detector/First Responder: Interactions between the Superior Colliculus-Pulvinar Pathway and Stimuli Relevant to Primates. Front. Neurosci. 11. 
Sommer, M.A., and Wurtz, R.H. (2004). What the Brain Stem Tells the Frontal Cortex. I. Oculomotor Signals Sent From Superior Colliculus to Frontal Eye Field Via Mediodorsal Thalamus. J. Neurophysiol. 91, 1381-1402.

Sommer, M.A., and Wurtz, R.H. (2008). Visual perception and corollary discharge. Perception 37, 408-418.

Sperry, R.W. (1950). Neural basis of the spontaneous optokinetic response produced by visual inversion. J. Comp. Physiol. Psychol. 43, 482-489.

Squatrito, S., and Maioli, M.G. (1996). Gaze field properties of eye position neurones in areas MST and 7a of the macaque monkey. Vis. Neurosci. 13, 385-398.

Stepniewska, I., Preuss, T.M., and Kaas, J.H. (1994). Thalamic connections of the primary motor cortex (M1) of owl monkeys. J. Comp. Neurol. 349, 558-582.

Stone, S.P., Halligan, P.W., and Greenwood, R.J. (1993). The incidence of neglect phenomena and related disorders in patients with an acute right or left hemisphere stroke. Age Ageing 22, 46-52.

Strumpf, H., Mangun, G.R., Boehler, C.N., Stoppel, C., Schoenfeld, M.A., Heinze, H.-J., and Hopf, J.-M. (2013). The role of the pulvinar in distractor processing and visual search. Hum. Brain Mapp. 34, 1115-1132.

Sudkamp, S., and Schmidt, M. (2000). Response characteristics of neurons in the pulvinar of awake cats to saccades and to visual stimulation. Exp. Brain Res. 133, 209-218.

Sultan, F., Augath, M., Hamodeh, S., Murayama, Y., Oeltermann, A., Rauch, A., and Thier, P. (2012). Unravelling cerebellar pathways with high temporal precision targeting motor and extensive sensory and parietal networks. Nat. Commun. 3.

Takayama, Y., Sugishita, M., Kido, T., Ogawa, M., Fukuyama, H., and Akiguchi, I. (1994). Impaired stereoacuity due to a lesion in the left pulvinar. J. Neurol. Neurosurg. Psychiatry 57, 652-654.

Tanaka, M. (2007). Spatiotemporal Properties of Eye Position Signals in the Primate Central Thalamus. Cereb. Cortex 17, 1504-1515.

Tanaka, M., and Kunimatsu, J. (2011). Contribution of the central thalamus to the generation of volitional saccades. Eur. J. Neurosci. 33, 2046-2057.

Treue, S., and Trujillo, J.C.M. (1999). Feature-based attention influences motion processing gain in macaque visual cortex. Nature $399,575-579$.

Ungerleider, L.G., and Haxby, J.V. (1994). 'What' and 'where' in the human brain. Curr. Opin. Neurobiol. 4, 157-165.

Ustinova, K., and Perkins, J. (2011). Gaze and viewing angle influence visual stabilization of upright posture. Brain Behav. 1, 19-25.

Vallar, G., and Perani, D. (1986). The anatomy of unilateral neglect after right-hemisphere stroke lesions. A clinical/CT-scan correlation study in man. Neuropsychologia 24, 609-622.

Van der Stigchel, S., Arend, I., van Koningsbruggen, M.G., and Rafal, R.D. (2010). Oculomotor integration in patients with a pulvinar lesion. Neuropsychologia 48, 3497-3504. 
Van Horn, S.C., Erisir, A., and Sherman, S.M. (2000). Relative distribution of synapses in the A-laminae of the lateral geniculate nucleus of the cat. J. Comp. Neurol. 416, 509-520.

Van Opstal, A.J., Hepp, K., Suzuki, Y., and Henn, V. (1995). Influence of eye position on activity in monkey superior colliculus. J. Neurophysiol. 74, 1593-1610.

Wang, X., Zhang, M., Cohen, I.S., and Goldberg, M.E. (2007). The proprioceptive representation of eye position in monkey primary somatosensory cortex. Nat. Neurosci. 10, 640-646.

Watanabe, K., and Funahashi, S. (2007). Prefrontal delay-period activity reflects the decision process of a saccade direction during a free-choice ODR task. Cereb Cortex 17 Suppl 1, i88100.

Watanabe, M., and Munoz, D.P. (2013). Effects of caudate microstimulation on spontaneous and purposive saccades. J. Neurophysiol. 110, 334-343.

Watanabe, Y., and Funahashi, S. (2004). Neuronal Activity Throughout the Primate Mediodorsal Nucleus of the Thalamus During Oculomotor Delayed-Responses. I. Cue-, Delay-, and Response-Period Activity. J. Neurophysiol. 92, 1738-1755.

Weyand, T.G., and Malpeli, J.G. (1993). Responses of neurons in primary visual cortex are modulated by eye position. J. Neurophysiol. 69, 2258-2260.

Wilke, M., Mueller, K.-M., and Leopold, D.A. (2009). Neural activity in the visual thalamus reflects perceptual suppression. Proc. Natl. Acad. Sci. 106, 9465-9470.

Wilke, M., Turchi, J., Smith, K., Mishkin, M., and Leopold, D.A. (2010). Pulvinar Inactivation Disrupts Selection of Movement Plans. J. Neurosci. 30, 8650-8659.

Wilke, M., Kagan, I., and Andersen, R.A. (2013). Effects of Pulvinar Inactivation on Spatial Decision-making between Equal and Asymmetric Reward Options. J. Cogn. Neurosci. 25, 1270-1283.

Wilke, M., Dechent, P., and Bähr, M. (2017). Sarcoidosis Manifestion Centered on the Thalamic Pulvinar Leading to Persistent Astasia. Mov. Disord. Clin. Pract. 4, 898-900.

Wilke, M., Schneider, L., Dominguez-Vargas, A.-U., Schmidt-Samoa, C., Miloserdov, K., Nazzal, A., Dechent, P., Cabral-Calderin, Y., Scherberger, H., Kagan, I., et al. (2018). Reach and grasp deficits following damage to the dorsal pulvinar. Cortex 99, 135-149.

Wurtz, R.H., Sommer, M.A., and Cavanaugh, J. (2005). Drivers from the deep: the contribution of collicular input to thalamocortical processing. Prog. Brain Res. 149, 207-225.

Wurtz, R.H., Joiner, W.M., and Berman, R.A. (2011a). Neuronal mechanisms for visual stability: progress and problems. Philos. Trans. R. Soc. B Biol. Sci. 366, 492-503.

Wurtz, R.H., McAlonan, K., Cavanaugh, J., and Berman, R.A. (2011b). Thalamic pathways for active vision. Trends Cogn. Sci. 15, 177-184.

Wyder, M.T., Massoglia, D.P., and Stanford, T.R. (2003). Quantitative Assessment of the Timing and Tuning of Visual-Related, Saccade-Related, and Delay Period Activity in Primate Central Thalamus. J. Neurophysiol. 90, 2029-2052. 
Wyder, M.T., Massoglia, D.P., and Stanford, T.R. (2004). Contextual Modulation of Central Thalamic Delay-Period Activity: Representation of Visual and Saccadic Goals. J. Neurophysiol. 91, 2628-2648.

Xu, B.Y., Karachi, C., and Goldberg, M.E. (2012). The Postsaccadic Unreliability of Gain Fields Renders It Unlikely that the Motor System Can Use Them to Calculate Target Position in Space. Neuron 76, 1201-1209.

Xu, Y., Wang, X., Peck, C., and Goldberg, M.E. (2011). The time course of the tonic oculomotor proprioceptive signal in area 3a of somatosensory cortex. J. Neurophysiol. 106, 71-77.

Yang, S., Heinen, S.J., and Missal, M. (2008). The Effects of Microstimulation of the Dorsomedial Frontal Cortex on Saccade Latency. J. Neurophysiol. 99, 1857-1870.

Yao, L., and Peck, C.K. (1997). Saccadic eye movements to visual and auditory targets. Exp. Brain Res. 115, 25-34.

Yeterian, E.H., and Pandya, D.N. (1989). Thalamic connections of the cortex of the superior temporal sulcus in the rhesus monkey. J Comp Neurol 282, 80-97.

Zhou, H., Schafer, R.J., and Desimone, R. (2016a). Pulvinar-Cortex Interactions in Vision and Attention. Neuron 89, 209-220.

Zhou, N., Maire, P.S., Masterson, S.P., and Bickford, M.E. (2017). The mouse pulvinar nucleus: Organization of the tectorecipient zones. Vis. Neurosci. 34.

Zhou, Y., Liu, Y., Lu, H., Wu, S., and Zhang, M. (2016b). Neuronal representation of saccadic error in macaque posterior parietal cortex.

Ziesche, A., and Hamker, F.H. (2011). A Computational Model for the Influence of Corollary Discharge and Proprioception on the Perisaccadic Mislocalization of Briefly Presented Stimuli in Complete Darkness. J. Neurosci. 31, 17392-17405.

Zipser, D., and Andersen, R.A. (1988). A back-propagation programmed network that simulates response properties of a subset of posterior parietal neurons. Nature 331, 679. 


\title{
7. Academic Resume
}

\author{
Lukas Schneider \\ Nationality: Austrian; Date of birth 1984/02/25. \\ Mobile Germany: +49 15771951540 \\ E-Mail: Lukas.schneider.m@gmail.com / Ischneider@dpz.eu
}

\section{Education:}

2013-2018 Decision and Awareness Group, Cognitive Neuroscience Laboratory, German Primate Center, Leibniz Institute for Primate Research (DPZ) Göttingen Graduate School for Neurosciences and Molecular Biosciences (GGNB), Systems Neuroscience Program, Göttingen Germany

2010-2012 Institut für Festkörperphysik, Technische Universität Wien Vienna, Austria (Institute for solid-state physics, technical university vienna)

2003-2012 Institut für Physik, Universität Wien

Vienna, Austria

(Faculty of nature sciences, university of Vienna)

Magister der Naturwissenschaften (Mag. Rer. Nat.)

\section{Research Experience}

2013-

Decision and Awareness Group, Cognitive Neuroscience Laboratory, German Primate Center, Leibniz Institute for Primate Research (DPZ). Göttingen Germany

Techniques Employed: Direct current microstimulation, extracellular recordings, pharmacological inactivation, psychophysics, eye tracking, MATLAB programming

2010-2012 Institut für Festkörperphysik, Technische Universität Wien Vienna, Austria (Institute for solid-state physics, technical university Vienna) Techniques Employed: Atomic force microscopy, Lithography, sputtering, Plasma enhanced chemical vapour deposition, Reactive ion etching AUTOCAD designing

\section{Publications}

2018 Wilke M, Schneider L, Dominguez-Vargas AU, Cabral-Calderin Y, Miloserdov K, Schmidt-Samoa C, Dechent P, Scherberger H, Kagan I and Bähr M Reach and grasp deficits following damage to the dorsal pulvinar Under review, Cortex 99 135-149

2017 Dominguez-Vargas A-U*, Schneider L*, Wilke M+, Kagan I+ (2017) Electrical Microstimulation of the Pulvinar Biases Saccade Choices and Reaction Times in a Time-Dependent Manner. The Journal of Neuroscience 37:2234-2257.

${ }^{*}$ Equal Contribution +Shared last authors

\section{Selected Abstracts}

2016 Annual Meeting of the Society for Neuroscience, San Diego, USA Poster: "Contribution of dorsal pulvinar to visuo-motor behavior and spatial decisionmaking" Kagan I., Domínguez Vargas A. U., Schneider L., Gibson L., Wilke M.

2014 Annual Meeting of the Society for Neuroscience, Washington, USA. Poster: "Time-dependent effects of pulvinar microstimulation on visuallyguided saccades and target selection" Domínguez Vargas A. U. *, Schneider L. *, Kagan I., Wilke $M$.

\section{*Equal Contribution}

2014 11th Göttingen meeting of the German Neuroscience Society, Göttingen, Germany 
Poster: "Time-dependent effects of pulvinar microstimulation on spatial target selection" Domínguez Vargas A. U. *, Schneider L. *, Kagan I., Wilke M.

\section{Selected Courses}

2015 FENS-IBRO-Hertie Winter School "The neuroscience of decision making" Obergurgl, Austria.

Poster: "Time-dependent effects of pulvinar microstimulation on visually-guided saccades and target selection“ Dominguez-Vargas A-U*, Schneider $L^{*}$, Wilke $M+$,

Kagan I+

${ }^{\star}$ Equal Contribution +Shared last authors

2014 EUPRIM-NET course on General Primate Biology - behavior, cognition and neurophysiology

Göttingen, Germany

2016 GTPN course "Primate Neurobiology methods: behavior, experiments, analysis, and ethics"

Tübingen, Germany

\section{Affiliation}

2013- Neurowissenschaftliche Gesellschaft (NWG)

(German Neuroscience Society)

Student Member

\section{Master students' laboratory rotation supervision}

2014 Alexander Kratzenberg

Developmental, Neural and Behavioral Biology, Faculty of Biology and Psychology, Georg-August-Universität Göttingen

\section{Master thesis supervision}

2015 Alexander Kratzenberg

"Intentional and perceptual aspects of spatial awereness and their neural correlates" Developmental, Neural and Behavioral Biology, Faculty of Biology and Psychology, Georg-August-Universität Göttingen 
8. Appendix

8.1 Appendix A 


\title{
Electrical Microstimulation of the Pulvinar Biases Saccade Choices and Reaction Times in a Time-Dependent Manner
}

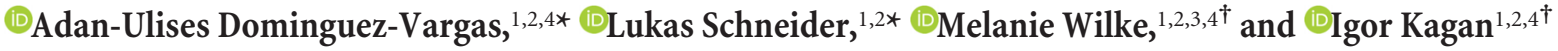 \\ ${ }^{1}$ Decision and Awareness Group, Cognitive Neuroscience Laboratory, German Primate Center, Leibniz Institute for Primate Research, Goettingen 37077, Germany, \\ ${ }^{2}$ Department of Cognitive Neurology, University of Goettingen, Goettingen 37075, Germany, ${ }^{3}$ Deutsche Forschungsgemeinschaft Center for Nanoscale Microscopy \\ and Molecular Physiology of the Brain, Göttingen 37075, Germany, and ${ }^{4}$ Leibnitz Science Campus Primate Cognition, Goettingen 37077, Germany
}

The pulvinar complex is interconnected extensively with brain regions involved in spatial processing and eye movement control. Recent inactivation studies have shown that the dorsal pulvinar (dPul) plays a role in saccade target selection; however, it remains unknown whether it exerts effects on visual processing or at planning/execution stages. We used electrical microstimulation of the dPul while monkeys performed saccade tasks toward instructed and freely chosen targets. Timing of stimulation was varied, starting before, at, or after onset of target(s). Stimulation affected saccade properties and target selection in a time-dependent manner. Stimulation starting before but overlapping with target onset shortened saccadic reaction times (RTs) for ipsiversive (to the stimulation site) target locations, whereas stimulation starting at and after target onset caused systematic delays for both ipsiversive and contraversive locations. Similarly, stimulation starting before the onset of bilateral targets increased ipsiversive target choices, whereas stimulation after target onset increased contraversive choices. Properties of dPul neurons and stimulation effects were consistent with an overall contraversive drive, with varying outcomes contingent upon behavioral demands. RT and choice effects were largely congruent in the visually-guided task, but stimulation during memory-guided saccades, while influencing RTs and errors, did not affect choice behavior. Together, these results show that the dPul plays a primary role in action planning as opposed to visual processing, that it exerts its strongest influence on spatial choices when decision and action are temporally close, and that this choice effect can be dissociated from motor effects on saccade initiation and execution.

Key words: choice; decision-making; electrophysiology; microstimulation; pulvinar; saccades

\section{Significance Statement}

Despite a recent surge of interest, the core function of the pulvinar, the largest thalamic complex in primates, remains elusive. This understanding is crucial given the central role of the pulvinar in current theories of integrative brain functions supporting cognition and goal-directed behaviors, but electrophysiological and causal interference studies of dorsal pulvinar (dPul) are rare. Building on our previous studies that pharmacologically suppressed dPul activity for several hours, here we used transient electrical microstimulation at different periods while monkeys performed instructed and choice eye movement tasks, to determine time-specific contributions of pulvinar to saccade generation and decision making. We show that stimulation effects depend on timing and behavioral state and that effects on choices can be dissociated from motor effects.

\section{Introduction}

The ability to decide flexibly between response options is a crucial attribute of adaptive behavior. One fundamental component of

Received June 20, 2016; revised Dec. 21, 2016; accepted Dec. 30, 2016.

Author contributions: A.-U.D.-V., L.S., M.W., and I.K. designed research; A.-U.D.-V., L.S., and I.K. performed research; A.-U.D.-V., L.S., and I.K. contributed unpublished reagents/analytic tools; A.-U.D.-V., L.S., and I.K. analyzed data; A.-U.D.-V., L.S., M.W., and I.K. wrote the paper.

This work was supported by the Hermann and Lilly Schilling Foundation, the German Research Foundation (Deutsche Forschungsgemeinschaft Grants WI 4046/1-1 and Research Unit FOR 1847 GA1475-B4, KA 3726/2-1), the Center for Nanoscale Microscopy and Molecular Physiology of the Brain Primate Platform, and the Cognitive Neuroscience Laboratory of the German Primate Center. We thank Lydia Gibson for collecting a subset of neuronal recordings; Sebastian Moeller for sharing the design of an MRI-compatible drive and many valuable suggestions; Hansjörg Scherberger for valuable discussions and suggestions regarding target selection equalization; Stefan Treue, Alexander Gail, members of the Decision and Awareness Group and the Cognitive Neuroscience Laboratory for helpful this process is the guidance of eye movements exploring spatial locations of potential interest. Representations of diverse variables contributing to saccadic decisions have been found in many discussions; Ira Panolias, Sina Plümer, Klaus Heisig, Dirk Prüße, and Ralf Brockhausen for technical support; and two anonymous reviewers for their fast, valuable, and constructive comments.

The authors declare no competing financial interests.

${ }^{*}$ A.-U.D.-V. and L.S. contributed equally to this work and are co-first authors.

${ }^{\dagger} \mathrm{M}$.W. and I.K. contributed equally to this work and are co-last authors.

Correspondence should be addressed to Igor Kagan, German Primate Center, Leibniz Institute for Primate Research, Kellnerweg 4, Goettingen 37077, Germany. E-mail: ikagan@dpz.eu.

DOI:10.1523/JNEUROSCI.1984-16.2016

Copyright $\odot 2017$ Dominguez-Vargas, Schneider et al.

This is an open-access article distributed under the terms of the Creative Commons Attribution License Creative Commons Attribution 4.0 International, which permits unrestricted use, distribution and reproduction in any medium provided that the original work is properly attributed. 
cortical and subcortical brain regions (Andersen and Cui, 2009; Shadlen and Kiani, 2013). Based on the extensive anatomical connectivity to those regions, the thalamic pulvinar has been suggested as a hub for the coordination of movements for goaldirected visually-guided behavior (Grieve et al., 2000; Wilke et al., 2010). In primates, the pulvinar forms the largest thalamic complex and can be coarsely subdivided into ventral and dorsal aspects (Kaas and Lyon, 2007; Preuss, 2007). The ventral aspect is organized retinotopically and is connected with striate and extrastriate visual cortices. The dorsal aspect does not seem to contain an orderly retinotopic topography and is interconnected reciprocally with areas that combine spatial attention and eye movement functions, such as the parietal, superior temporal, posterior cingulate, and prefrontal cortices (Seltzer et al., 1996; Gutierrez et al., 2000; Jones, 2012). Both the ventral (vPul) and dorsal (dPul) pulvinar receive input from the superior colliculus (SC): the vPul from the upper and the $\mathrm{dPul}$ from the lower and intermediate layers of the SC (Stepniewska, 2004; Berman and Wurtz, 2011). Therefore, anatomical connectivity of the pulvinar suggests that it is involved in the selection and planning of eye movements and spatial attention.

Converging evidence is also provided by electrophysiological and lesion/inactivation studies. Visually responsive pulvinar neurons enhance firing for stimuli that are attended and/or are target of an upcoming saccade (Petersen et al., 1985; Robinson and Petersen, 1992; Bender and Youakim, 2001; Saalmann et al., 2012; Zhou et al., 2016). In addition, many pulvinar neurons exhibit saccade-related activity, including spatially specific enhancement or suppression associated with the onset of the visual target and/or onset or offset of the saccade (Petersen et al., 1985; Robinson et al., 1986, 1990; Berman and Wurtz, 2011). Studies of neural responses in eye movement tasks in the nonretinotopic, dorsal part of the pulvinar are particularly sparse, but suggest a diversity of saccade-related properties, with neurons exhibiting spatially untuned or direction-dependent perisaccadic and/or postsaccadic discharges (Robinson et al., 1986; Benevento and Port, 1995). Some medial dPul neurons have two peak responses, one closely following the onset of the visual target and the other triggered to the onset or offset of the saccade (Benevento and Port, 1995).

Pulvinar lesions in humans or monkeys do not result in primary visual or saccade generation deficits (Bender and Butter, 1987; Bender and Baizer, 1990; Van der Stigchel et al., 2010; Wilke et al., 2010, 2013), although a modest lesion-induced increase of contralesional saccade latencies has been reported (Rafal et al., 2004; Wilke et al., 2013). More pronounced are "higher-order" spatial attention and decision-making impairments (Robinson and Petersen, 1992; Saalmann and Kastner, 2011). Specifically, structural and reversible lesions in the vPul and/or dPul impair the ability to shift visual attention toward the contralesional hemifield and result in an ipsilesional spatial exploration and saccade choice bias (Rafal and Posner, 1987; Karnath et al., 2002; Arend et al., 2008; Snow et al., 2009; Wilke et al., 2010, 2013; Zhou et al., 2016). Although these lesion/inactivation studies provide strong evidence that normal pulvinar functioning is crucial for the selection of saccade goals in the presence of competing targets, they cannot resolve at which processing stage pulvinar exerts its effect on saccade behavior.

The aim of the current study was to investigate putative pulvinar-driven interactions between target selection and saccade generation in a temporally specific manner. To this end, we applied transient electrical microstimulation in the pulvinar while macaque monkeys performed visually- or memory-guided saccades to single (instructed) targets or chose between two targets in opposite hemifields. Crucially, we varied the timing of microstimulation, starting it before, at, or after onset of the saccade target(s). Our results demonstrate a temporal-specific impact of the pulvinar on spatial choices and saccade generation, further elucidating its involvement in goal-directed behaviors.

\section{Materials and Methods}

\section{Procedures}

All experimental procedures were conducted in accordance with the European Directive 2010/63/EU, the corresponding German law governing animal welfare, and German Primate Center institutional guidelines. The procedures were approved by the responsible government agency (LAVES, Oldenburg, Germany).

\section{Animal preparation}

Two adult male rhesus monkeys (Macaca mulatta), Monkey C and Monkey L, weighing 8 and $9 \mathrm{~kg}$, respectively, were used. In an initial surgery, monkeys were implanted with an MRI-compatible polyetheretherketone (PEEK) head post embedded in a bone cement head cap (Palacos with gentamicin; BioMet) anchored by ceramic screws (Rogue Research) under general anesthesia and aseptic conditions. MR-visible markers were embedded in the head cap to aid the planning of the chamber in stereotaxic space (Monkey $\mathrm{C}$, right hemisphere: center at $0.5 \mathrm{~A} / 14.5 \mathrm{~L} \mathrm{~mm}$, tilted $-11 \mathrm{P} / 27 \mathrm{~L}$ degrees; Monkey $\mathrm{L}$, right hemisphere: center at -3.12 P/20.2 L mm, tilted: - $18 \mathrm{P} / 37 \mathrm{~L}$ degrees) with the MR-guided stereotaxic navigation software planner (Ohayon and Tsao, 2012). A separate surgery was performed to implant a PEEK MRI-compatible chamber (inside diameter $22 \mathrm{~mm}$ ) allowing access to the right pulvinar. After confirming chamber positioning with a postsurgical MRI, a partial craniotomy was made inside the chamber. The exposed dura was covered with a silicone elastomer (Kwik-sil; World Precision Instruments) to reduce the granulation tissue growth and dura thickening.

\section{MRI imaging}

Monkeys were scanned in a 3 T MRI scanner (Magnetom TIM Trio; Siemens). Full-head T1-weighted scans (3D magnetization-prepared rapid gradient-echo, MPRAGE, $0.5 \mathrm{~mm}$ isometric) were acquired before and after chamber implantation in an awake (Monkey C) or anesthetized (Monkey L) state using either built-in gradient body transmit coil and custom single loop receive coil or custom single loop transmit and fourchannel receive coil (Windmiller Kolster Scientific).

In addition to preimplantation and postimplantation scans, similar T1- and T2-weighted (rapid acquisition with relaxation enhancement, RARE, $0.25 \mathrm{~mm}$ in plane, $1 \mathrm{~mm}$ slice thickness) scans were acquired periodically during the course of experiments either in an awake (Monkey C) or sedated (Monkey L) state to confirm electrode positioning. T1- and T2-weighted scans were coregistered and transformed into "chamber normal" (aligned to the chamber vertical axis) and to AP-PC space for electrode targeting and visualization. These images were acquired with the chamber and the grid filled with gadolinium (Magnevist; Bayer)/saline solution (proportion 1:200) with tungsten rods inserted in predefined grid locations for alignment purposes.

\section{Pulvinar targeting}

The location of the electrode was estimated for every stimulation site based on anatomical MRI. Custom-made MR-compatible polyetherimide (Ultem) grids $(0.8 \mathrm{~mm}$ hole spacing, $0.45 \mathrm{~mm}$ hole diameter $)$ and custom-made plastic XYZ manipulator drives (design courtesy of Dr. Sebastian Moeller; Moeller et al., 2008) were used to position platinumiridium electrodes (FHC, see detailed specs in the next section) in the corresponding grid hole and estimated depth. During the penetration, the electrode was protected by a custom-made MRI-compatible fused silica guide tube (320 $\mu \mathrm{m}$ inner diameter, $430 \mu \mathrm{m}$ outer diameter; Polymicro Technologies) or a custom-made stainless steel guide tube (450 $\mu \mathrm{m}$ outer diameter, 27 gauge Spinocan; Braun Melsungen). A stopper (530 $\mu \mathrm{m}$ inner diameter, $665 \mu \mathrm{m}$ outer diameter, 23 gauge MicroFil; World Precision Instruments) ensured that the guide tube only penetrated the dura and minimally the cortex below. Before penetration, the 
electrode tip was aligned to the guide tube tip and was held in place by a drop of melted petroleum jelly. The guide tube was filled with sterile silicone oil before electrode insertion to ensure smooth electrode travel and to prevent backflow of CSF.

There are multiple parcellation schemes available for the pulvinar (Stepniewska, 2004; Jones, 2012). Here, the pulvinar was divided into $\mathrm{dPul}$ and vPul aspects using the brachium of SC as a landmark, as has been done in several studies (Gutierrez et al., 2000; Wilke et al., 2010; Komura et al., 2013). The dPul includes medial pulvinar and dorsal part of lateral pulvinar (also denoted as PLdm, or Pdm in earlier studies; Robinson and Petersen, 1992), whereas vPul contains inferior pulvinar and ventral part of lateral pulvinar (also denoted as PLvl; Robinson et al., 1986; Kaas and Lyon, 2007). Because currently available online and downloadable atlases use the traditional scheme segregating medial (MPul), lateral (LPul,) and inferior (IPul) (and sometimes anterior/oral) nuclei (Rohlfing et al., 2012; Calabrese et al., 2015), we adopted this scheme for the localization of stimulation and recording sites.

As can be seen in Figure 1, the stimulation sites in the main experiment corresponded to the $\mathrm{dPul}$, mostly to the MPul, but were also close to the dorsal aspect of the LPul. The brachium of the SC and other neighboring structures such as reticular thalamic nucleus and tail of the caudate nucleus were avoided.

\section{Electrical microstimulation}

An S88X dual output square pulse stimulator (Grass Products) triggered by a MATLAB-based task controller generated $200 \mathrm{~ms}$ trains of twin pulses at $300 \mathrm{~Hz}$, which in turn triggered a constant current stimulus isolator A365 (World Precision Instruments) to produce 60 biphasic pulses. The current (100-300 $\mu \mathrm{A}$, see below) was delivered using single monopolar electrodes ( $100 \mathrm{~mm}$ length platinum-iridium $125-\mu \mathrm{m}$-thick core, initial $2 \mathrm{~cm}$ glass-coated with an exposed tip of $40 \mu \mathrm{m}$, total thickness of $230 \mu \mathrm{m}$ including polyamide tubing coating, UEPLEFSS (UEIK1; FHC); a return (reference) tungsten rod was placed in the chamber filled with saline. Voltage drop across a $10 \mathrm{k} \Omega$ resistor in series with the electrode was monitored using a four-channel 1GS/s Tektronix TDS2004C oscilloscope.

The manufacturer-specified impedance of electrodes was 300-500 $\mathrm{k} \Omega$; the initial impedance measured at $1000 \mathrm{~Hz}$ before the experiment was $360-1300 \mathrm{k} \Omega$. Because the impedance dropped dramatically after a few stimulation trains were applied, before each session, 10 trains were delivered to the electrode immersed in saline using $300 \mu \mathrm{A}$ current to bring the electrode impedance to a more stable regime. After this procedure, the impedance ranged from 19 to $200 \mathrm{k} \Omega$ for electrodes used in Monkey $\mathrm{C}$ and from 11 to $100 \mathrm{k} \Omega$ in electrodes used in Monkey L (see Table 1).

\section{Electrophysiological recordings}

In 19 sessions in Monkey $\mathrm{C}$ and 28 sessions in Monkey L right dPul neuronal activity was recorded with up to three individually movable single platinum-tungsten (95-5\%) quartz glass-insulated electrodes with impedance ranging from 1 to $1.9 \mathrm{M} \Omega$ for Monkey $\mathrm{C}$ and from 1.3 to 3.5 $\mathrm{M} \Omega$ for Monkey L using a chamber-mounted five-channel Mini Matrix microdrive (Thomas Recording). The recording target locations were estimated similarly to the stimulation sessions using the same grids. Similar to microstimulation experiments, single custom-made stainless steel guide tubes (27 gauge) filled with the silicone oil (Thomas Recording) with a Spinocan funnel attached to the drive nozzle were used to protect electrodes during dura penetration. A reference tungsten rod or a silver wire were placed in the chamber filled with saline and were connected to the chassis of the drive. Neuronal signals were amplified ( $20 \times$ headstage, Thomas Recording; $5 \times, 128$ or 32 channel PZ2 preamplifier, Tucker-Davis Technologies), digitized at $24 \mathrm{kHz}$ and 16 bit resolution, and sent via fiber optics to an RZ2 BioAmp Processor (TuckerDavis Technologies) for online filtering, display, and storage on a hard drive together with behavioral and timing data streams.

\section{Behavioral tasks}

Monkeys sat in a dark room in custom-made primate chairs with their heads restrained $30 \mathrm{~cm}$ away from a 27-inch LED display $(60 \mathrm{~Hz}$ refresh rate, model $\mathrm{HN} 274 \mathrm{H}$; Acer). The gaze position of the right eye was monitored at $220 \mathrm{~Hz}$ using a MCU02 ViewPoint infrared eyetracker (Arrington Research). Monkey face and body were monitored with infrared cameras to ensure that microstimulation did not elicit abrupt movements or signs of discomfort. All stimulus presentation and behavioral control tasks were programmed in MATLAB (The MathWorks) and the Psychophysics Toolbox (Brainard, 1997).

Fixation task and evoked saccades. In each microstimulation session before the main visually-guided saccade task (see below), five to six blocks of 20 fixation trials (see Fig. $2 A$ ) were performed to determine the presence/absence of evoked saccades as a consequence of electrical stimulation. A dim red spot of $1^{\circ}$ diameter (luminance $9.4 \mathrm{~cd} / \mathrm{m}^{2}$ ) appeared in the center of the monitor $\left(0.16 \mathrm{~cd} / \mathrm{m}^{2}\right)$. Once the monkeys directed their gaze into a $5^{\circ}$ radius window surrounding the fixation spot, it became brighter $\left(33 \mathrm{~cd} / \mathrm{m}^{2}\right)$ to signal fixation acquisition. Monkeys were required to maintain their gaze position for a randomized period ranging from 1000 to $1300 \mathrm{~ms}$ to complete a trial successfully before receiving liquid reward. The intertrial interval (ITI) was 1000 to $2000 \mathrm{~ms}$. In half of the trials of each block, a stimulation was applied starting $500 \mathrm{~ms}$ after fixation was acquired. Each session started with a block of $100 \mu \mathrm{A}$ and, in each subsequent block, current was increased by $50 \mu \mathrm{A}$ until the $300 \mu \mathrm{A}$ limit was reached. This range of currents was selected to match related ongoing $\mathrm{fMRI} /$ microstimulation experiments in our laboratory. The presence or absence of evoked saccades in a given block was assessed by online monitoring and all sessions were characterized offline (see below). If no evoked saccades were observed with any of the currents, the following tasks were performed with $250 \mu \mathrm{A}$; otherwise, the current was set to $50 \mu \mathrm{A}$ below the lowest intensity that evoked saccades. If all current strengths evoked saccades and if, according to our MRI-based estimates, after moving the electrode, it still would be within $1 \mathrm{~mm}$ of the targeted pulvinar nucleus borders, the electrode was moved by 0.5 or $1 \mathrm{~mm}$ up or down and five blocks of fixation trials were run again ( 10 of 15 sessions in Monkey C, 0 of 15 in Monkey L). Alternatively, the highest current that did not evoke more saccades than the $100 \mu \mathrm{A}$ current was used (two of 15 sessions in Monkey C, zero of 15 in Monkey L). In five of 15 sessions in Monkey $\mathrm{C}$, the electrode was moved and the current was lowered below $250 \mu \mathrm{A}$ even after moving the electrode.

The offline analysis confirmed online observations. When data from all fixation trials were combined, Monkey L did not show any difference in amount of saccades during stimulation compared with the same period during control trials ( $4 \%$ and $4 \%$, respectively; $2 \%$ contraversive and $2 \%$ ipsiversive in each case). Monkey $\mathrm{C}$, which incidentally had more frequent "fixational" saccades within the $5^{\circ}$ radius fixation window even in control trials, exhibited predominantly contraversive saccades during the stimulation period (Fig. $2 C ; 60 \%$ of stimulation trials, $57 \%$ contraversive, $3 \%$ ipsiversive; $32 \%$ of control trials in the corresponding period, $22 \%$ contraversive, $10 \%$ ipsiversive). Contraversive saccades were typically followed by ipsiversive saccades (69\%) within up to $200 \mathrm{~ms}$ after stimulation offset. Because the monkey was required to maintain fixation during the stimulation and these ipsiversive saccades were directed back to the fixation spot, we call them "return" saccades ( $82 \%$ of return saccades were preceded by contraversive ones). For further analysis, we classified as evoked saccades only contraversive saccades during the stimulation period that were followed by return saccades. We normalized the probabilities of evoking saccades per current strength for each site by subtracting the mean values for each site and found that the normalized probability of evoking saccades correlated with the current strength (Spearman's $r=0.38, p<0.001$ ). A similar analysis for evoked saccade amplitudes also showed a positive correlation with the current strength (Spearman's $r=0.31, p=0.009$ ). Across all sites that were later used in the main experiment and across all tested currents, the probability of evoking saccades in Monkey C was 39\% (40.5\% for the currents selected for the main experiment; see Table 1; for comparison, only $2 \%$ of control trials would have been classified as "evoked" using the above approach). The amplitude of evoked saccades was $1.51 \pm 0.16^{\circ}$ (mean \pm SE) across sites $\left(1.9 \pm 1^{\circ}\right.$, mean $\pm \mathrm{SD}$ across trials), with a latency of $95 \pm 39 \mathrm{~ms}$ after stimulation onset. Similar effects (increased probability of contraversive movements during stimulation period) were observed in several sessions in which we delivered the stimulation during free-gaze exploration (Goldberg et al., 1986; Watanabe and Munoz, 2013), with the excep- 

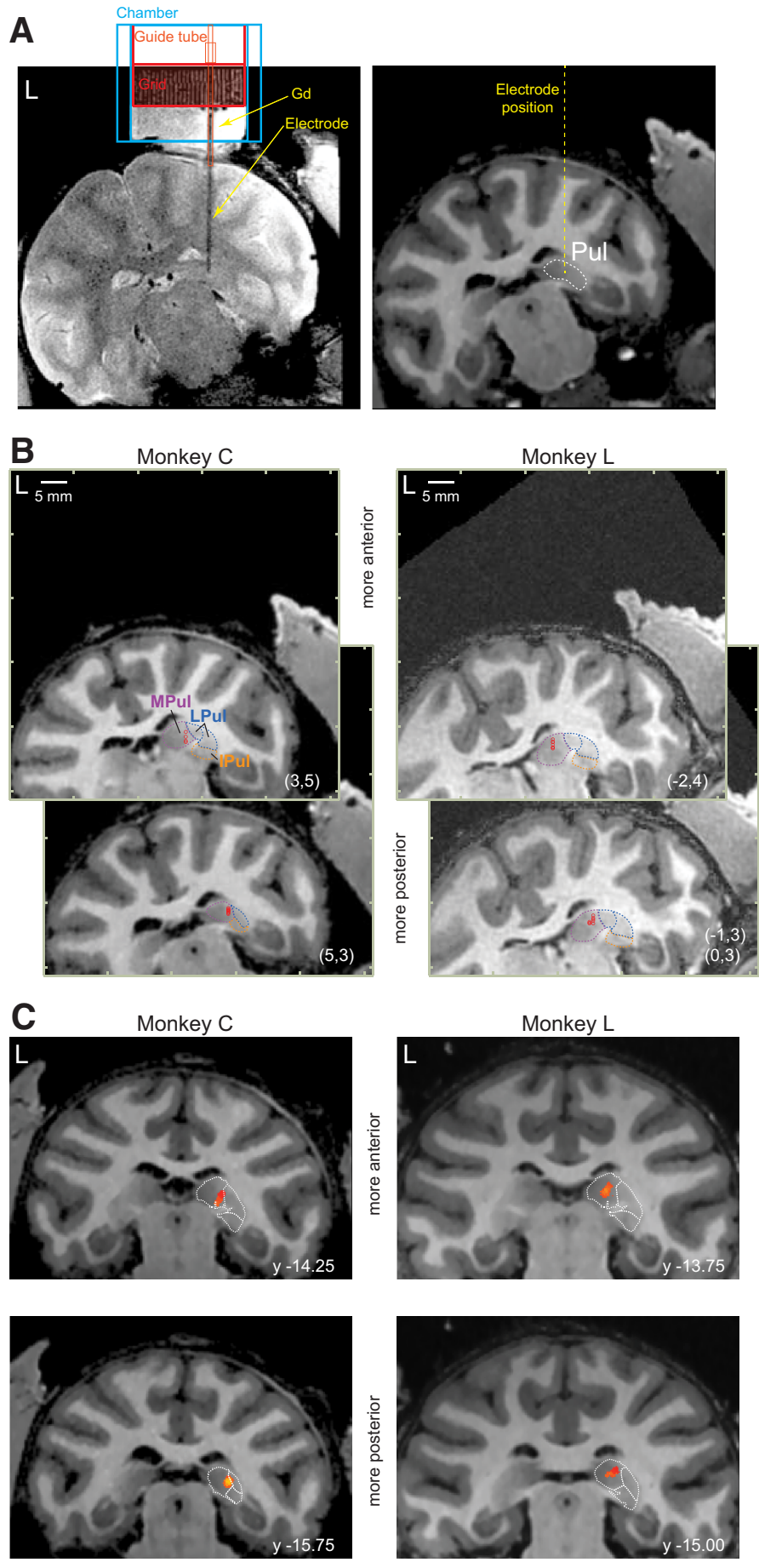

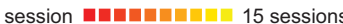
electrode tip probability map

Figure 1. Localization of stimulation sites in the dPul. $A$, Example scan of Monkey $($ with the stimulating electrode inserted in the pulvinar (grid location: $x=5, y=3$ ) with the chamber and the grid filled with the MRI contrast agent (gadolinium, Gd) (T2-weighted scan, left) and the corresponding section of the T1-weighted scan (right). $\boldsymbol{B}$, Electrode tip localization in individual stimulation sites (red circles) in chamber-normal coronal sections corresponding to specific grid locations $(x, y$; in parentheses) and depth. Pulvinar nuclei outlines (MPul, LPul, IPul) were adapted from the NeuroMaps atlas (Rohlfing et al., 2012), exported via the Scalable Brain Atlas (see https://scalablebrainatlas.incf.org/macaque/DB09 and https://scalablebrainatlas.incf.org/services/ rgbslice.php (Bakker et al., 2015), and LPul was further subdivided to PLdm and PLvI. C, Electrode tip localization probability maps in standard AC-PC space across all stimulation sites. The probability map was created by delineating a sphere of $0.5 \mathrm{~mm}$ radius around the tip in the chamber normal space for each stimulation session, transforming resulting volumes to $A C-P C$ space and converting volumes of interest (VOls) to a probability map using BrainVoyager VOI functions. Pulvinar nuclei outlines from the NeuroMaps atlas (dotted white) were scaled individually in the vertical and horizontal dimensions and overlaid on the corresponding anatomical sections. Right inset, Standard coronal sections (indicated by the $y$-coordinate) from the NeuroMaps atlas, going from anterior (top) to posterior (bottom). bsc, brachium of the SC; blv, body of the lateral ventricle. tion of not observing ipsiversive return saccades. Our observations are consistent with lateral posterior nucleus/pulvinar microstimulation studies in cats, which reported either absence of evoked saccades (Maldonado et al., 1980) or contraversive saccades with current strengths between 50 and $300 \mu \mathrm{A}$ (Crommelinck et al., 1977).

Although evoked saccades were present only in some sites, with $<50 \%$ probability and only in one monkey, we will briefly address relevant methodological considerations. Due to small amplitudes, the visual and positional consequences of these saccades are expected to be relatively minor (Carello and Krauzlis, 2004), although we cannot exclude the possibility of perceptual/attentional effects similar to consequences caused by fixational saccades (Hafed et al., 2015). Given the $24^{\circ}$ target eccentricity in the saccade tasks (see below), these displacements were not enough to land the gaze within the target window and did not seem to affect the ensuing choice. For example, during choice trials, when a small contraversive shift was apparent in the online display (and later during inspection of trial eye position traces), the monkey would often go on to select the ipsiversive target even though his gaze was already closer to the contraversive target.

At those sites where microstimulation evoked small saccades, required current strength was considerably higher than reported for SC, caudate nucleus or frontal eye fields (FEFs) (Robinson and Fuchs, 1969; Tehovnik et al., 1999; Yamamoto et al., 2012). Instead, the range of evoked saccade thresholds between 100 and 300 $\mu \mathrm{A}$ was more similar to required currents in visuomotor regions such as posterior parietal cortex (Shibutani et al., 1984; Thier and Andersen, 1996) and dorsomedial frontal cortex (Tehovnik et al., 1999).

Visually-guided saccade task. A trial started with the onset of the fixation spot. After the monkey acquired and held fixation within a $5^{\circ}$ radius for a randomized period ranging from 400 to $700 \mathrm{~ms}$, the fixation spot ( $1^{\circ}$ diameter $)$ was extinguished and either one target (instructed trials) or two targets (choice trials) appeared simultaneously (see Fig. 3A). This time point will be referred to as the "Go signal." Targets $\left(1^{\circ}\right.$ diameter) were presented in the left and/or right side(s) of the fixation spot, at $24^{\circ}$ eccentricity, with 3 potential angles relative to the horizontal axis: $0^{\circ}, 20^{\circ}$, or $-20^{\circ}\left(0^{\circ}, 8.2^{\circ}\right.$, and $-8.2^{\circ}$ vertical eccentricity). Monkeys had to make a saccade within $500 \mathrm{~ms}$ and keep their gaze position for $500 \mathrm{~ms}$ inside a $5^{\circ}$ radius window surrounding the target to complete a trial successfully and obtain a liquid reward after a delay of $200 \mathrm{~ms}$. In choice trials, monkeys were allowed to choose one of the targets freely; both choice targets were always presented at the same height and provided equal reward. The ITI for both successful and unsuccessful trials was 1000 or $2000 \mathrm{~ms}$. In seven of eight trials, a $200 \mathrm{~ms}$ stimulation train was applied at one of seven different periods in both instructed and choice trials. The trains started either before the Go signal $(-120 \mathrm{~ms},-80 \mathrm{~ms}$, or $-40 \mathrm{~ms}$; 
Table 1. Summary of 56 stimulation datasets

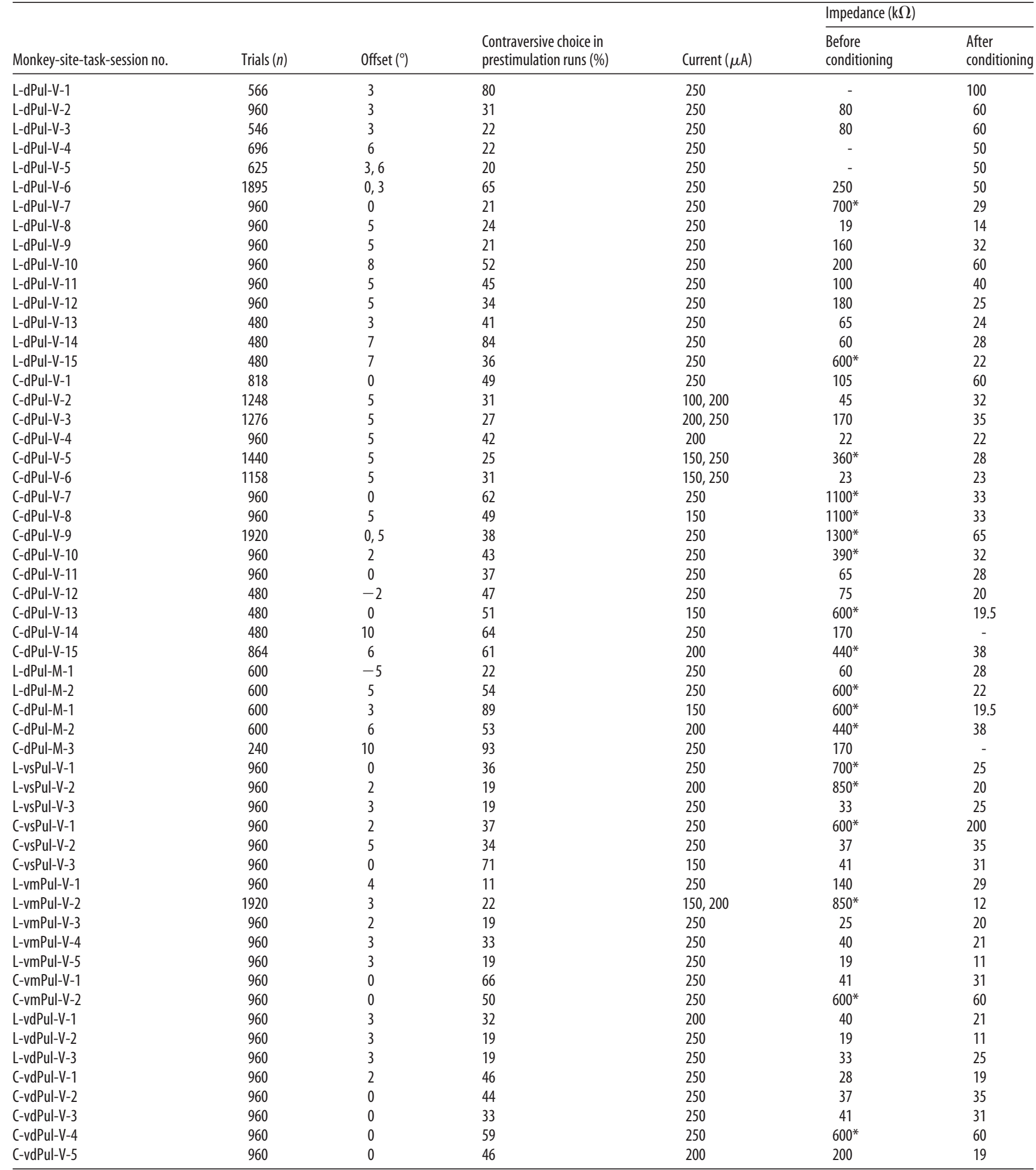

Offset is the horizontal shift of the entire stimulus array (fixation point and targets) from the center of the screen; positive values indicate shift to the right. Current is the current strength used in the visually-guided or memory-guided task. Impedance before conditioning refers to the electrode impedance before applying $10200 \mathrm{~ms} 300 \mu \mathrm{A}$ trains outside of the brain. First-time-use electrodes (out of the box) are marked with asterisks. Impedance after conditioning refers to resulting impedance after applying conditioning stimulation trains.

C, Monkey C, L, Monkey L; vs, ventral shallow; vm, ventral medium; vd, ventral deep; V, visually-guided task; M, memory-guided task.

early stimulation periods), simultaneously with the Go signal, or after the Go signal ( $+40 \mathrm{~ms},+80 \mathrm{~ms}$, or $+120 \mathrm{~ms}$; late stimulation periods). Note that because the train duration was $200 \mathrm{~ms}$, stimulation always ended after the Go signal. All trial types, target locations, and stimulation conditions were pseudorandomized. A minimum of 15 instructed trials per stimulation period and per hemifield were collected in each session (except in one session, where there was a minimum of 13 trials for the left hemifield and a session with 14 trials for the right hemifield).

Memory-guided saccade task. Similarly to the visually-guided saccade task, monkeys had to acquire and hold fixation for $400-700 \mathrm{~ms}$. Next, 
A

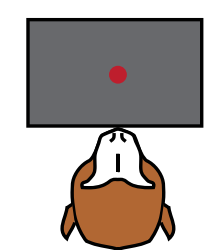

Fixation onset up to $500 \mathrm{~ms}$

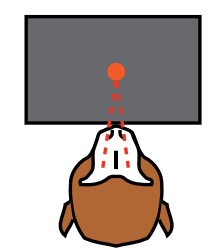

Fixation 1000 to $1300 \mathrm{~ms}$

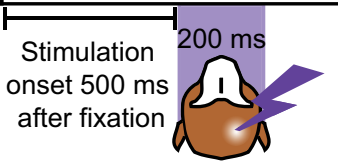

B

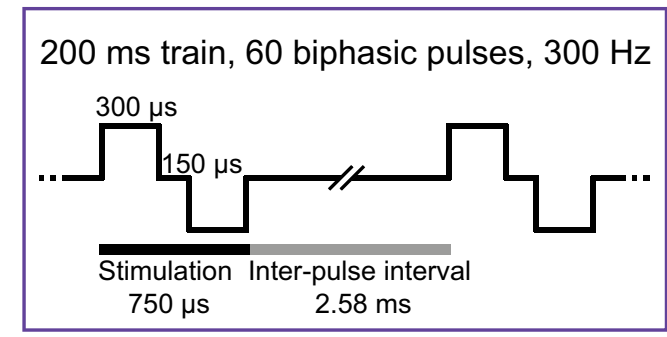

C
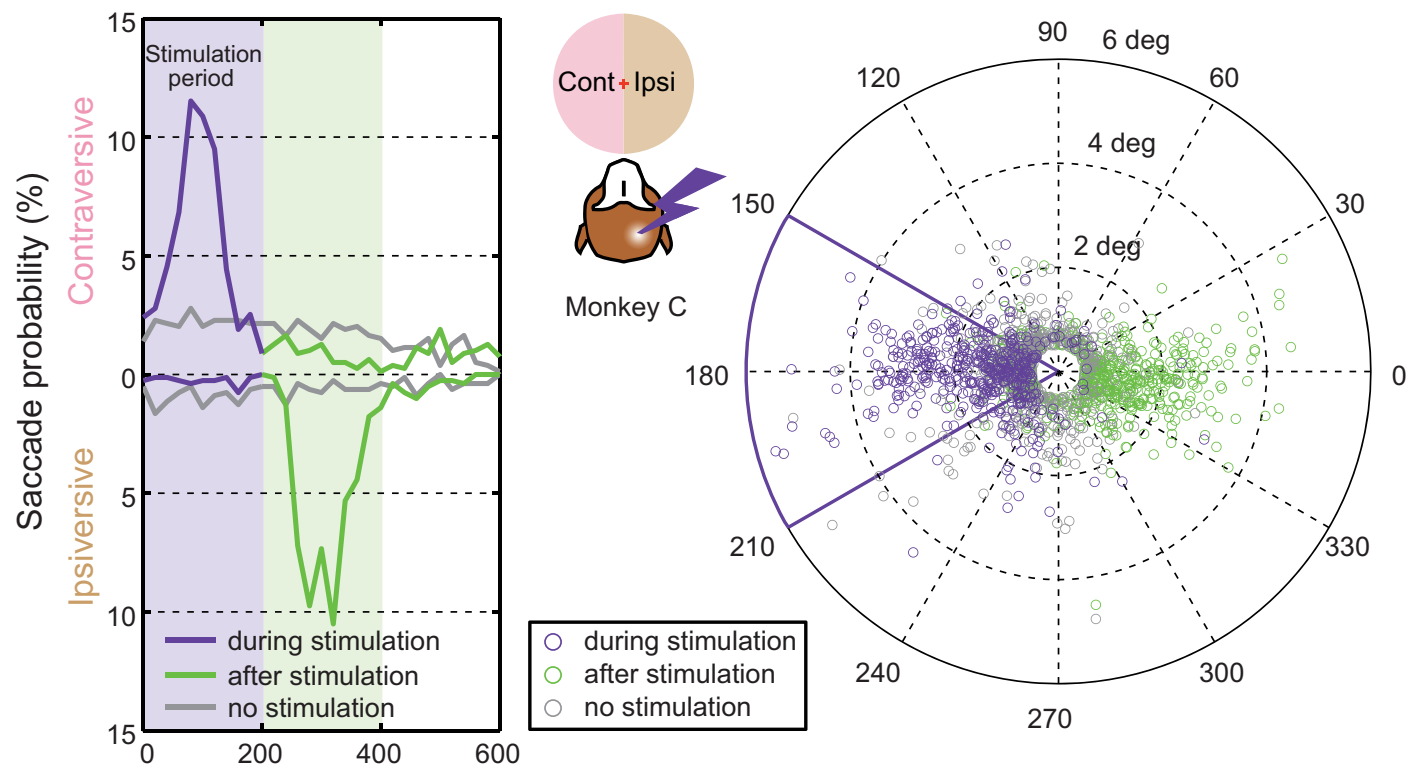

Time after stimulation onset [ms]

Figure 2. Fixation task and characterization of evoked saccades. $A$, Task layout. Monkeys fixated a central spot for a variable time to receive liquid reward. In half of the trials, we applied a train of biphasic electric pulses to characterize potential evoked saccades. B, Stimulation parameters. Each $200 \mathrm{~ms}$ stimulation train consisted of 60 biphasic pulses applied at $300 \mathrm{~Hz}$ (two pulses are shown). Each biphasic pulse started with a $300 \mu$ s positive phase, followed by $150 \mu$ s interphase interval and a $300 \mu$ s negative phase. There was a 2.58 ms interval between pulses. C, Saccade probability distribution as a function of time during and after stimulation (left) and corresponding saccade end points (right). Saccades that started during the stimulation period are shown in purple, saccades that started in the $200 \mathrm{~ms}$ window after stimulation in green, and saccades in trials without stimulation in gray. Plotted data are from 1580 fixation trials in Monkey C ( 15 stimulation sites). Only saccades with amplitudes $>0.5^{\circ}$ were included in this analysis. Left, Time axis relative to stimulation onset or a corresponding time in control trials. The probability of contraversive or ipsiversive saccades is shown as upward and downward histograms, respectively (bin $20 \mathrm{~ms}$ ). Right, Saccade direction and amplitude in the fixation task. End points are shown relative to each saccade starting position. We defined evoked saccades as saccades during the stimulation period that were followed by a saccade to the opposite side, returning to the fixation spot. The evoked saccades occurred mainly along the horizontal axis to the contraversive side: $83 \%$ were contained within a $30^{\circ}$ angle below and above the horizontal axis (solid purple sector outline).

one or two peripheral cues were displayed for $280 \mathrm{~ms}$ at the location(s) signaling the upcoming saccade target(s). These cues had the same spatial characteristics as the targets in the visually-guided task. Monkeys were required to maintain fixation throughout the cue period and also throughout the subsequent memory period (ranging from 200 to 400 $\mathrm{ms}$ ), after which the central fixation spot disappeared (Go signal), allowing monkeys to saccade to the instructed location or make a decision to go to one of the two cued locations. After the saccade to and fixation of the remembered target location for 100 to $200 \mathrm{~ms}$, the target became visible and, after an additional $500 \mathrm{~ms}$ of peripheral fixation, the trial was completed. We applied stimulation in four of five trials in one of four periods starting before or after the cue onset $(-80 \mathrm{~ms},+80 \mathrm{~ms})$ or before or after the Go signal ( $-80 \mathrm{~ms},+80 \mathrm{~ms})$.

Target selection equalization. During training, we consistently observed a strong selection bias to the right side of space in choice trials in both monkeys. This bias was potentially due to the fact that both monkeys were initially trained to perform reaches with their preferred right arm in the context of another experiment, in which they might have developed a strong rightward bias. To be able to assess potential target selection changes in both directions due to stimulation, we used a method similar to that used by Scherberger et al. (2003) to equalize the control target selection by shifting the entire stimulus array horizontally toward the preferred right hemifield without modifying the $24^{\circ}$ eccentricity from the fixation spot to the targets. The mean shift across visually-guided task sessions with stimulation in $\mathrm{dPul}$ was $3.2 \pm 0.8^{\circ}$ for Monkey C and $4.4 \pm$ $0.6^{\circ}$ for Monkey L to the right (mean \pm SE; see Table 1 ). These shifts resulted in the $44 \pm 3 \%$ and $40 \pm 6 \%$ leftward selection in prestimulation runs that were used for the equalization procedure (Monkeys $\mathrm{C}$ and $\mathrm{L}$, respectively, mean $\pm \mathrm{SE}$; see Table 1$)$. However, during the actual stimulation experiment, the leftward (contraversive) selection dropped to $29 \pm 5 \%$ and $26 \pm 5 \%$ in nonstimulation trials (Monkeys C and L, respectively). The same target positions were used for instructed trials. 
A

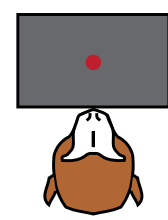

Fixation

onset

up to $500 \mathrm{~ms}$
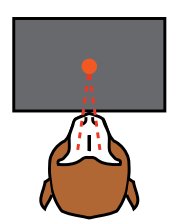

Fixation

$400-700 \mathrm{~ms}$
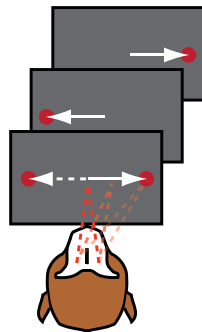

Target acquisition
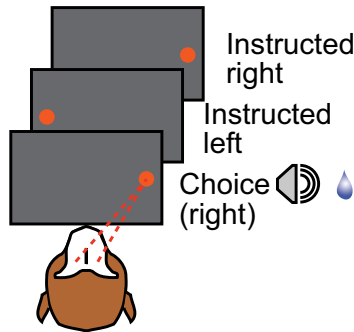

(right)

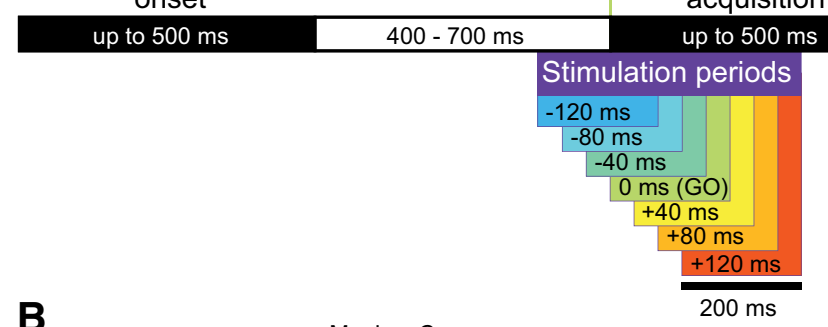

B

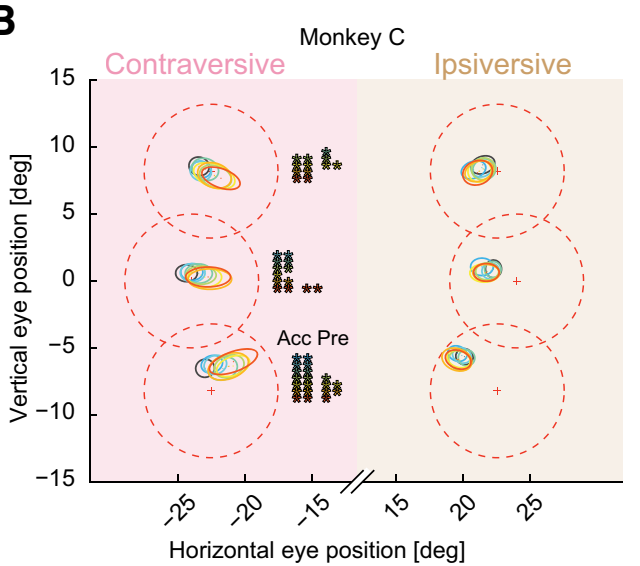

$200 \mathrm{~ms}$
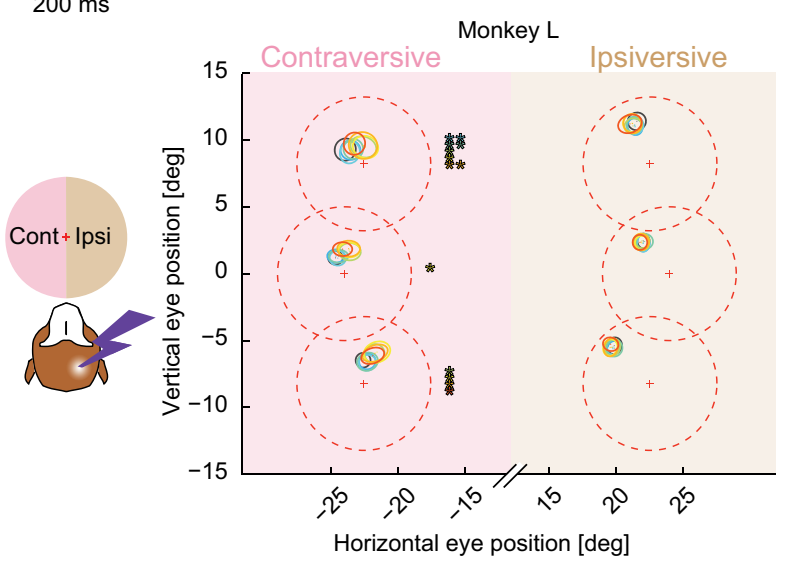

C
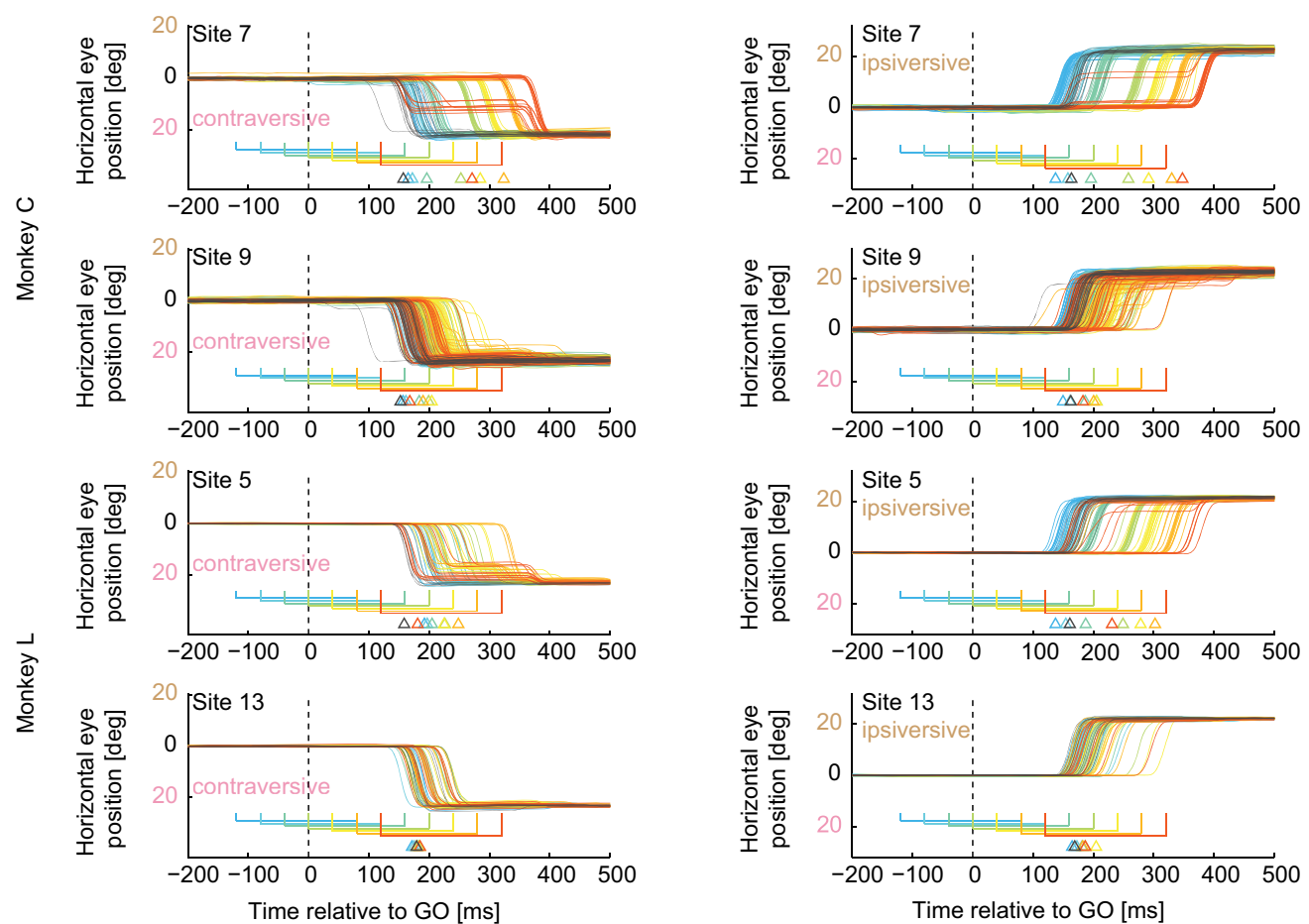

Figure 3. Visually-guided saccade task. $\boldsymbol{A}$, Task layout. Stimulation was delivered in one of seven different periods: starting before the target(s) onset ( $-120 \mathrm{~ms},-80 \mathrm{~ms}$, or $-40 \mathrm{~ms})$, at the $\mathrm{Go} \mathrm{signal,} \mathrm{or} \mathrm{after} \mathrm{the} \mathrm{Go} \mathrm{signal} \mathrm{(}+40 \mathrm{~ms},+80 \mathrm{~ms}$, or $+120 \mathrm{~ms}$ ). Trials without stimulation were interleaved as a control. The color code for each stimulation period is same for all following figures. $\boldsymbol{B}$, Saccade accuracy and end point scatter. Dashed red circles represent the allowed $5^{\circ}$ radius of target acquisition window; crosses represent the targets center. Half-axes of colored ellipses (control: gray) represent the means, across sessions, of SDs of radial and angular coordinates of end points; ellipse centers are means of mean end points across sessions. (Figure legend continues.) 
Table 2. Hit rates in the visually-guided saccade task, instructed trials, dorsal pulvinar stimulation (mean \pm SE across sessions)

\begin{tabular}{|c|c|c|c|c|c|c|}
\hline \multirow[b]{2}{*}{ Stimulation period onset } & \multicolumn{3}{|c|}{$\begin{array}{l}\text { Contraversive } \\
\text { hit rate }(\%)\end{array}$} & \multicolumn{3}{|c|}{$\begin{array}{l}\text { Ipsiversive } \\
\text { hit rate (\%) }\end{array}$} \\
\hline & $\begin{array}{l}\text { Both } \\
\text { monkeys }\end{array}$ & $\begin{array}{l}\text { Monkey } \\
\text { C }\end{array}$ & $\begin{array}{l}\text { Monkey } \\
\text { L }\end{array}$ & $\begin{array}{l}\text { Both } \\
\text { monkeys }\end{array}$ & $\begin{array}{l}\text { Monkey } \\
\text { C }\end{array}$ & $\begin{array}{l}\text { Monkey } \\
\text { L }\end{array}$ \\
\hline Control (no stimulation) & $99 \pm 0$ & $99 \pm 1$ & $100 \pm 0$ & $98 \pm 1$ & $100 \pm 0$ & $97 \pm 1$ \\
\hline-120 ms to $\mathrm{Go}$ & $100 \pm 0$ & $99 \pm 1$ & $100 \pm 0$ & $98 \pm 1$ & $98 \pm 1$ & $98 \pm 1$ \\
\hline$-80 \mathrm{~ms}$ to $\mathrm{Go}_{0}$ & $99 \pm 0$ & $99 \pm 1$ & $100 \pm 0$ & $99 \pm 0$ & $99 \pm 0$ & $99 \pm 1$ \\
\hline$-40 \mathrm{~ms}$ to $\mathrm{Go}_{0}$ & $99 \pm 0$ & $99 \pm 1$ & $100 \pm 0$ & $99 \pm 0$ & $100 \pm 0$ & $99 \pm 0$ \\
\hline Go (target onset) & $98 \pm 1$ & $98 \pm 1$ & $99 \pm 0$ & $97 \pm 1$ & $98 \pm 1$ & $96 \pm 1$ \\
\hline+40 ms from $\mathrm{Go}_{0}$ & $97 \pm 1$ & $96 \pm 1$ & $99 \pm 1$ & $98 \pm 0$ & $98 \pm 1$ & $98 \pm 1$ \\
\hline+80 ms from $\mathrm{Go}_{0}$ & $98 \pm 1$ & $97 \pm 2$ & $99 \pm 1$ & $97 \pm 1$ & $98 \pm 1$ & $97 \pm 1$ \\
\hline+120 ms from Go & $97 \pm 1$ & $95 \pm 2$ & $100 \pm 0$ & $96 \pm 1$ & $97 \pm 1$ & $95 \pm 2$ \\
\hline
\end{tabular}

Summary of the course of a session. After advancing the electrode to the desired location, the fixation task (with the fixation spot always in the center of the screen) was used to test for evoked saccades. This procedure defined the final electrode depth and the current strength. Next, a visually-guided saccade task was performed without stimulation and the stimulus array was shifted to find a regime in which the left and right target selection was approximately equalized (see "Target selection equalization" section above). After that, the control and stimulation data for the main visually-guided task was collected. For sessions in which monkeys also performed the memory-guided saccade task, target selection was equalized independently for the memory task because target preference differed between the two tasks.

\section{Data analysis}

Saccade definitions. Saccade velocity was calculated sample by sample as the square root of the sum of squared interpolated $(220 \mathrm{~Hz}$ to $1 \mathrm{kHz})$, smoothed (12 ms moving average rectangular window) horizontal and vertical eye position traces, and then smoothed again (12 ms moving average rectangular window). Saccade onset was defined as eye position change that exceeded a starting velocity threshold and the saccade offset as reaching an ending velocity threshold. For the fixation task, the starting and ending thresholds were $30 \% \mathrm{~s}$ and $15 \%$, respectively. For visuallyguided and memory-guided saccades, a starting velocity of $300 \%$ s and ending velocity of $50 \%$ sere used. For retrieving the saccade directions in error trials, the starting threshold was lowered to $150 \%$ secause eye position was not recorded after fixation breaks, so in some cases, the recorded velocity did not reach a high enough value before the trial and the recording were aborted. Saccade end point was defined as the eye position when the saccade velocity reached the ending threshold. In cases when several consecutive eye movements in the time interval from the Go signal until the target acquisition fitted the above criteria (e.g., due to interrupted saccades; see Results), the first saccade was selected for the reaction time (RT) analysis and the last one for the end point accuracy/ precision analysis.

Statistical analysis of behavioral data. All data analysis was performed using MATLAB R2012b. To test for changes in target selection preference within each session and the hit rates, Fisher's exact test was used. For all

(Figure legend continued.) Both monkeys showed reduced accuracy ("Acc," the distance from the target center to the mean end point) and increased end point scatter in the contraversive side of space (precision, "Pre," the radial component corresponding to ellipse major axis). The asterisks denote significant effects Acc and Pre separately for each target position $\left({ }^{*} p<0.05\right.$, ${ }^{* *} p<0.01$, Friedman with post hoc Wilcoxon signed-rank test, Bonferroni corrected). These accuracy and precision effects did not impair monkeys' ability to acquire any of the targets (Fisher's exact test within each session, Bonferroni corrected, $p>0.05$; Table 2). C, Horizontal eye position traces for the different stimulation periods in two example sessions in Monkey $\mathrm{C}$ (top two rows) and Monkey L (bottom two rows) for one pair of contraversive (left column) and ipsiversive (right column) targets, successful trials, aligned to the $\mathrm{Go} \mathrm{signal}(0 \mathrm{~ms}$, dotted vertical lines). The color of the traces represents corresponding stimulation periods, which are also shown as brackets below the traces (control: gray). The triangles below denote mean RT for each period. comparisons between two conditions across sessions, nonparametric tests were used. Whenever possible (i.e., same experimental conditions/ outcomes present in all stimulation periods and in all sessions), paired Friedman test with post hoc Wilcoxon signed-rank tests were used. Otherwise, Kruskal-Wallis test with post hoc Mann-Whitney U tests were used. Because the effects of multiple stimulation periods were tested against the control condition, for all post hoc tests and for Fisher's exact tests, the Bonferroni method was used to correct for multiple comparisons. To test for the relationship between two variables across sessions or across stimulation periods, Spearman's correlation coefficients were used. Statistical significance was reported at $p<0.05\left(^{*}\right)$ and $p<0.01$ $\left({ }^{*}\right)$. Specific statistical tests are listed for each individual analysis. In the figures and in the text, SD was used when averaging across trials and SE when averaging across sessions.

Analysis of neuronal activity. In the data from both monkeys, 230 single and multiunits for the visually-guided saccade task (140 Monkey C, 90 Monkey L) and 365 units for the memory-guided saccade task (251 Monkey C, 114 Monkey L) fulfilled analysis selection criteria (at least 50 spikes during the task periods; at least 60 instructed trials; typically 120 instructed trials, 10 instructed trials for each of the 12 targets). For recordings, the fixation hold period was $500 \mathrm{~ms}$, the memory period $1000 \mathrm{~ms}$, and the ITI period $1000 \mathrm{~ms}$; other parameters were same as in the stimulation runs. Target eccentricities were $12^{\circ}$ and $24^{\circ}$, arranged along the horizontal axis or at a $\pm 20^{\circ}$ angle from the horizontal axis. Spike sorting was done using Offline Sorter versions 4.0.0 and 2.8.8 (Plexon) for Monkeys $\mathrm{C}$ and L, respectively, using either a waveform template algorithm or a principle component analysis with k-means clustering algorithm.

For each trial and each epoch of interest, firing rates were computed by counting the spikes within the epoch and dividing the count by the epoch duration. The epochs analyzed in the visually-guided saccade task were "ITI" (400 to $100 \mathrm{~ms}$ before the onset of the central fixation spot), "fixation acquisition" (50 to $150 \mathrm{~ms}$ after acquiring central fixation), "fixation hold" (last $300 \mathrm{~ms}$ of central fixation), "target onset" (50 to $150 \mathrm{~ms}$ after target onset), "presaccadic" (100 to $10 \mathrm{~ms}$ before saccade onset), "perisaccadic" (10 ms before to $50 \mathrm{~ms}$ after saccade onset), "target acquisition" (50 to $120 \mathrm{~ms}$ after acquiring target fixation), and "target hold" (last $300 \mathrm{~ms}$ of fixating the peripheral target). For the memory-guided saccade task, "cue onset" (50-150 ms after onset of the cue) replaced the "target onset" and both will be referred to as "stimulus onset"; "target hold invisible" (first $100 \mathrm{~ms}$ of fixating the invisible peripheral target) replaced "target acquisition." Two additional epochs were also analyzed: "early memory" (first $200 \mathrm{~ms}$ of the memory period) and "late memory" (last $300 \mathrm{~ms}$ of the memory period).

For population analysis, data from six left and six right hemifield targets were combined. For each unit, a two-way ANOVA was performed across all firing rates in each of the respective epochs from successful instructed trials (same criteria as in "Behavioral tasks" section) using hemifield of the target position and epoch as factors for determining a main effect of epoch, hemifield, and interaction between the two. Spatial tuning in each epoch was determined by unpaired $t$ tests comparing firing rates in ipsilateral trials with firing rates in contralateral trials. The hemifield with the higher firing rate was marked if there was a significant difference. This analysis was performed only on units that showed either a main effect of hemifield or a hemifield $x$ epoch interaction.

Enhancement or suppression of neuronal activity (relative to fixation baseline, "fixation hold" epoch) in each subsequent epoch was defined by paired $t$ tests comparing firing rates for ipsilateral and contralateral trials independently. This analysis was only performed on units that showed either a main effect of epoch or hemifield $\times$ epoch interaction. Enhancement or suppression was reported if either ipsilateral, contralateral, or both types of trials showed a significant difference from fixation baseline. In rare cases in which one hemifield would show a significant enhancement while the other hemifield showed suppression, the unit was reported to have bidirectional response (example unit counts, memory-guided task, Monkey C/Monkey L: cue 2/1, perisaccade 2/0, target hold: 6/1; visually-guided task, Monkey C/Monkey L: target onset: $2 / 0$, perisaccade: $3 / 2$, target hold: $4 / 4$ )

For response field (RF) estimation, an independent one-way ANOVA was performed on firing rates during the stimulus onset epoch for each 


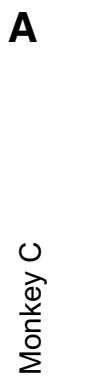

A

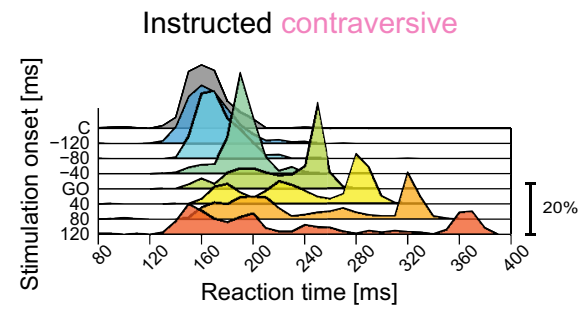

Instructed contra RT

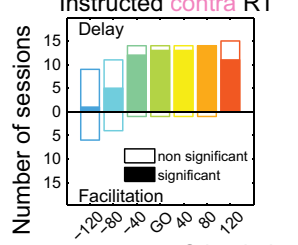

Stimulation onset [ms]
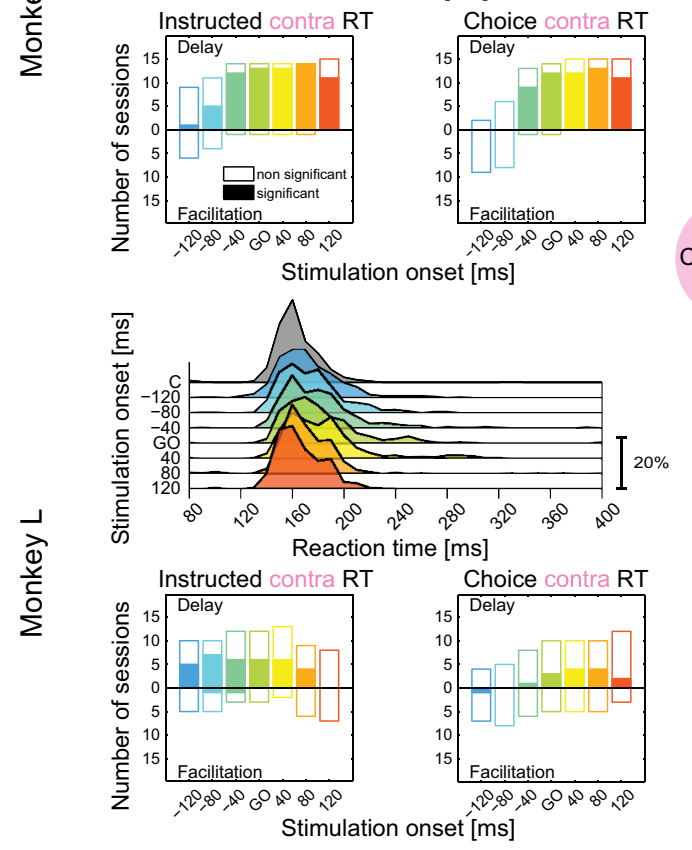

B
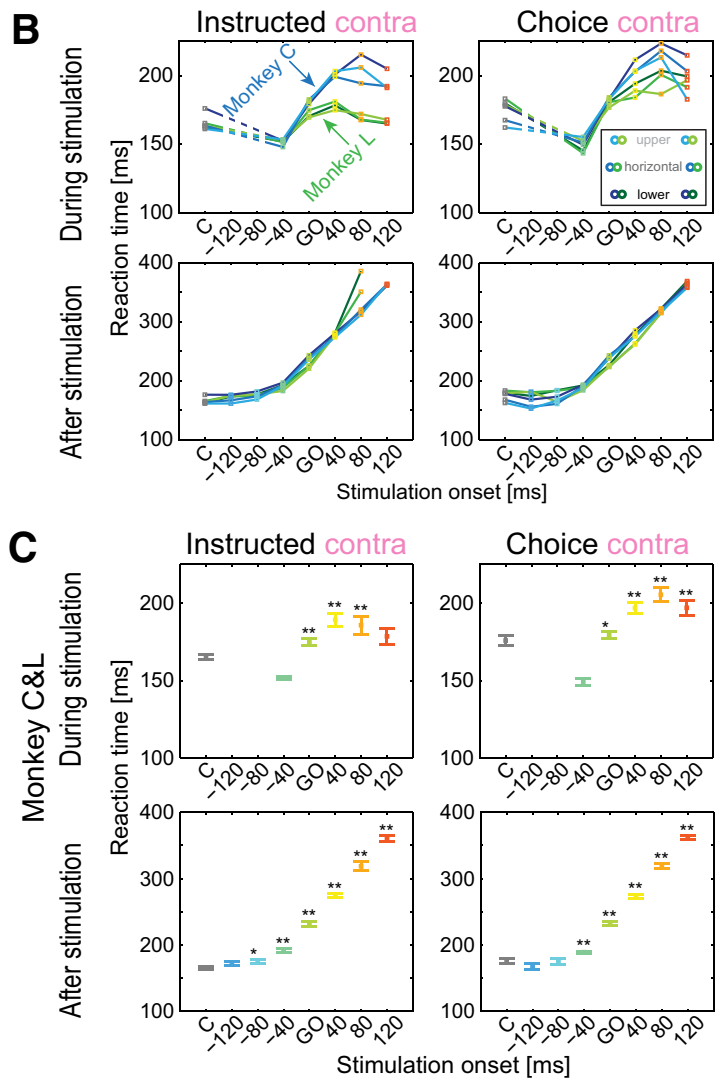
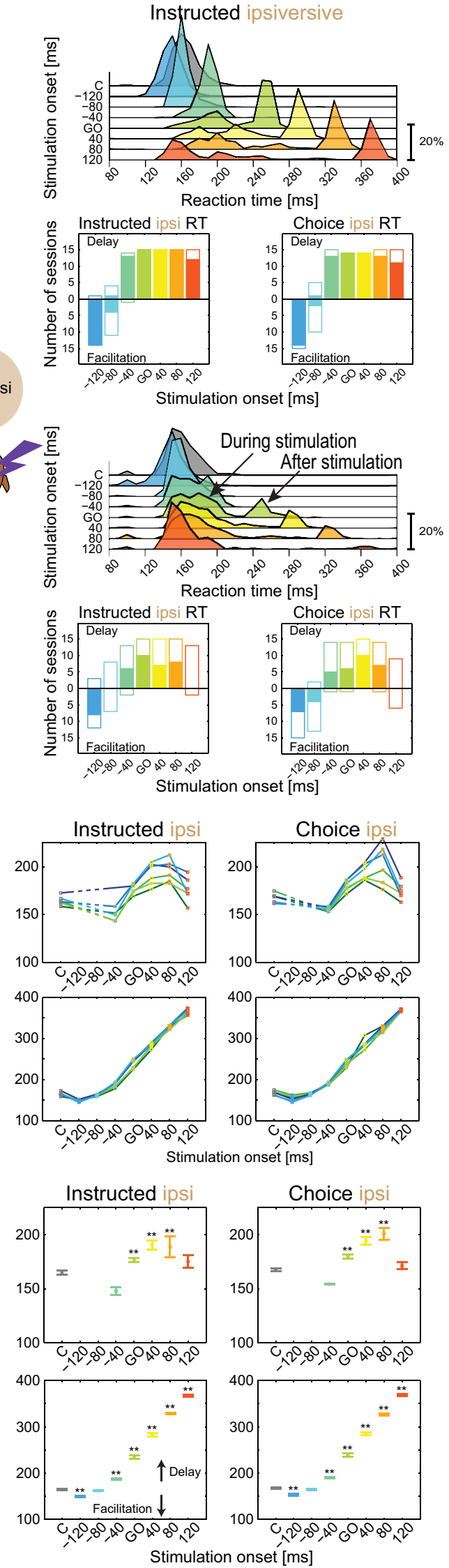

Figure 4. Effect of stimulation on RTs in the visually-guided saccade task. A, Stimulation effects on RTs for contraversive (left) and ipsiversive (right) saccades for Monkey C and Monkey L, respectively. Top, RT histograms (10 ms bin, normalized to $100 \%$ per condition) show data across all trials for each stimulation period. For stimulation onsets at or after the Go signal, the saccade initiation was delayed compared with control (gray). The saccade initiation was either delayed or arrested until the end of the stimulation period, resulting in a (Figure legend continues.) 
unit to determine the effect of target position. For defining a hemifield preference, the hemifield with the higher firing rate was marked if there was an effect of target position. For all units that showed an effect of target position, response modulation depth for each target position was calculated by averaging firing rates across trials, subtracting the lowest average firing rate across positions, and converting to percentage of maximal modulation depth. To estimate the center and size of the RFs, a 2D Gaussian was fitted to the modulation depth pattern. Six fitting parameters were determined using an iterative least-squares method ( 400 iterations), allowing elliptic RFs with peaks at the center. The size of the RF was defined by 2 SDs in each direction (semi-minor and semi-major axes). The fitting parameters were as follows: (1) the modulation depth in the center of the RF, (2) horizontal and (3) vertical location of the RF center, ( 4 and 5) ellipse major axis defined by 4 SDs and minor axis by aspect ratio, and (6) an angle of ellipse rotation. Importantly, the RF center was always kept within the dimensions of the target array $\left(-24^{\circ}\right.$ to $+24^{\circ}$ horizontally and $-8.2^{\circ}$ to $+8.2^{\circ}$ vertically). The amplitude was bounded by $50 \%$ and $150 \%$ of the original modulation depth, ellipse axes were bounded by $12^{\circ}$ (maximum horizontal distance between targets) and $48^{\circ}$ (target array extent), and maximum aspect ratio 4:1. Maximum modulation depth, the average of modulation-depth-weighted target positions (RF “center of mass"), intermediate major/minor axes $\left(30^{\circ} / 15^{\circ}\right)$, and a rotation of $0^{\circ}$ were used as starting values for the fits.

An averaged radius $(r)$ approximating the RF size was calculated by taking the square root of the product of the two axes of the elliptic RF. This way, $r^{2 \star} \pi$ always matches the area covered by the elliptic RF. RF size is reported as the diameter of the RF, $2^{\star} r$.

For target hold and "stimulus onset" epochs (cue onset for memoryguide saccades and target onset for visually-guided saccades) contralateral tuning indexes $(\mathrm{CI})$ for each unit were calculated as follows: $\mathrm{CI}=$ $\left(\mathrm{FR}_{\text {contra }}-\mathrm{FR}_{\mathrm{ipsi}}\right) /\left(\mathrm{FR}_{\text {contra }}+\mathrm{FR}_{\mathrm{ipsi}}\right)$, where $\mathrm{FR}_{\text {contra }}$ and $\mathrm{FR}_{\mathrm{ipsi}}$ are the average firing rate for all trials with targets in the contralateral and ipsilateral hemifield, respectively. Positive indexes indicate contralateral preference and negative indexes indicate ipsilateral preference.

To calculate population peristimulus time histograms, spike density functions of each trial, derived by convolution of the discrete spike arrival times with a Gaussian kernel (SD $20 \mathrm{~ms}$ ) were baseline corrected by subtracting the average ongoing firing rate in the late period of the ITI that immediately preceded the trial start (fixation spot onset). Average

$\leftarrow$

(Figure legend continued.) bimodal RT distribution for the late stimulation periods. In ipsiversive trials for the earliest stimulation period, $-120 \mathrm{~ms}$ to the Go signal, there was a facilitatory effect on saccade onsets. Bottom, Session by session directionality and significance of RT effect. For each stimulation period, each session RT that differed from control trials is shown as either a positive or negative bin, representing either delay or facilitation. Filled bins represent sessions in which the change from control was significant (Friedman followed by Mann-Whitney U test, Bonferroni corrected). It should be noted that, for ipsiversive saccades, the facilitation effects at the two early stimulation periods $(-120$ and -80$)$ were consistently present in both monkeys in both choice and instructed trials. B, Summary of RT effects for contraversive (left) and ipsiversive (right) saccades, separated by monkey (blue and green traces are for Monkey $C$ and Monkey L, respectively) and by vertical target position (light-, medium-, and dark-shaded traces denote upper, horizontal, and lower positions, see inset). Plots show saccades that started either during stimulation period (top row) or after stimulation offset (bottom row). Dashed lines in the top row connect control data with the next available stimulation data point (there were no correct saccades starting during -120 and -80 stimulation periods because this would abort fixation). C, Summary of RT effects for contraversive (left) and ipsiversive (right) saccades combined for the two monkeys and all vertical target positions (mean and SE across sessions). Top and bottom rows show saccades that started either during the stimulation period or after stimulation offset. Note that the $-40 \mathrm{~ms}$ period is a special case in which the separation into during and after stimulation does not provide meaningful information because the offset of this stimulation period ( $160 \mathrm{~ms}$ after the Go signal) happens at the same time as the mean onset of saccades in the control condition ( $165 \pm 2 \mathrm{~ms}$ and $165 \pm 2 \mathrm{~ms}$; contraversive and ipsiversive saccades, respectively, with both monkeys combined). Therefore, saccades that started during the $-40 \mathrm{~ms}$ stimulation period would by definition seem facilitated compared with control and saccades that started after stimulation would appear delayed. The RTs in all stimulation periods were compared with control trials (marked as " $\mathrm{C}^{\prime \prime}$ ) using Kruskal-Wallis followed by Mann-Whitney $U$ test, Bonferroni corrected $\left({ }^{*} p<0.05\right.$, ${ }^{* *} p<0.01$ ).

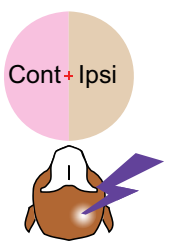

A

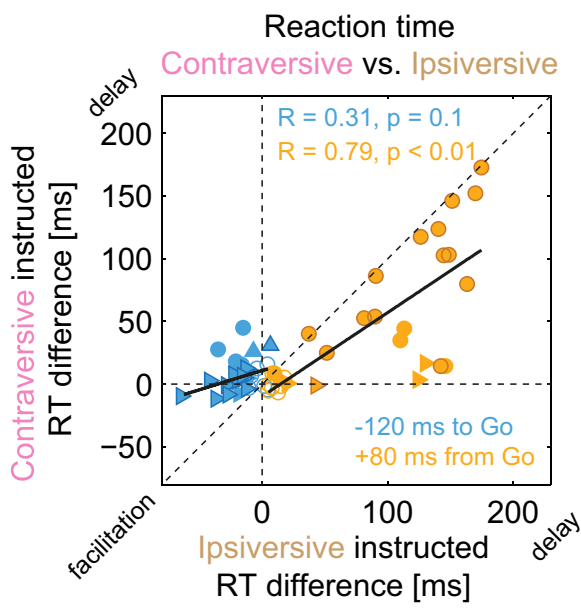

B

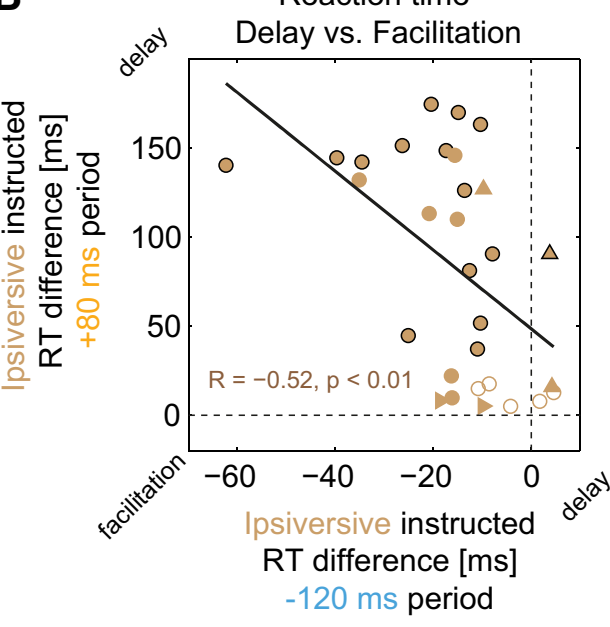

Figure 5. RT correlations across sessions. Two stimulations periods, -120 ms before and 80 ms after the Go signal, in which RT effects (facilitation and delay, respectively) were overall strongest and were selected for the correlation analysis. Data from both monkeys are combined in this and subsequent correlation plots. Filled circles indicate sessions in which both effects were significant, triangles indicate that only one of the effects was significant, and open circles indicate sessions with no significant change for any of them. $\boldsymbol{A}$, Contraversive versus ipsiversive RT difference (stimulation - control) in each session. For the early stimulation period $-120 \mathrm{~ms}$ before the Go signal (blue symbols), stronger facilitation (negative RT difference) in ipsiversive trials had an insignificant trend to correlate with shorter RTs in contraversive trials. For the late stimulation period +80 ms after the $G$ signal (orange symbols), delays (positive RT difference) in ipsiversive and contraversive trials were correlated, with most data points below the main diagonal (ipsiversive delay $>$ contraversive delay). $\boldsymbol{B}$, RT delay versus facilitation in ipsiversive saccades in each session. The facilitation due to stimulation in the $-120 \mathrm{~ms}$ period and the delay due to stimulation in the +80 period ms were correlated. Black lines show best linear fits. 


\section{A Spatial target selection modulation}
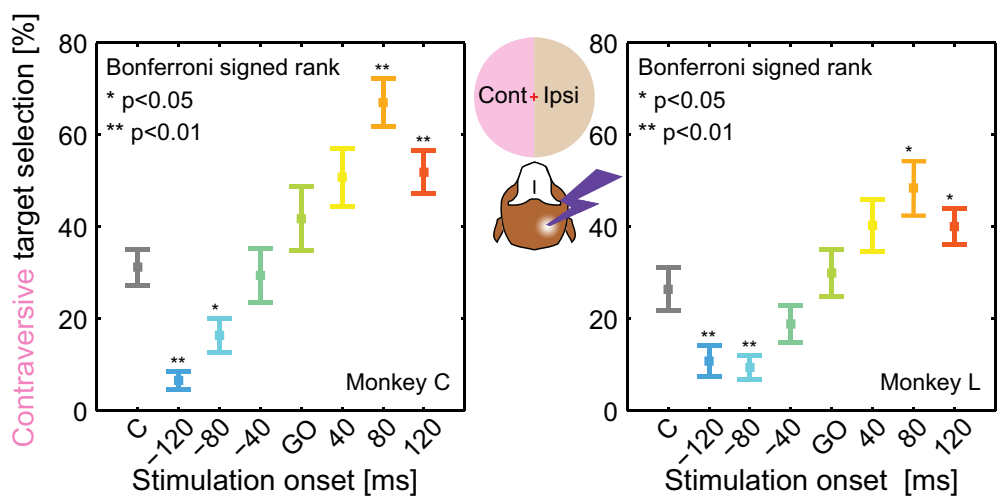

B Direction and significance of target selection modulation per session
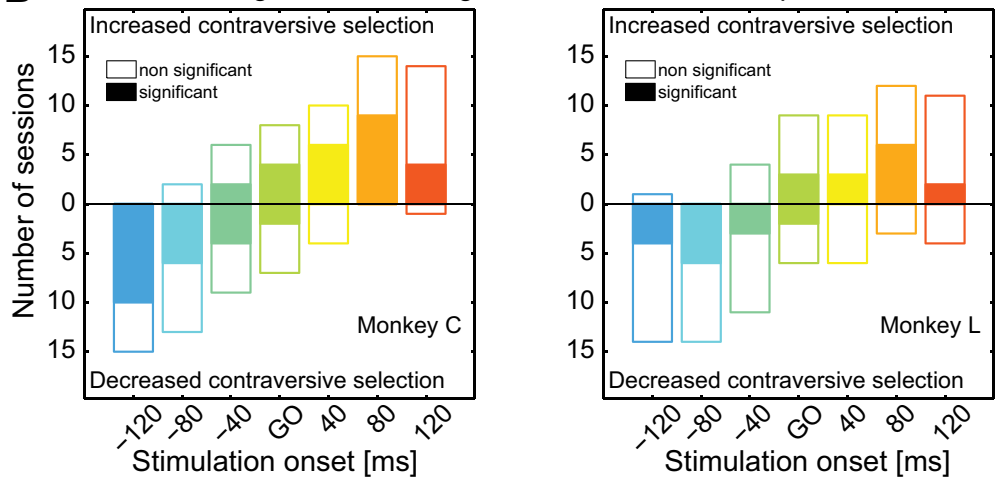

C Spatial target selection modulation per session
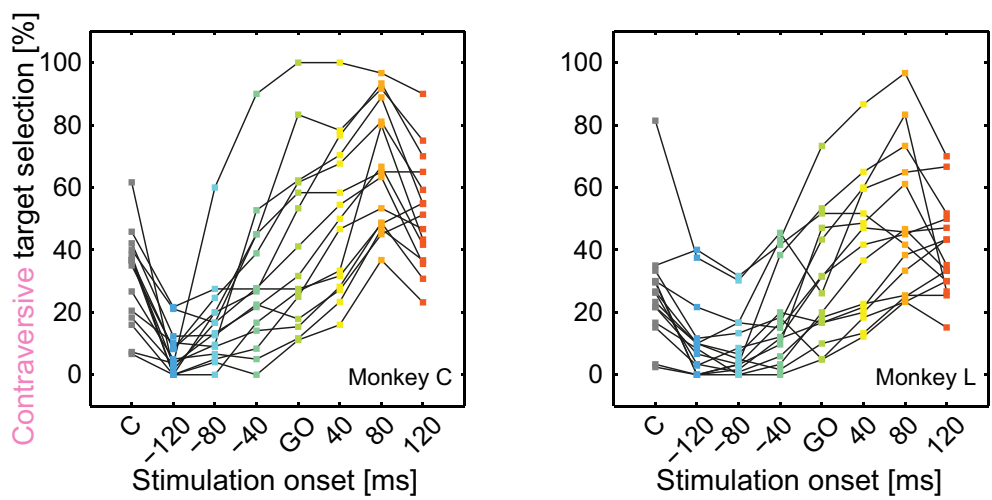

D Spatial target selection modulation per target position
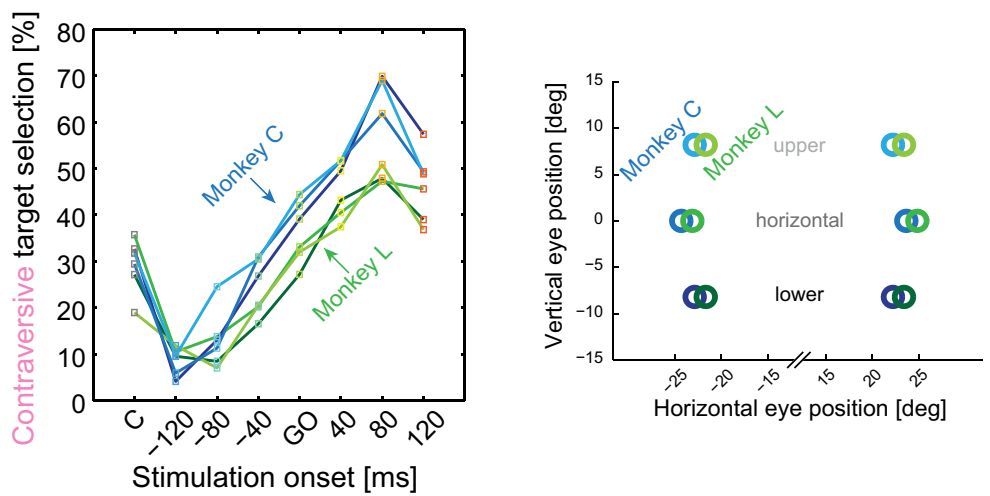

Figure 6. Effect of stimulation on target selection in visually-guided saccade task. $A$, Percentage of contraversive target selection as a function of stimulation periods. In control trials (marked as " $(")$, both monkeys showed an ipsiversive (right) target selection bias despite initial bias equalization (see Materials and Methods). In stimulation trials, current applied before the Go signal further decreased the selection of contraversive targets. Late stimulation periods increased contraversive target selection. responses for each unit were then derived by averaging the baseline-corrected spike density for each unit across all trials for the respective condition. Mean and SE of these baselinecorrected and averaged spike densities across units of a given subpopulation were calculated to display population responses. For better visualization of target position-dependent population cue response, instead of subtracting a baseline, the response was normalized by dividing each unit's response in all conditions by the same factor. That factor was defined as the peak firing rate during the cue onset epoch calculated across all trials (regardless of target position) in the preferred hemifield.

\section{Results}

Using an MRI-guided approach (see Materials and Methods), we stimulated the right dPul (Fig. 1, Table 1) and control sites in the vPul (see later section) in two monkeys performing three oculomotor tasks: fixation, visually-guided saccades, and memory-guided saccades (to instructed or chosen locations). The fixation task (Fig. 2A) was used to test for occurrence of evoked saccades and to characterize them if present. Monkey $\mathrm{L}$ did not exhibit evoked saccades in the regime tested; Monkey $\mathrm{C}$ showed predominantly small $\left(<2^{\circ}\right)$ contraversive saccades at $95 \pm 39 \mathrm{~ms}$ after the stimulation onset (Fig. 2C; Materials and Methods). The visually-guided task was used in the main experiment and the memory-guided task was used as a control for dissociating the cue processing, motor planning, and execution phases (see below). Both saccade tasks included $50 \%$ single-target-instructed trials and $50 \%$ choice trials between two equally rewarded targets located equidistantly from the central fixation spot at the same height.
Mean and SE across sessions, $p$-values from Friedman test followed by Wilcoxon signed-rank test, Bonferroni corrected $\left({ }^{*} p<0.05,{ }^{* *} p<0.01\right) . B$, Target selection modulation per session: direction and significance. For each session, we used Bonferroni-corrected Fisher's exact test to compare target selection in stimulation trials to control trials for each stimulation period. The direction of the effect is shown with a positive or negative vertical bar corresponding to increased or decreased contraversive selection; statistically significant sessions are filled. C, Percentage of contraversive target selection as a function of stimulation periods in individual sessions. Black lines connecting dots link data points from individual sessions. D, Target selection modulation per vertical position of left/right target pairs (right). The inset on the left shows the corresponding color code (Monkey C, blue; Monkey L, green; light-, medium-, and dark-shaded traces denote upper, horizontal, and lower target positions). 


\section{Visually-guided task: time-dependent RT facilitation and delay}

In the visually-guided saccade task (Materials and Methods), in stimulation trials, a $200 \mathrm{~ms}$ train was delivered at different periods relative to the target(s) onset and synchronous fixation spot offset, referred to as "Go" signal: before "Go" (early periods, blue-cyan colors), at "Go," or after "Go" (late periods, green-orange colors) (Fig. 3A). All trial conditions, instructed/choice, contraversive/ ipsiversive with respect to the stimulated right hemisphere (left hemifield/contraversive, right hemifield/ipsiversive), stimulation/no stimulation, and different stimulation periods, were interleaved randomly.

In instructed trials (single targets), the stimulation did not affect the hit rate (the fraction of successfully completed trials), which remained consistently high (Table 2). However, stimulation caused mildly hypometric saccades for contraversive locations: saccades were still initiated in the correct direction but often undershot, resulting in reduced end point accuracy and increased scatter along the saccade trajectory axis, predominantly in late stimulation periods during premovement and movement phases (Fig. 3B), similar to findings in SC (Schlag et al., 1989) and pre-SMA (Isoda, 2005).

The main effect of the stimulation on saccade performance consisted of changes in RTs. To illustrate this, we plotted the horizontal eye position as a function of time in the control (no stimulation) trials and in the different stimulation periods for two target positions in two example sessions in each monkey (Fig. $3 C)$. Three apparent effects of stimulation can be gleaned from these plots: (1) RT delay in most periods with saccade onsets either stereotypically deferred until after the stimulation offset (Fig. 3C, top example in each monkey) or delayed yet initiated during the stimulation (Fig. $3 C$, bottom example in each monkey) for saccades in both directions; (2) RT facilitation for ipsiversive saccades in early stimulation periods; and (3) occurrence of interrupted saccades (movement stopping in the mid-fly) in the late stimulation periods, especially for the contraversive targets.

Figure 4 quantifies RT effects across sessions. In control trials (gray), both monkeys had comparable RTs with unimodal distributions. The RT distributions for different stimulation periods confirmed that the stimulation predominately delayed the saccade initiation (Fig. 4A, top, in each monkey). The effect reached significance in a large proportion of individual sessions (Fig. $4 A$, bottom). Two distinct modes were evident upon inspection of RT distributions in Go and late period stimulation trials. As illustrated in examples shown in Figure $3 C$, the first mode contained saccades that started during the stimulation train, the second mode included saccades that started after the stimulation offset. Both effects were present in both monkeys, although Monkey L had fewer sessions in which the second mode was evident, especially for contraversive targets $(+40 \mathrm{~ms}$ period: three sessions in Monkey L, 14 sessions in Monkey C; +80 ms period: one session in Monkey L, nine sessions in Monkey C). Interestingly, there was a correlation between the depth of the microstimulation site and the probability of ipsiversive deferred saccades in both monkeys, suggesting that the occurrence of deferred saccades is site specific (but not monkey specific). In the subsequent analysis, we separated the saccades into these two categories ("during stimulation" and "after stimulation") and calculated a mean RT for each stimulation period across trials in each session and then across sessions (Fig. $4 B, C$ ). Figure $4 B$ plots the data separately for each monkey and for each vertical target position, demonstrating the consistency of RT effects. Monkey L showed weaker delays for saccades that started during stimulation (Fig. $4 B$, top row), espe- cially for contraversive instructed trials, but even in his data, the delay was significant across sessions (contraversive instructed: +40 ms simulation period, $p<0.05$; ipsiversive instructed: Go, $+40 \mathrm{~ms},+80 \mathrm{~ms}$ stimulation periods, $p<0.01$; Kruskal-Wallis followed by Bonferroni-corrected Mann-Whitney $U$ test). Sessions with saccades that were deferred until after stimulation offset were also present in both monkeys (Fig. 4B, bottom row). Therefore, in Figure 4C, we combined data from both monkeys.

Across all vertical target positions, saccades that started during stimulation were delayed by $10-26 \mathrm{~ms}$ (minimum to maximum) in the Go and late stimulation periods, with a maximal delay occurring in the $+40 \mathrm{~ms}$ or $+80 \mathrm{~ms}$ stimulation periods. The saccades with onsets that were deferred until the end of the stimulation were initiated $35 \pm 2 \mathrm{~ms}$ (contraversive) and $42 \pm 2 \mathrm{~ms}$ (ipsiversive) after the stimulation offset ( $37 \pm 2 \mathrm{~ms}$ and $48 \pm 1 \mathrm{~ms}$ in Monkey $\mathrm{C}$ and $30 \pm 6 \mathrm{~ms}$ and $33 \pm 3 \mathrm{~ms}$ in Monkey L)

The main difference between the effects in the two visual hemifields was the RT facilitation, present only for ipsiversive saccades in early stimulation periods $(-120 \mathrm{~ms}$ and to a lesser extent $-80 \mathrm{~ms}$ ), which all fell in the "after stimulation" category (Fig. 4C). This ipsiversive facilitation ( $16 \pm 3 \mathrm{~ms}$ in the $-120 \mathrm{~ms}$ period) was evident in the RT distributions (cf. gray and blue distributions), was significant in 14 of 15 sessions in Monkey C and in 8 of 15 sessions in Monkey L, and was significant in each monkey across sessions ( $p<0.01$ Monkey C, $p<0.05$ Monkey L, Friedman test with post hoc Wilcoxon signed-rank test). Another difference between the effects in the two hemifields was that, whereas RT delays followed a similar pattern for contraversive and ipsiversive saccades, the effect was stronger for the ipsiversive side (individually in each monkey, $p<0.05$ for all late stimulation periods, Friedman test with post hoc Wilcoxon signed-rank test, Bonferroni corrected). For example, in the $+80 \mathrm{~ms}$ stimulation period, the delay was $46 \pm 10 \mathrm{~ms}$ for contraversive and $83 \pm$ $11 \mathrm{~ms}$ for ipsiversive saccades $(p<0.01)$. This observation will be considered when looking at the choice behavior (see below).

The relationship of RT delays between contraversive and ipsiversive saccades is further illustrated by the scatter plot of ipsiversive versus contraversive delays in the $+80 \mathrm{~ms}$ stimulation period (RT difference, stimulation minus control) across sessions, showing a strong correlation between the two delays (Fig. 5A; Spearman's $r=0.79, p<0.01)$. No significant contraversiveipsiversive correlation was found for RT effects at $-120 \mathrm{~ms}$ stimulation period.

We also investigated whether there was a relationship between facilitation and delay effects across sessions in two representative early ( $-120 \mathrm{~ms})$ and late $(+80 \mathrm{~ms})$ stimulation periods for ipsiversive saccades that showed both effects. Indeed, there was a strong correlation between the facilitation and the delay (Fig. 5B; Spearman's $r=-0.52, p<0.01)$. Sessions that showed more facilitation in the early stimulation period also had more delay in the late stimulation period, indicating a shared influence of session-by-session variations in stimulation effectiveness. This relationship is in contrast to the opposite effect (less facilitation, more delay) found in the caudate nucleus (Watanabe and $\mathrm{Mu}-$ noz, 2011).

Finally, very similar effects on saccadic RTs were found for choice trials, including the facilitation of ipsiversive saccades in early stimulation periods (Fig. 4) and a larger delay for ipsiversive choices compared with contraversive choices (+80 ms stimulation period: $55 \pm 10 \mathrm{~ms}$ contraversive, $83 \pm 12 \mathrm{~ms}$ ipsiversive, $p<0.01$, Kruskal-Wallis with post hoc Mann-Whitney $U$ test, Bonferroni corrected). 


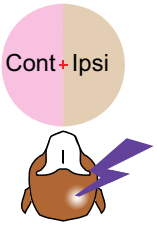

A

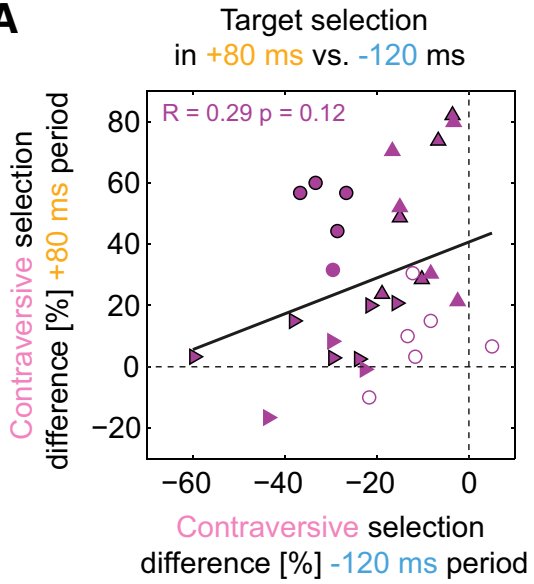

B Target selection vs. reaction time
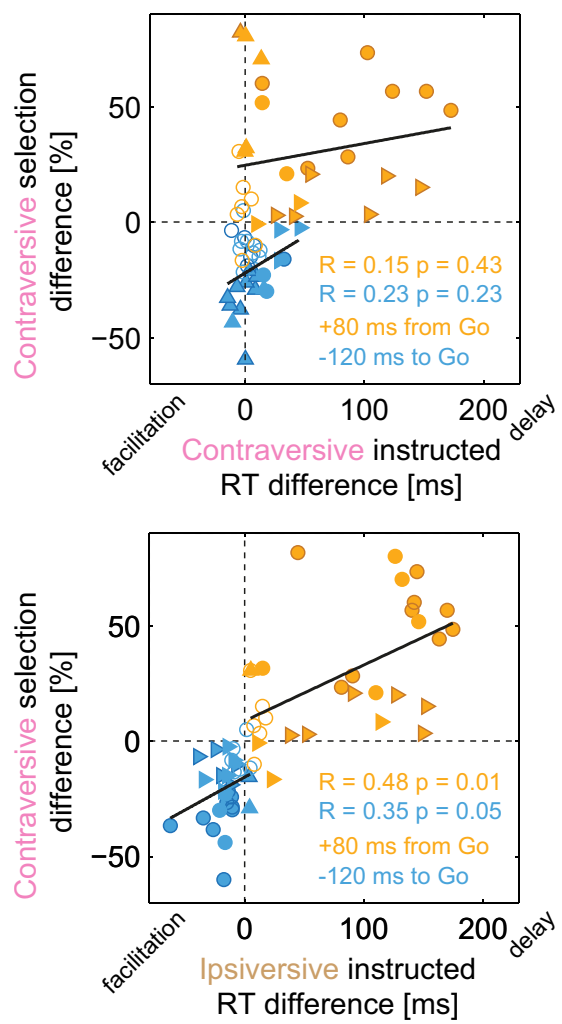

\begin{tabular}{|c|c|}
\hline \\
\hline \multicolumn{2}{|c|}{$\begin{array}{l}\text { Monkey } \\
\text { C L }\end{array}$} \\
\hline$\triangle \triangle$ & Significant session vertical axis \\
\hline$\triangleright>$ & Significant session horizontal axis \\
\hline 00 & Significant session in both axes \\
\hline 00 & Non significant \\
\hline
\end{tabular}

Figure 7. Target selection and RT correlations across sessions. $A$, Contraversive target selection difference (stimulation - control) in $-120 \mathrm{~ms}$ vs $+80 \mathrm{~ms}$ stimulation periods in each session. There was a weak, insignificant correlation between contraversive bias at the $+80 \mathrm{~ms}$ period and ipsiversive bias at the -120 ms period. $\boldsymbol{B}$, Contraversive target selection difference

\section{Visually-guided task: time-dependent spatial} choice modulation

In agreement with predictions from our previous pulvinar inactivation results (Wilke et al., 2010, 2013), dPul stimulation increased contraversive target selection, but only in late stimulation periods in which the train was delivered after the target onset/Go signal, during the decision and motor preparation phase (Fig. $6 A$ ). Surprisingly, stimulation in early periods, which started before but ended after the target onset, led to a decrease in contraversive selection (Fig. 6A). This biphasic modulation of spatial choice preference was a consistent pattern across sessions (Fig. $6 B, C)$, showing maximal ipsiversive bias in the $-120 \mathrm{~ms}$ or -80 ms periods and maximal contraversive bias in the $+80 \mathrm{~ms}$ period. Furthermore, this pattern was consistent across upper, horizontal, and lower vertical target positions (Fig. 6D).

Given the resemblance of the choice effect to the modulation of ipsiversive RTs (first facilitation, then delay), we investigated whether time courses of changes in RTs and target selection across stimulation periods were similar. To this end, we correlated the mean percentage of contraversive selection with the mean ipsiversive choice RT, across stimulation periods (the ipsiversive choice RT was chosen to comprise both delay and facilitation RT effects), and found a strong linear correlation (Spearman's $r=0.99, p<0.001$, Monkey C; $r=0.89, p=0.012$, Monkey L; a similar effect was found for the correlation with ipsiversive instructed RTs: $r=0.96, p=0.003$, Monkey C; $r=$ 0.93, $p<0.001$, Monkey L). This demonstrates the temporal congruency of choice and RT effects: in early stimulation periods, the ipsiversive choice bias was accompanied by the (ipsiversive) RT facilitation and, as stimulation onsets progressed toward the later decision and motor planning phases of a trial, the contraversive choice bias was accompanied by the RT delay.

To investigate whether ipsiversive bias in early stimulation periods and contraversive bias in late stimulation periods might represent manifestations of the same neural mechanism, we correlated the strength of both effects across sessions. For the early -120 ms period, we found only an insignificant tendency for a stronger ipsiversive bias to be associated with a weaker contraversive bias in the late $+80 \mathrm{~ms}$ period (Fig. 7A; Spearman's $r=0.29$, $p=0.12$ ). We also tested the $-80 \mathrm{~ms}$ period instead of $-120 \mathrm{~ms}$ period and found a stronger positive correlation (Spearman's $r=$ $0.65, p<0.01)$. Note that the positive correlation signifies an inverse relationship between strength of early ipsiversive and late contraversive bias. Therefore, at least on a session-by-session level, the relationship between the strength of the two effects is not straightforward, suggesting that factors other than overall stimulation effectiveness, for example, variations of sessionspecific spatial preferences, might play a role. Notably, this is the only aspect we found to be incongruent between directions of the across-sessions trends for RT and choice: recall that, for the ipsiversive RT, a stronger early facilitation was associated with a stronger, not weaker, late delay (cf. Fig. 5B).

It is important to emphasize, however, that most sessions exhibited a biphasic course of choice modulation (Fig. 6C) and, in five sessions, both effects reached significance even after the con-

$\leftarrow$

versus RT difference for the $-120 \mathrm{~ms}$ and $+80 \mathrm{~ms}$ stimulation periods for contraversive (top) and ipsiversive (bottom) saccades in each session. There was a weak, insignificant correlation between ipsiversive bias and RT changes in the $-120 \mathrm{~ms}$ period (blue symbols) for both contraversive and ipsiversive saccades and a strong correlation between contraversive bias and RT delay in the +80 ms period only for ipsiversive saccades (orange symbols). 


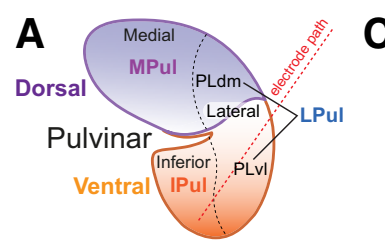

C
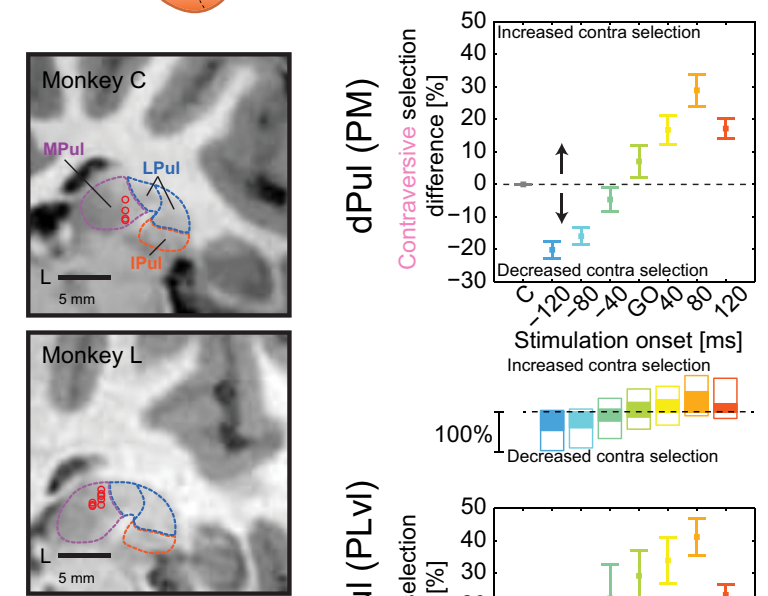

B
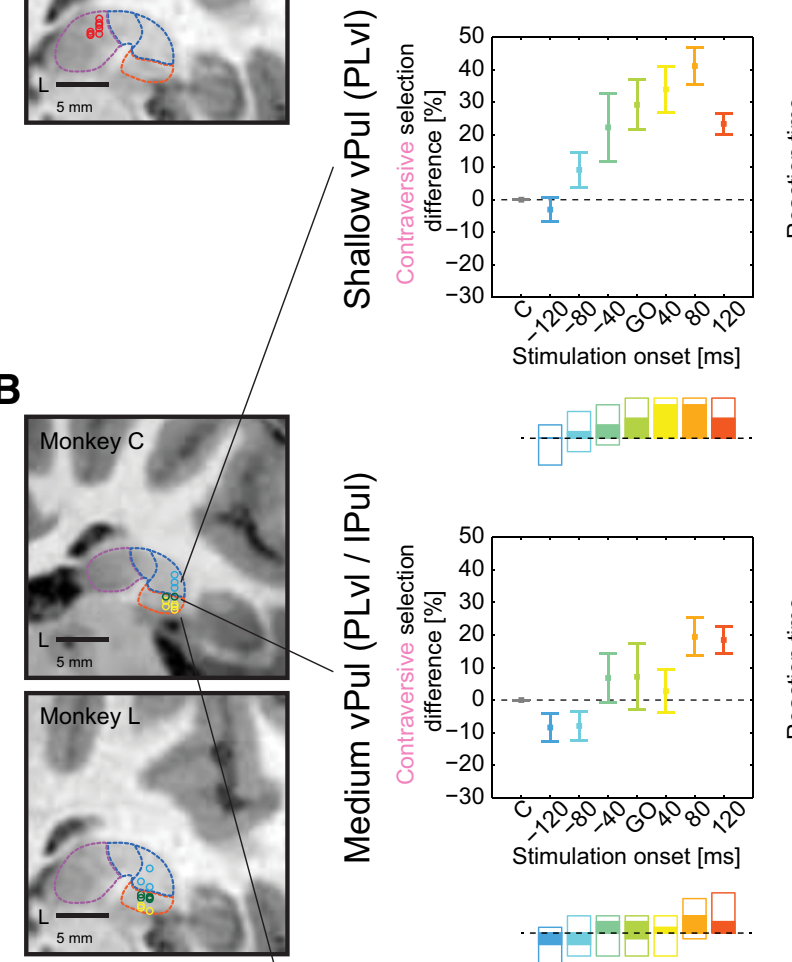

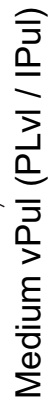
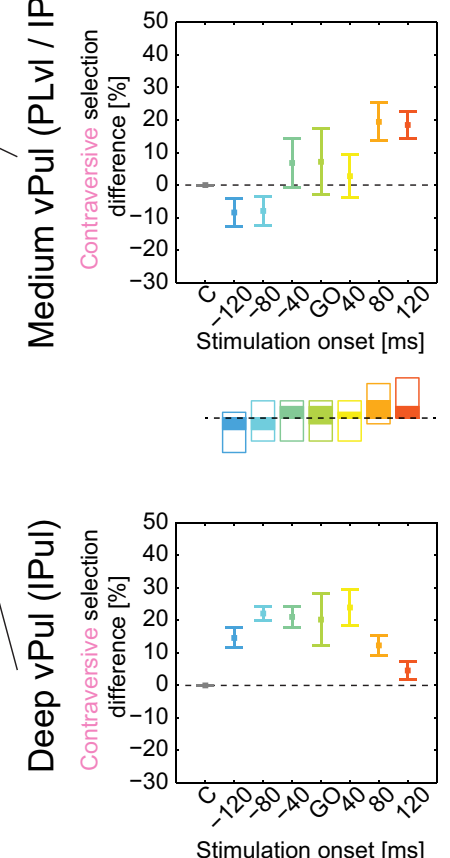

Stimulation onset [ms]

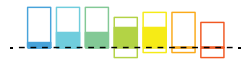

Cont + Ipsi

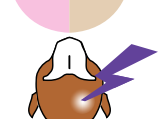

RT difference instructed

contraversive

ipsiversive
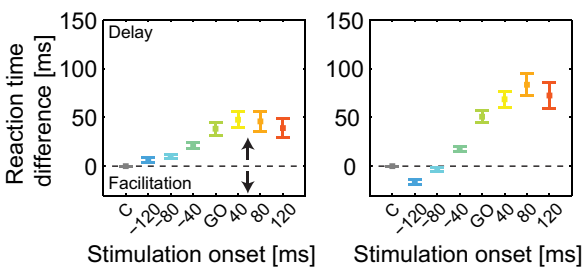

Stimulation onset [ms]
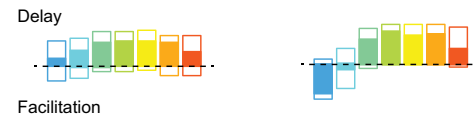

Facilitation
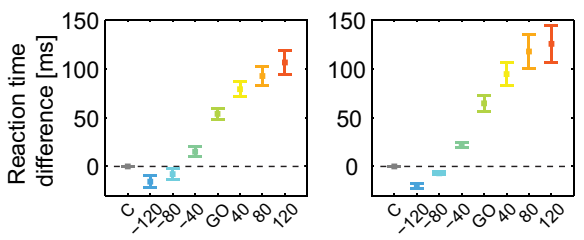

Stimulation onset [ms] Stimulation onset [ms]

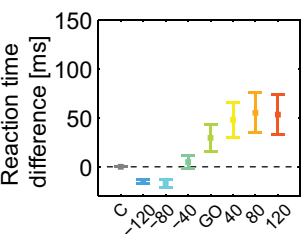

Stimulation onset [ms]

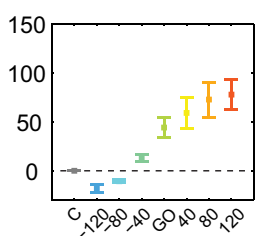

Stimulation onset $[\mathrm{ms}]$
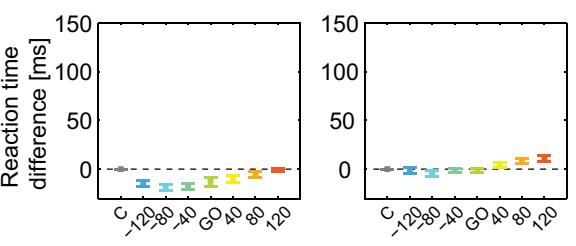

Stimulation onset $[\mathrm{ms}] \quad$ Stimulation onset $[\mathrm{ms}]$

Figure 8. Summary of stimulation effects in dPul and vPul. $A$, Top, Pulvinar parcellation schemes: dorsal versus ventral; medial, lateral, and inferior; with the lateral nucleus further subdivided to PLdm and PLvl. Bottom, Stimulation sites localization in the dPul (Monkey C: grid $y=5$; Monkey L: grid $y=3$ ), chamber normal coronal sections. B, Stimulation sites localization in the vPul (Monkey C: grid $y=4$; Monkey L: grid $y=3$ ), chamber normal coronal sections. C, In addition to 30 stimulation sites in dPul (top row), we performed control stimulations in 21 sites in vPul to assess the specificity of the stimulation effects on target selection and saccade generation. We binned these sites into three depth groups: shallow (six sessions, three in (Figure legend continues.) 
servative Bonferroni correction for all seven stimulation periods (Fig. 7A). Therefore, the biphasic choice modulation illustrated in Figure $6 \mathrm{~A}$ is not a consequence of averaging across sessions with either only early or only late period effects.

Similarly, to test the relationship between the choice bias and the RT effects, we correlated the stimulation-induced changes in the RT with the changes in choice preference across sessions. The increase of contraversive choices in the late $+80 \mathrm{~ms}$ period correlated with the RT delay for ipsiversive instructed saccades, but not with the RT delay for contraversive instructed saccades (Fig. $7 B$; Spearman's $r=0.15, p=0.43 ; r=0.48, p=0.01$, for contraversive instructed and ipsiversive instructed RT, respectively). The same dependency was observed for choice RT delay $(r=$ $0.09, p=0.64 ; r=0.50, p<0.01$, for contraversive choice and ipsiversive choice RT, respectively). Conversely, the decrease in contraversive choices in the early $-120 \mathrm{~ms}$ period was only weakly and insignificantly correlated with the RT changes in both contraversive and ipsiversive instructed saccades. Therefore, the contraversive choice bias in the late periods was associated with stronger, likely subjectively undesirable, ipsiversive delays. This reasoning is further supported by strong positive correlations between the contraversive selection increase versus ipsiversive contraversive RT difference in all but the two earliest stimulation periods (Spearman's $r>0.5, p<0.01$ for -40 , Go, +40 , and +120 periods, $r=0.44, p=0.02$ for +80 period).

We interpret the opposite direction effects in the early and the late stimulation periods as different manifestations of the same stimulation-induced mechanism. Decrease of contraversive choices and shortening of ipsiversive RTs suggest an ipsiversive orienting tendency due to the stimulation in the early periods starting before the Go signal. Conversely, increase of contraversive choices and stronger ipsiversive RT delay in the late stimulation periods point to a contraversive drive. To reconcile these findings, we propose that the effect of pulvinar activation is invariably contraversive and the apparent ipsiversive orienting is the consequence of a compensatory process that takes place due to behavioral task demands. In brief, when the stimulation is delivered in the early periods, while monkeys are tasked with maintaining fixation, they are (at least partially) suppressing or opposing the detrimental contraversive eye movements and this ipsiversive push-back against the stimulation-induced contraversive drive "spills over" beyond the stimulation offset to the interval after the Go signal when the decision and motor preparation take place. In agreement with this interpretation, but on a longer time scale across trials, in blocks of trials without stimulation, the contraversive target selection was higher than in the control (no stimulation) trials interleaved with the stimulation trials during stimulation blocks, suggesting that monkeys exhibited an ipsiversive tendency when "released" from stimulation (blocked-interleaved difference $14.6 \pm 5.3 \%, p<0.05$ for Mon-

$\leftarrow$

(Figure legend continued.) Monkey ( and three in Monkey L), medium (seven sessions, two in Monkey ( and five in Monkey L), and deep (eight sessions, five in Monkey C and three in Monkey L). Due to the angled chamber orientation, as we advanced deeper, we also targeted more medial and anterior parts of the vPul. One depth group is shown per row. For all rows, the left column, upper subpanel is the target selection difference from control, mean and SE across sessions and the bottom subpanel is the direction and significance of the stimulation effect per session normalized to the number of sessions ( $100 \%$ scale bar). The significance in each session was assessed using Fisher's exact test, Bonferroni corrected, filled colors $(p<0.05)$. Middle and right columns are data for instructed contraversive and ipsiversive RT effects, with significance in each session assessed using Kruskal-Wallis test followed by Mann-Whitney $U$ test, Bonferroni corrected. key C, $14.3 \pm 6.5 \%, p<0.01$ for Monkey L, mean \pm SE, Wilcoxon signed-rank test on differences). This and other alternative explanations are further considered in the Discussion.

\section{Visually-guided task: dPul versus vPul}

The effects of dPul stimulation were robust and consistent across multiple sites in both monkeys. To test for the site specificity of those effects, we conducted a series of control stimulation experiments in the vPul targeting different depths along the electrode track (Fig. $8 A, B$ ). Figure $8 C$ summarizes the results of the experiments as a function of electrode depth. The stimulation in shallow vPul sites, which correspond to the PLvl according to the parcellation of Kaas and Lyon (2007), resembled the patterns obtained in the main dPul experiment, with the exception of bilateral (not only ipsiversive) RT facilitation and no clear ipsiversive choice bias in the early stimulation periods. Deeper sites ("medium vPul") at the estimated border between the ventrolateral and inferior pulvinar showed similar but weaker stimulation effects. In contrast, deepest sites ("deep vPul") in the inferior pulvinar exhibited a distinct pattern: a contraversive bias in all but very late stimulation periods, a contraversive RT facilitation (no delay) in the same periods, and only very small RT effects for the ipsiversive saccades. It is worth noting that, in 3 cases for Monkey $\mathrm{C}$ and 4 cases for Monkey L at least 2 different electrode depths were used in the same penetration and, on such days, the neighboring sites were separated only by $1-2 \mathrm{~mm}$ but still elicited distinct behavioral patterns. This suggests that the effects of stimulation were markedly localized to specific portions of surrounding tissue.

\section{Memory-guided task: dissociating cue processing and motor planning phases}

The late stimulation periods in the visually-guided task started during the visual target presentation concurrently with the ensuing decision and saccade planning. To assess whether the stimulation effect on choices was due to affected visual, decision, or motor processing stages, we used a memory-guided saccade task and delivered the stimulation to the $\mathrm{dPul}$ in four different trial periods: before cue onset, after cue onset, before the Go signal, and after the Go signal (Fig. 9A). As shown in Figure 9B, there was no effect on target selection in any of the stimulation periods, although stimulation in the same sites and sessions during the visually-guided task elicited a consistent biphasic choice bias as described above (Fig. 9C). At the same time, and consistent with the visually-guided task, memory saccade RTs in the contraversive space were delayed with stimulation before and especially after the Go and the ipsiversive saccades were facilitated by the stimulation before the Go and strongly delayed by the stimulation after the Go (Fig. 9D).

In addition, the stimulation affected the memory-guided task hit rates (Tables 3, 4). Specifically, the two conditions in which the hit rate dropped $<80 \%$ in the stimulation trials in both monkeys were as follows: (1) instructed ipsiversive trials when the stimulation started before cue ( $-80 \mathrm{~ms}$ cue) and (2) instructed contraversive trials when the stimulation was applied after the Go signal (+80 ms from Go). Error trial eye position trajectories (Fig. 9E) showed that most errors in the instructed ipsiversive trials were fixation aborts due to saccades toward the ipsiversive cue $(79 \%$ of error trials with the stimulation onset before the cue), with a latency of $74 \pm 14 \mathrm{~ms}$ (mean \pm SD) after stimulation offset. The same effect was observed in choice trials; even in the presence of two opposite cues, monkeys tended to break fixation 


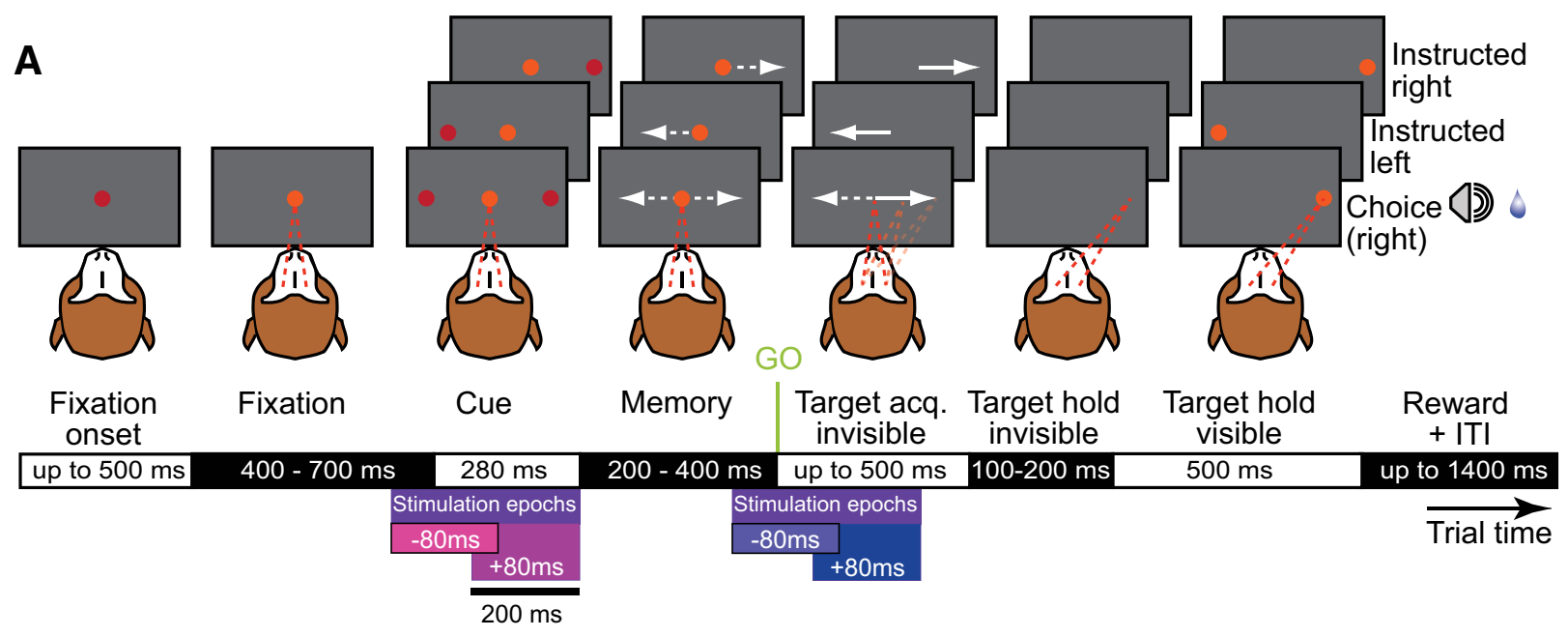

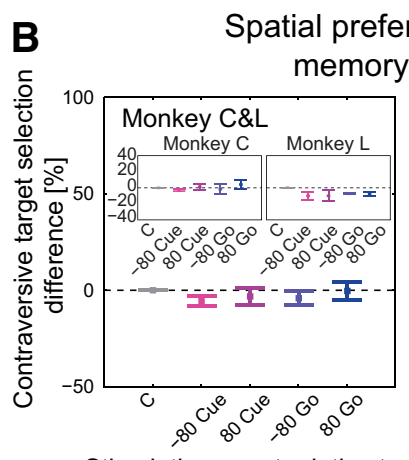

Stimulation onset relative to Cue and GO [ms]

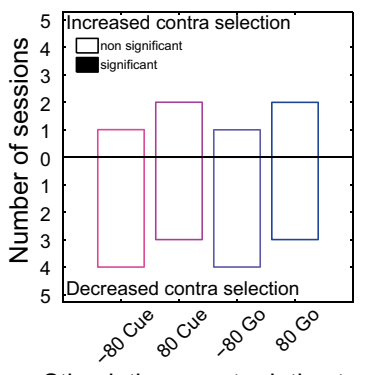

Stimulation onset relative to Cue and GO [ms]

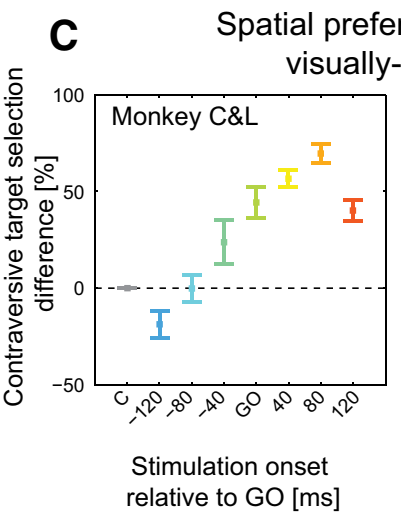

visually-guided task

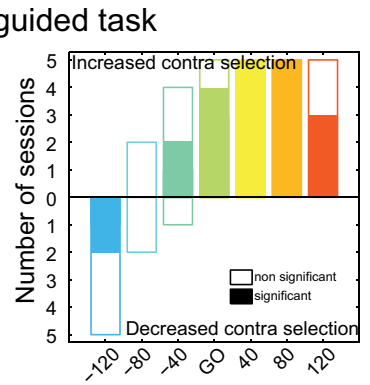

Stimulation onset relative to $\mathrm{GO}[\mathrm{ms}]$

D Reaction time difference, memory-guided task

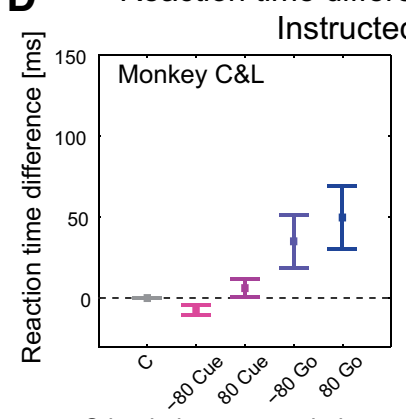

Stimulation onset relative to

Cue and GO [ms]

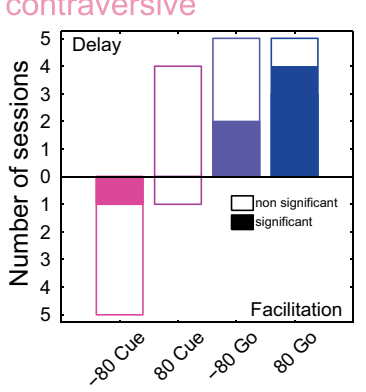

Stimulation onset relative to Cue and GO [ms]

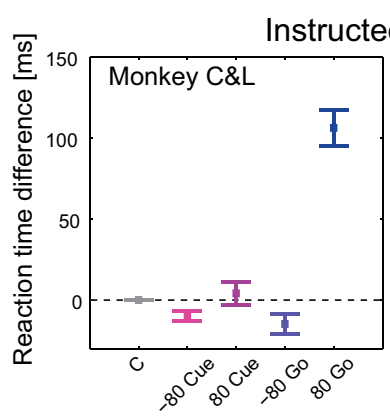

Stimulation onset relative to Cue and GO [ms]

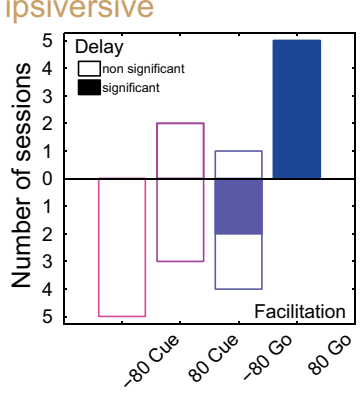

Stimulation onset relative to Cue and GO [ms]

\section{E Error trials in memory sessions}
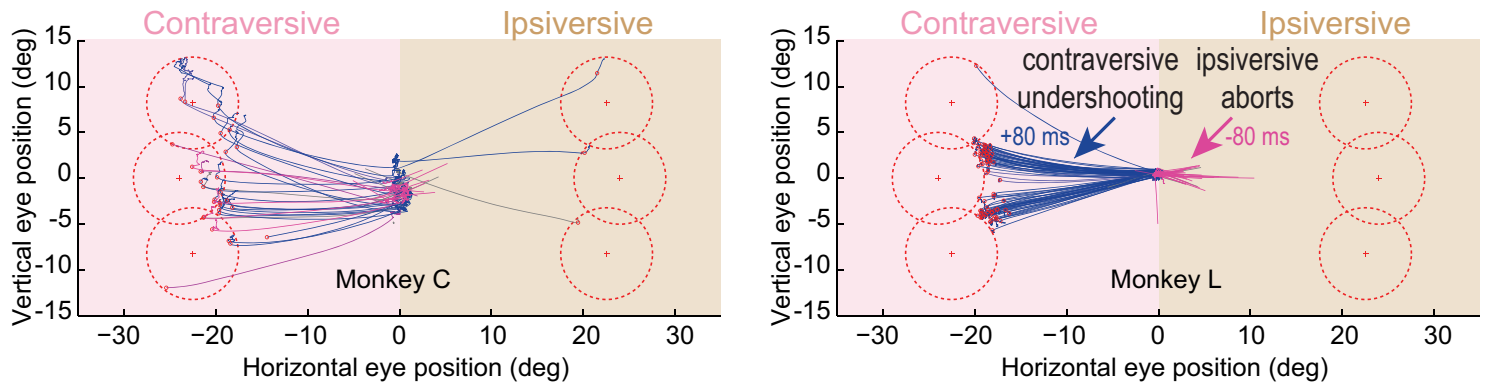

Figure 9. Stimulation effects in memory-guided saccade task (dPul). $\boldsymbol{A}$, Task layout. Stimulation was delivered in one of four periods: starting before onset of the visual cue(s) ( $-80 \mathrm{~ms}$ Cue), after the onset of the visual cue(s) ( $+80 \mathrm{~ms}$ (ue), before the $\mathrm{Go}$ signal ( $-80 \mathrm{~ms} \mathrm{Go}$ ), or after the $\mathrm{Go}$ signal ( $+80 \mathrm{~ms} \mathrm{Go}$ ). Trials without stimulation were interleaved as a control. $\boldsymbol{B}$, $\boldsymbol{C}$, Target selection in the memory-guided task (B) and in the visually-guided saccade task in the same sites and sessions ( $\boldsymbol{C}$ (five sessions and three sessions in Monkey $\boldsymbol{C}$ and two sessions in Monkey $\mathrm{L}$ ), mean and SE of contraversive selection difference from control, and direction and significance of preference change from control (Fisher's exact test, Bonferroni corrected). (Figure legend continues.) 
Table 3. Hit rates in the memory-guided saccade task, instructed trials, dorsal pulvinar stimulation (mean \pm SE across sessions)

\begin{tabular}{|c|c|c|c|c|c|c|}
\hline \multirow[b]{2}{*}{ Stimulation period onset } & \multicolumn{3}{|c|}{$\begin{array}{l}\text { Contraversive } \\
\text { hit rate }(\%) \\
\end{array}$} & \multicolumn{3}{|l|}{$\begin{array}{l}\text { Ipsiversive } \\
\text { hit rate (\%) } \\
\end{array}$} \\
\hline & $\begin{array}{l}\text { Both } \\
\text { monkeys }\end{array}$ & $\begin{array}{l}\text { Monkey } \\
\text { C }\end{array}$ & $\begin{array}{l}\text { Monkey } \\
\text { L }\end{array}$ & $\begin{array}{l}\text { Both } \\
\text { monkeys }\end{array}$ & $\begin{array}{l}\text { Monkey } \\
\text { C }\end{array}$ & $\begin{array}{l}\text { Monkey } \\
\text { L }\end{array}$ \\
\hline Control (no stimulation) & $96 \pm 2$ & $93 \pm 3$ & $100 \pm 0$ & $89 \pm 8.3$ & $82 \pm 13$ & $100 \pm 0$ \\
\hline-80 ms to Cue onset & $94 \pm 5$ & $90 \pm 7$ & $100 \pm 0$ & $73 \pm 9 *$ & $74 \pm 14$ & $71 \pm 11^{*}$ \\
\hline+80 ms from Cue onset & $96 \pm 2$ & $94 \pm 3$ & $98 \pm 2$ & $91 \pm 8$ & $84 \pm 13$ & $100 \pm 0$ \\
\hline-80 ms to $\mathrm{Go}_{0}$ & $89 \pm 5$ & $87 \pm 7$ & $92 \pm 8$ & $84 \pm 15$ & $74 \pm 25$ & $98 \pm 2$ \\
\hline+80 ms from $\mathrm{Go}_{0}$ & $53 \pm 14^{*}$ & $52 \pm 22^{*}$ & $54 \pm 21^{*}$ & $89 \pm 7$ & $84 \pm 12$ & $97 \pm 0$ \\
\hline
\end{tabular}

In two stimulation periods, $-80 \mathrm{~ms}$ to ipsiversive cue onset and $+80 \mathrm{~ms}$ from Go in contraversive trials, there was a drop in performance $<80 \%$ (in bold and with asterisk, significant in at least one session, Fisher's exact test, Bonferroni corrected, $p<0.05)$. Hit rate drops $<80 \%$ that did not reach significance are in italics.

Table 4. Hit rates (a fraction of successfully completed trials regardless of chosen hemifield) in the memory-guided saccade task, choice trials, dorsal pulvinar stimulation (mean \pm SE across sessions)

\begin{tabular}{|c|c|c|c|}
\hline \multirow[b]{2}{*}{ Stimulation period onset } & \multicolumn{3}{|l|}{ Hit rate (\%) } \\
\hline & Both monkeys & Monkey C & Monkey L \\
\hline Control (no stimulation) & $92 \pm 4$ & $89 \pm 6$ & $97 \pm 2$ \\
\hline$-80 \mathrm{~ms}$ to Cue onset & $76 \pm 4^{*}$ & $78 \pm 7^{*}$ & $72 \pm 1^{*}$ \\
\hline+80 ms from Cue onset & $93 \pm 6$ & $88 \pm 10$ & $99 \pm 1$ \\
\hline-80 ms to Go & $89 \pm 6$ & $85 \pm 9$ & $95 \pm 0$ \\
\hline$+80 \mathrm{~ms}$ from $\mathrm{G}_{0}$ & $74 \pm 15^{*}$ & $67 \pm 25^{*}$ & $86 \pm 4^{*}$ \\
\hline
\end{tabular}

Similar to instructed trials, in two stimulation periods, $-80 \mathrm{~ms}$ to cue onset and $+80 \mathrm{~ms}$ from $\mathrm{Go}$, there was a drop in performance (in bold and with asterisk, significant in at least one session, Fisher's exact test, Bonferroni corrected, $p<0.05$ ). Note that because these were two-target free-choice trials, we did not assign aborted, incomplete trials to left or right choices; therefore, here, the trials are not divided into contraversive and ipsiversive. However, the plot of eye position trajectories in error trials, similar to Figure $9 E$ for the instructed trials, demonstrated similar effects: saccades to the ipsiversive cue after the offset of early $-80 \mathrm{~ms}$ stimulation and contraversive undershooting in $+80 \mathrm{~ms}$ from Go late stimulation period (plot not shown).

by making saccades to the ipsiversive side ( $88 \%$ of error trials with the stimulation onset before the cue). Therefore, monkeys had difficulty suppressing reflexive saccades to the ipsiversive cues after stimulation offset, which is consistent with our interpretation of ipsiversive orienting in the early stimulation periods in the visually-guided task. Note that this does not contradict the absence of stimulation effect on choices: if the fixation was maintained and thus trials were not aborted, then subsequent choices were not affected.

Most errors in the second condition, the contraversive trials, were due to contraversive undershooting ( $85 \%$ of instructed and $64 \%$ of choice error trials with the stimulation onset after the Go signal). Monkeys were more severely affected by the stimulation during the motor preparation and response phase when there were no visible targets to guide it compared with the visuallyguided task, which showed milder hypometria with no drop in hit rates (cf. Fig. 3B, Table 2). But even taking into account those undershooting error choice trials that were directed toward con-

\footnotetext{
(Figure legend continued.) Insets in $\boldsymbol{B}$ for the memory-guided task show data for each monkey. $\boldsymbol{D}$, Effect of stimulation on RT in the memory-guided saccade task for instructed contraversive (left) and ipsiversive (right) trials. For both hemifields, panels on the left show the mean and SE across sessions and panels on the right show direction and significance of effect per session; significance in each session was assessed by Kruskal-Wallis test with post hoc MannWhitney $U$ test, Bonferroni corrected. E, Eye position traces during instructed memory-guided saccade error trials, with two sessions combined for each monkey (Monkey C on the left and Monkey L on the right). Trajectories are colored according to the period in which stimulation occurred; trials in which no stimulation was delivered are gray. There were two periods in which both monkeys showed a considerable decrease in the hit rate ( $<80 \%$; Table 3): before the Cue onset and after the Go signal. Errors in trials in which stimulation was delivered before the Cue onset were mostly fixation aborts toward the ipsiversive cue after the stimulation period ended. Errors after the Go signal were mostly hypometric saccades that did not reach the target window.
}

traversive targets, the choice was not significantly modulated by the stimulation in +80 from Go period in any of the sessions $(p>$ 0.05, Fisher's exact test).

Given the lack of choice effects, we considered the possibility that the delayed RTs are a consequence of stimulation interfering with the processing of fixation point offset (i.e., Go signal), but we deem it unlikely given the similarity to RT delays in the visually-guided task (where peripheral target onset coinciding with the fixation offset served as even more apparent Go signal) and the spatially specific difference between contralateral and ipsilateral RT delays (ipsiversive delay $>$ contraversive delay).

These results give rise to several important implications. First, the choice-relevant aspects of cue processing seem unaffected when they are temporally dissociated from the motor response. Similarly, the stimulation does not seem to affect the choice when the decision can be formed in advance of the action, neither in the cue/memory period nor just before or during the motor response. Third, the RT and the choice effects, which were largely congruent in the visually-guided task, were dissociated in the memory-guided task and thus might not depend critically on each other. This dissociation is reminiscent of recent perceptual decision study in the caudate (Ding and Gold, 2012). Like basal ganglia, the pulvinar is involved in multiple functional loops (Sherman and Guillery, 2002) and different populations or pathways might encode distinct processes. Together, the results of visually-guided and memory-guided tasks indicate that the transient pulvinar stimulation contributes to the spatial decision process only when the choice must be formed and executed close in time.

\section{Neuronal properties in the dPul}

To better understand the neural contribution of dPul to the behavioral effects of microstimulation, we analyzed the activity of $230 \mathrm{dPul}$ units recorded in the visually-guided saccade task and $365 \mathrm{dPul}$ units in the memory-guided saccade task in and around the same stimulation sites (see Materials and Methods). Dorsal pulvinar units predominantly showed low firing rates (mean firing rate across all task periods: 10 and 11 spikes/s, median: 6 and 7 spikes/s, SD: 10 and 11 spikes/s, for the visually-guided and memory-guided task, respectively).

Visual RFs were estimated offline using an array of 12 target positions $\left(12^{\circ}\right.$ and $24^{\circ}$ eccentricity). Cue responses for each target position in the memory-guided saccade task were fitted with a $2 \mathrm{D}$ Gaussian profile (see Materials and Methods). The position of the Gaussian peak and the area covered by two Gaussian SDs to each side defined center and size of the RF. Figure $10 \mathrm{~A}$ illustrates firing patterns and RF estimation in one example unit. Here, we refer to the (visual) RFs as those computed in the cue epoch, but RFs could also be computed during eye movements and peripheral fixation. As can be seen in the example for the peripheral target 
A

Contralateral trials Ipsilateral trials

Spike density, example unit
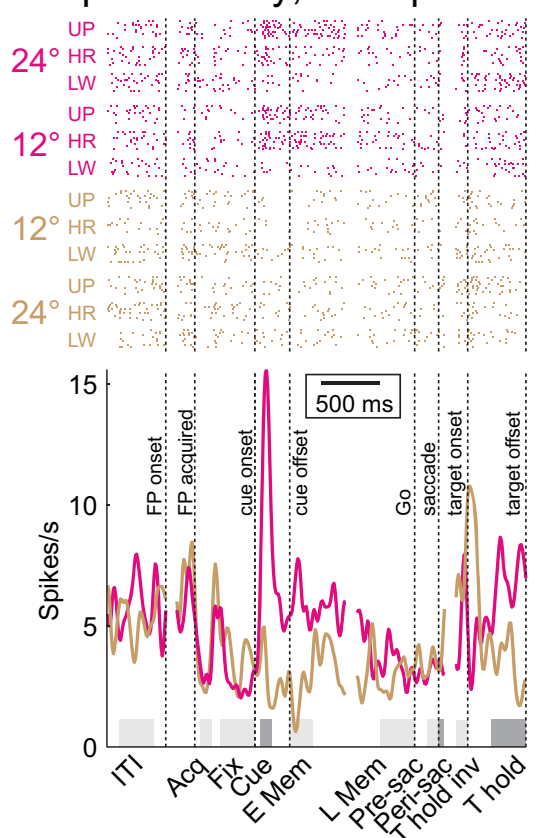

Cont+ + psi
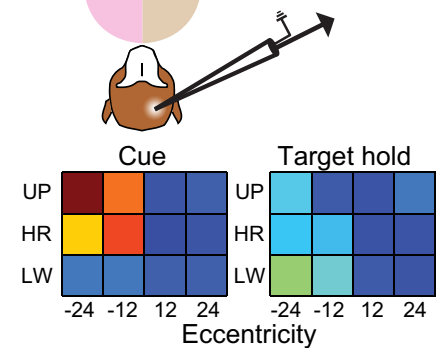

Spikes/s

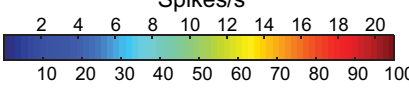

$\%$ of modulation depth

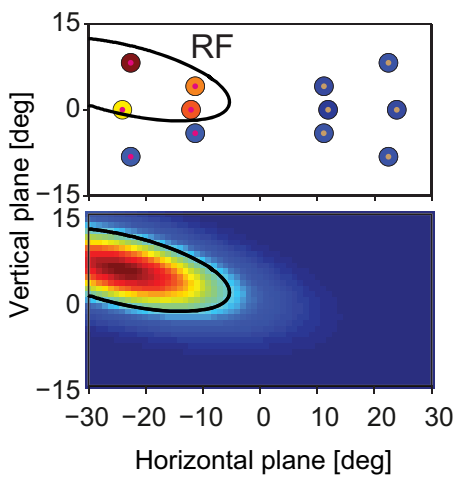

B

Contralateral population $(\mathrm{N}=58)$

Ipsilateral population $(\mathrm{N}=10)$

Population cue tuning

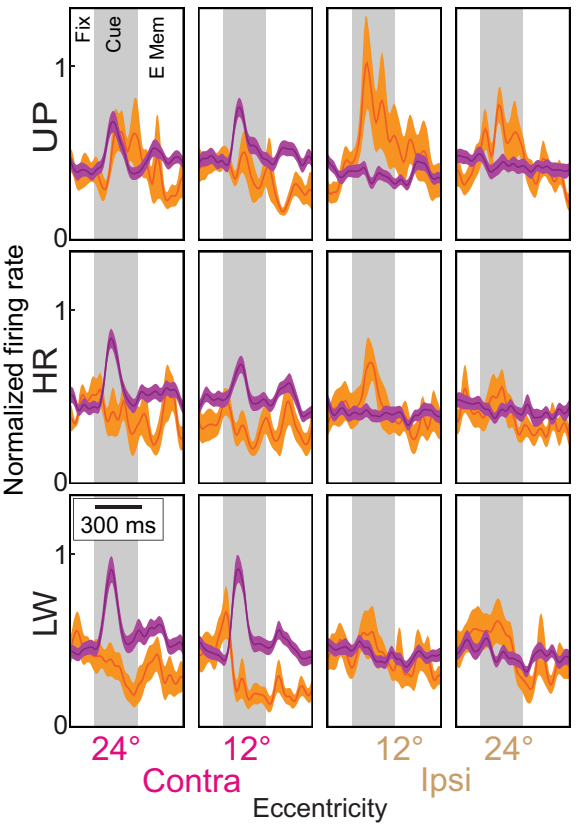

C Visual response fields, memory task $(\mathrm{N}=68)$

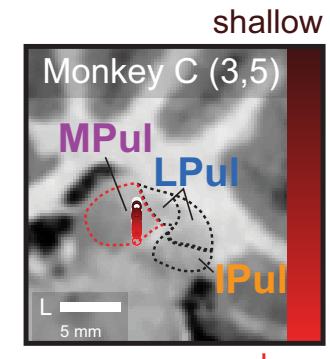

deep

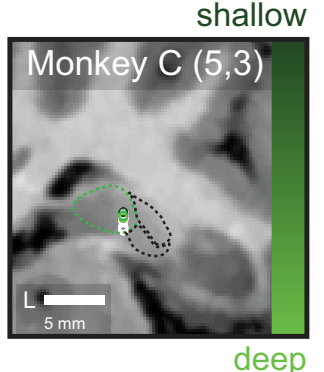

deep

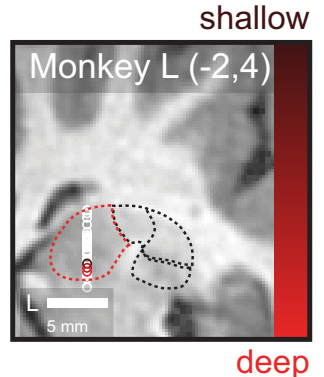

deep

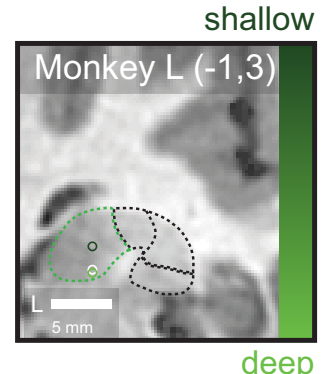

deep

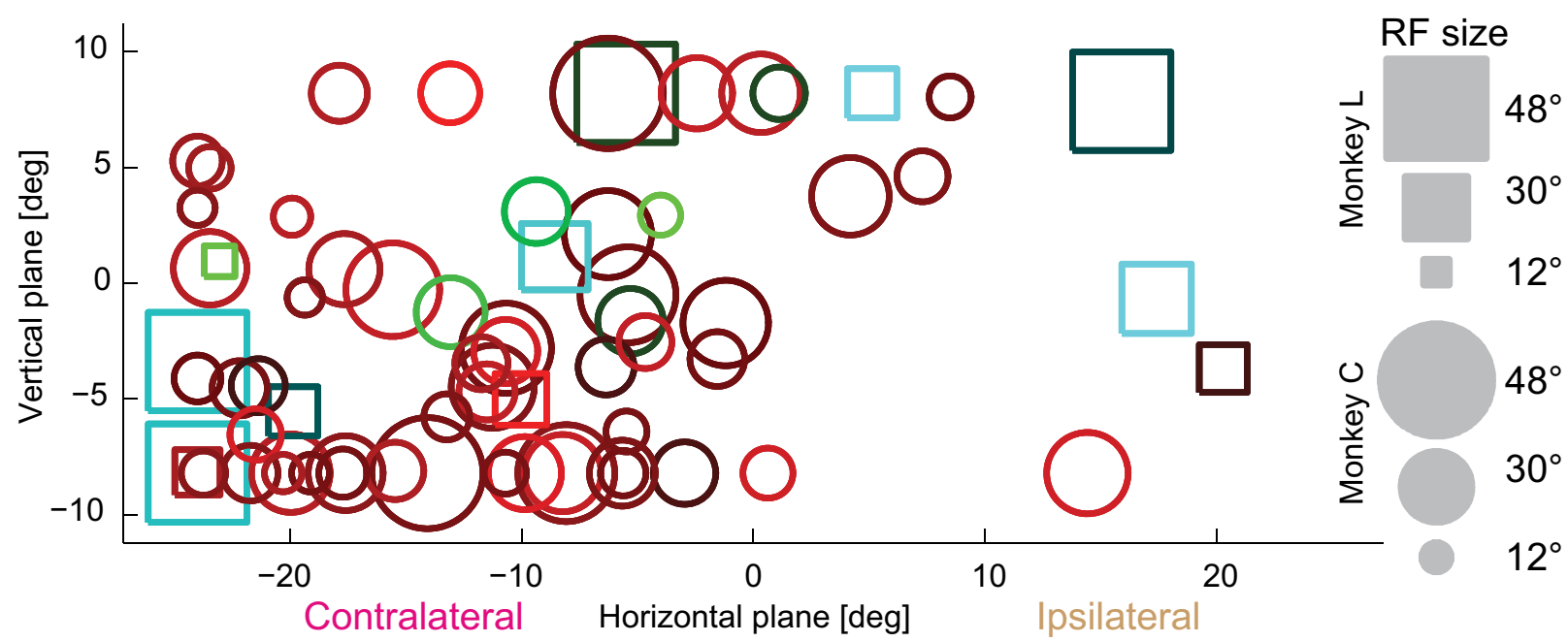

Figure 10. Spatial RF properties in the dPul. A, Offline RF estimation in an example unit (Cur_20150617_03). Left, Raster plot and resulting spike density functions (SDFs) shown separately for contralateral and ipsilateral trials in magenta and brown, respectively. Trials are grouped by hemifield, eccentricity, and vertical target position, upper (UP), horizontal (HR), and lower (LW). For the raster plot and SDF, black dotted lines denote events: fixation point onset ("FP onset"), acquiring fixation ("FP acquired"), cue onset, cue offset, and beginning of the memory period, offset of the central fixation point (which also served as the Go signal, "Go"), saccade onset, and onset and offset of the peripheral target. Discontinuous traces indicate gaps in (Figure legend continues.) 
hold epoch in Figure 10A, postsaccadic RFs can differ from visual RFs, as has been reported in the PLdm (Robinson et al., 1986).

The RF estimation was performed on all units showing a main ANOVA effect of stimulus position during the cue period (68 units). We separated this subset further by each unit's preferred hemifield and looked at the population cue response for each of the 12 target positions in contralaterally and ipsilaterally tuned subsets (Fig. 10B). Even though the contralateral subset was much larger (58 units) than the ipsilateral subset (10 units), contralateral population cue response was more time locked than ipsilaterally tuned responses. In addition, contralateral responses seemed to be more consistent across targets, with a small preference for lower and more peripheral targets.

Figure $10 C$ illustrates the estimated RFs at a scale of 1:10 in a plot representing the visual target array field, with colors representing different recording sites. RF centers were scattered across the entire tested visual field and their size varied substantially. Note that, because RF centers were constrained to the dimensions of the target array, it may seem as if many RFs are clustered along those borders. However, our mapping and fitting approach did not allow drawing conclusions about potential RF centers outside of the target array. Typically, RF estimates were large $\left(28 \pm 9^{\circ}\right)$ and most of them had their centers in the contralateral hemifield (mean eccentricity $=10^{\circ}$ in the contralateral hemifield, median $=11^{\circ}, \mathrm{SD}=11^{\circ}$ ), with a tendency for lower peripheral positions. We did not find a consistent topographical organization along the electrode recording tracts, like the previous $\mathrm{dPul}$ study (Petersen et al., 1985). The lack of retinotopic organization is consistent with fairly uniform microstimulation effects across sites and target positions. Furthermore, largely horizontal directions of small evoked saccades in Monkey $\mathrm{C}$ might have resulted from a vector summation of upward, horizontal, and downward RFs with a contralateral bias, possibly similar to a population coding in the deeper layers of the SC (Lee et al., 1988).

In the visually-guided task, there was also a stronger population response for contralateral stimulus onset (target onset epoch, "T onset"; Fig. 11A). In addition, population response showed a tran-

\footnotetext{
(Figure legend continued.) alignment to events: FP onset, cue onset, saccade onset, and target offset; the other event markers denote average onset relative to alignment events. Gray boxes above the time axis indicate analyzed epochs (see Materials and Methods). Top right, Average firing rates for all 12 target positions during the cue and target hold epochs (top part of the color scale). Bottom right, Modulation depth and Gaussian fit defining the RF for that unit. Percentage modulation depth of cue responses is displayed for each target location at its actual position on the screen (bottom part of the color scale). The size of the visual stimuli is indicated by the dot in the center of each target ( $0.5^{\circ}$ radius). The superimposed ellipse represents the boundaries of the Gaussian fit (2SDs to each side; see Materials and Methods). For this unit, the RF size was estimated as $21^{\circ}$. B. Mean population response and SE across units during fixation hold, cue, and early memory epochs for ipsilateral and contralateral subsets of units, shown in orange and purple, respectively, for each target position. The two subsets represent units that had a main effect of target position during the cue epoch (gray shaded area) and were sorted into contralateral and ipsilateral populations according to the preferred hemifield. Before averaging across units, the mean peak of each unit's activity during the cue epoch across all trials to the preferred hemifield was normalized to 1. C, Visual RFs in the memory-guided saccade task. Top, Electrode tip position in individual recording sites (circles) in chamber-normal coronal sections corresponding to specific grid location ( $x, y$; in parentheses). Recording sites where no spatially tuned units were found are denoted by white circles; recording sites that showed tuning are shown in red, green, and blue colors representing different grid locations, with dark-to-light shades denoting recording depth. Pulvinar nuclei outlines as in Figure 1, colored outlines are the MPul. Bottom, RF centers and sizes for all units showing a main effect of cue location. RF centers correspond to the center of markers: circles for Monkey C, and squares for Monkey L. The marker size represents RF size, scaled 10:1. The color of the markers indicates the recording sites corresponding to the site reconstruction panels above.
}

sient and then sustained enhancement after central fixation acquisition ("Fix") and transient postsaccadic peak ("Postsac"), which was stronger for contralateral than for ipsilateral targets. Note that the weak perisaccadic population response is due to different subsets showing either perisaccadic enhancement or suppression (see below).

Overall, $78 \%$ of units were modulated by the visually-guided task (main effect of epoch or epoch $\times$ hemifield interaction) and $56 \%$ showed spatial specificity in at least one epoch (main effect of hemifield or epoch $\times$ hemifield interaction). Epoch-specific enhancement or suppression, relative to the fixation baseline, was analyzed for the first subset (78\%) and epoch-specific spatial tuning was analyzed in the latter subset (56\%). The bottom panels in Figure $11 \mathrm{~A}$ summarize the main patterns of spatial tuning and enhancement/ suppression in the three epochs: "target onset," "perisaccadic," and "target hold." The significant tuning was predominantly to the contralateral hemifield in the "target onset" epoch, but became more equalized in "perisaccadic" and especially in "target hold" epochs. Spatially tuned units showed predominantly enhancement of firing relative to the fixation baseline (red outer sectors) for target onset epoch, whereas all other subsets had more equal proportions of enhancement and suppression.

Population response in the memory-guided saccade task (Fig. $11 B$ ) additionally revealed preference for contralateral trials during the memory period and in the postsaccadic peripheral fixation epoch in absence of visual stimulus, before target onset ("target hold invisible"). Similarly to in the visually-guided task, $84 \%$ of units were task modulated (main effect of epoch or epoch $\times$ hemifield interaction) and 55\% showed a main effect of hemifield or epoch $\times$ hemifield interaction. Epoch-specific enhancement or suppression relative to the fixation baseline was analyzed for the first subset (84\%) and the epoch-specific spatial tuning was analyzed in the latter subset (55\%). The bottom panels in Figure $11 B$ summarize the main patterns of spatial tuning and enhancement/suppression in the three epochs: "cue onset," "perisaccadic," and "target hold." Again, the significant tuning was predominantly to the contralateral hemifield in the "cue onset" epoch, but it became more equalized in "perisaccadic" and especially in "target hold" epochs. Units that were contralaterally tuned in the cue epoch predominantly showed enhancement of firing relative to the fixation baseline. In addition to spatially tuned responses, an additional 47 units (13\%) showed robust cue-related enhancement that was not spatially selective (no main effect of target position and no hemifield tuning). Nonspatially tuned units in the perisaccadic epoch showed predominantly suppression, whereas all other subsets had more equal proportions of enhancement and suppression (Fig. 11B).

To further assess the differences in spatial tuning in "cue onset" and "target hold" epochs, population responses for subsets that showed significant tuning in those epochs were derived (Fig. $11 C$, left and right columns). The units that were contralaterally tuned in the cue epoch on average did not show spatial tuning in the postsaccadic and the target hold epochs, suggesting that the tuning in the latter intervals can be congruent or incongruent with the visual cue tuning. This is further evidenced by weak contralateral cue tuning in both subsets that showed significantly tuned, either ipsilateral or contralateral, target hold response (Fig. 11C, right column).

A closer look at full-trial population responses for units showing perisaccadic suppression (110 units, 30\%; Fig. 11C, middle column, top row) revealed that many of these units increased firing during central fixation (58 of 110). Those responses might resemble so called "fixation cells" reported in the FEF and in the 
A

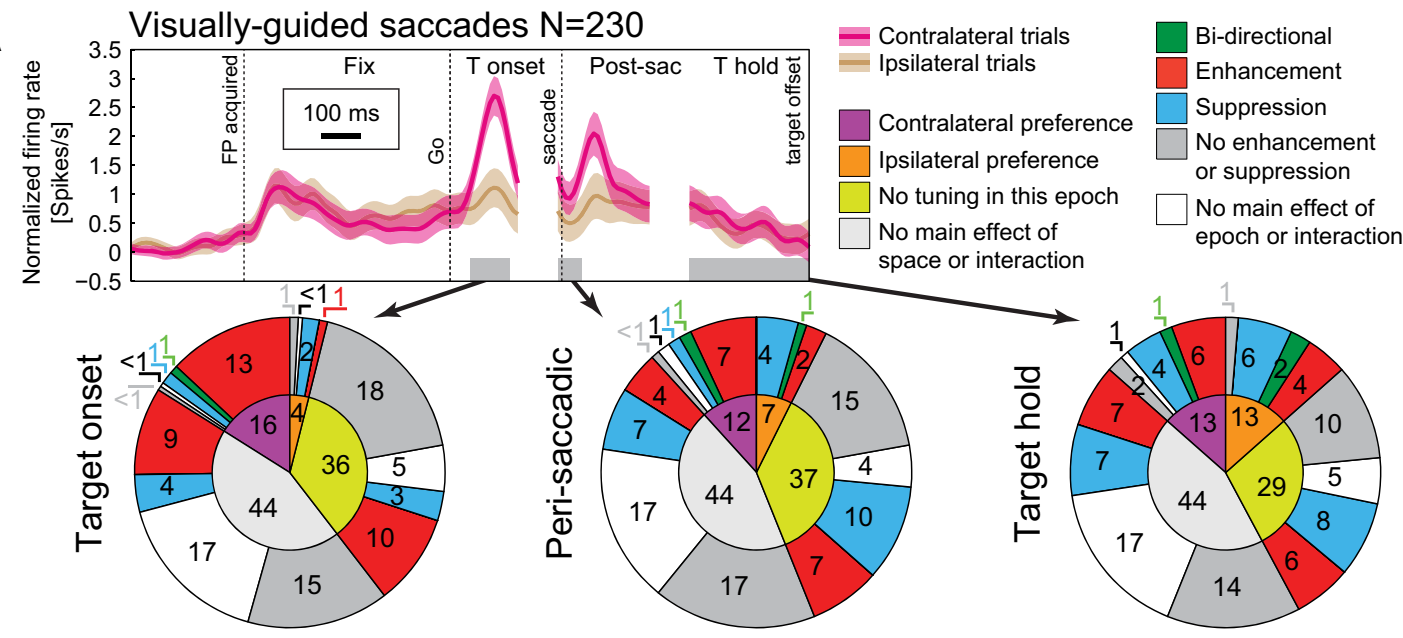

B

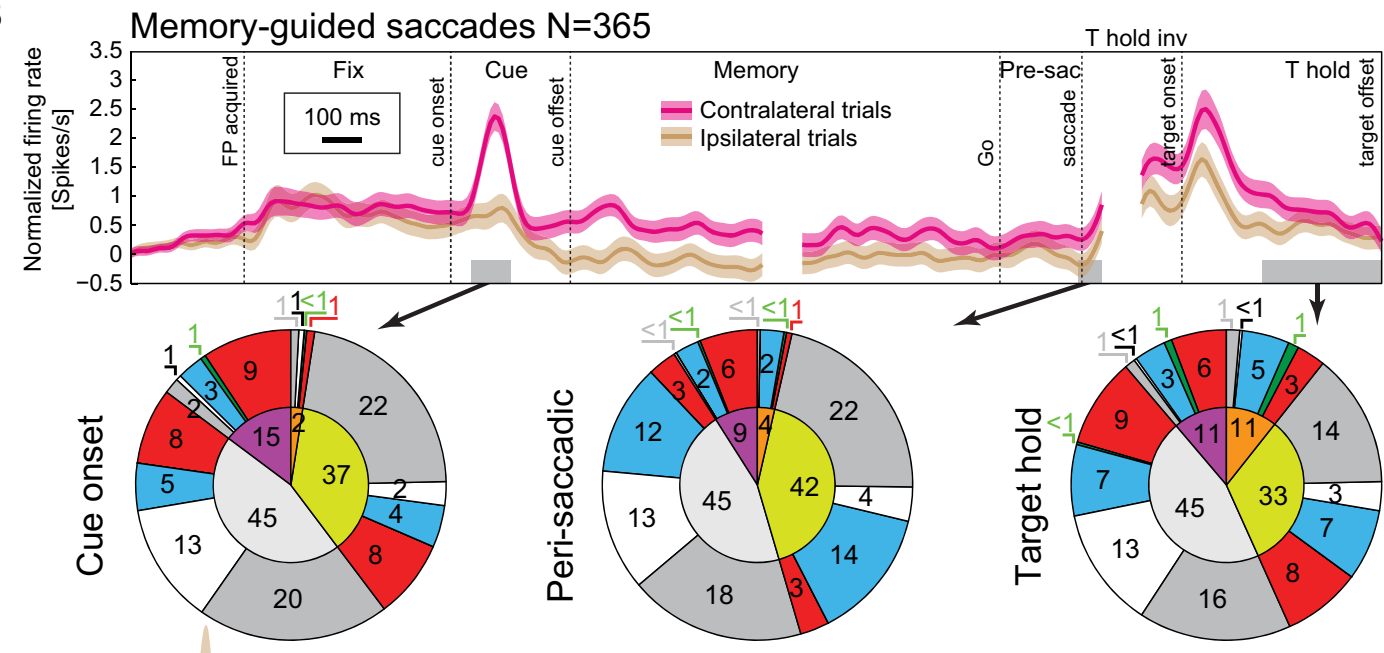

C
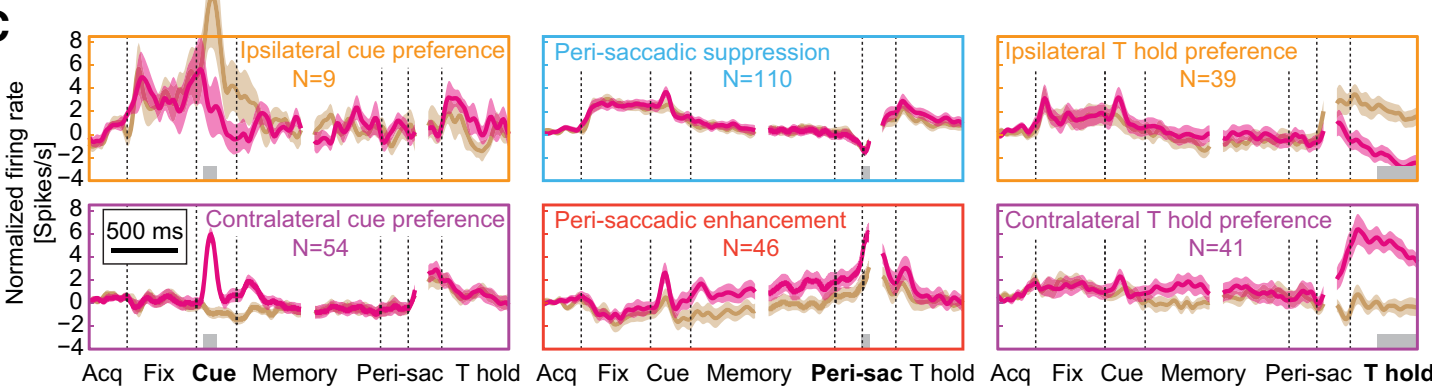

D Stimulus (target/cue) onset $(\mathrm{N}=121)$
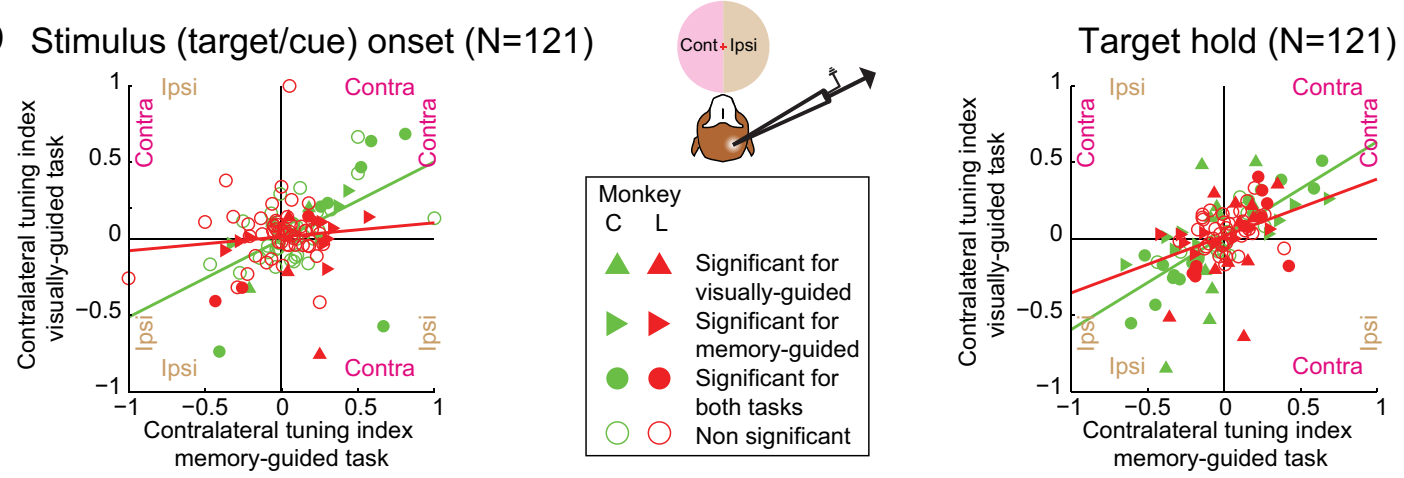

Figure 11. Neuronal population properties in the dPul. $A$, Units recorded during the visually-guided saccade task ( $n=230$ ). Top, Average baseline-corrected firing rate, mean (solid traces) and SE (shaded bands) across units, separately for contralateral and ipsilateral trials shown in magenta and brown, respectively. Same convention for alignment lines as in Figure 10A. Bottom, Spatial tuning and firing rate modulation in the three epochs (target onset, perisaccadic, and target hold). In each plot, sectors of the inner circle display the percentage of units that, in the respective epoch, preferred the ipsilateral hemifield (orange), contralateral hemifield (purple), were not tuned (light green), or were not tested for spatial tuning because they (Figure legend continues.) 
SC (Munoz and Wurtz, 1993a; Izawa et al., 2009). However, recent work by Hafed and Krauzlis (2012) demonstrated that, at the level of the SC, the tonic activity during fixation encodes fixational microsaccades or a retinal error by neurons tuned to foveal locations of very small eccentricity; it remains to be seen whether some pulvinar neurons might be similarly related to fixation maintenance. Furthermore, many "fixation response" cells in our sample showed a decreased firing during the memory delay compared with the initial fixation (45 of 100 units that showed enhanced firing during fixation relative to ITI), suggesting that spatially specific aspects such as visual memory or motor planning can modulate their activity (Fig. $11 C$, middle column, top row). Conversely, units that showed perisaccadic enhancement (Fig. 11C, middle column, bottom row) also exhibited an increased firing in contralateral trials from the cue onset and during the memory delay, ramping up before and peaking soon after the saccade, similar to visuomotor neurons in frontoparietal areas.

To summarize spatial tuning properties, we calculated contralateral tuning indexes (CIs) for stimulus onset and peripheral fixation ("target hold") responses for both tasks. Figure $11 D$ shows distribution of CIs for the subset of units that were recorded in both tasks for each monkey. For both tasks, across all recorded units CIs were significantly positive (i.e., contralateral) during "stimulus onset" epochs (visually-guided task: $0.07 \pm$ 0.24; memory-guided task: $0.09 \pm 0.26 ; p<0.001$, two-tailed one sample $t$ test); for the subset recorded in both tasks, only memoryguided task indexes were significantly positive (visually-guided task: $0.03 \pm 0.23, p=0.13$; memory-guided task: $0.05 \pm 0.26, p<$ $0.05)$. There was no significant tuning across the sample in the target hold epoch, reflecting nearly equal contralaterally and ipsilaterally tuned populations. For both epochs, there was a correlation between tuning indexes in the two tasks (Spearman's $r=$ $0.35, p<0.0001$ for "stimulus onset" and $r=0.59, p<0.0001$ for "target hold), indicating that the spatial response properties in these two epochs are largely consistent across the two tasks.

Although the full analysis of complex neuronal properties in the recorded population is beyond the scope of the present study, these data provide several points aiding the interpretation of the stimulation results. First, most recorded neurons were modulated by the visual and/or oculomotor contingencies of the two tasks. Second, the overall contralateral tuning in response to the visual stimulus (e.g., target and cue onset epochs), as well as in the presaccadic (data not shown) and the perisaccadic epochs, is con-

$\leftarrow$

(Figure legend continued.) showed neither a main ANOVA effect of hemifield nor an interaction of hemifield and epoch (light gray). The outer sectors display the percentage of units that showed enhancement or suppression in the respective epoch compared with the fixation hold epoch for each of the four aforementioned subsets separately: units showing enhancement for one hemifield and suppression for the other (green), only enhancement (red), only suppression (blue), neither enhancement nor suppression (dark gray), or neither a main ANOVA effect of epoch nor interaction of epoch and hemifield and thus not tested for enhancement or suppression (white). $\boldsymbol{B}$, Similar to $\boldsymbol{A}$, but for the memory-guided saccade task. Top, Average baselinecorrected firing rate. Bottom, Spatial tuning and firing rate modulation in the three epochs (cue onset, perisaccadic, and target hold). C, Average baseline-corrected PSTHs across different subsets of units in the memory-guided task. The subsets were defined by the classification in $\boldsymbol{B}$ : ipsilateral cue tuning (top left), contralateral cue tuning (bottom left), perisaccadic suppression (top center), perisaccadic enhancement (bottom center), ipsilateral tuning during target hold (top right), and contralateral tuning during target hold (bottom right). D, Cls (see Materials and Methods) for visually-guided versus memory-guided saccades for each unit where data for both tasks was available $(n=121)$. Filled markers denote units with significant tuning (see legend). Units recorded in Monkey C and L are in green and red, respectively. Left, Cls in "stimulus onset" ("target onset"/"cue onset") epochs. Right, (Is in "target hold" epoch. Lines indicate best linear fits. sistent with the contraversive drive elicited by the stimulation and the lack of topographic organization of RFs is consistent with similar stimulation effects across different sites and target positions. Third, the fact that the population tuning is still more contralateral than ipsilateral in the perisaccadic epochs suggests that the stronger RT delays for ipsiversive saccades in the late stimulation periods are not a direct consequence of disrupting ipsilaterally tuned populations more than contralaterally tuned ones. Fourth, a subset of units (cf. Fig. $11 A, B$, perisaccadic epochs, outer blue sectors corresponding to spatially nontuned populations and Fig. 11C, middle top panel) discharged vigorously during fixation intervals but paused firing in the perisaccadic period, potentially contributing to stimulation-induced saccade delays (Yang et al., 2008). Finally, many units had spatial tuning (both contralateral and ipsilateral) in the later part of the target hold period (starting at least $200 \mathrm{~ms}$ after the saccade offset, when the immediate postsaccadic effects are probably gone), suggesting a contribution of the dPul to the encoding of gaze, similar to the retinotopic inferior/lateral pulvinar (Robinson et al., 1990)

\section{Discussion}

Electrical microstimulation of the dPul influenced selection and execution of goal-directed saccades in a spatially and timedependent manner. This section focuses on the three main findings: (1) in the visually-guided task, stimulation starting before target onset (Go signal) reduced ipsiversive RTs, whereas stimulation at and after target onset caused a systematic increase in RTs for both ipsiversive and contraversive directions; (2) stimulation before the onset of targets increased ipsiversive choices and stimulation after onset of targets increased contraversive choices; and (3) in the memory-guided task, stimulation exerted effects on RT, but not on choices.

\section{Effects of microstimulation on saccade generation}

Bilateral RT delays with microstimulation after the Go signal have been reported for structures involved in saccade control, such as the dlPFC (Wegener et al., 2008), FEF (Izawa et al., 2004a), supplementary eye field (SEF) and pre-SMA (Isoda, 2005; Yang et al., 2008), caudate (Watanabe and Munoz, 2010, 2011), and rostral SC (Munoz and Wurtz, 1993b). In cortex, delays were typically stronger for ipsiversive saccades (Izawa et al., 2004b; Isoda, 2005; Wegener et al., 2008), whereas the opposite pattern was observed in SC and caudate (Munoz and Wurtz, 1993b; Watanabe and Munoz, 2013). The delay can be interpreted as suppression of gaze shifting, facilitation of gaze holding, and/or inhibition of a mechanism that switches between the two behavioral modes and might be explained by direct or indirect, uncrossed and crossed projections to substantia nigra pars reticulata, SC, and/or brainstem saccade generator nuclei (Izawa et al., 2004b; Isoda, 2005).

Unlike SC and caudate and similar to frontal cortical areas, ipsiversive RT delays were stronger in the $\mathrm{dPul}$, although ipsiversive and contraversive delays were correlated, suggesting a common mechanism; for example, the engagement of fixation neurons or nearly balanced recruitment of ipsilateral and contralateral populations, as well as untuned neurons.

In contrast to stimulation after the Go signal, stimulation starting before the Go shortened the ipsiversive RT, similarly to SEF and pre-SMA (Isoda, 2005; Yang et al., 2008) and caudate (Watanabe and Munoz, 2011). However, the above studies reported both contraversive and ipsiversive facilitation, indicating a general motor potentiation or release from gaze-holding signals. In caudate, release of SC/FEF from inhibition and subsequent rebound, or interplay between direct and indirect pathways, were suggested to explain the 
facilitation (Watanabe and Munoz, 2011). To account for the ipsiversive-specific RT facilitation in the $\mathrm{dPul}$, we propose a different, directional mechanism, which is the same as that for the ipsiversive choice bias (see below).

\section{Effects of microstimulation on choices}

Unlike the effects of microstimulation on saccade execution, interference on choices has been less studied, with most work focusing on perceptual decisions (Murasugi et al., 1993; Carello and Krauzlis, 2004; Hanks et al., 2006; Fetsch et al., 2014; Cicmil et al., 2015), but see (Opris et al., 2005; Mirpour et al., 2010). Therefore, one major question was whether and when pulvinar microstimulation influences free-choice target selection. Our previous work with pharmacological inactivation of dPul already implicated it in the spatial decision making (Wilke et al., 2010, 2013), but it was important to test whether the stimulation potentiates the "functioning" of the pulvinar, thus biasing choices in the direction opposite to the inactivation (i.e., contraversive vs inactivation-induced ipsilesional bias). Indeed, stimulation after the Go signal increased contraversive selection and, together with the contralateral neuronal tuning in the corresponding epoch, this suggests that it did not merely disrupt the normal functioning (Carello and Krauzlis, 2004). This is consistent with cortical and SC studies, which typically show a correspondence between the neuronal tuning and the direction of microstimulation effects (Clark et al., 2011).

However, even assuming a facilitatory activation by stimulation, the stimulation effects were not just a "mirror image" of inactivation. When stimulation started during fixation, before target onset, it caused ensuing ipsiversive bias, concurring with the ipsiversive RT facilitation. Such ipsiversive effects on target selection have been rarely reported. One study showed that microstimulation in upper layers of $\mathrm{V} 1$ biases choices away from the stimulated RFs (Tehovnik et al., 2002); similarly, caudate stimulation increased ipsiversive perceptual choices, away from contralateral RFs (Ding and Gold, 2012).

One hypothesis is that the ipsiversive selection is the manifestation of a stimulation-induced contraversive drive, which has to be counteracted during the fixation. Such a putative ipsiversive compensatory mechanism might be engaged until the end of the stimulation period and extend beyond the stimulation offset (after the Go signal) into the motor planning/execution epoch. Please note that this hypothesis does not necessarily imply that the monkeys were aware of the stimulation (Murphey and Maunsell, 2008) and compensated intentionally.

A related hypothesis is that the timing of stimulation offset relative to saccade RTs is important. Early stimulation periods ending $\sim 80$ ms before the typical control RTs $(\sim 160 \mathrm{~ms})$ led to an ipsiversive "advantage" in RTs and choices, whereas stimulation periods overlapping with the RTs led to a contraversive bias. Although the stimulation causes a contraversive drive, the offset of the stimulation per se might trigger a transient ipsiversive rebound regardless of task requirements. To resolve whether the timing of stimulation onset or the stimulation duration/offset is the crucial factor for the ipsiversive facilitation, the duration of the stimulation trains should be varied systematically in future studies.

An even more mechanistic explanation might be that the time course of stimulation on the evoked activity is initially excitatory and then inhibitory (Histed et al., 2013). Therefore, the initial contraversive drive would be suppressed by the end of the early stimulation periods, during target selection. Indeed, inhibitory consequences of the thalamic stimulation on cortical activity have been reported (Logothetis et al., 2010) and a pulvinar stimulation study in anesthetized tree shrews found that evoked ac- tivity in extrastriate cortex consists of early and late waves, with a gap 200 ms after the stimulation onset (Vanni et al., 2015).

Another explanation might be that the dPul stimulation engages a contraversive attentional shift, which acts as a "cue" in the inhibition of return phenomenon (Dorris et al., 2002). There is also a possibility that the pulvinar fulfills distinct functions in different behavioral states, for example, filtering out contralateral distractors and inhibiting reflexive contraversive saccades (Van der Stigchel et al., 2010), until cortical inputs signal the initiation of the active motor preparation phase. In this case, the potentiation of pulvinar activity during fixation would lead to a suppression of the currently irrelevant contraversive space. The presence of fixation-like neurons discharging persistently when monkeys maintained fixation supports this notion. However, the occurrence of contraversive evoked saccades during fixation challenges this interpretation unless the motor effects can be completely dissociated from the attentional/target selection signals.

Although a combination of contraversive facilitation drive and ipsiversive compensatory/rebound effect after the early stimulation offset seems most parsimonious explanation for the observed effects, the question whether a given stimulation protocol leads to functionally beneficial enhancement of "normal" neuronal activation, to a functionally detrimental disruption, or to replacement or "hijacking" (Cheney et al., 2013) is a long-standing debate, relevant for all stimulation studies (Desmurget et al., 2013). Some of our stimulation effects are consistent with the disruption of the (contraversive) pulvinar processing: ipsilateral facilitation in early stimulation periods and delayed saccades. However, this hypothesis is hard to reconcile with the contraversive choice facilitation in later stimulation periods unless the apparent contraversive facilitation is the consequence of "less contraversive disruption than ipsiversive disruption" during saccade generation. The latter possibility is consistent with stronger ipsiversive RT delays, although the neuronal tuning in pre/perisaccadic epochs was weakly contralateral. The contraversive disruption assumption also does not account for contraversive evoked saccades unless the main role of pulvinar is to help maintaining fixation and ignore contralateral hemifield.

Yet another possibility is that stimulating neurons with RFs away from the target but within the same hemifield is more detrimental than when the RFs and the target are in opposite hemifields, thus leading to ipsiversive facilitation. However, the reasons for contraversive facilitation in later stimulation periods remain unexplained under this assumption.

\section{Functional implications and future directions}

The effect on choices was present only in the visually-guided task, not the memory-guided task. Therefore, the choice bias is not driven by purely perceptual processing (otherwise, we would expect that the stimulation before or after visual cues affects subsequent choices) nor is it a purely motor consequence (otherwise, we should have seen effects before and after the Go signal). We suggest that the dPul contribution to the decision is crucial when the visuomotor contingencies have to be integrated rapidly and concomitantly with action selection. Alternatively, the pulvinar might affect the choices only when the target selection takes place in the presence of visual stimuli (which was not the case for memory-guided saccades); this conjecture needs to be tested in future experiments comparing memory-guided and visuallyguided delayed saccades.

The interpretation of the alleged contraversive drive due to pulvinar stimulation is still open. In the simplest scenario, it could relate to attentional/behavioral saliency vector in the reti- 
notopic reference frame. However, the spatial processing in the pulvinar, especially the dorsal part, might extend beyond purely visual aspects, contributing to gaze and postural encoding and perhaps to a prediction error (Grieve et al., 2000; Kanai et al., 2015). For example, the stimulation could affect the perceived direction of gaze relative to the head or the body or perceived body midline. Further experiments with manipulation of visuomotor and postural contingencies will address these possibilities. Another question is how general the biphasic choice effect is. VPul stimulation did not elicit an ipsiversive bias, but it would be interesting to test the same protocol in frontoparietal cortical areas interconnected with the dPul.

The inevitable conundrum of causal interference studies is to what extent the observed behavior depends on the functioning of the target area, as opposed to spread of in(activation) to neighboring structures and consequences of network effects. Sitespecific patterns (Fig. 8) and their dissimilarity from patterns in adjacent SC and caudate suggest fairly localized effects, but we cannot exclude some current spread through intercalated thalamocortical fibers, brachium of SC, or neighboring PIp/ $\mathrm{m} / \mathrm{cm}$ subdivisions of inferior pulvinar (Stepniewska, 2004; Rosenberg et al., 2009). The pulvinar stimulation with a similar protocol during fMRI activates an extensive visuomotor cortical circuitry in the stimulated hemisphere, with distinct patterns for dorsal versus ventral sites consistent with anatomical connectivity (L. Gibson, M. Wilke, I. Kagan, unpublished observations). Therefore, the observed effects can be mediated by predominantly contralaterally tuned cortical areas (Kagan et al., 2010; Wilke et al., 2012). Future work combining epoch-specific stimulation with $\mathrm{fMRI}$ and electrophysiological readouts should elucidate the neuronal basis of these effects.

\section{Notes}

Supplemental material for this article is available at http://www.dpz.eu/ dag/publications/jneurosci2016_supplemental. This material has not been peer reviewed.

\section{References}

Andersen RA, Cui H (2009) Intention, action planning, and decision making in parietal-frontal circuits. Neuron 63:568-583. CrossRef Medline

Arend I, Rafal R, Ward R (2008) Spatial and temporal deficits are regionally dissociable in patients with pulvinar lesions. Brain 131:2140-2152. CrossRef Medline

Bakker R, Tiesinga P, Kötter R (2015) The scalable brain atlas: instant webbased access to public brain atlases and related content. Neuroinformatics 13:353-366. CrossRef Medline

Bender DB, Baizer JS (1990) Saccadic eye movements following kainic acid lesions of the pulvinar in monkeys. Exp Brain Res 79:467-478. Medline

Bender DB, Butter CM (1987) Comparison of the effects of superior colliculus and pulvinar lesions on visual search and tachistoscopic pattern discrimination in monkeys. Exp Brain Res 69:140-154. Medline

Bender DB, Youakim M (2001) Effect of attentive fixation in macaque thalamus and cortex. J Neurophysiol 85:219-234. Medline

Benevento LA, Port JD (1995) Single neurons with both form/color differential responses and saccade-related responses in the nonretinotopic pulvinar of the behaving macaque monkey. Vis Neurosci 12:523-544. CrossRef Medline

Berman RA, Wurtz RH (2011) Signals conveyed in the pulvinar pathway from superior colliculus to cortical area MT. J Neurosci 31:373-384. CrossRef Medline

Brainard DH (1997) The Psychophysics Toolbox. Spat Vis 10:433-436. CrossRef Medline

Calabrese E, Badea A, Coe CL, Lubach GR, Shi Y, Styner MA, Johnson GA (2015) A diffusion tensor MRI atlas of the postmortem rhesus macaque brain. Neuroimage 117:408-416. CrossRef Medline

Carello CD, Krauzlis RJ (2004) Manipulating intent: evidence for a causal role of the superior colliculus in target selection. Neuron 43:575-583. CrossRef Medline

Cheney PD, Griffin DM, Van Acker GM 3rd (2013) Neural hijacking: action of high-frequency electrical stimulation on cortical circuits. Neuroscientist 19:434-441. CrossRef Medline

Cicmil, N., Cumming, B.G., Parker, A.J., and Krug, K (2015) Reward modulates the effect of visual cortical microstimulation on perceptual decisions. eLife e 07832 .

Clark KL, Armstrong KM, Moore T (2011) Probing neural circuitry and function with electrical microstimulation. Proc Biol Sci 278:1121-1130. CrossRef Medline

Crommelinck M, Roucoux A, Meulders M (1977) Eye movements evoked by stimulation of lateral posterior nucleus and puIvinar in the alert cat. Brain Res 124:361-366. CrossRef Medline

Desmurget M, Song Z, Mottolese C, Sirigu A (2013) Re-establishing the merits of electrical brain stimulation. Trends Cogn Sci 17:442-449. CrossRef Medline

Ding L, Gold JI (2012) Separate, causal roles of the caudate in saccadic choice and execution in a perceptual decision task. Neuron 75:865-874. CrossRef Medline

Dorris MC, Klein RM, Everling S, Munoz DP (2002) Contribution of the primate superior colliculus to inhibition of return. J Cogn Neurosci 14: 1256-1263. CrossRef Medline

Fetsch CR, Kiani R, Newsome WT, Shadlen MN (2014) Effects of cortical microstimulation on confidence in a perceptual decision. Neuron 83 : 797-804. CrossRef Medline

Goldberg ME, Bushnell MC, Bruce CJ (1986) The effect of attentive fixation on eye movements evoked by electrical stimulation of the frontal eye fields. Exp Brain Res 61:579-584. Medline

Grieve KL, Acuña C, Cudeiro J (2000) The primate pulvinar nuclei: vision and action. Trends Neurosci 23:35-39. CrossRef Medline

Gutierrez C, Cola MG, Seltzer B, Cusick C (2000) Neurochemical and connectional organization of the dorsal pulvinar complex in monkeys. J Comp Neurol 419:61-86. CrossRef Medline

Hafed ZM, Krauzlis RJ (2012) Similarity of superior colliculus involvement in microsaccade and saccade generation. J Neurophysiol 107:1904-1916. CrossRef Medline

Hafed ZM, Chen CY, Tian X (2015) Vision, perception, and attention through the lens of microsaccades: mechanisms and implications. Front Syst Neurosci 9:167. CrossRef Medline

Hanks TD, Ditterich J, Shadlen MN (2006) Microstimulation of macaque area LIP affects decision-making in a motion discrimination task. Nat Neurosci 9:682-689. CrossRef Medline

Histed MH, Ni AM, Maunsell JH (2013) Insights into cortical mechanisms of behavior from microstimulation experiments. Prog Neurobiol 103: 115-130. CrossRef Medline

Isoda M (2005) Context-dependent stimulation effects on saccade initiation in the presupplementary motor area of the monkey. J Neurophysiol 93:3016-3022. CrossRef Medline

Izawa Y, Suzuki H, Shinoda Y (2004a) Suppression of visually and memoryguided saccades induced by electrical stimulation of the monkey frontal eye field. II. Suppression of bilateral saccades. J Neurophysiol 92:22612273. CrossRef Medline

Izawa Y, Suzuki H, Shinoda Y (2004b) Suppression of visually and memoryguided saccades induced by electrical stimulation of the monkey frontal eye field. I. Suppression of ipsilateral saccades. J Neurophysiol 92:22482260. CrossRef Medline

Izawa Y, Suzuki H, Shinoda Y (2009) Response properties of fixation neurons and their location in the frontal eye field in the monkey. J Neurophysiol 102:2410-2422. CrossRef Medline

Jones EG (2012) The thalamus. New York: Springer.

Kaas JH, Lyon DC (2007) Pulvinar contributions to the dorsal and ventral streams of visual processing in primates. Brain Res Rev 55:285-296. CrossRef Medline

Kagan I, Iyer A, Lindner A, Andersen RA (2010) Space representation for eye movements is more contralateral in monkeys than in humans. Proc Natl Acad Sci U S A 107:7933-7938. CrossRef Medline

Kanai R, Komura Y, Shipp S, Friston K (2015) Cerebral hierarchies: predictive processing, precision and the pulvinar. Philos Trans R Soc Lond B Biol Sci 370: pii: 20140169. CrossRef Medline

Karnath HO, Himmelbach M, Rorden C (2002) The subcortical anatomy of 
human spatial neglect: putamen, caudate nucleus and pulvinar. Brain 125:350-360. CrossRef Medline

Komura Y, Nikkuni A, Hirashima N, Uetake T, Miyamoto A (2013) Responses of pulvinar neurons reflect a subject's confidence in visual categorization. Nat Neurosci 16:749-755. CrossRef Medline

Lee C, Rohrer WH, Sparks DL (1988) Population coding of saccadic eye movements by neurons in the superior colliculus. Nature 332:357-360. CrossRef Medline

Logothetis NK, Augath M, Murayama Y, Rauch A, Sultan F, Goense J, Oeltermann A, Merkle H (2010) The effects of electrical microstimulation on cortical signal propagation. Nat Neurosci 13:1283-1291. CrossRef Medline

Maldonado H, Joseph JP, Schlag J (1980) Types of eye movements evoked by thalamic microstimulation in the alert cat. Exp Neurol 70:613-625. CrossRef Medline

Mirpour K, Ong WS, Bisley JW (2010) Microstimulation of posterior parietal cortex biases the selection of eye movement goals during search. J Neurophysiol 104:3021-3028. CrossRef Medline

Moeller S, Freiwald WA, Tsao DY (2008) Patches with links: a unified system for processing faces in the macaque temporal lobe. Science 320:13551359. CrossRef Medline

Munoz DP, Wurtz RH (1993a) Fixation cells in monkey superior colliculus. I. Characteristics of cell discharge. J Neurophysiol 70:559-575. Medline

Munoz DP, Wurtz RH (1993b) Fixation cells in monkey superior colliculus. II. Reversible activation and deactivation. J Neurophysiol 70:576-589. Medline

Murasugi CM, Salzman CD, Newsome WT (1993) Microstimulation in visual area MT: effects of varying pulse amplitude and frequency. J Neurosci 13:1719-1729. Medline

Murphey DK, Maunsell JH (2008) Electrical microstimulation thresholds for behavioral detection and saccades in monkey frontal eye fields. Proc Natl Acad Sci U S A 105:7315-7320. CrossRef Medline

Ohayon S, Tsao DY (2012) MR-guided stereotactic navigation. J Neurosci Methods 204:389-397. CrossRef Medline

Opris I, Barborica A, Ferrera VP (2005) Microstimulation of the dorsolateral prefrontal cortex biases saccade target selection. J Cogn Neurosci 17:893-904. CrossRef Medline

Petersen SE, Robinson DL, Keys W (1985) Pulvinar nuclei of the behaving rhesus monkey: visual responses and their modulation. J Neurophysiol 54:867-886. Medline

Preuss, T.M (2007) Evolutionary specializations of primate brain systems. In: Primate origins: evolution and adaptations (Ravosa MJ, Dagosto M, eds). New York: Springer, p. 625-675.

Rafal RD, Posner MI (1987) Deficits in human visual spatial attention following thalamic lesions. Proc Natl Acad Sci U S A 84:7349-7353. CrossRef Medline

Rafal R, McGrath M, Machado L, Hindle J (2004) Effects of lesions of the human posterior thalamus on ocular fixation during voluntary and visually triggered saccades. J Neurol Neurosurg Psychiatry 75:1602-1606. CrossRef Medline

Robinson DA, Fuchs AF (1969) Eye movements evoked by stimulation of frontal eye fields. J Neurophysiol 32:637-648. Medline

Robinson DL, Petersen SE (1992) The pulvinar and visual salience. Trends Neurosci 15:127-132. CrossRef Medline

Robinson DL, Petersen SE, Keys W (1986) Saccade-related and visual activities in the pulvinar nuclei of the behaving rhesus monkey. Exp Brain Res 62:625-634. Medline

Robinson DL, McClurkin JW, Kertzman C (1990) Orbital position and eye movement influences on visual responses in the pulvinar nuclei of the behaving macaque. Exp Brain Res 82:235-246. Medline

Rohlfing T, Kroenke CD, Sullivan EV, Dubach MF, Bowden DM, Grant KA, Pfefferbaum A (2012) The INIA19 template and NeuroMaps atlas for primate brain image parcellation and spatial normalization. Front Neuroinform 6:27. CrossRef Medline

Rosenberg DS, Mauguière F, Catenoix H, Faillenot I, Magnin M (2009) Reciprocal thalamocortical connectivity of the medial pulvinar: a depth stimulation and evoked potential study in human brain. Cereb Cortex 19:1462-1473. CrossRef Medline

Saalmann YB, Kastner S (2011) Cognitive and perceptual functions of the visual thalamus. Neuron 71:209-223. CrossRef Medline

Saalmann YB, Pinsk MA, Wang L, Li X, Kastner S (2012) The pulvinar regulates information transmission between cortical areas based on attention demands. Science 337:753-756. CrossRef Medline

Scherberger H, Goodale MA, Andersen RA (2003) Target selection for reaching and saccades share a similar behavioral reference frame in the macaque. J Neurophysiol 89:1456-1466. Medline

Schlag J, Schlag-Rey M, Dassonville P (1989) Interactions between natural and electrically evoked saccades. Exp Brain Res 76:548-558. CrossRef Medline

Seltzer B, Cola MG, Gutierrez C, Massee M, Weldon C, Cusick CG (1996) Overlapping and nonoverlapping cortical projections to cortex of the superior temporal sulcus in the rhesus monkey: double anterograde tracer studies. J Comp Neurol 370:173-190. CrossRef Medline

Shadlen MN, Kiani R (2013) Decision making as a window on cognition. Neuron 80:791-806. CrossRef Medline

Sherman SM, Guillery RW (2002) The role of the thalamus in the flow of information to the cortex. Philos Trans R Soc Lond B Biol Sci 357:16951708. CrossRef Medline

Shibutani H, Sakata H, Hyvärinen J (1984) Saccade and blinking evoked by microstimulation of the posterior parietal association cortex of the monkey. Exp Brain Res 55:1-8. Medline

Snow JC, Allen HA, Rafal RD, Humphreys GW (2009) Impaired attentional selection following lesions to human pulvinar: evidence for homology between human and monkey. Proc Natl Acad Sci U S A 106:4054-4059. CrossRef Medline

Stepniewska, I (2004) The pulvinar complex. In: The primate visual system (Kaas JH, Collins CE, ed.), pp 53-80. Boca Raton, FL: CRC Press.

Tehovnik EJ, Slocum WM, Schiller PH (1999) Behavioural conditions affecting saccadic eye movements elicited electrically from the frontal lobes of primates. Eur J Neurosci 11:2431-2443. CrossRef Medline

Tehovnik EJ, Slocum WM, Schiller PH (2002) Differential effects of laminar stimulation of V1 cortex on target selection by macaque monkeys. Eur J Neurosci 16:751-760. CrossRef Medline

Thier P, Andersen RA (1996) Electrical microstimulation suggests two different forms of representation of head-centered space in the intraparietal sulcus of rhesus monkeys. Proc Natl Acad Sci U S A 93:4962-4967. CrossRef Medline

Van der Stigchel S, Arend I, van Koningsbruggen MG, Rafal RD (2010) Oculomotor integration in patients with a pulvinar lesion. Neuropsychologia 48:3497-3504. CrossRef Medline

Vanni MP, Thomas S, Petry HM, Bickford ME, Casanova C (2015) Spatiotemporal profile of voltage-sensitive dye responses in the visual cortex of tree shrews evoked by electric microstimulation of the dorsal lateral geniculate and pulvinar nuclei. J Neurosci 35:11891-11896. CrossRef Medline

Watanabe M, Munoz DP (2010) Saccade suppression by electrical microstimulation in monkey caudate nucleus. J Neurosci 30:2700-2709. CrossRef Medline

Watanabe M, Munoz DP (2011) Saccade reaction times are influenced by caudate microstimulation following and prior to visual stimulus appearance. J Cogn Neurosci 23:1794-1807. CrossRef Medline

Watanabe M, Munoz DP (2013) Effects of caudate microstimulation on spontaneous and purposive saccades. J Neurophysiol 110:334-343. CrossRef Medline

Wegener SP, Johnston K, Everling S (2008) Microstimulation of monkey dorsolateral prefrontal cortex impairs antisaccade performance. Exp Brain Res 190:463-473. CrossRef Medline

Wilke M, Turchi J, Smith K, Mishkin M, Leopold DA (2010) Pulvinar inactivation disrupts selection of movement plans. J Neurosci 30:8650-8659. CrossRef Medline

Wilke M, Kagan I, Andersen RA (2012) Functional imaging reveals rapid reorganization of cortical activity after parietal inactivation in monkeys. Proc Natl Acad Sci U S A 109:8274-8279. CrossRef Medline

Wilke M, Kagan I, Andersen RA (2013) Effects of pulvinar inactivation on spatial decision-making between equal and asymmetric reward options. J Cogn Neurosci 25:1270-1283. CrossRef Medline

Yamamoto S, Monosov IE, Yasuda M, Hikosaka O (2012) What and where information in the caudate tail guides saccades to visual objects. J Neurosci 32:11005-11016. CrossRef Medline

Yang SN, Heinen SJ, Missal M (2008) The effects of microstimulation of the dorsomedial frontal cortex on saccade latency. J Neurophysiol 99:18571870. CrossRef Medline

Zhou H, Schafer RJ, Desimone R (2016) Pulvinar-cortex interactions in vision and attention. Neuron 89:209-220. CrossRef Medline 
8.2 Appendix B 


\title{
Clinical neuroanatomy
}

\section{Reach and grasp deficits following damage to the dorsal pulvinar}

\author{
Melanie Wilke ${ }^{a, c, d, e, *}$, Lukas Schneider ${ }^{a, c}$, \\ Adan-Ulises Dominguez-Vargas ${ }^{a, c}$, Carsten Schmidt-Samoa ${ }^{a}$, \\ Kristina Miloserdov ${ }^{a, d}$, Ahmad Nazzal ${ }^{a}$, Peter Dechent ${ }^{a}$, \\ Yuranny Cabral-Calderin ${ }^{a, c}$, Hansjörg Scherberger ${ }^{c, e}$, Igor Kagan ${ }^{c, e}$ and \\ Mathias Bähr b,d \\ a Department of Cognitive Neurology, University Medicine Goettingen, Goettingen, Germany \\ ${ }^{\mathrm{b}}$ Department of Neurology, University Medicine Goettingen, Goettingen, Germany \\ c German Primate Center, Leibniz Institute for Primate Research, Goettingen, Germany \\ d DFG Center for Nanoscale Microscopy \& Molecular Physiology of the Brain (CNMPB), Germany \\ e Leibnitz ScienceCampus "Primate Cognition", Goettingen, Germany
}

\section{A R T I C L E I N F O}

Article history:

Received 26 January 2017

Reviewed 08 June 2017

Revised 17 July 2017

Accepted 2 October 2017

Action editor David Carey

Published online 8 November 2017

Keywords:

Pulvinar

Reaching

Grasping

Saccades

Visuomotor

Perceptual choice

Ataxia

\begin{abstract}
A B S T R A C T
Expansion of the dorsal pulvinar in humans and its anatomical connectivity suggests its involvement in higher-order cognitive and visuomotor functions. We investigated visuomotor performance in a 31 year old patient (M.B.) with a lesion centered on the medial portion of the dorsal pulvinar (left > right) due to an atypical Sarcoidosis manifestation. Unlike lesions with a vascular etiology, the lesion of M.B. did not include primary sensory or motor thalamic nuclei. Thus, this patient gave us the exceedingly rare opportunity to study the contribution of the dorsal pulvinar to visuomotor behavior in a human without confounding losses in primary sensory or motor domains. We investigated reaching, saccade and visual decision making performance. Patient data in each task was compared to at least seven age matched healthy controls. While saccades were hypometric towards both hemifields, the patient did not show any spatial choice bias or perceptual deficits. At the same time, he exhibited reach and grasp difficulties, which shared features with both, parietal and cerebellar damage. In particular, he had problems to form a precision grip and exhibited reach deficits expressed in decreased accuracy, delayed initiation and prolonged movement durations. Reach deficits were similar in foveal and extrafoveal viewing conditions and in both visual hemifields but were stronger with the right hand. These results suggest that dorsal pulvinar function in humans goes beyond its subscribed role in visual cognition and is critical for the programming of voluntary actions with the hands.

(C) 2017 The Author(s). Published by Elsevier Ltd. This is an open access article under the CC BY-NC-ND license (http://creativecommons.org/licenses/by-nc-nd/4.0/).
\end{abstract}

\footnotetext{
* Corresponding author. Department of Cognitive Neurology, University Medicine Goettingen, Robert-Koch-Str. 40, Goettingen 37075, Germany.

E-mail address: melanie.wilke@med.uni-goettingen.de (M. Wilke). 


\section{Introduction}

Evolutionary history and ontogenetic development together with fronto-parietal connectivity of the dorsal pulvinar suggest its contribution to higher cognitive functions and in particular to primate-specific abilities such as complex visuomotor transformations that require the integration of visual with eye and hand position information (Grieve, Acuna, \& Cudeiro, 2000; Preuss, 2007). The pulvinar is a typical association nucleus with strong reciprocal connections to a multitude of modality specific and multimodal cortical areas (Benarroch, 2015; Gutierrez, Cola, Seltzer, \& Cusick, 2000). The pulvinar is a heterogeneous structure for which different parcellations schemes have been proposed, depending on the anatomical techniques that were used such as cyto-, myelo- or chemoarchitecture (Jones, 2007). However, most authors agree on at least four major subdivisions in human and non-human primates, consisting of anterior pulvinar (PuA), medial pulvinar (PuM), lateral pulvinar (PuL) and inferior pulvinar (PuI). Together, the medial pulvinar and the dorsal portion of the lateral pulvinar form the so called 'dorsal pulvinar', which roughly occupies the region dorsal to the level of the brachium of the superior colliculus (BSc) (Gutierrez et al., 2000; Kaas \& Lyon, 2007). The majority of pulvinar studies have investigated its ventral aspect, which is retinotopically organized and is connected with striate and extrastriate visual cortices in monkeys and humans (Arcaro, Pinsk, \& Kastner, 2015; Saalmann \& Kastner, 2011). In contrast to the ventral pulvinar, the dorsal pulvinar portion does not contain an orderly retinotopic or visuomotor topography (Benevento \& Port, 1995) and is reciprocally interconnected with cortical regions that underlie the coordination of visuallyguided movements, such as posterior parietal and prefrontal cortices (Arcaro et al., 2015; Barron, Eickhoff, Clos, \& Fox, 2015; Gutierrez et al., 2000; Jones, 2007; Rosenberg, Mauguiere, Catenoix, Faillenot, \& Magnin, 2009). Response properties of dorsal pulvinar neurons resemble the complexity found in fronto-parietal cortices, i.e., neuronal firing correlates with visual attention, subjective perception, decision confidence as well as the planning and execution of eye- and hand movements (Bender \& Youakim, 2001; Benevento \& Port, 1995; Dominguez-Vargas, Schneider, Wilke, \& Kagan, 2017; Komura, Nikkuni, Hirashima, Uetake, \& Miyamoto, 2013; MagarinosAscone, Buno, \& Garcia-Austt, 1988; Wilke, Mueller, \& Leopold, 2009; Yirmiya \& Hocherman, 1987). There is also evidence from pulvinar lesion studies in monkeys (Komura et al., 2013; Robinson \& Petersen, 1992; Wilke, Kagan, \& Andersen, 2013; Wilke, Turchi, Smith, Mishkin, \& Leopold, 2010; Zhou, Schafer, \& Desimone, 2016) and humans (Arend, Rafal, \& Ward, 2008; Karnath, Himmelbach, \& Rorden, 2002; Rafal, McGrath, Machado, \& Hindle, 2004; Snow, Allen, Rafal, \& Humphreys, 2009; Van der Stigchel, Arend, van Koningsbruggen, \& Rafal, 2010; Ward, Danziger, \& Bamford, 2005; Zihl \& von Cramon, 1979) that the pulvinar is a critical contributor to a wide range of higher-order visual and oculomotor functions including attentional orienting, visual search, emotion recognition and saccadic decision making. At the same time, although an initial dorsal pulvinar inactivation study in monkeys suggests its critical contribution to the programming of reach and grasp movements (Wilke et al., 2010), there is a marked paucity of studies that tested basic visuomotor functions involving hand usage in humans (Benarroch, 2015; Bridge, Leopold, \& Bourne, 2016).

This is particularly surprising given that multimodal signals from a wide range of well-studied cortical visuomotor areas converge in the dorsal pulvinar and it has thus been proposed to facilitate cortico-spinal control over movements and possibly better parietal-premotor integration for the flexible control of goal-directed movements (Cappe, Morel, Barone, \& Rouiller, 2009; Grieve et al., 2000; Guillery \& Sherman, 2002). In the present study, we investigated visuomotor functions with a focus on reach performance in a patient with a circumscribed lesion centered on the medial portion of the dorsal pulvinar. This patient provided the unique opportunity to unravel the contribution of the pulvinar to proper visuomotor behavior without lesions in functionally pertinent first-order thalamic nuclei (Sherman, 2016), and without primary sensory or motor deficits. Based on our previous dorsal pulvinar inactivation study in monkeys (Wilke et al., 2010), we hypothesized that the patient would show reach inaccuracies and initiation delays, possibly with a stronger effect for the (right) hand and space located opposite to the more pronounced pulvinar lesion (left). From an optic ataxia we expected reaching errors to be stronger in extrafoveal as compared to foveal reaches (Andersen, Andersen, Hwang, \& Hauschild, 2014; Perenin \& Vighetto, 1988), a comparison not available from pulvinar lesion studies in monkeys.

\section{Material and methods}

\subsection{Participants}

\subsubsection{Patient M.B.}

Patient M.B. is a right-handed 31 year old male with an atypical cerebral manifestation of a systemic Sarcoidosis (Hoitsma, Drent, \& Sharma, 2010). Sarcoidosis is a rare disorder that shows CNS manifestations in $2-26 \%$ of the cases with many atypical lesion locations (Fritz, van de Beek, \& Brouwer, 2016). In patient M.B. the neural manifestation of the sarcoidosis affected the thalamic pulvinar on both sides. The patient's symptoms started with walking problems, headache and loss of appetite. These symptoms improved after an initial high dose corticosteroid therapy. Several weeks later, his symptoms relapsed and he was referred to our hospital. The diagnosis of Sarcoidosis was secured by thoracic biopsies together with pathological CD4/CD8 ratio in the bronchoalveolar-lavage and histopathological documentation of epithelioid-cell granulomas that followed the detection of suspicious lymph nodes in the abdominal-CT and FDG-PET-CT (Fritz et al., 2016). All examinations described in this paper were done in February 2016, within the two weeks when the disease cause was just diagnosed.

2.1.1.1. LOCALIZATION OF THE LESION. The pulvinar lesion was larger on the left side than on the right and included large portions of the medial pulvinar as well as a small portion of the anterior pulvinar. On the right side, initially only a small portion of the medial pulvinar was affected (Fig. 1). This right 
pulvinar hyperintensity (on T2-weighted FLAIR images) was faint in February 2016 (the first admission to the hospital and the period in which all our reported behavioral testing was done), but was repeatedly detected. Routine diagnostic MR examinations based on T1-weighted contrast (with and without contrast agent) and contrast agent-based brain perfusion did not show any other alteration. A bilateral (and stable circumscribed) pulvinar lesion was then visible in all repeated scans from May-November 2016. This supported our interpretation that the right pulvinar lesion has been already present in February. Lesions did not involve the ventral pulvinar or surrounding primary sensory or motor thalamic nuclei (e.g., ventral anterior or lateral thalamic nuclei). Brainstem nuclei, cerebellum and cortex were structurally intact as evidenced by MRI (Fig. 1, Supplementary Figs. S1 and S2).

To facilitate delineation of suspected lesion areas within the thalamus, these were mapped on the co-registered Morel atlas. While different pulvinar parcellation schemes exist (Benarroch, 2015; Jones, 2007), we here adopt the traditional terminology also used by the Morel atlas (Morel, Magnin, \& Jeanmonod, 1997), subdividing the pulvinar into anterior or oral pulvinar $(\mathrm{PuA})$, medial pulvinar (PuM), lateral pulvinar (PuL) and inferior pulvinar (PuI). In this scheme, the lesion of M.B. was centered on the medial pulvinar with an anterior extension into the anterior pulvinar on the left side and possibly also a small portion of the centromedian nucleus on the left side as well.

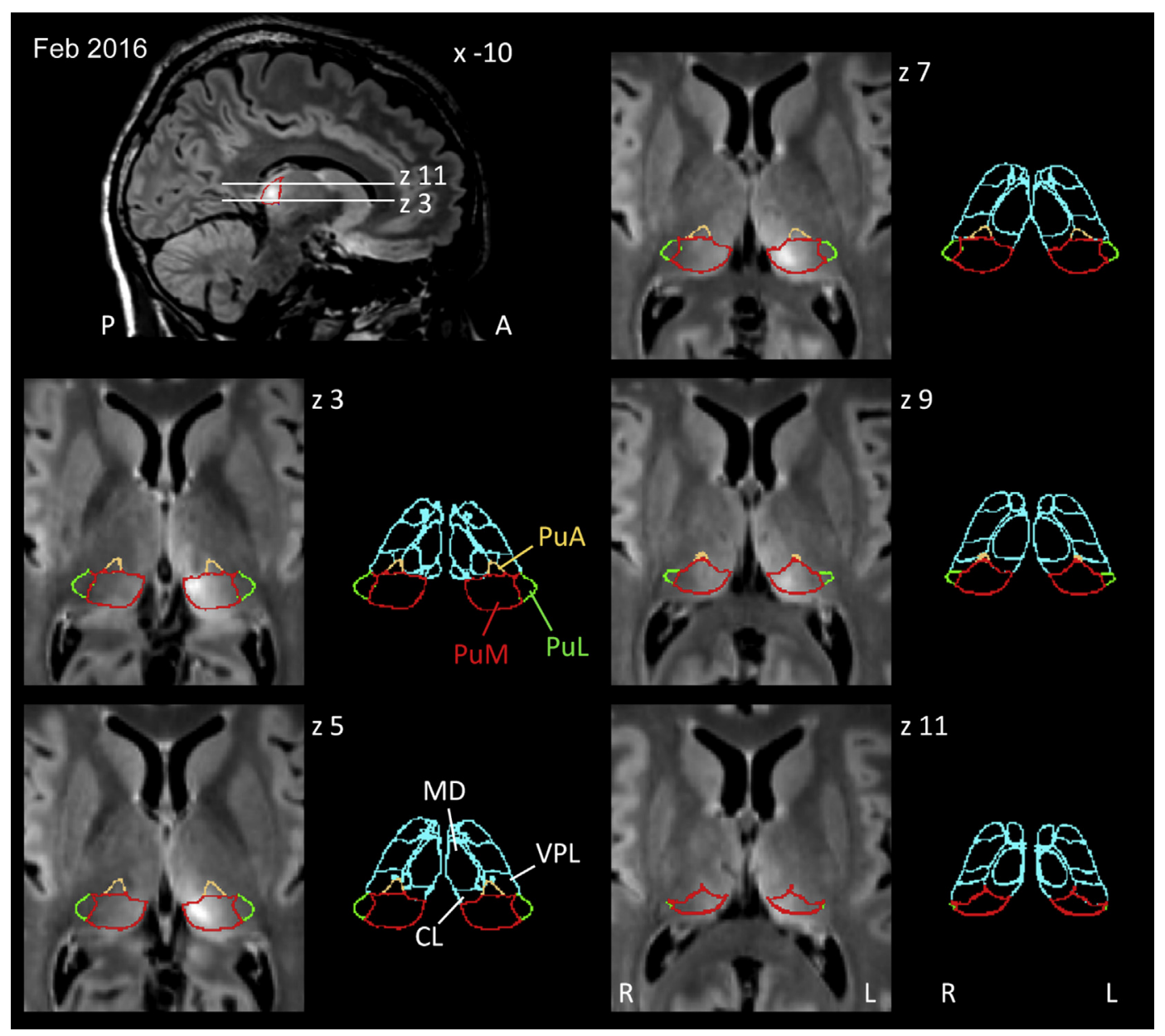

Fig. 1 - Lesion reconstruction of patient M.B. Magnified views of fluid-attenuated inversion recovery (FLAIR) MR images of patient M.B. in MNI-space, co-registered to the digital version of the Morel atlas. Images were acquired in February 2016 when the behavioral testing took place. The top left shows a sagittal section indicating the orientation of the axial crosssections. FLAIR images show hyperintensity in the medial pulvinar on both sides, stronger in the left pulvinar (radiological convention with left hemisphere shown on the right side). Lesions spared the ventral pulvinar portions, anterior thalamus, brainstem, cerebellum and surrounding cortices. Cross-sections show the lesioned thalamic regions based on the overlaid pulvinar regions defined by the Morel atlas. Corresponding sections of the Morel atlas with all regions are shown on the right to the FLAIR images. The thalamic regions from the Morel atlas are outlined in light blue, except for medial pulvinar (PuM, red), lateral pulvinar (PuL, green), and anterior pulvinar (PuA, orange). A: anterior; CL: central lateral nucleus; L: left; MD: mediodorsal nucleus; P: posterior; R: right; VPL: ventral posterior lateral nucleus. $\mathbf{x}, \mathbf{z}$ (in mm) denote the level of the cross-sections in MNI-space. 
2.1.1.2. NeURological AND NEUROPHySiological exAmination. The corresponding neurological and neurophysiological examination took place within the first two weeks after hospital admission in February 2016. At that time, the patient's cranial nerve examinations were normal except for a slight posturedependent down-beat nystagmus (while sitting upright but not while lying down in the horizontal position). His smooth pursuit was normal and there were no indications of gaze palsy. Further neurological and neurophysiological examinations showed normal vestibulo-ocular reflexes, no primary vestibular dysfunction with normal caloric tests, normal subjective visual vertical (SVV) and no visual impairment with normal visually evoked cortical potentials (VEP), visual acuity and visual field perimetry tests. The reflex-status, muscle tone and other sensory and muscle strength tests were normal. EEG, nerve conductance velocities, amplitudes and central motor conductance times were in the normal range. There was no indication of primary somatosensory or proprioceptive deficits with normal position and vibration sense and equal temperature and pain sensation on both sides. He did not display gait ataxia, wide-based stance and gait or other classical signs of cerebellar lesions such as impaired finger-to-nose test, heel-toshin test, checking response or rebound phenomenon. Nevertheless, the patient had severe problems with upright stance and walking resembling an unusual form of (thalamic) astasia (Masdeu \& Gorelick, 1988).

Over the course of several months after February, he developed a hand tremor with an unusual (dyskinetic-dystonic) hand posture as previously described as a delayed consequence of posterior thalamic lesions (Ghika, Bogousslavsky, Henderson, Maeder, \& Regli, 1994; Kim, 2001; Miwa, Hatori, Kondo, Imai, \& Mizuno, 1996). The development of dystonia, defined by sustained or repetitive muscle contractions resulting in twisting and repetitive movements or abnormal fixed postures (Albanese et al., 2013) with severe tremor prevented further (interpretable) testing of reach and grasp functions. Thus, all behavioral data presented in this paper were collected within two weeks in February 2016 when tremor and dystonia were not interfering with task performance.

\subsubsection{Neuropsychological testing of patient M.B}

Neuropsychological assessment revealed normal executive, memory and language functions apart from mild attentional impairments (Supplementary Table S1). Attentional functions were tested with the German equivalents of the Test of Attentional Performance (TAP) (Zimmermann \& Fimm, 2002). Executive functions were tested with the Stroop color and word test (FWIT) (Bäumler \& Stroop, 1985) and the Regensburger word fluency test (RWT) (Aschenbrenner, Tucha, \& Lange, 2000). Learning and memory were tested with the forward and backward span of the Wechsler Memory ScaleRevised (WMS-R) (Härting et al., 2000) and the verbal learning and memory test (VLMT) (Helmstaedter, Lendt, \& Lux, 2001). Based on the neuropsychological assessment, he exhibited normal executive, memory and language functions apart from a slight dysarthria. He showed mild attentional impairments in the subtests intrinsic and phasic alertness of the attentional performance battery (TAP) (Supplementary Table S1). There was no indication of spatial neglect or extinction, which was tested with the clinical confrontation method for visual auditory and somatosensory stimuli as well as with standard paper and pencil tasks (line bisection, line and apple cancellation test) (Fels \& Geissner, 1997; Manning, Halligan, \& Marshall, 1990; Mesulam, 1985). He showed deficits in the visuo-constructive mosaic subtest of the Wechsler Adult Intelligence Scale [WAIS -III (von Aster, 2006)], mostly because of his reach and grasp difficulties described below. While he was able to correctly describe location, shape and orientation of objects verbally, he typically adopted an abnormal hand posture when asked to grasp objects. At the same time, M.B. was able to perform tool use pantomime and imitate actions according to the Tulia-test (Vanbellingen et al., 2011), thus did not exhibit limb apraxia according to recent definitions (Osiurak \& Rossetti, 2017).

\subsubsection{Healthy control subjects}

For each of the behavioral tasks we compared M.B. with a group of 7-8 healthy, age-matched subjects. Details of each group are given in the subject description within the respective method section. Normal controls were recruited from the local community and entailed mostly university students and employees.

\subsection{MR-imaging}

\subsubsection{MRI acquisition}

MRI was performed in the same week as the behavioral tests using a 3 T MR system (Magnetom TIM Trio, Siemens Healthcare, Erlangen, Germany) with a standard 32-channel phased-array head coil. Three-dimensional (3D) anatomical datasets at $1 \mathrm{~mm}^{3}$ resolution were acquired with T1-weighting (turbo fast low angle shot (tFLASH), repetition time (TR): $2300 \mathrm{~ms}$, inversion time (TI): $900 \mathrm{~ms}$, echo time (TE): $2.96 \mathrm{~ms}$, flip angle $9^{\circ}$ ) and with T2-weighting (fluid-attenuated inversion recovery (FLAIR), TR: 5000 ms, TI: 1800 ms, TE: 394 ms, integrated parallel acquisition technique: factor 2).

\subsubsection{Lesion mapping}

Anatomical data were analyzed using the FMRIB software library (FSL 5.0.7, Center for Functional Magnetic Resonance Imaging of the Brain, University of Oxford, UK www.fmrib.ox. ac.uk/fsl). The T1-weighted tFLASH dataset was skull stripped (brain extraction tool BET) and registered to the standard brain template of the Montreal Neurologic Institute at $1 \mathrm{~mm}$ isotropic resolution (MNI152, provided with FSL), using the FMRIB's linear registration tool (FLIRT: 12 parameter affine transformation). The resulting transformation matrix was applied to the whole-head tFLASH dataset (without skull stripping) and the T2-weighted FLAIR dataset as well. In a second step, the linearly co-registered, whole-head tFLASH dataset was non-linearly registered to the MNI152 template using the FMRIB's non-linear registration tool (FNIRT). Again, the resulting transformation values were applied to the FLAIR dataset. Finally, FLIRT was used to sample the data to $.5 \mathrm{~mm}$ isotropic resolution using the MNI152 template at $.5 \mathrm{~mm}$ resolution. A digitalized version of the Morel atlas of the thalamus (Morel et al., 1997) provided by Krauth et al. (2010), registered to the high-resolution MNI 152 template allowed the visualization of the thalamic lesions in respect to the thalamic substructures detailed in the atlas. 


\subsection{Assessment of grasping performance}

Grasping was assessed qualitatively by scoring movies (at normal and reduced replay speed) recorded in a task that involved reaching to and picking up small objects from a table (two near (central) and two far (more peripheral left and right) positions), similar to our previous pulvinar inactivation experiments in monkeys (Wilke et al., 2010). For each trial, we separately evaluated the occurrence of 1) reach errors (i.e., first contact with the table noticeably off target) and 2) grasp errors (inappropriate wrist angle, lack of precision grip, or too wide grip aperture).

\subsection{Reaching experiments}

\subsubsection{Subjects}

Apart from patient M.B., seven neurologically intact and agematched subjects were tested (three males, mean age: 32.2 years, range: $26-39, \mathrm{SD}=4.5$ ). None of the subjects had a history of psychiatric illness and all had normal or correctedto-normal visual acuity.

\subsubsection{Experimental setup and stimulus presentation}

Subjects were sitting in a darkened room on a chair that was aligned to the center of the monitor with head and eyes facing straight ahead and with an eye-to-screen distance of $30 \mathrm{~cm}$. Their head was stabilized by a chin rest and locked tight into the position with bars pressing against both sides of the head (HeadLock $^{\mathrm{TM}}$ Ultra Precision Head Positioner TM, ViewPoint, Arrington Research, USA). Task controller and stimuli were programmed in MATLAB (The MathWorks, Inc. USA) using the Psychophysics Toolbox (Brainard, 1997). Stimuli were presented on a $27^{\prime \prime}$ LED display $(60 \mathrm{~Hz}$ refresh rate, model $\mathrm{HN} 274 \mathrm{H}$, Acer Inc. USA). Reaches were performed to a translucent surface acoustic wave touchscreen (IntelliTouch SCNIT-FLT27.8-001-006, ELO Touch Solutions Inc. USA) placed in front of the monitor. After a custom-made digital to analog conversion, horizontal and vertical finger touch coordinates were recorded at $100 \mathrm{~Hz}$ with an external data acquisition card (USB-1208FS, Measurement Computing Corporation, USA). Real-time eye tracking was performed with a ViewPoint system (Arrington Research Inc. USA) running on a separate PC, where a mini-IR sensitive camera placed below subjects' right eye continuously sampled their gaze position at $60 \mathrm{~Hz}$. The gaze position was then transferred to the task controlling PC using an Ethernet interface. Before the start of each experiment, the eye tracker was calibrated using the $4 \times 5$ point matrix from the ViewPoint software. An additional linear calibration implemented in the task controller guaranteed fast offset correction and gain refinement in case of slight head movements.

\subsubsection{Visually-guided delayed reaching tasks}

Before the start of the session and each block, the task and hand contingencies were explained to the subjects. M.B. and the control subjects performed the reach tasks in blocks in the same order. Task and hand varied as a function of block, which consisted of 20 successful trials. Each trial started with the onset of two dim fixation spots in the middle of the screen: a small $.5^{\circ}$ radius red circle (for eye fixation) and a larger $2^{\circ}$ radius green circle (for the hand). Subjects were required to look at the red circle and to touch the green circle. The circles would then brighten up and subjects would have to maintain fixation for $.5 \mathrm{sec}$ to start the contingency-specific part of the trial. A peripheral $2^{\circ}$ radius target stimulus at either $12^{\circ}$ or $24^{\circ}$ to the right or left of the central fixation circles cued the location of the movement. Subjects were instructed to start the reach when either one or both central circles disappeared after a delay of $1.28 \mathrm{sec}$. If only the central green circle disappeared, subjects had to make a reach while keeping their gaze at the red fixation spot (extrafoveal reaches). If both spots disappeared, subjects had to make a reach and were able to freely look for the remaining of the trial (foveal reaches). Once the hand was registered within a $5^{\circ}$ window around the target center, the targets would brighten up and subjects would have to maintain their gaze/hand position for $.5 \mathrm{sec}$ on the target. Subjects had $4 \mathrm{sec}$ to complete the movement and after each trial there was a 2 sec inter-trial interval.

\subsubsection{Reach definitions and statistical analysis}

Reach latency was defined as the time between fixation spot(s) offset and the moment when the hand lost contact with the touchscreen (reach onset). Reach duration was defined as the time from the reach onset to target acquisition. Reach endpoint was taken as the position of the first touchscreen contact after reach onset inside a $5^{\circ}$ (radius) window around the target center. For horizontal and vertical axes, the inaccuracy was calculated as mean (across trials) signed offset between the reach position and the target center, for each target. The horizontal and vertical inaccuracy values are reported in Supplementary Table S2. Note that for both hemifields, negative values for the horizontal inaccuracy denote undershooting. Additionally, we calculated the absolute (Euclidean distance) inaccuracy defined as the square root of the sum of squared offsets for each axis, reported in Supplementary Table S2 and in Fig. 3E and F. Similarly, reach imprecision (endpoint scatter) was defined as the square root of the sum of squared standard deviations (across trials) of the signed reach offsets for each axis. Unless noted otherwise, we analyzed successful trials as a function of hemifield and hand while combining the $12^{\circ}$ and $24^{\circ}$ target eccentricities (to increase statistical power since both eccentricities yielded similar results). All data analysis was performed using MATLAB R2012b and the Statistics Toolbox (The MathWorks, Inc. USA). M.B.'s data were analyzed by using univariate analyses of variance (ANOVA) with the factors Task (foveal reach us. extrafoveal reach), Space (left us. right hemifield) and Hand (left us. right hand). Statistical comparisons between M.B. and healthy controls were performed based on averaged data using Crawford's modified t-test for single case studies (Crawford \& Garthwaite, 2002). Means as well as t- and $p$-values for the comparisons of interest are reported in the text and listed in Supplementary Table S2.

\subsection{Visual spatial decisions}

\subsubsection{Subjects}

Apart from the patient M.B., eight neurologically intact subjects were tested (four males, mean age: 25.6 years, range: $20-30, \mathrm{SD}=1.8$ ). 


\subsubsection{Visual stimuli}

The stimuli consisted of trains of stereo flickers of $3 \mathrm{sec}$ duration that were presented on the horizontal plane of the screen at a horizontal eccentricity of $16^{\circ}$. Stimuli were drawn from a Poisson distribution. Each train had 6 flickers per second; each flicker duration was $16.7 \mathrm{~ms}$. Consecutive flickers had a minimum inter-pulse interval of $120 \mathrm{~ms}$ to minimize adaptation (Brunton, Botvinick, \& Brody, 2013). First and last flickers were presented bilaterally to prevent bias towards the side of the first or the last flicker presented. Stimuli were generated using MATLAB, version R2011b using custom scripts.

\subsubsection{Perceptual decision task}

Subjects were asked to perform a visual evidence accumulation task with two alternative forced choices, adapted from (Brunton et al., 2013) (Fig. 5A). Subjects had to determine whether more flickers were presented to the left or to the right side of the fixation spot. Each trial started with the presentation of a central red fixation cross, followed by a variable delay of 1-4 sec. The color of the fixation cross changed to green indicating the beginning of the trial. After a mandatory stable fixation period of $1 \mathrm{sec}$, stimuli were presented for 1-4 sec. Subjects were asked to respond with their right hand using the index finger to press key ' 1 ' for indicating that the trial had more stimuli on the left and key '2' with the middle finger when a trial had more stimuli on the right. No feedback was given to the subjects. The interval between trials was varied between 1 and $4 \mathrm{sec}$. Participants were asked to use the whole information presented to them in each trial to form their decision. Each participant completed one run with 48 trials.

\subsubsection{Behavioral data analysis}

We plotted the probability of a rightward choice as a function of number of flickers presented to the right minus the number of flickers presented to the left. We fit a 4-parameter sigmoidal function for each subject as follows:

$$
y(x)=y_{0}+\frac{a}{1+\exp \left(\frac{-\left(x-x_{0}\right)}{b}\right)}
$$

where $y_{0}$ is the left endpoint, $\left(y_{0}+a\right)$ is the right endpoint, $x_{0}$ is the bias, and $a / 4 b$ is the slope. Bias represents the inflection point of the sigmoidal curve in each subject. Fits were nonlinear least-square regressions calculated using the nlinfit function from MATLAB. Significant differences between M.B. and the healthy controls values of bias and slope were assessed with the Crawford modified t-test for single case studies.

\section{Results}

One apparent deficit of M.B. in daily life situations was an impairment in reach-grasp behavior, which appeared slowed and effortful. Fig. 2 illustrates his typical reach-grasp behavior under unconstrained viewing conditions. With the left hand, wrist rotation during the arm transport phase appeared abnormal and instead of a precision grip with the distal part of the fingers, he used the middle part of the index finger and the thumb to lift the object (Fig. 2A), or performed an even less accurate palm scooping movement with an abducted thumb
(Fig. 2B). When he attempted a precision grip (mostly with the right hand), his initial contact with the surface was typically off the target, and thumb-index finger aperture was too wide (Fig. 2C). The slowness of the grasp is indicated by the fact that he needed on average 3-4 sec to complete a given grasp as compared to typical $<1 \mathrm{sec}$ durations in healthy controls. Example reach-grasp movie sequences of patient M.B. are provided in the Supplementary Material.

Although it was not always possible to clearly dissociate misreaching from grasping deficits as in studies that specifically aimed to answer this question (Cavina-Pratesi, Ietswaart, Humphreys, Lestou, \& Milner, 2010), we estimated that $45 \%$ of trials contained only grasp impairments while the remaining contained reach as well as grasp errors. Impaired grip scaling was observed at closer and further table positions and with both hands.

\subsection{Reach performance}

The experiments described below aimed to quantify the reach aspect and to compare it with optic ataxia symptoms arising from parietal cortex lesions (Andersen et al., 2014; Perenin \& Vighetto, 1988). The reach data of M.B. were compared to a group of seven healthy subjects. We performed two types of reach tasks, measuring reach accuracy and timing. In the first (foveal reach) task, subjects were allowed to move their eyes to the target (Fig. 3A), while in the second (extrafoveal reach) task, subjects were not allowed to look at the target and were required to continue to fixate in the middle of the screen during the reach (Fig. 3B). From an optic ataxia we expected reach errors to be stronger in extrafoveal as compared to foveal reaches (Andersen et al., 2014; Perenin \& Vighetto, 1988).

\subsubsection{Reach endpoint accuracy and variability}

We first evaluated reach errors and their trial-by-trial endpoint variability as a function of hand usage and viewing condition. Fig. $3 \mathrm{C}$ and D displays the distribution of reach endpoints for the foveal and extrafoveal reach task in M.B. and the healthy controls. As compared with the average error of the healthy subjects, M.B's reach performance was less accurate and also more variable across trials with the left and right hand and to both sides of space (Supplementary Table S2). In the horizontal dimension, reaches were dysmetric (hypometric or hypermetric) without a systematic pattern. A more systematic pattern was revealed in respect to reach accuracy in the vertical dimension. Specifically, with the right hand M.B. misreached below the target in the left hemifield and above the target in the right hemifield, with similar reach errors for the foveal and extrafoveal reach condition (Fig. 3C-D). For statistical purposes, reach endpoint errors (inaccuracy) and variability (endpoint scatter) were grouped by hemifield, and data of M.B. were statistically compared with the healthy control (HC) group by the adjusted t-test (Crawford \& Garthwaite, 2002). In comparison to the healthy controls, M.B. exhibited significantly larger reaching errors when he used the right hand and the reach was toward the right hemifield (Fig. 3E-F). This higher magnitude of reach inaccuracy in M.B. was observed for both, the foveal and extrafoveal reach conditions [HC us. M.B., Foveal reach; $\mathrm{R}_{\text {hand }}$-RVF: . $7^{\circ}$ us. $2.2^{\circ}\left(\mathrm{t}_{(6)}=3.7, p<.01\right)$; Extrafoveal reach; $\mathrm{R}_{\text {hand }}$-RVF: . $6^{\circ}$ vs. $1.9^{\circ}$ 
A Hand posture during grasping
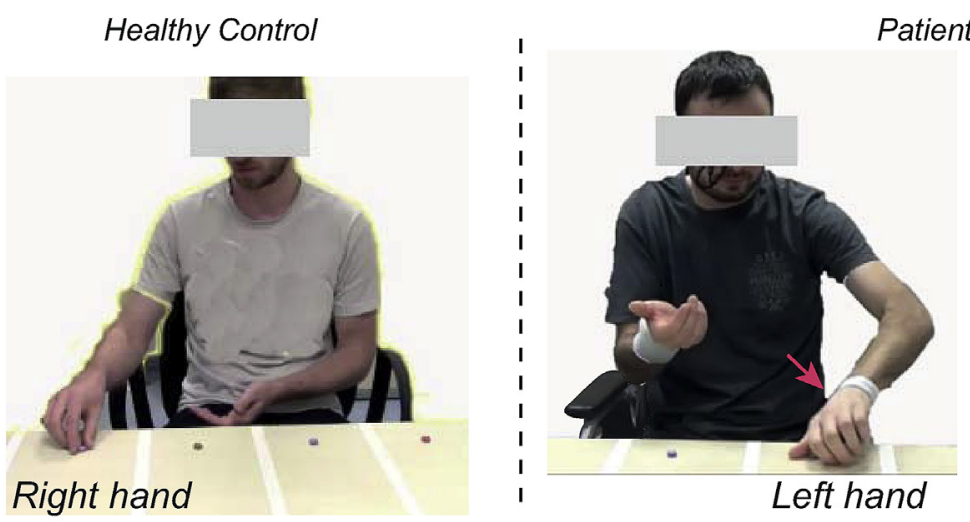

Patient M.B.

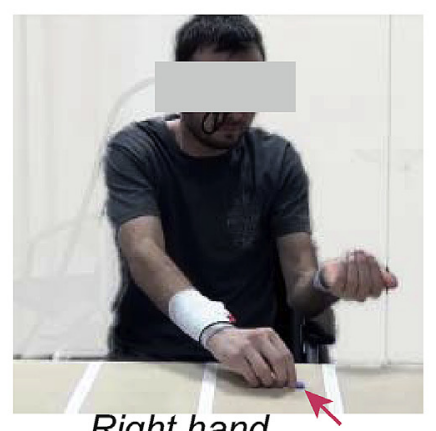

Right hand

\section{B Reach/Grasp Sequence: Left hand}

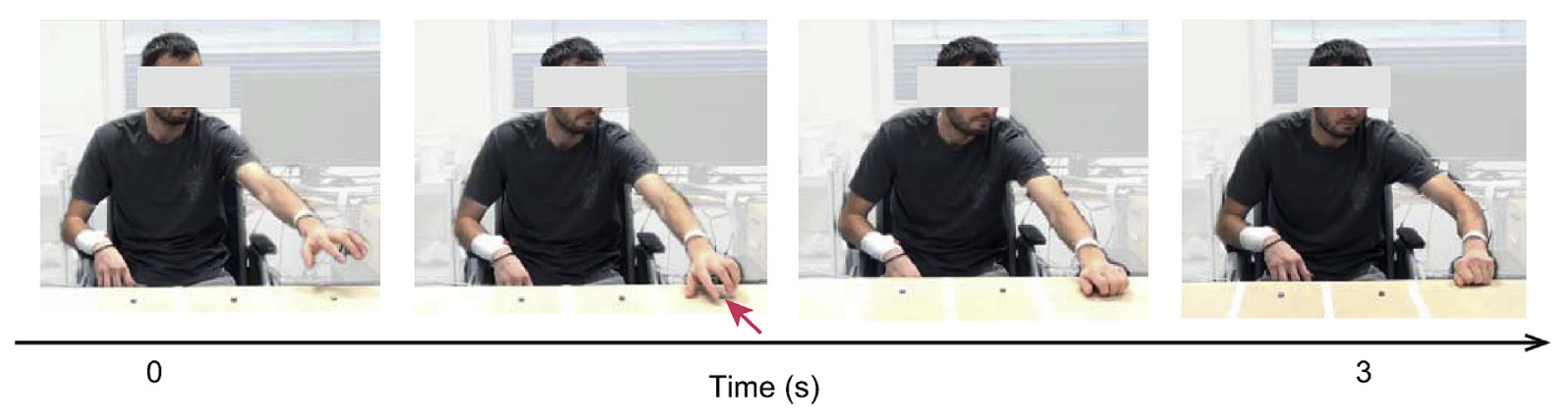

C Reach/Grasp Sequence: Right hand

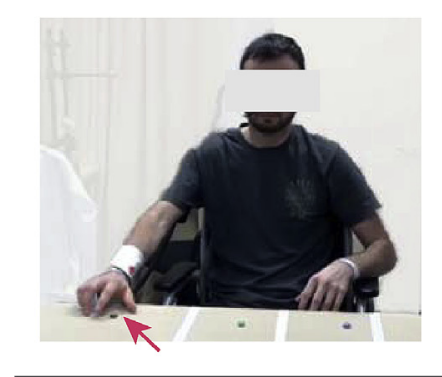

0

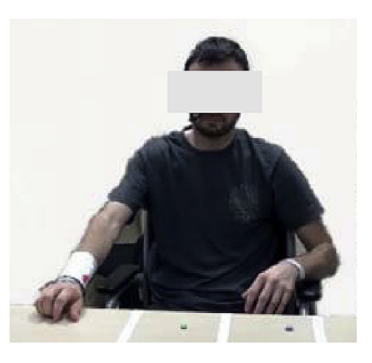

Time (s)

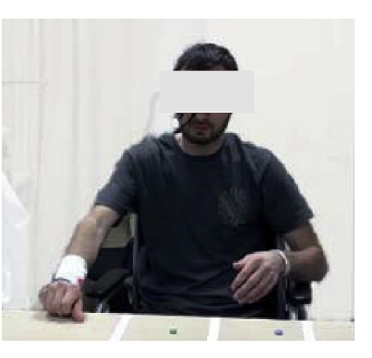

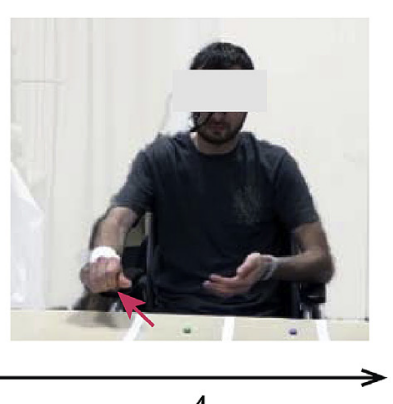

4

Fig. 2 - Reach-grasp deficits of M.B. (A) Typical grasp postures. Healthy control (left) and M.B. (right). Left hand: note the abnormal wrist angle (red arrow) and the absence of a precision grip. Right hand: note the attempted precision grip with the right hand, but off the target (red arrow). Also note that the non-acting hand is not dystonic but is used to hold the collected objects. (B) Typical reach-grasp sequence of M.B. with the left hand under unconstrained viewing conditions. Note the abduction of the thumb, absence of a precision grip (red arrow) and scooping movement for picking up the object. (C) Typical reach-grasp sequence of M.B. with the right hand. Note that scaling of the grip was too wide at the contact with the table and the object was squeezed between the index finger and the base of the abducted thumb (red arrows).

$\left.\left(\mathrm{t}_{(6)}=5.3, p<.01\right)\right]$ (Fig. 3E-F, Supplementary Table S2). None of the other comparisons with left hand and left visual field reached statistical significance with errors ranging between $.7^{\circ}$ and $1.5^{\circ}$ (all $\left.p>.1\right)$.

\subsubsection{Reach latencies and movement durations}

Next, we tested whether reaching deficits in M.B. would also be reflected in increased initiation times and movement durations, as has been observed in the recorded movies of unconstrained reach-to-grasp trials. To this end we compared reach initiation (lift of the hand after go-cue, central hand fixation offset) and reach durations (lift of the hand to target touch) between M.B. and the healthy controls. M.B.'s reach latencies towards both hemifields were delayed by 80-350 ms in comparison with the controls to either hemifield, with the longest latencies for right hand reaches (Fig. 4A 
A Foveal reach
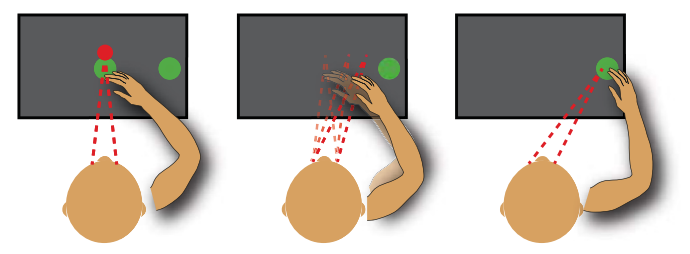

B

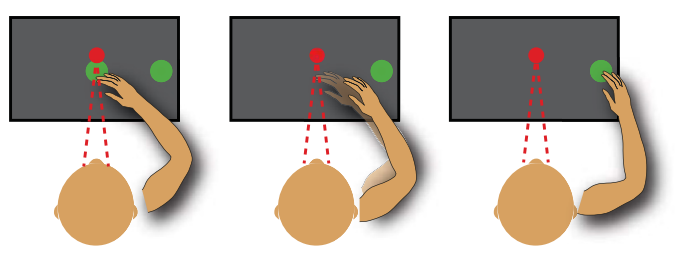

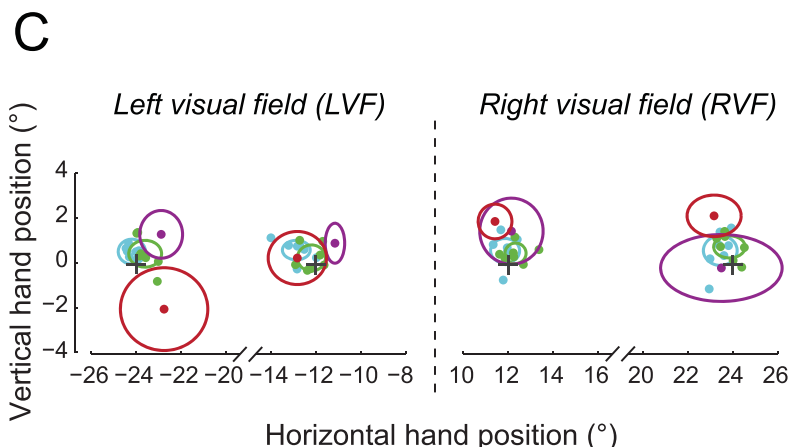

Horizontal hand position $\left({ }^{\circ}\right.$ )
D

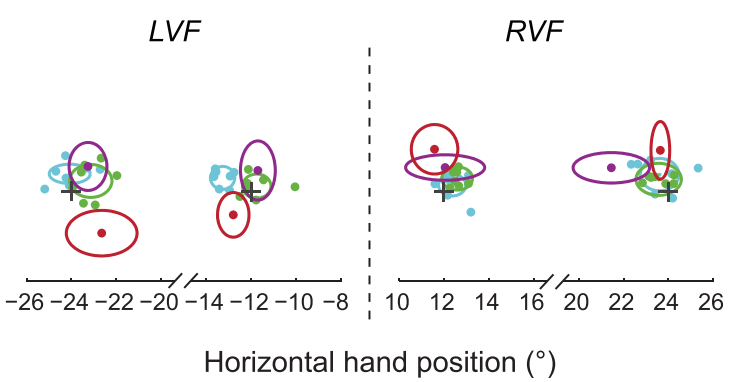

\section{Legend \\ Controls, left hand \\ Controls, right hand \\ M.B., left hand \\ M.B., right hand}

\section{E Reach endpoint errors}

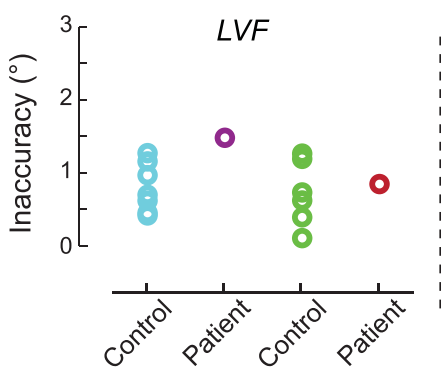

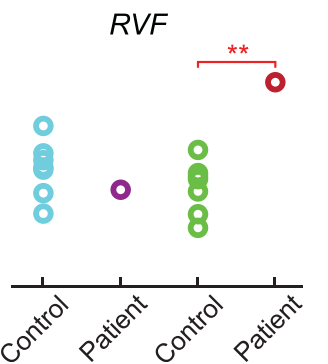

F
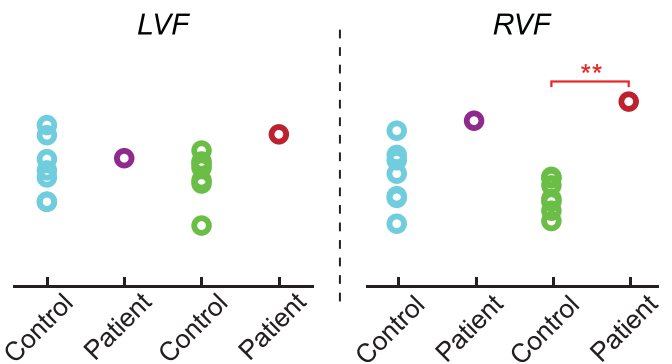

Fig. 3 - Reaching performance with foveal and extrafoveal viewing of the target. (A) Foveal reach task. (B) Extrafoveal reach task. (C,D) Endpoints of reaching movements in the foveal (left) and extrafoveal (right) reach tasks. Data are separated by visual hemifield (LVF and RVF) and hand (see Legend). Ellipses represent the horizontal and the vertical standard deviation over trials in M.B. and the mean standard deviation of the seven age-matched healthy control subjects. Note the larger endpoint variability in M.B. as compared to the healthy subjects, for reaches with both hands. (E,F) Absolute reaching inaccuracy (mean Euclidian distance from the reach target) for patient M.B. and controls as a function of hemifield, hand and reach task. Each dot represents the mean of a single subject, red connection lines indicate statistical significance of differences between M.B. and controls computed with the Crawford modified t-test, ${ }^{* *} p<.01$. LVF: left visual hemifield, RVF: right visual hemifield.

and B). This hand effect is also underlined by the ANOVA across trials in M.B., which revealed a main effect for hand $(F(1,72)=19.96, p<.0001)$, but not for space, hemifield or any interaction. M.B. not only started the reach movements later, but depending on the exact condition, he also needed between 500 and 1400 ms longer to complete the reach after initiation. In comparison with the healthy controls, this prolonged movement duration was statistically significant for both hands, hemifields as well as for foveal and extrafoveal reaches (two-tailed modified t-test, all $p<.05$, Fig. $4 \mathrm{C}$ and D; Supplementary Table S2). However, reach delays in M.B. were most pronounced with the right hand, which is also expressed in the ANOVA, revealing a significant main effect of hand $(\mathrm{F}(1,72)=26.6, p<.0001)$. The stronger reach deficit with the right hand might be explained by the more extensive lesion in his left pulvinar.

\subsection{Saccades and perceptual decision making}

The hand-specific effect in the reaching task and the generalization of his deficits across viewing conditions already suggests that M.B.'s reach difficulties cannot be solely due to deficient eye movements or perceptual impairments. Nonetheless, we conducted two additional control tasks that aimed 


\section{Foveal reach}

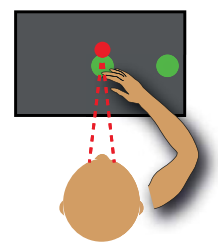

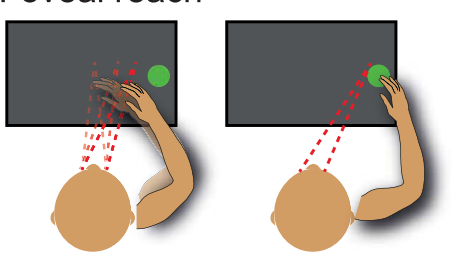
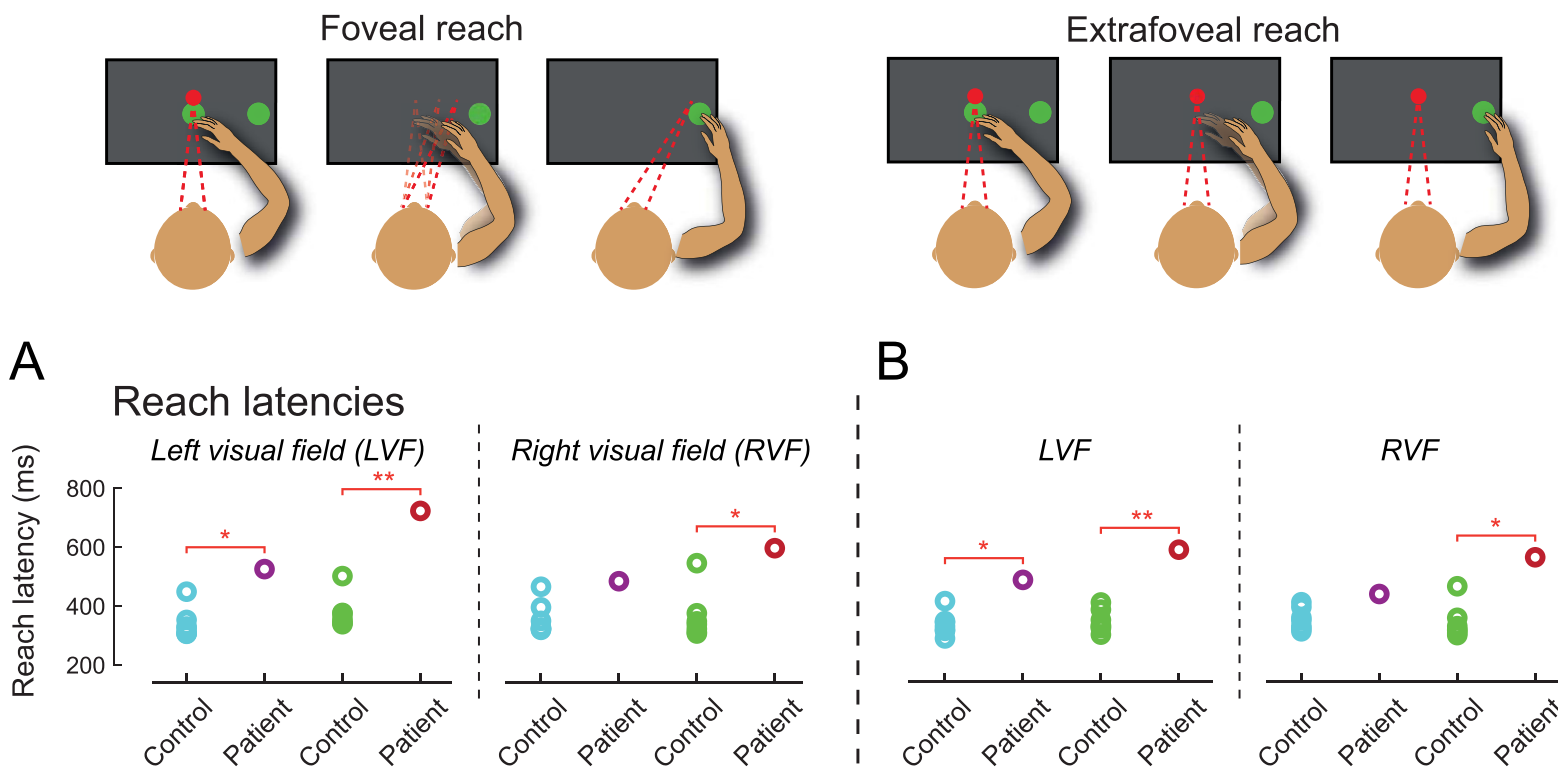

B
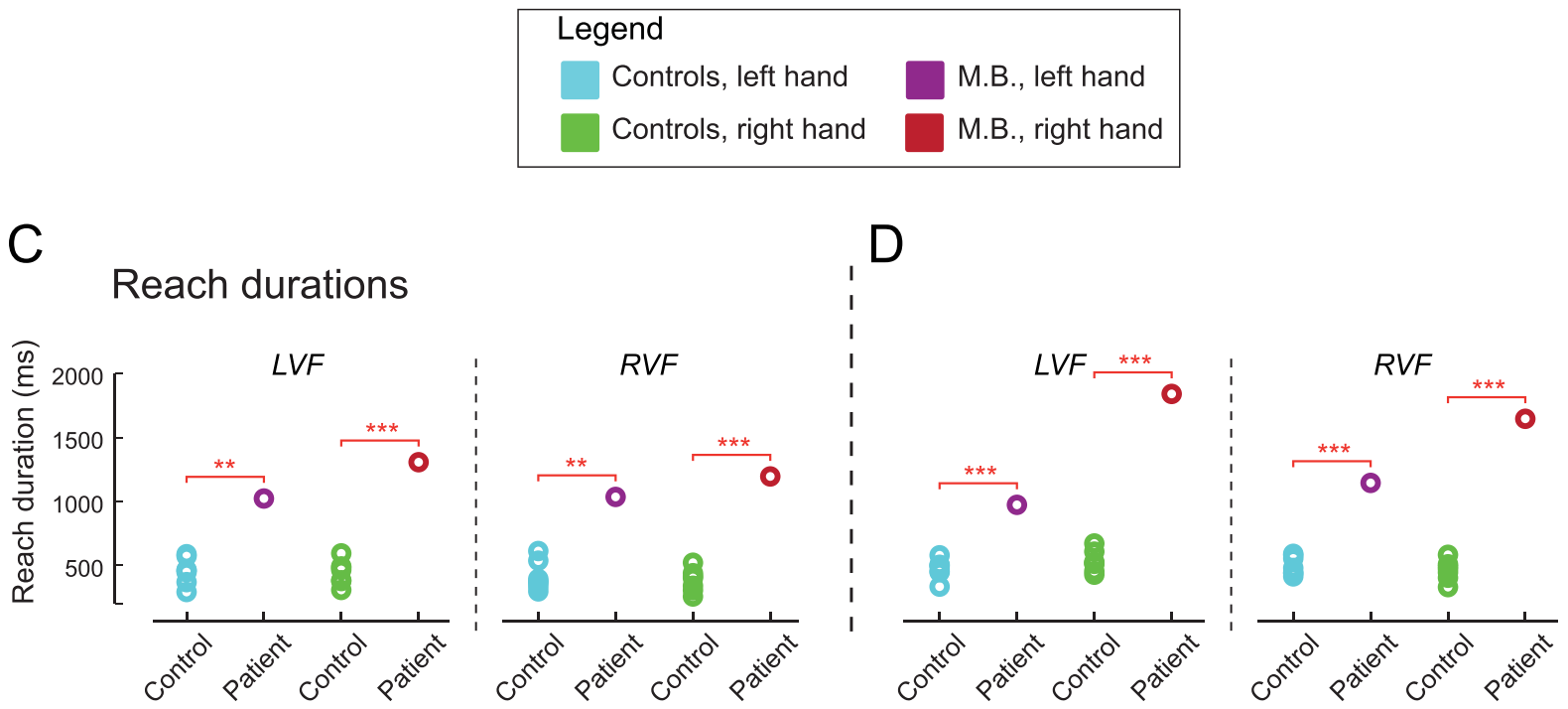

Fig. 4 - Reach latencies and durations in the foveal and extrafoveal reach task. (A,B) Reach latencies denoting the time between offset of the hand fixation spot and lift of the finger from the screen for correct trials, as a function of hand and hemifield, in the foveal (A) and extrafoveal (B) reach tasks. (C,D) Mean movement duration as a function of hand and hemifield in the foveal (C) and extrafoveal (D) reach tasks. Duration was computed from movement onset (lift of the finger from the touch screen) until target acquisition within the $5^{\circ}$ success window around the target. In (A-D) each dot represents the mean of a single subject, red connection lines indicate statistical significance of differences between M.B. and the controls computed with the Crawford modified t-test, ${ }^{*} p<.05,{ }^{* *} p<.01,{ }^{* * *} p<.001$. LVF: left visual hemifield, RVF: right visual hemifield.

to investigate saccade and perceptual performance without accompanying reaches.

\subsubsection{Saccades}

In order to assess eye movement performance, we tested M.B. in a visually-guided saccade task towards instructed and freely chosen targets at eccentricities of $10^{\circ}, 12.5^{\circ}$ or $15^{\circ}$ (Supplementary Material, Fig. S3A). Target locations were randomized across trials. In the instructed condition only one target was presented; in the choice condition two targets were presented at corresponding positions: one in the left and one in the right visual hemifield. In choice trials, subjects were asked to choose on every trial whether they wanted to perform a saccade to the left or to the right target (Supplementary Fig. S3A). In choice trials, M.B. selected in $39 \%$ of trials the right target and did thus not exhibit a significant spatial choice 
bias in comparison to the healthy controls $\left(\mathrm{t}_{(7)}=-.68, p=.52\right)$. This measure also confirms the neurological and neuropsychological assessment that M.B. did not exhibit spatial neglect or extinction (Supplementary Fig. S3B). Unlike the reaches described above, saccade latencies and durations did not significantly differ between M.B. and the healthy controls (all $p>$.2, Supplementary Fig. S3C and Table S3). A consistent impairment however was observed in saccade endpoints: in comparison to the healthy controls, M.B. exhibited hypometric saccades towards both hemifields and in both instructed and choice trials (all $p<.01$; Supplementary Fig. S3D). The saccade hypometria was demonstrated by a significantly lower gain of M.B's. saccades for both left and right targets as well as by the saccade endpoint inaccuracy (undershooting) as compared to the healthy control group (Supplementary Table S3; Supplementary Fig. S3E; saccade gain instructed: LVF: $\mathrm{t}_{(7)}=-7.7, p<.001 ;$ RVF: $\mathrm{t}_{(7)}=-3.9, p<.01$; choice: LVF: $\left.\mathrm{t}_{(7)}=-6.1, p<.001 ; \mathrm{RVF} \mathrm{t}_{(7)}=-5.8, p<.001\right)$. Since our target array was predominantly horizontal and did not contain purely vertical locations, we cannot independently evaluate the vertical saccade component, but the pattern of the endpoints with undershooting in both the horizontal and the vertical dimensions suggest hypometria along the target vector without a vertical tilt.

\subsubsection{Perceptual spatial decision making}

All tasks described above required a directed motor response. In order to assess perceptual performance in a more isolated manner, we tested the ability of M.B. to accumulate visual evidence from each hemifield without a directed motor response, i.e., reported with a button press. In this perceptual decision task (Brunton et al., 2013), different numbers of visual flickers were presented in each hemifield and subjects were required to accumulate the sensory evidence to decide which side contained more stimuli (Fig. 5A). As shown in the psychometric plots fitted with a four-parameter sigmoidal function to the rightward choices of each individual subject, performance of M.B. and the healthy subjects in this task was similar (Fig. 5B). Importantly, as compared to the healthy subjects, M.B. did not exhibit a significant decision bias towards either hemifield $\left(\mathrm{t}_{(7)}=-.51, p=.62\right)$ (Fig. 5C). We also did not find a significant perceptual accumulation deficit, indicated by the insignificant difference between slope estimates from patient data as compared to healthy controls $\left(\mathrm{t}_{(7)}=.19\right.$,

A Perceptual decision task

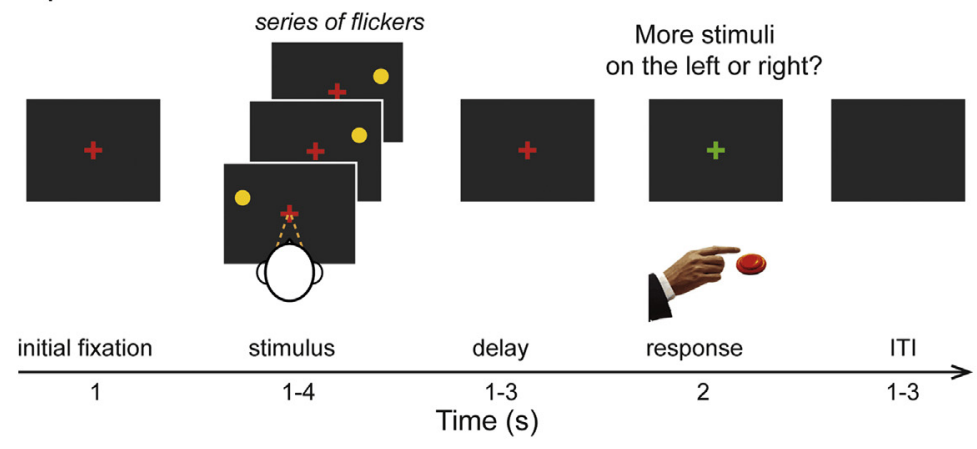

B Decisions in the visual accumulation task
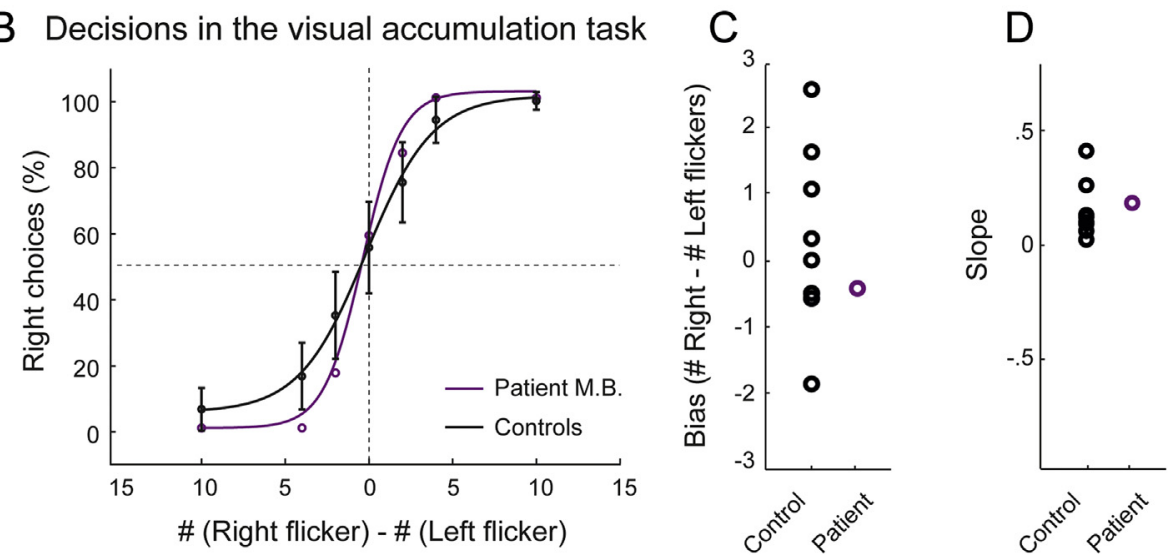

Fig. 5 - Perceptual evidence accumulation performance. (A) Visual decision task with two alternative forced choices. (B) Psychometric curves of \% rightward choices as a function of number of flickers presented to the right minus number of flickers to the left. Curves represent fitting of a four parameters sigmoid function, dots the unfitted raw percentages (purple: patient M.B., black: healthy controls). Note the similarity of the curves, indicating that M.B. was able to accumulate perceptual evidence from both sides of space and to form a correct decision. (C,D) Each dot represents the spatial bias defined as the inflection point of the sigmoidal curve (C), or the slope of each individual subject (D). Note that spatial bias and slope of M.B. were in the range of the healthy subjects. 
$p=.86$ ) (Fig. 5D). There were no reaction time differences between M.B. and controls (leftward: $\mathrm{t}_{(7)}=.5, p=.63$; rightward: $\left.\mathrm{t}_{(7)}=-.17, p=.87\right)$. Thus, data from the evidence accumulation task are in agreement with the free choice saccade task, indicating that M.B. did not suffer from a visual perception deficit.

\section{Discussion}

Taken together, our results indicate that the subcortical route through the medial pulvinar is critical for proper reach-grasp behavior. To our knowledge, this is the first study that systematically investigated reach behavior after a selective pulvinar lesion in humans. Possibly, this is because the known cases with thalamic lesions also affecting the pulvinar were of vascular etiology and thus entailed primary somatosensory and motor nuclei in the thalamus as well, resulting in respective deficits (Schmahmann, 2003; Schmahmann \& Pandya, 2008). The symptoms seen in M.B. shared features of (parietal) cortex and cerebellar lesions, without being identical with either of them. While his grasping deficits were reminiscent of patients with parietal lesions, he did not show optic ataxia which is alleviated with central object viewing (Andersen et al., 2014; Perenin \& Vighetto, 1988). In summary, our data indicate that the function of the human pulvinar goes well beyond its subscribed role in visual cognition and provide evidence that the medial pulvinar serves as an important hub for the control of limb movements.

\subsection{The pulvinar and reach-grasp behavior}

During object grasping under natural viewing conditions, M.B. often exhibited an abnormal wrist rotation during the arm movement, did not adapt a precision grasp, widened the grip aperture too much, and performed a scooping movement only after making contact with the object. In the quantified reaching task, M.B.'s reaching performance was less accurate and slower than in controls, in particular when he used the right hand and when the reach was towards the right hemifield.

Is it possible that the grasping deficit of M.B. reflected a secondary impairment due to positional insecurity and misreaching as has been previously reported in an optic ataxia patient with a posterior parietal lesion (Cavina-Pratesi et al., 2010)? Although we cannot exclude the possibility that some of the errors in the reach-to-grasp task might have been due to spatial mislocalization, this would not well account for the distorted hand posture, cumbersome approach trajectory, and the difficulty in picking up the object. Also, in contrast to the optic ataxia patient described in (Cavina-Pratesi et al., 2010) his grasp deficit occurred for close and peripheral object locations. Most importantly, grasp deficits were more pronounced with the left hand, while reach endpoint errors in the touchscreen task were more pronounced when he used the right hand and for reaches toward the right hemifield. It needs to be noted however that a clear limitation of our study is the absence of precise kinematic measurements to dissociate between proximal and distal components of the grasp movements (Jakobson, Archibald, Carey, \& Goodale, 1991).
Generally, the pattern of reach and grasp deficits resembled our previous observations with pharmacological inactivation of the dorsal pulvinar in monkeys, which also showed failures to form a precision grip and increased reach errors that were stronger for the contralesional hand and space (Wilke et al., 2010). In this monkey study however, reach behavior following pulvinar inactivation was only assessed under unconstrained viewing conditions. Thus, further monkey inactivation studies are needed to resolve whether the reach deficits in monkeys would be present for foveal as well as extrafoveal viewing conditions. To our knowledge, this is the first report examining reach and grasp deficits in a patient with a pulvinar lesion without accompanying lesions in any of the primary sensory or motor thalamic nuclei such as ventrolateral thalamus, lateral geniculate nucleus or white matter tracts running through the internal capsule. The sparing of those nuclei and tracts in our patient is most likely due to the fact that the lesion etiology of M.B. was a sarcoidosis and thus did not affect the vascular territories that are typically damaged by ischemia. Thus, the closest patient in the literature is the description of reach-grasping deficits in a patient with thalamic hemorrhage that was more extensive than in M.B. and entailed the right posterior thalamus (pulvinar, geniculate body), superior colliculus and adjacent fibers of the internal capsule as well as (Classen et al., 1995). In contrast to M.B. this patient had primary visual and somatosensory deficits as well. Similar to M.B. the patient described in this study (Classen et al., 1995) misreached with either hand and in both hemifields with and without central fixation and grasp kinematics were also impaired. The observed reach deficits of M.B. differed from optic ataxia observed following unilateral or bilateral parietal lesions in monkeys and humans as they were: 1) not alleviated when he was allowed to look at the target (foveal reach) and 2) the largest reach errors were observed in the vertical and not in the horizontal dimension (Battaglia-Mayer et al., 2013; Hwang, Hauschild, Wilke, \& Andersen, 2012; Karnath \& Perenin, 2005; Perenin \& Vighetto, 1988). Interestingly, some symptoms of M.B. also overlapped with descriptions of lesions in deep cerebellar nuclei, including the bilateral reach imprecision and hypometric saccades. However, in contrast to patients with a cerebellar lesion (Bastian \& Thach, 1995), movements of M.B. were not decomposed and appeared rather smooth. Some of the features such as the abnormal approach and object picking patterns, hand specificity and the fact that M.B. exhibited similar reach deficits whether he was allowed to look at the target and the hand or had to maintain central fixation, suggest that the problem was not 'just' on a cognitive or visuomotor transformation level. In this sense, one might consider at least part of the observed deficit as "motor". In the context of visuomotor tasks, dorsal pulvinar neurons show a variety of firing patterns ranging from purely visuospatial responses modulated by the task context to motor planning, execution, and post-execution signals, further modulated by postural effects (Acuna, Gonzalez, \& Dominguez, 1983; Dominguez-Vargas et al., 2017). Therefore, it is unlikely that the deficits observed after dorsal pulvinar lesion would be readily classified into visuospatial, transformational, or motor-only domains. Generally, reach and grasping movements are dependent on neural activity in a widely distributed 
network consisting of cortical regions such as motor, premotor, ventral supplementary motor area (SMA), superior parietal and dorsal occipital cortex as well as the cerebellum (Andersen et al., 2014; Castiello, 2005). All of those structures have reciprocal connections with the dorsal pulvinar as evidenced by anatomical tracer and microstimulation studies in monkeys (Baleydier \& Mauguiere, 1985; Gutierrez et al., 2000; Romanski, Giguere, Bates, \& Goldman-Rakic, 1997; Sultan et al., 2012; Trojanowski \& Jacobson, 1974), and functional imaging and microstimulation studies in humans (Arcaro et al., 2015; Barron et al., 2015; Rosenberg et al., 2009). The unique combination of M.B.'s symptoms might thus either be explained by the loss of integrative pulvinar functions and/or by a functional disconnection with and between those regions.

\subsection{Effects on saccade metrics and spatial decision behavior}

M.B. had normal saccade latencies to all screen positions tested, which is consistent with a previous posterior thalamus lesion study in humans (Rafal et al., 2004). There is convergent evidence from lesion studies in humans (Rafal et al., 2004; Van der Stigchel et al., 2010) and monkeys (Wilke et al., 2013; Wilke et al., 2010) that the dorsal pulvinar, including its medial portion, is a critical contributor to the selection of goaldirected eye movements but is less critical for saccade execution itself. This notion is also consistent with electrophysiological and microstimulation studies in monkeys, showing a diversity of saccade-related neural activity without a clear retinotopic or saccade direction organization and relatively high current thresholds for evoking saccades, unlike in the connected superior colliculus, frontal eye fields or lateral intraparietal area (Benevento \& Port, 1995; DominguezVargas et al., 2017). Albeit investigated in only few studies, pulvinar lesions in humans or monkeys do typically not result in primary oculomotor deficits and when such deficits have been reported in either humans (Rafal et al., 2004) or in monkeys performing memory saccades (Wilke et al., 2013), effect sizes were relatively small. However, saccade metrics were affected in M.B. as he did exhibit hypometric saccades towards both hemifields. This undershooting was not easily predicted from the current pulvinar literature, unless one assumes a Balint-like syndrome that can be found following bilateral parietal lesions (Andersen et al., 2014). Indeed, attention-related impairments in distractor filtering, spatial exploration and decision-making have been repeatedly reported following (unilateral) pulvinar lesions in humans (Karnath et al., 2002; Rafal et al., 2004; Van der Stigchel et al., 2010) and monkeys (Robinson \& Petersen, 1992; Wilke et al., 2013; Wilke et al., 2010; Zhou et al., 2016). Since the lesion in M.B. was more pronounced in the left pulvinar, we expected a saccade choice bias towards the left hemifield based on our monkey studies that used a similar task design (Wilke et al., 2013; Wilke et al., 2010). While M.B. did choose slightly more targets $(61 \%)$ in the left visual hemifield, this bias was in the range of the normal subjects. The absence of neglect and extinction might be due to the fact that the pulvinar lesion was most pronounced in the left hemisphere or could be due to the fact that the lesion was bilateral to some extent
(Rushmore, Valero-Cabre, Lomber, Hilgetag, \& Payne, 2006). Since the patient with a left medial pulvinar lesion described by Ward et al. did also not exhibit spatial neglect symptoms, we favor the interpretation that left dorsal pulvinar lesions in humans might not lead to a spatial bias (Ward et al., 2005). It is also worth noting that spatial attention and higher-order saccade selection deficits following pulvinar lesions were typically interpreted to reflect functional disruption in frontoparietal cortices (Arend, Machado, et al., 2008; Wilke et al., 2010). However, the pattern of intact saccade latencies in combination with hypometric saccade endpoints differ from left and right unilateral or bilateral parietal lesions in humans that cause deficits in both latencies and endpoints (PierrotDeseilligny, Rivaud, Gaymard, \& Agid, 1991; Ptak \& Muri, 2013). Also, the perceptual decisions reported by a button response were not impaired in M.B., which is consistent with recent dorsal pulvinar inactivation studies in monkeys, showing intact detection of large reward stimuli even in the contralesional hemifield (Wilke et al., 2013) and intact perceptual categorization performance (Komura et al., 2013). Since the error patterns in the reach tasks qualitatively differed from the saccade errors and since the perceptual task did not reveal deficits, we assume that the reach deficits might be related to an impaired integration of visual stimulus position, hand position and/or movement planning.

\subsection{Possible limitations}

Finally, we wondered whether the reaching deficits could be due to a lesion in structures other than the pulvinar. We have carefully assessed tracts and nuclei that could potentially lead to a similar picture as documented in our patient and discuss it together with the neurological assessment. The MR scans indicated that the lesion in M.B. did not involve those thalamic nuclei that are usually affected by ischemia-induced lesions: the posterior cerebral artery supplies not only the posterior thalamus but also the lateral nucleus and the ventral posterior nucleus. The posterior choroidal arteries supply not only the pulvinar but also the lateral and medial geniculate nucleus (Schmahmann, 2003; Schmahmann \& Pandya, 2008). Theoretically, a lesion in the rubro-thalamic tract could result in a similar picture. However, the rubro-thalamic tract runs far more anterior and lateral than the lesion in M.B. (Kwon et al., 2011). Patients with affection of the paramedian territory usually show somnolence, gaze abnormalities, display memory problems or hemiparesis. If the thalamogeniculate area or the posterior choroidal artery territories are affected, one expects neurological symptoms that were not present in M.B. such as hemiataxia, pain sensations and hemianopia. Tuberothalamic lesions come with facial paresis and hemisymptoms. As stated above, M.B. did not have abnormalities in his primary sensory qualities (e.g., position sense, touch, vibration) nor in his vestibular or cerebellar tests. Nevertheless, he showed deficits with reaching and grasping. We think that this can only be explained by the lesion pattern due to the atypical sarcoidosis manifestation that spares vascular territories and is restricted to the medial pulvinar. Is it possible that the visuomotor deficits in M.B. are due to damage of fibers that originate in neighboring thalamic nuclei but travel through the pulvinar? This is unlikely since (unlike the lateral pulvinar portion) there is no clear 
evidence for fiber tracts travelling through the core of the medial pulvinar (Rosenberg et al., 2009). Consideration must also be given to the fibers that travel within or along the posterior thalamus such as the posterior limb of the internal capsule and the brachium of the superior colliculus that connects the parietal cortex with the superior colliculus (Jones, 2007). However, no such damage was detected on the MRI. While the possibility remains that the patient had very small lesions that were below the spatial resolution of our structural imaging, we conclude based on his clinical symptoms, which clearly differed between lesions of the parietal cortex (optic ataxia) and from lesions of the posterior limb of the internal capsule (which lead to disturbances in primary sensory and motor functions), that the deficits observed in of M.B. are most parsimoniously explained by the pulvinar lesion.

\subsection{Ethics}

All subjects, including patient M.B. gave written informed consent to participate in the study and M.B. signed a video release form. The study was approved by the local Ethics Committee of the Georg-August-University Göttingen according to the Declaration of Helsinki.

Supplementary data related to this article can be found online at https://doi.org/10.1016/j.cortex.2017.10.011.

\section{Author contributions}

M.W. and M.B. designed the study, M.W., I.K. and M.B. wrote the paper. M.B. supervised and/or performed the clinical assessment, neurophysiological and neuropsychological examinations of the patient, M.W. and I.K. planned and supervised data collection and analysis. L.S. and A.D. programmed, performed and analyzed the reaching experiments. K.M., H.S. and M.W. planned, performed and analyzed the grasping data. P.D., C.S., Y.C. performed the imaging data acquisition and P.D. analyzed the structural data for lesion mapping. C.S. programmed, performed and analyzed the saccade experiment. A.N. performed and analyzed the perceptual accumulation task. All authors contributed figures, methods and result sections and critique to the manuscript.

\section{Acknowledgments}

We thank Dr. Jeffrey C. Erlich for providing the MATLAB script for stimulus generation for the perceptual accumulation tasks and help with the analysis, Severin Heumueller for excellent technical and computer support, Britta Perl and Ilona Pfahlert for assistance with the acquisition of the MRI data, Gunther Helms for the initial implementation of the digital Morel atlas and Sabine Nuhn for performing the neuropsychological testing. We thank Drs. Hans-Otto Karnath, Jörg Schultz and Kristina Nielsen for valuable discussions of the recorded patient movies. We are especially grateful to patient M.B. Supported by the Hermann and Lilly Schilling Foundation, German Research Foundation (DFG) grants: WI 4046/1-1 and Research
Unit GA1475-B4, KA 3726/2-1 and the Center for Nanoscale Microscopy \& Molecular Physiology of the Brain (CNMPB).

\section{Supplementary data}

Supplementary data related to this article can be found at https://doi.org/10.1016/j.cortex.2017.10.011.

\section{REFERENCES}

Acuna, C., Gonzalez, F., \& Dominguez, R. (1983). Sensorimotor unit activity related to intention in the pulvinar of behaving Cebus Apella monkeys. Experimental Brain Research, 52, $411-422$.

Albanese, A., Bhatia, K., Bressman, S. B., Delong, M. R., Fahn, S., Fung, V. S., et al. (2013). Phenomenology and classification of dystonia: A consensus update. Movement Disorders: Official Journal of the Movement Disorder Society, 28, 863-873.

Andersen, R. A., Andersen, K. N., Hwang, E. J., \& Hauschild, M. (2014). Optic ataxia: From Balint's syndrome to the parietal reach region. Neuron, 81, 967-983.

Arcaro, M. J., Pinsk, M. A., \& Kastner, S. (2015). The anatomical and functional organization of the human visual pulvinar. The Journal of Neuroscience: The Official Journal of the Society for Neuroscience, 35, 9848-9871.

Arend, I., Machado, L., Ward, R., McGrath, M., Ro, T., \& Rafal, R. D. (2008). The role of the human pulvinar in visual attention and action: Evidence from temporal-order judgment, saccade decision, and antisaccade tasks. Progress in Brain Research, 171, 475-483.

Arend, I., Rafal, R., \& Ward, R. (2008). Spatial and temporal deficits are regionally dissociable in patients with pulvinar lesions. Brain, 131, 2140-2152.

Aschenbrenner, S., Tucha, O., \& Lange, K. (2000). In Regensburger Wortflüssigkeitstest-RWT. Göttingen: Hogrefe Publishing.

von Aster, M. (2006). In Wechsler Intelligenztest für Erwachsene: WIE; Übersetzung und Adaption der WAIS-III. Harcourt Test Services.

Baleydier, C., \& Mauguiere, F. (1985). Anatomical evidence for medial pulvinar connections with the posterior cingulate cortex, the retrosplenial area, and the posterior parahippocampal gyrus in monkeys. Journal of Comparative Neurology, 232, 219-228.

Barron, D. S., Eickhoff, S. B., Clos, M., \& Fox, P. T. (2015). Human pulvinar functional organization and connectivity. Human Brain Mapping, 36, 2417-2431.

Bastian, A. J., \& Thach, W. T. (1995). Cerebellar outflow lesions: A comparison of movement deficits resulting from lesions at the levels of the cerebellum and thalamus. Annals of Neurology, 38, 881-892.

Battaglia-Mayer, A., Ferrari-Toniolo, S., Visco-Comandini, F., Archambault, P. S., Saberi-Moghadam, S., \& Caminiti, R. (2013). Impairment of online control of hand and eye movements in a monkey model of optic ataxia. Cerebral Cortex, 23, 2644-2656.

Bäumler, G., \& Stroop, J. R. (1985). In Farbe-Wort-Interferenztest nach JR Stroop (FWIT). Hogrefe: Verlag für Psychologie.

Benarroch, E. E. (2015). Pulvinar: Associative role in cortical function and clinical correlations. Neurology, 84, 738-747.

Bender, D. B., \& Youakim, M. (2001). Effect of attentive fixation in macaque thalamus and cortex. Journal of Neurophysiology, 85, 219-234.

Benevento, L. A., \& Port, J. D. (1995). Single neurons with both form/color differential responses and saccade-related 
responses in the nonretinotopic pulvinar of the behaving macaque monkey. Visual Neuroscience, 12, 523-544.

Brainard, D. H. (1997). The psychophysics toolbox. Spatial Vision, 10, 433-436.

Bridge, H., Leopold, D. A., \& Bourne, J. A. (2016). Adaptive pulvinar circuitry supports visual cognition. Trends in Cognitive Sciences, 20, 146-157.

Brunton, B. W., Botvinick, M. M., \& Brody, C. D. (2013). Rats and humans can optimally accumulate evidence for decisionmaking. Science, 340, 95-98.

Cappe, C., Morel, A., Barone, P., \& Rouiller, E. M. (2009). The thalamocortical projection systems in primate: An anatomical support for multisensory and sensorimotor interplay. Cerebral Cortex, 19, 2025-2037.

Castiello, U. (2005). The neuroscience of grasping. Nature Reviews Neuroscience, 6, 726-736.

Cavina-Pratesi, C., Ietswaart, M., Humphreys, G. W., Lestou, V., \& Milner, A. D. (2010). Impaired grasping in a patient with optic ataxia: Primary visuomotor deficit or secondary consequence of misreaching? Neuropsychologia, 48, 226-234.

Classen, J., Kunesch, E., Binkofski, F., Hilperath, F., Schlaug, G., Seitz, R. J., et al. (1995). Subcortical origin of visuomotor apraxia. Brain, 118(Pt 6), 1365-1374.

Crawford, J. R., \& Garthwaite, P. H. (2002). Investigation of the single case in neuropsychology: Confidence limits on the abnormality of test scores and test score differences. Neuropsychologia, 40, 1196-1208.

Dominguez-Vargas, A. U., Schneider, L., Wilke, M., \& Kagan, I. (2017). Electrical microstimulation of the pulvinar biases saccade choices and reaction times in a time-dependent manner. The Journal of Neuroscience, 37, 2234-2257.

Fels, M., \& Geissner, E. (1997). Neglect-Test (NET) Ein Verfahren zur Erfassung visueller Neglectphänomene. Göttingen: Hogrefe, (Chapter Chapter).

Fritz, D., van de Beek, D., \& Brouwer, M. C. (2016). Clinical features, treatment and outcome in neurosarcoidosis: Systematic review and meta-analysis. BMC Neurology, 16, 220.

Ghika, J., Bogousslavsky, J., Henderson, J., Maeder, P., \& Regli, F. (1994). The "jerky dystonic unsteady hand": A delayed motor syndrome in posterior thalamic infarctions. Journal of Neurology, 241, 537-542.

Grieve, K. L., Acuna, C., \& Cudeiro, J. (2000). The primate pulvinar nuclei: Vision and action. Trends in Neurosciences, 23, 35-39.

Guillery, R. W., \& Sherman, S. M. (2002). The thalamus as a monitor of motor outputs. Philosophical Transactions of Royal Society of London B: Biological Sciences, 357, 1809-1821.

Gutierrez, C., Cola, M. G., Seltzer, B., \& Cusick, C. (2000). Neurochemical and connectional organization of the dorsal pulvinar complex in monkeys. Journal of Comparative Neurology, $419,61-86$

Härting, C., Markowitsch, H. J., Neufeld, H., Calabrese, P., Deisinger, K., Kessler, J. H., et al. (2000). In Wechsler Gedächtnis Test-Revidierte Fassung (WMS-R). Bern: Huber.

Helmstaedter, C., Lendt, M., \& Lux, S. (2001). In VLMT: Verbaler Lern-und Merkfähigkeitstest. Göttingen: Hogrefe.

Hoitsma, E., Drent, M., \& Sharma, O. P. (2010). A pragmatic approach to diagnosing and treating neurosarcoidosis in the 21st century. Current Opinion in Pulmonary Medicine, 16, 472-479.

Hwang, E. J., Hauschild, M., Wilke, M., \& Andersen, R. A. (2012). Inactivation of the parietal reach region causes optic ataxia, impairing reaches but not saccades. Neuron, 76, 1021-1029.

Jakobson, L. S., Archibald, Y. M., Carey, D. P., \& Goodale, M. A. (1991). A kinematic analysis of reaching and grasping movements in a patient recovering from optic ataxia. Neuropsychologia, 29, 803-809.
Jones, E. G. (2007). The lateral posterior and pulvinar nuclei. In The thalamus (2nd ed., pp. 1009-1071). Cambridge: Cambridge University Press.

Kaas, J. H., \& Lyon, D. C. (2007). Pulvinar contributions to the dorsal and ventral streams of visual processing in primates. Brain Research Reviews, 55, 285-296.

Karnath, H. O., Himmelbach, M., \& Rorden, C. (2002). The subcortical anatomy of human spatial neglect: Putamen, caudate nucleus and pulvinar. Brain, 125, 350-360.

Karnath, H. O., \& Perenin, M. T. (2005). Cortical control of visually guided reaching: Evidence from patients with optic ataxia. Cerebral Cortex, 15, 1561-1569.

Kim, J. S. (2001). Delayed onset mixed involuntary movements after thalamic stroke: Clinical, radiological and pathophysiological findings. Brain, 124, 299-309.

Komura, Y., Nikkuni, A., Hirashima, N., Uetake, T., \& Miyamoto, A. (2013). Responses of pulvinar neurons reflect a subject's confidence in visual categorization. Nature Neuroscience, 16, 749-755.

Krauth, A., Blanc, R., Poveda, A., Jeanmonod, D., Morel, A., \& Szekely, G. (2010). A mean three-dimensional atlas of the human thalamus: Generation from multiple histological data. NeuroImage, 49, 2053-2062.

Kwon, H. G., Hong, J. H., Hong, C. P., Lee, D. H., Ahn, S. H., \& Jang, S. H. (2011). Dentatorubrothalamic tract in human brain: Diffusion tensor tractography study. Neuroradiology, 53, 787-791.

Magarinos-Ascone, C., Buno, W., Jr., \& Garcia-Austt, E. (1988). Monkey pulvinar units related to motor activity and sensory response. Brain Research, 445, 30-38.

Manning, L., Halligan, P. W., \& Marshall, J. C. (1990). Individual variation in line bisection: A study of normal subjects with application to the interpretation of visual neglect. Neuropsychologia, 28, 647-655.

Masdeu, J. C., \& Gorelick, P. B. (1988). Thalamic astasia: Inability to stand after unilateral thalamic lesions. Annals of Neurology, 23, 596-603.

Mesulam, M. M. (1985). In Principles of behavioral neurology. Philadelphia: F.A. Davis.

Miwa, H., Hatori, K., Kondo, T., Imai, H., \& Mizuno, Y. (1996). Thalamic tremor: Case reports and implications of the tremorgenerating mechanism. Neurology, 46, 75-79.

Morel, A., Magnin, M., \& Jeanmonod, D. (1997). Multiarchitectonic and stereotactic atlas of the human thalamus. The Journal of Comparative Neurology, 387, 588-630.

Osiurak, F., \& Rossetti, Y. (2017). Definition: Limb apraxia. Cortex, 93, 228.

Perenin, M. T., \& Vighetto, A. (1988). Optic ataxia: A specific disruption in visuomotor mechanisms. I. Different aspects of the deficit in reaching for objects. Brain, 111(Pt 3), 643-674.

Pierrot-Deseilligny, C., Rivaud, S., Gaymard, B., \& Agid, Y. (1991). Cortical control of reflexive visually-guided saccades. Brain, 114(Pt 3), 1473-1485.

Preuss, T. M. (2007). Evolutionary specializations of primate brain systems. In M. J. Ravosa, \& M. Dagosto (Eds.), Primate origins: evolution and adaptations (pp. 625-675). New York: Springer.

Ptak, R., \& Muri, R. M. (2013). The parietal cortex and saccade planning: Lessons from human lesion studies. Frontiers in Human Neuroscience, 7, 254.

Rafal, R., McGrath, M., Machado, L., \& Hindle, J. (2004). Effects of lesions of the human posterior thalamus on ocular fixation during voluntary and visually triggered saccades. Journal of Neurology, Neurosurgery, and Psychiatry, 75, 1602-1606.

Robinson, D. L., \& Petersen, S. E. (1992). The pulvinar and visual salience. Trends in Neurosciences, 15, 127-132.

Romanski, L. M., Giguere, M., Bates, J. F., \& Goldman-Rakic, P. S. (1997). Topographic organization of medial pulvinar 
connections with the prefrontal cortex in the rhesus monkey. Journal of Comparative Neurology, 379, 313-332.

Rosenberg, D. S., Mauguiere, F., Catenoix, H., Faillenot, I., \& Magnin, M. (2009). Reciprocal thalamocortical connectivity of the medial pulvinar: A depth stimulation and evoked potential study in human brain. Cerebral Cortex, 19, 1462-1473.

Rushmore, R. J., Valero-Cabre, A., Lomber, S. G., Hilgetag, C. C., \& Payne, B. R. (2006). Functional circuitry underlying visual neglect. Brain, 129, 1803-1821.

Saalmann, Y. B., \& Kastner, S. (2011). Cognitive and perceptual functions of the visual thalamus. Neuron, 71, 209-223.

Schmahmann, J. D. (2003). Vascular syndromes of the thalamus. Stroke, 34, 2264-2278.

Schmahmann, J. D., \& Pandya, D. N. (2008). Disconnection syndromes of basal ganglia, thalamus, and cerebrocerebellar systems. Cortex, 44, 1037-1066.

Sherman, S. M. (2016). Thalamus plays a central role in ongoing cortical functioning. Nature Neuroscience, 19, 533-541.

Snow, J. C., Allen, H. A., Rafal, R. D., \& Humphreys, G. W. (2009). Impaired attentional selection following lesions to human pulvinar: Evidence for homology between human and monkey. Proceedings of the National Academy of Sciences of the United States of America, 106, 4054-4059.

Sultan, F., Augath, M., Hamodeh, S., Murayama, Y., Oeltermann, A., Rauch, A., et al. (2012). Unravelling cerebellar pathways with high temporal precision targeting motor and extensive sensory and parietal networks. Nature Communications, 3, 924.

Trojanowski, J. Q., \& Jacobson, S. (1974). Medial pulvinar afferents to frontal eye fields in rhesus monkey demonstrated by horseradish peroxidase. Brain Research, 80, 395-411.
Vanbellingen, T., Kersten, B., Van de Winckel, A., Bellion, M., Baronti, F., Muri, R., et al. (2011). A new bedside test of gestures in stroke: The apraxia screen of TULIA (AST). Journal of Neurology, Neurosurgery, and Psychiatry, 82, 389-392.

Van der Stigchel, S., Arend, I., van Koningsbruggen, M. G., \& Rafal, R. D. (2010). Oculomotor integration in patients with a pulvinar lesion. Neuropsychologia, 48, 3497-3504.

Ward, R., Danziger, S., \& Bamford, S. (2005). Response to visual threat following damage to the pulvinar. Current Biology, 15, 571-573.

Wilke, M., Kagan, I., \& Andersen, R. A. (2013). Effects of pulvinar inactivation on spatial decision-making between equal and asymmetric reward options. Journal of Cognitive Neuroscience, 25, 1270-1283.

Wilke, M., Mueller, K. M., \& Leopold, D. A. (2009). Neural activity in the visual thalamus reflects perceptual suppression. Proceedings of the National Academy of Sciences of the United States of America, 106, 9465-9470.

Wilke, M., Turchi, J., Smith, K., Mishkin, M., \& Leopold, D. A. (2010). Pulvinar inactivation disrupts selection of movement plans. Journal of Neuroscience, 30, 8650-8659.

Yirmiya, R., \& Hocherman, S. (1987). Auditory- and movementrelated neural activity interact in the pulvinar of the behaving rhesus monkey. Brain Research, 402, 93-102.

Zhou, H., Schafer, R. J., \& Desimone, R. (2016). Pulvinar-cortex interactions in vision and attention. Neuron, 89, 209-220.

Zihl, J., \& von Cramon, D. (1979). The contribution of the 'second' visual system to directed visual attention in man. Brain, 102, 835-856.

Zimmermann, P., \& Fimm, B. (2002). In P. Z. M. Leclercq (Ed.), A test battery for attentional performance. Theory, diagnosis and rehabilitation. London: Psychology Press. 